Carina Tetal

Analyse

von Deliktsähnlichkeiten 


\section{Kriminologische Forschungsberichte}

Herausgegeben von Hans-Jörg Albrecht und Günther Kaiser

Band K 141 


\title{
Analyse von Deliktsähnlichkeiten auf der Basis von Individualdaten der Freiburger Kohortenstudie
}

\author{
Carina Tetal
}

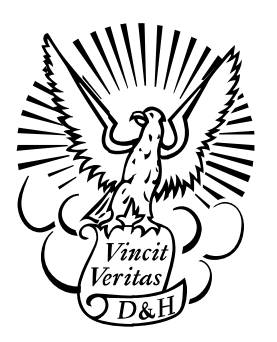

Duncker \& Humblot • Berlin 
Bibliografische Information der Deutschen Nationalbibliothek

Die Deutsche Nationalbibliothek verzeichnet diese Publikation in der Deutschen Nationalbibliografie; detaillierte bibliografische Daten sind im Internet über http://dnb.d-nb.de abrufbar.

DOI https://doi.org/10.30709/978-3-86113-093-2

Alle Rechte vorbehalten

(C) 2008 Max-Planck-Gesellschaft zur Förderung der Wissenschaften e.V. c/o Max-Planck-Institut für ausländisches und internationales Strafrecht

Günterstalstraße 73, 79100 Freiburg i.Br.

http://www.mpicc.de

Vertrieb in Gemeinschaft mit Duncker \& Humblot GmbH, Berlin

http://www.duncker-humblot.de

Umschlaggestaltung: Carina Tetal

Druck: Stückle Druck und Verlag, Stückle-Straße 1, 77955 Ettenheim

Printed in Germany

ISSN $1861-5937$

ISBN 978-3-86113-093-2 (Max-Planck-Institut)

ISBN 978-3-428-12968-3 (Duncker \& Humblot)

Gedruckt auf alterungsbeständigem (säurefreiem) Papier

entsprechend ISO 9706 


\section{Vorwort}

Die vorliegende Arbeit wurde im Wintersemester 2007/2008 von der Philosophischen Fakultät der Albert-Ludwigs-Universität Freiburg als Dissertation angenommen. Sie entstand am Max-Planck-Institut für ausländisches und internationales Strafrecht in Freiburg. Die Analysen dieser Arbeit beruhen auf Daten der Freiburger Kohortenstudie, deren Datenmaterial für die kriminologische Forschung von großer Bedeutung ist.

Mein Dank gilt zunächst meinem Doktorvater, Herrn Prof. Dr. Dr. h.c. HansJörg Albrecht, Direktor der kriminologischen Abteilung des Max-Planck-Institutes für ausländisches und internationales Strafrecht. Insbesondere durch seinen Anstoß kam diese Doktorarbeit zustande. Der Max-Planck-Gesellschaft bin ich für die Finanzierung zu Dank verpflichtet.

Herrn Prof. Dr. Baldo Blinkert danke ich für die Betreuung im Kolloquium und für die Erstellung des Zweitgutachtens. Er gab mir wertvolle Anregungen und unterstützte mich bei der Umsetzung meiner Forschungsabsichten.

Ganz besonders herzlich danken möchte ich Dr. Volker Grundies. Sowohl seine fachliche Unterstützung als auch inhaltliches Interesse und persönliche Anteilnahme trugen grundlegend zur Entstehung und Fertigstellung dieser Arbeit bei.

Danken möchte ich ebenfalls Dr. Claudia Dorsch, die zu den Anfängen der Arbeit meine Zimmerkollegin war und somit die Entstehung der Arbeit direkt mitbekam. Die Arbeitsatmosphäre, aber auch unsere gemeinsamen Freizeitaktionen, insbesondere unsere sportlichen Aktivitäten, die bis heute noch stattfinden, trugen auf ihre Art sehr zum Gelingen der Arbeit bei. Darüber hinaus möchte ich ihr für das Korrekturlesen von Teilen des Manuskriptes trotz eigener Arbeitsbelastung danken.

Viele Kolleginnen und Kollegen des Max-Planck-Institutes haben in unterschiedlicher Weise zum erfolgreichen Abschluss dieses Projektes beigetragen, sei es als Ansprechpartner, beim Mittagessen oder bei der Freizeitgestaltung. Erwähnen möchte ich hier Juliane Laule und Susanne Forster: unsere Gespräche, das gemeinsame Mittagessen, die gemeinsamen sportlichen Aktivitäten, die gemeinsamen Feste möchte ich nicht missen. Tim Lukas danke ich zusätzlich für die Durchsicht von Teilen des Manuskriptes sowie für seine konstruktiven Anmerkungen. Ein Dank geht ebenfalls an Harald Arnold, der jederzeit als Ansprechpartner zur Ver- 
fügung stand. Auch möchte ich den Kolleginnen und Kollegen aus EDV, Bibliothek, Verwaltung und Verlag danken wie all den anderen Ungenannten in und außerhalb des Instituts, die auf verschiedene Weise einen Beitrag geleistet haben.

Dank schulde ich nicht zuletzt Klaus Amann, nicht nur für die Durchsicht des Manuskripts, sondern besonders dafür, dass er mich während der ganzen Zeit unterstützte und immer für mich da war.

Freiburg, im September 2008

Carina Tetal 


\section{Inhaltsverzeichnis}

Seite

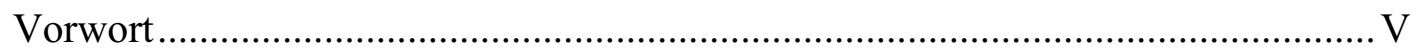

Inhaltsverzeichnis .................................................................................... VII

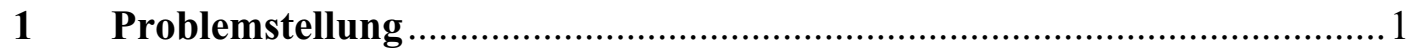

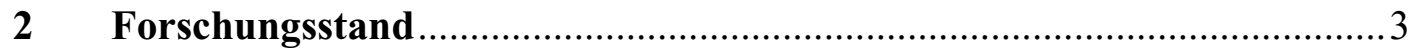

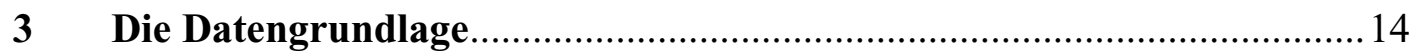

3.1 Die Freiburger Kohortenstudie ...................................................... 14

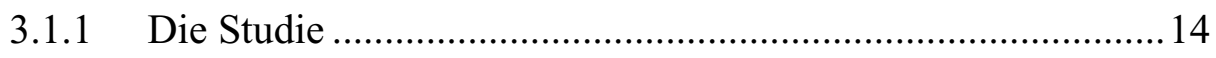

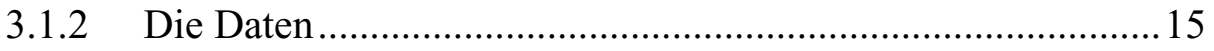

3.1.3 Die Bundeszentralregisterdaten .......................................... 16

3.2 Relevante Variablen für die Analyse .................................................20

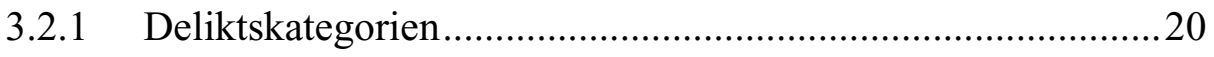

3.2.2 Soziodemografische Merkmale ...........................................25

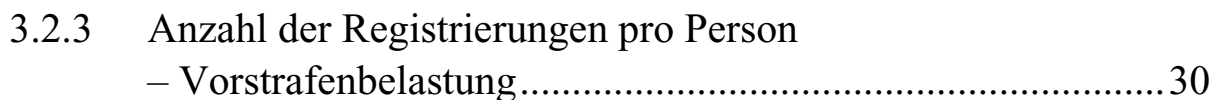

3.3 Besonderheiten und Probleme der Datengrundlage.............................. 33

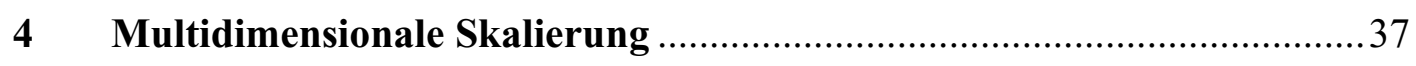

4.1 Was ist Multidimensionale Skalierung ….......................................... 37

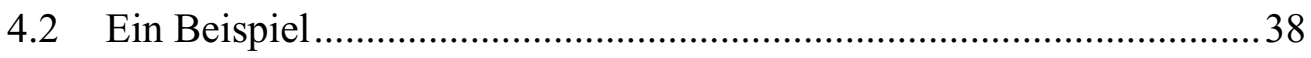

4.3 Beurteilungskriterien ................................................................... 41

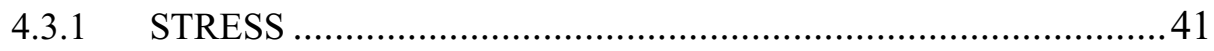

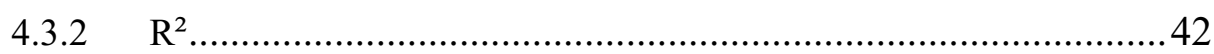

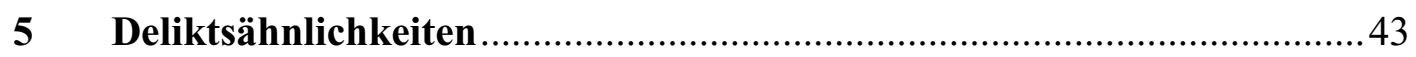




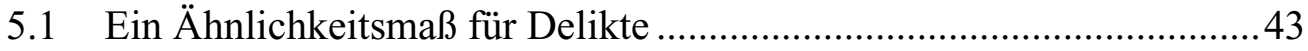

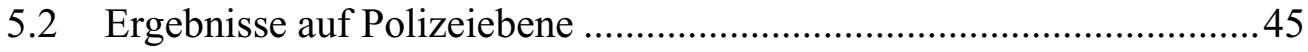

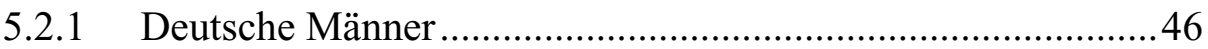

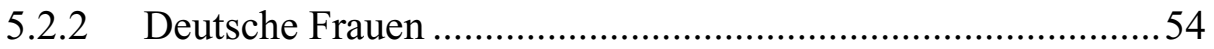

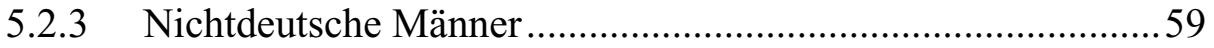

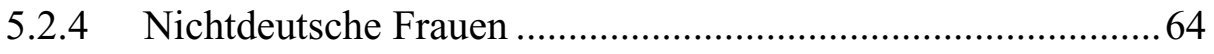

5.2.5 Spezialfall: Deliktskombination einer Person an

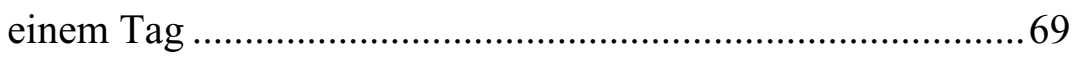

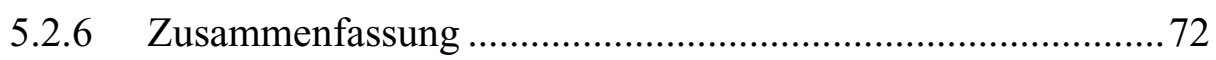

5.3 Ergebnisse auf justizieller Ebene................................................ 73

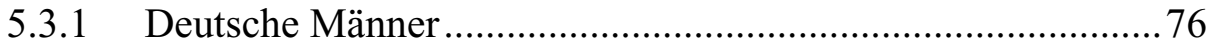

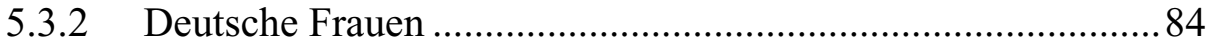

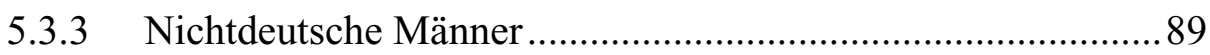

5.3.4 Nichtdeutsche Frauen ..............................................................94

5.3.5 Problem Tateinheit und Tatmehrheit ......................................99

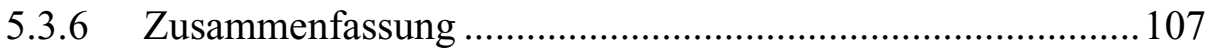

5.4 Berücksichtigung der Dauer zwischen den Straftaten ....................... 109

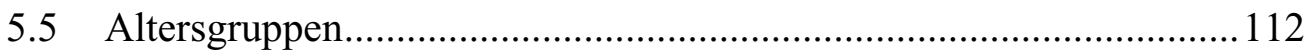

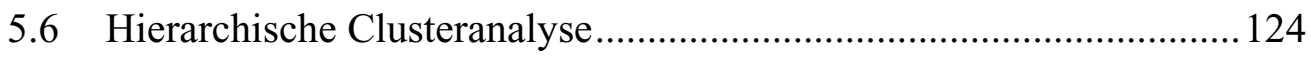

5.7 Zusammenfassung der Ergebnisse ................................................ 131

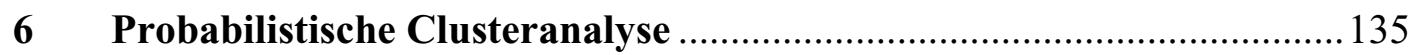

6.1 Probabilistische Clusteranalyse für justiziell Registrierte des Geburtsjahrgangs 1970

6.1.1 Clusteranalyse justizieller Registrierungen deutscher

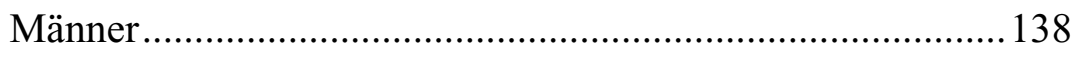

6.1.2 Spezialisierung, Wechsel und Abbruch im Lebenslauf

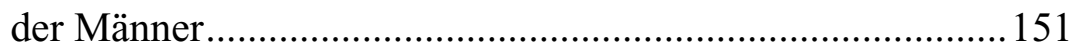

6.1.3 Clusteranalyse justizieller Registrierungen deutscher

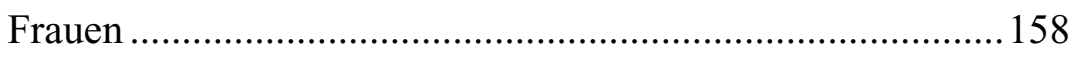

6.1.4 Spezialisierung, Wechsel und Abbruch im Lebenslauf der Frauen. 
6.2 Probabilistische Clusteranalyse für polizeilich Registrierte des Geburtsjahrgangs 1970

6.2.1 Clusteranalyse polizeilicher Registrierungen deutscher Männer..

6.2.2 Spezialisierung, Wechsel und Abbruch im Lebenslauf der Männer.

6.2.3 Clusteranalyse polizeilicher Registrierungen deutscher Frauen.

6.2.4 Spezialisierung, Wechsel und Abbruch im Lebenslauf der Frauen .

6.3 Zusammenfassung der Ergebnisse.

7 Spezialisierung auf einzelne Deliktskategorien

8 Zusammenfassung der Ergebnisse und Schlussfolgerungen

Literaturverzeichnis

Abkürzungsverzeichnis

Tabellenverzeichnis

Abbildungsverzeichnis

Anhang 



\section{Problemstellung}

In der Kriminologie wird meist untersucht, wie häufig Straftaten, allgemein oder differenziert nach einzelnen Deliktskategorien, vorkommen. Muster von Straftaten werden aber seltener analysiert. Jedoch könnte gerade die Kenntnis von Mustern von Straftaten bei der Vorhersage und beim Verstehen der Ursachen von Straftaten helfen und zu einer Verbesserung der Prävention beitragen.

Ziel dieser Studie ist, die Ähnlichkeit von verschiedenen Straftaten zu bestimmen, wobei zuerst die Ähnlichkeitskriterien zu hinterfragen und festzulegen sind. So können die Ähnlichkeiten von Delikten z.B. über den Ansatz einer aus der Sanktionierung bestimmten Deliktsschwere oder über verschiedene andere Kriterien wie Gewaltanwendungen, Schadenshöhe oder entsprechende Informationen, die die Straftat betreffen, definiert werden. In dieser Studie soll jedoch keine dieser A-priori-Kategorisierungen verwendet werden. Vielmehr soll die Ähnlichkeit von Delikten empirisch bestimmt werden, und zwar mittels des gemeinsamen Auftretens von Delikten innerhalb der kriminellen Karriere von Personen. Die Delikte werden über den Angelpunkt Individuum miteinander verknüpft, dem damit ein bestimmtes psychosoziales Motiv für sein abweichendes Verhalten unterstellt wird. In dieser Untersuchung werden somit Gemeinsamkeiten zwischen Delikten erfasst, die auf eine länger andauernde Verhaltensphase zurückzuführen sind. Gleichwohl ist es bei der Untersuchung auch möglich zu analysieren, ob die Ähnlichkeit von Delikten altersspezifisch variiert.

Ausgegangen wird davon, dass Delikte, die von ein und derselben Person begangen wurden, Ähnlichkeiten aufweisen. Als Ähnlichkeitsmaß wird die Häufigkeit von Deliktspaaren bei Personen benutzt, die mit dem erwarteten Vorkommen unter der Annahme einer zufälligen Verteilung verglichen wird (Adjusted Standardized Residual). Von Interesse sind also zuerst die möglichen Deliktskombinationen jeder Person.

Der Analyse werden die Daten der Freiburger Kohortenstudie zugrunde gelegt. Es handelt sich dabei um Längsschnittdaten der Geburtsjahrgänge 1970, 1973, 1975, 1978, 1985 und 1988 der Personenauskunftsdatei (PAD) des Landeskriminalamts Baden-Württemberg und des Bundeszentralregisters (BZR). Bei den Bundeszentralregisterdaten werden für die Analyse nur die Daten der Personen benutzt, die mindestens eine gerichtliche Entscheidung in Baden-Württemberg haben. Damit werden sowohl die polizeilichen als auch die justiziellen Registrierungen einer Person erfasst. Anhand dieser Daten ist es möglich, einer Person alle registrierten Delikte zuzuordnen. Während bei den PAD-Daten jede Registrierung genau ein Delikt beinhaltet, umfassen die BZR-Registrierungen häufig mehrere Delikte.

Für die Auswertungen sind nur die Personen relevant, die mindestens zwei Straftaten begangen haben. 
Gegenstand der vorliegenden Arbeit ist die Analyse von Deliktsähnlichkeiten. Dazu wird im zweiten Abschnitt auf den Stand der Forschung eingegangen. Im dritten Abschnitt wird das Datenmaterial näher dargestellt und im vierten Abschnitt wird die hier verwendete Methode der Multidimensionalen Skalierung beschrieben. Mit Hilfe der Methode der Multidimensionalen Skalierung ist es möglich, die Ähnlichkeiten von Delikten grafisch darzustellen. Die Ergebnisse der Multidimensionalen Skalierung werden im fünften Abschnitt vorgestellt. Dazu werden die analysierten Deliktsähnlichkeiten beschrieben und spezielle Auswertungen zu Straftaten dargestellt, die von einer Person an einem Tag begangen wurden. Hierdurch kann der Einfluss von tateinheitlicher Begehungsweise geprüft werden. Analysiert wird auch, ob die Dauer zwischen zwei Straftaten Einfluss auf das Ergebnis der Deliktsähnlichkeiten hat. Außerdem wird untersucht, ob in verschiedenen Altersgruppen verschiedene Deliktsähnlichkeiten auftreten. Mit Hilfe der Ähnlichkeitsdaten wird des Weiteren eine Clusteranalyse durchgeführt, was eine weitere optische Darstellung der Deliktsgruppierungen liefert. Im sechsten Abschnitt werden mittels eines probabilistischen Clusteranalyseverfahrens die Delikte gruppiert. Außerdem werden mit dem Ergebnis der probabilistischen Clusteranalyse weiterführende Untersuchungen zu Spezialisierung und zur kriminellen Laufbahn durchgeführt. Im siebten Abschnitt wird speziell der Grad der Spezialisierung auf einzelne Deliktskategorien analysiert. Den Abschluss der Arbeit bildet eine Zusammenfassung und Diskussion der gefundenen Ergebnisse. 


\section{Forschungsstand}

„Die Erscheinungsformen der Kriminalität sind mannigfaltig. Daher besteht das Bedürfnis, sie zu ordnen. Die Einteilung von Verhaltensweisen nach dem strafrechtlich vorgegebenen Verbrechensbegriff erscheint oft zu eng und zu wenig aussagekräftig. Deshalb ist seit langem in der Wissenschaft versucht worden, umfassendere Strukturen zu entdecken."1

Strukturen von Kriminalität weisen auf Tätertypen und Rückfallverhalten hin. Somit wäre bei Kenntnis der Strukturen eine bessere Prognose von Kriminalität und damit auch eine bessere Prävention möglich.

Schon im 19. Jh. typologisierte Lombroso Täter aufgrund einer langjährigen medizinisch-anthropologischen Untersuchung. ${ }^{2}$ Anhand der Kopfform bestimmte er „Verbrechertypen“.3 Außerdem vertrat er die Theorie des „geborenen Verbrechers“ (delinquente nato). ${ }^{4}$,Waren seine Forschungsmethoden - nach heutigen Maßstäben - auch mangelhaft, so bleibt es gleichwohl sein Verdienst, die kriminologische Forschung auf die Untersuchung des Rechtsbrechers ausgerichtet und auf empirische Grundlagen gestellt zu haben."5

Abgesehen von den sehr frühen Arbeiten, ist es seit den 1950er Jahren in der Kriminologie üblich, Tätergruppen zu unterscheiden. Die Geschichte kriminologischer Typologien kann in zwei Gruppen eingeteilt werden: frühe Arbeiten, die sich damit beschäftigen, Täter anhand ihrer kriminellen Aktivitäten einzuteilen, und neuere Arbeiten, die sich mehr mit der Häufigkeit von Delikten und kriminellen Karrieren befassen. ${ }^{6}$ Seelig und Weindler ${ }^{7}$ unterschieden 1949 zwischen 8 Haupttypen ,von Kriminellen:

1. Berufsverbrecher aus Arbeitsscheu

2. Vermögensverbrecher aus geringer Widerstandskraft

3. Aggressive Gewalttäter

4. Verbrecher aus sexueller Unbeherrschtheit

5. Krisenverbrecher

6. Primitivreaktive Verbrecher

7. Überzeugungsverbrecher

8. Verbrecher aus Mangel an Gemeinschaftsdisziplin."8

1 Kaiser 1993, S. 242.

2 Vgl. Schwind 2007, S. 91 ff., Schöch 1993, S. 215, Lombroso 2006 (neue englische Übersetzung mit einer Einleitung von Mary Gibson und Nicole Hahn Rafter).

3 Vgl. Schwind 2007, S. 92.

4 Vgl. Lombroso 2006, S. 1.

5 Schneider 1987, S. 927.

6 Vgl. Francis, Soothill \& Fligelstone 2004, S. 48.

7 Seelig \& Weindler 1949.

8 Seelig \& Weindler 1949, S. 19. 
Eine Zusammenfassung zu Tätertypen der 1960er Jahre findet man bei Ferdinand (1966), der zwischen "empirical typologies" und "ideal typologies", also zwischen empirischer und theoretischer Typenbildung, unterscheidet. ${ }^{9}$ Ferdinand beschreibt soziologische und psychologische Theorien seiner Zeit. Er kommt zu dem Schluss, dass die einzelnen Theorien gemischt werden müssen und dass zur Bestimmung von kriminellem Verhalten sowohl Theorie wie Empirie, und zwar Empirie in Form von Feldforschung, nötig ist. ${ }^{10}$

Weitere kriminologische Arbeiten zur Typisierung von kriminellem Verhalten gab es in den späten 1960er und frühen 1970er Jahren. Clinard und Quinney beschäftigten sich mit den Mustern von kriminellem Verhalten. ${ }^{11} \mathrm{Ihr}$ Buch zu diesem Thema wurde erstmals 1967 veröffentlicht. Clinard und Quinney unterschieden zwischen "legalistic", "individualistic" und "social" Typologien. Ihre eigene Einteilung war eine Mischung dieser Typologievarianten und ergab neun Typen, darunter befanden sich Gewaltkriminalität, seltene Eigentumskriminalität, politische Kriminalität und professionelle Kriminalität sowie fünf weitere Typen kriminellen Verhaltens. Kriterien von Taten und Tätern wurden bei dieser Einteilung vermischt. Eine ähnliche Einteilung, aber mit mehr Kategorien, bildete Gibbons im Jahr 1972.12 Gibbons unterschied zwischen 20 sogenannten "Role-Careers". Diese Typologie von Rechtsbrechern basierte einerseits auf der Straftat und andererseits auf dem sozialen Kontext der Täter. So unterschied Gibbons z.B. zwischen professionellen Dieben, halbprofessionellen Eigentumskriminellen und amateurhaften Ladendieben.

Roebuck bestimmte anhand einer empirischen Untersuchung im Jahr 1963 kriminelle Typologien. ${ }^{13}$ Er bildete vier Hauptgruppen anhand von 400 Strafgefangenen:

1. “Single Pattern”. Das Kriterium dieser Gruppe ist, dass der Täter mindestens dreimal wegen desselben Deliktes sanktioniert wurde, somit ein Spezialist ist.

2. "Multiple Pattern". Ein Täter hat verschiedene Delikte begangen, die jeweils mehrfach vorkommen.

3. “Mixed Pattern". Ein Täter wurde drei- oder mehrmals sanktioniert wegen verschiedener Taten.

4. “No Pattern”. Ein Täter wurde nur ein- oder zweimal sanktioniert. ${ }^{14}$

\footnotetext{
9 Ferdinand 1966.

10 Vgl. Ferdinand 1966, S. 205 ff.

11 Clinard \& Quinney 1994.

12 Gibbons 1992.

13 Roebuck 1963 und Roebuck 1967.

14 Vgl. Roebuck 1963, S 51.
} 
Diese Hauptgruppen wurden weiter durch die Straftaten unterschieden, was 13 Kategorien ergab. Die größte Kategorie war "Mixed Pattern" ( $n=71)$, die zweitgrößte Gruppe war "Double Pattern" mit schwerem und einfachem Diebstahl $(\mathrm{n}=64)$ und die drittgrößte Kategorie war "Single Pattern" mit Betäubungsmitteldelikten $(\mathrm{n}=50) .15$

Chaiken und Chaiken teilten in den 1980er Jahren knapp 2200 Strafgefangene anhand ihrer selbstberichteten Delinquenz der letzten zwei Jahre in zehn Tätergruppen ein. ${ }^{16}$ Für die Einteilung benutzten sie sieben Deliktskategorien: Raub, Körperverletzung, Einbruch, Diebstahl, Fälschung, Betrug und Drogenhandel. ${ }^{17} \mathrm{Ihr}$ Ergebnis der Typisierung von kriminellem Verhalten war, dass sich die größte Tätergruppe zusammensetzte aus Gewalttätern, die auch Drogenhandel begangen hatten. Weitere Gruppen waren Täter, die hauptsächlich Raub und Körperverletzung begingen, außerdem die Gruppen Raub und Drogenhandel, Raub, Körperverletzung, Einbruch und Drogenhandel, Einbruch, Eigentumsdelikte und Drogenhandel, Eigentumsdelikte und eine Gruppe, die nur Drogenhändler umfasste. ${ }^{18}$

Diese Art der Zusammenfassung der Täter nach Typen wurde von Kriminologen auch kritisiert. Gibbons, obwohl er selbst schon in den 1960er Jahren Täter typologisierte, kritisierte später, dass viele Täter nicht in das theoretische Schema passen. ${ }^{19}$ Auch Figlio kritisierte diese Typologien als zu statisch. Zeitliche Veränderungen der kriminellen Aktivitäten können nicht mit einbezogen werden. ${ }^{20}$ Figlio untersuchte kriminelle Karrieren mit Hilfe von Markow-Ketten. ${ }^{21}$ Er kam zu dem Ergebnis, dass die Kenntnis der Deliktsvorgeschichte nicht bei der Vorhersage weiterer Delikte hilft. ${ }^{22}$

Blumstein und Cohen untersuchten 1979 die Deliktsstruktur von Erwachsenen, die 1973 in Washington wegen eines schweren Deliktes, d.h. eines Tötungsdeliktes, eines Sexualdeliktes, wegen Raubes, schwerer Körperverletzung oder schwerem Diebstahl inhaftiert waren. ${ }^{23}$ Sie fanden heraus, dass es zwar nur eine geringe Spezialisierung nach Deliktstypen gibt, sich aber eine Tendenz feststellen lässt,

\footnotetext{
15 Vgl. Roebuck 1963, S 51.

16 Chaiken \& Chaiken 1984.

17 Vgl. Chaiken und Chaiken 1984, S. 207.

18 Vgl. Chaiken und Chaiken 1984, S. 207.

19 Vgl. Gibbons 1992, S. 213.

20 Figlio 1981, S. 25.

21 Figlio 1981, Markow-Ketten dienen der Analyse und/oder der Prognose von zukünftigen Ereignissen. Mit der Kenntnis der Vorgeschichte werden Wahrscheinlichkeiten für zukünftige Ereignisse berechnet. Ausgangspunkt bei Figlio ist die Übergangsmatrix vom ersten zum zweiten Delikt (Transitionsmatrix), wobei die Delikte in Gruppen eingeteilt

22 Vgl. Figlio 1981, S. 37.

23 Blumstein \& Cohen 1979.
} sind. 
ähnliche Straftaten zu begehen. Insbesondere traten häufig Eigentumsdelikte und Betäubungsmitteldelikte zusammen innerhalb einer kriminellen Karriere auf. ${ }^{24}$

Ähnlich wie Figlio führten Rojek und Erickson 1982 eine Studie zu Deliktsspezialisierung mit Hilfe von Markow-Ketten durch. ${ }^{25}$ Dazu wurden zuerst die Transitionsmatrizen der Deliktsübergänge betrachtet. ${ }^{26}$ Die Transitionsmatrix wurde zuerst in der Philadelphia Studie von Wolfgang, Figlio und Sellin 1972 benutzt. ${ }^{27}$ Bei der Analyse mit Transitionsmatrizen ist die chronologische Abfolge der Straftaten von Bedeutung. Rojek und Erickson untersuchten die justiziellen Registrierungen von knapp 1200 Jugendlichen in Arizona. ${ }^{28}$ Die Delikte wurden in fünf Kategorien eingeteilt: Gewaltdelikte, Eigentumsdelikte, Drogendelikte, Ausreißen und sonstige Delikte. ${ }^{29}$ Die Auswertung der Transitionsmatrizen zeigte eine geringe Spezialisierung auf einzelne Deliktskategorien, die überlagert wird von einer großen Anzahl von vielfältigen Deliktsverläufen. ${ }^{30}$ Des Weiteren wurden Untersuchungen zu Deliktsspezialisierung durchgeführt, indem Adjusted Standardized Residuals ${ }^{31}$ (ASR) berechnet wurden. Das Residuum besagt, ob unter der Annahme einer zufälligen Verteilung, mehr oder weniger Ereignisse eintreten als erwartet. Bei der Analyse mit Residuen werden im Unterschied zur Analyse mit Transitionsmatrizen auch die unterschiedlichen Größen der Deliktskategorien mit einbezogen. Die Auswertung der Residuen ergab, dass der Spezialisierungsgrad bei Eigentumsdelikten und Ausreißen deutlich höher ist als bei Gewaltdelikten, Drogendelikten und sonstigen Delikten. ${ }^{32}$

Smith und Smith untersuchten 1984 mit Hilfe von Markow-Ketten und ASR die justiziellen Registrierungen von 767 schwerkriminellen männlichen Jugendlichen. ${ }^{33}$ Auch ihr Ergebnis war, dass es Anhaltspunkte für Spezialisierung gibt, dass die Deliktsmuster aber eher durch Vielfältigkeit charakterisiert werden. ${ }^{34}$ Spezialisierung zeigte sich am ehesten in der Deliktskategorie Raub. ${ }^{35}$ Sie betrachteten auch die Kombinationen verschiedener Deliktskategorien miteinander und stellten einen Zusammenhang zwischen Raub und Körperverletzung fest. Für Täter, die

\footnotetext{
24 Vgl. Blumstein \& Cohen 1979, S. 585.

25 Rojek \& Erickson 1982.

26 Die Transitionsmatrix gibt an, wie häufig ein folgendes Delikt bei einem bestimmten Ausgangsdelikt vorkommt (Zeilenprozente).

27 Vgl. Kyvsgaard 2003, S.153.

28 Vgl. Rojek \& Erickson 1982, S. 9 f.

29 Vgl. Rojek \& Erickson 1982, S. 10.

30 Vgl. Rojek \& Erickson 1982, S. 15.

31 Siehe Fußnote 145.

32 Vgl. Rojek \& Erickson 1982, S. 16.

33 Smith \& Smith 1984.

34 Vgl. Smith \& Smith 1984, S. 155 f.

35 Vgl. Smith \& Smith 1984, S. 155.
} 
wegen Raubes oder Körperverletzung inhaftiert waren, war die Wahrscheinlichkeit groß, in ihrer späteren kriminellen Karriere wieder wegen Raubes oder Körperverletzung inhaftiert zu werden. ${ }^{36}$ Die Auswertungen ergaben zwei unterschiedliche Gruppen von jugendlichen Straftätern. Eine Gruppe umfasste jugendliche Straftäter, die weniger schwere Straftaten, großteils Eigentumsdelikte, begingen. Die andere Gruppe Jugendlicher beging großteils schwere Straftaten, insbesondere Raub und Körperverletzung. Smith und Smith folgerten daraus, dass der Begriff der Spezialisierung nicht zu eng gefasst werden darf. Es soll nicht nur nach Spezialisierung von einzelnen Deliktskategorien geforscht werden, sondern darüber hinaus sollen auch die Zusammenhänge der Deliktskategorien analysiert werden. ${ }^{37}$

Klein verglich im Jahr 198433 Studien aus den Jahren 1963 bis 1982, die sich mit Deliktsspezialisierung und Deliktsvielfalt bei Jugendlichen beschäftigten. ${ }^{38} 21$ dieser Studien kamen zum Ergebnis, dass es keine Spezialisierung gibt. In 8 Studien wurde die Tendenz zu Spezialisierung weder bestätigt noch widerlegt. Vier Studien fanden eindeutige Hinweise auf Spezialisierung. ${ }^{39}$ Die Studien waren sehr unterschiedlicher Art. ${ }^{40}$ Die Datengrundlage waren Befragungen, Beobachtungen, Interviews, Polizeidaten oder justizielle Daten. Die Stichproben setzten sich u.a. zusammen aus Schülern, Studenten, Erstregistrierten, Mehrfachstraftätern oder Schwerkriminellen. Auch wurden jeweils verschiedene Deliktskategorien verwendet. Als Methoden wurden u.a. deskriptive Analyse, Faktorenanalyse, Multidimensionale Skalierung, Korrelation, Clusteranalyse oder die Berechnung von Übergangswahrscheinlichkeiten bei Transitionsmatrizen verwendet. Die häufigste Methode der 1960er und 1970er Jahre war die Faktorenanalyse. Im Unterschied zu Analysen mit Transitionsmatrizen spielt bei der Faktorenanalyse die chronologische Abfolge keine Rolle. Alle vier Studien, die Spezialisierung feststellten, waren Befragungen, die mit Faktorenanalyse ausgewertet wurden. ${ }^{41}$ Es gab aber auch sieben Studien mit Befragungen und Faktorenanalyse, die keine Spezialisierung feststellten. An den Daten und an der Methode konnte somit nicht festgemacht werden, ob Deliktsspezialisierung vorlag oder nicht. Klein folgert daraus, dass Deliktsspezialisierung bei Jugendlichen nicht nachweisbar ist, wobei vier Studien von dieser Regel abweichen. ${ }^{42}$

Farrington führte 1986 Berechnungen zur Spezialisierung in der kriminellen Karriere durch. Dafür unterteilte er die Delikte in drei Kategorien und berechnete die

\footnotetext{
36 Vgl. Smith \& Smith 1984, S. 155 f.

37 Vgl. Smith \& Smith 1984, S. 156.

38 Klein 1984.

39 Vgl. Klein 1984, S. 187.

$40 \mathrm{Vgl}$. Klein 1984, S. 186 f.

41 Vgl. Klein 1984, S. 187 f.

42 Vgl. Klein 1984, S. 188.
} 
Spezialisierung mit dem Forward Specialization Coefficient (FSC). ${ }^{43}$ Farrington fand heraus, dass der Spezialisierungsgrad bei Erwachsenen höher ist als bei Jugendlichen und Heranwachsenden. ${ }^{44}$

1988 führten Farrington, Snyder und Finnegan eine Studie zu Spezialisierung bei Jugendlichen mit US-amerikanischen Gerichtsdaten durch. ${ }^{45}$ Untersucht wurden fast 70.000 Jugendliche. Die Delikte wurden in 21 Kategorien eingeteilt. ${ }^{46}$ Farrington, Snyder und Finnegan kamen hierbei zu folgenden Ergebnissen: Deliktsspezialisierung ist nachweisbar, wird aber überlagert von einer großen Anzahl von vielfältigen Deliktsverläufen. ${ }^{47}$ Der Grad der Spezialisierung stimmt bei Männern und Frauen in etwa überein. ${ }^{48}$ Am häufigsten kommt es bei den Delikten Einbruch (schwerer Diebstahl), Autodiebstahl, Alkoholvergehen und Drogendelikten zu Spezialisierungen. Fast 20\% der Jugendlichen wurden als Spezialisten identifiziert. 49

Viele Studien kamen zum Ergebnis, dass Deliktsverläufe eher vielfältig als spezialisiert sind. ${ }^{50}$ Dennoch zeigten sich auch in einigen dieser Studien Hinweise auf Spezialisierung, insbesondere wenn die einzelnen Deliktskategorien groß gewählt wurden. In den Studien von Peterson et al. und Petersilia et al. zeigte sich Spezialisierung sowohl bei Eigentumsdelikten als auch bei Gewaltdelikten. ${ }^{51}$

Albrecht und Moitra untersuchten 1988 die Muster von kriminellen Karrieren anhand von Markow-Ketten. ${ }^{52}$ Die Analyse wurde mit justiziellen Registrierungen aus Baden-Württemberg durchgeführt. ${ }^{53}$ Ergebnis der Analyse war, dass Deliktstypen in der Vergangenheit einer Person einen geringen Einfluss auf Deliktstypen in der Zukunft haben. ${ }^{54}$ Des Weiteren wurden Untersuchungen zu Spezialisierung durchgeführt, indem die Adjusted Standardized Residuals berechnet wurden. Albrecht und Moitra kamen zu dem Ergebnis, dass bei Betrug, Eigentumsdelikten und Verkehrsdelikten Spezialisierung sichtbar war, bei Gewaltdelikten aber nicht.

1988 analysierten Blumstein, Cohen, Das und Moitra die kriminelle Karriere erwachsener Strafgefangener in den USA. ${ }^{55}$ Untersucht wurden mehr als 32.000

\footnotetext{
43 Farrington 1986, S. 227.

44 Vgl. Farrington 1986, S. 226 f.

45 Farrington, Snyder \& Finnegan 1988.

46 Vgl. Farrington, Snyder \& Finnegan 1988, S. 461.

47 Vgl. Farrington, Snyder \& Finnegan 1988, S. 483.

48 Vgl. Farrington, Snyder \& Finnegan 1988, S. 472.

49 Vgl. Farrington, Snyder \& Finnegan 1988, S. 483.

50 Kempf 1987, Kobrin, Hellum \& Peterson 1980, Petersilia, Greenwood \& Lavin 1977, Peterson, Braiker \& Polich 1981.

51 Petersilia, Greenwood \& Lavin 1977, Peterson, Braiker \& Polich 1981.

52 Albrecht \& Moitra 1988.

53 Vgl. Albrecht \& Moitra 1988, S. 116.

54 Vgl. Albrecht \& Moitra 1988, S. 127, S.134, S. 135.

55 Blumstein, Cohen, Das \& Moitra 1988.
} 
Strafgefangene, die zwischen 1974 und 1977 wegen schwerer Straftaten inhaftiert waren. ${ }^{56}$ Die Delikte wurden in 12 Kategorien eingeteilt, wobei für die Analyse zehn Deliktskategorien betrachtet wurden. ${ }^{57}$ Für die Auswertung wurden Transitionsmatrizen erstellt und Adjusted Standardized Residuals sowie Forward Specialization Coefficients berechnet. Ein Ergebnis der Studie im Vergleich zu einer früheren Studie war, dass der Spezialisierungsgrad Erwachsener höher war als der Spezialisierungsgrad Jugendlicher. Auch innerhalb der Gruppe der Erwachsenen waren die Älteren spezialisierter als die Jüngeren. ${ }^{58}$ Weitere Ergebnisse der Studie ergaben, dass sich bzgl. der Deliktskategorien Unterschiede im Grad der Spezialisierung zeigten. Der Spezialisierungsgrad war bei Drogendelikten, Betrug und Autodiebstahl am höchsten. Bei Gewaltkriminalität war der Spezialisierungsgrad deutlich geringer. ${ }^{59}$ Analysiert wurde auch, ob systematische Deliktsmuster innerhalb der kriminellen Karriere vorkamen. Systematische Deliktsmuster bedeutet einerseits Spezialisierung in dem Sinne, dass dieselbe Deliktskategorie wiederholt auftritt, andererseits aber auch das Vorkommen von Gruppen von ähnlichen Delikten. ${ }^{60}$ Blumstein et al. untersuchten zudem die Ähnlichkeit von verschiedenen Delikten, indem die Adjusted Standardized Residuals der Übergangsmatrizen für verschiedene Deliktspaare berechnet wurden. Diese Untersuchung ergab zwei unterschiedliche Deliktsgruppen: die Gruppe der Eigentumsdelikte (schwerer Diebstahl, einfacher Diebstahl, Betrug) und die Gruppe der Gewaltdelikte (Tötungsdelikte, Vergewaltigung, schwere Körperverletzung). ${ }^{61}$ Deliktswechsel der Täter waren häufiger innerhalb einer dieser Deliktsgruppen als zwischen den beiden Deliktsgruppen. ${ }^{62}$

In einer kanadischen Längsschnittstudie befragten Blanc und Fréchette 1684 Männer im Alter zwischen 12 und 19 Jahren (Dunkelfeld) und 470 justiziell registrierte Männer im Alter zwischen 13 und 25 Jahren. Die Auswertung war hauptsächlich deskriptiv. Sie stellten fest, dass das Deliktsspektrum der Jugendlichen vielfältiger ist als das der Erwachsenen. Das heißt aber noch nicht, dass Erwachsene spezialisierter sind, auch Erwachsene wechseln zwischen verschiedenen Delikten. 63

Die Studie von Stander, Farrington, Hill und Altham im Jahr 1989 zeigte Spezialisierung bei Sexualdelikten und bei Betrug. Weniger Spezialisierung zeigte sich

\footnotetext{
56 Vgl. Blumstein, Cohen, Das \& Moitra 1988, S. 307 f.

57 Vgl. Blumstein, Cohen, Das \& Moitra 1988, S. 343, S. 311.

58 Vgl. Blumstein, Cohen, Das \& Moitra 1988, S. 303.

59 Vgl. Blumstein, Cohen, Das \& Moitra 1988, S. $341 \mathrm{f}$.

60 Vgl. Blumstein, Cohen, Das \& Moitra 1988, S. $341 \mathrm{f}$.

61 Vgl. Blumstein, Cohen, Das \& Moitra 1988, S. 326 f.

62 Vgl. Blumstein, Cohen, Das \& Moitra 1988, S. 342.

63 Blanc \& Fréchette 1989, S. 132 f.
} 
bei Gewaltdelikten und Diebstahl. ${ }^{64}$ Untersucht wurden 698 englische männliche Strafgefangene im Alter von 21 Jahren oder älter. Die Delikte waren in sechs Deliktskategorien eingeteilt. ${ }^{65}$ Die Analyse wurde durchgeführt mit Hilfe von Markow-Ketten und der Berechnung des Forward Specialization Coefficient. Die Analyse von Stander et al. ergab, dass die Kenntnis von früheren Straftaten bei der Vorhersage von Straftaten hilfreich ist. ${ }^{66}$ Des Weiteren wurden Deliktstripel ausgewertet, d.h. drei zeitlich aufeinanderfolgende Delikte einer Person. Keines der Deliktstripel bestand aus drei verschiedenen Deliktskategorien. ${ }^{67}$ Auch damit zeigte sich eine gewisse Art von Spezialisierung.

Auch eine Analyse von Farrington im Jahr 1992 mit Längsschnittdaten der Cambridge Studie 68 (Alter 8 bis 32 Jahre) zeigte keine Spezialisierung bei Gewaltdelikten. 69

Gottfredson und Gottfredson veröffentlichten 1994 die Ergebnisse einer Studie über mehr als 6000 Männer, die in den frühen 1960er Jahren in Kalifornien inhaftiert waren. ${ }^{70} 1988$ wurden die weiteren Inhaftierungen dieser Männer erfasst und damit eine Spanne von über 25 Jahren betrachtet. Für ihre Analyse verwendeten sie Transitionsmatrizen sowie Adjusted Standardized Residuals und Forward Specialization Coefficients. Die Delikte waren in sechs Deliktskategorien eingeteilt. Ein Ergebnis der Studie war, dass es zwar Hinweise auf Deliktsspezialisierung gibt, diese aber überlagert wird von sehr vielen vielfältigen Deliktsverläufen. ${ }^{71}$ Des Weiteren wurde das Vorkommen von Kombinationen von zwei Deliktskategorien in der kriminellen Laufbahn untersucht. Gottfredson und Gottfredson fanden heraus, dass bestimmte Deliktskombinationen häufig auftreten, andere dafür sehr selten. Häufig sind die Deliktspaare Eigentumsdelikte und "nuisance" ("nuisance" umfasst die Delikte leichte Betäubungsmitteldelikte, Trunkenheit im Verkehr und Verstöße gegen Bewährungsauflagen). Selten ist die Kombination Betrug und "nuisance". 72

Kyvsgaard untersuchte 2003 die kriminelle Karriere mit dänischen Längsschnittdaten. Die Daten umfassten die justiziellen Registrierungen von knapp 45.000 Per-

\footnotetext{
64 Vgl. Stander, Farrington, Hill \& Altham 1989, S. 327.

65 Vgl. Stander, Farrington, Hill \& Altham 1989, S. 321.

66 Vgl. Stander, Farrington, Hill \& Altham 1989, S. 324.

67 Vgl. Stander, Farrington, Hill \& Altham 1989, S. 325.

${ }^{6}$ Bei der Cambridge Studie wurden 411 männliche Jugendliche aus London der Geburtsjahrgänge 1954/55 in regelmäßigen Abständen in Bezug auf eigene Delinquenz befragt, und zusätzlich wurden ihre polizeilichen Registrierungen in die Untersuchung mit einbezogen. Vgl. Schwind 2007, S. 158.

69 Vgl. Farrington 1992, S. 21.

70 Gottfredson \& Gottfredson 1994.

71 Vgl. Gottfredson \& Gottfredson 1994, S. 460.

72 Vgl. Gottfredson \& Gottfredson 1994, S. 465.
} 
sonen im Alter von 15 Jahren und älter. Unter anderem berechnete sie Spezialisierungsgrade mit dem Forward Specialization Coefficient. ${ }^{73}$ Sie stellte fest, dass der Koeffizient abhängig von der Wahl der Deliktskategorien ist. ${ }^{74}$ Bei der Beschreibung früherer Studien bemerkte sie, dass die Anzahl der Deliktskategorien in den verschiedenen Studien zu Spezialisierung und Deliktsvielfalt sehr variiert. Häufig werden fünf bis acht Kategorien verwendet, selten mehr als zehn. ${ }^{75}$ Kyvsgaard unterteilte die Delikte in elf Deliktskategorien. ${ }^{76}$ Folgende Ergebnisse ergaben ihre Untersuchungen zu Spezialisierung:

- Je kürzer das Zeitintervall zwischen den einzelnen Delikten ist, desto höher ist der Spezialisierungsgrad.

- Frauen sind häufiger spezialisiert als Männer.

- $\quad$ Ältere haben einen höheren Spezialisierungsgrad als Jüngere.

- Bei Tätern mit vielen Registrierungen und verschiedenen Delikten ist die Vielfalt der Deliktstypen oft auf wenige beschränkt.

- Es ist ein Mythos und nicht empirisch bewiesen, dass Sexualdelikte und Gewaltdelikte zum gleichen Deliktstyp gehören.

- Es ist wichtig, Spezialisierung nicht zu überinterpretieren, weil dies die Arbeit der Kriminalpolizei falsch lenken könnte. ${ }^{77}$

Das Landeskriminalamt Nordrhein-Westfalen führte 2005 eine Untersuchung von jungen Mehrfachtatverdächtigen in Nordrhein-Westfalen durch. ${ }^{78}$ Ausgewertet wurde die Polizeiliche Kriminalstatistik von 1994 bis 2003. Es wurden unter anderem auch Analysen zum Spezialisierungsgrad mit Hilfe des FSC durchgeführt. Dafür wurden die Delikte in zehn Deliktskategorien eingeteilt. Den höchsten Spezialisierungsgrad gab es bei den Deliktskategorien Betrug und Betäubungsmitteldelikte.

Moffitt ${ }^{79}$ analysierte kriminelle Karrieren mit einem entwicklungsdynamischen Ansatz. Er unterschied zwischen zwei Gruppen von Individuen, die "adolescencelimited", diejenigen die nur während der Jugendzeit auffällig sind, und die "lifecourse-persistend", diejenigen die über die gesamte Lebensspanne auffällig sind. Die Gruppen unterscheiden sich ätiologisch, im Entwicklungsablauf, in der Prognose und bei der Verhaltenseinordnung, und zwar entweder normativ oder pathologisch. Moffitt stellt fest, dass vier Fünftel aller Jugendlichen polizeilich auffällig

\footnotetext{
${ }^{73}$ Kyvsgaard 2003, S. 154. Es handelt sich um eine dänische Längsschnittstudie, die justizielle Registrierungen von 1979 bis 1991 umfasst.

74 Vgl. Kyvsgaard 2003, S. 241.

75 Vgl. Kyvsgaard 2003, S. 149.

76 Vgl. Kyvsgaard 2003, S. 150.

77 Vgl. Kyvsgaard 2003, S. 242 f.

78 Landeskriminalamt Nordrhein-Westfalen 2005.

79 Moffitt 1993.
} 
werden. Die Gruppe der "adolescence-limited" begeht hauptsächlich Delikte, mit denen sie die jugendliche Gier nach Anerkennung und Privilegien befriedigen will: Diebstahl, Sachbeschädigung, Straftaten gegen die öffentliche Ordnung und Betäubungsmitteldelikte. ${ }^{80}$ Die kleine Gruppe der "life-course-persistend" fängt ihre kriminelle Karriere früher, schon in der Kindheit an, sie begeht andere Delikte als die Gruppe der "adolescence-limited" und sie setzt ihre kriminelle Karriere im Erwachsenenalter fort. Die Delikte der "life-course-persistend" verändern sich mit dem Alter: mit 4 Jahren beißen und schlagen, mit 10 Jahren Ladendiebstahl und Schule schwänzen, mit 16 Jahren Betäubungsmitteldelikte und Autodiebstahl ${ }^{81}$, mit 22 Jahren Raub und Vergewaltigung und mit 30 Jahren Betrug und Kindesmissbrauch. ${ }^{82}$

Das Landeskriminalamt Nordrhein-Westfalen schrieb 2005 dazu: „Die Jugendkriminalität gilt als episodenhaft und ubiquitär, d. h. die überwiegende Mehrzahl der jugendlichen Tatverdächtigen begeht eine oder mehrere Straftaten innerhalb einer kurzen Altersspanne. Die in der Jugendzeit auftretende Kriminalität wird in der Regel auch ohne Intervention nicht fortgesetzt. Daneben hat die kriminologische Forschung eine kleine Gruppe tatverdächtiger Jugendlicher identifiziert, die über einen wesentlich längeren Zeitraum hinweg wiederholt straffällig wird. Bei dieser Gruppe wird die Gefahr einer Verfestigung kriminellen Verhaltens bis in das Erwachsenenalter hinein gesehen. "83

Francis, Soothill und Fligelstone ${ }^{84}$ analysierten im Jahr 2004 Deliktstypen mit einem probabilistischen Clusteranalyseverfahren ${ }^{85}$. Sie legten besonderen Wert auf die Beschreibung der Methode. Francis, Soothill und Fligelstone benutzten Gerichtsdaten aus England und Wales des Geburtsjahrganges 1953 bis zum Alter von 40 Jahren. Die Daten wurden in 5-Jahresabschnitte eingeteilt, wobei innerhalb dieser Abschnitte die Delikte 0/1 kodiert wurden, d.h. wenn z.B. das Delikt Ladendiebstahl bei einer Person in einem Alterabschnitt auftrat, wurde eine Eins notiert, andernfalls eine Null. Die Häufigkeit spielte also keine Rolle, es war nur von Bedeutung, ob das Delikt vorkam oder nicht. Die einzelnen Altersabschnitte der Personen wurden Clustern zugeordnet. Für jedes Cluster wurden die Wahrscheinlichkeiten berechnet, dass die zugehörigen Altersabschnitte die einzelnen Delikte enthalten. Für die Männer ergab die optimale Lösung neun Cluster: verschiedene Straftaten, schwerer Diebstahl, Betrug, Gewaltdelikte, einfacher Diebstahl, ein

\footnotetext{
80 Vgl. Moffitt 1993, S. 691.

81 Autodiebstahl von Jugendlichen, nur für kurze Spritztouren, kommt in den USA deutlich häufiger vor als in Deutschland.

82 Vgl. Moffitt 1993, S. 679.

${ }^{83}$ Landeskriminalamt Nordrhein-Westfalen 2005, S. 1.

${ }^{84}$ Francis, Soothill \& Fligelstone 2004.

${ }^{85}$ Francis, Soothill \& Fligelstone berechnen die binäre latent class analysis mit dem Programm Latent Gold.
} 
Cluster mit Eigentums-, Gewalt- und Verkehrsdelikten, Autodiebstahl, Körperverletzung und Ladendiebstahl. Für die Frauen ergaben sich 3 Cluster: verschiedene Straftaten, Ladendiebstahl und Untreue. Des Weiteren wurde die Altersverteilung der Cluster untersucht und weiterführende Untersuchungen zu Spezialisierung und krimineller Laufbahn durchgeführt. ${ }^{86}$

In den angesprochenen Studien zeigen sich große Unterschiede bzgl. der Daten, der Stichprobengröße, der Methode und der Einteilung der Delikte in Deliktskategorien. Auch der Begriff „Spezialisierung“ ist nicht eindeutig. Sich spezialisieren heißt, „sich auf ein Teilgebiet beschränken“87. Kyvsgaard schrieb dazu, dass es sich bei einem Spezialisten um ein Individuum handelt, welches dieselbe Straftat mehrmals auf dieselbe Art und Weise begeht. ${ }^{88}$ Aber was heißt dieselbe Straftat und was heißt dieselbe Art und Weise? Wenn diese Einteilungen sehr fein gewählt werden, kommt Spezialisierung nie vor, da jede Straftat ein Einzelfall ist. Daher ist Kategorisierung notwendig. Die Kategorisierung der Straftaten baut zwangsläufig auf den polizeilichen und justiziellen Deliktstypen auf. Die Anzahl und die Größe der Deliktskategorien beeinflusst das Ergebnis der Analysen zur Spezialisierung. Je größer die Deliktskategorie, desto wahrscheinlicher ist es, dass das folgende Delikt derselben Kategorie angehört. Nachdem die Deliktskategorien gebildet wurden, ist zudem auch von Bedeutung, ob ein Spezialist nur jemand ist, der immer Delikte derselben Kategorie begeht, oder ob es sich bei jemandem, der grundsätzlich nur Delikte aus zwei Kategorien oder aus einer Gruppe von ähnlichen Deliktskategorien begeht, auch um einen Spezialisten handelt. Angenommen die Delikte sind in zehn Kategorien eingeteilt, darunter Diebstahl und Betäubungsmitteldelikte und acht weitere Kategorien. Ein Individuum, das immer nur Delikte der Deliktskategorien Diebstahl und Betäubungsmitteldelikte begeht, ist auch ein Spezialist. In diesem Fall, der Spezialisierung auf Delikte mehrerer Deliktskategorien, aber auch bei Spezialisierung auf Delikte einer Deliktskategorie, wird hier von Deliktsähnlichkeiten gesprochen.

${ }^{86}$ Die Methode wird im Abschnitt „Probabilistische Clusteranalyse erläutert“ und Ergebnisse der Studie befinden sich im Anhang.

87 Duden, Fremdwörterbuch 1982, S. 719.

88 Vgl. Kyvsgaard 2003, S. 147. 


\section{Die Datengrundlage}

In diesem Abschnitt werden die Daten, mit denen die Analysen dieser Arbeit durchgeführt wurden, vorgestellt. Hierbei wird zunächst die Freiburger Kohortenstudie einschließlich der verwendeten Datenquellen präsentiert. Im Detail werden sodann die für die Untersuchung benutzten Variablen, insbesondere die Deliktskategorien, beschrieben. Hierbei werden auch die soziodemografischen Variablen erläutert, die in den Daten vorhanden sind und die für die Auswertungen benutzt wurden. Danach werden schließlich Probleme des Datenmaterials angesprochen.

\subsection{Die Freiburger Kohortenstudie}

\subsubsection{Die Studie}

Am Max-Planck-Institut für ausländisches und internationales Strafrecht in Freiburg wird seit 1986 eine Kohortenstudie durchgeführt. Bei der Freiburger Kohortenstudie werden in einem Längsschnittdesign Sekundärdaten ${ }^{89}$ analysiert. Bei den Kohorten handelt es sich um Geburtsjahrgänge. Personen der Jahrgänge 1970, 1973, 1975, 1978, 1985 und 1988 werden hinsichtlich ihrer Registrierungen in amtlichen Datenbeständen verfolgt. ${ }^{90}$ Verwendung finden dabei Informationen zur polizeilichen Registrierung aus der Personenauskunftsdatei (PAD) Baden-Württemberg und zur justiziellen Registrierung aus dem Bundeszentralregister (BZR) ${ }^{91}$. In den BZR-Daten der Freiburger Kohortenstudie befinden sich Personen, die mindestens eine gerichtliche Registrierung in Baden-Württemberg haben. Das Design der Studie gewährleistet durch jährliche Datenziehung, dass alle Registrierungen der bestimmten Geburtskohorten vorhanden sind, auch die, die in PAD und BZR gelöscht wurden. Die offiziellen kriminellen Karrieren der genannten Geburtskohorten sind komplett vorhanden. Der aktuelle Datenbestand für die Polizeidaten 2003 und für die justiziellen Daten 2004 ist Tabelle $1 \mathrm{zu}$ entnehmen.

\footnotetext{
89 Bei Sekundärdaten handelt es sich um Daten, die für einen anderen als den Untersuchungszweck erhoben wurden.

90 Zum Design der Studie vgl. Grundies 1998, Höfer 2003, sowie insbesondere zu den datenschutzrechtlichen Fragen Schneider, Sutterer \& Karger 1988.

${ }_{91}$ Die BZR-Daten werden seit 1989 jährlich geliefert. Dadurch sind alle offiziellen Registrierungen der ausgewählten Geburtskohorten vorhanden. Die BZR-Daten umfassen auch die Eintragungen im Erziehungsregister. Eine Person kann frühestens mit 14 Jahren justiziell abgeurteilt werden ( $\$ 19$ StGB), d.h. die BZR-Daten beinhalten die Registrierungen von Personen ab 14 Jahren. Polizeiliche Registrierungen sind schon ab einem Alter von 7 Jahren möglich. In den PAD-Daten fehlen teilweise die ersten Registrierungen der älteren Kohorten wegen Löschungen (Kohorte 70/Alter 7-13, 73/7-11, 75/7-9 nicht vollständig). Bis auf diese wenigen fehlenden sind auch hier alle offiziellen Registrierungen vorhanden.
} 
Tabelle 1: $\quad$ Datenbestand der Freiburger Kohortenstudie (Stand PAD 2003, BZR 2004)

\begin{tabular}{lrrrrrrrr}
\hline & \multicolumn{9}{c}{ PAD } & \multicolumn{5}{c}{ BZR } \\
\cline { 2 - 8 } Kohorte & Registr. & $\%$ & TV & $\%$ & Registr. & $\%$ & Täter & $\%$ \\
\hline 1970 & 191.215 & 28,8 & 56.583 & 27,6 & 105.603 & 27,8 & 44.145 & 25,1 \\
1973 & 158.610 & 23,9 & 45.750 & 22,3 & 82.446 & 21,7 & 34.792 & 19,8 \\
1975 & 140.659 & 21,2 & 42.173 & 20,5 & 75.079 & 19,8 & 32.699 & 18,6 \\
1978 & 115.234 & 17,3 & 35.411 & 17,3 & 65.943 & 17,4 & 30.410 & 17,3 \\
1985 & 44.146 & 6,6 & 17.257 & 8,4 & 36.486 & 9,6 & 22.475 & 12,8 \\
1988 & 15.123 & 2,3 & 8.086 & 3,9 & 14.173 & 3,7 & 11.276 & 6,4 \\
Total & 664.987 & 100 & 205.260 & 100 & 379.730 & 100 & 175.797 & 100 \\
\hline
\end{tabular}

Der Datenbestand im Jahr 2003 der Polizeidaten der Freiburger Kohortenstudie umfasst 205.260 Tatverdächtige mit 664.987 Straftaten, was im Schnitt 3,2 Straftaten pro Person ausmacht.

Der Datenbestand der BZR-Daten bis zum Jahr 2004 der Freiburger Kohortenstudie mit mindestens einer Registrierung in Baden-Württemberg umfasst 175.797 Straftäter mit 379.730 justiziellen Entscheidungen; dies entspricht im Schnitt 2,2 Entscheidungen pro Person.

75\% der Personen, die im BZR registriert sind und mindestens eine Registrierung in Baden-Württemberg haben, sind auch in den Polizeidaten Baden-Württembergs registriert. $9251 \%$ der Personen, die polizeilich registriert sind, sind auch justiziell registriert. 93

\subsubsection{Die Daten}

Für die weiteren Berechnungen werden die Daten der Personenauskunftsdatei (PAD) des Landeskriminalamtes Baden-Württemberg und des Bundeszentralregisters (BZR) benutzt. Bei der PAD handelt es sich um einen Teil des polizeilichen Informationssystems zur präventiven und repressiven Kriminalitätsbekämpfung. Gespeichert werden Personen- und Falldaten zu Verdächtigen und Beschuldigten im Rahmen eines strafrechtlichen Ermittlungsverfahrens. ${ }^{94}$ Die Daten der PAD bilden eine der Grundlagen für die Erstellung der Polizeilichen Kriminalstatistik.

92 Ohne Berücksichtigung der Personen im BZR, die ausschließlich wegen StVG Delikten registriert sind.

${ }_{93}$ Auch hier ohne Berücksichtigung der Personen im BZR, die ausschließlich wegen StVG Delikten registriert sind.

94 Vgl. Informationssystem der Polizei in Baden-Württemberg 1992, S. PAD / 6. 
Die Daten der Freiburger Kohortenstudie sind anonymisiert; sie enthalten nicht den Geburtsnamen und nicht das vollständige Geburtsdatum, wohl aber das Geburtsjahr. Zur Personenkennung gibt es einen Personenschlüssel, der sowohl für die Daten der PAD wie für die Daten des BZR gilt.

Während bei den PAD-Daten jede Registrierung genau ein Delikt beinhaltet, umfassen die BZR-Registrierungen häufig mehrere Delikte. ${ }^{95}$

Für diese Untersuchung werden Datenlieferungen und Aufbereitungen bis zum Jahr 2003 für die PAD-Daten und bis zum Jahr 2004 für die BZR-Daten benutzt. ${ }^{96}$ Bei den PAD-Daten wurde das Tatjahr 2002 nicht mehr in die Untersuchung mit einbezogen, da es zum Zeitpunkt der Datenlieferung 2003 noch nicht vollständig vorlag. Die Dauer bis zur Eintragung in die jeweiligen Daten ist teilweise relativ lang.

Bei den PAD-Daten sind alle Registrierungen bis einschließlich 2001 in die Untersuchung einbezogen, bei den BZR-Daten alle Registrierungen bis einschließlich 2003.

\subsubsection{Die Bundeszentralregisterdaten}

Das Bundeszentralregister enthält das Zentralregister (§§ 3-58 BZRG), das Erziehungsregister ( $\S 59-64$ BZRG) und Teile des Strafregisters der Deutschen Demokratischen Republik (§§ 64a, 64b BZRG). „In das Bundeszentralregister werden überwiegend Justizdaten eingetragen, die im Zusammenhang mit dem Abschluss von Strafverfahren anfallen. "97 Im Zentralregister eingetragen werden rechtskräftig strafgerichtlich Verurteilte, Vermerke über Schuldunfähigkeit (§11 BZRG) und nachträgliche Entscheidungen, die sich auf eine frühere Eintragung beziehen. ${ }^{98}$ Nicht registriert werden Freisprüche und Verfahrenseinstellungen nach den $\S \S 153$ ff. StPO. Nur schwere, hart sanktionierte Straftaten Jugendlicher werden im Zentralregister eingetragen, ${ }^{99}$ die übrigen Anordnungen und Entscheidungen gegenüber Jugendlichen erfolgen im Erziehungsregister. ${ }^{100}$ Im Erziehungsregister wird die Anordnung von Erziehungsmaßregeln oder Zuchtmitteln, Nebenstrafen oder Ne-

95 In den PAD-Daten sind die Delikte anhand von Straftatenschlüsseln zusammengefasst. Siehe dazu Landeskriminalamt Baden-Württemberg 2002, S. 4 und INPOL-BW, TSH.

96 Die letzten Datenlieferungen, die für diese Arbeit benutzt wurden, stammen bei den PAD Daten vom Februar 2003, bei den BZR Daten vom Dezember 2004.

97 Veith 1999, S. 112.

98 Vgl. Veith 1999, S. 114.

99 Vgl. $§ 5$ Abs. 2 BZRG.

100 Vgl. § 60 BZRG. Ein Unterschied zwischen Zentralregister und Erziehungsregister besteht darin, dass Eintragungen im Zentralregister im Führungszeugnis ( $\$ 30$ BZRG) aufgeführt werden, Eintragungen des Erziehungsregisters erscheinen nicht im Führungszeugnis. 
benfolgen allein oder in Verbindung miteinander eingetragen ( $\$ 60$ Abs. 1 Nr. 2 BZRG). Außerdem wird der Freispruch und die Einstellung des Verfahrens wegen mangelnder Reife eingetragen ( $\$ 60$ Abs. 1 Nr. 6 BZRG). Im Gegensatz zum allgemeinen Strafrecht und zu den Registrierungen im Zentralregister wird im Erziehungsregister auch die Einstellung des Verfahrens ( $\S 45,47 \mathrm{JGG})$ eingetragen (§60 Abs. 1 Nr. 7 BZRG). Bei straffällig gewordenen Jugendlichen und Heranwachsenden steht der Erziehungsgedanke und die soziale Integration im Mittelpunkt der Bemühungen. ${ }^{101}$

Wenn hier von Bundeszentralregisterdaten gesprochen wird, so sind die Daten des Zentralregisters einschließlich des Erziehungsregisters gemeint. D.h., die Daten beinhalten Straftaten von Verurteilten und bei Jugendlichen zusätzlich auch die Straftaten, bei denen das Verfahren eingestellt wurde. ${ }^{102}$ Bei den hier verwendeten Daten sind bei den Jugendlichen $61 \%$ und bei den Heranwachsenden 35\% der Entscheidungen eingestellt worden.

Die Bundeszentralregisterdaten werden als Rohdaten geliefert. ${ }^{103}$ In jeder Zeile stehen die Daten einer Person. Die Zeilen haben unterschiedliche Längen, da sie unterschiedlich viele Registrierungen enthalten. Auch die einzelnen Registrierungen haben nicht die gleiche Länge. Variablen wie Geburtstag, Geburtsname, Familienname und Aktenzeichen, die im Bundeszentralregister gespeichert sind, werden aus datenschutztechnischen Gründen nicht geliefert. In Tabelle 2 sind die Variablen aufgeführt, die jeder Datensatz enthält. Mit Textkennzahlen werden normierte Texte kodiert, die Angaben zur Tat enthalten, und weitere Angaben, die im Zusammenhang mit dem Abschluss des Strafverfahrens anfallen, wie Nebenfolgen, Maßregeln und Entscheidungen, die im Zusammenhang mit der Vollstreckung ergehen. Je nach Textkennzahl gibt es in diesem Feld noch weitere Angaben wie Datum, Dauer, freier Text oder keine weiteren Angaben. ${ }^{104}$ Jede Registrierung kann unterschiedlich viele Textkennzahlen enthalten. Die Textkennzahlen, die in allen relevanten Erstmitteilungen vorkommen, sind „Tatbezeichnung“ und ,angewendete Vorschriften“, beides als Freitextfeld. Drei Beispiele:

Tatbezeichnung: Diebstahl

Angewendete Vorschriften: StGB $\S 242$ Abs. 1, JGG $\S 1, \S 2, \S 3, \S 14, \S 15$ Abs. 1 Nr. 3

101 Vgl. Götz \& Tolzmann 2000, S. 297 (§ 59 Rdnr. 4).

102 Das Verfahren kann nach § 45 JGG vom Staatsanwalt oder nach $\S 47$ JGG vom Jugendrichter eingestellt werden. Das Verfahren wird hier nicht mangels Beweisen eingestellt, sondern nur bei hinreichendem Tatverdacht.

103 Genauere Informationen zu den Daten des Bundeszentralregisters vgl. Uhlig 1985 und vgl. Veith 1999. Den Inhalt der Eintragungen regelt § 5 BZRG.

104 Beispiele für Textkennzahlen sind: 3212: Verwarnung (gemeint ist damit die Verwarnung nach § $14 \mathrm{JGG}$ ), 2145: 210195 (Rest der Jugendstrafe zur Bewährung ausgesetzt bis: TTMMJJ ( $88 \mathrm{JGG})$, dies ist meistens eine nachträgliche Mitteilung. 
Tatbezeichnung: Widerstand gegen Vollstreckungsbeamte, Hausfriedensbruch, Erschleichen von Leistungen, Beleidigung in 4 Fällen

Angewendete Vorschriften: StGB § 265a, § 248a, § 185, § 194, § 123, § 113, $\S 53, \S 55$

Tatbezeichnung: schwere räuberische Erpressung in Tateinheit mit Vergewaltigung sowie Beleidigung in 5 Fällen, davon ein Fall in Tateinheit mit sexueller Nötigung, ein weiterer Fall in Tateinheit mit versuchter sexueller Nötigung und ein dritter Fall in Tateinheit mit Bedrohung

Angewendete Vorschriften: StGB $\S 177, \S 253, \S 255, \S 250$ Abs. 1 Nr. 2 und Abs. $2, \S 178, \S 185, \S 241, \S 22, \S 23, \S 47, \S 49, \S 52, \S 53, \S 54$

Aus dem Paragrafenfeld wird für die statistische Auswertung einerseits das schwerste Delikt ausgewählt und des Weiteren werden die Delikte Deliktskategorien zugeordnet. ${ }^{105}$ Die Schwereeinstufung richtet sich nach dem gesetzlichen Strafrahmen. Diese Einstufung wird auch in der Strafverfolgungsstatistik angewendet. Da es seit Beginn der Kohortenstudie immer wieder Änderungen des Strafrechts gab, die letzte große Änderung war das Sechste Gesetz zur Reform des Strafrechts vom 31. Januar 1998, gibt es je nach Tatdatum für die Schwereeinstufung drei Zeitpunkte, 1.1.1988, 1.1.1995, 1.1.1999. Die Schwereeinstufung wir anhand einer Deliktsschweretabelle vorgenommen. ${ }^{106}$

105 Der Umfang der Daten erfordert eine maschinelle Aufbereitung der Daten. Die Daten werden mit einem FORTRAN Programm aufbereitet. 1988 wurde dazu an der Universität Konstanz (Lehrstuhl Prof. Heinz) KOSIMA (Konstanzer System zur Inhaltsanalyse und maschinenlesbaren Aufbereitung von Bundeszentralregisterdaten) entwickelt. Dies Programm wurde 1989 dem Max-Planck-Institut zur Verfügung gestellt und seitdem hier weiterentwickelt. Inzwischen werden die Daten in einer ORACLE Datenbank gespeichert und verwaltet.

106 Die Deliktsschweretabelle wurde ursprünglich in Konstanz von Peter Sutterer erstellt und muss laufend auf den aktuellen Stand gebracht werden. 
Tabelle 2: $\quad$ Satzaufbau eines BZR-Datensatzes mit den Variablen, die der Freiburger Kohortenstudie geliefert werden

\begin{tabular}{|c|c|}
\hline Feldname & Feldinhalt \\
\hline \multicolumn{2}{|l|}{ Personennummer } \\
\hline \multicolumn{2}{|l|}{ Geburtsjahr } \\
\hline \multicolumn{2}{|l|}{ Geschlecht } \\
\hline Personenschlüssel & $\begin{array}{l}\text { Schlüssel, gebildet aus Geburtsname und Ge- } \\
\text { burtsdatum (identischer Schlüssel wie in den } \\
\text { PAD-Daten) }\end{array}$ \\
\hline \multicolumn{2}{|l|}{ Vorname } \\
\hline \multicolumn{2}{|l|}{ Geburtsort } \\
\hline \multicolumn{2}{|l|}{ Staatsangehörigkeit } \\
\hline \multicolumn{2}{|l|}{ Registrierungsnummer } \\
\hline \multirow[t]{2}{*}{ Belegart } & E: Erstmitteilung \\
\hline & N: Nachträgliche Mitteilung \\
\hline Entscheidungsdatum & Datum des ersten Urteils \\
\hline Behördenkennzeichen der erkennenden Stelle & $\begin{array}{l}\text { Stelle, die die Entscheidung erlassen hat } \\
\text { (z.B. AG Freiburg) }\end{array}$ \\
\hline \multicolumn{2}{|l|}{ Rechtskraftdatum } \\
\hline Tatdatum & Datum der letzten Tat \\
\hline Strafart & Art der Freiheitsentziehung \\
\hline Dauer der Strafe & In Jahren, Monaten, Wochen und Tagen \\
\hline Strafvorbehalt & Verwarnung mit Strafvorbehalt (§ 59 StGB) \\
\hline \multicolumn{2}{|l|}{ Anzahl Tagessätze } \\
\hline \multicolumn{2}{|l|}{ Höhe Tagessatz } \\
\hline Sperre der Fahrerlaubnis & Datum des Ablaufs \\
\hline Schuldspruch & Nach $\S 27$ JGG \\
\hline Bewährungszeit & In Jahren, Monaten, Wochen und Tagen \\
\hline Gewerbezusammenhang & $\mathrm{X}$, wenn ja \\
\hline Fahrverbot & Dauer \\
\hline Textkennzahl & $\begin{array}{l}\text { Kennzahl mit normiertem Text, die weitere In- } \\
\text { formation, die im Zusammenhang mit dem Ab- } \\
\text { schluss des Strafverfahrens steht, liefert }\end{array}$ \\
\hline \multicolumn{2}{|l|}{ weitere Textkennzahlen } \\
\hline \multicolumn{2}{|l|}{ weitere Registrierungen einer Person: } \\
\hline \multicolumn{2}{|l|}{ Vorname } \\
\hline \multicolumn{2}{|l|}{ Geburtsort } \\
\hline \multicolumn{2}{|l|}{ Staatsangehörigkeit } \\
\hline \multicolumn{2}{|l|}{ Registrierungsnummer } \\
\hline Belegart & \\
\hline$\ldots$ & \\
\hline
\end{tabular}




\subsection{Relevante Variablen für die Analyse}

\subsubsection{Deliktskategorien}

Bei der Einteilung der Delikte in Kategorien besteht die Diskrepanz, dass es einerseits gut wäre, alle Delikte von vornherein einzeln zu betrachten, und erst nach der Analyse Kategorien zu bilden, andererseits ist dies aber praktisch nicht möglich, weil sehr viele der einzelnen Deliktskategorien dann zu klein wären und es viel zu viele Kombinationen gäbe, was eine sinnvolle Auswertung nicht mehr gestatten würde. Hier werden die Delikte der polizeilichen Registrierungen in $28 \mathrm{Ka}$ tegorien eingeteilt und die der Bundeszentralregister-Registrierungen in 31 Kategorien. Die Deliktskategorien, wenn nicht einzelne Delikte speziell betrachtet werden, entsprechen großteils den Abschnitten im besonderen Teil des StGB. Ansonsten sind alle Betäubungsmitteldelikte und alle Vergehen gegen das Ausländergesetz und Asylverfahrensgesetz zusammengefasst. Die Deliktskategorien „StGB Rest“ und „Nebengesetze Rest" werden in die weiteren Auswertungen nicht mit einbezogen. Verkehrsdelikte werden in den Polizeidaten nicht registriert, dazu zählen die Deliktskategorien Verkehrsdelikte nach StGB, StVG und PflVG. Für die Auswertungen der justiziellen Registrierungen bilden diese drei Kategorien die Deliktskategorie Verkehrsdelikte. Die Delikte fahrlässige Körperverletzung und fahrlässige Tötung werden bei den Auswertungen der Polizeidaten nicht miteinbezogen, da hier die Fallzahlen zu klein sind. Dies liegt daran, dass diese Delikte hauptsächlich in Kombination mit Verkehrsdelikten vorkommen. Auch Vollrausch wird wegen der geringen Fallzahl bei den polizeilichen Auswertungen nicht berücksichtigt. Somit werden für die folgenden Analysen die polizeilichen Registrierungen in 25 Deliktskategorien eingeteilt und die justiziellen Registrierungen in 29.107

In Tabelle 3 sind die einzelnen Deliktskategorien aufgeführt. Die zweite Spalte enthält bei den Delikten nach StGB den Abschnitt des besonderen Teils des StGB. Die dritte Spalte enthält die Paragrafen des StGB mit dem Stand nach dem 6. Strafrechtsreformgesetz vom 26.1.1998. Gesetzesänderungen seit 1984 sind auf diesen Stand hin angepasst. In der letzten Spalte sind die Straftaten-Schlüssel (TSH) der PAD eingetragen. Auch die Straftaten-Schlüssel verändern sich im Laufe der Jahre. Der Datenbestand der Polizei wird bei Veränderungen der Straftaten-Schlüssel automatisch auf den neuesten Stand gebracht. D.h. der Straftaten-Schlüssel hier ist der des Jahres 2003. In den Kohortendaten befinden sich aber auch Datensätze, die in den Polizeidaten nicht mehr enthalten sind. Diese in den Polizeidaten gelöschten Daten befinden sich auch in dem hier benutzten Datensatz, da sie aber nicht im aktuellen PAD-Bestand enthalten sind, haben sie bei Veränderungen der StraftatenSchlüssel den alten Straftaten-Schlüssel. In der Spalte mit den Straftaten-Schlüs

107 Die justiziellen Deliktskategorien umfassen die polizeilichen Deliktskategorien plus Verkehr (Verkehrsdelikte nach StGB, StVG, PflVG), Vollrausch, fahrlässige Körperverletzung und fahrlässige Tötung. 
seln sind die alten und aktuellen TSH aufgeführt. So hatte z.B. Hausfriedensbruch bis 2001 den TSH 6200001 und seit 1.1.2001 den TSH 6221001.

Das Kriterium für die Deliktskategorienbildung ist einerseits eine genügend große Fallzahl, andererseits aber auch die Spezialität des Vergehens. Gerade schwere Delikte sind bedeutend, haben aber nur eine kleine Fallzahl.

Tabelle 3: Deliktskategorien

\begin{tabular}{|c|c|c|c|}
\hline Deliktskategorie & Abschnitt bes. Teil StGB & $\S \S$ StGB & PAD TSH \\
\hline $\begin{array}{l}\text { Gefährdung des demokrati- } \\
\text { schen Rechtsstaates }\end{array}$ & 1 & $80-90 \mathrm{~b}$ & $9900001-9900014,9900053$ \\
\hline $\begin{array}{l}\text { Widerstand gegen die Staats- } \\
\text { gewalt }\end{array}$ & 6 & $111-122$ & $621 *$ \\
\hline $\begin{array}{l}\text { Straftaten gegen die öffentliche } \\
\text { Ordnung (ohne Hausfriedens- } \\
\text { bruch, Unerlaubtes Entfernen } \\
\text { vom Unfallort) }\end{array}$ & 7 ohne $\S \S 123,142$ & 124-145d ohne 142 & $\begin{array}{l}62 *, 9900056 \\
\text { nicht } 621^{*}, 6200001,6221^{*}\end{array}$ \\
\hline Hausfriedensbruch & in 7 & 123 & $6200001,6221 *$ \\
\hline Fälschung & $8,23(+\S 348$ aus 30$)$ & 146-152a, 267-281, 348 & $54^{*}, 55^{*}, 6500013,6550008$ \\
\hline Meineid & 9 & $153-163$ & 6700001-6 \\
\hline Falsche Verdächtigung & 10 & 164 & 6700007 \\
\hline Sexualdelikte & 13 & 177,178 & $111^{*}, 1120003-1120007$ \\
\hline Sonstige Sexualdelikte & 13 & $\begin{array}{l}174-175,176,176 a \\
179-184\end{array}$ & $110^{*}, 113^{*}, 12^{*}, 13^{*}, 14^{*}$ \\
\hline Beleidigung & 14 & $185-199$ & $673^{*}, 9900048$ \\
\hline Tötungsdelikte & 16 & $211-217,220 a, 221$ & $\begin{array}{l}0 *, 9900049,6700019 \\
\text { nicht 300001, 04* }\end{array}$ \\
\hline fahrlässige Tötung & 16 & 222 & 300001 \\
\hline fahrlässige Körperverletzung & $\S 229$ aus 17 & $229,(230$ alt $)$ & alt 2200002 , neu $225^{*}$ \\
\hline Körperverletzung & $\S 223$ aus 17 & 223 & $224 *$ \\
\hline schwere Körperverletzung & $\begin{array}{l}17 \text { ohne } \S \S 223,229 \\
(+\S 340)\end{array}$ & $\begin{array}{l}224-227,231,340, \\
(223 \mathrm{a} \text { alt })\end{array}$ & $\begin{array}{l}22 *, 6551^{*} \\
\text { nicht } 2200002,224^{*}\end{array}$ \\
\hline $\begin{array}{l}\text { Straftaten gegen die persönli- } \\
\text { che Freiheit }\end{array}$ & 18 & $234-241 \mathrm{a}$ & $\begin{array}{l}23 *, 9900050,9900051 \\
\text { nicht } 235^{*}\end{array}$ \\
\hline einfacher Diebstahl & 19 & $\begin{array}{l}242,246,247,248 b \\
248 c\end{array}$ & $3^{*}, 53^{*}$ \\
\hline schwerer Diebstahl & 19 & $243-244 a$ & $4 *$ \\
\hline Raub & $20(+\S 316 a$ aus 28$)$ & $249-255,316 a$ & $21^{*}, 61^{*}$ \\
\hline Hehlerei & 21 & $257-261$ & $63 *$ \\
\hline Betrug & 22 ohne $\S 265 \mathrm{a}$ & $263-266 \mathrm{~b}$ ohne $265 \mathrm{a}$ & $51^{*}, 52^{*}$, ohne $515^{*}$ \\
\hline
\end{tabular}




\begin{tabular}{|c|c|c|c|}
\hline Deliktskategorie & Abschnitt bes. Teil StGB & $\S \S$ StGB & PAD TSH \\
\hline Erschleichen von Leistungen & in 22 & $265 a$ & $515^{*}$ \\
\hline Sachbeschädigung & 27 & $303-305 a$ & $674 *$ \\
\hline Gemeingefährliche Straftaten & $\begin{array}{l}28 \\
\text { ohne } \S \S 315 \mathrm{~b}, 315 \mathrm{c}, \\
316,316 \mathrm{a}\end{array}$ & $\begin{array}{l}\text { 306-323c ohne } 315 b \\
315 c, 316,316 a, 323 a\end{array}$ & $\begin{array}{l}64 *, 6700023,6700025-26 \\
6700028-34,6700036 \\
6700037,6700050-52, \\
675^{*}, 6770001-2,2350001\end{array}$ \\
\hline Vollrausch & aus 28 & $323 a$ & 67000035 \\
\hline $\begin{array}{l}\text { Gefährliche Eingriffe in den } \\
\text { Straßenverkehr }\end{array}$ & aus 28 & $315 b$ & $6700027(\S 315 b)$ \\
\hline BTM-Delikte & & BtMG & $73 *$ \\
\hline AuslG & & AuslG & $7250^{*}-7254^{*}, 7257^{*}, 7259^{*}$ \\
\hline AsylVfG & & AsylVfG & $7255^{*}, 7256^{*}$ \\
\hline StGB Verkehrsdelikte & aus 7,28 & $142,315 \mathrm{c}, 316$ & \\
\hline StVG & & StVG & \\
\hline PflVG & & PflVG & \\
\hline StGB Rest (Landesverrat, & $2-5,11,12,15$ & 93-109, 166-173, & $9900015-46$ \\
\hline Straft. gg. ausl. Staaten, & $24-26,29$ & 201-206, 218-219b, & $\begin{array}{l}04^{*}, 235^{*}, 56^{*}, 65^{*}, 66^{*}, \\
67^{*} .\end{array}$ \\
\hline Straft. gg. Verfassungsorgane, & 30 (ohne $\S \S 340,348$ ) & 283-302a, & 9900054, (nicht 6700001-7) \\
\hline Straft. gg. Landesverteidigung, & $\S \S 218-219 \mathrm{~b}$ aus 16 & 306-357 ohne $315 b$ & nicht 2350001 , \\
\hline Straft. bzgl. Religion, & & $315 \mathrm{c}, 316,316 \mathrm{a}$ & nicht 6500013,6550008 , \\
\hline Straft. gg. Personenstand, & & $323 a, 340,348$ & nicht 6700019,6700023 , \\
\hline Verletzg. pers. Geheimbereich, & & & nicht 6700025-37, \\
\hline Abtreibung, Konkurs, & & & nicht 6700050-52, \\
\hline Eigennutz, Wettbewerb, & & & nicht $673^{*}, 674^{*}, 675^{*}$ \\
\hline Umwelt, Straft. im Amt) & & & nicht $6770001-2$ \\
\hline $\begin{array}{l}\text { Nebengesetze Rest }(\mathrm{NG} \text {, die in } \\
\text { der PAD registriert sind) }\end{array}$ & & & $7 *$, nicht $73^{*}, 725^{*}$ \\
\hline
\end{tabular}

Die Häufigkeiten der Deliktskategorien sind in Tabelle 4 dargestellt. Die Prozentzahlen geben den Anteil an allen Straftaten an. Zum Beispiel sind 3,9\% aller in der PAD registrierten Straftaten Körperverletzung. Bei den BZR-Daten können pro Entscheidung mehrere Delikte vorkommen, aber pro Entscheidung wird jede Deliktskategorie maximal einmal gezählt. Die Tabelle gibt die Anzahl der Delikte wieder, nicht die Anzahl der Entscheidungen. Bei den BZR-Daten gibt es zwei verschiedene Anteilspalten. Die erste Spalte bezieht sich nur auf Delikte, die auch in der PAD registriert sind. Die zweite Spalte bezieht sich auf alle im BZR registrierten Delikte. Der Grund hierfür ist, dass Verkehrsdelikte nicht in den Polizeidaten registriert werden. 
Tabelle 4: Häufigkeitsverteilung der Delikte in PAD und BZR (alle Registrierungen PAD bis 2001, BZR bis 2003)

\begin{tabular}{lrrrrr}
\hline & \multicolumn{2}{c}{ PAD } & & \multicolumn{3}{c}{ BZR } \\
\cline { 2 - 6 } Delikt & $\mathbf{N}$ & $\mathbf{\%}$ & $\mathbf{N}$ & \% (ohne Verk.) & \% \\
\hline einfacher Diebstahl & 169.829 & 27,4 & 101.616 & 28,0 & 21,8 \\
schwerer Diebstahl & 79.593 & 12,9 & 19.930 & 5,5 & 4,3 \\
BTM-Delikte & 63.618 & 10,3 & 30.382 & 8,4 & 6,5 \\
AuslG108 & 53.879 & 8,7 & 20.670 & 5,7 & 4,4 \\
Betrug & 42.436 & 6,9 & 24.559 & 6,8 & 5,3 \\
Sachbeschädigung & 27.138 & 4,4 & 14.697 & 4,1 & 3,2 \\
Leistungserschleichung & 25.297 & 4,1 & 19.125 & 5,3 & 4,1 \\
schwere Körperverletzung & 24.901 & 4,0 & 13.224 & 3,6 & 2,8 \\
Körperverletzung & 24.326 & 3,9 & 28.838 & 8,0 & 6,2 \\
Fälschung & 14.811 & 2,4 & 12.862 & 3,5 & 2,8 \\
Beleidigung & 13.173 & 2,1 & 9.856 & 2,7 & 2,1 \\
Straft. gg. d. pers. Freiheit & 12.944 & 2,1 & 8.286 & 2,3 & 1,8 \\
Raub & 10.126 & 1,6 & 5.121 & 1,4 & 1,1 \\
Hehlerei & 9.922 & 1,6 & 6.767 & 1,9 & 1,5 \\
Hausfriedensbruch & 7.282 & 1,2 & 4.353 & 1,2 & 0,9 \\
öffentliche Ordnung & 6.144 & 1,0 & 3.466 & 1,0 & 0,7 \\
sonstige Sexualdelikte & 3.384 & 0,5 & 1.624 & 0,4 & 0,3 \\
Wid. gg. Staatgewalt & 3.346 & 0,5 & 3.264 & 0,9 & 0,7 \\
gemeingef. Straftaten & 2.790 & 0,5 & 1.330 & 0,4 & 0,3 \\
Sexualdelikte & 1.938 & 0,3 & 878 & 0,2 & 0,2 \\
Meineid & 1.704 & 0,3 & 1.504 & 0,4 & 0,3 \\
Falsche Verdächtigung & 1.613 & 0,3 & 1.132 & 0,3 & 0,2 \\
Gefährdung dem. Rechtsstaat & 1.454 & 0,2 & 824 & 0,2 & 0,2 \\
gef. Eingr. in den Straßenverk. & 659 & 0,1 & 1.016 & 0,3 & 0,2 \\
Tötungsdelikte & 628 & 0,1 & 304 & 0,1 & 0,1 \\
Vollrausch & 84 & 0,0 & 1.089 & 0,3 & 0,2 \\
fahrlässige Körperverletzung & 392 & 0,1 & 14.636 & 4,0 & 3,1 \\
fahrlässige Tötung & 42 & 0,0 & 549 & 0,2 & 0,1 \\
StGB Rest & 4.199 & 0,7 & 2.101 & 0,6 & 0,5 \\
NG Rest & 11.744 & 1,9 & 8.369 & 2,3 & 1,8 \\
StGB Verkehr & & & 43.984 & & 9,4 \\
StVG & & & 46.290 & & 9,9 \\
PflVG & & & 13.367 & & 2,9 \\
\hline Summe & & & & & \\
\hline Summe ohne Verkehrsdelikte & & & & & 100 \\
\hline
\end{tabular}

108 In „AuslG“ sind Vergehen gegen AuslG und AsylVfG zusammengefasst. 
Der einfache Diebstahl bildet sowohl bei den polizeilichen als auch bei den justiziellen Registrierungen die größte Kategorie mit 27,4\% bei den polizeilichen Registrierungen und 28\% bei den BZR-Registrierungen bezogen auf Delikte, die auch in der PAD registriert sind. Die zweitstärkste Kategorie in den Polizeidaten bildet der schwere Diebstahl (12,9\%). Bei den justiziellen Registrierungen nimmt der schwere Diebstahl einen deutlich niedrigeren Anteil ein, 5,5\%, bei Betrachtung der Delikte, die auch in den Polizeidaten registriert werden. Einfacher und schwerer Diebstahl zusammen belegen über $40 \%$ der registrierten Polizeieinträge.

Beim Delikt Diebstahl gibt es bei den BZR-Daten eine Besonderheit. Dies hängt damit zusammen, dass in einer BZR-Registrierung häufig mehrere Delikte eingetragen sind. Immer wenn $\S 243$, schwerer Diebstahl, eingetragen ist, wird grundsätzlich auch $\S 242$, Diebstahl, eingetragen. Es ist also anhand des Textfeldes, in dem die Paragrafen eingetragen sind, nicht möglich, zu unterscheiden, ob mit dieser Registrierung ein schwerer und ein einfacher Diebstahl abgeurteilt wurde oder nur ein schwerer Diebstahl. Alle diese Fälle werden hier nur als schwerer Diebstahl gezählt, deshalb fehlen in den BZR-Daten einfache Diebstähle, und der Anteil des einfachen Diebstahls ist tatsächlich noch höher als hier angegeben.

Die Deliktskategorie der Verkehrsdelikte, dazu gehören Verkehrsdelikte nach StGB, Straftaten des StVG und PflVG, welche nur im BZR registriert werden, ist ebenso groß wie die Deliktskategorie einfacher Diebstahl. Fahrlässige Körperverletzung, die meist in Zusammenhang mit Verkehrsdelikten steht, nimmt bei BZRRegistrierungen einen wesentlich höheren Anteil ein, als bei PAD-Registrierungen.

Der Anteil an Vergehen gegen Ausländergesetze ist mit 8,7\% bei den Polizeiregistrierungen und 5,7\% bei justiziellen Registrierungen relativ hoch, insbesondere deshalb weil bei diesem Delikt bedacht werden muss, dass diese Straftaten fast nur von Nichtdeutschen begangen werden und deren Anteil weniger als die Hälfte aller polizeilich Registrierten und nur etwa ein Drittel aller justiziell Registrierten ausmacht.

Weitere Delikte, die eine relativ große Kategorie bilden, sind Betäubungsmitteldelikte mit einem Anteil von über $10 \%$ bei den polizeilichen Registrierungen und über $8 \%$ bei den justiziell Registrierten.

Alle Gewaltdelikte ${ }^{109}$ zusammen, dazu gehören Körperverletzung, schwere Körperverletzung, Raub, Tötungsdelikte, Sexualdelikte, sonstige Sexualdelikte und

109 Es gibt ganz unterschiedliche Definitionen von Gewalt. Zu den Gewaltdelikten werden hier Straftaten gegen die Person, einschließlich Sexualdelikten gezählt. Fahrlässige Körperverletzung und fahrlässige Tötung gehören nicht zu den Gewaltdelikten. Auch Straftaten gegen Sachen (Sachbeschädigung, Hausfriedensbruch) gehören nach dieser Definition nicht zu den Gewaltdelikten. Ausführlich zum Begriff Gewaltkriminalität siehe Kürzinger 1993, S. 171 ff. Die PKS benutzt einen sehr viel engeren Begriff für Gewaltkriminalität (siehe Landeskriminalamt Baden-Württemberg 2002, S. 5). Bei der PKS gehören einfache Körperverletzung, sexueller Missbrauch, Bedrohung und Nötigung nicht zu den Gewaltdelikten. 
Straftaten gegen die persönliche Freiheit, haben einen Anteil von 12,5\% bei den polizeilichen Registrierungen. Bei den justiziellen Registrierungen ist der Anteil der Gewaltdelikte mit 16,1\% höher. Bezieht man, wie die PKS es tut, die einfache Körperverletzung bei den Gewaltdelikten nicht mit ein, ist der Anteil bei polizeilichen und justiziellen Registrierungen gleich (8\%). Bei justiziellen Registrierungen ist der Anteil der einfachen Körperverletzung deutlich höher als bei polizeilichen Registrierungen.

\subsubsection{Soziodemografische Merkmale}

Als soziodemografische Merkmale stehen sowohl aus den PAD-Daten wie aus den BZR-Daten die Variablen Geschlecht, Staatsangehörigkeit und das Datum der Tatzeit und damit das Alter zur Tatzeit zur Verfügung. Den Datenbestand der polizeilichen Registrierungen, getrennt nach Geschlecht und Staatsangehörigkeit, deutsch - nichtdeutsch, zeigt Tabelle 5.

Tabelle 5: Datenbestand der Freiburger Kohortenstudie bis 2001 (PAD) 110

\begin{tabular}{lrcccccccc}
\hline & \multicolumn{1}{c}{ PAD } \\
\hline & \multicolumn{3}{c}{ männl. } & \multicolumn{9}{c}{ weibl. } & \multicolumn{3}{c}{ Summe } \\
\cline { 2 - 11 } & TV & Taten & Tat/TV & TV & Taten & Tat/TV & TV & Taten & Tat/TV \\
\hline dt. & 76.065 & 281.946 & 3,7 & 27.630 & 56.626 & 2,0 & 103.695 & 338.572 & 3,3 \\
nicht-dt. & 70.609 & 245.354 & 3,5 & 20.009 & 34.609 & 1,7 & 90.618 & 279.963 & 3,1 \\
Summe & 146.674 & 527.300 & 3,6 & 47.639 & 91.235 & 1,9 & 194.313 & 618.617 & 3,2 \\
\hline
\end{tabular}

Der Datenbestand der Polizeidaten der Freiburger Kohortenstudie einschließlich des Tatjahres 2001 umfasst 194.313 Tatverdächtige mit 618.617 Straftaten, was im Schnitt 3,2 Straftaten pro Person ausmacht.

Erwartungsgemäß dominiert das männliche Geschlecht. Dies gilt für die Ebene der Personen und noch mehr für die der Registrierungen. 75\% der Tatverdächtigen sind Männer und sie begehen $85 \%$ der Straftaten.

Bei den Männern sind im Schnitt 3,6 Straftaten pro Person registriert, bei den Frauen 1,9. 53\% aller Tatverdächtigen sind Deutsche, und sie begehen 55\% der Taten. Es gibt keinen deutlichen Unterschied bei der Anzahl der Straftaten pro Person bei Deutschen und Nichtdeutschen.

Tabelle 6 zeigt den Datenbestand der BZR-Daten nach Geschlecht und Staatsangehörigkeit getrennt.

110 Die Summe der Taten stimmt nicht mit der Anzahl der Delikte aus Tabelle 4 überein, da es bei den Variablen Geschlecht und Nationalität fehlende Werte gibt. 779 der gesamten Registrierungen haben entweder einen fehlenden Wert beim Geschlecht oder bei der Nationalität. 
Der Datenbestand der BZR-Daten umfasst 170.533 Straftäter mit 368.627 justiziellen Entscheidungen und 465.848 Delikten, was im Schnitt 2,2 Entscheidungen pro Person ergibt bzw. 2,7 Delikte.

Auch hier dominieren die Männer. 77\% der Straftäter sind Männer und 84\% der justiziellen Entscheidungen betreffen männliche Straftäter.

Bei den Männern sind im Schnitt 2,4 Entscheidungen pro Person registriert, bei den Frauen 1,5.

$65 \%$ aller Straftäter sind Deutsche und auch 65\% aller Registrierungen sind von Deutschen.

Tabelle 6: $\quad$ Datenbestand der Freiburger Kohortenstudie bis 2003 (BZR)111

\begin{tabular}{lrrrrrrrrr}
\hline & \multicolumn{1}{c}{ BZR } \\
\hline & \multicolumn{3}{c}{ männl. } & \multicolumn{3}{c}{ weibl. } & \multicolumn{3}{c}{ Summe } \\
\cline { 2 - 10 } & Täter & Registr. & Delikte & Täter & Registr. & Delikte & Täter & Registr. & Delikte \\
\hline dt. & 83.505 & 199.335 & 258.728 & 26.897 & 42.067 & 48.267 & 110.402 & 241.402 & 306.995 \\
nicht-dt. & 48.143 & 110.081 & 139.507 & 11.988 & 17.144 & 19.346 & 60.131 & 127.225 & 158.853 \\
Summe & 131.648 & 309.416 & 398.235 & 38.885 & 59.211 & 67.613 & 170.533 & 368.627 & 465.848 \\
\hline
\end{tabular}

Neben den Variablen Geschlecht und Staatsangehörigkeit steht auch die Variable Alter zur Verfügung. Die Altersspanne reicht bei den Polizeidaten von 7 Jahren bis maximal 31 Jahren und bei den BZR-Daten von 14 Jahren bis maximal 33 Jahren. ${ }^{112}$

Tabelle 7: Altersspannen in PAD und BZR

\begin{tabular}{lcc}
\hline Kohorte & Altersspanne PAD & Altersspanne BZR \\
\hline 1970 & $7-31$ & $14-33$ \\
1973 & $7-28$ & $14-30$ \\
1975 & $7-26$ & $14-28$ \\
1978 & $7-23$ & $14-25$ \\
1985 & $7-16$ & $14-18$ \\
1988 & $7-13$ & $14-15$ \\
\hline
\end{tabular}

111 Die Summe der Delikte stimmt nicht mit der Summe aus Tabelle 4 überein, da es bei der Variable Geschlecht 165 fehlende Werte gibt.

112 Polizeiliche Registrierungen sind ab einem Alter von 7 Jahren möglich. Eintragungen im Bundeszentralregister bzw. Erziehungsregister erfolgen aufgrund der Strafmündigkeit erst ab dem Alter von 14 Jahren. 
Das Geburtsdatum steht aus Datenschutzgründen nicht zur Verfügung, sondern nur das Geburtsjahr. Damit sind die Altersangaben nicht exakt. Ist zum Beispiel das Datum der Tat der 23. August 1984 und das Geburtsjahr 1970, so ist das Alter 14 Jahre, wenn der Geburtstag zwischen Januar und dem 23. August liegt, ansonsten 13 Jahre. Allgemeiner ausgedrückt heißt das, wenn hier im Weiteren von einem Alter von 14 Jahren gesprochen wird, liegt das Alter im Schnitt zwischen 131/2 und 141/2 Jahren. Bei einer Altersangabe von 18 Jahren sind auch 17-Jährige dabei.

In Abbildung 1 wird die Häufigkeitsverteilung der polizeilichen Registrierungen nach dem Alter dargestellt und in Abbildung 2 ebendiese Verteilung der justiziellen Registrierungen. $\mathrm{Zu}$ sehen ist jeweils der gesamte Verlauf sowie die Einzeldarstellungen getrennt nach Geschlecht und Nationalität. Die Altersverteilung ist nicht repräsentativ, da die Kohorten mit unterschiedlichen Altersspannen vertreten sind. ${ }^{113}$ Wie in Tabelle $7 \mathrm{zu}$ sehen ist, sind nur bis zum Alter von 13 Jahren alle Kohorten vertreten. Der Rückgang im Alter von 17 Jahren hängt damit zusammen, dass hier die Kohorte 1985 nicht mehr vertreten ist. Ebenso ist zu berücksichtigen, dass die Häufigkeitszahlen nicht auf die Wohnbevölkerung bezogen sind. Der Verlauf, besonders bei den Nichtdeutschen, kann auch damit zusammenhängen, dass die Altersgruppen in der Bevölkerung nicht gleichmäßig verteilt sind.

\section{Abbildung 1: Häufigkeitsverteilung der polizeilichen Registrierungen nach Alter}

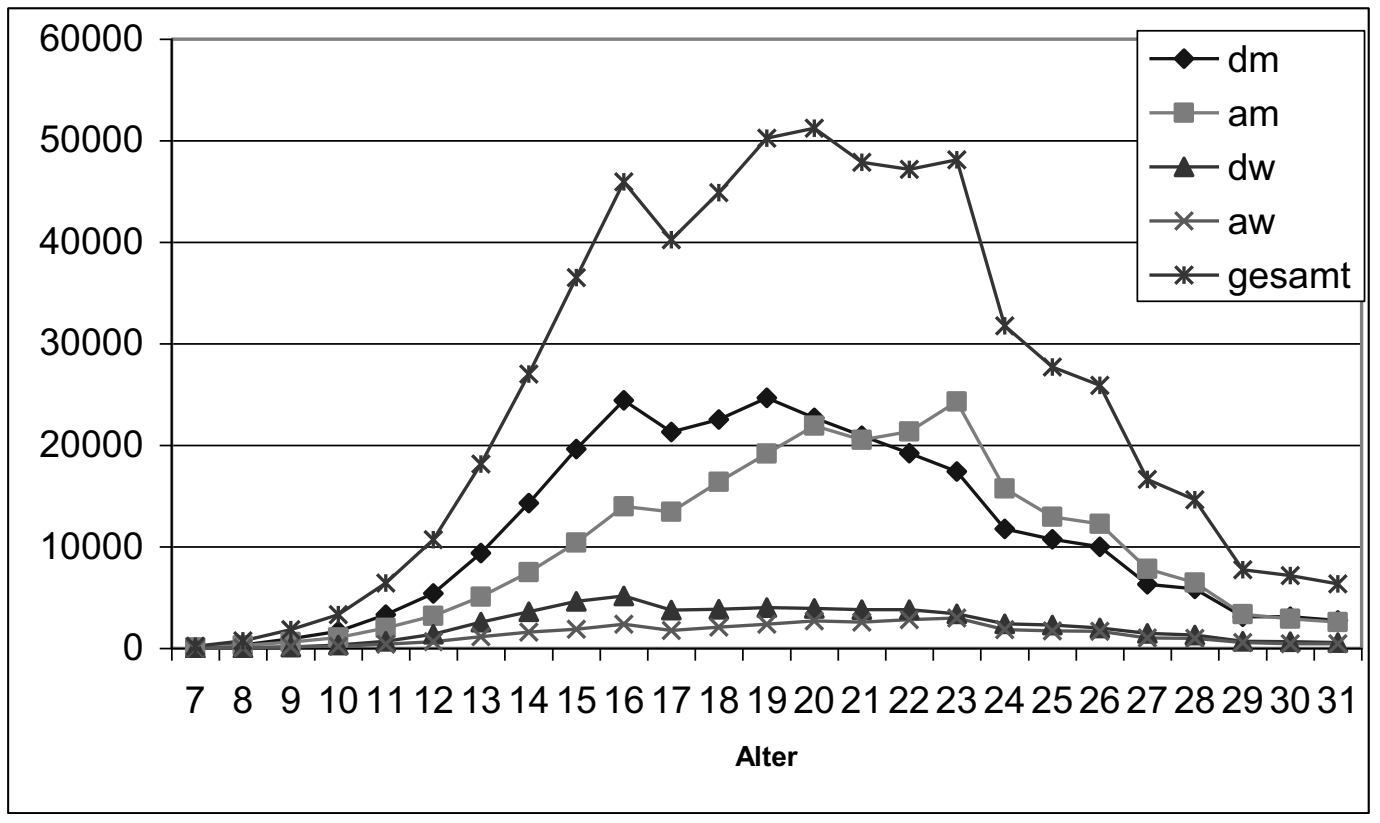

In den Kohortendaten sind die meisten polizeilichen Registrierungen im Alter von 16 bis 23. Das Maximum liegt beim Alter 20 mit über 50.000 Registrierungen.

113 Zur Altersverteilung der polizeilichen Registrierungen siehe auch Grundies, Höfer \& Tetal 2002, S. 91. 
Bei den deutschen Männern (dm) sind die meisten Registrierungen im Alter von 16 bis 19 Jahren, danach nimmt die Zahl der Registrierungen ab. Bei den deutschen Frauen (dw) liegt das Maximum schon im Alter von 16 Jahren mit etwa 5.000 Registrierungen.

In Abbildung 2 wird die Häufigkeitsverteilung der justiziellen Registrierungen nach dem Alter dargestellt. Bei den justiziellen Registrierungen sind die meisten Einträge im Alter von 15 bis 18 Jahren. Auch bei dieser Grafik ist zu berücksichtigen, dass je nach Altersstufe unterschiedlich viele Kohorten einbezogen sind. Nur bis zum Alter von 15 Jahren sind alle Kohorten vertreten, bis zum Alter von 18 Jahren sind fünf der sechs Kohorten vertreten. Des Weiteren ist zu berücksichtigen, dass die Häufigkeitszahlen nicht auf die Wohnbevölkerung bezogen sind. Der Verlauf, hier besonders zu sehen bei den nichtdeutschen Männern (am), hängt auch damit zusammen, dass die Altersgruppen in der Bevölkerung nicht gleichmäßig verteilt sind. Besonders bei Nichtdeutschen gibt es Wellen von Zuzügen bzw. auch Wegzügen.

Abbildung 2: Häufigkeitsverteilung der justiziellen Entscheidungen nach Alter

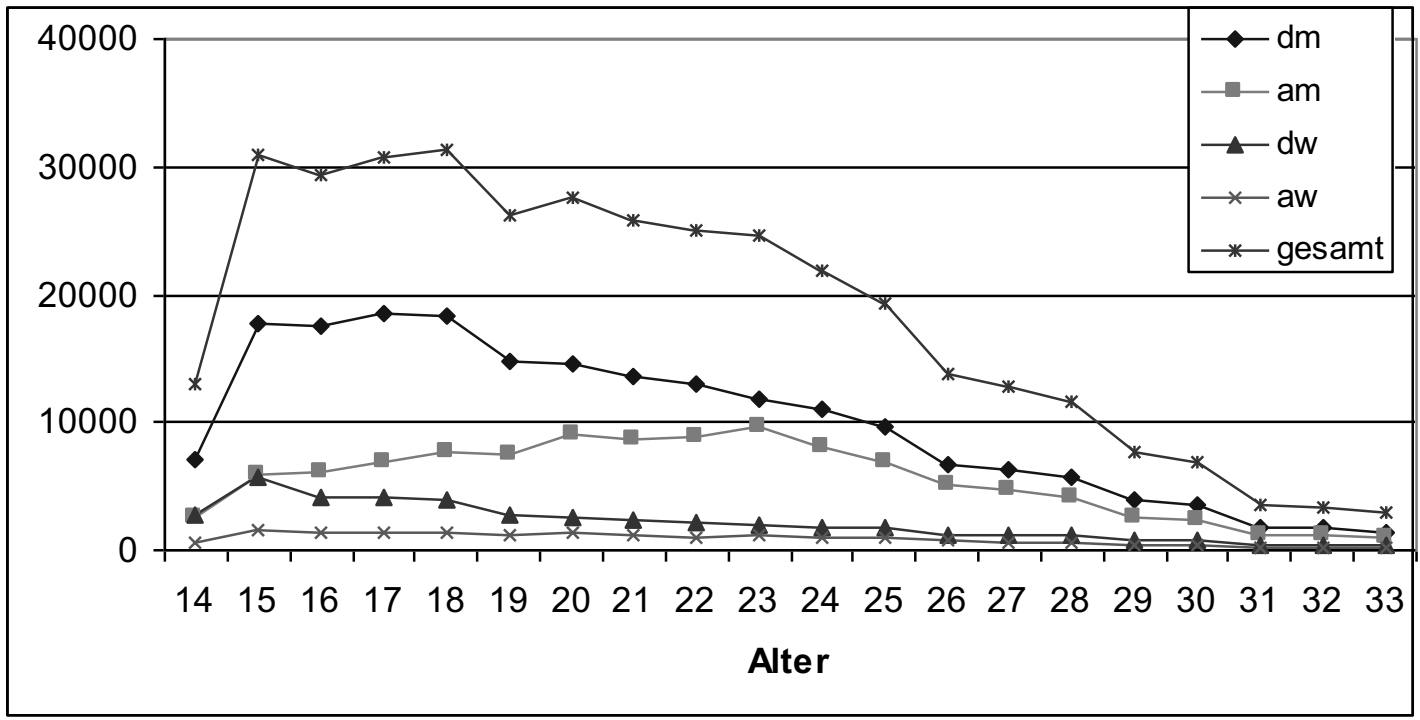

Abbildung 3 zeigt die Deliktsverteilung nach Alter bei deutschen Männern bei Polizeiregistrierungen. Die gestreiften Flächen unten zeigen die Gewaltdelikte. Ihr Anteil nimmt konstant mit dem Alter zu, auf einen Anteil von insgesamt 23\% im Alter von 31 Jahren. Die größte Fläche ist der einfache Diebstahl, er hat im Alter von 12 Jahren einen Anteil von über 50\%. In der Grafik darüber befindet sich der schwere Diebstahl. Das Maximum des schweren Diebstahls, anteilsmäßig zu den anderen Delikten, liegt im Alter von 17 Jahren bei 26\%. Der Anteil der Sachbeschädigung ist bei Kindern relativ groß mit einem Anteil von $19 \%$ bei den 9Jährigen, nimmt dann ab und liegt bei den 21- bis 31-Jährigen konstant bei $4 \%$. 
Auch bei gemeingefährlichen Straftaten ist der Anteil bei Kindern relativ groß, liegt aber schon bei den Jugendlichen unter einem Prozent und bleibt verschwindend gering. Im Gegensatz dazu nimmt der Anteil von Betrug im Erwachsenenalter zu. Im Alter von 18 liegt der Anteil von Betrug bei 4\% und wächst mit dem Alter auf über 15\%. Der Anteil der Betäubungsmitteldelikte nimmt im Jugendalter zu, erreicht bei den jungen Erwachsenen einen Anteil von etwa 20\% und nimmt danach wieder ab. Der Anteil bei Beleidigung steigt stetig mit dem Alter. Der Anstieg verhält sich ebenso wie bei den Gewaltdelikten.

Abbildung 3: Deliktsverteilung nach Alter bei deutschen Männern (PAD) 114

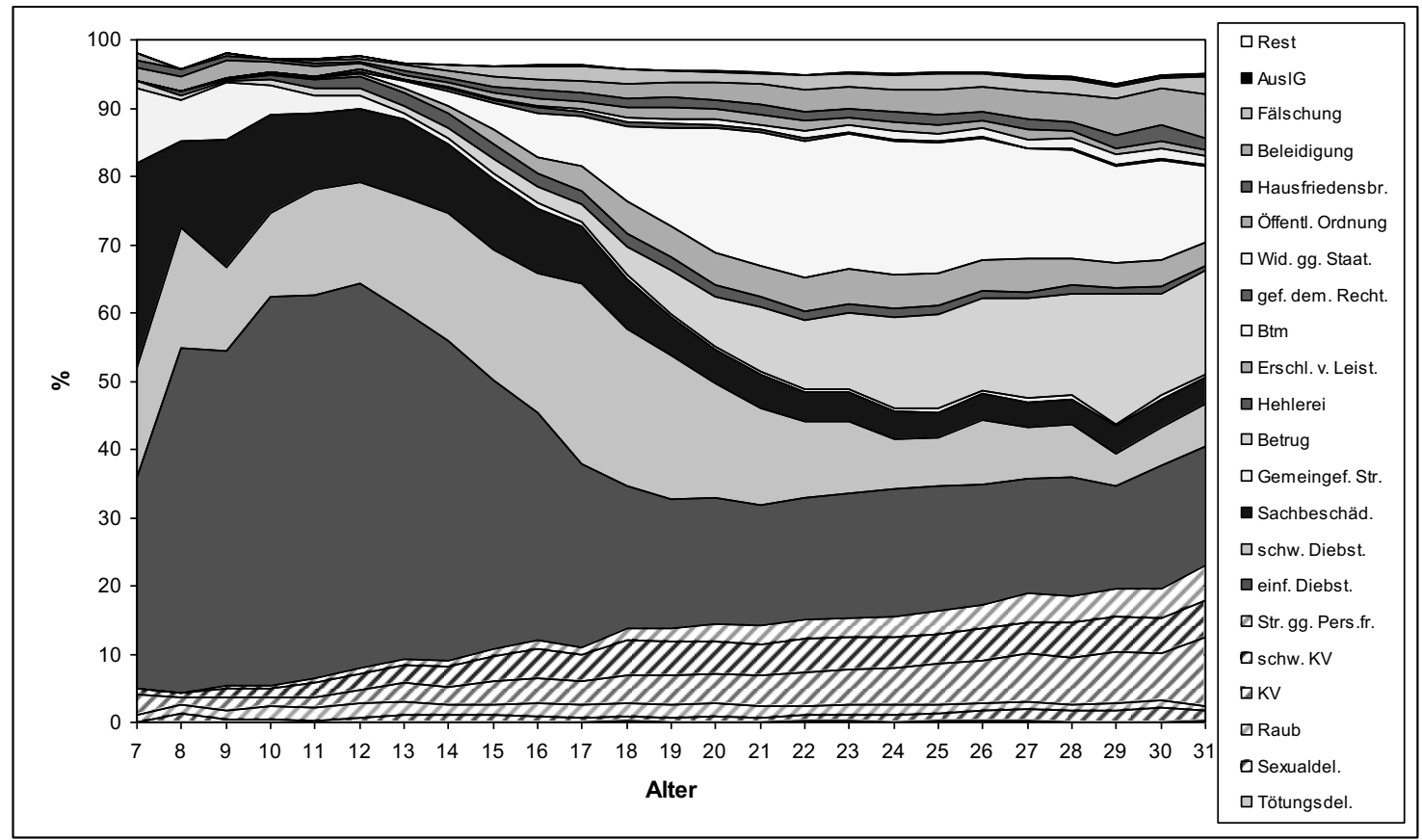

Abbildung 4 zeigt die Deliktsanteile bei justiziellen Registrierungen. Bei den BZR-Delikten ist zu beachten, dass die Registrierung erst mit dem Alter 14 beginnt, deshalb ist die Darstellung, die sich auf den ersten Blick grundsätzlich von den Polizeidaten unterscheidet, nicht so anders. Auffällig ist, dass der Anteil der Gewaltdelikte über das Alter hin konstant bleibt. Weiter sieht man ab einem Alter von 16 Jahren den großen Anteil an Verkehrsdelikten und auch einen deutlichen Anteil von fahrlässiger Körperverletzung und fahrlässiger Tötung.

114 Sexualdelikte sind einschließlich der Kategorie „sonstige Sexualdelikte“. 
Abbildung 4: $\quad$ Deliktsverteilung nach Alter bei deutschen Männern (BZR) ${ }^{115}$

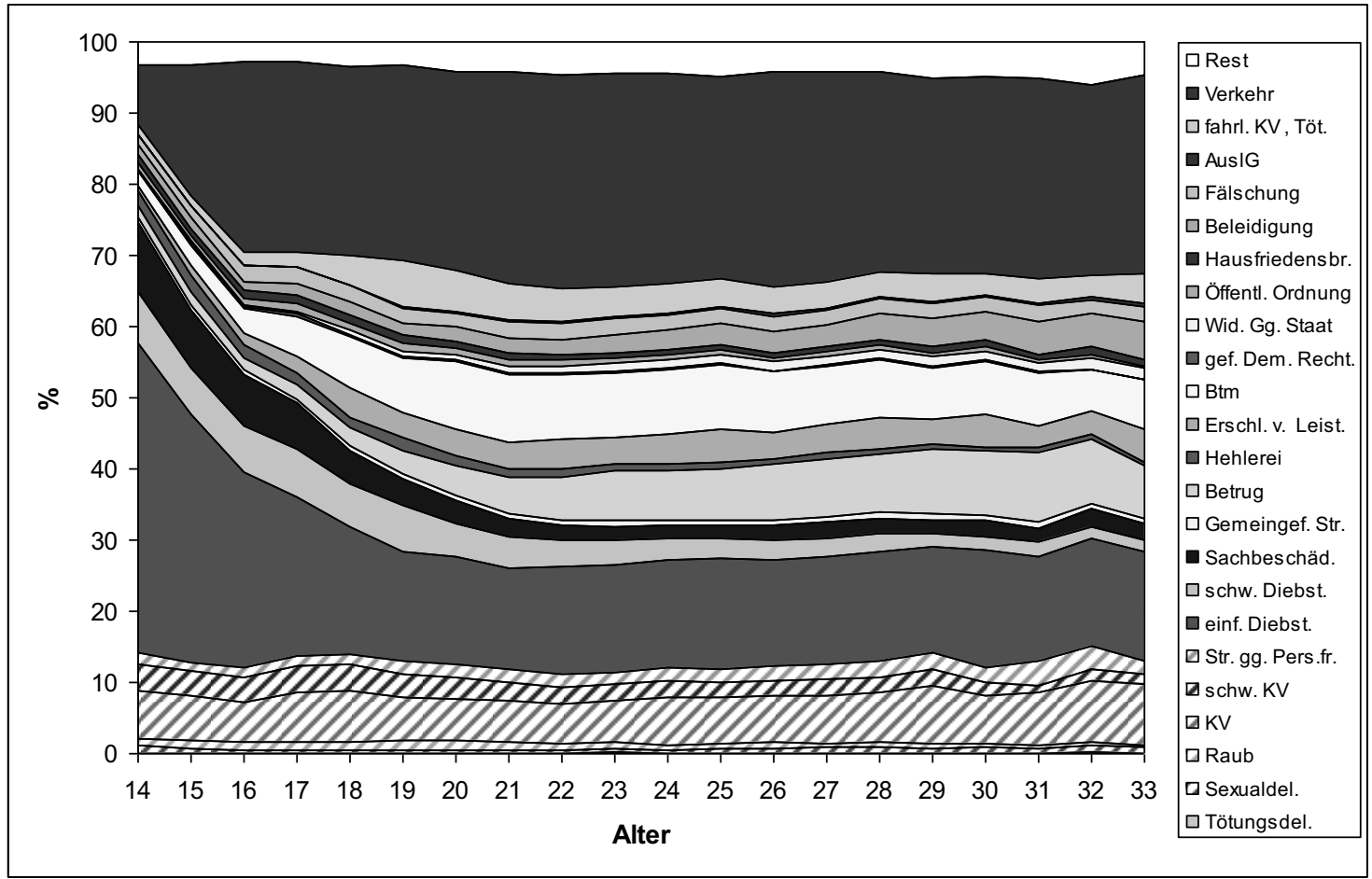

In diesen Abbildungen sieht man die Veränderungen der Häufigkeiten des Auftretens der Delikte mit dem Alter. Damit können aber nicht Zusammenhänge zwischen Delikten bestimmt werden, da man nicht weiß, welche Delikte von einer Person begangen wurden. Ziel dieser Arbeit ist, Zusammenhänge zwischen Delikten aufzuzeigen.

\subsubsection{Anzahl der Registrierungen pro Person - Vorstrafenbelastung}

Das Ziel ist, Ähnlichkeiten von Delikten empirisch zu bestimmen, und zwar mittels des gemeinsamen Auftretens von Delikten innerhalb der kriminellen Karriere von Personen. Von Bedeutung sind damit die Deliktskombinationen einer Person. Relevant für die Auswertung von Deliktsähnlichkeiten auf Personenebene sind somit nur Personen, bei denen zwei oder mehr Straftaten registriert sind. Je nach Anzahl der Registrierungen gibt es unterschiedlich viele Kombinationsmöglichkeiten.

115 Sexualdelikte sind einschließlich der Kategorie „sonstige Sexualdelikte“. 
Tabelle 8: Anzahl der Kombinationen je nach Anzahl der Registrierungen

\begin{tabular}{cc}
\hline Anzahl Registrierungen & Anzahl Kombinationen \\
\hline 2 & 1 \\
3 & 3 \\
4 & 6 \\
5 & 10 \\
6 & 15 \\
7 & 21 \\
8 & 28 \\
\hline
\end{tabular}

Anhand eines Beispiels wird die Bestimmung der Anzahl der Kombinationen erklärt. Eine Person ist mit drei Delikten - einfacher Diebstahl, Körperverletzung und Betrug - registriert. Damit ergeben sich die drei Kombinationen: 1. einfacher Diebstahl - Körperverletzung, 2. einfacher Diebstahl - Betrug und 3. Betrug - Körperverletzung. Die Formel zur Berechnung der Kombinationen lautet: $N^{*}(\mathrm{~N}-1) / 2$. Dazu ein Beispiel: Eine Person hat 4 Registrierungen, $N=4$, dann gibt es unter diesen Registrierungen $4 * 3 / 2=6$ Kombinationsmöglichkeiten. ${ }^{116}$

Von Bedeutung ist die Anzahl der Registrierungen einer Person. Tabelle 9 zeigt die Anzahl der Tatverdächtigen mit der jeweiligen Zahl der Registrierungen unterschieden nach Frauen und Männern. Die Tabelle zeigt, dass bei weniger als 50\% der tatverdächtigen Männer zwei oder mehr Straftaten registriert sind. Bei diesen Männern mit zwei oder mehr Straftaten sind aber 85\% der Taten registriert. 30\% der tatverdächtigen Frauen haben zwei oder mehr Straftaten begangen. Auch hier ist der Anteil der Straftaten hoch. Bei diesen 30\% der Frauen sind 63\% der von Frauen begangenen Straftaten registriert. In absoluten Zahlen heißt das: Etwa 69.000 tatverdächtige Männer haben zwei oder mehr Straftaten begangen und sind mit insgesamt etwa 450.000 Straftaten registriert. Bei den Frauen sind es deutlich weniger. Bei etwas mehr als 14.000 tatverdächtigen Frauen mit zwei oder mehr Straftaten sind etwas weniger als 58.000 Straftaten registriert.

116 Hierzu zwei Beispiele: Bei einer Person sind die Delikte Diebstahl, BTM, Körperverletzung und Raub registriert. Damit gibt es die 6 Kombinationen: Diebstahl-BTM, Diebstahl-Körperverletzung, Diebstahl-Raub, BTM-Körperverletzung, BTM-Raub, Körperverletzung-Raub. Sind bei einer Person die 5 Delikte schwerer Diebstahl, schwerer Diebstahl, Hehlerei, schwerer Diebstahl, schwerer Diebstahl registriert, ergibt sich 4-mal die Kombination schwerer Diebstahl-Hehlerei und 6-mal die Kombination schwerer Diebstahl-schwerer Diebstahl, insgesamt 10 Kombinationen. 
Tabelle 9: PAD: Tatverdächtige und Taten (alle Registrierungen bis 2001)

\begin{tabular}{lrrrrrrrr}
\hline & \multicolumn{9}{c}{ männl. } \\
\cline { 2 - 9 } Anz. Registr. & TV & $\%$ & Registr. & $\%$ & TV & $\%$ & Registr. & $\%$ \\
\hline 1 & 78.047 & 53,2 & 78.047 & 14,8 & 33.474 & 70,2 & 33.474 & 36,6 \\
2 & 24.542 & 16,7 & 49.084 & 9,3 & 7.305 & 15,3 & 14.610 & 16,0 \\
3 & 11.574 & 7,9 & 34.722 & 6,6 & 2.644 & 5,5 & 7.932 & 8,7 \\
4 & 6.813 & 4,6 & 27.252 & 5,2 & 1.270 & 2,7 & 5.080 & 5,6 \\
5 und mehr & 25.806 & 17,6 & 338.472 & 64,1 & 2.992 & 6,3 & 30.265 & 33,1 \\
Summe & 146.782 & 100 & 527.577 & 100 & 47.685 & 100 & 91.361 & 100 \\
\hline
\end{tabular}

Tabelle 10 zeigt die Anzahl der justiziell registrierten Täter mit der jeweiligen Zahl Registrierungen. Unterschieden wird nach Frauen und Männern. Bei den justiziell Registrierten haben 45\% der Männer zwei oder mehr Registrierungen, was $77 \%$ der Registrierungen entspricht. Bei $24 \%$ der Frauen sind zwei oder mehr Entscheidungen registriert, bei 50\% der Registrierungen bei Frauen gibt es mindestens eine weitere Registrierung. 59.000 männliche Täter haben zwei oder mehr Registrierungen, was etwa 237.000 justiziellen Registrierungen entspricht. Bei den Frauen sind es deutlich weniger. Auf 9.368 Frauen mit zwei oder mehr Registrierungen entfallen 29.694 justizielle Entscheidungen.

Tabelle 10: BZR: Täter, Registrierungen-männlich-weiblich (alle Reg. bis 2003)

\begin{tabular}{lrrrrrrrr}
\hline & \multicolumn{3}{c}{ männl. } \\
\cline { 2 - 9 } Anz. Reg. & Täter & \% & Registr. & \% & Täter & \% & Registr. & \% \\
\hline 1 & 72.251 & 54,9 & 72.251 & 23,4 & 29.517 & 75,9 & 29.517 & 49,9 \\
2 & 24.196 & 18,4 & 48.392 & 15,6 & 5.291 & 13,6 & 10.582 & 17,9 \\
3 & 11.957 & 9,1 & 35.871 & 11,6 & 1.810 & 4,7 & 5.430 & 9,2 \\
4 & 6.869 & 5,2 & 27.476 & 8,9 & 822 & 2,1 & 3.288 & 5,6 \\
\multirow{2}{*}{5 und mehr } & 16.375 & 12,4 & 125.426 & 40,5 & 1.445 & 3,7 & 10.394 & 17,6 \\
\multirow{2}{*}{ Summe } & 131.648 & 100 & 309.416 & 100 & 38.885 & 100 & 59.211 & 100 \\
\hline
\end{tabular}

Tabelle 11 zeigt die Anzahl der justiziell registrierten Täter mit der jeweiligen Zahl der begangenen Delikte, d.h. es werden nicht nur die Registrierungen gezählt, sondern auch die darin abgeurteilten Delikte, wobei aber jedes Delikt nur einmal gezählt wird, auch wenn es mehrmals in einer Entscheidung vorkommt. Unterschieden wird nach Frauen und Männern. Für die weitere Auswertung sind auch hier nur Täter mit zwei oder mehr Delikten relevant. Bei den justiziell Registrierten 
sind das 53\% der Männer, mit 85\% der Delikte und 31\% der justiziell registrierten Frauen mit $60 \%$ der bei Frauen registrierten Delikte. Etwa 70.000 männliche Täter haben zwei oder mehr Straftaten begangen und sind insgesamt mit etwa 340.000 Delikten registriert. Bei den Frauen sind es deutlich weniger. Auf 12.000 Frauen mit zwei oder mehr registrierten Delikten entfallen 41.000 Delikte.

Tabelle 11: BZR: Täter, Delikte - männlich-weiblich (alle Registrierungen bis 2003)

\begin{tabular}{lrrrrrrrr}
\hline & \multicolumn{3}{c}{ männl. } & \multicolumn{5}{c}{ weibl. } \\
\cline { 2 - 9 } Anz. Del. & Täter & $\%$ & Delikte & $\%$ & Täter & $\%$ & Delikte & $\%$ \\
\hline 1 & 61.617 & 46,8 & 61.617 & 15,5 & 26.880 & 69,1 & 26.880 & 39,8 \\
2 & 25.445 & 19,3 & 50.890 & 12,8 & 6.344 & 16,3 & 12.688 & 18,8 \\
3 & 13.374 & 10,2 & 40.122 & 10,1 & 2.451 & 6,3 & 7.353 & 10,9 \\
4 & 8.001 & 6,1 & 32.004 & 8,0 & 1.140 & 2,9 & 4.560 & 6,7 \\
5 und mehr & 23.211 & 17,6 & 213.602 & 53,6 & 2.070 & 5,3 & 16.132 & 23,9 \\
Summe & 131.648 & 100 & 398.235 & 100 & 38.885 & 100 & 67.613 & 100 \\
\hline
\end{tabular}

\subsection{Besonderheiten und Probleme der Datengrundlage}

Bei den Daten dieser Studie handelt es sich um offizielle Daten. Nur die Delikte von polizeilich bzw. justiziell erfassten Straftaten sind vorhanden. Offiziell nicht erfasste Straftaten verbleiben im Dunkelfeld ${ }^{117}$.

„Kriminalität ist kein Sachverhalt, der einfach gemessen werden könnte, wie etwa die Länge, das Gewicht oder die Temperatur eines Gegenstandes. Kriminalität ist vielmehr ein von Struktur und Intensität strafrechtlicher Sozialkontrolle abhängiger Sachverhalt. Die Bezeichnung als ,Kriminalität' ist einerseits das Ergebnis vorgängiger gesellschaftlicher Festlegungen, andererseits die Folge von zumeist mehrstufig verlaufenden Prozessen der Wahrnehmung von Sachverhalten und deren Bewertung.

Von der Menge der Ereignisse, die Opfer oder Tatzeugen wahrnehmen und als ,kriminell' bewerten, wird nur ein Teil den Behörden gemeldet (Hellfeld), der größte Teil verbleibt im - aus Sicht der Polizei - Dunkelfeld. Die Anzeigewahrscheinlichkeit ist nicht für alle Delikt- bzw. Tätergruppen gleich hoch; sie ist vielmehr nach Deliktart und -schwere, nach Täter- und Opfermerkmalen, nach Täter-Opfer-Beziehungen, nach Einschätzung polizeilicher Aufklärungswahrscheinlichkeit usw. höchst unter-

117 „Unter Dunkelfeld versteht man die Summe der Straftaten, die zwar tatsächlich begangen, den Strafverfolgungsbehörden (Polizei, Justiz) aber nicht bekannt geworden sind und die deshalb nicht in der offiziellen Kriminalstatistik erscheinen.“, Schneider 1987, S. 182. Einen ausführlichen Überblick über die verschiedenen Definitionen des Begriffs Dunkelfeld gibt Kreuzer 1993, S. 14 f. 
schiedlich. Über die Zeit hinweg unterliegt sie überdies dem Wandel. Registrierte Kriminalität ist deshalb kein repräsentativer Ausschnitt aus der Gesamtmenge aller gegen Strafrechtsnormen verstoßenden Handlungen. Die amtlichen Statistiken geben vermutlich weder den Umfang noch die Struktur noch die differenzielle Betroffenheit unterschiedlicher Bevölkerungsgruppen adäquat wieder."118

„Die Kriminal- und Strafrechtspflegestatistiken messen nicht ,das' Hellfeld der Kriminalität, sondern sie messen jeweils die Ergebnisse der Tätigkeit von Polizei, Staatsanwaltschaft oder Gericht. Sie spiegeln - eingeschränkt - die hier stattfindenden Prozesse der Wahrnehmung und Registrierung, Ausfilterung und der Bewertungsänderung wider."119

Nicht jeder anzeigbare Vorfall wird angezeigt, nicht jeder angezeigte Sachverhalt wird aufgeklärt, nicht jeder Tatverdächtige wird angeklagt und nicht jeder Angeklagte wird auch verurteilt. ${ }^{120}$

„Die informelle Sozialkontrolle, die in Familie, Schule, Betrieb, Nachbarschaft und Gruppen von Gleichaltrigen (Peer-Groups) ausgeübt wird, bewertet alles Verhalten gleichfalls mit Blick auf Strafbarkeit, wendet aber häufig zunächst informelle Sanktionen an. Wenn Taten außerhalb solcher Zusammenhänge begangen werden, gegen Gruppen bzw. gegen Firmen, gegen private oder öffentliche Einrichtungen usw., spielen entsprechende Entscheidungs- und Reaktionsprozesse dieser Opfer/Betroffenen ebenfalls eine ausschlaggebende Rolle."121

Die Opfer und Beobachter von Straftaten entscheiden, ob sie gar nichts unternehmen, ob sie es bei einer informellen Reaktion belassen oder aber ob sie die Straftat anzeigen. So bestimmen die Opfer und Beobachter von Straftaten, welche Delikte und welche Täter offiziell registriert werden, wo genau im Einzelnen also die Grenze zwischen Hellfeld (der registrierten Kriminalität) und dem Dunkelfeld verläuft. ${ }^{122}$ Auch die Polizei selbst beeinflusst, wie viele und welche Delikte registriert werden. Je nachdem, wie hoch der Arbeitseinsatz ist, welche Orte kontrolliert werden und welche Straftaten verfolgt werden.

Wenn in letzter Zeit in den Medien davon gesprochen wird, dass immer mehr Gewaltstraftaten stattfinden, ist nicht bekannt, ob tatsächlich mehr Gewalttaten stattgefunden haben, ob diese Taten nur häufiger angezeigt und damit häufiger registriert werden bzw. ob die Polizei ihren Arbeitseinsatz erhöht hat.

118 Bundesministerium des Innern und Bundesministerium der Justiz (Hg.) 2006. Zweiter Periodischer Sicherheitsbericht, S. 9.

119 Bundesministerium des Innern und Bundesministerium der Justiz (Hg.) 2006. Zweiter Periodischer Sicherheitsbericht, S. 9.

$120 \mathrm{Vgl}$. Bundesministerium des Innern und Bundesministerium der Justiz (Hg.) 2006. Zweiter Periodischer Sicherheitsbericht, S. 10.

121 Bundesministerium des Innern und Bundesministerium der Justiz (Hg.) 2006. Zweiter Periodischer Sicherheitsbericht, S. 11.

$122 \mathrm{Vgl}$. Bundesministerium des Innern und Bundesministerium der Justiz (Hg.) 2006. Zweiter Periodischer Sicherheitsbericht, S. 11. 
Dennoch haben polizeilich und justiziell registrierte Straftaten eine Aussagekraft. Polizeidaten und Bundeszentralregisterdaten sind ein wichtiges Erkenntnismittel für die registrierte Kriminalität. ${ }^{123}$ Die Analysen, die in dieser Arbeit durchgeführt werden, beziehen sich auf offiziell registrierte Straftaten.

Abgesehen von diesen Besonderheiten der Registrierung von Kriminalität allgemein muss im Folgenden aber auch auf Besonderheiten der hier verwendeten Daten hingewiesen werden. Alle Personen, die jemals registriert waren, sind in den Daten enthalten. Dies ist in den offiziellen Daten nicht der Fall, dort werden regelmäßig Löschungen vorgenommen. In den Kohortendaten sind diese Personen und ihre Registrierungen aber weiterhin in den Daten. Problematisch ist nun herauszufinden, ob es sich bei einer „neuen“ Person auch wirklich um eine neu registrierte Person handelt oder ob es sich um eine gelöschte Person handelt, die wieder neu registriert worden ist. ${ }^{124}$ D.h. zwei Fehlerquellen sollten möglichst vermieden werden: 1. Eine Person wird als neue Person gewertet, obwohl sie schon erfasst ist. 2. Unterschiedliche Personen werden als identische Person erfasst.

Aus Datenschutzgründen enthält der Datensatz aber keinen Familien- oder Geburtsnamen. Personen werden anhand eines Keys zusammengeführt. Der Key wird aus Geburtsnamen und aus Geburtsdatum gebildet. Der Key ist nicht immer eindeutig. Es kommen gelegentlich unterschiedliche Personen mit identischen Keys vor. Dies ist etwas häufiger bei Nichtdeutschen der Fall, da es hier auch vorkommt, dass das exakte Geburtsdatum nicht bekannt ist und dafür der 1. Januar eingetragen wurde. Deshalb werden die Keys zusätzlich noch über Vorname und Geburtsort abgeglichen. Dieser Abgleich erfolgt maschinell. Bei unterschiedlichen Schreibweisen des Vornamens oder des Geburtsortes kann es passieren, dass das Programm nicht erkennen kann, ob Personen identisch sind oder nicht. Diese Entscheidungen müssen ,,von Hand“ getroffen werden.

Besondere Schwierigkeiten ergeben sich bei der Aufbereitung der Delikte in den BZR-Daten. Im Paragrafenfeld (siehe Abschnitt 3.1.3) sind alle angewendeten Vorschriften einer Entscheidung aufgeführt. Es ist aber nicht immer eindeutig, ob es sich um mehrere Straftaten handelt oder ob es sich um eine Straftat handelt, die durch mehrere Paragrafen beschrieben wird. Ein Beispiel dafür ist, dass bei der Registrierung eines schweren Diebstahls (§ 243 StGB) immer auch Diebstahl ( 242 StGB) eingetragen wird. Hier ist nicht ersichtlich, ob es sich nur um einen schweren Diebstahl oder um eine beliebige Zahl von schweren und einfachen Diebstählen handelt. Zweideutigkeiten ergeben sich auch bei § 248a StGB (Diebstahl und Unterschlagung geringwertiger Sachen). Normalerweise ist dieser Paragraf in Kombination mit $\S 242$ (Diebstahl), $§ 246$ (Unterschlagung) oder $\S 265 \mathrm{a}$

123 Vgl. Bundesministerium des Innern und Bundesministerium der Justiz (Hg.) 2006. Zweiter Periodischer Sicherheitsbericht, S. 11.

124 In den BZR Daten haben die Personen eine Personennummer. Wird eine Person gelöscht und wird später wieder registriert, erhält sie eine neue Personennummer. 
(Erschleichen von Leistungen) eingetragen und bezeichnet keine eigene Straftat. Es gibt aber auch Fälle in denen nur $\S 248$ a eingetragen ist; hier muss dieser Paragraf als Diebstahl gezählt werden. Grundsätzlich kann $§ 248$ a aber nicht als Diebstahl gezählt werden, denn oft wird $\S 248 \mathrm{a}$ gleichzeitig mit $\S 265 \mathrm{a}$ (Leistungserschleichung) eingetragen, ist dann aber kein Diebstahl.

Probleme ergeben sich auch durch die im Laufe der Untersuchung durchgeführten Gesetzesänderungen. Hier insbesondere, wenn ein vorhandener Paragraf nach der Änderung eine andere inhaltliche Bedeutung erhält. In einer Übergangszeit nach der Änderung sind beide Bedeutungen eines Paragrafen möglich. Hierzu ein Beispiel: Bevor die Änderungen des 6. Strafrechtsreformgesetzes ${ }^{125}$ in Kraft traten, war $\S 230$ StGB die fahrlässige Körperverletzung. Nach der Änderung normiert $\S 230 \mathrm{StGB}$ nunmehr den Strafantrag bei Körperverletzung. Bei maschineller Bearbeitung ist nicht zu erkennen, ob es sich um eine Körperverletzung und eine fahrlässige Körperverletzung handelt oder ob es sich alleine um Körperverletzung handelt. In diesen Fällen wurde für die Zeit nach dem 1.4.1998 davon ausgegangen, dass hier eine Körperverletzung registriert ist.

Ein weiteres Problem bereiten unterschiedliche Schreibweisen von Gesetzen und Vorschriften. ${ }^{126}$ Diese Fälle müssen, wenn sie auffallen, nachbearbeitet werden.

Trotz der erwähnten Einschränkungen und Schwierigkeiten bietet das Datenmaterial aber eine gute Basis für die Analyse von Deliktsähnlichkeiten.

125 Am 1.4.1998 ist das sechste Gesetz zur Reform des Strafrechts in Kraft getreten. Seitdem ist $\S 229$ fahrlässige Körperverletzung. Vor dem 1.4.1998 war die Bedeutung von $\S 229$ Vergiftung.

126 Z.B. gibt es zahlreiche Varianten der Schreibweise des Pflichtversicherungsgesetzes. 


\section{$4 \quad$ Multidimensionale Skalierung}

\subsection{Was ist Multidimensionale Skalierung}

Multidimensionale Skalierung (MDS) ${ }^{127}$ ist die Bezeichnung für eine Gruppe von Methoden, die Ähnlichkeiten bzw. Unähnlichkeiten zwischen Daten räumlich repräsentieren. ${ }^{128}$ Ziel der multidimensionalen Skalierung ist die grafische Darstellung von Objekten im mehrdimensionalen Raum, wobei aus Anschaulichkeitsgründen möglichst wenige Dimensionen verwendet werden.

Das Ziel ist, Zusammenhänge zwischen Delikten aufzuzeigen. Wenn es gelingt, ein Ähnlichkeitsmaß für Deliktspaare zu bestimmen, ist Multidimensionale Skalierung eine viel versprechende Methode, um Konfigurationen von Delikten darzustellen.

MDS basiert auf dem paarweisen Vergleich der Ähnlichkeiten der einzelnen Merkmalsträger zueinander derart, dass die Ähnlichkeiten in numerische Distanzen umgerechnet werden: 129

- geringe Unterschiede / Unähnlichkeiten $\quad \Rightarrow \quad$ kleine Distanz

- große Unterschiede / Unähnlichkeiten $\quad \Rightarrow \quad$ große Distanz

Ein Ähnlichkeitsmaß ist z.B. der Abstand zwischen zwei Punkten. Ähnlichkeitsmaße gibt es in verschiedenster Form.

Weitere Beispiele von Ähnlichkeitsmaßen sind:

- Entfernung von Städtepaaren in Kilometer

- Ähnlichkeitseinschätzungen von Objektpaaren mit einer Skala 1...n

- multiple Rangordnungen

- Korrelationen

- Entscheidungs- und Reaktionszeiten

- usw.

Das Ziel ist, die Objekte so in einem x-dimensionalen Raum zu positionieren, dass sie optimal verteilt sind, d.h. dass die Distanzen zwischen den Objekten möglichst gut eingehalten werden. Den Objekten werden durch ein iteratives Verfahren Koordinatenpaare im Raum zugewiesen, und die sich ergebenden Distanzen wer-

127 Einen Überblick über MDS geben die Schriften von Coxon 1982, Ahrens 1974, Borg \& Groenen 1997, Kruskal \& Wish 1978 und das Kapitel über MDS in Backhaus, Erichson, Plinke \& Weiber 2003, S. 605-671.

128 Vgl. Coxon 1982, S. 1.

129 Vgl. Ahrens 1974, S. 73. 
den mit einem Algorithmus so lange mit den empirisch ermittelten Abständen verglichen, bis die Positionierung optimal ist. ${ }^{130}$

\subsection{Ein Beispiel}

Das Konzept der MDS lässt sich sehr gut anhand eines Beispiels verdeutlichen, bei dem das Ergebnis der Analyse schon bekannt ist. ${ }^{131}$ Die verfügbaren Informationen sind die paarweisen Distanzen von 10 Städten. In Tabelle 12 sind die Entfernungsangaben (Luftlinie) von 10 Städten untereinander in Kilometern angegeben. ${ }^{132}$

Tabelle 12: Entfernung zwischen 10 Städten in km (Luftlinie)

\begin{tabular}{l|r|r|r|r|r|r|r|r|r}
\hline & $\begin{array}{r}\text { Lon- } \\
\text { don }\end{array}$ & $\begin{array}{r}\text { Stock- } \\
\text { holm }\end{array}$ & $\begin{array}{r}\text { Lissa- } \\
\text { bon }\end{array}$ & Madrid & Paris & $\begin{array}{r}\text { Am- } \\
\text { sterd. }\end{array}$ & Berlin & Prag & Rom \\
\hline London & & & & & & & & & \\
\hline Stockh. & 1418 & & & & & & & & \\
\hline Lissab. & 1593 & 2957 & & & & & & & \\
\hline Madrid & 1256 & 2579 & 479 & & & & & & \\
\hline Paris & 351 & 1539 & 1431 & 1053 & & & & & \\
\hline Amster. & 365 & 1121 & 1836 & 1485 & 432 & & & & \\
\hline Berlin & 932 & 810 & 2282 & 1870 & 879 & 587 & & & \\
\hline Prag & 1033 & 1040 & 2221 & 1775 & 878 & 716 & 270 & & \\
\hline Rom & 1445 & 1944 & 1863 & 1384 & 1100 & 1289 & 1161 & 911 & \\
\hline Dublin & 459 & 1620 & 1640 & 1472 & 790 & 749 & 1323 & 1458 & 1890 \\
\hline
\end{tabular}

Mit Hilfe der MDS wird nun aus den vorhandenen paarweisen Distanzen die relative Lage aller Orte zueinander, d.h. die Konfiguration der 10 Städte ermittelt. ${ }^{133}$ Das Ergebnis ist die Skizze einer Landkarte, das die Lage der 10 Städte abbildet. Die Koordinaten für die grafische Darstellung zeigt Tabelle 13.

130 Vgl. Backhaus, Erichson, Plinke \& Weiber 2003, S. 621 ff.

131 Vgl. Backhaus, Erichson, Plinke \& Weiber 2003, S. 606 ff. Hier wird anschaulich erklärt, wie das Ergebnis einer Multidimensionalen Skalierung erzeugt wird.

132 Gemessen wurde die Entfernung in einem Atlas.

133 Die MDS wird hier mit der Prozedur PROXSCAL aus SPSS berechnet. 
Tabelle 13: Koordinaten

\begin{tabular}{l|cc}
\hline & Dimension 1 & Dimension 2 \\
\hline London & 0,06 & 0,15 \\
Stockholm & $-0,82$ & 0,50 \\
Lissabon & 1,03 & 0,27 \\
Madrid & 0,81 & $-0,16$ \\
Paris & 0,06 & $-0,02$ \\
Amsterdam & $-0,18$ & 0,01 \\
Berlin & $-0,60$ & $-0,17$ \\
Prag & $-0,53$ & $-0,29$ \\
Rom & 0,06 & $-0,82$ \\
Dublin & 0,10 & 0,52 \\
\hline
\end{tabular}

Abbildung 5 zeigt das Ergebnis der MDS. Auf den ersten Blick ist die gewohnte Landkarte nicht zu erkennen. Aber zu sehen ist z.B., dass Berlin und Prag nah beieinander liegen und Stockholm weit von Rom entfernt ist. Dies entspricht den Entfernungsangaben. Bei der Darstellung in Abbildung 5 stimmt die Himmelsrichtung der Städte nicht, aber die Entfernungen entsprechen den Angaben aus Tabelle 12. Durch Rotation und Spiegelung an der y-Achse erhält man die Darstellung in Abbildung 6. Hier erkennt man die gewohnte Landkarte. Die Lösungen in Abbildung 5 und 6 sind von ihrer Aussage her identisch.

Abbildung 5: Durch MDS gewonnene Konfiguration von 10 Städten (vor Rotation und Spiegelung)

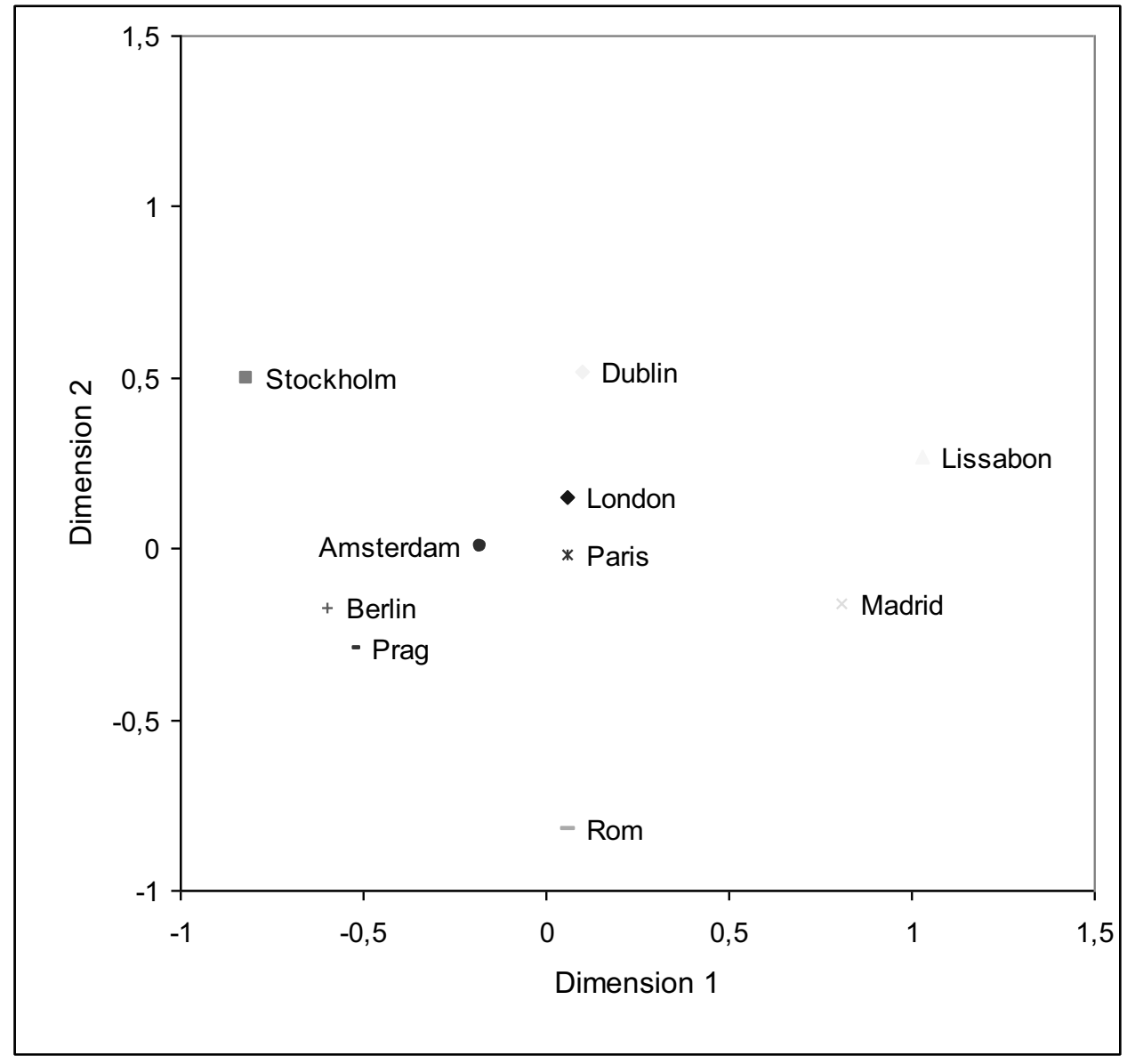


Für den Aussagegehalt der MDS ist es nicht von Bedeutung, ob ein Punkt oben, unten, links oder rechts liegt, sondern nur, wie dicht beieinander die Punkte liegen. Je näher die Objekte beieinander liegen, desto ähnlicher sind die Objekte. Das Ergebnis wird durch Spiegelung und Drehung nicht verändert. 134

\section{Abbildung 6: $\quad$ Konfiguration der Städte nach Rotation und Spiegelung}

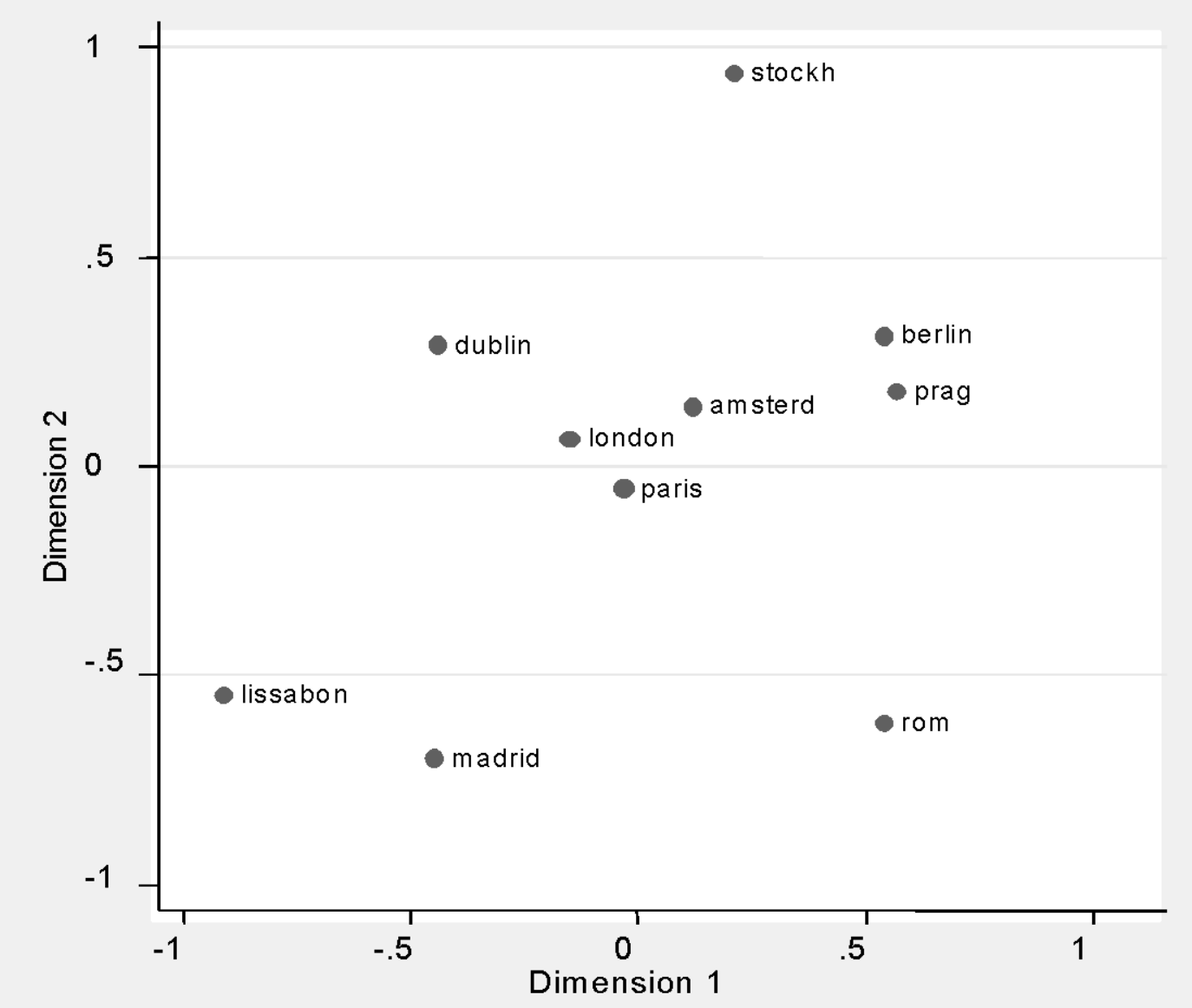

„Das Verfahren der MDS lässt sich wie folgt umreißen: Aus vorgegebenen Ähnlichkeiten bzw. Unähnlichkeiten $\mathrm{u}_{\mathrm{kl}}$ (für Objekte $\mathrm{k}$ und 1 ) ist in einem Raum mit möglichst geringer Dimensionalität eine Konfiguration zu ermitteln, deren Distanzen $\mathrm{d}_{\mathrm{kl}}$ möglichst gut die folgende Monotoniebedingung erfüllen sollten:

Wenn $\mathrm{u}_{\mathrm{kl}}>\mathrm{u}_{\mathrm{ij}}$, dann $\mathrm{d}_{\mathrm{kl}}>\mathrm{d}_{\mathrm{ij}}{ }{ }{ }^{135}$

D.h. wenn die Unähnlichkeit von zwei Objekten größer ist als die Unähnlichkeit von zwei anderen Objekten, dann soll auch die Distanz größer sein.

134 Bei der MDS geht es darum, die relative Position der Objekte zueinander adäquat abzubilden. Die Entfernung der Objekte zueinander ist von Bedeutung. Die Objektkonfiguration ist unabhängig von Spiegelung und Drehung der Objekte, vgl. Backhaus, Erichson, Plinke \& Weiber 2003, S. $608 \mathrm{f}$.

135 Backhaus, Erichson, Plinke \& Weiber 2003, S. 621. 
„In der gesuchten Konfiguration sollte also die Rangfolge der Distanzen zwischen den Objekten möglichst gut die Rangfolge der vorgegebenen Unähnlichkeiten wiedergeben. Eine perfekte Erfüllung der Monotoniebedingung ist i.d.R. nicht möglich.“136

MDS kann mit verschiedenen Dimensionen durchgeführt werden. Aus praktischen Erwägungen beschränkt man sich normalerweise auf zwei oder drei Dimensionen, „um eine grafische Darstellung der Ergebnisse zu ermöglichen und so die inhaltliche Interpretation zu erleichtern." 137 Hier wird die Analyse mit zweidimensionaler MDS durchgeführt, da hier die Ergebnisse am besten grafisch darstellbar sind.

Im Städtebeispiel ist schon die Ausgangsähnlichkeit bzw. -unähnlichkeit der Objekte eine Distanz. Dies ist nicht notwendig. Es gibt auch ganz andere Ähnlichkeitsmaße, z.B. direkte Ähnlichkeitseinschätzung von Objektpaaren oder Reaktionszeiten. 138

\subsection{Beurteilungskriterien}

Um zu beurteilen, wie gut das Ergebnis ist, das durch die MDS erstellt wurde, werden üblicherweise zwei Kriterien benutzt. Zum einen wird STRESS als Gütekriterium benutzt und zum anderen $\mathrm{R}^{2}$. STRESS ist ein methodenspezifisches Kriterium. $\mathrm{R}^{2}$ ist ein Beurteilungskriterium, welches auch bei vielen anderen Methoden benutzt wird. Beide Beurteilungskriterien können getrennt voneinander verwendet werden.

\subsubsection{STRESS}

STRESS ist ein Maß für die Güte der Lösung.

"Das STRESS-Maß misst, wie gut (genauer gesagt, wie schlecht) eine Konfiguration die Monotoniebedingung erfüllt. Je größer STRESS ausfällt, desto schlechter ist die Anpassung der Distanzen an die Ähnlichkeiten (badness of fit)." "139

STRESS beschreibt die normierte Summe der Differenzen des empirischen ${ }^{140}$ und des errechneten Abstandes zwischen den Objekten, im Beispiel zwischen den

136 Backhaus, Erichson, Plinke \& Weiber 2003, S. 621.

137 Backhaus, Erichson, Plinke \& Weiber 2003, S. 632.

138 Rangwerte, ordinalskalierte Daten, sind bereits ausreichend, um eine Konfiguration mit MDS zu erstellen. Dies bezieht sich nur auf die Input-Daten, die Ergebnisse sind immer metrisch, siehe Backhaus, Erichson, Plinke \& Weiber 2003, S. 610 f.

139 Backhaus, Erichson, Plinke \& Weiber 2003, S. 626. Die Monotoniebedingung ist auf Seite 40 angegeben.

140 Der empirische Abstand zwischen zwei Objekten wird Disparität genannt, siehe Backhaus, Erichson, Plinke \& Weiber 2003, S. 624. 
Städten. Es existieren unterschiedliche Varianten, um das STRESS-Maß auf Werte zwischen 0 und $1 \mathrm{zu}$ normieren. Je kleiner STRESS ist, d.h. je näher bei 0, desto besser ist die Lösung. ${ }^{141}$ Bei einer guten Lösung ist das STRESS-Maß kleiner 0,2. ${ }^{142}$ Beim Städtebeispiel kommt es zu einer vollkommenen Lösung mit STRESS 0,001. Bei diesem optimalen Ergebnis sind empirischer und errechneter Abstand gleich.

\subsection{2 $R^{2}$}

$\mathrm{R}^{2}$ ist die quadrierte Korrelation zwischen dem empirischen und dem errechneten Abstand zwischen den Objekten, hier die Abstände zwischen den Städten in der Grafik. $\mathrm{R}^{2}$ kann interpretiert werden als das Maß, das angibt, wie viel der Varianz erklärt wird. Im Städtebeispiel ist $\mathrm{R}^{2}=1$, was bedeutet, dass $100 \%$ der Varianz erklärt werden. Das Ergebnis ist also sehr gut. Das hängt damit zusammen, dass die Entfernungen aus einem Atlas und damit schon aus einer 2-dimensionalen Darstellung entnommen wurden. Wäre die Entfernungen an einem Globus gemessen worden, wäre das Ergebnis nicht mit 100\%iger Varianzerklärung zustande gekommen. Die Kartografen haben hier die Fehlerkorrektur schon übernommen. ${ }^{143}$

${ }^{141}$ Das im Weiteren benutzte STRESS-Maß ist STRESS-I aus der SPSS Prozedur PROXSCAL. Weitere Informationen zu diesem STRESS-Maß siehe SPSS 14.0 Statistical Algorithms, PROXSCAL.pdf, S. 11. Allgemeine Informationen zum STRESS-Maß siehe Backhaus, Erichson, Plinke \& Weiber 2003, S. 626.

142 Vgl. Backhaus, Erichson, Plinke \& Weiber 2003, S. 630.

143 Das Ergebnis der MDS im Beispiel mit Städteentfernungen ist auch deshalb sehr gut, weil das Ähnlichkeitsmaß schon eine Distanz ist. 


\section{Deliktsähnlichkeiten}

In diesem Abschnitt werden die Analyse der Daten bezüglich der Ähnlichkeit von Delikten dargestellt und die Ergebnisse präsentiert.

Wie ist die in Abschnitt 4 vorgestellte Methode bei Delikten anzuwenden?

Das Ziel ist, von paarweisen Ähnlichkeiten von Delikten auf Deliktskonfigurationen zu schließen, was mit Multidimensionaler Skalierung möglich ist. Dazu muss zuerst ein Ähnlichkeitsmaß für Delikte festgelegt werden. Dies wird in Abschnitt 5.1 beschrieben.

Die grafische Darstellung der Multidimensionalen Skalierung soll Deliktskonfigurationen aufzeigen. Die Analyse soll folgende Fragen beantworten:

- Gibt es Deliktskombinationen, die sehr häufig vorkommen (ähnliche Delikte)?

- Gibt es Deliktskombinationen, die fast nie vorkommen (unähnliche Delikte)?

- $\quad$ Sind Schlüsse möglich wie: Eine Person, die Delikt A begangen hat, begeht mit hoher Wahrscheinlichkeit auch Delikt B? Bzw. umgekehrt: Hat eine Person, die Delikt $\mathrm{C}$ begangen hat, schon mit hoher Wahrscheinlichkeit Delikt D begangen?

- Gibt es in unterschiedlichen Altersstufen, auch unterschiedliche Deliktskonfigurationen?

- Verändert sich das Ergebnis, wenn bei der Auswertung die Dauer von einer Straftat zur nächsten berücksichtigt wird?

Die Auswertung wird für Personen mit polizeilichen Registrierungen und für Personen mit justiziellen Registrierungen durchgeführt. Die Personen sind in die Subpopulationen deutsche Männer, deutsche Frauen, nichtdeutsche Männer und nichtdeutsche Frauen unterteilt.

\subsection{Ein Ähnlichkeitsmaß für Delikte}

Das Ziel ist, Delikte nicht nur paarweise zu vergleichen, sondern eine Deliktskonfiguration zu erhalten. Eine Möglichkeit dies zu erreichen, ist die Methode Multidimensionale Skalierung. Dafür wird ein Ähnlichkeitsmaß für die einzelnen Objektpaare benötigt. Entsprechend diesen Ähnlichkeiten werden die Distanzen der Objekte (Delikte) festgelegt. Im Städtebeispiel waren direkt die Kilometerentfernungen von einer Stadt zur anderen und damit die Distanzen angegeben.

Um zu einem Ähnlichkeitsmaß bei Delikten zu kommen, werden zunächst die Anzahl der Deliktskombinationen betrachtet (siehe Tabelle 15). Die Häufigkeiten zeigen, welche Deliktspaare häufig innerhalb von kriminellen Karrieren auftreten und welche selten. Im Unterschied zu Transitionsmatrizen, die jeweils eine Straftat mit der zeitlich direkt folgenden erfassen, werden hier alle Deliktskombinationen einer kriminellen Karriere ge- 
zählt. Die Chronologie des Auftretens der Straftaten spielt hierbei keine Rolle, von Bedeutung ist alleine die Deliktskombination. Alle Delikte einer Person werden erfasst. Damit können insbesondere Deliktszusammenhänge von Mehrfachstraftätern erfasst werden. ${ }^{144}$ Als Ähnlichkeitsmaß kann die Häufigkeit der Kombinationen aber nicht direkt verwendet werden, da die Häufigkeiten immer dann groß sind, wenn ein Delikt oft registriert ist. Die Häufigkeiten müssen normiert werden. Dafür eignet sich das Adjusted Standardized Residual ${ }^{145}$ (ASR) von Deliktspaaren (Tabelle 16). ${ }^{146}$ Das ASR besagt, ob unter der Annahme einer zufälligen Verteilung mehr oder weniger Ereignisse als erwartet eintreten.

Ein positives Residuum besagt, dass das Deliktspaar häufiger vorkommt als erwartet, bei einem negativen Residuum ist die Häufigkeit des Deliktspaars seltener als erwartet. Zwei Delikte sind sich ähnlich, wenn sie häufiger vorkommen als erwartet, unähnlich, wenn sie seltener vorkommen als erwartet. Ein positives ASR besagt, dass diese Deliktskombinationen ähnlich sind, ein negatives ASR besagt, dass diese Deliktskombinationen unähnlich sind. Je größer der Wert des ASR, des-

144 Werden nur die zeitlich aufeinanderfolgenden Straftaten betrachtet, sind bei schweren Straftaten Kombinationen mit leichten Straftaten häufig, da innerhalb von kriminellen Karrieren vor der nächsten schweren Straftat häufig auch leichte Straftaten auftreten. Zusammenhänge zwischen schweren Straftaten lassen sich so schlecht zeigen.

145 Die Formel zur Berechnung des Adjusted Standardized Residual (korrigierten standardisierten Residuums) lautet:

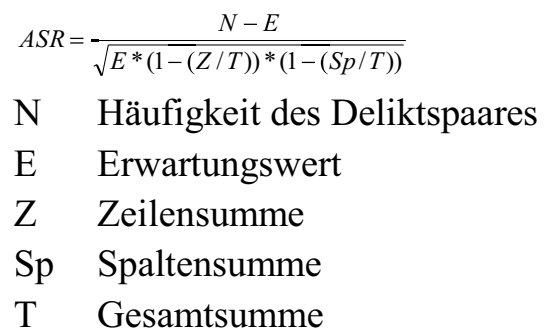

Der Erwartungswert ist der Wert, der sich unter der Annahme einer zufälligen Verteilung ergibt. Berechnet wird der Erwartungswert bei einer $\mathrm{n} \times \mathrm{m}$ Matrix (hier Tabelle) mit: Zeilensumme * Spaltensumme / Gesamtsumme der Beobachtungen. Hier ist die Matrix eine Dreiecksmatrix. Um den Erwartungswert und um das korrigierte standardisierte Residuum zu berechnen, wird die Dreiecksmatrix symmetrisiert, d.h. links und rechts der Diagonale werden die jeweiligen $\mathrm{N} / 2$ eingetragen $\left(\mathrm{N}_{\mathrm{ij}}=\mathrm{N}_{\mathrm{ji}}\right)$. Es ist nicht von Bedeutung, ob Delikt $\mathrm{i}$ oder Delikt $\mathrm{j}$ zuerst vorkam, das Deliktspaar $\mathrm{i}-\mathrm{j}$ ist identisch mit dem Deliktspaar j - i. Das Adjusted Standardized Residual beinhaltet das Residuum einer Zelle dividiert durch eine Schätzung des Standardfehlers der erwarteten Zellhäufigkeit. (Vgl. SPSS Base 14.0 User's Guide, S. 346)

Blumstein, Cohen, Das \& Moitra 1988 und Rojek \& Erickson 1982, S. 15 f. führten mit Hilfe des ASR Untersuchungen zur Deliktsspezialisierung durch.

146 Die Berechnung der Deliktsähnlichkeiten wurde auch mit anders konstruierten Ähnlichkeitsmaßen (z.B. E/N und standardisiertes Residuum) durchgeführt. Dabei stellte sich das ASR als das am besten geeignete Ähnlichkeitsmaß heraus. Wie schon in einer Fußnote im Abschnitt 4.2 im Beispiel zur MDS erklärt, sind ordinalskalierte Daten als Input-Daten ausreichend. Die Annahme, dass die Werte des ASR ordinalskaliert seien, ändert nichts am Ergebnis. 
to ähnlicher das Deliktspaar und umgekehrt je kleiner der Wert des ASR (großer negativer Wert), desto unähnlicher. Beim ASR handelt es sich somit um ein Similaritätsmaß.

\subsection{Ergebnisse auf Polizeiebene}

Für die Auswertung von Deliktsähnlichkeiten auf Personenebene sind nur Personen relevant, bei denen zwei oder mehr Straftaten registriert sind, da die Kombinationen von Straftaten ausgewertet werden. Die Delikte sind in 25 Kategorien unterteilt. In Tabelle 14 sind die Häufigkeiten der einzelnen Deliktskategorien getrennt nach Geschlecht und Staatsangehörigkeit aufgeführt von Tatverdächtigen mit zwei oder mehr Straftaten.

Der einfache Diebstahl ist in allen Subpopulationen (deutsche Männer, nichtdeutsche Männer, deutsche Frauen, nichtdeutsche Frauen) am häufigsten vertreten. Bei den deutschen Männern mit zwei oder mehr Registrierungen sind über $25 \%$ der Registrierungen einfacher Diebstahl, bei den nichtdeutschen Männern 24\% und sogar 28\%, wenn Vergehen gegen Ausländergesetze nicht berücksichtigt werden. Bei den Frauen ist dieser Anteil noch deutlich höher, fast 40\%; und wenn bei den nichtdeutschen Frauen die Vergehen gegen Ausländergesetze nicht berücksichtigt werden, so beträgt der Anteil sogar fast 50\%. Die zweitgrößte Deliktskategorie der Männer ist schwerer Diebstahl, die der Frauen Betrug. Eine weitere große Kategorie bilden die Vergehen gegen Betäubungsmitteldelikte. Der Anteil liegt bei über 10\%, nur bei den nichtdeutschen Frauen liegt der Anteil unter 5\%.

Bei den deutschen Männern mit zwei oder mehr Registrierungen sind 14\% Gewaltdelikte $^{147}$, bei den nichtdeutschen Männern ohne Berücksichtigung der Ausländervergehen sind 18\% Gewaltdelikte, bei den Frauen 9\%. Eine große Deliktskategorie ist bei den Nichtdeutschen Vergehen gegen Ausländergesetze mit 16\%.

Bei Einfachregistrierten ist der Anteil von einfachem Diebstahl höher, dafür gibt es seltener Registrierungen von schwerem Diebstahl, Betäubungsmitteldelikten und Gewaltdelikten.

147 Tötungsdelikte, Sexualdelikte, sonstige Sexualdelikte, Raub, schwere Körperverletzung, Körperverletzung, Straftaten gegen die persönliche Freiheit. 
Tabelle 14: Häufigkeitsverteilung der Deliktskategorien bei Tatverdächtigen mit 2 oder mehr Straftaten (Registrierungen bis 2001)

\begin{tabular}{|c|c|c|c|c|c|c|c|c|}
\hline & \multicolumn{4}{|c|}{ männl. } & \multicolumn{4}{|c|}{ weibl. } \\
\hline & deut. & $\%$ & nichtdt. & $\%$ & deut. & $\%$ & nichtdt. & $\%$ \\
\hline Tötungsdelikte & 213 & 0,1 & 275 & 0,1 & 43 & 0,1 & 11 & 0,1 \\
\hline Sexualdelikte & 789 & 0,3 & 903 & 0,4 & 4 & 0,0 & 1 & 0,0 \\
\hline sonst. Sexualdelikte & 1.336 & 0,6 & 723 & 0,4 & 496 & 1,3 & 164 & 0,8 \\
\hline Raub & 4.224 & 1,8 & 4.598 & 2,3 & 334 & 0,9 & 169 & 0,8 \\
\hline schw. Körperverl. & 10.500 & 4,5 & 9.440 & 4,6 & 972 & 2,6 & 361 & 1,8 \\
\hline Körperverletzung & 10.712 & 4,6 & 8.596 & 4,2 & 1.192 & 3,2 & 603 & 3,0 \\
\hline $\begin{array}{l}\text { Str. gg. pers. Frei- } \\
\text { heit }\end{array}$ & 5.317 & 2,3 & 5.410 & 2,7 & 461 & 1,2 & 243 & 1,2 \\
\hline Einfacher Diebstahl & 59.490 & 25,4 & 47.958 & 23,6 & 13.896 & 37,5 & 7.948 & 39,9 \\
\hline schwerer Diebstahl & 43.471 & 18,6 & 28.972 & 14,2 & 1.724 & 4,6 & 1.014 & 5,1 \\
\hline Sachbeschädigung & 15.778 & 6,7 & 5.673 & 2,8 & 934 & 2,5 & 228 & 1,1 \\
\hline $\begin{array}{l}\text { gemeingef. Strafta- } \\
\text { ten }\end{array}$ & 1.385 & 0,6 & 469 & 0,2 & 106 & 0,3 & 29 & 0,1 \\
\hline Hehlerei & 3.933 & 1,7 & 3.396 & 1,7 & 632 & 1,7 & 256 & 1,3 \\
\hline Betrug & 16.791 & 7,2 & 10.838 & 5,3 & 5.336 & 14,4 & 1.891 & 9,5 \\
\hline Leistungserschl. & 9.494 & 4,1 & 8.244 & 4,1 & 2.765 & 7,5 & 1.065 & 5,3 \\
\hline $\begin{array}{l}\text { Gef. dem. Rechts- } \\
\text { staat }\end{array}$ & 1.066 & 0,5 & 108 & 0,1 & 49 & 0,1 & 11 & 0,1 \\
\hline $\begin{array}{l}\text { Wid. gg. Staatsge- } \\
\text { walt }\end{array}$ & 1.702 & 0,7 & 1.228 & 0,6 & 150 & 0,4 & 56 & 0,3 \\
\hline $\begin{array}{l}\text { Öffentliche Ord- } \\
\text { nung }\end{array}$ & 2.857 & 1,2 & 1.310 & 0,6 & 520 & 1,4 & 173 & 0,9 \\
\hline Hausfriedensbruch & 3.220 & 1,4 & 2.569 & 1,3 & 496 & 1,3 & 215 & 1,1 \\
\hline Beleidigung & 5.779 & 2,5 & 3.796 & 1,9 & 1.150 & 3,1 & 486 & 2,4 \\
\hline Fälschung & 4.191 & 1,8 & 6.437 & 3,2 & 647 & 1,7 & 751 & 3,8 \\
\hline Meineid & 635 & 0,3 & 464 & 0,2 & 185 & 0,5 & 81 & 0,4 \\
\hline $\begin{array}{l}\text { falsche Verdächti- } \\
\text { gung }\end{array}$ & 640 & 0,3 & 374 & 0,2 & 290 & 0,8 & 107 & 0,5 \\
\hline BTM-Delikte & 29.942 & 12,8 & 17.942 & 8,8 & 4.515 & 12,2 & 786 & 3,9 \\
\hline $\begin{array}{l}\text { gef. Eingr. } \\
\text { Str.Verkehr }\end{array}$ & 351 & 0,1 & 77 & 0,0 & 13 & 0,0 & 4 & 0,0 \\
\hline AuslG & 229 & 0,1 & 33.625 & 16,5 & 185 & 0,5 & 3.274 & 16,4 \\
\hline Gesamt & 234.045 & 100,0 & 203.425 & 100,0 & 37.095 & 100 & 19.927 & 100 \\
\hline
\end{tabular}

\subsubsection{Deutsche Männer}

Während in Tabelle 14 die Häufigkeiten der Deliktskategorien dargestellt wurden, werden nun in Tabelle 15 die Deliktskombinationen betrachtet. Die Delikte, zunächst von tatverdächtigen deutschen Männern, werden paarweise gezählt. In der Tabelle werden die Häufigkeiten von Deliktspaaren aus den oben beschriebenen 24 
Deliktskategorien ${ }^{148}$ dargestellt. Die Tabelle zeigt nicht die Anzahl der Personen, bei denen diese Deliktspaare vorkommen, sondern die Anzahl der Deliktspaare, d.h. bei einer Person kann ein bestimmtes Deliktspaar mehrmals vorkommen. Der Zusammenhang zwischen der Häufigkeit der Delikte und der Anzahl der Deliktspaare wurde im Abschnitt 3.2.3 erläutert.

Insgesamt gibt es bei den deutschen Männern 2.400.000 Deliktspaare. Das häufigste Paar ist schwerer Diebstahl - schwerer Diebstahl. Es tritt 365.000-mal auf. Am zweithäufigsten ist einfacher Diebstahl - schwerer Diebstahl (311.000), erst danach kommt einfacher Diebstahl - einfacher Diebstahl (212.000) und das, obwohl einfacher Diebstahl das häufigste Delikt ist (siehe Tabelle 14). Betrachtet man die Summe aller Kombinationen mit schwerem Diebstahl und vergleicht diese mit der Summe aller Kombinationen mit einfachem Diebstahl, so zeigt sich, dass es mehr Kombinationen mit schwerem Diebstahl gibt. Die Häufigkeiten der Delikte in Deliktspaaren sind andere als die Häufigkeiten der Registrierungen. Das häufige Auftreten von schwerem Diebstahl in Deliktspaaren ist ein Hinweis darauf, dass das Delikt schwerer Diebstahl besonders häufig ein Delikt von Mehrfachtatverdächtigen ist.

Tabelle 15: Anzahl der Deliktspaare (deutsche Männer)

\begin{tabular}{|c|c|c|c|c|c|c|c|c|}
\hline & $\begin{array}{l}\text { Tötungsde- } \\
\text { likte }\end{array}$ & $\begin{array}{l}\text { Sexual- } \\
\text { delikte }\end{array}$ & sonst. Sex. & Raub & schw. KV & $\mathrm{KV}$ & $\begin{array}{l}\text { Str. gg. } \\
\text { Pers. Fr. }\end{array}$ & einf. Dieb. \\
\hline Tötungsdelikte & 36 & & & & & & & \\
\hline Sexualdelikte & 23 & 431 & & & & & & \\
\hline sonst. Sexualdel. & 7 & 549 & 1.347 & & & & & \\
\hline Raub & 128 & 378 & 270 & 3.227 & & & & \\
\hline schw. KV & 249 & 693 & 660 & 4.192 & 6.882 & & & \\
\hline Körperverletzung & 187 & 664 & 724 & 3.818 & 10.985 & 6.889 & & \\
\hline Str. gg. Pers. Fr. & 101 & 430 & 406 & 2.368 & 5.359 & 6.061 & 2.106 & \\
\hline einf. Diebstahl & 683 & 2.491 & 2.807 & 21.788 & 29.314 & 31.722 & 17.338 & 211.955 \\
\hline schw. Diebstahl & 695 & 2.055 & 2.186 & 22.681 & 25.827 & 27.149 & 15.063 & 311.035 \\
\hline Sachbeschäd. & 259 & 590 & 664 & 4.309 & 10.552 & 10.085 & 5.490 & 51.015 \\
\hline gemeingef. Str. & 89 & 38 & 65 & 340 & 533 & 571 & 374 & 4.807 \\
\hline Hehlerei & 38 & 142 & 148 & 1.201 & 2.125 & 1.998 & 1.110 & 16.259 \\
\hline Betrug & 214 & 705 & 884 & 4.378 & 5.750 & 7.300 & 5.022 & 68.370 \\
\hline Erschl. v. Leist. & 130 & 314 & 440 & 3.228 & 5.056 & 4.994 & 2.857 & 45.717 \\
\hline Gef. dem. Recht. & 36 & 14 & 59 & 207 & 1.194 & 675 & 519 & 1.786 \\
\hline Wid. gg. Staat. & 37 & 93 & 77 & 698 & 2.080 & 2.200 & 991 & 6.515 \\
\hline öff. Ordnung & 58 & 79 & 166 & 832 & 2.183 & 1.701 & 1.151 & 8.145 \\
\hline Hausfriedensbr. & 35 & 220 & 219 & 1.225 & 2.718 & 3.011 & 1.592 & 17.654 \\
\hline Beleidigung & 74 & 451 & 684 & 1.790 & 5.353 & 6.691 & 4.064 & 17.336 \\
\hline Fälschung & 41 & 232 & 297 & 1.200 & 1.753 & 2.103 & 1.396 & 22.400 \\
\hline Meineid & 7 & 11 & 22 & 176 & 368 & 334 & 185 & 1.877 \\
\hline falsche Verd. & 8 & 40 & 56 & 230 & 351 & 447 & 309 & 2.638 \\
\hline BTM-Delikte & 323 & 948 & 1.026 & 10.273 & 13.703 & 14.034 & 6.951 & 116.132 \\
\hline gef. Eingr. Str.V. & 5 & 4 & 4 & 26 & 112 & 107 & 59 & 454 \\
\hline
\end{tabular}

148 Die Deliktskategorie AuslG / AsylVfG wird bei Deutschen nicht berücksichtigt. 


\begin{tabular}{|c|c|c|c|c|c|c|c|c|}
\hline & $\begin{array}{l}\text { schw. } \\
\text { Dieb. }\end{array}$ & Sachb. & $\begin{array}{l}\text { gemein- } \\
\text { gef. Str. }\end{array}$ & Hehlerei & Betrug & $\begin{array}{l}\text { Erschl. v. } \\
\text { Leist. }\end{array}$ & $\begin{array}{l}\text { Gef. dem. } \\
\text { Recht. }\end{array}$ & $\begin{array}{l}\text { Wid. gg. } \\
\text { Staat. }\end{array}$ \\
\hline schw. Diebstahl & 365.023 & & & & & & & \\
\hline Sachbeschädig. & 48.618 & 19.756 & & & & & & \\
\hline gemeingef. Str. & 5.657 & 2.012 & 925 & & & & & \\
\hline Hehlerei & 16.708 & 2.525 & 226 & 969 & & & & \\
\hline Betrug & 53.922 & 8.397 & 966 & 3.215 & 60.012 & & & \\
\hline Erschl. v. Leist. & 27.981 & 7.666 & 592 & 1.752 & 10.063 & 18.314 & & \\
\hline Gef. dem. Recht. & 1.760 & 1.453 & 101 & 103 & 512 & 421 & 600 & \\
\hline Wid. gg. Staat. & 5.517 & 2.273 & 122 & 428 & 1.145 & 1.204 & 180 & 369 \\
\hline öff. Ordnung & 6.782 & 2.600 & 272 & 526 & 3.606 & 1.367 & 673 & 406 \\
\hline Hausfriedensbr. & 10.243 & 4.325 & 201 & 759 & 2.724 & 3.954 & 225 & 757 \\
\hline Beleidigung & 12.509 & 6.191 & 377 & 1.020 & 4.326 & 3.613 & 642 & 1.481 \\
\hline Fälschung & 21.840 & 2.823 & 264 & 1.236 & 9.440 & 2.114 & 155 & 429 \\
\hline Meineid & 1.754 & 352 & 51 & 164 & 1.089 & 217 & 20 & 58 \\
\hline falsche Verd. & 2.085 & 468 & 61 & 162 & 1.089 & 351 & 30 & 74 \\
\hline BTM-Delikte & 89.786 & 17.856 & 1.438 & 7.238 & 20.124 & 20.884 & 736 & 3.468 \\
\hline \multirow[t]{2}{*}{ gef. Eingr. Str.V. } & 408 & 241 & 12 & 28 & 68 & 52 & 8 & 24 \\
\hline & öff. Ord. & Hausfr. & $\begin{array}{l}\text { Beleidi- } \\
\text { gung }\end{array}$ & Fälschung & Meineid & $\begin{array}{l}\text { falsche } \\
\text { Verd. }\end{array}$ & $\begin{array}{c}\text { BTM- } \\
\text { Delikte }\end{array}$ & $\begin{array}{c}\text { gef. Eingr. } \\
\text { Str.V. }\end{array}$ \\
\hline öff. Ordnung & 638 & & & & & & & \\
\hline Hausfriedensbr. & 594 & 1.839 & & & & & & \\
\hline Beleidigung & 1.299 & 2.266 & 2.751 & & & & & \\
\hline Fälschung & 738 & 749 & 1.142 & 3.008 & & & & \\
\hline Meineid & 90 & 86 & 165 & 191 & 37 & & & \\
\hline falsche Verd. & 185 & 124 & 285 & 206 & 47 & 29 & & \\
\hline BTM-Delikte & 2.892 & 8.224 & 7.889 & 6.324 & 952 & 1.109 & 58.919 & \\
\hline gef. Eingr. Str.V. & 37 & 19 & 78 & 24 & 1 & 2 & 139 & 21 \\
\hline
\end{tabular}

Betrachtet man Männer mit zwei oder mehr polizeilichen Registrierungen, so sind fast $90 \%$ der registrierten schweren Diebstähle von Männern mit fünf oder mehr Registrierungen begangen worden. Dies erklärt die vielen Kombinationen mit schwerem Diebstahl. Das häufige Auftreten der Kombination schwerer Diebstahl schwerer Diebstahl weist auf eine gewisse Spezialisierung bei diesem Delikt hin. Im Gegensatz dazu ist einfacher Diebstahl sehr häufig eine Einzelstraftat.

Um Deliktsähnlichkeiten mit Hilfe von MDS zu bestimmen, wird ein Ähnlichkeitsmaß für Delikte benötigt. Wie oben beschrieben, sind die Häufigkeiten kein $\mathrm{Maß}$ für Ähnlichkeiten, wohl aber die Adjusted Standardized Residuals (ASR) von Deliktspaaren (Tabelle 16). ${ }^{149}$

Alle Werte der Diagonale sind positiv. Dies bedeutet, dass die Kombinationen gleicher Delikte überzufällig häufig auftreten. Spezialisierung auf einzelne Delikts-

149 Deliktspaare mit dem Delikt ,gefährlicher Eingriff in den Straßenverkehr“ kommen sehr selten vor, deshalb wird dieses Delikt hier für weitere Auswertungen nicht benutzt. Die Fallzahlen sind zu klein. 
kategorien zeigt sich dadurch. Anders ausgedrückt heißt das, dass auch diese Methode Deliktsähnlichkeiten zu bestimmen, zu dem Ergebnis kommt, dass alle Delikte zu sich selbst ähnlich sind. Damit ist eine Voraussetzung der Multidimensionalen Skalierung, dass alle Objekte selbstähnlich sind, erfüllt. Insbesondere wird die Eigenähnlichkeit von Betrug und schwerem Diebstahl deutlich und gibt damit einen Hinweis auf Spezialisierung.

Ansonsten fallen die negativen Residuen im Zusammenhang mit schwerem Diebstahl auf. D.h. der schwere Diebstahl hat keine Ähnlichkeit mit anderen Delikten. Obwohl das Deliktspaar einfacher Diebstahl - schwerer Diebstahl von der Zahl her oft vorkommt, wie in der vorherigen Tabelle zu sehen war, ist das ASR hoch negativ. D.h. die Kombination einfacher Diebstahl - schwerer Diebstahl kommt deutlich seltener vor als erwartet.

Positive Residuen gibt es u.a. bei den Deliktspaaren schwere Körperverletzung Straftaten gegen die persönliche Freiheit, Körperverletzung - Straftaten gegen die persönliche Freiheit, schwere Körperverletzung - Beleidigung, Körperverletzung Beleidigung, Straftaten gegen die persönliche Freiheit - Beleidigung.

Tabelle 16: Adjusted Standardized Residuals der Deliktspaare (deutsche Männer)150

\begin{tabular}{lrrrrrrrr}
\hline & $\begin{array}{c}\text { Tötungs- } \\
\text { delikte }\end{array}$ & $\begin{array}{c}\text { Sexual- } \\
\text { del. }\end{array}$ & $\begin{array}{r}\text { sonst. } \\
\text { Sex. }\end{array}$ & Raub & $\begin{array}{r}\text { schw. } \\
\text { KV }\end{array}$ & KV & $\begin{array}{c}\text { Str. gg. } \\
\text { Pers. Fr. }\end{array}$ & einf. Dieb. \\
\hline Tötungsdelikte & $\mathbf{3 0 , 8}$ & & & & & & & \\
Sexualdelikte & 3,4 & $\mathbf{1 0 7 , 4}$ & & & & & & \\
sonst. Sexualdel. & $-0,9$ & $\mathbf{5 8 , 9}$ & $\mathbf{2 7 2 , 1}$ & & & & & \\
Raub & 5,3 & 6,9 & $-0,8$ & $\mathbf{8 0 , 2}$ & & & & \\
schw. KV & $\mathbf{1 0 , 0}$ & $\mathbf{1 2 , 5}$ & 6,9 & $\mathbf{1 9 , 4}$ & $\mathbf{1 0 3 , 6}$ & & & \\
Körperverletzung & 5,3 & $\mathbf{1 0 , 5}$ & 8,2 & $\mathbf{1 2 , 3}$ & $\mathbf{6 9 , 3}$ & $\mathbf{9 5 , 3}$ & & \\
Str. gg. Pers. Fr. & 3,7 & $\mathbf{1 0 , 9}$ & 6,3 & $\mathbf{1 3 , 8}$ & $\mathbf{4 1 , 1}$ & $\mathbf{4 8 , 5}$ & $\mathbf{5 2 , 3}$ & \\
einf. Diebstahl & $-5,7$ & $-8,5$ & $-13,8$ & $-9,1$ & $-32,7$ & $-28,8$ & $-22,1$ & $\mathbf{1 9 1 , 7}$ \\
schw. Diebstahl & $-9,3$ & $-21,9$ & $-29,6$ & $-25,8$ & $-72,8$ & $-73,8$ & $-53,8$ & $-90,9$ \\
Sachbeschäd. & 5,1 & 0,4 & $-1,6$ & $-1,2$ & $\mathbf{3 1 , 9}$ & $\mathbf{2 4 , 5}$ & $\mathbf{1 7 , 3}$ & $-26,3$ \\
gemeingef. Str. & $\mathbf{1 3 , 4}$ & $-1,4$ & $-0,1$ & $-2,3$ & $-2,9$ & $-2,6$ & 0,3 & $-6,1$ \\
Hehlerei & $-0,7$ & $-0,6$ & $-2,3$ & 0,6 & 4,8 & 1,2 & 1,1 & 4,7 \\
Betrug & $-1,3$ & $-3,3$ & $-3,8$ & $-18,7$ & $-32,0$ & $-23,3$ & $-7,5$ & $-48,1$ \\
Erschl. v. Leist. & $-0,1$ & $-4,8$ & $-4,0$ & $-3,2$ & $-4,2$ & $-7,1$ & $-3,8$ & $-2,0$ \\
Gef. dem. Recht. & 6,2 & $-2,2$ & 2,1 & $-1,7$ & $\mathbf{2 9 , 8}$ & 9,9 & $\mathbf{1 4 , 3}$ & $-20,9$ \\
Wid. gg. Staat. & 2,2 & 1,2 & $-1,5$ & 3,0 & $\mathbf{2 7 , 0}$ & $\mathbf{2 8 , 2}$ & $\mathbf{1 4 , 0}$ & $-12,7$ \\
öff. Ordnung & 4,1 & $-1,1$ & 3,1 & 2,9 & $\mathbf{2 2 , 4}$ & $\mathbf{1 0 , 8}$ & $\mathbf{1 3 , 9}$ & $-12,1$ \\
Hausfriedensbr. & $-1,3$ & 3,1 & 0,6 & $-0,7$ & $\mathbf{1 2 , 0}$ & $\mathbf{1 5 , 0}$ & 9,6 & 6,1 \\
Beleidigung & 1,1 & $\mathbf{1 1 , 6}$ & $\mathbf{1 8 , 1}$ & 2,7 & $\mathbf{3 9 , 8}$ & $\mathbf{5 6 , 1}$ & $\mathbf{4 8 , 4}$ & $-24,4$ \\
Fälschung & $-1,8$ & 1,2 & 1,6 & $-7,1$ & $-10,9$ & $-7,3$ & $-0,9$ & 7,0 \\
Meineid & 0,3 & $-1,5$ & $-0,6$ & 0,9 & 5,3 & 3,2 & 2,4 & $-4,2$ \\
falsche Verd. & 0,1 & 1,9 & 2,9 & 1,5 & 1,5 & 4,7 & 6,8 & $-0,2$ \\
BTM-Delikte & $-0,8$ & $-5,0$ & $-8,8$ & 9,8 & $-3,1$ & $-4,9$ & $-10,1$ & $-9,0$ \\
\hline
\end{tabular}

150 Aus Gründen der Übersichtlichkeit wurden die ASR größer oder gleich 10 fett und die ASR kleiner oder gleich 10 kursiv gedruckt. 


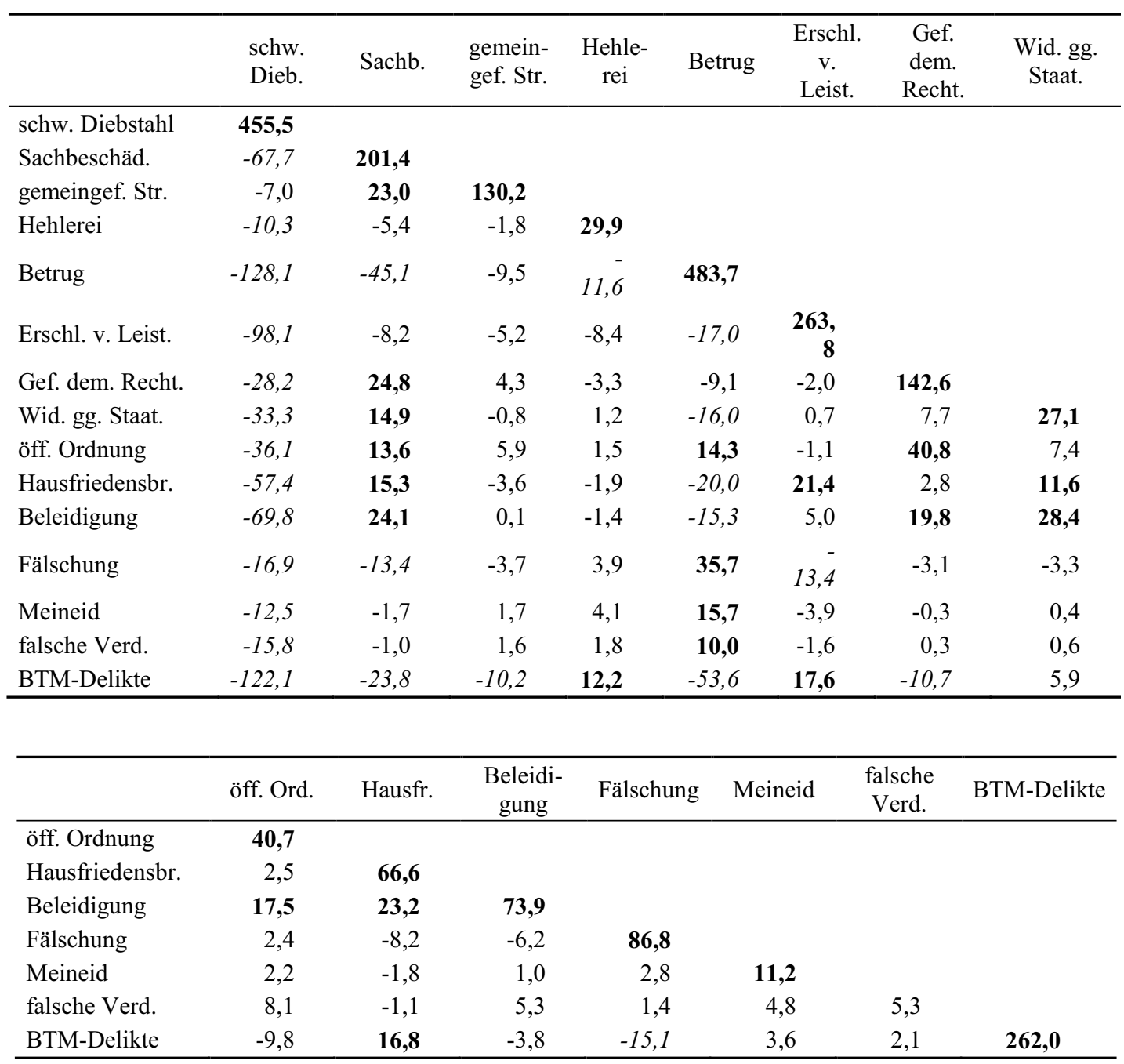

Dies überdurchschnittlich häufige Auftreten dieser Deliktspaare kann aber auch daher stammen, dass von einem Tatverdächtigen eine Handlung begangen wurde, wobei diese eine Handlung mehrere Strafgesetze verletzt. Beispiele dafür sind Körperverletzung mit Beleidigung oder Schläge mit gleichzeitiger Androhung von mehr Schlägen, wenn der Raum verlassen wird. Registriert wird im ersten Fall sowohl Körperverletzung wie Beleidigung, im zweiten Fall sowohl Körperverletzung wie die Straftat gegen die persönliche Freiheit. Betrachtet man speziell diese Deliktspaare, zeigt sich, dass die Kombinationen häufig innerhalb einer Tat vorkommen (an einem Tag), dass diese Kombinationen aber deutlich häufiger auch an verschiedenen Tattagen einer Person vorkommen. Ausführlich werden diese Fälle im Abschnitt 5.2.5, Spezialfall: Deliktskombination einer Person an einem Tag, untersucht.

Je größer das ASR, desto ähnlicher sind die beiden Delikte. Das größte ASR bei verschiedenen Delikten ist beim Deliktspaar Körperverletzung - schwere Körperverletzung mit 69,3. Der nächstgrößte Wert ist beim Deliktspaar sonstige Sexualdelikte - Sexualdelikte mit 58,9, der nächste Wert ist 56,1 bei Beleidigung - Körper- 
verletzung. Dies bedeutet, dass das Deliktspaar Körperverletzung - schwere Körperverletzung am ähnlichsten ist, am zweitähnlichsten ist sonstige Sexualdelikte Sexualdelikte, danach folgt Beleidigung - Körperverletzung.

Ähnlichkeiten zeigen sich auch bei Tötungsdelikten mit weiteren Gewaltdelikten, insbesondere mit schwerer Körperverletzung. Dies ist insofern erwähnenswert, da bei Tötungsdelikten oft von singulären Straftaten gesprochen wird. 51\% der Tötungsdelikte der deutschen Männer in der PAD passieren im familiären Kreis, darunter befinden sich aber auch häufig Einfachtatverdächtige. ${ }^{151}$ Hier werden nur Personen mit mindestens zwei Registrierungen betrachtet. Wenn in der kriminellen Laufbahn einer Person zusätzlich zum Tötungsdelikt eine weitere Straftat registriert ist, ist dies häufig ein anderes Gewaltdelikt.

Am unähnlichsten anhand der ASR ist Betrug - schwerer Diebstahl mit dem Wert -128,1, als nächstes unähnliches Paar folgt die Deliktskombination BTMDelikt - schwerer Diebstahl mit -122,1 und das nächste unähnliche Paar ist Erschleichen von Leistungen - schwerer Diebstahl $(-98,1)$.

Mit dem Adjusted Standardized Residual werden paarweise Ähnlichkeiten von Delikten aufgezeigt, aber wünschenswert wäre es auch, die gesamte Deliktskonfiguration zu erhalten. Wie schon oben beschrieben, bietet Multidimensionale Skalierung ${ }^{152}$ eine Möglichkeit, dies zu erreichen, und das dafür benötigte Ähnlichkeitsmaß der Deliktspaare ist das ASR.

Die Werte der Diagonale in der Tabelle der ASR, die Eigenkombinationen, werden bei der Multidimensionalen Skalierung nicht mit einbezogen. Es ist eine Voraussetzung der MDS, dass die Objekte mit sich selbst ähnlich sind.

Mit Multidimensionaler Skalierung erhält man die Gesamtdarstellung der Deliktskategorien (Abbildung 7). Dies ist das zweidimensionale Ergebnis der Multidimensionalen Skalierung. Es ist auch möglich, sich Ergebnisse mit mehr Dimensionen berechnen zu lassen, was von der Güte der Lösung her besser sein kann. Je mehr Dimensionen zur Verfügung stehen, desto mehr Möglichkeiten sind vorhanden, die Objekte im Raum optimal anzuordnen. Geht man von drei Objekten aus, so liegen diese grundsätzlich in einer Ebene (2-dimensional), fügt man ein viertes Objekt hinzu, hat man im dreidimensionalen Raum mehr Möglichkeiten, dieses Objekt anzuordnen, als im zweidimensionalen Raum. Die Lösung im zweidimensionalen Raum ergibt nicht das beste, aber das anschaulichste Ergebnis.

151 Vgl. Grundies \& Titterington 2007

152 Die Multidimensionale Skalierung wird mit dem SPSS Programm PROXSCAL berechnet. Bei PROXSCAL lässt sich die Multidimensionale Skalierung sowohl mit Dissimilaritäten wie auch mit Similaritäten berechnen. Die Ähnlichkeitswerte können bei der Eingabe bei PROXSCAL auch negativ sein (Distanzen sind immer positiv), denn ein Ähnlichkeitsmaß verändert sich nicht, wenn eine Konstante addiert wird. 
Von Bedeutung bei dieser Darstellung sind nur die Entfernungen der einzelnen Punkte untereinander, oben, unten, links, rechts spielt für sich betrachtet keine Rolle. Wenn die Grafik gedreht wäre, der schwere Diebstahl nicht ganz rechts, sondern unten in der Abbildung wäre, würde sich am Ergebnis nichts ändern. Von Bedeutung ist nur, wie nahe die Punkte, d.h. die Objekte, beieinander liegen. Je näher die Punkte beieinander liegen, desto ähnlicher sind die Delikte. In der Abbildung gibt ein Abstand von 0,7 Einheiten ein ASR von Null wieder. ${ }^{153}$ Dies gilt für alle Abbildungen dieser Studie. Damit sind Deliktskategorien, die etwa in einer Entfernung von 0,7 Einheiten voneinander entfernt liegen, weder ähnlich noch unähnlich. Sind die Punkte der Deliktskategorien näher als 0,7 Einheiten beieinander, ähneln sich diese Kategorien, sind die Abstände größer als 0,7 Einheiten, sind die Deliktskategorien unähnlich.

Schwerer Diebstahl hat einen relativ weiten Abstand zu anderen Delikten und ist damit mit allen Delikten unähnlich. Auch einfacher Diebstahl liegt abseits und hat damit keine besonderen Ähnlichkeiten mit anderen Delikten. Insbesondere sind einfacher und schwerer Diebstahl einander unähnlich. Auch Betrug befindet sich am Rand der Abbildung und ist zu den meisten Delikten unähnlich. Besonders unähnlich sind Betrug und schwerer Diebstahl sowie Betrug und einfacher Diebstahl. Dies würde heißen, dass eine Person selten Vermögensdelikte und Eigentumsdelikte begeht, sondern entweder Vermögensdelikte oder Eigentumsdelikte.

Ähnlich sind, d.h. nah beieinander liegen, schwere Körperverletzung, Beleidigung, Körperverletzung, Straftaten gegen die persönliche Freiheit. Sexualdelikte liegen in relativer Nähe zu Straftaten gegen die persönliche Freiheit und damit auch zu Körperverletzung. Auch Tötungsdelikte befinden sich in der Nähe anderer Gewaltdelikte. D. h. die Ähnlichkeit dieser Gewaltdelikte zeigt sich.

Bei Betäubungsmitteldelikten zeigt sich keine Ähnlichkeit zu schwerem Diebstahl, Betrug und sonstigen Sexualdelikten. Ähnlichkeiten zeigen sich bei Betäubungsmitteldelikten zu Leistungserschleichung, Hehlerei, Raub und Hausfriedensbruch. Diese Delikte gehören somit zur Beschaffungskriminalität zur Finanzierung der Sucht. Ein Zusammenhang von Drogendelikten und Beschaffungsprostitution zeigt sich bei den Männern nicht.

Mit den empirischen und den berechneten Distanzen, die auch ein Ergebnis der MDS sind, lässt sich die erklärte Varianz berechnen. ${ }^{154}$ Die erklärte Varianz $\mathrm{R}^{2}$ der

153 Exakt gilt dies nur, wenn die Güte der Abbildung optimal ist, d.h. bei einem STRESS-Wert von Null und einer erklärten Varianz von 100\%. In Abbildung 7 müssten z.B. Betrug und Fälschung näher beieinander liegen, da das ASR dieser Kombination 35,7 ist. Somit wird die Darstellung dieser Deliktskategorien in der 2-dimensionalen Darstellung nicht optimal gelöst.

154 Zur Berechnung der erklärten Varianz für die einzelnen Delikte wird eine Korrelation zwischen distances (Distanzen) und transformed proximities (Disparitäten, empirisch ermittelte Distanz) berechnet. Die Daten der Distanzen und der Disparitäten werden mit PROXSCAL berechnet. 
gesamten Darstellung beträgt $81 \%$, die Darstellung ist damit sehr gut. Das STRESS-Maß liegt bei 0,21, was auch noch ein relativ guter Wert für dieses Beurteilungskriterium ist.

Abbildung 7: $\quad$ Ergebnis der MDS (deutsche Männer) ${ }^{155}$

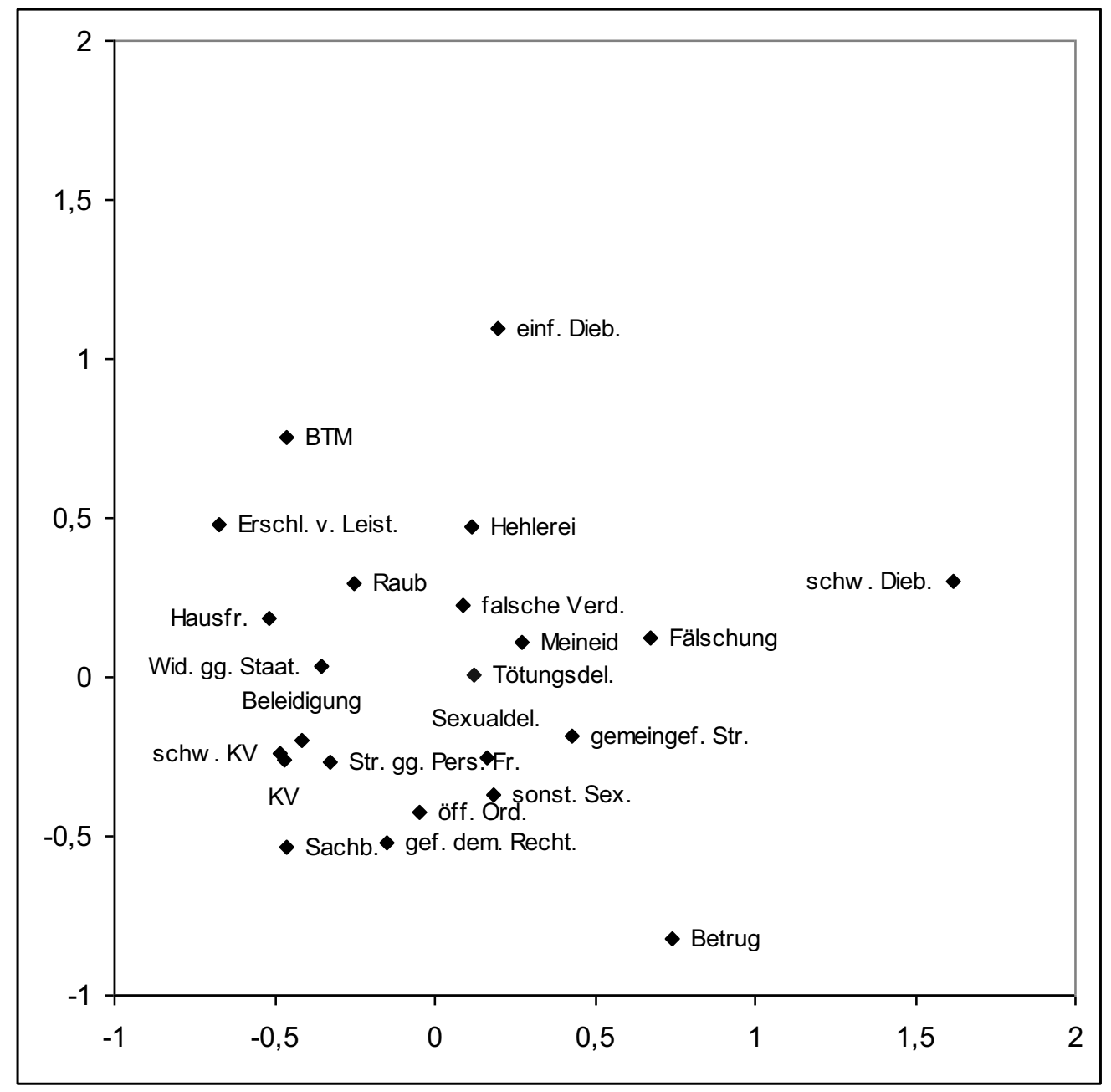

Trotz einer insgesamt sehr guten Darstellung können einzelne Delikte innerhalb der zweidimensionalen Darstellung schlechter repräsentiert werden. Tabelle 17 zeigt die erklärte Varianz für die einzelnen Deliktskategorien. Je näher die erklärte Varianz bei $100 \%$ liegt, desto sicherer ist die Lage des Objekts, hier Delikt, im 2dimensionalen Raum. Die Deliktskategorien, die im Vergleich zur Gesamtdarstellung eine höhere erklärte Varianz aufweisen, sind in der Tabelle fett gedruckt.

155 Ein Abstand von 0,7 Einheiten entspricht einem ASR von Null. 
Tabelle 17: Erklärte Varianz (deutsche Männer)

\begin{tabular}{lclc}
\hline & $\mathrm{R}^{2}$ in $\%$ & $\mathrm{R}^{2}$ in \% \\
\hline Tötungsdelikte & 55 & Betrug & 58 \\
Sexualdelikte & 67 & Erschl. von Leistungen & 79 \\
sonstige Sexualdelikte & 77 & Gefährdung des dem. Rechtsstaates & $\mathbf{8 6}$ \\
Raub & 79 & Widerstand gg. Staatsgewalt & $\mathbf{9 1}$ \\
schwere Körperverletzung & $\mathbf{9 3}$ & öffentliche Ordnung & 69 \\
Körperverletzung & $\mathbf{9 2}$ & Hausfriedensbruch & $\mathbf{8 2}$ \\
Straftaten gg. pers. Freiheit & $\mathbf{8 9}$ & Beleidigung & $\mathbf{9 6}$ \\
einfacher Diebstahl & 58 & Fälschung & 54 \\
schwerer Diebstahl & 53 & Meineid & 29 \\
Sachbeschädigung & $\mathbf{8 6}$ & falsche Verdächtigung & 42 \\
Gemeingefährliche Straftaten & 44 & BTM-Delikte & $\mathbf{8 6}$ \\
Hehlerei & 54 & & \\
\hline
\end{tabular}

Von den 23 Deliktskategorien sind 9 überdurchschnittlich gut dargestellt, die erklärte Varianz liegt über $81 \%$. Nur bei drei Delikten, gemeingefährliche Straftaten, falsche Verdächtigung und Meineid, liegt die erklärte Varianz unter 50\%. Besonders Meineid ist in der zweidimensionalen Abbildung nicht gut dargestellt. Bei schwerem Diebstahl sind 53\% der Varianz erklärt, damit ist die Aussage, dass schwerer Diebstahl zu allen anderen Delikten unähnlich ist, bestätigt. Sehr gute Ergebnisse gibt es bei den ähnlichen Delikten schwere Körperverletzung $\left(\mathrm{R}^{2}=93 \%\right)$, Körperverletzung $\left(\mathrm{R}^{2}=92 \%\right)$, Straftaten gegen die persönliche Freiheit $\left(\mathrm{R}^{2}=89 \%\right)$ und Beleidigung $\left(\mathrm{R}^{2}=96 \%\right)$. Sehr gute Ergebnisse gibt es auch beim Delikt Widerstand gegen die Staatsgewalt mit 91\% erklärter Varianz und bei BTM-Delikten mit $86 \%$.

Das Ergebnis der zweidimensionalen Darstellung der Multidimensionalen Skalierung zeigt, dass insbesondere Gewaltdelikte und auch Beleidigung und Sachbeschädigung ähnlich zueinander sind. Diese Delikte kommen häufig gemeinsam innerhalb der kriminellen Karriere von Personen vor. Es zeigt sich auch ein $\mathrm{Zu}-$ sammenhang von Betäubungsmitteldelikten und den zur Beschaffungskriminalität zählenden Delikten Leistungserschleichung, Raub, Hehlerei und Hausfriedensbruch. Besonders unähnlich zu anderen Delikten ist schwerer Diebstahl.

\subsubsection{Deutsche Frauen}

Ebenso wie bei den deutschen Männern beschrieben, soll hier die Deliktskonfiguration der deutschen Frauen untersucht werden. Dafür werden zuerst alle individuellen Deliktskombinationen gezählt.

In Tabelle 62 im Anhang sind die Häufigkeiten der Deliktspaare der deutschen Frauen dargestellt. Die Tabelle zeigt die Häufigkeit von Deliktspaaren von 24 De- 
liktskategorien. Bei den deutschen Frauen gibt es insgesamt 196.000 Deliktspaare und damit deutlich weniger als bei den Männern.

Bei den deutschen Frauen ist das häufigste Paar einfacher Diebstahl - einfacher Diebstahl. Es tritt 28.167-mal auf. Am zweithäufigsten ist Betrug - Betrug (18.684), am dritthäufigsten sind BTM-Delikte - einfacher Diebstahl (16.922). Schon hier sieht man einen Unterschied zu den Männern, bei denen die Paare schwerer Diebstahl - schwerer Diebstahl und schwerer Diebstahl - einfacher Diebstahl am häufigsten sind. Einfacher Diebstahl - einfacher Diebstahl kommt bei den Männern erst an dritter Stelle. Dafür sind bei Frauen Betrugskombinationen häufiger. Sexualdelikte sind bei den Frauen so selten, dass sie für weitere Auswertungen nicht verwendet werden, ebenso das Delikt gefährlicher Eingriff in den Straßenverkehr.

Tabelle 18: Adjusted Standardized Residuals (deutsche Frauen) ${ }^{156}$

\begin{tabular}{lccrrrrr}
\hline & $\begin{array}{r}\text { Tötungs- } \\
\text { delikte }\end{array}$ & sonst. Sex. & Raub & $\begin{array}{r}\text { schw. } \\
\text { KV }\end{array}$ & KV & $\begin{array}{r}\text { Str. gg. } \\
\text { Pers. Fr. }\end{array}$ & $\begin{array}{r}\text { einf. } \\
\text { Dieb. }\end{array}$ \\
\hline Tötungsdelikte & $\mathbf{2 7 , 8}$ & & & & & & \\
sonst. Sexualdel. & $-1,3$ & $\mathbf{9 1 , 0}$ & & & & & \\
Raub & 3,0 & $-3,7$ & $\mathbf{2 1 , 7}$ & & & & \\
schw. KV & 2,2 & $-2,1$ & 6,0 & $\mathbf{1 9 , 9}$ & & & \\
Körperverletzung & 3,4 & $-4,6$ & 3,4 & $\mathbf{1 7 , 3}$ & $\mathbf{2 2 , 8}$ & & \\
Str. gg. Pers. Fr. & 2,4 & 0,5 & 2,4 & $\mathbf{1 2 , 9}$ & $\mathbf{1 2 , 0}$ & $\mathbf{1 2 , 9}$ & \\
einf. Diebstahl & $-2,2$ & $-14,6$ & $-2,6$ & $-5,8$ & $-5,5$ & $-5,4$ & $\mathbf{7 5 , 1}$ \\
schw. Diebstahl & $-1,5$ & $-10,4$ & 0,9 & $-6,3$ & $-5,8$ & $-5,4$ & $-7,8$ \\
Sachbeschäd. & 2,5 & $-1,0$ & 1,9 & 7,6 & 8,3 & 7,3 & $-2,6$ \\
gemeingef. Str. & 2,1 & $-3,6$ & 0,1 & 2,4 & 1,7 & $-0,0$ & 0,4 \\
Hehlerei & $-0,3$ & $-2,8$ & 0,2 & 1,4 & 1,1 & 1,9 & 1,8 \\
Betrug & $-3,5$ & $-33,7$ & $-8,5$ & $-15,5$ & $-16,1$ & $-11,1$ & $-51,9$ \\
Erschl. v. Leist. & $-1,1$ & 8,8 & $-2,3$ & 1,9 & $-2,2$ & $-0,7$ & $-9,6$ \\
Gef. dem. Recht. & 3,1 & $-2,5$ & $-0,1$ & 2,1 & 2,1 & 0,5 & $-3,3$ \\
Wid. gg. Staat. & 0,4 & $-0,3$ & $-0,5$ & 5,8 & 7,6 & 5,0 & $-5,1$ \\
öff. Ordnung & 2,5 & $-4,0$ & 0,9 & 0,2 & 0,5 & 1,3 & $-4,3$ \\
Hausfriedensbr. & 0,3 & 1,1 & $-0,9$ & $-0,6$ & 1,7 & 1,6 & 6,3 \\
Hausfr. & 2,5 & $-1,1$ & 0,5 & $\mathbf{1 3 , 2}$ & $\mathbf{2 1 , 6}$ & $\mathbf{1 7 , 0}$ & $-9,4$ \\
Beleidigung & $-1,8$ & $-7,8$ & 0,7 & $-1,9$ & $-2,2$ & $-1,9$ & 2,3 \\
Fälschung & $-0,1$ & $-2,3$ & $-1,0$ & 0,5 & 1,2 & 1,0 & $-5,5$ \\
Meineid & $-0,7$ & $-0,1$ & $-1,0$ & 1,0 & 0,7 & 2,7 & $-0,8$ \\
falsche Verd. & 0,3 & $\mathbf{1 4 , 2}$ & 4,2 & $-3,4$ & $-4,4$ & $-5,0$ & $-12,1$ \\
BTM-Delikte & & & & & & & \\
\hline & & & & & &
\end{tabular}

156 Aus Gründen der Übersichtlichkeit wurden die ASR größer oder gleich 10 fett und die ASR kleiner oder gleich 10 kursiv gedruckt. 


\begin{tabular}{|c|c|c|c|c|c|c|c|c|}
\hline & $\begin{array}{l}\text { schw. } \\
\text { Dieb. }\end{array}$ & Sachb. & $\begin{array}{l}\text { gemein- } \\
\text { gef. Str. }\end{array}$ & Hehlerei & Betrug & $\begin{array}{l}\text { Erschl. v. } \\
\text { Leist. }\end{array}$ & $\begin{array}{l}\text { Gef. } \\
\text { dem. } \\
\text { Recht. }\end{array}$ & $\begin{array}{l}\text { Wid. } \\
\text { gg. } \\
\text { Staat. }\end{array}$ \\
\hline schw. Diebstahl & 120,7 & & & & & & & \\
\hline Sachbeschäd. & $-0,2$ & 18,1 & & & & & & \\
\hline gemeingef. Str. & $-0,3$ & 3,4 & 36,0 & & & & & \\
\hline Hehlerei & 3,1 & $-0,6$ & $-0,4$ & 11,1 & & & & \\
\hline Betrug & $-24,4$ & $-12,4$ & $-3,3$ & $-9,7$ & 213,1 & & & \\
\hline Erschl. v. Leist. & $-15,1$ & $-3,3$ & $-2,8$ & $-3,9$ & $-40,8$ & 90,1 & & \\
\hline $\begin{array}{l}\text { Gef. dem. } \\
\text { Recht. }\end{array}$ & 2,0 & 4,4 & 6,2 & $-0,4$ & $-1,8$ & $-1,0$ & 29,9 & \\
\hline Wid. gg. Staat. & $-3,0$ & 6,1 & 0,4 & 1,4 & $-9,4$ & 0,9 & 2,2 & 7,9 \\
\hline öff. Ordnung & $-3,4$ & 1,8 & 0,9 & 2,1 & 7,8 & $-1,8$ & 7,4 & 1,4 \\
\hline Hausfriedensbr. & $-6,1$ & 5,0 & $-0,7$ & $-1,2$ & $-19,6$ & 6,7 & $-0,6$ & 3,7 \\
\hline Beleidigung & $-8,2$ & 10,2 & $-0,5$ & 1,6 & $-11,4$ & $-1,4$ & 3,0 & 11,6 \\
\hline Fälschung & $-2,7$ & $-3,1$ & $-0,4$ & 1,4 & 11,2 & $-7,5$ & $-0,5$ & $-0,7$ \\
\hline Meineid & $-3,4$ & 0,2 & 0,1 & 1,6 & 13,9 & $-3,9$ & 0,0 & $-0,2$ \\
\hline falsche Verd. & $-1,9$ & 0,6 & $-0,3$ & 2,7 & $-2,6$ & $-1,1$ & $-1,0$ & 0,9 \\
\hline BTM-Delikte & $-10,6$ & $-4,3$ & 0,1 & 3,2 & $-56,0$ & $-3,4$ & $-2,9$ & $-0,2$ \\
\hline
\end{tabular}

\begin{tabular}{lccccccc}
\hline & öff. Ord. & Hausfr. & $\begin{array}{c}\text { Beleidi- } \\
\text { gung }\end{array}$ & $\begin{array}{c}\text { Fäl- } \\
\text { schung }\end{array}$ & Meineid & $\begin{array}{c}\text { falsche } \\
\text { Verd. }\end{array}$ & $\begin{array}{c}\text { BTM- } \\
\text { Delikte }\end{array}$ \\
\hline offf. Ordnung & 8,6 & & & & & & \\
$\begin{array}{l}\text { Hausfrie- } \\
\text { densbr. }\end{array}$ & $-1,5$ & $\mathbf{2 0 , 3}$ & & & & & \\
Beleidigung & 1,8 & 1,4 & $\mathbf{2 4 , 1}$ & & & & \\
Fälschung & 0,8 & $-2,9$ & $-2,4$ & $\mathbf{2 0 , 6}$ & & & \\
Meineid & 2,9 & $-2,1$ & $-0,4$ & $-0,1$ & 6,6 & & \\
falsche Verd. & 6,4 & $-0,4$ & 4,3 & 1,5 & 2,2 & $\mathbf{1 4 , 2}$ & \\
BTM-Delikte & $-4,9$ & 1,3 & $-6,9$ & $-5,5$ & $-3,5$ & $-3,7$ & $\mathbf{8 8 , 6}$ \\
\hline
\end{tabular}

Die ASR der Deliktspaare der deutschen Frauen sind in Tabelle 18 dargestellt. Wie bei den Männern sind auch bei den Frauen die Werte der Diagonale immer positiv. Die Kombination gleicher Delikte tritt überzufällig häufig bei Personen auf. Besonders häufig ist die Eigenkombination von Betrug $(213,1)$ und von schwerem Diebstahl $(120,7)$.

Ebenso wie bei den Männern ist auch bei den Frauen sowohl einfacher wie schwerer Diebstahl zu vielen anderen Delikten unähnlich, das ASR ist negativ. Aber im Gegensatz zu den Männern gibt es bei den Frauen Kombinationen mit schwerem Diebstahl, die häufiger als erwartet vorkommen. Dabei handelt es sich um die Kombinationen von schwerem Diebstahl mit den Deliktskategorien Hehlerei, Gefährdung des demokratischen Rechtsstaates und Raub. Auch bei einfachem Diebstahl gibt es Kombinationen die häufiger vorkommen als erwartet, und zwar die Kombinationen von einfachem Diebstahl mit den Deliktskategorien Hausfriedensbruch, Fälschung und Hehlerei. 
Auch Betrug ist zu den meisten Delikten unähnlich. Am unähnlichsten von allen Deliktspaaren ist die Kombination Betrug - Betäubungsmitteldelikte (-56,0). Diese Kombination kommt in Bezug zu den erwarteten Werten sehr selten vor bei Frauen. Das zweitunähnlichste Deliktspaar ist das Paar Betrug - einfacher Diebstahl $(-51,9)$, dann folgt Betrug - Erschleichen von Leistungen $(-40,8)$. Es zeigt sich eine deutliche Unähnlichkeit zwischen dem Eigentumsdelikt Diebstahl und dem Vermögensdelikt Betrug.

Deutlich mehr Deliktspaare als erwartet gibt es bei den Deliktskombinationen Beleidigung - Körperverletzung (21,6), Körperverletzung - schwere Körperverletzung (17,3), Beleidigung - Straftaten gegen die persönliche Freiheit $(17,0)$, BTMDelikte - sonstige Sexualdelikte ${ }^{157}(14,2)$, Meineid - Betrug $(13,9)$, Beleidigung schwere Körperverletzung $(13,2)$, Straftaten gegen die persönliche Freiheit schwere Körperverletzung $(12,9)$, Straftaten gegen die persönliche Freiheit - Körperverletzung (12,0), Beleidigung - Widerstand gegen die Staatsgewalt (11,6), Fälschung - Betrug $(11,2)$ und Beleidigung - Sachbeschädigung $(10,2)$. Auch die Kombination Erschleichen von Leistungen - sonstige Sexualdelikte $(8,8)$ tritt relativ häufig auf.

Wie auch schon bei den Männern wird zur Darstellung der Deliktskonfiguration eine MDS durchgeführt. Das ASR wird dafür als Ähnlichkeitsmaß der Deliktskategorien verwendet. Abbildung 8 zeigt die zweidimensionale Deliktskonfiguration der deutschen Frauen. Wie bei den Männern entspricht auch in dieser Abbildung ein Abstand von 0,7 Einheiten einem ASR von Null. Betrug, schwerer Diebstahl und einfacher Diebstahl liegen am Rand der Darstellung, in relativ großer Entfernung zu anderen Deliktskategorien und sind demnach jeweils unähnlich zu fast allen anderen Delikten. Nah beieinander und somit ähnlich sind Körperverletzung, schwere Körperverletzung, Beleidigung und Straftaten gegen die persönliche Freiheit. Die Ähnlichkeit dieser Delikte zeigte sich auch bei den deutschen Männern. Anders als bei den Männern liegt die Deliktskategorie Erschleichen von Leistungen in der Nähe der „sonstigen Sexualdelikte“ und nicht in der Nähe der Betäubungsmitteldelikte. Bei den Frauen zeigt sich ein Zusammenhang von Drogendelikten und Beschaffungsprostitution (sonst. Sex.) mit einem ASR von 14,2.

Besonders unähnlich sind die Deliktskategorien Diebstahl und Leistungserschleichung sowie Diebstahl und Betrug. D.h. wie bei den Männern zeigt sich, dass Vermögensdelikte zusammen mit Eigentumsdelikten selten von einer Person begangen werden, sondern entweder Vermögensdelikte oder Eigentumsdelikte.

157 Unter sonstigen Sexualdelikten verbirgt sich bei Frauen hauptsächlich das Delikt verbotene Prostitution, bei den Männern hauptsächlich sexueller Missbrauch und exhibitionistische Handlungen. 
Abbildung 8: $\quad$ Ergebnis der MDS (deutsche Frauen) ${ }^{158}$

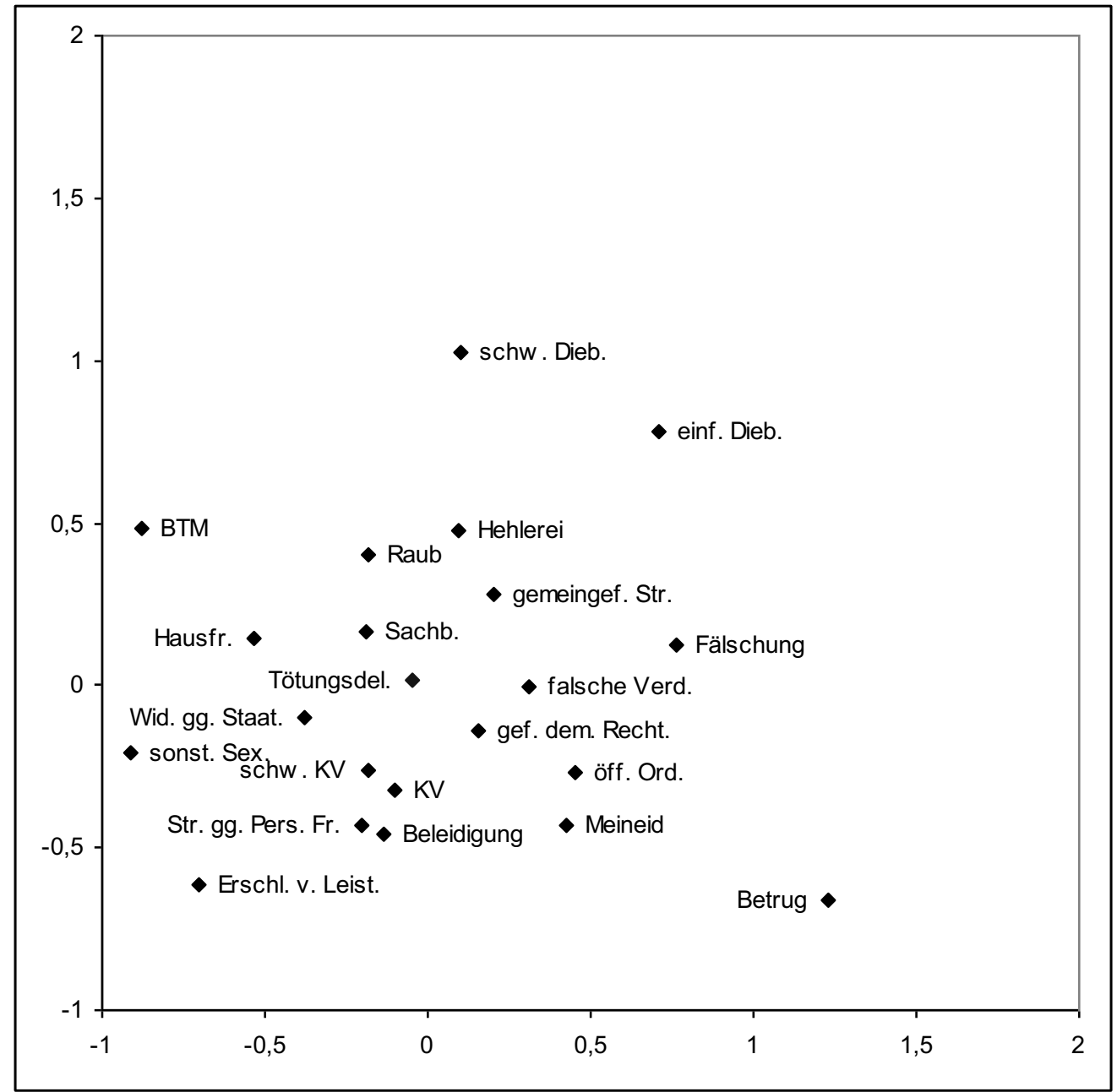

Die erklärte Varianz der Gesamtdarstellung beträgt $77 \%$ und ist damit etwas schlechter als die der deutschen Männer, aber auch sehr gut. Der etwas schlechtere Wert hängt wohl hauptsächlich mit den geringeren Fallzahlen zusammen. Das STRESS-Maß beträgt 0,22.

In Tabelle 19 ist die erklärte Varianz der einzelnen Deliktskategorien dargestellt. Bei 10 der 22 Deliktskategorien ist die erklärte Varianz höher oder gleich $77 \%$. Nur bei einer Deliktskategorie, falsche Verdächtigung, ist die erklärte Varianz kleiner 50\%. Besonders hoch ist die erklärte Varianz bei den ähnlichen Deliktskategorien Körperverletzung, schwere Körperverletzung, Beleidigung, Widerstand gegen die Staatsgewalt und Straftaten gegen die persönliche Freiheit. Ebenso gut sind die Werte von Erschleichen von Leistungen und Betrug.

158 Ein Abstand von 0,7 Einheiten entspricht einem ASR von Null. 
Tabelle 19: Erklärte Varianz (deutsche Frauen) 159

\begin{tabular}{lclc}
\hline & $\mathrm{R}^{2}$ in $\%$ & & $\mathrm{R}^{2}$ in \% \\
\hline Tötungsdelikte & $\mathbf{7 9}$ & Betrug & $\mathbf{8 4}$ \\
sonstige Sexualdelikte & $\mathbf{7 7}$ & Erschl. von Leistungen & $\mathbf{8 2}$ \\
Raub & 52 & Gefährd. d. dem. Rechtsstaates & 62 \\
schwere Körperverletzung & $\mathbf{8 9}$ & Widerstand gg. Staatsgewalt & $\mathbf{8 3}$ \\
Körperverletzung & $\mathbf{8 7}$ & öffentliche Ordnung & $\mathbf{7 8}$ \\
Straftaten gg. pers. Freiheit & $\mathbf{8 4}$ & Hausfriedensbruch & 59 \\
einfacher Diebstahl & 50 & Beleidigung & $\mathbf{9 0}$ \\
schwerer Diebstahl & 61 & Fälschung & 70 \\
Sachbeschädigung & 71 & Meineid & 69 \\
Gemeingefährliche Straftaten & 64 & falsche Verdächtigung & 43 \\
Hehlerei & 56 & BTM-Delikte & 69 \\
\hline
\end{tabular}

Das Ergebnis der zweidimensionalen Darstellung der Multidimensionalen Skalierung zeigt, dass, wie schon bei den Männern, auch bei den Frauen insbesondere Gewaltdelikte, Beleidigung und Sachbeschädigung ähnlich zueinander sind. Diese Delikte kommen häufig gemeinsam innerhalb der kriminellen Karriere von Personen vor. Es zeigt sich auch ein Zusammenhang von Betäubungsmitteldelikten und den zur Beschaffungskriminalität zählenden Delikten Raub und Hausfriedensbruch. Eine gewisse Ähnlichkeit zeigt sich bei den Frauen auch zwischen Betäubungsmitteldelikten und verbotener Prostitution (sonstige Sexualdelikte). Besonders unähnlich zu anderen Delikten ist Betrug. Insbesondere zeigt sich wie auch schon bei den Männern keine Ähnlichkeit von Vermögens- und Eigentumsdelikten.

\subsubsection{Nichtdeutsche Männer}

Wie oben für die deutschen Männer beschrieben, soll hier die Deliktskonfiguration der nichtdeutschen Männer untersucht werden. Dafür werden zuerst alle individuellen Deliktskombinationen gezählt.

In Tabelle 63 im Anhang sind die Häufigkeiten der Deliktspaare der nichtdeutschen Männer dargestellt. Die Tabelle zeigt die Häufigkeit von Deliktspaaren von 25 Deliktskategorien. Insgesamt sind es 1.664.000 Deliktspaare.

Das häufigste Deliktspaar bei den nichtdeutschen Männern ist, wie bei den deutschen Männern, schwerer Diebstahl - schwerer Diebstahl. Es tritt 234.691-mal auf. Ebenso wie bei den deutschen Männern ist das zweithäufigste Deliktspaar schwerer Diebstahl - einfacher Diebstahl (145.700) und das dritthäufigste einfacher Diebstahl - einfacher Diebstahl (137.710). Einfacher Diebstahl ist bei den nichtdeutschen Männern sowohl das häufigste Delikt bei Betrachtung der Häufigkeiten als auch bei Betrachtung aller Deliktskombinationen mit einfachem Diebstahl.

159 Die Deliktskategorien, die im Vergleich zur Gesamtdarstellung, eine höhere erklärte Varianz aufweisen, sind in der Tabelle fett gedruckt. 
Vergehen gegen das Ausländergesetz bzw. das Asylverfahrensgesetz (AuslG) treten bei den nichtdeutschen Männern oft auf. Auch die Eigenkombination AuslG - AuslG (86.480) ist besonders häufig.

In Tabelle 20 sind die Adjusted Standardized Residuals der Deliktspaare der nichtdeutschen Männer dargestellt. ${ }^{160}$ Auch hier sind die Werte der Diagonale immer positiv, was heißt, dass die Kombinationen gleicher Delikte überzufällig häufig bei Personen auftreten. Besonders häufig ist die Eigenkombination von AuslG mit einem ASR von 544,7 und von schwerem Diebstahl mit einem ASR von 509,5.

Tabelle 20: Adjusted Standardized Residuals (nichtdeutsche Männer) ${ }^{161}$

\begin{tabular}{lrrrrrrrr}
\hline & $\begin{array}{c}\text { Tötungs- } \\
\text { delikte }\end{array}$ & $\begin{array}{c}\text { Sexu- } \\
\text { aldel. }\end{array}$ & $\begin{array}{c}\text { sonst. } \\
\text { Sex. }\end{array}$ & Raub & $\begin{array}{r}\text { schw. } \\
\text { KV }\end{array}$ & KV & $\begin{array}{c}\text { Str. gg. } \\
\text { Pers. Fr. }\end{array}$ & $\begin{array}{c}\text { einf. } \\
\text { Dieb. }\end{array}$ \\
\hline Tötungsdelikte & $\mathbf{1 1 , 7}$ & & & & & & & \\
Sexualdelikte & 0,6 & $\mathbf{5 1 , 9}$ & & & & & & \\
sonst. Sexualdel. & 0,5 & $\mathbf{1 9 , 0}$ & $\mathbf{9 4 , 6}$ & & & & & \\
Raub & 4,9 & 7,9 & 0,9 & $\mathbf{1 1 5 , 1}$ & & & & \\
schw. KV & 9,1 & $\mathbf{1 4 , 3}$ & 4,6 & $\mathbf{2 2 , 4}$ & $\mathbf{8 6 , 2}$ & & & \\
Körperverletzg. & 5,1 & $\mathbf{1 3 , 4}$ & 8,8 & 8,8 & $\mathbf{5 9 , 7}$ & $\mathbf{8 4 , 5}$ & & \\
Str. gg. Pers. Fr. & 5,2 & $\mathbf{2 2 , 8}$ & 5,3 & 9,8 & $\mathbf{4 3 , 8}$ & $\mathbf{5 2 , 1}$ & $\mathbf{5 4 , 4}$ & \\
einf. Diebstahl & $-4,1$ & $-7,1$ & $-3,2$ & $-7,4$ & $-24,3$ & $-23,4$ & $-23,3$ & $\mathbf{2 1 9 , 1}$ \\
schw. Diebstahl & $-7,1$ & $-16,1$ & $-13,4$ & $-19,5$ & $-59,8$ & $-60,1$ & $-49,0$ & $-106,0$ \\
Sachbeschäd. & 4,1 & 5,9 & 5,8 & 5,2 & $\mathbf{3 0 , 9}$ & $\mathbf{2 8 , 8}$ & $\mathbf{1 9 , 5}$ & $-2,2$ \\
gemeingef. Str. & 2,0 & 1,2 & 1,7 & 2,3 & 3,0 & 2,4 & 0,8 & 0,3 \\
Hehlerei & $-1,5$ & 0,3 & $-1,2$ & 1,9 & 5,7 & 5,4 & 3,4 & 4,9 \\
Betrug & $-2,0$ & $-4,4$ & 2,3 & $-8,1$ & $-10,2$ & $-0,3$ & 4,7 & $-20,6$ \\
Erschl. v. Leist. & 0,2 & $-0,8$ & 0,1 & $-3,9$ & $-3,6$ & $-5,7$ & $-4,8$ & 7,8 \\
Wid. gg. Staat. & 3,2 & $-0,4$ & $-0,4$ & $-2,2$ & $\mathbf{1 3 , 7}$ & $\mathbf{1 9 , 3}$ & 8,1 & $-11,7$ \\
öff. Ordnung & 2,5 & 4,4 & 3,8 & 8,5 & $\mathbf{1 7 , 4}$ & $\mathbf{1 2 , 0}$ & 9,8 & $-8,1$ \\
Hausfriedensbr. & $-0,7$ & 1,8 & $-1,3$ & $-2,6$ & 6,7 & 6,3 & 8,8 & 9,6 \\
Beleidigung & $-0,3$ & $\mathbf{1 0 , 7}$ & 9,4 & 1,3 & $\mathbf{3 8 , 7}$ & $\mathbf{5 1 , 5}$ & $\mathbf{4 5 , 7}$ & $-20,7$ \\
Fälschung & $-1,3$ & $-0,9$ & $-0,8$ & $-13,9$ & $-12,0$ & $-10,3$ & $-4,4$ & $-21,0$ \\
Meineid & 0,0 & 0,5 & 0,4 & 0,0 & 4,8 & 4,7 & 5,1 & $-8,1$ \\
falsche Verd. & 2,3 & 1,3 & 1,3 & $-0,5$ & 4,0 & 4,1 & 6,8 & $-0,6$ \\
BTM-Delikte & 0,4 & $-6,3$ & $-2,4$ & $\mathbf{1 0 , 8}$ & 3,3 & $-0,0$ & $-5,0$ & $-24,6$ \\
AuslG & $-0,98$ & $-12,35$ & $-12,34$ & $-47,72$ & $-41,85$ & $-45,52$ & $-27,59$ & $-49,42$ \\
\hline
\end{tabular}

160 Deliktskombinationen mit ,gefährlicher Eingriff in den Straßenverkehr” und mit „Gefährdung des demokratischen Rechtsstaates" sind so selten, dass sie hier für weitere Auswertungen nicht benutzt werden.

161 Aus Gründen der Übersichtlichkeit wurden die ASR größer oder gleich 10 fett und die ASR kleiner oder gleich 10 kursiv gedruckt. 


\begin{tabular}{|c|c|c|c|c|c|c|c|c|}
\hline & $\begin{array}{l}\text { schw. } \\
\text { Dieb. }\end{array}$ & Sachb. & $\begin{array}{l}\text { gemein } \\
\text { gef. Str }\end{array}$ & & Hehlerei & Betrug & $\begin{array}{l}\text { Erschl. v. } \\
\text { Leist. }\end{array}$ & $\begin{array}{l}\text { Wid. gg. } \\
\text { Staat. }\end{array}$ \\
\hline schw. Diebstahl & 509,5 & & & & & & & \\
\hline Sachbeschäd. & $-30,6$ & 55,9 & & & & & & \\
\hline gemeingef. Str. & $-7,4$ & 13,9 & 59,2 & & & & & \\
\hline Hehlerei & $-14,8$ & 1,3 & 0,8 & & 35,5 & & & \\
\hline Betrug & $-59,6$ & $-7,6$ & $-1,1$ & & 0,8 & 270,8 & & \\
\hline Erschl. v. Leist. & $-71,6$ & $-0,6$ & $-0,1$ & & $-5,9$ & $-1,6$ & 132,8 & \\
\hline Wid. gg. Staat. & $-23,8$ & 9,4 & 1,1 & & $-0,7$ & $-7,8$ & 3,3 & 18,5 \\
\hline öff. Ordnung & $-21,0$ & 10,5 & 4,3 & & 4,7 & 13,1 & $-1,1$ & 4,4 \\
\hline Hausfriedensbr. & $-38,1$ & 6,1 & 0,0 & & $-0,1$ & $-11,2$ & 5,8 & 7,7 \\
\hline Beleidigung & $-45,7$ & 21,3 & 0,7 & & 3,1 & $-1,0$ & 2,2 & 16,5 \\
\hline Fälschung & $-46,2$ & $-10,5$ & 0,1 & & $-1,3$ & 21,0 & $-6,9$ & $-2,5$ \\
\hline Meineid & $-5,1$ & $-0,4$ & 1,4 & & 4,9 & 13,3 & $-2,9$ & 0,3 \\
\hline falsche Verd. & $-4,8$ & 2,8 & 1,5 & & 2,6 & 6,1 & $-2,0$ & $-0,3$ \\
\hline BTM-Delikte & $-101,6$ & 2,6 & 0,2 & & 13,5 & $-18,1$ & 16,7 & 8,0 \\
\hline \multirow[t]{2}{*}{ AuslG } & $-197,8$ & $-43,4$ & $-12,4$ & & $-21,5$ & $-53,4$ & 15,4 & 3,9 \\
\hline & $\begin{array}{l}\text { öff. } \\
\text { Ord. }\end{array}$ & $\begin{array}{l}\text { Haus- } \\
\text { fr.br. }\end{array}$ & $\begin{array}{l}\text { Beleidi- } \\
\text { gung }\end{array}$ & $\begin{array}{c}\text { Fäl- } \\
\text { schung }\end{array}$ & $\begin{array}{l}\text { Mein- } \\
\text { eid }\end{array}$ & $\begin{array}{l}\text { falsche } \\
\text { Verd. }\end{array}$ & BTM-Del. & AuslG \\
\hline öff. Ordnung & 20,5 & & & & & & & \\
\hline Hausfriedensbr. & $-0,9$ & 65,8 & & & & & & \\
\hline Beleidigung & 7,5 & 11,2 & 57,3 & & & & & \\
\hline Fälschung & 1,0 & $-8,1$ & $-8,9$ & 223,8 & & & & \\
\hline Meineid & 1,2 & $-1,3$ & 0,9 & 1,3 & 7,4 & & & \\
\hline falsche Verd. & 8,5 & 0,2 & 3,4 & 1,2 & 4,0 & 7,8 & & \\
\hline BTM-Delikte & 2,9 & 2,0 & 2,2 & $-16,4$ & 8,6 & 2,1 & 229,2 & \\
\hline AuslG & $-17,2$ & 1,4 & $-32,9$ & 28,9 & $-9,9$ & $-14,3$ & $-40,5$ & 544,7 \\
\hline
\end{tabular}

Wie bei den deutschen Männern, fallen die negativen Residuen in Kombination mit dem schweren Diebstahl auf, d.h. das Delikt schwerer Diebstahl hat wenig Ähnlichkeiten mit anderen Delikten, aber wie der große positive Wert der Eigenkombination zeigt, gibt es häufig Personen, die mehrere schwere Diebstähle begehen. Dies ist ein Hinweis auf Spezialisierung und darauf, dass schwerer Diebstahl insbesondere ein Delikt von Mehrfachtatverdächtigen ist. Deutlich seltener als erwartet treten die Deliktspaare AuslG - schwerer Diebstahl (-197,8), schwerer Diebstahl - einfacher Diebstahl (-106,0) und BTM - schwerer Diebstahl $(-101,6)$ auf.

Ähnlich wie beim schweren Diebstahl sieht es auch bei der Deliktskategorie Vergehen gegen Ausländergesetze aus. Bei dieser Deliktskategorie gibt es kaum Ähnlichkeiten mit anderen Delikten, die meisten Residuen sind negativ. Ausnahmen bilden hier die Deliktskombinationen von Vergehen gegen das Ausländergesetz oder Asylverfahrensgesetz mit Hausfriedensbruch, Erschleichen von Leistungen, Widerstand gegen die Staatsgewalt und insbesondere „Fälschung“. Die Deliktskombination „Fälschung“ und Vergehen gegen Ausländergesetze weist Ähnlichkeiten auf, der Wert des ASR ist relativ groß $(28,9)$. Althoff und de Haan untersuchten im Jahr 2004 Asylbewerber in den Niederlanden hinsichtlich ihres krimi- 
nellen Verhaltens. ${ }^{162}$ Sie fanden heraus, dass es sich bei den Straftaten der Asylbewerber häufig um Ladendiebstahl sowie Schwarzfahren und Graufahren (Fahren mit gefälschten Karten) handelt. ${ }^{163}$ D.h. bei „Fälschung“ handelt es sich neben der erwarteten Urkundenfälschung häufig auch um Wertzeichenfälschung (Fahrkartenfälschung). Auch die Eigenkombination der Ausländervergehen hat einen großen positiven Wert. Personen, die gegen Ausländergesetze verstoßen, verstoßen häufig mehrmals dagegen.

Die Deliktspaare Körperverletzung - schwere Körperverletzung (59,7), Körperverletzung - Straftaten gegen die persönliche Freiheit $(52,1)$ und Körperverletzung - Beleidigung $(51,5)$ treten wie schon bei den Deutschen überzufällig häufig auf.

Wie auch bei den Deutschen, wird zur Darstellung der Deliktskonfiguration eine MDS durchgeführt, wobei das ASR als Ähnlichkeitsmaß benutzt wird. In Abbildung 9 wird das Ergebnis der zweidimensionalen Multidimensionalen Skalierung dargestellt. Die Abbildung zeigt, dass wie auch bei den deutschen Männern Körperverletzung, Beleidigung, Straftaten gegen die persönliche Freiheit und schwere Körperverletzung ähnlich sind. Diese Delikte liegen nah beieinander. Auch ähnlich zu diesen Delikten sind Sachbeschädigung, Hehlerei und Raub. Sexualstraftaten sind etwas abseits dieser Kategorien. Bei Sexualdelikten zeigen sich Ähnlichkeiten zu Tötungsdelikten, Meineid und „sonstigen Sexualdelikten“. Vergehen gegen Ausländergesetze liegen abseits von allen anderen Delikten, abgesehen von „Fälschung“ und Leistungserschleichung. Schwerer Diebstahl ist zu allen Delikten unähnlich, ebenso einfacher Diebstahl.

Die erklärte Varianz der Gesamtdarstellung liegt bei 72\%. Das STRESS-Maß beträgt 0,26. Die Werte sind etwas schlechter als bei den deutschen Männern. Tabelle 21 zeigt die erklärte Varianz für die einzelnen Deliktskategorien. Von den 23 Deliktskategorien haben 10 einen überdurchschnittlichen Wert der erklärten Varianz, $72 \%$ oder darüber. Nur bei Betrug ist die erklärte Varianz mit $16 \%$ nicht besonders gut. D.h. die zweidimensionale Darstellung stellt die Ähnlichkeit der Delikte, abgesehen von Betrug, gut dar. Sehr gut dargestellt sind die Delikte Raub, schwere Körperverletzung, Körperverletzung, Straftaten gegen die persönliche Freiheit, schwerer Diebstahl, Sachbeschädigung, Beleidigung und Vergehen gegen Ausländergesetze.

162 Althoff \& de Haan 2004.

163 Vgl. Althoff \& de Haan 2004, S.445. 
Abbildung 9: $\quad$ Ergebnis der MDS (nichtdeutsche Männer) 164

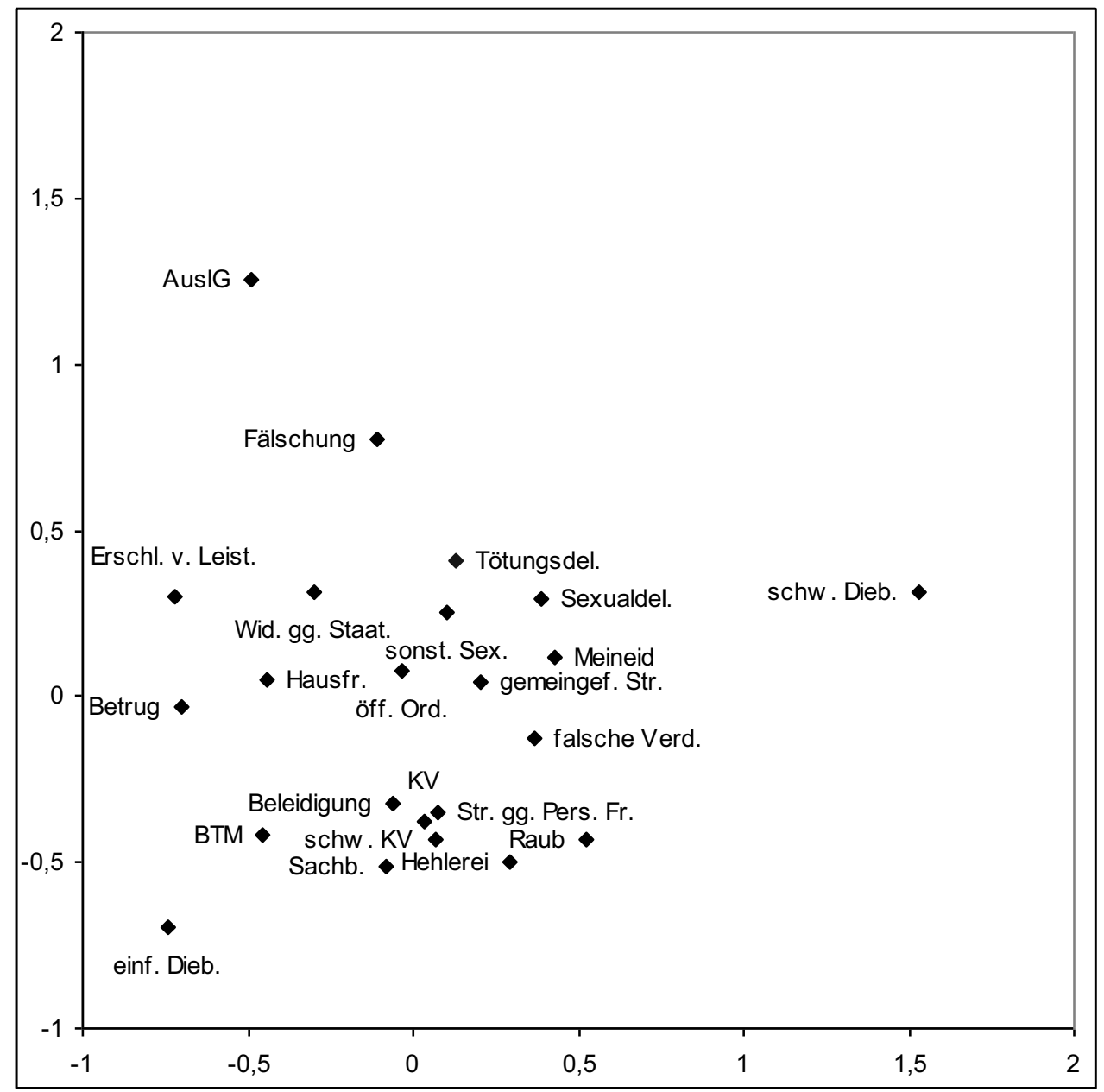

Das Ergebnis der zweidimensionalen Darstellung der Multidimensionalen Skalierung zeigt, dass wie auch bei den Deutschen insbesondere Gewaltdelikte und auch Beleidigung und Sachbeschädigung ähnlich zueinander sind. Diese Delikte kommen häufig gemeinsam innerhalb der kriminellen Karriere von Personen vor. Anhand des ASR zeigt sich auch ein Zusammenhang von Betäubungsmitteldelikten und den zur Beschaffungskriminalität zählenden Delikten Leistungserschleichung, Raub und Hehlerei. Besonders unähnlich zu anderen Delikten ist schwerer Diebstahl. Auch Vergehen gegen Ausländergesetze sind, abgesehen von der Kombination mit „Fälschung“ und Leistungserschleichung, was hauptsächlich auf Urkundenfälschung, Wertzeichenfälschung (Fahrkarten) und Beförderungserschleichung hinweist, unähnlich zu allen anderen Delikten.

164 Ein Abstand von 0,7 Einheiten entspricht einem ASR von Null. 
Tabelle 21: Erklärte Varianz (nichtdeutsche Männer) ${ }^{165}$

\begin{tabular}{lclc}
\hline & $\mathrm{R}^{\mathbf{2}}$ in \% & $\mathrm{R}^{\mathbf{2}}$ in \% \\
\hline Tötungsdelikte & 47 & Betrug & 16 \\
Sexualdelikte & 61 & Erschl. von Leistungen & 66 \\
sonstige Sexualdelikte & 60 & Widerstand gg. Staatsge- & 49 \\
Raub & $\mathbf{7 7}$ & walt & öffentliche Ordnung \\
schwere Körperverletzung & $\mathbf{8 8}$ & Hausfriedensbruch & $\mathbf{7 3}$ \\
Körperverletzung & $\mathbf{9 0}$ & Beleidigung & 40 \\
Straftaten gg. pers. Freiheit & $\mathbf{8 3}$ & Fälschung & $\mathbf{8 8}$ \\
einfacher Diebstahl & 46 & Meineid & 40 \\
schwerer Diebstahl & $\mathbf{8 2}$ & falsche Verdächtigung & 53 \\
Sachbeschädigung & $\mathbf{8 1}$ & BTM-Delikte & 54 \\
Gemeingefährliche Straftaten & $\mathbf{7 8}$ & AuslG & 56 \\
Hehlerei & 66 & & $\mathbf{8 5}$ \\
\hline
\end{tabular}

\subsubsection{Nichtdeutsche Frauen}

Wie schon für die anderen Gruppen beschrieben, soll hier die Deliktskonfiguration der nichtdeutschen Frauen untersucht werden. Dazu werden zuerst alle Deliktskombinationen betrachtet, die innerhalb der kriminellen Karrieren der nichtdeutschen Frauen auftreten. In Tabelle 64 im Anhang sind die Häufigkeiten der Deliktspaare dargestellt. Bei den nichtdeutschen Frauen ergeben sich 65.000 Deliktspaare und damit nochmals deutlich weniger als bei den deutschen Frauen. In Relation zu den anderen Gruppierungen sind nichtdeutsche Frauen selten polizeilich registriert und bei sehr wenigen nichtdeutschen Frauen sind mehrere Straftaten registriert.

Wie bei den deutschen Frauen ist auch bei den nichtdeutschen Frauen das häufigste Deliktspaar einfacher Diebstahl - einfacher Diebstahl (15.206). Das zweithäufigste Deliktspaar ist Vergehen gegen Ausländergesetze - einfacher Diebstahl (5.365), danach folgt Betrug - einfacher Diebstahl (3.336).

In Tabelle 22 sind die Adjusted Standardized Residuals der Deliktspaare der nichtdeutschen Frauen dargestellt. ${ }^{166}$ Mit Ausnahme des Delikts gemeingefährliche Straftaten, diese Eigenkombination kommt bei nichtdeutschen Frauen nicht vor, sind die Werte der Diagonale positiv, also auch hier treten die Kombinationen gleicher Delikte überzufällig häufig auf. Besonders häufig treten die Kombinationen

165 Die Deliktskategorien, die im Vergleich zur Gesamtdarstellung, eine höhere erklärte Varianz aufweisen, sind in der Tabelle fett gedruckt.

166 Deliktskombinationen mit Tötungsdelikten, Sexualdelikten und dem Delikt Gefährdung des demokratischen Rechtsstaates sind so selten, dass sie hier für weitere Analysen nicht verwendet werden. 
„sonstige Sexualdelikte“, Betrug, schwerer Diebstahl und Vergehen gegen das Ausländergesetz oder Asylverfahrensgesetz jeweils mit sich selbst auf. Straftaten in Kombination mit Vergehen gegen Ausländergesetze haben negative Residuen, d.h. es gibt zwischen Vergehen gegen Ausländergesetze und anderen Delikten keine Ähnlichkeiten, mit Ausnahme von der Kombination mit Fälschungsdelikten.

Auch bei den Kombinationen mit schwerem Diebstahl gibt es keine Ähnlichkeiten, Ausnahmen bilden hier Raub und Hehlerei. Das unähnlichste Deliktspaar ist Betrug - einfacher Diebstahl (-27,7). Auch bei den deutschen Frauen kommt diese Kombination selten in polizeilichen Registrierungen vor.

Relativ häufige Deliktskombinationen sind: Beleidigung - Körperverletzung $(17,6)$, wie schon erwähnt, Fälschung - Vergehen gegen Ausländergesetze (14,3), Körperverletzung - schwere Körperverletzung (12,0), Straftaten gegen die persönliche Freiheit Körperverletzung $(11,9)$ und Beleidigung - schwere Körperverletzung $(11,4)$.

Tabelle 22: Adjusted Standardized Residuals (nichtdeutsche Frauen) ${ }^{167}$

\begin{tabular}{lrrrrrrr}
\hline & $\begin{array}{r}\text { sonst. } \\
\text { Sex. }\end{array}$ & Raub & schw. KV & KV & $\begin{array}{c}\text { Str. gg. } \\
\text { Pers. Fr. }\end{array}$ & $\begin{array}{c}\text { einf. } \\
\text { Dieb. }\end{array}$ & $\begin{array}{c}\text { schw. } \\
\text { Dieb }\end{array}$ \\
\hline sonst. Sexualdel. & $\mathbf{1 0 0 , 9}$ & & & & & & \\
Raub & $-1,9$ & $\mathbf{1 5 , 6}$ & & & & & \\
schw. KV & $-3,4$ & 5,1 & $\mathbf{1 3 , 3}$ & & & & \\
Körperverletzung & $-3,9$ & 4,6 & $\mathbf{1 2 , 0}$ & $\mathbf{1 2 , 9}$ & & & \\
Str. gg. Pers. Fr. & $-2,5$ & 3,4 & 7,1 & $\mathbf{1 1 , 9}$ & $\mathbf{1 0 , 0}$ & & \\
einf. Diebstahl & $-10,6$ & $-2,3$ & $-7,0$ & $-5,6$ & $-7,8$ & $\mathbf{5 8 , 2}$ & \\
schw. Diebstahl & $-6,3$ & 2,2 & $-3,1$ & $-5,4$ & $-2,8$ & $-18,8$ & $\mathbf{8 9 , 2}$ \\
Sachbeschäd. & $-1,5$ & 0,3 & 6,3 & 8,3 & 6,7 & $-5,9$ & $-1,4$ \\
gemeingef. Str. & $-0,9$ & $-0,8$ & 0,3 & 0,5 & 0,1 & $-0,6$ & 0,7 \\
Hehlerei & $-3,2$ & $-0,9$ & 0,6 & $-0,1$ & 0,1 & $-3,1$ & 2,0 \\
Betrug & $-8,9$ & $-3,7$ & $-1,4$ & $-3,1$ & 0,0 & $-26,7$ & $-14,4$ \\
Erschl. v. Leist. & $-2,6$ & $-2,6$ & 1,1 & $-1,1$ & $-0,7$ & $-6,4$ & $-10,0$ \\
Wid. gg. Staat. & 1,8 & 5,1 & 2,8 & 5,2 & 2,8 & $-4,4$ & $-2,5$ \\
off. Ordnung & $-2,0$ & 0,8 & 0,4 & 0,3 & 3,0 & $-2,9$ & $-1,8$ \\
Hausfriedensbr. & 1,9 & $-1,9$ & 0,6 & $-0,0$ & 1,8 & 5,3 & $-5,1$ \\
Beleidigung & $-4,4$ & 0,9 & $\mathbf{1 1 , 4}$ & $\mathbf{1 7 , 6}$ & $\mathbf{1 0 , 7}$ & $-9,0$ & $-5,3$ \\
Fälschung & $-4,0$ & $-3,1$ & $-0,4$ & $-1,5$ & $-2,4$ & $-11,5$ & $-4,3$ \\
Meineid & 1,4 & 0,6 & 1,3 & $-1,5$ & 0,4 & $-3,8$ & $-2,8$ \\
falsche Verd. & $-2,1$ & 2,4 & 1,9 & 3,7 & 6,2 & $-4,2$ & $-2,37$ \\
BTM-Delikte & 6,1 & $-1,3$ & $-2,1$ & $-1,9$ & $-2,6$ & $-11,6$ & $-5,33$ \\
AuslG & $-2,6$ & $-2,0$ & $-7,2$ & $-8,5$ & $-4,8$ & $-10,0$ & $-6,29$ \\
\hline
\end{tabular}

167 Aus Gründen der Übersichtlichkeit wurden die ASR größer oder gleich 10 fett und die ASR kleiner oder gleich 10 kursiv gedruckt. 


\begin{tabular}{|c|c|c|c|c|c|c|c|}
\hline & Sachb. & $\begin{array}{l}\text { gemein- } \\
\text { gef. Str. }\end{array}$ & Hehlerei & Betrug & $\begin{array}{l}\text { Erschl. v. } \\
\text { Leist. }\end{array}$ & $\begin{array}{l}\text { Wid. gg. } \\
\text { Staat. }\end{array}$ & öff. Ord. \\
\hline Sachbeschäd. & 8,6 & & & & & & \\
\hline gemeingef. Str. & 1,9 & $-0,2$ & & & & & \\
\hline Hehlerei & 1,4 & $-0,1$ & 10,5 & & & & \\
\hline Betrug & $-2,5$ & $-0,8$ & 1,8 & 89,9 & & & \\
\hline Erschl. v. Leist. & 1,8 & $-0,6$ & $-2,3$ & $-4,3$ & 46,3 & & \\
\hline Wid. gg. Staat. & 5,0 & $-0,4$ & $-0,5$ & $-1,1$ & $-1,0$ & 4,9 & \\
\hline öff. Ordnung & 2,9 & 2,8 & $-0,1$ & 3,3 & $-0,3$ & 0,4 & 16,1 \\
\hline Hausfriedensbr. & 1,7 & $-0,3$ & $-1,1$ & $-4,0$ & 1,4 & 2,3 & 0,0 \\
\hline Beleidigung & 8,7 & 0,4 & 0,5 & $-2,2$ & 1,4 & 6,2 & 1,7 \\
\hline Fälschung & $-1,0$ & $-1,1$ & 1,8 & 6,5 & $-3,1$ & $-1,3$ & 0,2 \\
\hline Meineid & $-0,6$ & $-0,3$ & 2,1 & 7,3 & $-1,2$ & $-0,7$ & 1,7 \\
\hline falsche Verd. & 3,6 & $-0,5$ & $-0,3$ & 2,0 & $-1,5$ & 1,4 & 9,2 \\
\hline BTM-Delikte & 1,6 & 2,8 & 4,1 & $-7,6$ & 2,7 & 0,8 & $-2,0$ \\
\hline \multirow[t]{2}{*}{ AuslG } & $-8,1$ & $-0,9$ & $-3,7$ & $-17,4$ & $-7,4$ & $-2,9$ & $-5,0$ \\
\hline & Hausfr. & $\begin{array}{l}\text { Beleidi- } \\
\text { gung }\end{array}$ & $\begin{array}{l}\text { Fäl- } \\
\text { schung }\end{array}$ & Meineid & $\begin{array}{l}\text { falsche } \\
\text { Verd. }\end{array}$ & $\begin{array}{l}\text { BTM- } \\
\text { Delikte }\end{array}$ & AuslG \\
\hline Hausfriedensbr. & 4,1 & & & & & & \\
\hline Beleidigung & 3,0 & 15,5 & & & & & \\
\hline Fälschung & $-2,5$ & $-0,3$ & 17,2 & & & & \\
\hline Meineid & $-0,3$ & 0,0 & $-0,1$ & 10,4 & & & \\
\hline falsche Verd. & $-1,5$ & 2,4 & $-0,0$ & 1,3 & 5,2 & & \\
\hline BTM-Delikte & 1,8 & 0,7 & 0,5 & 2,1 & $-2,0$ & 59,8 & \\
\hline AuslG & $-5,2$ & $-9,8$ & 14,3 & $-2,5$ & $-1,0$ & $-19,1$ & 73,2 \\
\hline
\end{tabular}

Wie für die anderen Gruppen wird auch für die ausländischen Frauen mit Multidimensionaler Skalierung die Deliktskonfiguration analysiert. In Abbildung 10 ist das Ergebnis der zweidimensionalen Multidimensionalen Skalierung dargestellt. Die Abbildung zeigt, wie auch schon für die anderen Gruppen, die Ähnlichkeit von Körperverletzung, schwerer Körperverletzung, Straftaten gegen die persönliche Freiheit, Beleidigung und Sachbeschädigung. Deutlich zu sehen ist die Ähnlichkeit von Betrug und „Fälschung“. Die Deliktskategorie Vergehen gegen Ausländergesetze liegt am Rand der Abbildung und zeigt damit Unähnlichkeiten zu fast allen übrigen Deliktskategorien. 
Abbildung 10: Ergebnis der MDS (nichtdeutsche Frauen) ${ }^{168}$

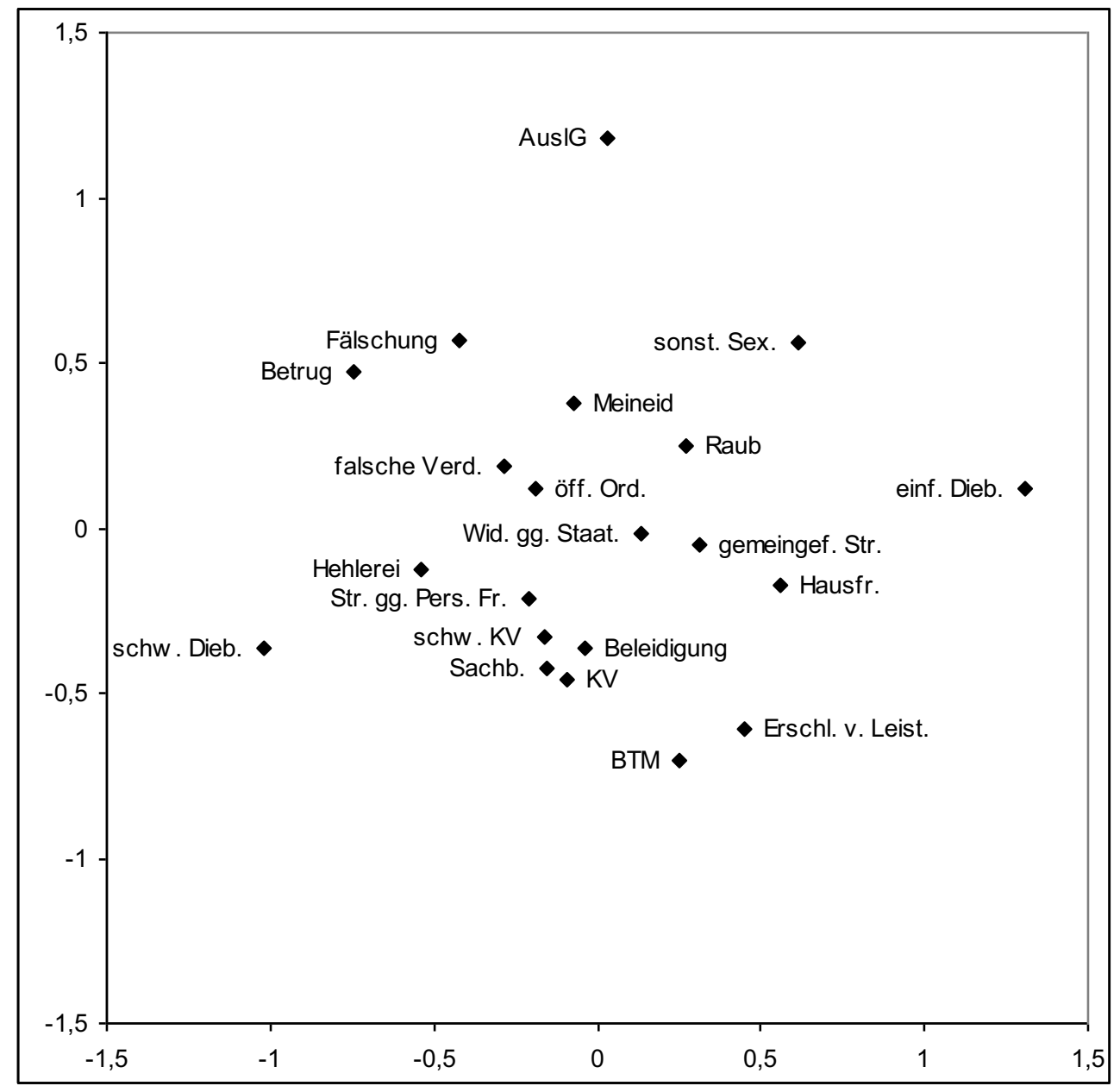

Die erklärte Varianz der Gesamtdarstellung beträgt 68\% und das STRESS-Maß ist 0,24. Die Güte der zweidimensionalen Darstellung ist somit etwas schlechter als die der anderen Gruppen.

168 Ein Abstand von 0,7 Einheiten entspricht einem ASR von Null. 
Tabelle 23: Erklärte Varianz (nichtdeutsche Frauen) ${ }^{169}$

\begin{tabular}{lclc}
\hline & $\mathrm{R}^{2}$ in $\%$ & & $\mathrm{R}^{2}$ in \% \\
\hline sonstige Sexualdelikte & 28 & Erschl. von Leistungen & $\mathbf{7 2}$ \\
Raub & 13 & Widerstand gg. Staatsgewalt & $\mathbf{7 4}$ \\
schwere Körperverletzung & $\mathbf{8 5}$ & öffentliche Ordnung & $\mathbf{7 3}$ \\
Körperverletzung & $\mathbf{8 4}$ & Hausfriedensbruch & $\mathbf{7 4}$ \\
Straftaten gg. pers. Freiheit & $\mathbf{8 2}$ & Beleidigung & $\mathbf{8 9}$ \\
einfacher Diebstahl & 56 & Fälschung & 41 \\
schwerer Diebstahl & 38 & Meineid & 42 \\
Sachbeschädigung & $\mathbf{9 4}$ & falsche Verdächtigung & $\mathbf{8 8}$ \\
Gemeingefährliche Straftaten & 26 & BTM-Delikte & 44 \\
Hehlerei & $\mathbf{7 9}$ & AuslG & 63 \\
Betrug & $\mathbf{7 3}$ & & \\
\hline
\end{tabular}

Tabelle 23 gibt die erklärte Varianz der einzelnen Deliktskategorien der nichtdeutschen Frauen wieder. Bei 12 von 21 Delikten ist die erklärte Varianz höher als die erklärte Varianz der Gesamtdarstellung (68\%). Bei sieben Delikten ist die erklärte Varianz kleiner 50\%. Besonders hoch ist die erklärte Varianz bei den ähnlichen Delikten Körperverletzung, schwere Körperverletzung, Beleidigung, Widerstand gegen die Staatsgewalt und Straftaten gegen die persönliche Freiheit. Diese Delikte sind in jeder Gruppe gut dargestellt. Ebenso gut sind die Werte von Sachbeschädigung und falsche Verdächtigung. Die Delikte Erschleichen von Leistungen und Betrug, die bei den deutschen Frauen sehr gut dargestellt sind, sind auch bei den nichtdeutschen Frauen sehr gut dargestellt. Bei den nichtdeutschen Frauen sind insbesondere sieben Delikte nicht gut dargestellt. Die erklärte Varianz von Raub ist nur 13\% und auch „sonstige Sexualdelikte“ (hauptsächlich verbotene Prostitution) und gemeingefährliche Straftaten sind nicht besonders gut dargestellt, die erklärte Varianz ist $28 \%$ bzw. 26\%. Auch die erklärte Varianz von schwerem Diebstahl, „Fälschung“, Meineid und Betäubungsmitteldelikten ist kleiner als 50\%.

Die relativ schlechten Werte der erklärten Varianz einzelner Deliktskategorien der nichtdeutschen Frauen hängen wohl hauptsächlich mit der geringeren Häufigkeit von Deliktskombinationen zusammen. Die geringeren Häufigkeiten von Deliktskombinationen der nichtdeutschen Frauen erklären sich dadurch, dass relativ wenige Frauen registriert sind und diese zudem selten mehrfach. 72\% der nichtdeutschen Registrierten sind mit nur einer Straftat registriert und weniger als 5\% der nichtdeutschen Registrierten sind mit 5 und mehr Straftaten registriert. Die seltene Mehrfachregistrierung nichtdeutscher Frauen kann auch damit zusammen hängen, dass bei nichtdeutschen Frauen die Mobilität höher ist als bei deutschen Frauen. Wenn eine weitere Registrierung vorliegt, liegt diese nicht unbedingt in Baden-Württemberg vor.

169 Die Deliktskategorien, die im Vergleich zur Gesamtdarstellung, eine höhere erklärte Varianz aufweisen, sind in der Tabelle fett gedruckt. 
Das Ergebnis der zweidimensionalen Darstellung der Multidimensionalen Skalierung zeigt, dass wie auch bei den übrigen Gruppen insbesondere Gewaltdelikte und auch Beleidigung und Sachbeschädigung ähnlich zueinander sind. Diese Delikte kommen häufig gemeinsam innerhalb der kriminellen Karriere von Personen vor. Vergehen gegen Ausländergesetze sind wie auch bei den Männern, abgesehen von der Kombination mit „Fälschung“, unähnlich zu allen anderen Deliktskategorien.

\subsubsection{Spezialfall: Deliktskombination einer Person an einem Tag}

Wie schon im Abschnitt 5.2.1 angesprochen, kann es vorkommen, dass eine Straftat, mehrfach in der PAD registriert wird. Ein Beispiel dafür ist, dass eine Person jemanden gleichzeitig schlägt und beleidigt und somit Körperverletzung und Beleidigung registriert wird. Um herauszufinden, ob Deliktspaare, die aus einer Handlung heraus resultieren, einen besonderen Einfluss auf die Deliktskonfiguration haben, wurden diese gesondert untersucht. Dazu wurden die Deliktskategorien speziell im Hinblick auf Deliktskombinationen, die an einem Tag stattfinden betrachtet. ${ }^{170}$ Hierzu sind in Tabelle 24 die Häufigkeiten der Delikte dargestellt und dazu die Anteile mit und ohne ein weiteres Delikt am selben Tag bei einem Tatverdächtigen. Bei Kombinationen wurde unterschieden zwischen demselben Delikt und einem anderen Delikt. Es wurden nur die Delikte betrachtet, die von Tatverdächtigen mit mindestens zwei Registrierungen stammen.

Generell kann gesagt werden, dass es häufig mehrere Registrierungen bei einer Person an einem Tag gibt. 65\% der Registrierungen sind ohne eine weitere Registrierung bei derselben Person am selben Tag, bei 30\% der Registrierungen ist am selben Tag beim selben Tatverdächtigen ein weiteres Delikt einer anderen Deliktskategorie registriert. In knapp 6\% der Fälle wird dasselbe Delikt registriert.

Das Delikt Widerstand gegen die Staatsgewalt tritt besonders häufig in Kombination mit einem anderen Delikt am selben Tag auf. In 60,8\% der Fälle wird ein weiteres Delikt registriert, meist Körperverletzung oder Beleidigung. Besonders selten wird bei Erschleichen von Leistungen und bei schwerem Diebstahl ein anderes Delikt mitregistriert, wobei Erschleichen von Leistungen besonders häufig einzeln registriert wird (80\% der Fälle). Bei schwerem Diebstahl wird besonders häufig ein weiterer schwerer Diebstahl mitregistriert (19\% der Fälle), aber, wie oben erwähnt, selten ein anderes Delikt. Auch bei Beleidigung wird in über 50\% der Fälle ein weiteres Delikt registriert.

170 Weitere Variablen, die auf „Tateinheit“ hinweisen, wie z.B. Tagebuchnummer in Kombination mit Polizeidienststelle, werden hier nicht benutzt, da Berechnungen gezeigt haben, dass hierdurch keine entscheidenden Veränderungen eintreten. 
Tabelle 24: Delikt mit oder ohne weitere Delikte am selben Tag

\begin{tabular}{|c|c|c|c|c|}
\hline \multirow[b]{2}{*}{ Delikt } & \multirow[b]{2}{*}{$\begin{array}{l}\text { Registrie- } \\
\text { rungen* }\end{array}$} & \multicolumn{2}{|c|}{$\begin{array}{c}\text { Kombination mit weiterem Delikt } \\
\text { am selben Tag in } \%\end{array}$} & \multirow{2}{*}{$\begin{array}{l}\text { einzeln } \\
\text { in \% }\end{array}$} \\
\hline & & anderes Delikt & selbes Delikt & \\
\hline Tötungsdelikte & 628 & 39,0 & 1,3 & 59,7 \\
\hline Sexualdelikte & 1.938 & 31,6 & 2,0 & 66,5 \\
\hline sonst. Sexualdel. & 3.384 & 28,6 & 3,9 & 67,5 \\
\hline Raub & 10.126 & 26,7 & 5,3 & 68,1 \\
\hline schw. KV & 24.901 & 32,2 & 1,4 & 66,5 \\
\hline Körperverletzung & 24.326 & 34,5 & 0,9 & 64,6 \\
\hline Str. gg. Pers. Fr. & 12.944 & 45,6 & 0,7 & 53,6 \\
\hline einf. Diebstahl & 169.829 & 31,7 & 4,7 & 63,5 \\
\hline schw. Diebstahl & 79.593 & 16,8 & 19,0 & 64,2 \\
\hline Sachbeschäd. & 27.138 & 40,0 & 4,0 & 56,0 \\
\hline gemeingef. Str. & 2.790 & 43,7 & 1,9 & 54,4 \\
\hline Hehlerei & 9.922 & 31,0 & 1,5 & 67,5 \\
\hline Betrug & 42.436 & 29,4 & 3,7 & 66,9 \\
\hline Erschl. v. Leist. & 25.297 & 18,8 & 1,5 & $\mathbf{7 9 , 7}$ \\
\hline gef. dem. Recht. & 1.454 & 44,8 & 1,2 & 54,0 \\
\hline Wid. gg. Staat. & 3.346 & 60,8 & 0,4 & 38,9 \\
\hline öff. Ordnung & 6.144 & 43,0 & 0,9 & 56,2 \\
\hline Hausfriedensbr. & 7.282 & 42,5 & 0,6 & 56,9 \\
\hline Beleidigung & 13.173 & 50,5 & 0,8 & 48,7 \\
\hline Fälschung & 14.811 & 36,9 & 1,5 & 61,6 \\
\hline Meineid & 1.704 & 25,8 & 0,9 & 73,3 \\
\hline falsche Verd. & 1.613 & 36,3 & 0,7 & 62,9 \\
\hline BTM-Delikte & 63.618 & 21,4 & 3,4 & 75,1 \\
\hline gesamt & 548.397 & 29,5 & 5,6 & 65,0 \\
\hline
\end{tabular}

Tabelle 25 zeigt den Anteil der Deliktskombinationen, die von einer Person an einem Tag begangen wurden, in Relation zu den im Datensatz überhaupt vorkommenden Deliktspaaren. Betrachtet werden hier nur die Kombinationen verschiedener Deliktskategorien, da es um die Auswirkung von den in einer Handlung ausgeübten Straftaten auf die Deliktsähnlichkeiten geht. Zum Beispiel werden 9,3\% der Deliktspaare schwere Körperverletzung - Straftaten gegen die persönliche Freiheit von einer Person an einem Tag begangen und könnten somit eine Handlung sein. ${ }^{171}$ 5,7\% der Deliktspaare Sexualdelikt - schwere Körperverletzung und 5,5\% der Deliktspaare Sexualdelikt - Körperverletzung werden an einem Tag begangen, d.h. etwa 95\% dieser Paare sind unabhängige Handlungen.

171 Natürlich ist es auch möglich, dass eine Person an einem Tag zwei strafbare Handlungen begeht. Mit der zusätzlichen Benutzung der Variablen Tagebuchnummer wurde geprüft, ob sich die Ergebnisse damit wesentlich verändern. Dies war nicht der Fall. 
Bei den Deliktspaaren ist zu unterscheiden zwischen Paaren wie Sexualdelikt Tötungsdelikt, diese Kombination besteht, auch wenn sie zur selben Zeit passiert, aus zwei eigenständigen Straftaten und zwischen Kombinationen wie Widerstand gegen die Staatsgewalt - Körperverletzung, dies kann eine Handlung sein.

Berechnungen haben gezeigt, dass sich die Ergebnisse nicht grundlegend ändern, wenn statt allen Delikten, bei Mehrfachauftreten von Delikten einer Person an einem Tag, nur das schwerste Delikt ${ }^{172}$ zur Berechnung von Deliktsähnlichkeiten benutzt wurde. Zum Beispiel ist das ASR vom Deliktspaar Beleidigung - Körperverletzung auch dann deutlich positiv, wenn die Deliktspaare nur mit dem schwersten Delikt eines Tages einer Person berechnet wurden und somit die Kombinationen an einem Tag wegfallen.

Tabelle 25: Anteil Deliktspaare mit gleichem Tatdatum bei einer Person an allen Deliktspaaren in \%

\begin{tabular}{lrrrrrrrr}
\hline & $\begin{array}{c}\text { Tötungs- } \\
\text { delikte }\end{array}$ & $\begin{array}{c}\text { Sexual- } \\
\text { del. }\end{array}$ & $\begin{array}{c}\text { sonst. } \\
\text { Sex. }\end{array}$ & Raub & $\begin{array}{c}\text { schw. } \\
\text { KV }\end{array}$ & KV & $\begin{array}{c}\text { Str. gg. } \\
\text { Pers. Fr. }\end{array}$ & $\begin{array}{c}\text { einf. } \\
\text { Dieb. }\end{array}$ \\
\hline Sexualdelikte & 13,5 & & & & & & & \\
sonst. Sexual- & 0,0 & 6,7 & & & & & & \\
del. & 11,5 & 7,1 & 2,1 & & & & & \\
Raub & 11,3 & 5,7 & 1,7 & 4,8 & & & & \\
schw. KV & 2,3 & 5,5 & 2,6 & 2,5 & 3,2 & & & \\
Körperverlet- & & & & & & & & \\
zung & 6,3 & 11,0 & 4,7 & 4,7 & 9,3 & 9,7 & & \\
Str. gg. Pers. & & & & & & \\
Fr. & 0,7 & 0,5 & 0,4 & 1,2 & 0,7 & 1,0 & 1,3 & \\
einf. Diebstahl & 1,8 & 0,3 & 0,3 & 0,8 & 0,4 & 0,2 & 0,5 & 1,4 \\
schw. Diebstahl & 1,6 & 1,2 & 1,4 & 1,6 & 6,9 & 7,5 & 8,0 & 2,3 \\
Sachbeschäd. & 5,6 & 0,0 & 1,8 & 3,6 & 1,8 & 2,0 & 1,4 \\
gemeingef. Str. & 18,1 & 0,0 & 0,0 & 0,3 & \\
Hehlerei & 4,1 & 0,0 & 0,3 & 1,7 & 0,3 & 0,4 & 0,7 & 2,6 \\
Betrug & 1,1 & 0,3 & 0,4 & 1,7 & 0,6 & 0,4 & 0,9 & 2,1 \\
Erschl. v. Leist. & 0,0 & 0,3 & 0,0 & 0,2 & 0,4 & 1,2 & 1,1 & 0,4 \\
gef. dem. & 3,8 & 2,7 & 6,3 & 0,0 & 5,5 & 4,2 & 6,5 & 2,2 \\
Recht. & 12,3 & 2,5 & 1,4 & 2,8 & 9,4 & 17,1 & 11,4 & 1,3 \\
Wid. gg. Staat. & 1,9 & 1,0 & 4,1 & 3,2 & 6,4 & 2,3 & 5,6 & 1,8 \\
off. Ordnung & 8,9 & & & & \\
Hausfriedensbr. & 1,2 & 1,6 & 0,6 & 1,5 & 4,4 & 7,4 & 9,6 & 2,2 \\
Beleidigung & 3,4 & 2,9 & 4,2 & 2,2 & 8,8 & 13,5 & 18,5 & 0,9 \\
Fälschung & 0,0 & 0,4 & 1,5 & 1,3 & 0,6 & 0,3 & 0,8 & 2,9 \\
Meineid & 0,0 & 0,0 & 1,4 & 0,0 & 0,3 & 0,4 & 0,2 & 0,1 \\
falsche Verd. & 4,2 & 1,4 & 0,6 & 2,4 & 2,6 & 2,1 & 5,0 & 1,5 \\
BTM-Delikte & 1,6 & 1,8 & 1,0 & 1,7 & 0,7 & 0,7 & 0,9 & 0,6 \\
\hline
\end{tabular}

172 Die Schwereeinstufung der Delikte wurde anhand des Strafrahmens gebildet. 


\begin{tabular}{lcccccccc}
\hline & $\begin{array}{c}\text { schw. } \\
\text { Dieb. }\end{array}$ & Sachb. & $\begin{array}{l}\text { gemein- } \\
\text { gef. Str. }\end{array}$ & Hehlerei & Betrug & $\begin{array}{c}\text { Erschl. v. } \\
\text { Leist. }\end{array}$ & $\begin{array}{c}\text { gef. dem. } \\
\text { Recht. }\end{array}$ & $\begin{array}{c}\text { Wid. gg. } \\
\text { Staat. }\end{array}$ \\
\hline Sachbeschäd. & 2,7 & & & & & & & \\
gemeingef. Str. & 2,3 & 5,6 & & & & & & \\
Hehlerei & 1,2 & 0,7 & 0,6 & & & & & \\
Betrug & 1,1 & 0,9 & 0,9 & 2,2 & & & & \\
Erschl. v. Leist. & 0,3 & 0,5 & 1,1 & 0,4 & 0,7 & & & \\
gef. dem. Recht. & 2,9 & 13,1 & 3,5 & 0,9 & 0,5 & 0,2 & & \\
Wid. gg. Staat. & 1,4 & 11,3 & 5,7 & 2,0 & 1,2 & 1,5 & 8,8 & \\
off. Ordnung & 1,1 & 6,5 & 6,5 & 4,5 & 3,7 & 0,8 & 14,5 & 13,6 \\
Hausfriedensbr. & 1,1 & 11,9 & 4,0 & 0,5 & 0,9 & 0,7 & 9,1 & 6,6 \\
Beleidigung & 0,4 & 9,3 & 1,4 & 0,4 & 0,7 & 2,3 & 10,0 & 23,8 \\
Fälschung & 1,2 & 0,7 & 0,7 & 3,9 & 8,0 & 2,2 & 1,6 & 2,6 \\
Meineid & 0,0 & 0,2 & 0,0 & 12,1 & 1,0 & 0,3 & 0,0 & 2,9 \\
falsche Verd. & 0,5 & 2,3 & 1,2 & 5,8 & 1,6 & 0,9 & 0,0 & 6,1 \\
BTM-Delikte & 0,5 & 0,6 & 1,8 & 1,5 & 0,6 & 0,2 & 0,5 & 3,9 \\
\hline
\end{tabular}

\begin{tabular}{lcccccc}
\hline & öff. Ord. & Hausfr. & $\begin{array}{c}\text { Beleidi- } \\
\text { gung }\end{array}$ & Fälschung & Meineid & $\begin{array}{c}\text { falsche } \\
\text { Verd. }\end{array}$ \\
\hline Hausfriedensbruch & 2,7 & & & & & \\
Beleidigung & 5,7 & 9,0 & & & & \\
Fälschung & 2,4 & 0,8 & 0,8 & & & \\
Meineid & 1,9 & 0,0 & 1,0 & 2,4 & & \\
falsche Verd. & 28,3 & 0,0 & 7,0 & 4,9 & 12,4 & \\
BTM-Delikte & 1,0 & 0,6 & 0,9 & 1,9 & 0,1 & 2,5 \\
\hline
\end{tabular}

\subsubsection{Zusammenfassung}

Um die Ähnlichkeiten von Delikten zu analysieren, werden Kombinationen von polizeilich registrierten Straftaten einer Person betrachtet. Die häufigste Kombination ist bei den Männern schwerer Diebstahl - schwerer Diebstahl, die zweithäufigste einfacher Diebstahl - schwerer Diebstahl und die dritthäufigste einfacher Diebstahl - einfacher Diebstahl. D. h., obwohl einfacher Diebstahl das häufigste Delikt ist bei Betrachtung der Registrierungen, kommt schwerer Diebstahl häufiger in Deliktskombinationen vor. Dies ist ein Hinweis darauf, dass das Delikt schwerer Diebstahl besonders häufig ein Delikt von Mehrfachtätern ist und dass es beim Delikt schwerer Diebstahl häufiger zu Spezialisierung kommt als bei anderen Delikten. Bei den Frauen, sowohl bei den deutschen wie bei den nichtdeutschen, ist die häufigste Kombination einfacher Diebstahl - einfacher Diebstahl. Die zweithäufigste Kombination bei deutschen Frauen ist Betrug - Betrug. Bei nichtdeutschen Frauen ist die zweithäufigste Kombination einfacher Diebstahl Verstöße gegen Ausländergesetze und die dritthäufigste Kombination einfacher Diebstahl - Betrug.

Zur Darstellung der Deliktskonfiguration mit Multidimensionaler Skalierung wird als Ähnlichkeitsmaß das Adjusted Standardized Residual der Deliktskombinationen benutzt. Ein positives ASR gibt an, dass Deliktspaare überzufällig häufig 
vorkommen, ein negatives ASR zeigt, dass diese Deliktskombinationen selten in Bezug zum erwarteten Wert sind und somit unähnlich sind.

Deliktskombinationen mit demselben Delikt sind immer positiv und damit überzufällig häufig. Besonders häufig und damit ein Hinweis auf Spezialisierung sind die Eigenkombinationen von Betrug und schwerem Diebstahl. Bei Frauen kommt auch die Eigenkombination von ,sonstigen Sexualdelikten“ (verbotene Prostitution) und bei Nichtdeutschen die Eigenkombination von Vergehen gegen das Ausländergesetz oder Asylverfahrensgesetz mit hohen überdurchschnittlichen Werten vor.

Bei allen Gruppen, sowohl bei Männern wie bei Frauen, bei Deutschen wie bei Nichtdeutschen ist das ASR der Deliktskombinationen Körperverletzung - schwere Körperverletzung, Körperverletzung - Beleidigung und Körperverletzung - Straftaten gegen die persönliche Freiheit hoch positiv. Diese Deliktskombinationen sind überzufällig häufig bei Personen polizeilich registriert. Beim schweren Diebstahl zeigen sich bei allen Gruppen Unähnlichkeiten zu allen anderen Deliktskategorien, d.h. Kombinationen mit schwerem Diebstahl treten seltener auf als erwartet. Bei allen Gruppen zeigt sich auch die Unähnlichkeit von Betrug und einfachem Diebstahl. Eigentums- und Vermögensdelikte kommen relativ selten innerhalb der polizeilich registrierten kriminellen Karriere gleichzeitig vor. Bei den Nichtdeutschen zeigt sich die Unähnlichkeit von Vergehen gegen das Ausländergesetz oder Asylverfahrensgesetz mit fast allen anderen Delikten. Nur die Kombinationen von „Fälschung“ und Leistungserschleichung mit Vergehen gegen das Ausländergesetz oder Asylverfahrensgesetz kommen überzufällig häufig vor. Vergehen gegen das Ausländergesetz oder Asylverfahrensgesetz gehen häufig mit Urkundenfälschung einher. Eine häufige Straftat von Asylbewerbern ist Schwarzfahren (Leistungserschleichung) und Graufahren (Fahren mit gefälschten Karten und damit Wertzeichenfälschung). ${ }^{173}$

Die Ergebnisse der zweidimensionalen Darstellung der Multidimensionalen Skalierung zeigen, dass sich insbesondere Gewaltdelikte und auch Beleidigung und Sachbeschädigung durch hohe Ähnlichkeiten auszeichnen, d.h. sie kommen häufig als Deliktskombination bei Personen vor. Bei Betäubungsmitteldelikten zeigt sich ein Zusammenhang zu den zur Beschaffungskriminalität zählenden Delikten Raub und Hausfriedensbruch. Bei Frauen wird auch der Zusammenhang von Drogendelikten und Beschaffungsprostitution (sonstige Sexualdelikte) sichtbar.

\subsection{Ergebnisse auf justizieller Ebene}

Wie schon für polizeilich Registrierte im vorhergehenden Abschnitt beschrieben, sollen hier für justiziell Registrierte Ähnlichkeiten von Delikten untersucht werden. Betrachtet werden Täter, die wegen zwei oder mehr Straftaten registriert sind. D.h. eine Person muss mindestens zweimal justiziell registriert sein oder innerhalb einer

173 Vgl. Althoff \& de Haan 2004, S. 445. 
justiziellen Registrierung müssen mindestens zwei verschiedene Straftaten registriert sein. Analysiert werden die Kombinationen von Straftaten einer Person, wobei die Straftaten in Deliktskategorien eingeteilt sind. Bei Deliktskombinationen bei justiziellen Registrierungen werden sowohl die Deliktskombinationen von Personen betrachtet, die innerhalb einer Entscheidung wegen verschiedener Straftaten abgeurteilt wurden, wie die Kombinationen mehrerer Entscheidungen. Innerhalb einer Entscheidung wird jede Deliktskategorie nur einmal gezählt, auch wenn das Delikt mehrfach begangen wurde. Die Tatbezeichnung lautet z.B. „Diebstahl in zwei Fällen, unerlaubter Besitz von Betäubungsmitteln in zwei Fällen“. Den angewendeten Vorschriften ist nicht zu entnehmen, wie häufig eine Straftat abgeurteilt wurde, und auch nicht immer, ob es sich um Tateinheit oder Tatmehrheit handelt. ${ }^{174}$ (Angewendete Vorschriften des Beispiels: StGB $\S 242, \S 248 \mathrm{a}, \S 21, \S 53$, BtMG $\S 1$ Abs. 1, 33 Abs. 1, Nr. 1, § 29 Abs. 1 Nr. 3.) Die Deliktskombination ist hier einfacher Diebstahl - Betäubungsmitteldelikte, einmal. ${ }^{175}$ Wenn es bei diesem Täter zusätzliche Entscheidungen gibt, gibt es auch weitere Kombinationen. Nur bei zwei oder mehr Entscheidungen sind Eigenkombinationen möglich, innerhalb einer Entscheidung nicht. Mehrere Delikte einer Deliktskategorie innerhalb einer Entscheidung werden für die Auswertungen nicht berücksichtigt. ${ }^{176}$

In Tabelle 26 sind die Häufigkeiten und prozentualen Anteile der einzelnen Deliktskategorien für Täter mit zwei oder mehr Straftaten getrennt nach Geschlecht und Nationalität (deutsch/nichtdeutsch) aufgeführt. Bei den justiziellen Registrierungen gibt es im Vergleich zu den polizeilichen Registrierungen drei zusätzliche Deliktskategorien: Vollrausch, fahrlässige Körperverletzung/fahrlässige Tötung und Verkehrsdelikte. Die Delikte fahrlässige Körperverletzung und fahrlässige Tötung sind zu einer Deliktskategorie zusammengefasst, weil fahrlässige Tötung sehr selten vorkommt und die beiden Delikte vom selben Deliktstyp sind. Zur Deliktskategorie der Verkehrsdelikte gehören Vergehen gegen StVG, PflVG und StGB Verkehrsdelikte ( $\S 142,315 c, 316)$. Verkehrsdelikte werden polizeilich nicht registriert.

174 Dazu wurde von mir ein Programm entwickelt, welches die Tatbezeichnung erfasst und die Häufigkeit der Straftaten kodiert. Viele Fälle müssen aber dennoch zusätzlich bearbeitet werden wegen vielfältiger Schreibformen bzw. Schreibfehlern. Deshalb gibt es diese Angaben nur für einen Teil der Daten und sie werden hier nicht für die Auswertung benutzt.

Weitere Beispiele zu Tatbezeichnung und angewendeten Vorschriften siehe Seite 18. Probleme mit dem Datenmaterial siehe Abschnitt 3.3 und zu Tateinheit/Tatmehrheit siehe Abschnitt 5.3.5.

175 Wenn innerhalb von zwei Entscheidungen jeweils die Delikte einfacher Diebstahl und Betäubungsmitteldelikt vorkommen, gibt es 6 Kombinationen.

Entscheidung 1: einfacher Diebstahl (ED), Betäubungsmitteldelikt (BTM)

Entscheidung 2: einfacher Diebstahl (ED), Betäubungsmitteldelikt (BTM)

Kombinationen: ED - BTM, ED - ED, ED - BTM, BTM - ED, BTM - BTM, ED - BTM.

176 Anders als bei der vom statistischen Bundesamt herausgegebenen Strafverfolgungsstatistik werden aber alle Deliktskategorien einer Entscheidung in die Auswertung mit einbezogen und nicht nur die schwerste Straftat. 
Verkehrsdelikte haben einen besonders großen Anteil an justiziellen Registrierungen, besonders bei den deutschen Männern, hier sind mehr als ein Viertel aller Straftaten Verkehrsdelikte. Ansonsten bildet wie bei den polizeilichen Registrierungen der einfache Diebstahl die größte Deliktskategorie. Bei den deutschen Männern ist der einfache Diebstahl die zweitgrößte Deliktskategorie nach den Verkehrsdelikten. Besonders bei den Frauen ist der Anteil des einfachen Diebstahls sehr groß, bei den deutschen Frauen 32\% und bei den nichtdeutschen Frauen 38\%.

Tabelle 26: Häufigkeitsverteilung der Deliktskategorien bei Tätern mit 2 oder mehr Straftaten (Registrierungen bis 2003)

\begin{tabular}{lrrrrrrrr} 
& \multicolumn{9}{c}{ männl. } & \multicolumn{7}{c}{ weibl. } \\
\cline { 2 - 9 } & deut. & \multicolumn{1}{c}{$\%$} & nichtdt. & $\%$ & deut. & $\%$ & nichtdt. & $\%$ \\
\hline Tötungsdelikte & 124 & 0,1 & 119 & 0,1 & 19 & 0,1 & 3 & 0,0 \\
Sexualdelikte & 449 & 0,2 & 355 & 0,3 & 1 & 0,0 & 1 & 0,0 \\
sonst. Sexualdelikte & 736 & 0,3 & 313 & 0,3 & 169 & 0,6 & 39 & 0,4 \\
Raub & 2.688 & 1,3 & 1.937 & 1,7 & 187 & 0,6 & 64 & 0,6 \\
schw. Körperverletzung & 7.394 & 3,5 & 4.501 & 3,9 & 650 & 2,2 & 198 & 1,9 \\
Körperverletzung & 16.047 & 7,5 & 8.729 & 7,6 & 1.739 & 5,9 & 466 & 4,4 \\
Str. gg. pers. Freiheit & 4.312 & 2,0 & 3.027 & 2,6 & 284 & 1,0 & 107 & 1,0 \\
Einfacher Diebstahl & 36.095 & 16,9 & 22.021 & 19,2 & 9.459 & 31,9 & 4.068 & 38,0 \\
Schwerer Diebstahl & 11.718 & 5,5 & 5.444 & 4,7 & 516 & 1,7 & 180 & 1,7 \\
Sachbeschädigung & 9.090 & 4,2 & 2.380 & 2,1 & 498 & 1,7 & 101 & 0,9 \\
Gemeingef. Str. & 791 & 0,4 & 219 & 0,2 & 46 & 0,2 & 9 & 0,1 \\
Hehlerei & 3.097 & 1,4 & 2.099 & 1,8 & 434 & 1,5 & 140 & 1,3 \\
Betrug & 11.113 & 5,2 & 5.494 & 4,8 & 3.300 & 11,1 & 995 & 9,3 \\
Leistungserschleichung & 8.088 & 3,8 & 4.819 & 4,2 & 2.538 & 8,6 & 819 & 7,7 \\
Gefährd. dem. Recht. & 690 & 0,3 & 34 & 0,0 & 25 & 0,1 & 1 & 0,0 \\
Wid. gg. Staatsgewalt & 1.994 & 0,9 & 997 & 0,9 & 164 & 0,6 & 54 & 0,5 \\
Öffentliche Ordnung & 1.922 & 0,9 & 539 & 0,5 & 322 & 1,1 & 73 & 0,7 \\
Hausfriedensbruch & 2.232 & 1,0 & 1.142 & 1,0 & 305 & 1,0 & 100 & 0,9 \\
Beleidigung & 5.524 & 2,6 & 2.558 & 2,2 & 661 & 2,2 & 211 & 2,0 \\
Fälschung & 5.315 & 2,5 & 4.476 & 3,9 & 1.031 & 3,5 & 517 & 4,8 \\
Meineid & 625 & 0,3 & 399 & 0,3 & 197 & 0,7 & 57 & 0,5 \\
falsche Verdächtigung & 471 & 0,2 & 220 & 0,2 & 217 & 0,7 & 71 & 0,7 \\
BTM-Delikte & 16.279 & 7,6 & 7.233 & 6,3 & 1.938 & 6,5 & 267 & 2,5 \\
gef. Eingr. Str.V. & 619 & 0,3 & 262 & 0,2 & 18 & 0,1 & 3 & 0,0 \\
AuslG & 393 & 0,2 & 13.328 & 11,6 & 114 & 0,4 & 1.029 & 9,6 \\
Vollrausch & 845 & 0,4 & 121 & 0,1 & 34 & 0,1 & 4 & 0,0 \\
fahrl. Tötung/KV & 7.894 & 3,7 & 2.454 & 2,1 & 1.113 & 3,8 & 221 & 2,1 \\
Verkehrsdelikte & 57.351 & 26,8 & 19.664 & 17,1 & 3.691 & 12,4 & 898 & 8,4 \\
Gesamt & 213.896 & 100 & 114.884 & 100 & 29.670 & 100 & 10.696 & 100 \\
\hline & & & & & & & &
\end{tabular}

Der Anteil des einfachen Diebstahls könnte noch etwas größer sein, denn wie schon in Abschnitt 3.2.1 beschrieben, wird bei der Registrierung von schwerem Diebstahl im Bundeszentralregister, automatisch auch einfacher Diebstahl einge- 
tragen. Diese Fälle des einfachen Diebstahls werden nicht berücksichtigt. Dennoch ist es möglich, dass ein Täter wegen eines schweren und eines einfachen Diebstahls verurteilt wird. Dies ist den Daten nicht zu entnehmen und diese Fälle des einfachen Diebstahls fehlen.

Die nächstgrößten Deliktskategorien bei den Männern nach Verkehrsdelikten und einfachem Diebstahl sind Körperverletzung und Betäubungsmitteldelikte. Bei den deutschen Frauen ist die zweitgrößte Deliktskategorie die der Verkehrsdelikte, dann folgen Betrug und Leistungserschleichung. Bei den nichtdeutschen Frauen ist die zweitgrößte Deliktskategorie Betrug, dann Verkehrsdelikte und Leistungserschleichung (ohne Berücksichtigung von Vergehen gegen das Ausländergesetz und das Asylverfahrensgesetz). Betäubungsmitteldelikte bilden auch bei den deutschen Frauen eine große Deliktskategorie. Fasst man alle Gewaltdelikte ${ }^{177}$ zusammen, bilden auch diese eine große Deliktskategorie. Bei den deutschen Männern mit zwei oder mehr Delikten sind 15\% aller Delikte Gewaltdelikte, bei den nichtdeutschen Männern 16,5\%, bei den deutschen Frauen 10,4\% und bei den nichtdeutschen Frauen 7,3\%. Straftaten innerhalb der speziellen Ausländergesetze bilden bei den Nichtdeutschen eine große Deliktskategorie, $12 \%$ bei den Männern und 10\% bei den Frauen. Die Deliktskategorie schwerer Diebstahl ist im Vergleich zu den polizeilichen Registrierungen in allen Gruppen deutlich kleiner.

Vergleicht man die Häufigkeitsverteilung der Delikte bei Mehrfachstraftätern mit der Häufigkeitsverteilung der Einfachstraftäter, so zeigt sich, dass es bei Mehrfachstraftätern anteilsmäßig deutlich mehr Registrierungen von Gewaltdelikten, schwerem Diebstahl und Betrug gibt und dafür deutlich weniger Registrierungen von einfachem Diebstahl und Verkehrsdelikten.

\subsubsection{Deutsche Männer}

Ebenso wie in Abschnitt 5.2 für polizeilich Registrierte beschrieben, soll hier die Deliktskonfiguration der justiziell registrierten deutschen Männer untersucht werden. Dafür werden zuerst alle individuellen Deliktskombinationen betrachtet.

Tabelle 65 im Anhang zeigt die Häufigkeit der Deliktskombinationen innerhalb der justiziell registrierten kriminellen Karriere deutscher Männer. Die Tabelle sagt nichts aus über die Anzahl der Personen, sondern gibt die Anzahl der Deliktspaare wieder. Bei einer Person sind je nach Deliktsanzahl mehrere Deliktspaare vorhanden. Bei den deutschen Männern mit justizieller Registrierung gibt es etwa 858.000 Deliktspaare, bei den deutschen Männern mit polizeilicher Registrierung sind es 2.400.000 Deliktspaare. Der Unterschied in der Häufigkeit liegt hauptsächlich daran, dass es bei den polizeilichen Registrierungen deutlich mehr Personen gibt mit fünf und mehr Straftaten als bei den justiziellen Registrierungen. Delikte, die in Se-

177 Tötungsdelikte, Sexualdelikte, sonstige Sexualdelikte, Raub, schwere Körperverletzung, Körperverletzung, Straftaten gegen die persönliche Freiheit. 
rie begangen werden, z.B. Serieneinbruch, werden polizeilich mehrfach registriert, justiziell aber meist in einer Entscheidung erledigt.

Das häufigste Deliktspaar bei justiziell Registrierten ist Verkehrsdelikte - Verkehrsdelikte, diese Kombination kommt 72.081-mal vor. Das zweithäufigste Paar ist Verkehrsdelikte - einfacher Diebstahl (57.283), danach kommt einfacher Diebstahl - einfacher Diebstahl (32.182). Häufig kommen auch die Paare schwerer Diebstahl - Verkehrsdelikte, Körperverletzung - Verkehrsdelikte und einfacher Diebstahl - Betäubungsmitteldelikte vor.

Als Ähnlichkeitsmaß bei Delikten werden, wie in Abschnitt 5.1 beschrieben, die Adjusted Standardized Residuals ${ }^{178}$ (ASR) der Deliktspaare benutzt (Tabelle 27). Ein positives Residuum besagt, dass das Deliktspaar häufiger vorkommt als erwartet; bei einem negativen Residuum ist die Häufigkeit des Deliktspaars seltener als erwartet.

Die Adjusted Standardized Residuals der Deliktspaare der gleichen Deliktskategorien miteinander sind alle positiv, mit Ausnahme von Tötungsdelikte - Tötungsdelikte und falsche Verdächtigung - falsche Verdächtigung. Diese Kombinationen kommen nicht, bzw. sehr selten, vor. Besonders groß ist das ASR bei den Kombinationen Leistungserschleichung - Leistungserschleichung und Verkehrsdelikte Verkehrsdelikte. Leistungserschleichung und Verkehrsdelikte werden bei Personen besonders häufig mehrfach registriert. Alle Eigenkombinationen kommen überdurchschnittlich häufig vor, jedes Delikt ist damit mit sich selbst ähnlich. Besonders häufig mehrfach offiziell registriert sind auch Betäubungsmitteldelikte und Betrug.

Bei den Deliktspaaren unterschiedlicher Delikte gibt es große positive ASR bei Körperverletzung - schwere Körperverletzung (80,3), Betrug - Fälschung (37,3), Verkehrsdelikte - fahrlässige Tötung/Körperverletzung (36,7), Straftaten gegen die persönliche Freiheit - Beleidigung (30,4), Körperverletzung - Beleidigung (27,2), Sexualdelikte - sonstige Sexualdelikte $(27,0)$ und Fälschung - Verkehrsdelikte $(26,7)$. Diese Deliktskombinationen kommen deutlich häufiger als erwartet bei Tätern vor. Diese Deliktspaare sind somit besonders ähnlich. Mit der Größe des Wertes kann auch eine Aussage über die Stärke der Ähnlichkeit gemacht werden. Verkehrsdelikte - fahrlässige Tötung/Körperverletzung ist ähnlicher als Verkehrsdelikte - Fälschung, weil das ASR größer ist. Die Kombination Verkehrsdelikte - fahrlässige Tötung/Körperverletzung kommt in der kriminellen Laufbahn von Personen häufiger vor in Relation zu erwartetem Wert als die Kombination Verkehrsdelikte Fälschung.

\footnotetext{
178 Siehe Fußnote 145.
} 
Tabelle 27: Adjusted Standardized Residuals der Deliktspaare (deutsche Männer) ${ }^{179}$

\begin{tabular}{|c|c|c|c|c|c|c|c|c|}
\hline & $\begin{array}{l}\text { Tötungs- } \\
\text { delikte }\end{array}$ & $\begin{array}{c}\text { Sexual- } \\
\text { delikte }\end{array}$ & $\begin{array}{l}\text { sonst. } \\
\text { Sex. }\end{array}$ & Raub & $\begin{array}{l}\text { schw. } \\
\text { KV }\end{array}$ & KV & $\begin{array}{l}\text { Str. gg. } \\
\text { Pers. Fr. }\end{array}$ & $\begin{array}{l}\text { einf. } \\
\text { Dieb. }\end{array}$ \\
\hline Tötungsdelikte & $-0,5$ & & & & & & & \\
\hline Sexualdelikte & 1,7 & 10,5 & & & & & & \\
\hline $\begin{array}{l}\text { sonst. Sexual- } \\
\text { del. }\end{array}$ & $-0,3$ & 27,0 & 54,2 & & & & & \\
\hline Raub & 5,5 & 4,1 & $-0,2$ & 9,8 & & & & \\
\hline schw. KV & 5,3 & 4,3 & $-1,7$ & 15,3 & 19,5 & & & \\
\hline $\begin{array}{l}\text { Körperverlet- } \\
\text { zung }\end{array}$ & 2,5 & 4,7 & $-0,7$ & 12,6 & 80,3 & 30,8 & & \\
\hline $\begin{array}{l}\text { Str. gg. Pers. } \\
\text { Fr. }\end{array}$ & 1,9 & 6,3 & 1,8 & 8,0 & 16,2 & 24,6 & 11,0 & \\
\hline einf. Diebstahl & $-1,4$ & $-1,4$ & 1,2 & 0,6 & $-18,7$ & $-25,7$ & $-13,0$ & 60,1 \\
\hline schw. Diebstahl & $-0,4$ & 1,8 & $-0,1$ & 10,2 & $-8,0$ & $-13,7$ & $-5,3$ & 0,9 \\
\hline Sachbeschäd. & 0,9 & 0,0 & $-0,4$ & $-0,0$ & 9,5 & 14,2 & 6,7 & $-1,8$ \\
\hline gemeingef. Str & 6,3 & 1,6 & $-0,7$ & $-0,3$ & $-1,5$ & $-3,0$ & 0,1 & $-1,6$ \\
\hline Hehlerei & 0,3 & $-0,2$ & $-1,7$ & 0,7 & $-2,7$ & $-5,0$ & $-2,5$ & 7,9 \\
\hline Betrug & $-1,7$ & $-1,9$ & $-0,1$ & $-6,5$ & $-19,8$ & $-25,3$ & $-6,1$ & 17,7 \\
\hline Erschl. v. Leist. & $-1,3$ & $-4,2$ & $-2,4$ & $-3,8$ & $-11,2$ & $-16,2$ & $-7,1$ & 14,3 \\
\hline $\begin{array}{l}\text { gef. dem. } \\
\text { Recht. }\end{array}$ & 0,9 & $-0,6$ & $-0,4$ & 0,4 & 11,8 & 9,8 & 3,9 & $-8,7$ \\
\hline Wid. gg. Staat. & 0,7 & $-1,1$ & $-1,2$ & $-1,0$ & 8,4 & 20,7 & 5,7 & $-12,4$ \\
\hline öff. Ordnung & 1,2 & $-0,9$ & $-0,5$ & 0,1 & 2,5 & 0,4 & 3,0 & $-3,7$ \\
\hline Hausfriedensbr. & $-0,8$ & $-1,9$ & $-1,4$ & 0,4 & 1,2 & 3,1 & 4,7 & 7,2 \\
\hline Beleidigung & $-1,0$ & 1,1 & 1,9 & $-2,7$ & 12,5 & 27,2 & 30,4 & $-15,9$ \\
\hline Fälschung & $-1,1$ & $-1,2$ & $-2,1$ & $-4,9$ & $-14,7$ & $-19,7$ & $-8,2$ & $-1,2$ \\
\hline Meineid & $-0,4$ & $-0,0$ & 0,8 & $-0,9$ & $-0,8$ & $-1,6$ & $-0,6$ & $-2,6$ \\
\hline falsche Verd. & 0,7 & $-0,5$ & 0,7 & $-1,8$ & $-2,9$ & $-3,0$ & 0,4 & 0,9 \\
\hline BTM-Delikte & $-2,2$ & $-4,6$ & $-4,3$ & 1,5 & $-10,9$ & $-15,7$ & $-9,6$ & 18,6 \\
\hline $\begin{array}{l}\text { gef. Eingr. } \\
\text { Str.V. }\end{array}$ & 0,6 & $-0,3$ & $-1,4$ & $-0,3$ & 1,5 & 1,5 & 9,7 & $-5,6$ \\
\hline Vollrausch & 0,9 & $-0,5$ & $-0,5$ & $-2,0$ & 2,4 & 4,1 & 0,3 & $-2,2$ \\
\hline $\begin{array}{l}\text { fahrl. Tö- } \\
\text { tung/KV }\end{array}$ & $-1,4$ & $-1,2$ & $-0,2$ & $-7,8$ & $-7,6$ & 25,3 & $-2,8$ & $-16,3$ \\
\hline Verkehrsdel. & $-2,8$ & $-5,5$ & $-4,4$ & $-17,5$ & $-32,2$ & $-32,9$ & $-19,5$ & $-35,7$ \\
\hline
\end{tabular}

179 Aus Gründen der Übersichtlichkeit wurden die ASR größer oder gleich 10 fett und die ASR kleiner oder gleich 10 kursiv gedruckt. 


\begin{tabular}{|c|c|c|c|c|c|c|c|c|}
\hline & $\begin{array}{l}\text { schw. } \\
\text { Dieb. }\end{array}$ & Sachb. & $\begin{array}{l}\text { gemein- } \\
\text { gef. Str. }\end{array}$ & Hehlerei & Betrug & $\begin{array}{l}\text { Erschl. v. } \\
\text { Leist. }\end{array}$ & $\begin{array}{l}\text { gef. dem. } \\
\text { Recht. }\end{array}$ & $\begin{array}{l}\text { Wid. gg. } \\
\text { Staat. }\end{array}$ \\
\hline schw. Diebstahl & 46,3 & & & & & & & \\
\hline Sachbeschäd. & 6,0 & 17,4 & & & & & & \\
\hline gemeingef. Str. & 4,3 & 7,8 & 11,3 & & & & & \\
\hline Hehlerei & 13,1 & $-2,9$ & $-0,6$ & 5,6 & & & & \\
\hline Betrug & $-5,3$ & $-16,9$ & $-2,2$ & $-0,7$ & 91,1 & & & \\
\hline Erschl. v. Leist. & $-4,9$ & $-3,4$ & $-4,2$ & $-6,8$ & $-2,9$ & 159,6 & & \\
\hline $\begin{array}{l}\text { gef. dem. } \\
\text { Recht. }\end{array}$ & $-4,8$ & 15,2 & 2,5 & $-2,8$ & $-5,8$ & $-2,0$ & 46,4 & \\
\hline Wid. gg. Staat. & $-4,7$ & 8,0 & $-0,0$ & $-3,3$ & $-12,8$ & $-4,4$ & 1,9 & 13,0 \\
\hline öff. Ordnung & $-2,3$ & 4,0 & 5,9 & $-1,1$ & 5,8 & $-2,7$ & 14,8 & 0,8 \\
\hline Hausfriedensbr. & $-2,2$ & 15,7 & $-1,9$ & $-0,8$ & $-8,7$ & 5,1 & 0,7 & 5,2 \\
\hline Beleidigung & $-11,8$ & 14,7 & $-1,3$ & $-4,7$ & $-12,1$ & $-1,1$ & 10,6 & 32,7 \\
\hline Fälschung & 4,5 & $-11,6$ & $-2,4$ & 1,7 & 37,3 & $-8,8$ & $-5,2$ & $-7,7$ \\
\hline Meineid & 0,1 & $-1,7$ & $-0,1$ & 14,2 & 5,9 & $-5,2$ & 0,1 & $-1,1$ \\
\hline falsche Verd. & $-0,2$ & $-0,2$ & 2,3 & 2,3 & 2,5 & $-1,1$ & $-0,4$ & $-1,0$ \\
\hline BTM-Delikte & 2,3 & $-4,9$ & $-4,4$ & 5,4 & $-21,3$ & 5,2 & $-8,1$ & $-5,2$ \\
\hline $\begin{array}{l}\text { gef. Eingr. } \\
\text { Str.V. }\end{array}$ & 0,0 & 2,2 & 11,6 & $-2,7$ & $-1,9$ & $-5,1$ & $-1,9$ & 2,8 \\
\hline Vollrausch & $-3,7$ & 6,0 & 0,9 & $-2,5$ & $-6,2$ & $-2,3$ & 2,6 & 6,0 \\
\hline $\begin{array}{l}\text { fahrl. Tö- } \\
\text { tung/KV }\end{array}$ & $-12,2$ & $-5,8$ & 1,6 & $-3,7$ & $-8,9$ & $-16,1$ & $-3,2$ & $-1,8$ \\
\hline Verkehrsdel. & $-9,8$ & $-22,6$ & 0,0 & $-6,2$ & $-9,4$ & $-53,6$ & $-12,1$ & $-11,9$ \\
\hline
\end{tabular}

\begin{tabular}{lcccccccc}
\hline & öff. Ord. & Hausfr. & Beleidigung & Fälschung & $\begin{array}{c}\text { Mein- } \\
\text { eid }\end{array}$ & $\begin{array}{c}\text { falsche } \\
\text { Verd. }\end{array}$ & $\begin{array}{c}\text { BTM- } \\
\text { Delikte }\end{array}$ & $\begin{array}{c}\text { gef. } \\
\text { Eingr. } \\
\text { Str.V. }\end{array}$ \\
\hline öff. Ordnung & 6,2 & & & & & & & \\
Hausfriedensbr. & $-0,3$ & $\mathbf{2 3 , 0}$ & & & & & & \\
Beleidigung & 2,8 & $\mathbf{1 0 , 9}$ & $\mathbf{2 8 , 1}$ & & & & & \\
Fälschung & $-0,7$ & $-7,7$ & $-11,9$ & $\mathbf{1 1 , 6}$ & & & & \\
Meineid & 0,2 & $-1,4$ & $-0,9$ & 0,5 & 4,6 & & & \\
falsche Verd. & 5,0 & $-1,3$ & 1,0 & 1,9 & 5,5 & $-0,4$ & & \\
BTM-Delikte & $-7,8$ & 2,2 & $-8,7$ & $-10,9$ & 0,6 & 1,0 & $\mathbf{9 9 , 9}$ & \\
gef. Eingr. & $-0,1$ & $-2,7$ & 2,6 & $-1,1$ & $-1,1$ & $-0,1$ & -5, & 2,9 \\
Str.V. & 0,8 & 3,2 & 6,1 & $-5,0$ & $-1,5$ & $-0,3$ & $-3,3$ & 0,5 \\
Vollrausch & $-2,7$ & $-6,3$ & $-4,5$ & $-4,7$ & 0,1 & $-0,8$ & $-10,7$ & 6,8 \\
fahrl. Tö- & $-1,4$ & $-22,0$ & $-26,1$ & $\mathbf{2 6 , 7}$ & $-0,0$ & $-1,3$ & $-33,5$ & 1,9 \\
tung/KV & & & & & & & & \\
Verkehrsdel. & $-1,2,9$ &
\end{tabular}




\begin{tabular}{lccc}
\hline & Vollrausch & $\begin{array}{c}\text { fahrl. Tö- } \\
\text { tung/KV }\end{array}$ & Verkehrsdel. \\
\hline Vollrausch & $\mathbf{2 1 , 8}$ & & \\
fahrl. Tö- & $-1,6$ & $\mathbf{1 5 , 4}$ & \\
tung/KV & $-1,4$ & $\mathbf{3 6 , 7}$ & $\mathbf{1 5 0 , 2}$ \\
Verkehrsdel. & & & \\
\hline
\end{tabular}

Das überdurchschnittlich häufige Auftreten eines Deliktspaares könnte auch dadurch zustande kommen, dass häufig „Tateinheit“ zwischen Delikten besteht. Eine Untersuchung dieses Problems ergab, dass das Ergebnis der Deliktsähnlichkeiten durch „Tateinheit" nicht beeinflusst wird. Näheres dazu wird in Abschnitt 5.3.5 beschrieben. Außerdem kommt es im Bundeszentralregister vor, dass bei bestimmten Straftaten ,automatisch“ mehrere Vorschriften registriert werden. Immer ist dies der Fall bei schwerem Diebstahl; hier wird immer bei $\S 243$ StGB auch $\S 242$ StGB (Diebstahl) registriert. Aber auch bei schwerer Körperverletzung wird häufig, aber nicht immer, auch Körperverletzung registriert. Der angewendeten Vorschrift ist nicht $\mathrm{zu}$ entnehmen, ob es tatsächlich eine schwere Körperverletzung und eine Körperverletzung gab oder ob die einfache Körperverletzung zusätzlich zur schweren Körperverletzung ,automatisch“ in die angewendeten Vorschriften geschrieben wurde. Das ASR des Deliktpaars schwere Körperverletzung - Körperverletzung ist somit sicher etwas zu groß. Da aber alle Entscheidungen einer Person betrachtet werden und zwischen verschiedenen Entscheidungen dieses Problem nicht vorhanden ist, gilt die Aussage, dass schwere Körperverletzung und Körperverletzung ähnlich sind.

Besonders unähnliche Deliktspaare, d. h. Deliktspaare mit großen negativen ASR, sind Verkehrsdelikte - Erschleichen von Leistungen $(-53,6)$, Verkehrsdelikte - einfacher Diebstahl (-35,7), Verkehrsdelikte - Betäubungsmitteldelikte $(-33,5)$, Verkehrsdelikte - Körperverletzung $(-32,9)$, Verkehrsdelikte - schwere Körperverletzung (-32,2), Verkehrsdelikte - Beleidigung (-26,1), einfacher Diebstahl - Körperverletzung $(-25,7)$ und Körperverletzung - Betrug $(-25,3)$. An der Unähnlichkeit von Verkehrsdelikte - einfacher Diebstahl ist zu sehen, dass alleine über die Häufigkeit von Deliktspaaren keine Aussage über die Ähnlichkeit möglich ist, denn dieses Paar ist sehr häufig, weil die Delikte oft vorkommen, aber die Kombination wird noch häufiger erwartet, als sie dann tatsächlich vorhanden ist.

Die Unähnlichkeit von einfachem Diebstahl und Betrug, die sich bei den polizeilich Registrierten zeigt, ist bei den justiziell Registrierten nicht zu beobachten. Dies hängt damit zusammen, dass der Anteil von einfachem Diebstahl bei polizeilichen Registrierungen deutlich höher ist als bei justiziellen Registrierungen. Dies hängt wiederum einerseits damit zusammen, dass einfacher Diebstahl eine leichte Straftat ist und dementsprechend häufig das Verfahren eingestellt wird, wenn keine weiteren Straftaten vorliegen. Andererseits wird einfacher Diebstahl häufig in Serie begangen. Diese Seriendiebstähle werden mehrfach polizeilich registriert, aber justi- 
ziell in einer Verhandlung abgeurteilt. Somit zeigt sich bei Betrachtung der ASR bei polizeilich registriertem einfachem Diebstahl eine hohe Selbstähnlichkeit und eine Unähnlichkeit von einfachem Diebstahl und anderen Delikten. Bei justiziell registriertem einfachem Diebstahl ist die Selbstähnlichkeit nicht so groß und die Unähnlichkeit zu anderen Delikten wird nicht sichtbar.

Die Unähnlichkeit des schweren Diebstahls mit anderen Delikten, die bei den polizeilichen Registrierungen zu sehen ist, zeigt sich bei den justiziellen Registrierungen nicht. Erklärt werden kann dies damit, dass wie schon beim einfachen Diebstahl schwerer Diebstahl (Einbruch) häufig in Serie begangen und dementsprechend häufig polizeilich registriert wird. Justiziell wird diese „Einbruchserie“ innerhalb einer Entscheidung abgehandelt und als ein schwerer Diebstahl gezählt. Somit ist bei polizeilichen Registrierungen das ASR von schwerer Diebstahl schwerer Diebstahl sehr groß und damit die ASR von schwerem Diebstahl mit anderen Deliktskategorien klein. Bei justiziell registriertem schwerem Diebstahl ist die Selbstähnlichkeit nicht so groß wie bei polizeilich registriertem und Ähnlichkeiten mit anderen Deliktskategorien zeigen sich.

Das ASR der Deliktspaare schwerer Diebstahl - Hehlerei, -Raub, - Sachbeschädigung, - Fälschung, - Betäubungsmitteldelikte, - gemeingefährliche Straftaten, Sexualdelikte, - einfacher Diebstahl ist positiv. Diese Deliktspaare sind ähnlich, sie kommen überdurchschnittlich häufig bei Tätern vor. Das ASR vom Deliktspaar schwerer Diebstahl - einfacher Diebstahl ist etwas zu niedrig, weil nicht alle einfachen Diebstähle dieser Kombination erfasst werden können. Das Deliktspaar einfacher Diebstahl - schwerer Diebstahl innerhalb einer justiziellen Entscheidung kommt in diesen Auswertungen nicht vor, obwohl es solche Fälle sicher gibt. Aber innerhalb der kriminellen Karriere einer Person ist diese Kombination bei mehreren Entscheidungen natürlich möglich und hier auch berücksichtigt. Im Gegensatz zu polizeilichen Registrierungen zeigt sich bei justiziellen Registrierungen eine Ähnlichkeit von einfachem und schwerem Diebstahl trotz des Fehlens von einigen Registrierungen von einfachem Diebstahl in dieser Kombination.

Das Ziel ist, Delikte nicht nur paarweise zu vergleichen, sondern eine Darstellung der Deliktskonfiguration zu erhalten. Eine Möglichkeit dies zu erreichen ist, wie schon im Abschnitt der polizeilichen Registrierungen beschrieben, die Methode Multidimensionale Skalierung ${ }^{180}$, wobei als Ähnlichkeitsmaß das ASR benutzt wird.

Abbildung 11 zeigt die Gesamtdarstellung der Deliktskonfiguration als zweidimensionales Ergebnis der Multidimensionalen Skalierung. Wie schon im Abschnitt der Ergebnisse auf Polizeiebene beschrieben, sind in der Darstellung nur die Entfernungen der einzelnen Punkte untereinander von Bedeutung, oben, unten, links,

180 Die Multidimensionale Skalierung wird mit dem SPSS Programm PROXSCAL berechnet. 
rechts spielt keine Rolle. Je näher die Punkte beieinander liegen, desto ähnlicher sind die Delikte, und je größer die Entfernung zwischen den Delikten ist, desto unähnlicher sind die Delikte.

Abbildung 11: Ergebnis der MDS (deutsche Männer) ${ }^{181}$

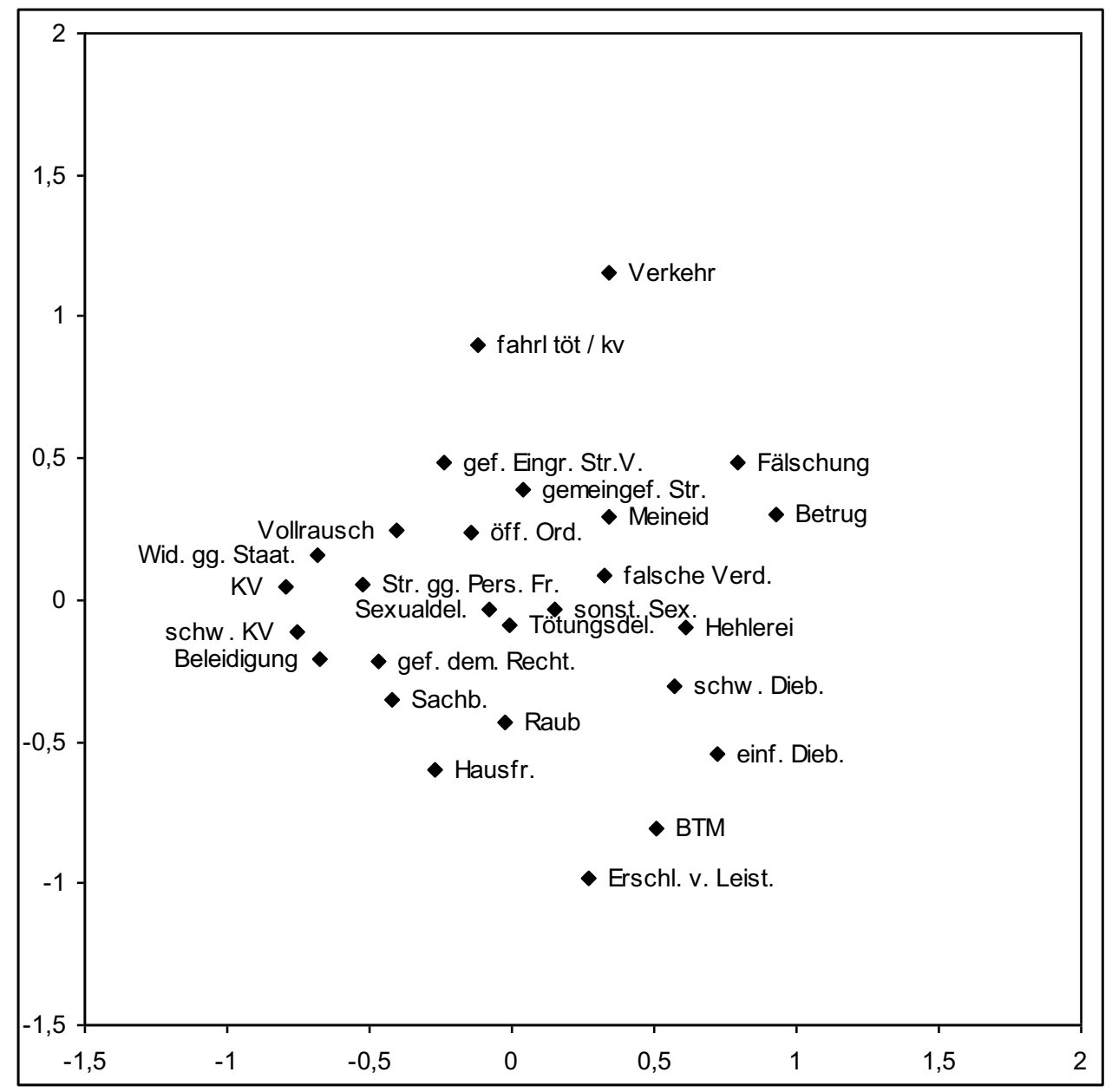

Die Ähnlichkeiten bzw. Unähnlichkeiten, die sich schon in der Tabelle der ASR zeigten, sind hier grafisch abgebildet. Die Unähnlichkeit von Verkehrsdelikten und Erschleichen von Leistungen zeigt sich dadurch, dass die Delikte in der Grafik einander gegenüber liegen, der Abstand ist sehr groß.

Ebenso liegen einfacher Diebstahl und Verkehrsdelikte sowie einfacher Diebstahl und Körperverletzung weit auseinander. Besonders unähnlich sind auch Betrug und Körperverletzung. Nah beieinander liegen Betäubungsmitteldelikte und Erschleichen von Leistungen und auch Betäubungsmitteldelikte und einfacher Diebstahl. Somit zeigt sich der Zusammenhang von Drogendelikten und Beschaf-

181 Ein Abstand von 0,7 Einheiten entspricht einem ASR von Null. 
fungskriminalität. Auch Beleidigung und schwere Körperverletzung liegen nah beieinander. Es zeigt sich, dass alle Gewaltdelikte einschließlich Sexualdelikten relativ nah beieinander liegen und in deren Nähe auch Beleidigung, Widerstand gegen die Staatsgewalt, Vollrausch, Hausfriedensbruch, Gefährdung des demokratischen Rechtsstaates und Sachbeschädigung. Fahrlässige Körperverletzung/Tötung und Gewaltdelikte sind einander nicht ähnlich.

Schwerer Diebstahl liegt in der Nähe von Hehlerei, diese Delikte sind ähnlich. Auch einfacher Diebstahl ist ähnlich zu schwerem Diebstahl, diese Ähnlichkeit zeigte sich bei polizeilichen Registrierungen nicht. Verkehrsdelikte liegen wie erwartet in der Nähe von fahrlässiger Körperverletzung und fahrlässiger Tötung. Ähnlich sind auch die Deliktskategorien „Fälschung“ und Betrug. Wenn eine Person wegen eines Betrugsdeliktes justiziell registriert ist, ist sie auch häufig wegen eines Fälschungsdeliktes registriert.

Die Güte der Darstellung kann mit zwei Werten geprüft werden. Die erklärte Varianz $\mathrm{R}^{2}$ der gesamten Darstellung beträgt 76\%. Das STRESS-Maß ist 0,21. Die Güte der Darstellung entspricht der, der Multidimensionalen Skalierung der polizeilichen Registrierungen. Mit den empirischen und den berechneten Distanzen, die ein Ergebnis der Multidimensionalen Skalierung sind, lässt sich die erklärte Varianz für die einzelnen Deliktskategorien bezogen auf die zweidimensionale Darstellung berechnen.

Tabelle 28: Erklärte Varianz (deutsche Männer) ${ }^{182}$

\begin{tabular}{lclc}
\hline & $\mathrm{R}^{2}$ in $\%$ & & $\mathrm{R}^{2}$ in $\%$ \\
\hline Tötungsdelikte & 60 & Gefährdung. d. dem. Rechtsstaates & $\mathbf{8 2}$ \\
Sexualdelikte & 58 & Widerstand gg. Staatsgewalt & $\mathbf{8 7}$ \\
sonstige Sexualdelikte & 33 & öffentliche Ordnung & 38 \\
Raub & 52 & Hausfriedensbruch & 75 \\
schwere Körperverletzung & $\mathbf{8 9}$ & Beleidigung & $\mathbf{8 2}$ \\
Körperverletzung & $\mathbf{8 4}$ & Fälschung & $\mathbf{9 0}$ \\
Straftaten gg. pers. Freiheit & $\mathbf{8 6}$ & Meineid & 47 \\
einfacher Diebstahl & $\mathbf{8 4}$ & falsche Verdächtigung & 41 \\
schwerer Diebstahl & 63 & BTM-Delikte & 69 \\
Sachbeschädigung & $\mathbf{8 3}$ & Gef. Eingriff i. d. Straßenverkehr & 59 \\
Gemeingefährliche Straftaten & 56 & Vollrausch & 75 \\
Hehlerei & 70 & fahrlässige Tötung/Körperverletzung & 72 \\
Betrug & 67 & Verkehrsdelikte & $\mathbf{9 2}$ \\
Erschl. von Leistungen & 66 & & \\
\hline
\end{tabular}

Die erklärte Varianz für die einzelnen Deliktskategorien ist in Tabelle 28 aufgeführt. Der Wert der erklärten Varianz gibt eine Aussage darüber, wie sicher die Lage des Delikts in der zweidimensionalen Darstellung ist. Von den 27 Deliktskategorien haben 10

182 Die Deliktskategorien, die im Vergleich zur Gesamtdarstellung, eine höhere erklärte Varianz aufweisen, sind in der Tabelle fett gedruckt. 
eine erklärte Varianz größer als die erklärte Varianz der Gesamtdarstellung von 76\%. Nur bei vier Deliktskategorien, sonstige Sexualdelikte, öffentliche Ordnung, Meineid, falsche Verdächtigung, liegt die erklärte Varianz unter 50\%. Besonders ,sonstige Sexualdelikte" sind in der zweidimensionalen Darstellung nicht gut dargestellt. Sehr gute Ergebnisse gibt es bei den meisten Gewaltdelikten, schwere Körperverletzung, Körperverletzung, Straftaten gegen die persönliche Freiheit und auch bei den Delikten, Sachbeschädigung, Beleidigung, Hausfriedensbruch, Gefährdung des demokratischen Rechtsstaates und Widerstand gegen die Staatsgewalt. Sehr gut dargestellt sind auch die Eigentumsdelikte einfacher Diebstahl und „Fälschung“. Ebenfalls sehr gut dargestellt sind Vollrausch und Verkehrsdelikte. Die Werte der erklärten Varianz bestätigen die Ähnlichkeit der Gewaltdelikte miteinander und auch ihre Ähnlichkeit mit Beleidigung und Sachbeschädigung.

Bis auf die Lage von schwerem und einfachem Diebstahl stimmen die Ergebnisse auf justizieller Ebene mit den Ergebnissen auf polizeilicher Ebene überein. Gewaltdelikte liegen eng beieinander und auch Ähnlichkeiten von Gewaltdelikten zu Beleidigung und Sachbeschädigung zeigen sich. Auch bei Sexualdelikten lässt sich ein Zusammenhang zu Gewaltdelikten feststellen. Die Deliktskategorien Fälschung und Betrug sind sich ähnlich. Es zeigt sich ein Zusammenhang von Betäubungsmitteldelikten und den zur Beschaffungskriminalität zählenden Delikten Leistungserschleichung und einfacher Diebstahl. Die Unähnlichkeit von Eigentumsdelikten und Vermögensdelikten, die sich bei polizeilich Registrierten zeigt, wird hier nicht sichtbar. Betrug und Diebstahl werden justiziell auch gemeinsam registriert. Schwerer Diebstahl ist auf polizeilicher Ebene zu allen anderen Delikten unähnlich. Auf justizieller Ebene befindet sich der schwere Diebstahl in der Nähe von Hehlerei; somit zeigt sich die Ähnlichkeit von schwerem Diebstahl und Hehlerei und auch die Ähnlichkeit von schwerem und einfachem Diebstahl wird deutlich.

\subsubsection{Deutsche Frauen}

In diesem Abschnitt wird beschrieben, was die Analyse der Deliktsähnlichkeiten der justiziell registrierten deutschen Frauen ergibt. In Tabelle 66 im Anhang sind die Häufigkeiten der Deliktspaare der deutschen Frauen dargestellt. Insgesamt gibt es 69.000 Deliktspaare, dies sind deutlich weniger als bei den Männern (858.000). Das häufigste Deliktspaar bei den Frauen ist wie auch bei den polizeilich Registrierten einfacher Diebstahl - einfacher Diebstahl (9.114). Das zweithäufigste Paar ist einfacher Diebstahl - Betrug (4.503). Kombinationen mit Verkehrsdelikten kommen nicht so häufig vor wie bei Männern.

Wie schon beschrieben, wird das Adjusted Standardized Residual als Ähnlichkeitsmaß für die Multidimensionale Skalierung benutzt. In Tabelle $29^{183}$ sind die

183 Die Deliktskategorien Sexualdelikte und gefährlicher Eingriff in den Straßenverkehr kommen bei Frauen so selten vor, dass sie hier für weitere Auswertungen nicht verwendet werden 
ASR der Deliktspaare der deutschen Frauen dargestellt. Die Residuen der Diagonale sind, mit Ausnahme der Deliktskategorie „Gefährdung des demokratischen Rechtsstaates“ (diese Eigenkombination kommt bei Frauen kaum vor), alle positiv. D.h. alle Delikte sind mit sich selbst ähnlich. Besonders ähnlich sind die Eigenkombinationen von „sonstigen Sexualdelikten“ und von Leistungserschleichung.

Hohe negative Werte gibt es bei den Kombinationen Erschleichen von Leistungen Verkehrsdelikte $(-18,3)$, einfacher Diebstahl - Verkehrsdelikte $(-13,6)$ und Betrug - Betäubungsmitteldelikte $(-13,3)$, diese Deliktskombinationen sind einander unähnlich.

Starke Ähnlichkeiten zeigen sich bei den Deliktspaaren Körperverletzung - schwere Körperverletzung (35,0), Körperverletzung - fahrlässige Tötung/Körperverletzung $(33,7)$ und Betrug - Fälschung $(25,9)$. Betrug ist zu vielen Delikten unähnlich, außer wie schon erwähnt zur Deliktskategorie Fälschung und auch zu Meineid.

Tabelle 29: Adjusted Standardized Residuals (deutsche Franen) ${ }^{184}$

\begin{tabular}{lrrrrrrrr}
\hline & $\begin{array}{c}\text { Tötungs- } \\
\text { delikte }\end{array}$ & $\begin{array}{c}\text { sonst. } \\
\text { Sex. }\end{array}$ & Raub & schw. KV & KV & $\begin{array}{c}\text { Str. gg. } \\
\text { Pers. Fr. }\end{array}$ & $\begin{array}{c}\text { einf. } \\
\text { Dieb. }\end{array}$ & $\begin{array}{r}\text { schw. } \\
\text { Dieb. }\end{array}$ \\
\hline Tötungsdelikte & 6,7 & & & & & & & \\
sonst. Sexualdel. & $-0,7$ & $\mathbf{4 3 , 8}$ & & & & & & \\
Raub & 4,5 & 0,8 & 2,1 & & & & & \\
schw. KV & 1,3 & $-1,0$ & 6,5 & 3,6 & & & & \\
Körperverl. & $-0,3$ & $-4,2$ & 5,2 & $\mathbf{3 5 , 0}$ & 7,1 & & & \\
Str. gg. Pers. Fr. & 1,8 & $-0,1$ & 5,1 & 7,7 & $\mathbf{1 0 , 2}$ & 3,3 & & \\
einf. Diebstahl & $-1,6$ & $-3,1$ & $-0,6$ & $-7,6$ & $-12,2$ & $-5,4$ & $\mathbf{3 1 , 7}$ & \\
schw. Diebstahl & 0,8 & 0,3 & 3,9 & $-1,3$ & $-1,7$ & 0,2 & 1,0 & 7,5 \\
Sachbeschäd. & $-0,1$ & $-0,6$ & 0,7 & 2,3 & 4,6 & 2,9 & $-3,4$ & 5,5 \\
gemeingef. Str & 2,5 & $-0,9$ & 0,6 & 0,1 & 0,3 & 1,1 & $-1,3$ & $-0,2$ \\
Hehlerei & $-0,0$ & $-2,0$ & $-0,8$ & $-0,6$ & $-1,8$ & $-1,2$ & 1,0 & 3,1 \\
Betrug & 0,8 & $-6,9$ & $-4,5$ & $-7,5$ & $-9,6$ & $-2,8$ & $-2,8$ & $-2,2$ \\
Erschl. v. Leist. & $-1,1$ & 4,6 & $-2,6$ & $-3,4$ & $-7,9$ & $-4,5$ & $-6,1$ & $-3,0$ \\
gef. dem. Recht. & 2,6 & $-0,9$ & $-0,7$ & 1,6 & 1,0 & 0,5 & $-0,4$ & 0,3 \\
Wid. gg. Staat. & $-0,6$ & $-0,5$ & 0,9 & 4,0 & $\mathbf{1 0 , 3}$ & 2,1 & $-6,1$ & $-0,5$ \\
öff. Ordnung & 1,1 & $-0,2$ & 1,2 & $-0,7$ & $-1,0$ & 2,9 & $-2,7$ & 0,4 \\
Hausfriedensbr. & $-0,7$ & $-0,6$ & $-0,2$ & $-0,8$ & $-1,4$ & 1,1 & 0,5 & $-1,0$ \\
Beleidigung & 1,3 & $-1,1$ & 0,8 & 7,1 & $\mathbf{1 5 , 2}$ & 9,4 & $-6,3$ & $-0,9$ \\
Fälschung & $-0,4$ & $-3,7$ & $-2,2$ & $-4,1$ & $-5,4$ & $-2,3$ & $-0,7$ & $-0,5$ \\
Meineid & 0,6 & 0,8 & $-0,5$ & $-1,3$ & $-2,2$ & $-0,2$ & $-1,9$ & $-0,8$ \\
falsche Verd. & 1,4 & 0,2 & $-0,8$ & $-2,0$ & $-1,8$ & 3,7 & 0,1 & $-0,4$ \\
BTM-Delikte & $-1,3$ & 6,5 & 1,9 & $-3,5$ & $-5,5$ & $-3,3$ & 1,7 & 2,9 \\
Vollrausch & $-0,2$ & $-1,2$ & 1,1 & $-0,4$ & 1,9 & 1,5 & $-1,2$ & $-0,3$ \\
fahrl. Tötung/KV & $-0,8$ & $-3,5$ & $-1,9$ & $-1,9$ & $\mathbf{3 3 , 7}$ & $-1,7$ & $-10,8$ & $-3,3$ \\
Verkehrsdel. & $-0,1$ & $-6,7$ & $-3,9$ & $-5,8$ & $-4,9$ & $-2,2$ & $-13,6$ & $-2,6$ \\
\hline & & & & & & & &
\end{tabular}

184 Aus Gründen der Übersichtlichkeit wurden die ASR größer oder gleich 10 fett und die ASR kleiner oder gleich 10 kursiv gedruckt. 


\begin{tabular}{|c|c|c|c|c|c|c|c|c|c|c|c|c|}
\hline & Sachb. & \multicolumn{2}{|c|}{$\begin{array}{l}\text { Gemein- } \\
\text { gef. Str. }\end{array}$} & \multicolumn{2}{|c|}{ Hehlerei } & \multicolumn{2}{|c|}{ Betrug } & \multicolumn{2}{|c|}{$\begin{array}{l}\text { Erschl. } \\
\text { v. Leist. }\end{array}$} & $\begin{array}{l}\text { gef. dem. } \\
\text { Recht. }\end{array}$ & \multirow[t]{2}{*}{$\begin{array}{l}\text { Wid. gg. } \\
\text { Staat. }\end{array}$} & \multirow[t]{2}{*}{$\begin{array}{l}\text { öff. } \\
\text { Ord. }\end{array}$} \\
\hline Sachbeschäd. & 3, & & & & & & & & & & & \\
\hline gemeingef. Str & 1, & 4,1 & & & & & & & & & & \\
\hline Hehlerei & -1, & 0,2 & & 0,8 & & & & & & & & \\
\hline Betrug & -4, & $-1,4$ & & $-2,0$ & & 38 & & & & & & \\
\hline Erschl. v. Leist. & $-0, \varepsilon$ & $-0,7$ & & $-2,8$ & & -12 & & 61 , & & & & \\
\hline gef. dem. Recht. & 4, & 2,0 & & 0,2 & & -1, & & -1 , & & $-0,2$ & & \\
\hline Wid. gg. Staat. & 2, & 0,0 & & $-0,4$ & & -5 & & -2 & & 0,8 & 3,7 & \\
\hline öff. Ordnung & 0, & 0,6 & & $-0,0$ & & 1 , & & -2 & & 4,9 & $-0,6$ & 5,8 \\
\hline Hausfriedensbr. & 6, & 1,0 & & 1,7 & & -2 & & 1 , & & 0,5 & 0,2 & 1,4 \\
\hline Beleidigung & 7, & 1,3 & & $-0,2$ & & -6 , & & -4 & & 3,8 & 16,4 & 1,9 \\
\hline Fälschung & -3, & $-1,5$ & & $-0,8$ & & 25 , & & -4 & & $-1,1$ & $-2,4$ & 0,4 \\
\hline Meineid & $-1,2$ & 0,3 & & 9,8 & & 3 , & & -3 & & $-0,6$ & $-1,2$ & 0,6 \\
\hline falsche Verd. & $-0,2$ & 0,1 & & 1,3 & & -0 & & 0 , & & $-0,6$ & 1,0 & 2,6 \\
\hline BTM-Delikte & -1, & 0,5 & & 4,1 & & -13 & & -2 , & & $-1,7$ & $-1,0$ & $-2,5$ \\
\hline Vollrausch & 5, & $-0,3$ & & $-1,2$ & & -2 & & -0, & & 1,5 & 3,6 & 0,7 \\
\hline fahrl. Tötung/KV & -2, & 0,5 & & $-2,5$ & & -4 & & -8 & & $-1,0$ & $-0,9$ & $-1,2$ \\
\hline \multirow[t]{2}{*}{ Verkehrsdel. } & -1, & 1,2 & & 0,0 & & -0 & & -18 & & $-1,1$ & $-1,1$ & 2,4 \\
\hline & Hausfr. & $\begin{array}{l}\text { Beleidi- } \\
\text { gung }\end{array}$ & $\begin{array}{r}\text { Fä } \\
\text { schl }\end{array}$ & $\begin{array}{l}1- \\
\text { ang }\end{array}$ & Mein & & $\begin{array}{r}\text { fals } \\
\mathrm{Ve}\end{array}$ & & $\begin{array}{l}\text { BTM- } \\
\text { Delikte }\end{array}$ & $\begin{array}{c}\text { Voll- } \\
\text { e rausch }\end{array}$ & $\begin{array}{c}\text { fahrl. } \\
\text { Tö- } \\
\text { tung/KV }\end{array}$ & $\begin{array}{c}\text { Ver- } \\
\text { kehrs- } \\
\text { del. }\end{array}$ \\
\hline Hausfriedensbr. & 5,4 & & & & & & & & & & & \\
\hline Beleidigung & 2,2 & 8,2 & & & & & & & & & & \\
\hline Fälschung & $-1,9$ & $-4,4$ & 2,1 & & & & & & & & & \\
\hline Meineid & $-0,3$ & $-0,9$ & $-1,3$ & & 1,3 & & & & & & & \\
\hline falsche Verd. & 0,8 & 1,3 & $-0,9$ & & 3,8 & & 0 , & & & & & \\
\hline BTM-Delikte & 0,2 & $-2,5$ & $-3,9$ & & 0,6 & & -1 , & & 29,1 & & & \\
\hline Vollrausch & 1,8 & 3,1 & $-1,4$ & & $-0,7$ & & 0 , & & $-0,7$ & 2,3 & & \\
\hline $\begin{array}{l}\text { fahrl. Tö- } \\
\text { tung/KV }\end{array}$ & $-2,5$ & $-2,3$ & $-3,6$ & & $-0,7$ & & -1 & & $-4,9$ & $-0,5$ & 7,7 & \\
\hline Verkehrsdel. & $-3,0$ & $-5,0$ & $-1,4$ & & 2,4 & & -0 & & $-4,5$ & $-0,3$ & 20,1 & 54,1 \\
\hline
\end{tabular}

Zur Darstellung der Deliktskonfiguration wird auch für die deutschen Frauen eine Multidimensionale Skalierung durchgeführt. Abbildung 12 zeigt die zweidimensionale Darstellung der Deliktskonfiguration der deutschen Frauen. Die Unähnlichkeiten, die sich in den Residuen zeigten, zeigen sich hier optisch. Erschleichen von Leistungen und Verkehrsdelikte liegen jeweils am anderen Ende der Abbildung und haben demzufolge keine Ähnlichkeit miteinander. Ebenso unähnlich sind einfacher Diebstahl und Körperverletzung. 
Abbildung 12: Ergebnis der MDS (deutsche Frauen) $)^{185}$

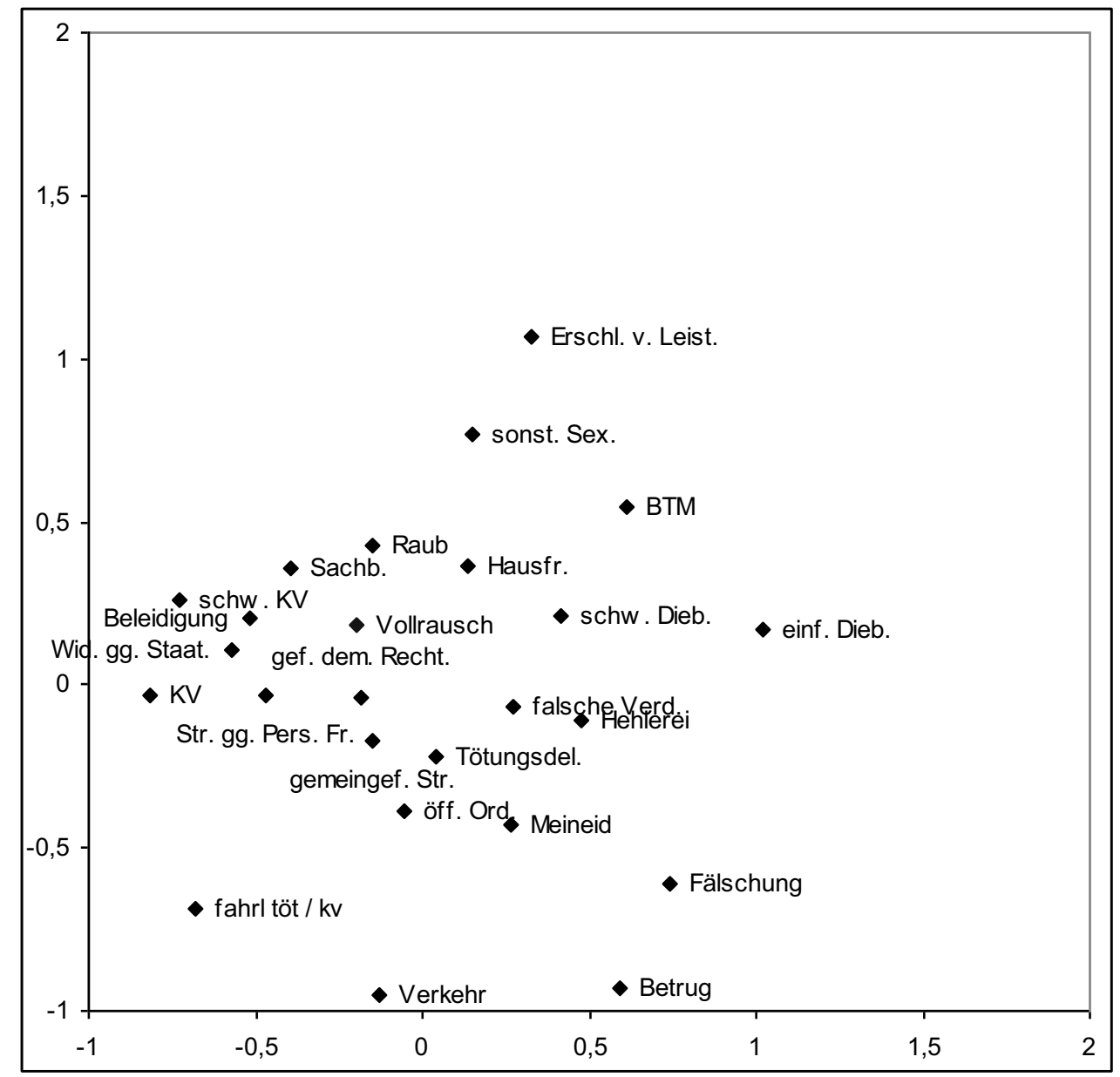

Ähnlichkeiten zeigen sich bei den Delikten fahrlässige Tötung/Körperverletzung Verkehrsdelikte, Betrug - Fälschung, sonstige Sexualdelikte (verbotene Prostitution) Erschleichen von Leistungen und auch sonstige Sexualdelikte - Betäubungsmitteldelikte. Ähnlich sind schwerer Diebstahl und Hehlerei und auch einfacher Diebstahl und Betäubungsmitteldelikte zeigen Ähnlichkeiten zu diesen Delikten. Ähnlichkeiten zeigen sich auch für schwere Körperverletzung, Beleidigung, Widerstand gegen die Staatsgewalt, Körperverletzung, Straftaten gegen die persönliche Freiheit und auch Sachbeschädigung. Auch Sachbeschädigung, Vollrausch, Hausfriedensbruch und Raub sind einander ähnlich. Die Deliktsähnlichkeiten entsprechen großteils denen der Männer. Der deutlichste Unterschied zwischen Männern und Frauen wird bei „,sonstigen Sexualdelikten“ deutlich. Dies liegt daran, dass sich darunter bei Männern und Frauen verschiedene Delikte verbergen. Bei den Frauen sind ,sonstige Sexualdelikte“ hauptsächlich verbotene Prostitution, bei den Männern sexueller Missbrauch. Bei den Frauen zeigen sich Ähnlichkeiten zwischen verbotener Prostitution, Betäubungsmitteldelikten und auch Leistungserschleichung und somit der Zusammenhang zwischen Dogendelikten, Beschaf-

185 Ein Abstand von 0,7 Einheiten entspricht einem ASR von Null. 
fungsprostitution und Beschaffungskriminalität. Bei den Männern zeigen sich Ähnlichkeiten zwischen sexuellem Missbrauch (sonstige Sexualdelikte) und Vergewaltigung/sexuelle Nötigung (Sexualdelikte). Sexualdelikte kommen bei Frauen kaum vor.

Im Vergleich zu den Deliktsähnlichkeiten nach polizeilichen Registrierungen zeigen sich wie schon bei den Männern erwähnt Unterschiede bei schwerem und einfachem Diebstahl.

Die Ähnlichkeiten von schwerer Körperverletzung, Beleidigung, Sachbeschädigung und Widerstand gegen die Staatsgewalt zeigt sich sowohl bei polizeilichen wie bei justiziellen Registrierungen.

Die erklärte Varianz der Gesamtdarstellung ist mit $74 \%$ etwa so gut wie bei den Männern. Der STRESS-Wert beträgt 0,21. Die Beurteilungskriterien geben einen Hinweis darauf, dass die zweidimensionale Darstellung insgesamt betrachtet gut ist. Die erklärte Varianz der einzelnen Deliktskategorien (Tabelle 30) zeigt, welche Deliktskategorie wie gut dargestellt ist. Bei 11 von 25 Kategorien ist die erklärte Varianz überdurchschnittlich gut im Vergleich zur Gesamtdarstellung. Bei den Deliktskategorien Meineid und falsche Verdächtigung ist die erklärte Varianz kleiner als 50\%. Diese Deliktskategorien werden bei Frauen sehr selten registriert. Gut dargestellt sind „,sonstige Sexualdelikte“, schwere Körperverletzung, Körperverletzung, Straftaten gegen die persönliche Freiheit, einfacher Diebstahl, Sachbeschädigung, Betrug, Widerstand gegen die Staatsgewalt, Beleidigung, Fälschung, Betäubungsmitteldelikte Vollrausch, fahrlässige Tötung/ Körperverletzung und Verkehrsdelikte.

Tabelle 30: Erklärte Varianz (deutsche Frauen) ${ }^{186}$

\begin{tabular}{lclc}
\hline & $\mathrm{R}^{2}$ in $\%$ & & $\mathrm{R}^{2}$ in \% \\
\hline Tötungsdelikte & 51 & Gefährdung. d. dem. Rechtsstaates & 68 \\
sonstige Sexualdelikte & 68 & Widerstand gg. Staatsgewalt & $\mathbf{8 9}$ \\
Raub & 53 & öffentliche Ordnung & 54 \\
schwere Körperverletzung & $\mathbf{8 8}$ & Hausfriedensbruch & 54 \\
Körperverletzung & $\mathbf{9 1}$ & Beleidigung & $\mathbf{8 7}$ \\
Straftaten gg. pers. Freiheit & $\mathbf{8 1}$ & Fälschung & $\mathbf{8 0}$ \\
einfacher Diebstahl & $\mathbf{8 4}$ & Meineid & 47 \\
schwerer Diebstahl & 56 & falsche Verdächtigung & 32 \\
Sachbeschädigung & $\mathbf{8 7}$ & BTM-Delikte & 69 \\
Gemeingefährliche Straftaten & 58 & Vollrausch & 65 \\
Hehlerei & 57 & fahrlässige Tötung/Körperverletzung & $\mathbf{8 2}$ \\
Betrug & $\mathbf{8 9}$ & Verkehrsdelikte & $\mathbf{8 4}$ \\
Erschl. von Leistungen & 61 & & \\
\hline
\end{tabular}

Das Ergebnis der zweidimensionalen Darstellung der Multidimensionalen Skalierung zeigt, dass wie schon bei den Männern und bei den polizeilich registrierten

186 Die Deliktskategorien, die im Vergleich zur Gesamtdarstellung, eine höhere erklärte Varianz aufweisen, sind in der Tabelle fett gedruckt. 
Frauen auch bei den justiziell registrierten Frauen insbesondere Gewaltdelikte ${ }^{187}$ und auch Beleidigung und Sachbeschädigung ähnlich zueinander sind. Diese Delikte kommen häufig gemeinsam innerhalb der kriminellen Karriere von Personen vor. Es zeigt sich auch ein Zusammenhang von Betäubungsmitteldelikten und den zur Beschaffungskriminalität zählenden Delikten Raub, Diebstahl und Hausfriedensbruch. Eine gewisse Ähnlichkeit zeigt sich bei den Frauen auch zwischen Betäubungsmitteldelikten und verbotener Prostitution (sonstige Sexualdelikte). Besonders unähnlich zu anderen Delikten ist Betrug mit Ausnahme der Deliktsähnlichkeit von Betrug und „Fälschung“. Auch Straßenverkehrsdelikte sind unähnlich zu allen anderen Delikten mit Ausnahme der Ähnlichkeit zu fahrlässiger Körperverletzung und fahrlässiger Tötung.

\subsubsection{Nichtdeutsche Männer}

Wie schon für die anderen Gruppen, werden in diesem Abschnitt die Deliktsähnlichkeiten der nichtdeutschen Männer dargestellt. ${ }^{188}$ Tabelle 67 im Anhang zeigt die Häufigkeit der Deliktskombinationen innerhalb der justiziell registrierten kriminellen Karriere nichtdeutscher Männer. Bei den nichtdeutschen Männern sind knapp 370.000 Deliktspaare vorhanden, darunter befinden sich mehr als 62.000 Deliktskombinationen mit Vergehen gegen das Ausländergesetz oder Asylverfahrensgesetz. Bei den deutschen Männern sind es 858.000 Deliktspaare.

Das häufigste Deliktspaar ist Verkehrsdelikte - Verkehrsdelikte; diese Kombination kommt 19.511-mal vor. Das zweithäufigste Paar ist einfacher Diebstahl - einfacher Diebstahl (18.448), gleich danach folgt einfacher Diebstahl - Verkehrsdelikte (18.218). Häufig kommen auch weitere Kombinationen mit einfachem Diebstahl vor, darunter besonders häufig das Deliktspaar einfacher Diebstahl - AuslG (12.809). Auch das Deliktspaar AuslG -AuslG (10.381) kommt häufig vor.

Die Adjusted Standardized Residuals der nichtdeutschen Männer sind in Tabelle 31 dargestellt. Die Residuen der Deliktspaare der gleichen Deliktskategorien miteinander sind alle positiv. Das zeigt, dass Kombinationen gleicher Delikte überzufällig häufig auftreten. Besonders häufig sind insbesondere die Eigenkombinationen von Vergehen gegen das Ausländergesetz oder Asylverfahrensgesetz und von Verkehrsdelikten.

Für die Untersuchung der Deliktskonfiguration sind die Residuen von verschiedenen Delikten von Bedeutung. Auffällig sind die negativen Residuen bei Kombinationen mit Vergehen gegen das Ausländergesetz oder Asylverfahrensgesetz. Vergehen gegen das Ausländergesetz oder Asylverfahrensgesetz sind zu fast allen Delikten unähnlich, eine Ausnahme bilden die Deliktskategorien „Fälschung“ und

187 Sexualdelikte kommen bei den Frauen kaum vor. Zu sonstigen Sexualdelikten gehört bei den Frauen hauptsächlich verbotene Prostitution und ist somit kein Gewaltdelikt.

188 Die Kombinationen von ,Gefährdung des demokratischen Rechtsstaates“ mit anderen Delikten kommen so selten vor, dass sie hier für weitere Auswertungen nicht benutzt werden. 
einfacher Diebstahl. „Fälschung“ und einfacher Diebstahl wird häufig bei Personen registriert, die wegen Vergehen gegen das Ausländergesetz oder Asylverfahrensgesetz verurteilt sind.

Den größten positiven Wert nimmt das Residuum beim Deliktspaar schwere Körperverletzung - Körperverletzung $(64,3)$ ein, danach folgt Betrug - Fälschung $(25,4)$ und der nächstgrößte Wert ist bei Beleidigung - Straftaten gegen die persönliche Freiheit (24,6). Diese Deliktspaare sind besonders ähnlich. Besonders unähnlich sind die Deliktspaare Vergehen gegen das Ausländergesetz oder Asylverfahrensgesetz - Körperverletzung $(-32,8)$, einfacher Diebstahl - Verkehrsdelikte $(-28,9)$ und Erschleichen von Leistungen - Verkehrsdelikte $(-26,5)$.

Tabelle 31: Adjusted Standardized Residuals (nichtdeutsche Männer)189

\begin{tabular}{lcrrrrrrr}
\hline & $\begin{array}{c}\text { Tötungs- } \\
\text { delikte }\end{array}$ & $\begin{array}{c}\text { Sexual- } \\
\text { delikte }\end{array}$ & $\begin{array}{c}\text { sonst. } \\
\text { Sex. }\end{array}$ & Raub & $\begin{array}{r}\text { schw. } \\
\text { KV }\end{array}$ & KV & $\begin{array}{c}\text { Str. gg. } \\
\text { Pers. Fr. }\end{array}$ & $\begin{array}{c}\text { einf. } \\
\text { Dieb. }\end{array}$ \\
\hline Tötungsdelikte & 5,1 & & & & & & & \\
Sexualdelikte & 2,6 & 1,6 & & & & & & \\
sonst. Sexualdel. & 0,9 & $\mathbf{1 4 , 5}$ & $\mathbf{1 8 , 9}$ & & & & & \\
Raub & 4,4 & 2,2 & 0,1 & 9,9 & & & & \\
schw. KV & 6,1 & 2,8 & 1,1 & $\mathbf{1 7 , 0}$ & 9,3 & & & \\
Körperverletzung & 3,5 & 6,2 & 2,4 & $\mathbf{1 5 , 4}$ & $\mathbf{6 4 , 3}$ & $\mathbf{1 7 , 9}$ & & \\
Str. gg. Pers. Fr. & 0,3 & 6,9 & 3,5 & 6,0 & $\mathbf{1 4 , 4}$ & $\mathbf{2 4 , 5}$ & 9,2 & \\
einf. Diebstahl & $-2,1$ & $-2,5$ & $-0,6$ & $-3,6$ & $-17,8$ & $-22,4$ & $-13,9$ & $\mathbf{6 6 , 8}$ \\
schw. Diebstahl & $-0,5$ & 0,9 & $-1,0$ & $\mathbf{1 0 , 2}$ & $-3,2$ & $-5,5$ & $-5,5$ & $-3,7$ \\
Sachbeschäd. & 0,0 & 0,2 & 0,1 & 0,5 & 7,7 & $\mathbf{1 2 , 2}$ & 7,8 & $-5,8$ \\
gemeingef. Str. & 2,3 & 0,5 & $-1,0$ & 1,5 & 1,4 & 1,0 & 0,7 & $-4,3$ \\
Hehlerei & $-0,5$ & $-0,5$ & $-0,1$ & 1,7 & $-1,1$ & $-2,5$ & $-2,5$ & 5,4 \\
Betrug & $-2,0$ & $-0,6$ & $-1,0$ & $-2,7$ & $-9,5$ & $-9,3$ & $-3,8$ & 3,4 \\
Erschl. v. Leist. & $-1,2$ & $-2,5$ & $-0,7$ & $-3,5$ & $-9,5$ & $-10,9$ & $-6,2$ & $\mathbf{1 0 , 8}$ \\
Wid. gg. Staat. & $-0,1$ & $-0,0$ & $-1,3$ & $-1,5$ & 3,1 & $\mathbf{1 3 , 4}$ & 5,4 & $-9,1$ \\
öff. Ordnung & 0,7 & $-0,1$ & 0,2 & $-1,4$ & 2,3 & 1,7 & 2,7 & $-3,6$ \\
Hausfriedensbr. & $-0,8$ & $-0,3$ & 1,5 & $-1,4$ & $-0,5$ & 1,2 & 5,1 & 6,7 \\
Beleidigung & $-0,3$ & 0,1 & 2,3 & 0,1 & 8,0 & $\mathbf{2 1 , 5}$ & $\mathbf{2 4 , 6}$ & $-13,8$ \\
Fälschung & $-1,2$ & $-1,6$ & $-2,1$ & $-6,0$ & $-12,6$ & $-15,6$ & $-7,2$ & $-0,7$ \\
Meineid & $-1,1$ & $-0,7$ & $-0,2$ & $-1,2$ & 1,4 & 1,4 & 0,1 & $-4,2$ \\
falsche Verd. & $-0,8$ & $-0,9$ & 0,3 & 0,2 & $-0,1$ & 0,5 & 2,6 & $-3,1$ \\
BTM-Delikte & $-0,3$ & $-1,8$ & $-0,4$ & 5,9 & $-4,7$ & $-7,9$ & $-6,9$ & $-6,3$ \\
gef. Eingr. Str.V. & $-0,9$ & 1,0 & 0,1 & 0,6 & 1,9 & 2,3 & 7,6 & $-6,1$ \\
AuslG & $-1,7$ & $-5,0$ & $-4,9$ & $-18,6$ & $-24,7$ & $-32,8$ & $-16,4$ & $\mathbf{1 0 , 5}$ \\
Vollrausch & $-0,7$ & 0,6 & $-0,4$ & $-1,4$ & 1,5 & 4,1 & 0,8 & $-1,0$ \\
fahrl. Tötung/KV & 0,8 & 0,1 & 1,0 & $-3,8$ & $-2,1$ & $\mathbf{1 5 , 4}$ & $-0,7$ & $-11,2$ \\
Verkehrsdel. & $-1,7$ & $-2,7$ & $-2,8$ & $-12,2$ & $-14,9$ & $-15,7$ & $-7,9$ & $-28,9$ \\
\hline & & & & & & & &
\end{tabular}

189 Aus Gründen der Übersichtlichkeit wurden die ASR größer oder gleich 10 fett und die ASR kleiner oder gleich 10 kursiv gedruckt. 


\begin{tabular}{lrrrrrrrr}
\hline & $\begin{array}{l}\text { schw. } \\
\text { Dieb. }\end{array}$ & Sachb. & $\begin{array}{c}\text { Gemein- } \\
\text { gef. Str. }\end{array}$ & Hehlerei & Betrug & $\begin{array}{c}\text { Erschl. v. } \\
\text { Leist. }\end{array}$ & $\begin{array}{c}\text { Wid. gg. } \\
\text { Staat. }\end{array}$ & $\begin{array}{c}\text { öff. } \\
\text { Ord. }\end{array}$ \\
\hline schw. Diebstahl & $\mathbf{4 0 , 1}$ & & & & & & & \\
Sachbeschäd. & 4,3 & 9,8 & & & & & & \\
gemeingef. Str & 4,8 & 4,5 & 0,4 & & & & & \\
Hehlerei & $\mathbf{1 2 , 8}$ & $-0,5$ & $-0,0$ & 5,6 & & & & \\
Betrug & $-1,4$ & $-3,0$ & $-0,6$ & 0,1 & $\mathbf{3 4 , 1}$ & & & \\
Erschl. v. Leist. & $-3,7$ & $-2,4$ & $-1,5$ & $-4,7$ & 3,7 & $\mathbf{7 3 , 9}$ & & \\
Wid. gg. Staat. & $-2,7$ & 7,0 & 2,8 & $-3,2$ & $-5,2$ & $-0,8$ & 9,7 & \\
öff. Ordnung & $-0,7$ & 3,3 & 1,5 & $-0,1$ & 4,6 & $-1,2$ & 1,4 & 3,1 \\
Hausfriedensbr. & $-1,5$ & 8,2 & $-1,4$ & $-1,7$ & $-5,5$ & 3,2 & 2,9 & $-0,5$ \\
Beleidigung & $-4,9$ & 9,6 & $-0,4$ & $-2,7$ & $-4,4$ & $-1,3$ & $\mathbf{1 7 , 7}$ & 0,4 \\
Fälschung & $-0,3$ & $-6,5$ & 0,0 & $-0,9$ & $\mathbf{2 5 , 4}$ & $-3,3$ & $-3,7$ & $-0,3$ \\
Meineid & 0,4 & 0,1 & 0,2 & $\mathbf{1 1 , 4}$ & 4,1 & $-3,1$ & $-2,3$ & 0,2 \\
falsche Verd. & 1,2 & 0,7 & 0,6 & 2,4 & 2,4 & $-2,1$ & $-1,7$ & 3,4 \\
BTM-Delikte & 4,1 & $-1,2$ & $-1,5$ & 4,2 & $-6,6$ & 5,9 & 0,9 & $-1,4$ \\
gef. Eingr. Str.V. & 0,7 & 2,9 & $\mathbf{1 4 , 6}$ & $-0,7$ & $-0,5$ & $-4,6$ & 2,3 & 0,6 \\
AuslG & $-20,3$ & $-17,3$ & $-5,4$ & $-10,4$ & $-17,0$ & $-2,7$ & $-5,6$ & $-7,8$ \\
Vollrausch & $-0,7$ & 3,6 & $-0,2$ & $-0,5$ & $-1,6$ & $-1,5$ & 3,2 & $-0,2$ \\
fahrl. Tötung/KV & $-5,2$ & $-2,3$ & 1,6 & $-1,6$ & $-0,9$ & $-7,3$ & $-1,0$ & 1,4 \\
Verkehrsdel. & $-2,4$ & $-7,3$ & 0,3 & $-2,9$ & 0,8 & $-26,5$ & $-6,9$ & 2,5 \\
\hline
\end{tabular}

\begin{tabular}{|c|c|c|c|c|c|c|c|c|}
\hline & Hausfr. & $\begin{array}{l}\text { Beleidi- } \\
\text { gung }\end{array}$ & Fälschung & Meineid & $\begin{array}{l}\text { falsche } \\
\text { Verd. }\end{array}$ & $\begin{array}{l}\text { BTM- } \\
\text { Delikte }\end{array}$ & $\begin{array}{c}\text { gef. Eingr. } \\
\text { Str.V. }\end{array}$ & AuslG \\
\hline Hausfriedensbr. & 15,8 & & & & & & & \\
\hline Beleidigung & 5,5 & 14,6 & & & & & & \\
\hline Fälschung & $-4,7$ & $-8,8$ & 6,9 & & & & & \\
\hline Meineid & $-2,2$ & $-1,3$ & $-0,2$ & 2,9 & & & & \\
\hline falsche Verd. & $-0,9$ & 0,1 & 0,2 & 5,2 & 3,0 & & & \\
\hline BTM-Delikte & $-0,1$ & $-1,6$ & $-6,8$ & 2,7 & 0,2 & 72,6 & & \\
\hline gef. Eingr. Str.V. & $-1,4$ & 1,5 & $-0,7$ & $-0,2$ & 1,6 & $-3,6$ & 0,3 & \\
\hline AuslG & $-4,3$ & $-18,8$ & 14,5 & $-5,2$ & $-5,3$ & $-16,2$ & $-6,4$ & 164,6 \\
\hline Vollrausch & 1,0 & 2,4 & $-1,7$ & $-1,1$ & $-0,7$ & $-0,5$ & $-0,4$ & $-4,3$ \\
\hline fahrl. Tötung/KV & $-3,0$ & $-0,2$ & $-4,3$ & 1,8 & 0,8 & $-5,5$ & 2,6 & $-10,4$ \\
\hline Verkehrsdel. & $-10,4$ & $-8,2$ & 14,3 & 0,6 & 1,8 & $-17,7$ & 4,8 & $-22,0$ \\
\hline
\end{tabular}

\begin{tabular}{lccc}
\hline & $\begin{array}{c}\text { Voll- } \\
\text { rausch }\end{array}$ & $\begin{array}{c}\text { fahrl. Tö- } \\
\text { tung/KV }\end{array}$ & Verkehrsdel. \\
\hline Vollrausch & 3,3 & & \\
fahrl. Tötung/KV & $-1,4$ & $\mathbf{1 0 , 1}$ & \\
Verkehrsdel. & 0,9 & $\mathbf{2 2 , 4}$ & $\mathbf{9 7 , 9}$ \\
\hline
\end{tabular}


Abbildung 13: Ergebnis der MDS (nichtdeutsche Männer) ${ }^{190}$

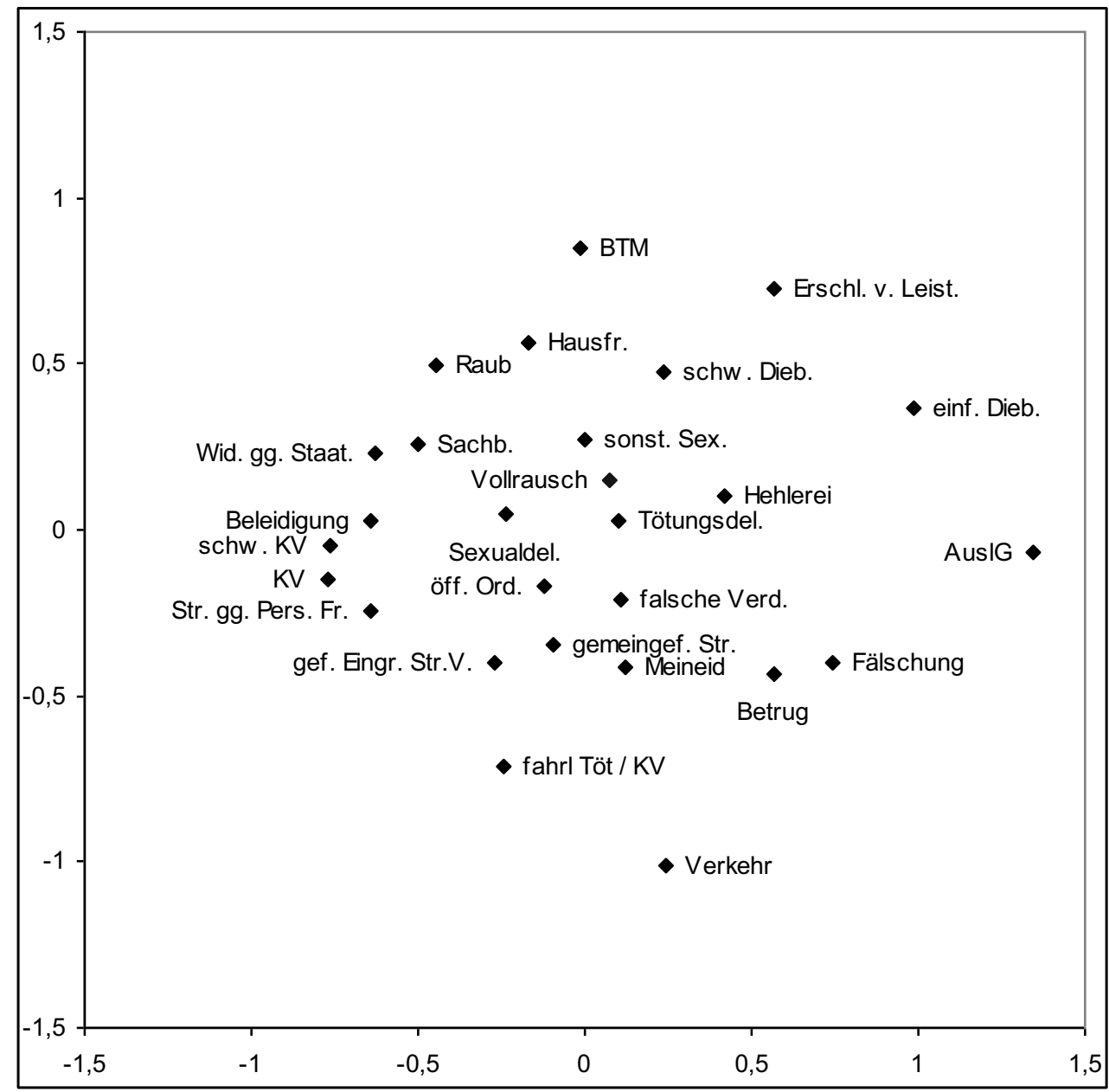

Abbildung 13 zeigt das Ergebnis der zweidimensionalen Multidimensionalen Skalierung. Je näher die Delikte beieinander liegen, desto ähnlicher sind sie. Ähnlichkeiten zeigen sich auch hier bei den Gewaltdelikten (einschließlich Sexualdelikten) untereinander sowie auch mit Beleidigung, Widerstand gegen die Staatsgewalt und Sachbeschädigung. Vergehen gegen das Ausländergesetz oder Asylverfahrensgesetz sind zu vielen anderen Delikten unähnlich. Besonders unähnlich sind Gewaltdelikte und Ausländervergehen. Auch hier zeigt sich die Ähnlichkeit von Vergehen gegen das Ausländergesetz oder Asylverfahrensgesetz und „Fälschung“, aber auch die Ähnlichkeit von einfachem Diebstahl und Vergehen gegen Ausländergesetze. Verkehrsdelikte befinden sich in einer Randlage und sind somit zu vielen Delikten unähnlich. Ähnlich sind Verkehrsdelikte und fahrlässige Tötung/Körperverletzung. Betrug und „Fälschung“ liegen sehr nah beieinander; damit sind diese Delikte ähnlich, was heißt, dass diese Kombination oft in der kriminellen Karriere einer Person vorkommt.

190 Ein Abstand von 0,7 Einheiten entspricht einem ASR von Null. 
Die erklärte Varianz der Gesamtdarstellung beträgt 72\%. Der STRESS-Wert ist 0,23. Damit ist die Güte dieser zweidimensionalen Darstellung ähnlich der der deutschen Männer.

Tabelle 32 zeigt die erklärte Varianz der einzelnen Deliktskategorien. Von den 27 Deliktskategorien haben 11 eine erklärte Varianz größer als die erklärte Varianz der Gesamtdarstellung von $72 \%$. Bei 7 Deliktskategorien ist die erklärte Varianz kleiner 50\%. Wobei nur die erklärte Varianz von Tötungsdelikten mit 28\% und von Vollrausch mit $26 \%$ gering ist. Besonders gut ist die erklärte Varianz von Gewaltdelikten (mit Ausnahme von Tötungsdelikten und „sonstigen Sexualdelikten“), Widerstand gegen die Staatsgewalt, Sachbeschädigung, Beleidigung, Fälschung, einfachem Diebstahl, Betäubungsmitteldelikten, Verkehrsdelikten, gefährlichem Eingriff in den Straßenverkehr und fahrlässiger Tötung/Körperverletzung.

Tabelle 32: Erklärte Varianz (nichtdeutsche Männer) ${ }^{191}$

\begin{tabular}{lclc}
\hline & $\mathrm{R}^{2}$ in $\%$ & & $\mathrm{R}^{2}$ in \% \\
\hline Tötungsdelikte & 28 & Widerstand gg. Staatsgewalt & 71 \\
Sexualdelikte & $\mathbf{7 9}$ & öffentliche Ordnung & 59 \\
sonstige Sexualdelikte & 49 & Hausfriedensbruch & 57 \\
Raub & 66 & Beleidigung & $\mathbf{8 7}$ \\
schwere Körperverletzung & $\mathbf{8 6}$ & Fälschung & $\mathbf{8 5}$ \\
Körperverletzung & $\mathbf{9 1}$ & Meineid & 42 \\
Straftaten gg. pers. Freiheit & $\mathbf{8 7}$ & falsche Verdächtigung & 33 \\
einfacher Diebstahl & $\mathbf{7 8}$ & BTM-Delikte & 70 \\
schwerer Diebstahl & 38 & gef. Eingriff i. d. Straßenverkehr & $\mathbf{7 4}$ \\
Sachbeschädigung & $\mathbf{8 7}$ & AuslG & 54 \\
Gemeingefährliche Straftaten & 62 & Vollrausch & 26 \\
Hehlerei & 42 & fahrl. Tötung/Körperverletzung & $\mathbf{7 9}$ \\
Betrug & 63 & Verkehrsdelikte & $\mathbf{8 4}$ \\
Erschl. von Leistungen & 43 & & \\
\hline
\end{tabular}

Vergleicht man das Ergebnis der justiziellen Registrierungen mit dem Ergebnis auf Polizeiebene, sieht man wie schon bei den deutschen Männern die unterschiedliche Lage von schwerem Diebstahl. Schwerer Diebstahl ist bei polizeilichen Registrierungen unähnlich zu allen anderen Delikten. Bei justiziellen Registrierungen zeigen sich diese eindeutigen Unähnlichkeiten des schweren Diebstahls nicht. Auch beim einfachen Diebstahl zeigen sich andere Deliktsähnlichkeiten als bei polizeilichen Registrierungen. Bei den polizeilich Registrierten sind die Delikte einfacher Diebstahl und Vergehen gegen Ausländergesetze unähnlich, nach justiziellen Registrierungen sind sie ähnlich. Die unterschiedlichen Ähnlichkeiten des einfachen und schweren Diebstahls weisen auf die unterschiedlichen Registrierungen von einfachem und schwerem Diebstahl hin. Beides sind Delikte, die deutlich

191 Die Deliktskategorien, die im Vergleich zur Gesamtdarstellung, eine höhere erklärte Varianz aufweisen, sind in der Tabelle fett gedruckt. 
häufiger polizeilich registriert sind. Die Unähnlichkeit von Vergehen gegen das Ausländergesetz oder Asylverfahrensgesetz mit vielen anderen Delikten zeigt sich bei polizeilichen wie bei justiziellen Registrierungen. Sowohl bei justiziellen wie bei polizeilichen Registrierungen zeigt sich die Ähnlichkeit von Gewaltdelikten einschließlich Beleidigung und Sachbeschädigung. Auch der Zusammenhang von Drogendelikten und den zur Beschaffungskriminalität zählenden Delikten Raub, Hausfriedensbruch, schwerer Diebstahl und Leistungserschleichung wird sichtbar.

\subsubsection{Nichtdeutsche Frauen}

In diesem Abschnitt werden die Deliktsähnlichkeiten der nichtdeutschen Frauen dargestellt. Tabelle 68 im Anhang zeigt die Häufigkeit der Deliktskombinationen innerhalb der justiziell registrierten kriminellen Karriere nichtdeutscher Frauen. Bei den nichtdeutschen Frauen gibt es knapp 19.000 Deliktspaare und damit deutlich weniger als in allen anderen Gruppen. 2.126 der Deliktspaare sind Kombinationen mit Vergehen gegen das Ausländergesetz oder Asylverfahrensgesetz. Es gibt sehr wenige nichtdeutsche Frauen, die mehrfach justiziell registriert sind bzw. wegen mehrerer Straftaten justiziell registriert sind.

Wie bei den deutschen Frauen ist das häufigste Deliktspaar einfacher Diebstahl einfacher Diebstahl (3.550), gefolgt vom Deliktspaar einfacher Diebstahl - Betrug (1.237). Auch die Deliktspaare einfacher Diebstahl - Leistungserschleichung, einfacher Diebstahl - Vergehen gegen das Ausländergesetz oder Asylverfahrensgesetz und einfacher Diebstahl - Verkehrsdelikte treten relativ häufig auf.

Ebenso wie auf Polizeiebene sind Deliktskombinationen mit Tötungsdelikten, Sexualdelikten und dem Delikt Gefährdung des demokratischen Rechtsstaates so selten, dass sie hier für weitere Analysen nicht verwendet werden; Gleiches gilt für die Delikte gefährlicher Eingriff in den Straßenverkehr sowie Vollrausch.

Tabelle 33: Adjusted Standardized Residuals (nichtdeutsche Frauen) ${ }^{192}$

\begin{tabular}{lrrrrrrrr}
\hline & $\begin{array}{c}\text { sonst. } \\
\text { Sex. }\end{array}$ & Raub & $\begin{array}{c}\text { schw. } \\
\text { KV }\end{array}$ & KV & $\begin{array}{c}\text { Str. gg. } \\
\text { Pers. Fr. }\end{array}$ & $\begin{array}{c}\text { einf. } \\
\text { Dieb. }\end{array}$ & $\begin{array}{l}\text { schw. } \\
\text { Dieb. }\end{array}$ & Sachb. \\
\hline sonst. Sexualdel. & $\mathbf{4 6 , 5}$ & & & & & & & \\
Raub & 3,8 & 3,3 & & & & & & \\
schw. KV & $-1,2$ & 6,6 & 2,4 & & & & \\
Körperverletzung & $-1,8$ & 3,6 & $\mathbf{2 2 , 5}$ & 5,0 & & & \\
Str. gg. Pers. Fr. & $-0,9$ & 1,5 & 6,2 & 7,3 & 0,6 & & & \\
einf. Diebstahl & $-4,2$ & $-3,1$ & $-7,1$ & $-11,8$ & $-3,8$ & $\mathbf{2 5 , 9}$ & & \\
schw. Diebstahl & $-0,1$ & 0,5 & $-1,0$ & $-2,4$ & $-1,5$ & 3,2 & 9,0 &
\end{tabular}

192 Aus Gründen der Übersichtlichkeit wurden die ASR größer oder gleich 10 fett und die ASR kleiner oder gleich 10 kursiv gedruckt. 


\begin{tabular}{lcccccccc}
\hline & sonst. & Raub & $\begin{array}{c}\text { schw. } \\
\text { KV }\end{array}$ & KV & $\begin{array}{c}\text { Str. gg. } \\
\text { Pers. Fr. }\end{array}$ & $\begin{array}{c}\text { einf. } \\
\text { Dieb. }\end{array}$ & $\begin{array}{c}\text { schw. } \\
\text { Dieb. }\end{array}$ & Sachb. \\
\hline Sachbeschäd. & $-0,2$ & $-0,0$ & 2,7 & 4,6 & 4,1 & $-3,6$ & 2,2 & 0,9 \\
gemeingef. Str. & $-0,2$ & $-0,4$ & 1,1 & 0,9 & 1,7 & $-0,9$ & 1,0 & 0,8 \\
Hehlerei & $-1,0$ & $-0,1$ & $-0,4$ & $-0,5$ & $-1,0$ & 1,4 & 1,8 & 1,6 \\
Betrug & $-2,4$ & $-1,7$ & $-4,5$ & $-4,4$ & $-0,9$ & $-1,0$ & $-1,6$ & $-0,9$ \\
Erschl. v. Leist. & $-0,8$ & 0,6 & $-1,1$ & $-2,5$ & $-1,8$ & $-2,6$ & $-2,9$ & $-0,7$ \\
Wid. gg. Staat. & $-0,8$ & 0,6 & 3,8 & 9,4 & 1,8 & $-6,9$ & $-1,4$ & 2,0 \\
öff. Ordnung & $-0,7$ & 0,3 & 1,0 & 0,6 & 0,6 & $-0,7$ & 0,5 & 1,0 \\
Hausfriedensbr. & 0,0 & $-1,2$ & $-0,3$ & 0,1 & 0,4 & 2,3 & 6,6 & 3,0 \\
Beleidigung & $-1,0$ & $-0,1$ & 7,6 & $\mathbf{1 4 , 0}$ & 6,0 & $-6,8$ & $-1,9$ & 3,5 \\
Fälschung & $-1,0$ & $-0,8$ & $-2,9$ & $-3,3$ & $-1,6$ & $-4,9$ & $-0,5$ & $-1,2$ \\
Meineid & 1,4 & $-0,1$ & $-0,8$ & $-1,8$ & 0,1 & $-1,4$ & $-0,9$ & $-0,8$ \\
falsche Verd. & $-0,7$ & $-0,5$ & $-1,0$ & $-1,1$ & 2,8 & $-2,5$ & $-0,5$ & $-0,7$ \\
BTM-Delikte & 7,2 & 1,0 & $-2,8$ & $-1,4$ & $-1,3$ & $-3,4$ & $-1,3$ & 0,7 \\
AuslG & 0,6 & $-0,1$ & $-2,9$ & $-4,4$ & $-3,5$ & $-0,8$ & $-0,3$ & $-2,9$ \\
fahrl. Tötung/KV & $-0,9$ & $-0,7$ & 0,2 & $\mathbf{1 5 , 9}$ & 0,0 & $-7,1$ & $-2,3$ & $-1,5$ \\
Verkehrsdel. & $-1,4$ & $-1,6$ & $-3,2$ & $-1,4$ & $-0,2$ & $-10,9$ & $-3,5$ & $-0,9$ \\
\hline
\end{tabular}

\begin{tabular}{lcccccccc}
\hline & $\begin{array}{l}\text { gemein- } \\
\text { gef. Str. }\end{array}$ & Hehlerei & Betrug & $\begin{array}{c}\text { Erschl. v. } \\
\text { Leist. }\end{array}$ & $\begin{array}{c}\text { Wid. gg. } \\
\text { Staat. }\end{array}$ & öff. Ord. & Hausfr. & $\begin{array}{c}\text { Beleidi- } \\
\text { gung }\end{array}$ \\
\hline gemeingef. Str. & $-0,1$ & & & & & & & \\
Hehlerei & $-0,5$ & 1,3 & & & & & & \\
Betrug & 0,0 & $-1,3$ & $\mathbf{1 0 , 0}$ & & & & & \\
Erschl. v. Leist. & $-0,8$ & $-2,1$ & $-0,1$ & $\mathbf{2 6 , 3}$ & & & & \\
Wid. gg. Staat. & 2,3 & $-0,1$ & $-2,2$ & $-1,2$ & 7,7 & & & \\
offf. Ordnung & 1,1 & $-0,3$ & 1,7 & $-0,6$ & 0,8 & 3,0 & & \\
Hausfriedensbr. & $-0,5$ & 0,4 & $-2,5$ & $-0,8$ & $-0,5$ & $-0,3$ & $\mathbf{1 3 , 0}$ & \\
Beleidigung & 0,9 & $-0,7$ & $-2,5$ & $-2,7$ & $\mathbf{1 2 , 7}$ & $-0,6$ & $-0,6$ & 5,0 \\
Fälschung & $-0,3$ & $-0,6$ & $\mathbf{1 3 , 9}$ & $-2,5$ & $-1,8$ & $-0,5$ & $-2,6$ & $-2,1$ \\
Meineid & $-0,2$ & 4,1 & 3,4 & $-1,5$ & $-0,8$ & $-0,7$ & $-0,5$ & $-0,7$ \\
falsche Verd. & $-0,3$ & 1,7 & 2,1 & 0,3 & $-0,0$ & 4,3 & $-1,0$ & 0,9 \\
BTM-Delikte & $-0,8$ & 1,0 & $-2,8$ & 0,1 & $-1,6$ & $-1,4$ & $-0,9$ & $-1,2$ \\
AuslG & $-0,6$ & $-0,9$ & $-5,4$ & $-5,8$ & $-1,1$ & $-1,9$ & $-2,3$ & $-3,1$ \\
fahrl. Tö- & 2,8 & 0,1 & $-2,4$ & $-3,0$ & $-0,3$ & 0,6 & $-1,6$ & $-0,9$ \\
tung/KV & $-1,2$ & $-0,4$ & $-0,9$ & $-4,8$ & $-0,4$ & $-0,8$ & $-4,5$ & $-0,3$ \\
Verkehrsdel. & & & & & & &
\end{tabular}




\begin{tabular}{llllllll}
\hline & $\begin{array}{l}\text { Fäl- } \\
\text { schung }\end{array}$ & Meineid & $\begin{array}{l}\text { falsche } \\
\text { Verd. }\end{array}$ & $\begin{array}{l}\text { BTM- } \\
\text { Delikte }\end{array}$ & AuslG & $\begin{array}{l}\text { fahrl. Tö- } \\
\text { tung/KV }\end{array}$ & Verkehrsdel. \\
\hline Fälschung & 1,2 & & & & & & \\
Meineid & $-0,1$ & 1,4 & & & & & \\
falsche Verd. & 0,3 & 5,9 & 1,3 & & & & \\
BTM-Delikte & $-0,9$ & 1,5 & $-0,1$ & $\mathbf{2 3 , 1}$ & & & \\
AuslG & 8,5 & $-0,3$ & $-1,6$ & $-5,1$ & $\mathbf{2 9 , 0}$ & & \\
fahrl. Tötung/KV & $-2,7$ & $-0,9$ & $-0,5$ & $-0,6$ & $-2,2$ & 0,0 & \\
Verkehrsdel. & $-0,9$ & $-0,3$ & 0,5 & 1,7 & $-3,4$ & $\mathbf{1 4 , 2}$ & $\mathbf{3 0 , 9}$ \\
\hline
\end{tabular}

Wie schon beschrieben wird das Adjusted Standardized Residual als Ähnlichkeitsmaß benutzt. In Tabelle 33 sind die ASR der Deliktspaare der nichtdeutschen Frauen dargestellt. Mit Ausnahme der Deliktskategorie ,gemeingefährliche Straftaten" (diese Eigenkombination kommt bei nichtdeutschen Frauen nicht vor) sind alle ASR der Diagonale positiv. D.h. alle Delikte sind mit sich selbst ähnlich. Einen Hinweis auf Spezialisierung wegen eines großen ASR gibt es bei ,sonstigen Sexualdelikten" (verbotener Prostitution). Relativ hohe ASR sind auch bei den Eigenkombinationen von Verkehrsdelikten und Vergehen gegen das Ausländergesetz oder Asylverfahrensgesetz vorhanden.

Besonders ähnlich (mit hohem positivem ASR) sind die Deliktspaare Körperverletzung - schwere Körperverletzung (22,5), Körperverletzung - fahrlässige Tötung/Körperverletzung $(15,9)$, Verkehrsdelikte - fahrlässige Tötung/Körperverletzung $(14,2)$, Körperverletzung - Beleidigung $(14,0)$ und Betrug - Fälschung $(13,9)$.

Hohe negative Werte gibt es bei den Kombinationen einfacher Diebstahl - Körperverletzung $(-11,8)$ und einfacher Diebstahl - Verkehrsdelikte $(-10,9), d$. h. diese Deliktskombinationen sind einander unähnlich. Vergehen gegen das Ausländergesetz oder Asylverfahrensgesetz sind zu fast allen Delikten unähnlich, mit Ausnahme von „Fälschung“; hier zeigt sich eine Ähnlichkeit. Einfacher Diebstahl ist, auBer zu Hausfriedensbruch, Hehlerei und schwerem Diebstahl, zu allen Delikten unähnlich. Auch Betrug ist zu vielen Delikten unähnlich.

In Abbildung 14 ist die Deliktskonfiguration der nichtdeutschen Frauen dargestellt. Die zweidimensionale Darstellung ist nicht so gut wie die der Männer und der deutschen Frauen und in etwa so wie das Ergebnis der nichtdeutschen Frauen auf polizeilicher Ebene. Die erklärte Varianz der Gesamtdarstellung beträgt $70 \%$ und der STRESS-Wert ist 0,24.

Die Darstellung zeigt die Randlage der Vergehen gegen das Ausländergesetz oder Asylverfahrensgesetz. Vergehen gegen das Ausländergesetz oder Asylverfahrensgesetz sind mit den meisten Delikten unähnlich. Eine Ausnahme bildet „Fälschung", diese Deliktskategorie kommt gemeinsam mit Vergehen gegen das Aus- 
ländergesetz oder Asylverfahrensgesetz in der kriminellen Karriere nichtdeutscher Frauen vor. Wie bei den anderen Gruppen auch, zeigt sich die Ähnlichkeit von Körperverletzung, schwerer Körperverletzung, Straftaten gegen die persönliche Freiheit, gemeinsam mit Beleidigung, Widerstand gegen die Staatsgewalt und Sachbeschädigung. „sonstige Sexualdelikte“ (verbotener Prostitution) zeigen keine Ähnlichkeit mit anderen Gewaltdelikten, aber es stellt sich die Ähnlichkeit von „sonstigen Sexualdelikten“ und Betäubungsmitteldelikten heraus. Deutlich zeigt sich auch, dass sowohl einfacher Diebstahl, wie auch Leistungserschleichung kaum Ähnlichkeiten mit anderen Delikten aufweist. D. h. Leistungserschleichung wird innerhalb der kriminellen Karriere einer nichtdeutschen Frau selten in Kombination mit einem anderen Delikt registriert. Gleiches gilt für einfachen Diebstahl, hier aber mit der Ausnahme, dass sich beim Deliktspaar einfacher Diebstahl - schwerer Diebstahl Ähnlichkeiten zeigen.

Abbildung 14: Ergebnis der MDS (nichtdeutsche Frauen)193

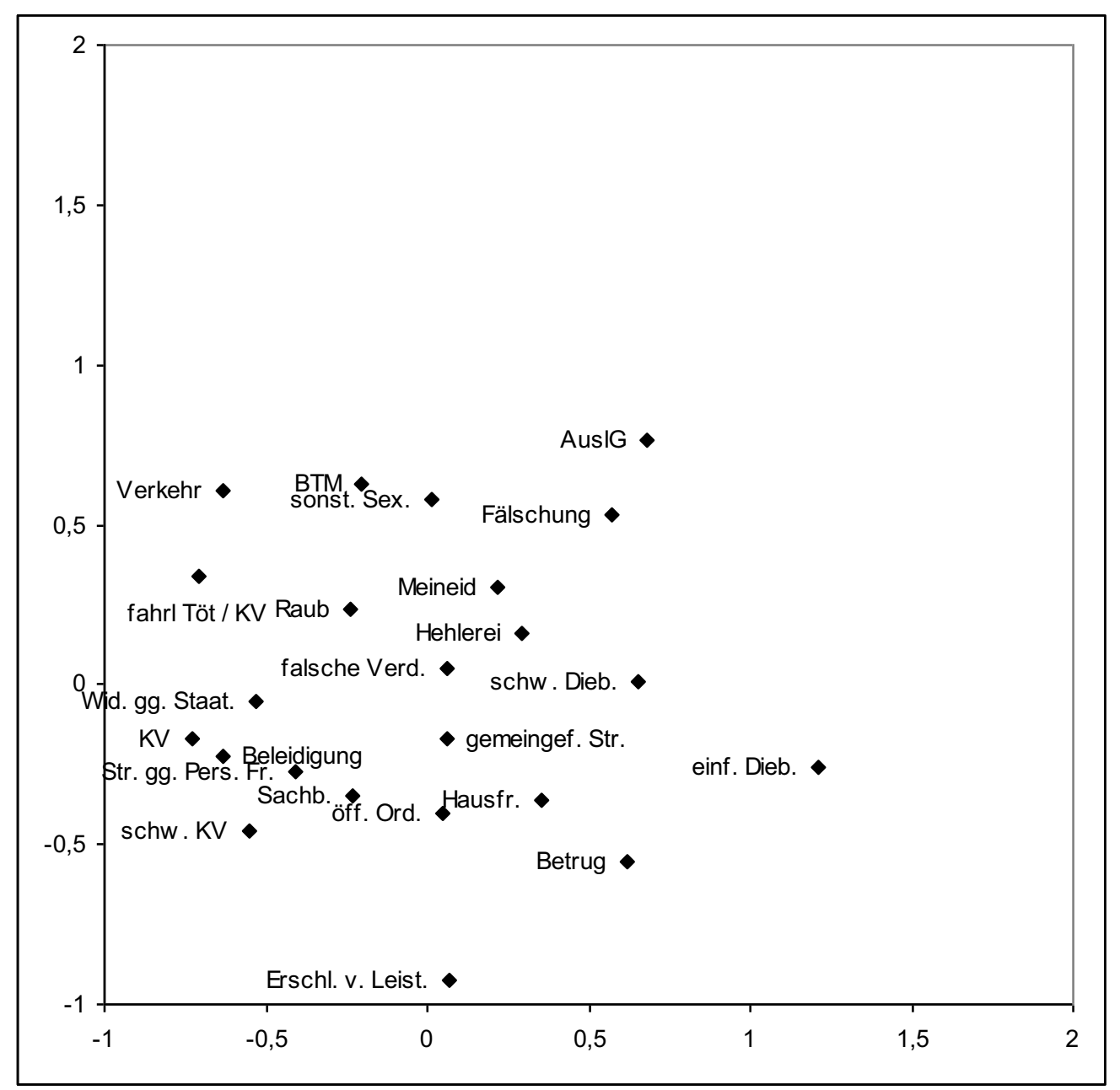

193 Ein Abstand von 0,7 Einheiten entspricht einem ASR von Null. 
In Tabelle 34 ist die erklärte Varianz der einzelnen Deliktskategorien aufgeführt. Bei 8 der 23 Deliktskategorien ist die erklärte Varianz größer oder gleich 70\%, was der erklärten Varianz der Gesamtdarstellung entspricht. Bei acht Deliktskategorien ist die erklärte Varianz kleiner $50 \%$. Schlecht ist die erklärte Varianz bei Betrug, Hausfriedensbruch und gemeingefährlichen Straftaten. Diese Delikte sind im Zweidimensionalen nicht gut dargestellt. Sehr gute Werte gibt es bei den meisten Gewaltdelikten, Beleidigung, Widerstand gegen die Staatsgewalt, einfacher Diebstahl, schwerer Diebstahl, Sachbeschädigung, fahrlässige Tötung/Körperverletzung, Verkehrsdelikte und Vergehen gegen das Ausländergesetz oder Asylverfahrensgesetz. Die Darstellung dieser Delikte ist im zweidimensionalen Raum sehr gut.

Tabelle 34: Erklärte Varianz (nichtdeutsche Frauen) ${ }^{194}$

\begin{tabular}{lclc}
\hline & $\mathrm{R}^{2}$ in $\%$ & & $\mathrm{R}^{2}$ in $\%$ \\
\hline sonstige Sexualdelikte & 56 & Widerstand gg. die Staatsgewalt & $\mathbf{8 1}$ \\
Raub & 39 & öffentliche Ordnung & 57 \\
schwere Körperverletzung & $\mathbf{7 9}$ & Hausfriedensbruch & 16 \\
Körperverletzung & $\mathbf{8 9}$ & Beleidigung & $\mathbf{8 9}$ \\
Straftaten gg. pers. Freiheit & $\mathbf{8 9}$ & Fälschung & 47 \\
einfacher Diebstahl & $\mathbf{8 4}$ & Meineid & 54 \\
schwerer Diebstahl & 60 & falsche Verdächtigung & 37 \\
Sachbeschädigung & 68 & BTM-Delikte & 50 \\
Gemeingefährliche Straftaten & 19 & AuslG & $\mathbf{7 0}$ \\
Hehlerei & 24 & fahrl. Tötung/Körperverletzung & $\mathbf{7 7}$ \\
Betrug & 19 & Verkehrsdelikte & 61 \\
Erschleichen von Leistungen & 35 & & \\
\hline
\end{tabular}

Viele Deliktskombinationen sind bei nichtdeutschen Frauen sehr selten. Nur relativ wenige nichtdeutsche Frauen sind mehrfach registriert. Deutlich zu sehen ist aber auch hier die Ähnlichkeit der Gewaltdelikte Körperverletzung, schwere Körperverletzung, Straftaten gegen die persönliche Freiheit mit Widerstand gegen die Staatsgewalt, Beleidigung und Sachbeschädigung. Wie sich schon bei den polizeilich registrierten Nichtdeutschen und auch bei den justiziell registrierten nichtdeutschen Männern zeigte, sind Vergehen gegen Ausländergesetze unähnlich zu allen anderen Delikten mit der Ausnahme der Kombination Vergehen gegen Ausländergesetze - Fälschung.

Zwischen den einzelnen Deliktskategorien konnten Deliktsähnlichkeiten und Unähnlichkeiten festgestellt werden. Es stellt sich die Frage, ob es sich bei den Ähnlichkeiten um „echte“ Ähnlichkeiten handelt oder ob diese Ähnlichkeiten nur festgestellt wurden, weil zwei verschiedene Deliktskategorien bei einer justiziellen

194 Die Deliktskategorien, die im Vergleich zur Gesamtdarstellung, eine höhere erklärte Varianz aufweisen, sind in der Tabelle fett gedruckt. 
Registrierung häufig gleichzeitig registriert werden, obwohl es sich nur um eine Straftat handelt. Dies soll im nächsten Abschnitt untersucht werden.

\subsubsection{Problem Tateinheit und Tatmehrheit}

In einer justiziellen Entscheidung sind häufig mehrere Straftaten registriert. Bei mehreren Straftaten in einer Entscheidung kann es sich um „Tateinheit“, „Tatmehrheit" oder auch um eine Kombination aus beidem handeln.

Hierzu die Begriffsbestimmung und einige Beispiele aus den Bundeszentralregisterdaten:

Tatmehrheit: Jemand hat mehrere Straftaten begangen, die gleichzeitig abgeurteilt werden ( $§ 53 \mathrm{StGB})$

Zwei Beispiele für Tatmehrheit:

1. Tatbezeichnung: Diebstahl in 2 Fällen

Angewendete Vorschriften: StGB § 242, § 53

Es handelt sich um Tatmehrheit, weil zwei Fälle und nicht nur ein Fall abgeurteilt werden.

2. Tatbezeichnung: Vorsätzliche Körperverletzung, Sachbeschädigung Angewendete Vorschriften: StGB $\S 223, \S 232, \S 303$ Abs. 1, § 303c, § 53, JGG $\S 105$ (Tatjahr 1992)

Es handelt sich um Tatmehrheit, weil verschiedene Straftaten abgeurteilt werden.

Tateinheit: Dieselbe Handlung verletzt mehrere Strafgesetze oder dasselbe Strafgesetz mehrmals ( $§ 52$ StGB)

Beispiel für Tateinheit:

3. Tatbezeichnung: Hausfriedensbruch mit Beleidigung Angewendete Vorschriften: StGB § 123, § 185, § 52

Beispiel für eine Kombination aus Tateinheit und Tatmehrheit:

4. Tatbezeichnung: schwere räuberische Erpressung in Tateinheit mit Vergewaltigung sowie Beleidigung in 5 Fällen, davon ein Fall in Tateinheit mit sexueller Nötigung, ein weiterer Fall in Tateinheit mit versuchter sexueller Nötigung und ein dritter Fall in Tateinheit mit Bedrohung

Angewendete Vorschriften: StGB § 177, § 253, § 255, § 250 Abs. 1 Nr. 2 und Abs. $2, \S 178, \S 185, \S 241, \S 22, \S 23, \S 47, \S 49, \S 52, \S 53, \S 54$ 
Beispiel mit möglicher Tateinheit, die aber weder in der Tatbezeichnung noch in den angewendeten Vorschriften vermerkt ist:

5. Tatbezeichnung: Widerstand gegen Vollstreckungsbeamte, Hausfriedensbruch, Erschleichen von Leistungen, Beleidigung in 4 Fällen

Angewendete Vorschriften: StGB $\S 265 \mathrm{a}, \S 248 \mathrm{a}, \S 185, \S 194, \S 123, \S 113$, $\S 53, \S 55$

Warum mehrere Straftaten in einer justiziellen Entscheidung registriert sind, warum es Tateinheit und Tatmehrheit gibt, erklärt folgendes Zitat aus einem juristischen Lehrbuch.

„Den $§ § 52-55$ liegt die Erwägung zugrunde, dass bei einem Zusammentreffen mehrerer Gesetzesverletzungen die Addition aller in Betracht kommender Freiheitsstrafen das Maß der Schuld des Täters übersteigen würde. Um eine derartige Summierung von Freiheitsstrafen zu vermeiden, stellt das Gesetz in Form der Tateinheit (§ 52) und der Tatmehrheit ( $\$ 53)$ zwei unterschiedliche Methoden für eine dem Täter vorteilhafte Kombination der betreffenden Strafdrohungen zur Verfügung. Bei den als Tateinheit erfassten Gesetzesverletzungen wird im Prinzip nur auf eine Strafe erkannt, die sich nach dem Gesetz bestimmt, das die schwerste Strafe androht (§ 52 I, II). Bei den als Tatmehrheit zu beurteilenden Gesetzesverletzungen hält das Gesetz eine höhere Schuld für gegeben, der es durch eine selbstständigere Anwendung der einschlägigen Strafdrohungen wie folgt Rechnung trägt: Die hier verwirkten Einzelstrafen werden (nach besonderen Regeln) auf eine Gesamtstrafe zurückgeführt, die durch eine Erhöhung der ihrer Art nach schwersten Strafe gebildet wird und die Summe der Einzelstrafen nicht erreichen darf (§§ 53, 54).“195

Die $\S \S 52,53$ StGB (Tateinheit und Tatmehrheit) sind nicht bei allen Registrierungen in den angewendeten Vorschriften korrekt vermerkt. Beispiel 4 zeigt die Problematik, dass auch wenn Tateinheit vermerkt ist, den angewendeten Vorschriften nicht entnommen werden kann, auf welche Straftaten sich die Tateinheit bezieht. Deshalb ist es nicht möglich, in die Auswertung die Kriterien Tateinheit und Tatmehrheit mit einzubeziehen. Eine Möglichkeit, diesem Problem aus dem Weg zu gehen, wäre, immer nur das schwerste Delikt einer Entscheidung zu verwenden, dadurch würden aber viele Straftaten verloren gehen.

195 Wessels \& Beulke 1999, S. 249. 
Tabelle 35: Häufigkeit von Delikten ohne weiteres Delikt in selber Registrierung (BZR-Registrierungen bis 2003)

\begin{tabular}{|c|c|c|c|}
\hline \multirow[b]{2}{*}{ Delikt } & \multirow[b]{2}{*}{ Registrierungen* } & \multicolumn{2}{|c|}{$\begin{array}{l}\text { Delikte ohne weiteres Delikt } \\
\text { in selber Registrierung }\end{array}$} \\
\hline & & $\mathrm{N}$ & $\%$ \\
\hline Tötungsdelikte & 265 & 101 & 38,1 \\
\hline Sexualdelikte & 806 & 291 & 36,1 \\
\hline sonstige Sexualdelikte & 1.257 & 860 & 68,4 \\
\hline Raub & 4.879 & 1.723 & 35,3 \\
\hline schwere Körperverletzung & 12.747 & 1.479 & 11,6 \\
\hline Körperverletzung & 26.993 & 7.072 & 26,2 \\
\hline Str. gg. persönliche Freiheit & 7.731 & 2.004 & 25,9 \\
\hline Einfacher Diebstahl & 71.669 & 55.488 & 77,4 \\
\hline Schwerer Diebstahl 196 & 17.864 & 11.114 & 62,2 \\
\hline Sachbeschädigung & 12.074 & 6.277 & 52,0 \\
\hline Gemeingefährliche Str. & 1.065 & 426 & 40,0 \\
\hline Hehlerei & 5.772 & 2.541 & 44,0 \\
\hline Betrug & 20.910 & 11.188 & 53,5 \\
\hline Erschleichen von Leistungen & 16.270 & 13.395 & 82,3 \\
\hline Gefährdung dem. Rechtsstaat & 750 & 342 & 45,6 \\
\hline Wid. gg. Staatsgewalt & 3.210 & 478 & 14,9 \\
\hline Öffentliche Ordnung & 2.856 & 1.318 & 46,1 \\
\hline Hausfriedensbruch & 3.779 & 1.812 & 47,9 \\
\hline Beleidigung & 8.956 & 3.305 & 36,9 \\
\hline Fälschung & 11.343 & 2.280 & 20,1 \\
\hline Meineid & 1.279 & 682 & 53,3 \\
\hline falsche Verdächtigung & 979 & 418 & 42,7 \\
\hline BTM-Delikte & 25.722 & 21.594 & 84,0 \\
\hline gef. Eingr. Str.V. & 902 & 251 & 27,8 \\
\hline AuslG & 14.867 & 11.817 & 79,5 \\
\hline Vollrausch 197 & 1.004 & 688 & 68,5 \\
\hline fahrlässige Tötung & 423 & 77 & 18,2 \\
\hline fahrlässige Körperverletzung & 11.263 & 2.905 & 25,8 \\
\hline Verkehrsdelikte & 81.636 & 44.359 & 54,3 \\
\hline
\end{tabular}

* Registrierungen von Personen mit zwei oder mehr Registrierungen

$196 \S 242 \mathrm{StGB}$, der grundsätzlich auch bei schwerem Diebstahl registriert wird, ist hier nicht berücksichtigt.

197 Bei Schuldunfähigkeit wegen Vollrausch wird die tatsächlich begangene Straftat nicht registriert (§ 323a StGB). 
Im Folgenden werden Analysen bezüglich dem mehrfachen Auftreten von Straftaten innerhalb einer Entscheidung durchgeführt, um zu untersuchen, ob „Tateinheit" künstliche Deliktsähnlichkeiten verursacht. Dazu wird auch das Verhältnis von Deliktspaaren innerhalb einer Entscheidung zu allen Deliktspaaren einer Person untersucht.

Tabelle 35 zeigt die Häufigkeitsverteilung von Deliktskategorien aller Täter mit zwei oder mehr Straftaten. Die dritte Spalte gibt an, bei wie vielen dieser Registrierungen keine weitere Straftat in derselben Entscheidung registriert ist. Die letzte Spalte zeigt den prozentualen Anteil der Straftaten ohne eine weitere Straftat.

In den justiziellen Registrierungen befinden sich 265 Tötungsdelikte; bei 101 dieser Delikte ist keine weitere Straftat innerhalb derselben Entscheidung registriert; das entspricht 38,1\%. Umgekehrt gesagt ist bei $61,9 \%$ der registrierten Tötungsdelikte ein weiteres Delikt registriert. Besonders häufig sind bei den Delikten schwere Körperverletzung und Widerstand gegen die Staatsgewalt weitere Straftaten registriert. Als weitere Straftaten zählen hier nur Straftaten einer anderen Deliktskategorie, Straftaten derselben Deliktskategorie werden nicht gezählt.

Betäubungsmitteldelikte, einfacher Diebstahl, schwerer Diebstahl, Leistungserschleichung, Vergehen gegen das Ausländergesetz oder Asylverfahrensgesetz und sonstige Sexualdelikte sind dagegen häufig allein in einer Entscheidung registriert.

Tabelle 36 gibt den Anteil der Deliktspaare innerhalb einer Entscheidung an allen Deliktspaaren an. 53,8\% aller Deliktspaare Sexualdelikte - Tötungsdelikte befinden sich innerhalb einer Entscheidung. Besonders häufig innerhalb einer Entscheidung ist das Deliktspaar Sexualdelikte - sonstige Sexualdelikte; hier befinden sich drei Viertel aller dieser Deliktspaare innerhalb einer Entscheidung. Knapp $50 \%$ der Deliktspaare Körperverletzung - schwere Körperverletzung befinden sich innerhalb einer Entscheidung. 10\% der Deliktspaare Hausfriedensbruch - schwerer Diebstahl befinden sich in einer Entscheidung, hier hätte man einen höheren Anteil erwarten können. Bei den ähnlichen Deliktspaaren Beleidigung - Körperverletzung und Beleidigung - schwere Körperverletzung befindet sich jedes vierte Deliktspaar, bzw. weniger als jedes fünfte innerhalb einer Entscheidung. 
Tabelle 36: Anteil Deliktspaare innerhalb einer Entscheidung an allen Deliktspaaren (BZR) in \%

\begin{tabular}{lrrrrrrrr}
\hline & $\begin{array}{c}\text { Tötungs- } \\
\text { delikte }\end{array}$ & $\begin{array}{c}\text { Sexual- } \\
\text { delikte }\end{array}$ & $\begin{array}{r}\text { sonst. } \\
\text { Sex. }\end{array}$ & Raub & $\begin{array}{r}\text { schw. } \\
\text { KV }\end{array}$ & KV & $\begin{array}{c}\text { Str. gg. } \\
\text { Pers. Fr. }\end{array}$ & $\begin{array}{c}\text { einf. } \\
\text { Dieb. }\end{array}$ \\
\hline Sexualdelikte & $\mathbf{5 3 , 8}$ & & & & & & & \\
sonst. Sexualdel. & 0,0 & $\mathbf{7 5 , 4}$ & & & & & & \\
Raub & 57,6 & 31,7 & 15,4 & & & & & \\
schw. KV & 46,9 & 35,7 & 14,8 & 37,6 & & & & \\
Körperverletzung & 28,9 & 36,5 & 18,0 & 28,6 & $\mathbf{4 9 , 2}$ & & & \\
Str. gg. Pers. Fr. & 15,9 & 45,3 & 27,8 & 27,6 & 31,7 & 30,3 & & \\
einf. Diebstahl & 3,6 & 3,8 & 3,2 & 8,5 & 5,5 & 5,8 & 8,0 & \\
schw. Diebstahl & 9,0 & 8,3 & 6,5 & 16,1 & 9,7 & 7,8 & 8,9 & 0,0198 \\
Sachbeschäd. & 9,2 & 8,4 & 5,7 & 11,8 & 17,6 & 17,8 & 23,5 & 10,3 \\
gemeingef. Str. & 84,6 & 3,6 & 15,4 & 25,4 & 20,8 & 16,3 & 24,8 & 6,9 \\
Hehlerei & 0,0 & 5,7 & 3,3 & 13,7 & 6,9 & 6,1 & 8,6 & 16,6 \\
Betrug & 9,2 & 4,1 & 3,9 & 12,6 & 5,3 & 4,9 & 5,9 & 17,6 \\
Erschl. V. Leist. & 0,0 & 2,5 & 1,1 & 3,1 & 3,0 & 3,8 & 5,1 & 5,5 \\
gef. Dem. Recht. & 42,9 & 16,7 & 14,3 & 8,0 & 12,6 & 9,9 & 14,5 & 5,1 \\
Wid. gg. Staat. & 20,8 & 9,1 & 12,5 & 11,1 & 20,3 & 34,4 & 26,2 & 5,8 \\
öff. Ordnung & 23,8 & 2,7 & 8,2 & 15,8 & 17,9 & 12,3 & 19,9 & 9,1 \\
Hausfriedensbr. & 0,0 & 6,8 & 10,2 & 8,4 & 12,9 & 14,6 & 23,4 & 8,8 \\
Beleidigung & 8,7 & 15,3 & 18,6 & 8,1 & $\mathbf{1 7 , 7}$ & $\mathbf{2 5 , 1}$ & 36,7 & 4,6 \\
Fälschung & 8,1 & 3,9 & 4,1 & 10,9 & 6,1 & 5,7 & 6,5 & 14,8 \\
Meineid & 0,0 & 12,5 & 7,7 & 7,5 & 6,3 & 5,0 & 13,3 & 2,9 \\
falsche Verd. & 0,0 & 22,2 & 11,8 & 5,4 & 4,8 & 8,4 & 29,0 & 8,7 \\
BTM-Delikte & 2,2 & 4,3 & 4,1 & 8,1 & 4,4 & 4,0 & 5,3 & 3,3 \\
gef. Eingr. Str.V. & 25,0 & 22,2 & 0,0 & 16,9 & 35,2 & 25,7 & 60,0 & 4,9 \\
AuslG & 0,0 & 3,2 & 18,9 & 4,1 & 3,6 & 4,1 & 4,9 & 7,4 \\
Vollrausch & 25,0 & 4,5 & 4,5 & 7,5 & 10,5 & 10,0 & 8,3 & 3,9 \\
fahrl. Tötung/KV & 11,5 & 10,5 & 5,1 & 9,5 & 9,2 & 55,9 & 14,3 & 3,5 \\
Verkehrsdel. & 5,8 & 4,3 & 2,3 & 6,3 & 4,3 & 7,8 & 6,8 & 5,1 \\
\hline & & & & & & & & \\
& & & & & & \\
& & & & & & \\
\end{tabular}

198 Das Deliktspaar einfacher Diebstahl - schwerer Diebstahl kommt hier nie innerhalb einer Entscheidung vor, weil bei einer Eintragung von $\S 243$ StGB grundsätzlich auch $\S 242 \mathrm{StGB}$ eingetragen wird. Da dies immer der Fall ist, wird hier $\S 242$ nicht berücksichtigt. 


\begin{tabular}{|c|c|c|c|c|c|c|c|c|}
\hline & $\begin{array}{l}\text { schw. } \\
\text { Dieb. }\end{array}$ & Sachb. & $\begin{array}{l}\text { nein- } \\
\text { Str. }\end{array}$ & Hehlerei & Betrug & $\begin{array}{l}\text { Erschl. } \\
\text { v. Leist. }\end{array}$ & $\begin{array}{l}\text { gef. dem. } \\
\text { Recht. }\end{array}$ & $\begin{array}{l}\text { Wid. gg. } \\
\text { Staat. }\end{array}$ \\
\hline Sachbeschäd. & 19,1 & & & & & & & \\
\hline gemeingef. Str. & 23,4 & 33,5 & & & & & & \\
\hline Hehlerei & 22,5 & 11,6 & 4,3 & & & & & \\
\hline Betrug & 11,5 & 5,6 & 0,7 & 11,9 & & & & \\
\hline Erschl. v. Leist. & 3,9 & 3,9 & 2,6 & 4,7 & 12,1 & & & \\
\hline gef. dem. Recht. & 6,5 & 32,6 & 3,7 & 2,0 & 3,1 & 0,8 & & \\
\hline Wid. gg. Staat. & 9,2 & 20,0 & 2,6 & 8,9 & 5,4 & 4,7 & 10,1 & \\
\hline öff. Ordnung & 12,1 & 19,1 & 2,3 & 21,2 & 17,0 & 2,4 & 38,7 & 21,2 \\
\hline Hausfriedensbr. & 10,0 & 31,2 & 2,1 & 6,2 & 6,8 & 7,7 & 8,5 & 16,6 \\
\hline Beleidigung & 5,8 & 18,2 & 0,6 & 5,2 & 4,2 & 7,1 & 21,0 & 47,1 \\
\hline Fälschung & 15,2 & 7,0 & 1,8 & 17,1 & 41,1 & 15,5 & 8,6 & 8,9 \\
\hline Meineid & 4,4 & 2,2 & 4,3 & 67,1 & 15,2 & 1,3 & 0,0 & 1,5 \\
\hline falsche Verd. & 9,6 & 13,5 & 9,4 & 39,6 & 7,8 & 5,6 & 16,7 & 11,9 \\
\hline BTM-Delikte & 6,6 & 2,9 & 5,7 & 6,6 & 6,0 & 1,9 & 2,7 & 9,1 \\
\hline gef. Eingr. Str.V. & 12,4 & 31,0 & 3,5 & 4,6 & 7,0 & 0,7 & 20,0 & 56,9 \\
\hline AuslG & 8,6 & 4,6 & 0,0 & 8,0 & 9,2 & 9,4 & 0,0 & 7,4 \\
\hline Vollrausch & 6,3 & 9,0 & 3,2 & 4,9 & 4,2 & 2,3 & 1,9 & 19,6 \\
\hline fahrl. Tötung/KV & 5,0 & 6,7 & 5,2 & 3,3 & 2,5 & 2,0 & 5,0 & 22,3 \\
\hline Verkehrsdel. & 9,5 & 5,8 & 4,1 & 7,1 & 4,0 & 1,8 & 3,9 & 11,4 \\
\hline & öff. Ord. & Hausfr. & $\begin{array}{l}\text { Belei- } \\
\text { digung }\end{array}$ & $\begin{array}{c}\text { Fäl- } \\
\text { schung }\end{array}$ & $\begin{array}{c}\text { Mein- } \\
\text { eid }\end{array}$ & $\begin{array}{l}\text { falsche } \\
\text { Verd. }\end{array}$ & $\begin{array}{l}\text { BTM- } \\
\text { Delikte }\end{array}$ & $\begin{array}{c}\text { gef. Eingr. } \\
\text { Str.V. }\end{array}$ \\
\hline Hausfriedensbr. & 8,5 & & & & & & & \\
\hline Beleidigung & 15,4 & 21,7 & & & & & & \\
\hline Fälschung & 14,7 & 5,3 & 4,9 & & & & & \\
\hline Meineid & 16,4 & 2,8 & 2,3 & 10,3 & & & & \\
\hline falsche Verd. & 36,2 & 8,3 & 18,6 & 18,1 & 71,9 & & & \\
\hline BTM-Delikte & 5,0 & 3,1 & 3,8 & 7,5 & 6,8 & 8,3 & & \\
\hline gef. Eingr. Str.V. & 14,0 & 6,7 & 26,8 & 11,6 & 7,1 & 25,0 & 4,7 & \\
\hline AuslG & 7,6 & 7,7 & 3,0 & 29,2 & 6,8 & 2,7 & 5,5 & 1,9 \\
\hline Vollrausch & 6,4 & 6,9 & 11,5 & 3,0 & 0,0 & 8,7 & 2,8 & 3,6 \\
\hline fahrl. Tötung/KV & 14,0 & 7,2 & 11,2 & 8,0 & 2,3 & 18,8 & 2,5 & 42,6 \\
\hline \multirow[t]{2}{*}{ Verkehrsdel. } & 13,3 & 4,1 & 6,4 & 23,9 & 2,6 & 14,4 & 3,7 & 11,4 \\
\hline & AuslG & Vollrausch & \multicolumn{2}{|c|}{$\begin{array}{l}\text { fahrl. Tö- } \\
\text { tung/KV }\end{array}$} & & & & \\
\hline Vollrausch & 4,3 & & & & & & & \\
\hline $\begin{array}{l}\text { fahrl. Tö- } \\
\text { tung/KV }\end{array}$ & 2,3 & 4,8 & & & & & & \\
\hline Verkehrsdel & 6,7 & 4,7 & 36 , & 5,2 & & & & \\
\hline
\end{tabular}


Tabelle 37: Anteil Deliktspaare innerhalb einer Entscheidung getrennt nach Deliktskategorien (BZR)

\begin{tabular}{lc}
\hline & $\begin{array}{c}\text { Anteil Deliktspaare innerhalb einer } \\
\text { Entscheidung (\%) }\end{array}$ \\
\hline Tötungsdelikte & 17,4 \\
Sexualdelikte & 16,2 \\
sonstige Sexualdelikte & 8,7 \\
Raub & $\mathbf{1 4 , 5}$ \\
schwere Körperverletzung & 19,2 \\
Körperverletzung & 17,7 \\
Straftaten gg. pers. Freiheit & 16,9 \\
einfacher Diebstahl & 6,7 \\
schwerer Diebstahl & 7,9 \\
Sachbeschädigung & 11,9 \\
Gemeingefährliche Straftaten & 13,1 \\
Hehlerei & 11,8 \\
Betrug & 11,6 \\
Erschleichen von Leistungen & 13,8 \\
Gefährdung dem. Rechtsstaat & 11,4 \\
Widerstand gg. Staatsgewalt & 17,9 \\
offentliche Ordnung & 12,9 \\
Hausfriedensbruch & 10,5 \\
Beleidigung & 13,4 \\
Fälschung & 18,9 \\
Meineid & 9,3 \\
falsche Verdächtigung & 12,7 \\
BTM-Delikte & 4,1 \\
gef. Eingriff i. d. Straßenverkehr & 20,1 \\
AuslG & 8,6 \\
Vollrausch & 15,0 \\
fahrlässige Tötung / Körperverletzung & 14,8 \\
Verkehrsdelikte & 8,5 \\
\hline &
\end{tabular}

Tabelle 37 zeigt den Anteil von Deliktspaaren innerhalb einer Entscheidung an allen Deliktspaaren getrennt nach Deliktskategorie. 14,5\% aller Deliktskombinationen mit Raub befinden sich innerhalb einer Entscheidung und damit sind 85,5\% aller Deliktskombinationen mit Raub in verschiedenen justiziellen Registrierungen einer Person. Die Tabelle zeigt, dass immer $80 \%$ oder mehr (oft über 90\%) der Deliktspaare mit Straftaten aus verschiedenen Registrierungen einer Person gebildet werden. D.h. einzelne besondere Deliktspaare kommen häufig innerhalb einer Entscheidung vor. Betrachtet man aber alle Deliktskombinationen eines Deliktes, befinden sich die meisten Delikte eines Deliktspaares in verschiedenen Entscheidungen. 
Abbildung 15: Ergebnis der MDS (deutsche Männer, nur schwerstes Delikt) ${ }^{199}$

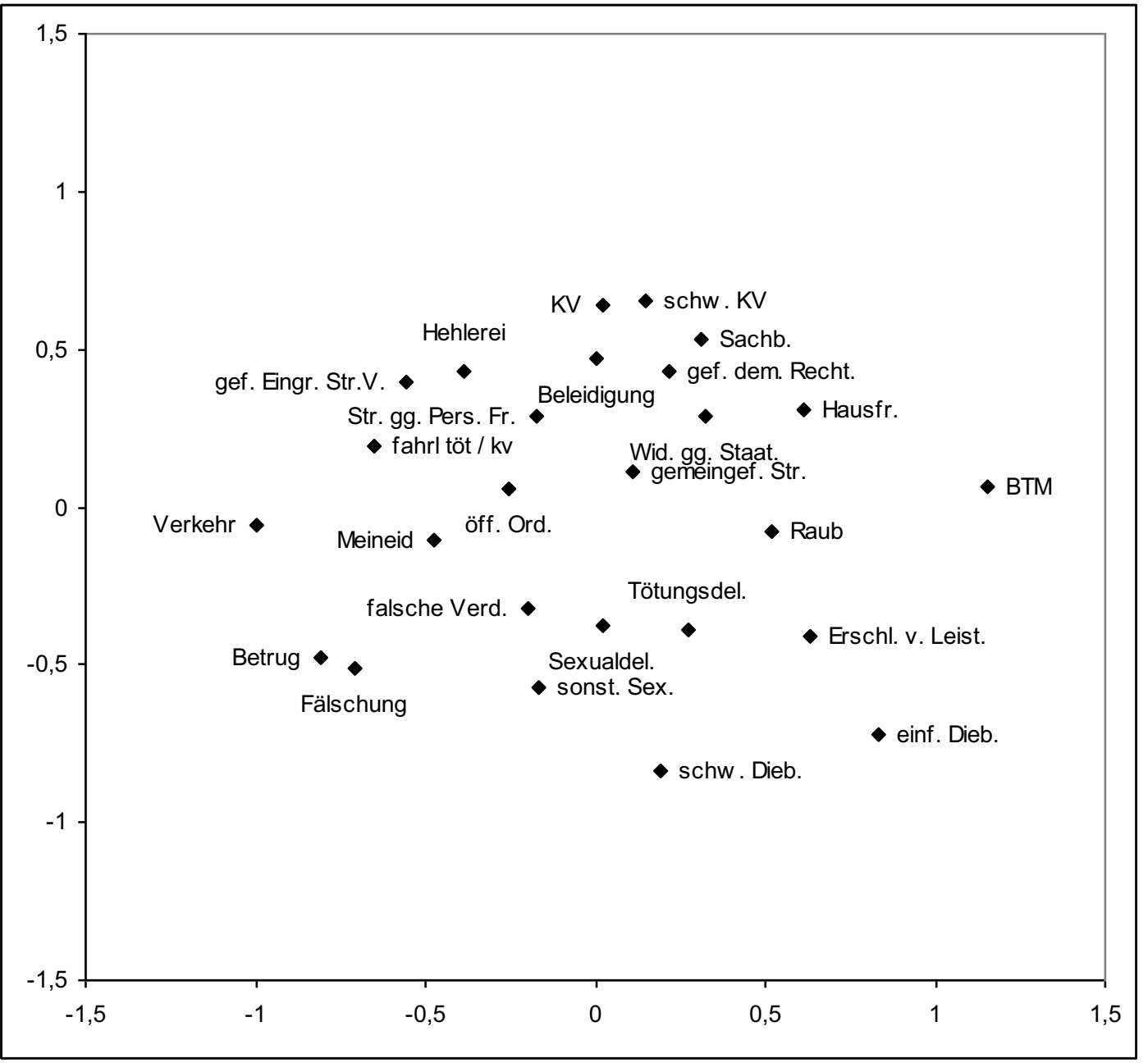

Um zu kontrollieren, ob es künstliche Deliktsähnlichkeiten gibt, d.h. ob es Deliktspaare gibt, die häufig innerhalb einer Entscheidung registriert sind, aber im eigentlichen Sinne nur eine Straftat sind, wird die Deliktsähnlichkeit für die deutschen Männer nur mit dem schwersten Delikt ${ }^{200}$ jeder Entscheidung berechnet. Es werden also nicht alle Straftaten einer Entscheidung in die Analyse mit einbezogen, sondern nur die schwerste Straftat.

199 Ein Abstand von 0,7 Einheiten entspricht einem ASR von Null.

200 Die Schwereeinstufung der Delikte wurde anhand des Strafrahmens gebildet. Diese Schwereeinstufung wird auch bei der vom statistischen Bundesamt herausgegeben Strafverfolgungsstatistik benutzt. Die Strafverfolgungsstatistik erscheint jährlich und bezieht sich immer auf die Entscheidungen eines Jahres. Die hier benutzten Daten umfassen Entscheidungen von 1984 bis 2003. Innerhalb dieser Zeit gab es mehrere Änderungen der Strafrahmen und damit auch bei der Einteilung der Deliktsschwere. Für die Einteilung der Deliktsschwere wurden hier drei Zeitpunkte benutzt, 1988, 1995 und 1998 (hier sind die Änderungen des Sechsten Gesetzes zur Reform des Strafrechts berücksichtigt). 
Berechnet man die Multidimensionale Skalierung nur mit dem schwersten Delikt einer Entscheidung (Abbildung 15), ergibt sich das gleiche Ergebnis wie bei der Berechnung mit allen Delikten einer Entscheidung (Abbildung 11) ${ }^{201}$. Eine Ausnahme bildet Hehlerei bei den schwersten Delikten, aber hier ist die erklärte Varianz sehr schlecht und damit die Lage beim Ergebnis der Multidimensionalen Skalierung nicht gesichert. Dadurch dass, abgesehen von Hehlerei, die gleichen Deliktsähnlichkeiten bestimmt werden, einerseits bei Verwendung nur des schwersten Delikts und andererseits bei Verwendung aller Delikte einer Entscheidung, zeigt sich, dass das Ergebnis durch die Nichtberücksichtigung von „Tateinheit“ nicht verändert wird. Das Ergebnis wird durch künstliche Deliktsähnlichkeiten nicht verfälscht.

\subsubsection{Zusammenfassung}

Um Deliktsähnlichkeiten zu analysieren, werden zunächst die Kombinationen von Straftaten einer Person betrachtet. Bei justiziellen Registrierungen werden sowohl die Deliktskombinationen einer Personen betrachtet, die innerhalb einer Entscheidung wegen verschiedener Straftaten abgeurteilt wurden, wie die Kombinationen mehrerer Entscheidungen. Innerhalb einer Entscheidung wird jede Deliktskategorie nur einmal gezählt, auch wenn das Delikt mehrfach begangen wurde. Bei den Männern ist die häufigste Deliktskombination Verkehrsdelikte - Verkehrsdelikte, bei den Frauen einfacher Diebstahl - einfacher Diebstahl. Weitere häufige Kombination bei den Männern sind Verkehrsdelikte - einfacher Diebstahl und einfacher Diebstahl - einfacher Diebstahl. Bei den Frauen ist die zweithäufigste Kombination einfacher Diebstahl - Betrug. Die Kombination schwerer Diebstahl schwerer Diebstahl, die bei den polizeilichen Registrierungen der Männer besonders häufig ist, ist bei den justiziellen Registrierungen deutlich seltener zu finden. Kombinationen mit Verkehrsdelikten sind bei den Männern deutlich häufiger als bei den Frauen.

Um Deliktsähnlichkeiten darzustellen, wird zunächst ein Maß für die Ähnlichkeit von Delikten benötigt. Das hier benutzte Maß ist das Adjusted Standardized Residual. Ein positives Residuum gibt an, dass Deliktspaare überzufällig häufig vorkommen, ein negatives zeigt, dass diese Kombinationen selten in Bezug zum erwarteten Wert und deshalb unähnlich sind. Deliktskombinationen mit demselben Delikt sind überzufällig häufig. Bei allen Gruppen, sowohl bei Männern wie bei Frauen, bei Deutschen wie Nichtdeutschen, ist das Residuum von Verkehrsdelikte - Verkehrsdelikte besonders groß. Verkehrsdelikte sind überzufällig häufig mehrfach justiziell registriert. Ansonsten fällt bei allen Gruppen das häufige Auftreten der Kombination Körperverletzung - schwere Körperverletzung auf. Besonders

201 Drehungen und Spiegelungen sind in der Darstellung der MDS nicht von Bedeutung. D.h., es spielt keine Rolle, ob Verkehrsdelikte oben oder rechts in der Abbildung dargestellt sind. Nur die Entfernung der Deliktskategorien untereinander spielt eine Rolle. 
häufig und damit ähnlich ist die Kombination der Deliktskategorien Betrug und Fälschung. Bei den Nichtdeutschen ist die häufige Kombination von AuslG AuslG auffällig.

Unähnlich sind die Deliktspaare Leistungserschleichung - Verkehrsdelikte und einfacher Diebstahl - Körperverletzung. Bei den nichtdeutschen Männern und Frauen sind auch die meisten Kombinationen anderer Deliktskategorien mit Vergehen gegen das Ausländergesetz oder Asylverfahrensgesetz sehr selten. Eine Ausnahme bildet hier das Deliktspaar Vergehen gegen das Ausländergesetz oder Asylverfahrensgesetz - Fälschung. Wie schon im Abschnitt der polizeilichen Registrierungen erwähnt, gehen Vergehen gegen das Ausländergesetz oder Asylverfahrensgesetz häufig mit Urkundenfälschung einher. Außerdem ist eine häufige Straftat von Asylbewerbern Schwarzfahren (Leistungserschleichung) bzw. Graufahren (Fahren mit gefälschten Karten und damit Wertzeichenfälschung). ${ }^{202}$

Die Ergebnisse der zweidimensionalen Darstellung der Multidimensionalen Skalierung zeigen, dass insbesondere Gewaltdelikte untereinander und auch in Kombination mit Beleidigung ähnlich sind; diese Delikte kommen häufig gemeinsam in der kriminellen Karriere einer Person vor. Bei den Männern zeigt sich auch ein Zusammenhang von Gewaltdelikten und Sexualdelikten.

Ein Zusammenhang zeigt sich auch zwischen Drogendelikten und Beschaffungskriminalität und bei den Frauen auch zwischen Drogendelikten und Beschaffungsprostitution.

Auch die Deliktskategorien Betrug und Fälschung sind ähnlich, diese Deliktskategorien liegen in allen Darstellungen nah beieinander. Die Unähnlichkeit von Betrug und einfachem Diebstahl, wie sie bei polizeilichen Registrierungen sichtbar war, zeigt sich bei justiziellen Registrierungen nicht. Dies hängt damit zusammen, dass der Anteil von einfachem Diebstahl bei polizeilichen Registrierungen deutlich höher ist als bei justiziellen Registrierungen. Dies hängt wiederum einerseits damit zusammen, dass einfacher Diebstahl eine leichte Straftat ist und dementsprechend häufig das Verfahren eingestellt wird, wenn keine weiteren Straftaten vorliegen. Andererseits wird einfacher Diebstahl häufig in Serie begangen. Diese Seriendiebstähle werden mehrfach polizeilich registriert, aber justiziell in einer Verhandlung abgeurteilt. Somit zeigt sich bei Betrachtung der ASR bei polizeilich registriertem einfachem Diebstahl eine hohe Selbstähnlichkeit und eine Unähnlichkeit von einfachem Diebstahl und anderen Delikten. Bei justiziell registriertem einfachem Diebstahl ist die Selbstähnlichkeit nicht so groß und die Unähnlichkeit zu anderen Delikten wird nicht sichtbar.

Die Unähnlichkeit des schweren Diebstahls mit anderen Delikten, die bei den polizeilichen Registrierungen zu sehen ist, zeigt sich bei den justiziellen Registrierungen nicht. Erklärt werden kann dies damit, dass wie schon beim einfachen Dieb

202 Vgl. Althoff \& de Haan 2004, S. 445. 
stahl schwerer Diebstahl (Einbruch) häufig in Serie begangen und dementsprechend häufig polizeilich registriert wird. Justiziell wird diese „Einbruchserie“ innerhalb einer Entscheidung abgehandelt und wird als ein schwerer Diebstahl gezählt. Somit ist bei polizeilichen Registrierungen das ASR von schwerer Diebstahl - schwerer Diebstahl sehr groß und damit die ASR von schwerem Diebstahl mit anderen Deliktskategorien klein. Bei justiziell registriertem schwerem Diebstahl ist die Selbstähnlichkeit nicht so groß wie bei polizeilich registriertem und Ähnlichkeiten mit anderen Deliktskategorien zeigen sich.

Besonders unähnlich sind die Delikte Verkehrsdelikte - Erschleichen von Leistungen, sie liegen in allen Darstellungen jeweils am gegenüberliegenden Rand. Beim Ergebnis der nichtdeutschen Männer und Frauen ist deutlich zu sehen, dass Vergehen gegen das Ausländergesetz oder Asylverfahrensgesetz zu vielen Delikten unähnlich sind, insbesondere zu Gewaltdelikten.

Eine Kontrollrechnung nur mit dem schwersten Delikt zeigt, dass es sich bei den Ähnlichkeiten nicht um Scheinähnlichkeiten handelt.

\subsection{Berücksichtigung der Dauer zwischen den Straftaten}

Bis jetzt wurde die Ähnlichkeit von Delikten unabhängig von der Dauer von einer Straftat zur nächsten analysiert. Alle Paare innerhalb der offiziellen kriminellen Karriere einer Person wurden kombiniert. Jedes Deliktspaar wurde gezählt, unabhängig davon, ob zwischen den Straftaten drei Jahre oder eine Woche liegt. In diesem Abschnitt wird untersucht, ob die Berücksichtigung der Dauer zwischen den Straftaten Einfluss auf die Deliktsähnlichkeiten hat. Dabei soll ein Deliktspaar mehr Gewicht haben, wenn die Dauer von einer Straftat zur nächsten kürzer ist und weniger Gewicht bei längerem Abstand zwischen den Straftaten. Dafür werden die Deliktspaare mit folgender Formel gewichtet gezählt:

$$
\begin{aligned}
& \text { gew }=\frac{1}{\text { dauer }+1} \\
& \text { dauer: Abstand in Monaten zwischen den Delikten eines Deliktspaares }
\end{aligned}
$$

Bei jedem Deliktspaar wird die Dauer zwischen den beiden Tatzeiten berücksichtigt. Waren beide Tatzeitpunkte der beiden Delikte eines Deliktspaares im gleichen Monat, wird das Deliktspaar als 1 gezählt, bei einem Monat Abstand wird das Paar als $1 / 2$ gezählt, bei zwei Monaten Abstand als 1/3, usw. Die Deliktspaare werden symmetrisch betrachtet, d.h. Beleidigung - Körperverletzung und Körperverletzung - Beleidigung wird gleich gezählt. Es wird nicht berücksichtigt welches Delikt zuerst registriert war.

Analysiert wurden die polizeilichen und justiziellen Registrierungen der deutschen Männer. Zuerst werden die Deliktsähnlichkeiten bei polizeilichen Registrierungen betrachtet. Bei der nach Dauer zwischen den Straftaten gewichteten Zählung der Deliktspaare ergeben sich 419.000 Deliktspaare, ohne Berücksichtigung der Dauer sind es 2.400.000. 
Das Ähnlichkeitsmaß, das für die Multidimensionale Skalierung benötigt wird, wird nun aus diesen gewichteten Häufigkeiten ermittelt, indem die Adjusted Standardized Residuals der Deliktspaare entsprechend berechnet werden.

Abbildung 16: Ergebnis der MDS (deutsche Männer PAD, gewichtet nach Dauer von einer Straftat zur nächsten) ${ }^{203}$

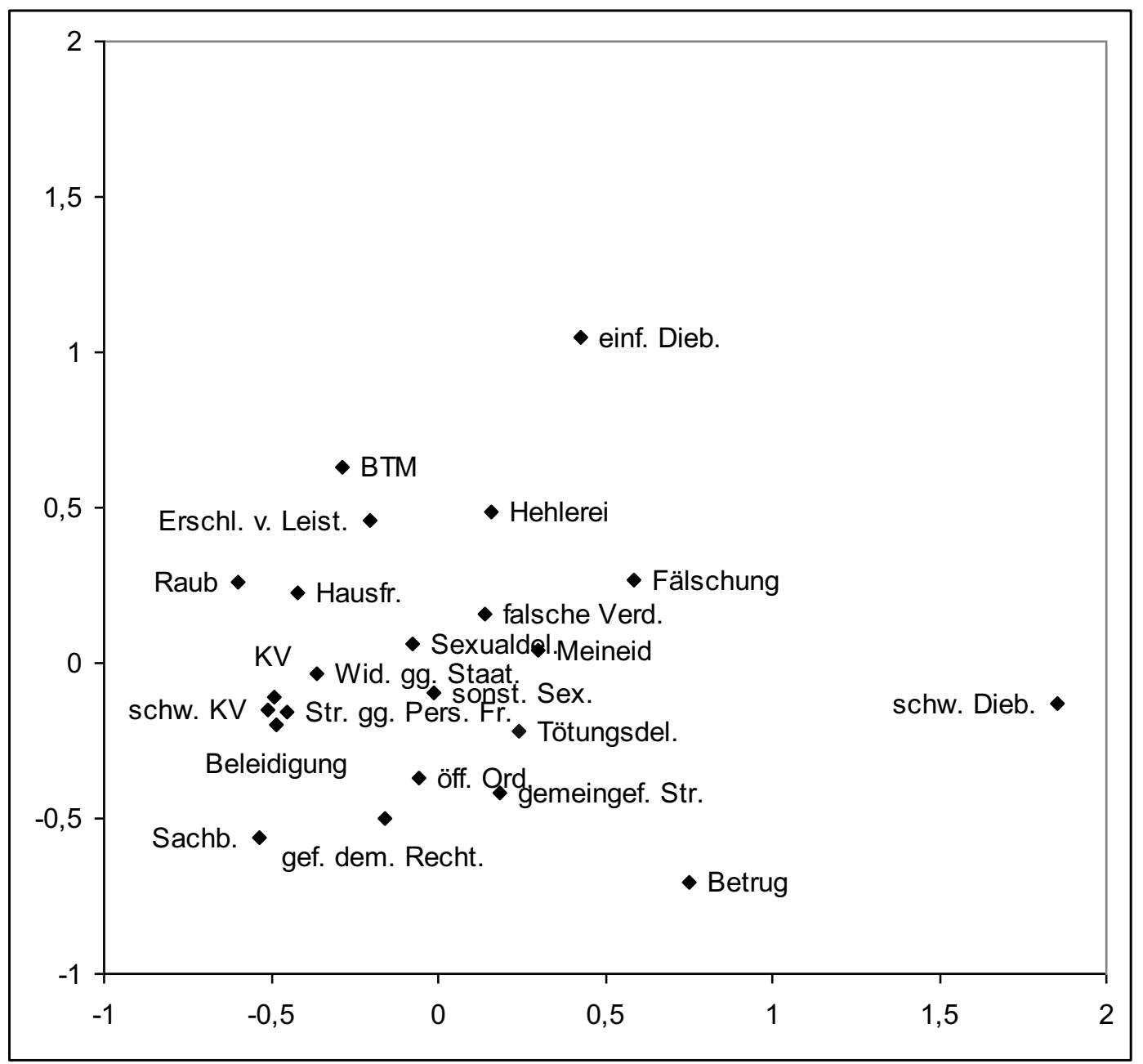

Abbildung 16 zeigt das Ergebnis der Multidimensionalen Skalierung der polizeilich registrierten deutschen Männer, wobei bei der Bestimmung des Ähnlichkeitsmaßes der Deliktspaare die Dauer von einer Straftat zur nächsten berücksichtigt wurde. Vergleicht man diese Abbildung mit der Abbildung der polizeilich registrierten deutschen Männer ohne Berücksichtigung der Dauer zwischen den Straftaten (Abbildung 7), so zeigen sich keine deutlichen Unterschiede. Die zweidimensionalen Darstellungen mit und ohne Berücksichtigung der Dauer zwischen den Straftaten sind einander sehr ähnlich. Die Einbeziehung der Dauer zwischen den Straftaten hat keinen Einfluss auf das Ergebnis der Deliktsähnlichkeiten. Auch die

${ }^{203}$ Ein Abstand von 0,7 Einheiten entspricht einem ASR von Null. 
Güte von beiden Darstellungen ist gleich, der STRESS-Wert ist jeweils 0,21. Die erklärte Varianz der einzelnen Delikte in Abbildung 16 ist größer als 50\% mit Ausnahme von schwerem Diebstahl und Betrug. Die erklärte Varianz von Gewaltdelikten, Beleidigung und Betäubungsmitteldelikten ist größer als $80 \%$.

Auch für die justiziell Registrierten wird untersucht, ob die Dauer einen Einfluss auf Deliktsähnlichkeiten hat. Bei justiziellen Registrierungen wird zur Bestimmung der Dauer das Entscheidungsdatum benutzt, da das Tatdatum bei Bundeszentralregister-Einträgen häufig nicht vorhanden ist. ${ }^{204}$ Bei der nach Dauer zwischen den Entscheidungen gewichteten Zählung der Deliktspaare ergeben sich 127.000 Deliktspaare, ohne Berücksichtigung der Dauer 858.000 Deliktspaare.

\section{Abbildung 17: Ergebnis der MDS (deutsche Männer BZR, gewichtet nach Dauer von einer Straftat zur nächsten)205}

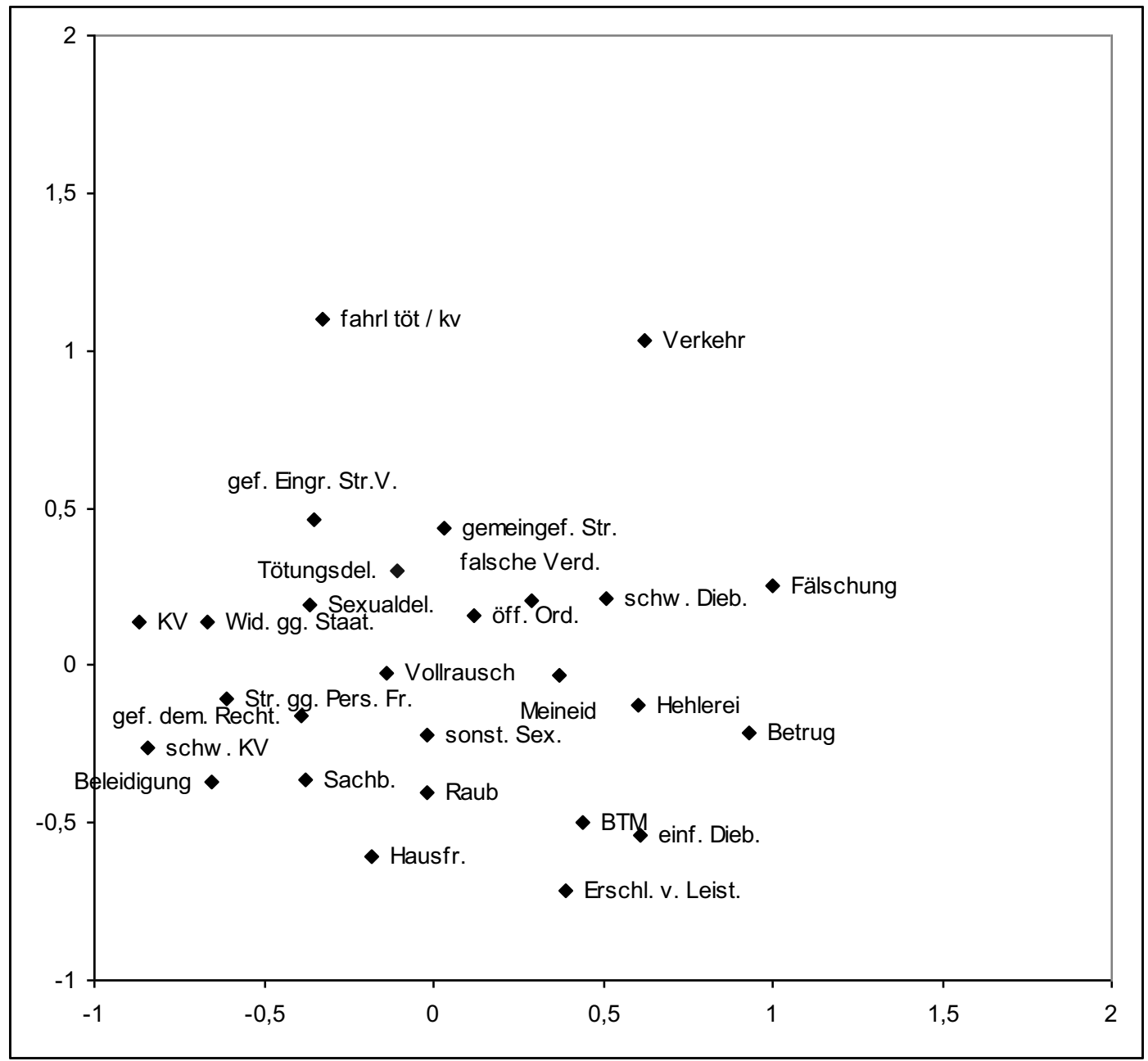

${ }^{204}$ Das Tatdatum in einer Entscheidung im Bundeszentralregister ist, bei der Aburteilung mehrerer Straftaten, das Tatdatum der letzten Tat.

205 Ein Abstand von 0,7 Einheiten entspricht einem ASR von Null. 
Abbildung 17 zeigt das Ergebnis der Multidimensionalen Skalierung der justiziell registrierten deutschen Männer, wobei bei der Bestimmung des Ähnlichkeitsmaßes der Deliktspaare die Dauer von einer Entscheidung zur nächsten berücksichtigt wurde. Vergleicht man diese Abbildung mit der Abbildung der justiziell registrierten deutschen Männer ohne Berücksichtigung der Dauer zwischen den Entscheidungen (Abbildung 11) zeigen sich wie auch schon bei den polizeilich Registrierten keine deutlichen Unterschiede. Die zweidimensionalen Darstellungen mit und ohne Berücksichtigung der Dauer zwischen den Entscheidungen zeigen viele Übereinstimmungen. Die Einbeziehung der Dauer zwischen den Entscheidungen hat keinen Einfluss auf das Ergebnis der Deliktsähnlichkeiten. Auch die Güte der Darstellung mit und ohne Einbezug der Dauer stimmt überein, der STRESS-Wert mit Einbezug der Dauer beträgt 0,22 und ohne Einbezug der Dauer 0,21. Die erklärte Varianz der einzelnen Delikte in Abbildung 17 ist bei 18 der 29 Delikte besser als $60 \%$, nur bei zwei Delikten, Straftaten gegen die öffentliche Ordnung und falsche Verdächtigung, ist die erklärte Varianz kleiner als 40\%. Die Güte der Darstellung ist gut.

Die Einbeziehung der Dauer zwischen den Straftaten einer kriminellen Laufbahn beeinflusst somit das Ergebnis der Deliktsähnlichkeiten nicht.

\subsection{Altersgruppen}

Das Vorkommen von Kriminalität ist nicht gleichmäßig über das gesamte Lebensalter verteilt. ${ }^{206}$ Auch die Deliktsverteilung zeigt altersspezifische Unterschiedene, wie schon in Abbildung 3 und 4 zu sehen war. Moffitt ${ }^{207}$ unterscheidet zwischen zwei Gruppen von Individuen, einerseits den "adolescence-limited", denjenigen, die nur während der Jugendzeit auffällig sind und andererseits den "lifecourse-persistend", denjenigen, die über die gesamte Lebensspanne auffällig sind. Beide Gruppen unterscheiden sich in den Deliktstypen, die sie begehen, und auch die verschiedenen Altersabschnitte der "life-course-persistend" unterscheiden sich bzgl. der Zusammensetzung der Delikte. Die Gruppe der "adolescence-limited" begeht hauptsächlich Delikte, mit denen sie die jugendliche Gier nach Anerkennung und Privilegien befriedigen wollen: Diebstahl, Sachbeschädigung, Straftaten gegen die öffentliche Ordnung und Betäubungsmitteldelikte. ${ }^{208}$ Die kleine Gruppe der "life-course-persistend" fängt ihre kriminelle Karriere früher, schon in der Kindheit an, sie begeht andere Delikte als die Gruppe der "adolescence-limited" und sie setzt ihre kriminelle Karriere im Erwachsenenalter fort. ${ }^{209}$

\footnotetext{
206 Vgl. Kaiser 1997, S. 261 ff.

207 Moffitt 1993.

208 Vgl. Moffitt 1993, S. 691.

209 Vgl. Moffitt 1993, S. 679.
} 
Hier sollen die Deliktsähnlichkeiten getrennt nach Altersgruppen ${ }^{210}$ untersucht werden. Das Lebensalter wird eingeteilt in die Abschnitte:

7 bis 13 Jahre

14 bis 17 Jahre

18 bis 21 Jahre

22 bis 25 Jahre

26 bis 31 (PAD), 33 (BZR) Jahre
Kinder

Jugendliche

Heranwachsende

junge Erwachsene

Erwachsene

Untersucht werden soll, wie sich die Deliktsähnlichkeiten in den verschiedenen Lebensphasen unterscheiden. Die Analyse wird durchgeführt für die polizeilichen und die justiziellen Registrierungen der deutschen Männer. Die Methode ist dieselbe, die auch für alle deutschen Männer verwendet wurde. Die Abbildungen 18 bis 22 zeigen die Ergebnisse der Multidimensionalen Skalierung der polizeilich registrierten deutschen Männer der einzelnen Altersgruppen (Alter zur Tatzeit). Bei der zweidimensionalen Darstellung aller Altersgruppen zeigt sich insbesondere die Ähnlichkeit der Gewaltdelikte. Die Distanz zwischen Körperverletzung, schwerer Körperverletzung, Straftaten gegen die persönliche Freiheit und auch Beleidigung, Sachbeschädigung und Hausfriedensbruch ist in allen Altersgruppen klein. Auch die Ähnlichkeit der Deliktskategorien Betrug und Fälschung zeigt sich in allen Altersgruppen. Der schwere Diebstahl befindet sich bei allen Abbildungen am Rand der Abbildung und zeigt somit seine Unähnlichkeit zu allen anderen Delikten. Allerdings variiert dieser Abstand in den verschiedenen Altersgruppen. Er ist bei den Kindern relativ klein und bei den Jugendlichen am größten.

Abbildung 18 zeigt die Deliktskonfiguration der Kinder. Bei den Kindern ergeben sich 54.000 Deliktspaare. Die Delikte Meineid und Widerstand gegen die Staatsgewalt sind bei Kindern polizeilich nicht registriert. Der STRESS-Wert der Abbildung beträgt 0,21 und entspricht damit dem aller deutschen Männer. Die Darstellung der einzelnen Delikte ist gut mit Ausnahme von Tötungsdelikten und Straftaten gegen die öffentliche Ordnung, bei denen die erklärte Varianz kleiner als $40 \%$ ist.

210 Von den Registrierten in den Polizeidaten Baden-Württembergs und im Bundeszentralregister ist nur das Geburtsjahr bekannt, nicht der Geburtsmonat und der Geburtstag. Deshalb sind die Altersangaben nicht exakt. Unter den „21-Jährigen“ befinden sich etwa zur Hälfte 20-Jährige. Die Altersgruppen $14-17,18-21$ und $22-25$ sind auch unter dem Aspekt gewählt, dass sie die gleiche Dauer umfassen. Allgemein bezeichnet man als Heranwachsende die 18- bis 20-Jährigen. Die Gruppen stimmen hier nur in etwa. In den hier benutzten Datensätzen sind die ältesten Registrierten der Polizei 31 Jahre alt, die des Bundeszentralregisters 33. Die Berechnungen sind mit allen Geburtsjahrgängen durchgeführt worden. 
Bei den Kindern zeigen sich in der zweidimensionalen Darstellung im Vergleich zu allen Männern zwei Unterschiede. Bei allen Männern sind Betäubungsmitteldelikte und Leistungserschleichung ähnlich. Bei den Kindern zeigt sich diese Ähnlichkeit nicht, sondern hier ergibt sich ein Zusammenhang zwischen Gewaltdelikten und Betäubungsmitteldelikten. Wohl aber wird die Ähnlichkeit von Betrug und einfachem Diebstahl sichtbar, die sich über alle Altersgruppen nicht zeigt.

Abbildung 18: Ergebnis der MDS (deutsche Männer PAD, Alter 7 bis 13) 11

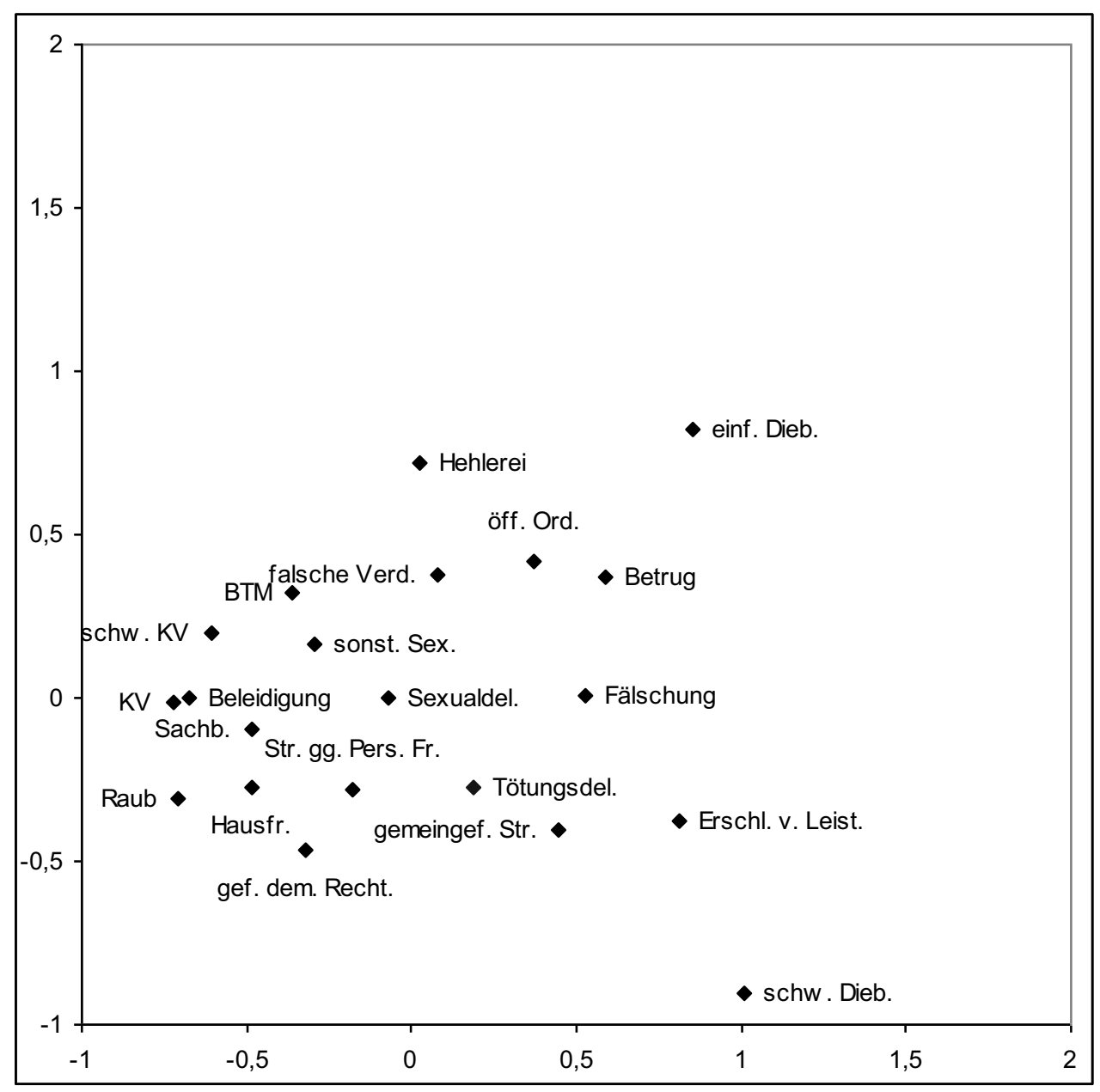

Abbildung 19 zeigt die Deliktskonfiguration der Jugendlichen. Bei den Jugendlichen gibt es 338.000 Deliktspaare. Der STRESS-Wert der Darstellung ist 0,20. Alle Delikte sind in der zweidimensionalen Darstellung gut dargestellt. Die erklärte Varianz der einzelnen Delikte reicht von 59\% bis $96 \%$.

Besonders unähnlich zu allen anderen Delikten ist der schwere Diebstahl bei den Jugendlichen. Der deutliche Zusammenhang zwischen Sexualdelikten und ,sonsti-

211 Ein Abstand von 0,7 Einheiten entspricht einem ASR von Null. 
gen Sexualdelikten“ hängt bei Jugendlichen damit zusammen, dass bei Sexualdelikten von Jugendlichen das Opfer häufig jünger als 14 Jahre ist und es sich somit um einen Fall von sexuellem Missbrauch von Kindern ( $§ 176 \mathrm{StGB})$ handelt, was in die Deliktskategorie „sonstige Sexualdelikte“ fällt. Dieselbe Tat fällt in die Deliktskategorie „Sexualdelikte“, wenn das Opfer 14 Jahre oder älter ist. Wie bei den Kindern, aber im Gegensatz zum Ergebnis aller deutschen Männer, zeigt sich auch bei den Jugendlichen eine Ähnlichkeit von Betrug und einfachem Diebstahl. Ansonsten stimmt die zweidimensionale Darstellung der Delikte mit der aller deutschen Männer überein.

Abbildung 19: Ergebnis der MDS (deutsche Männer PAD, Alter 14 bis 17)212

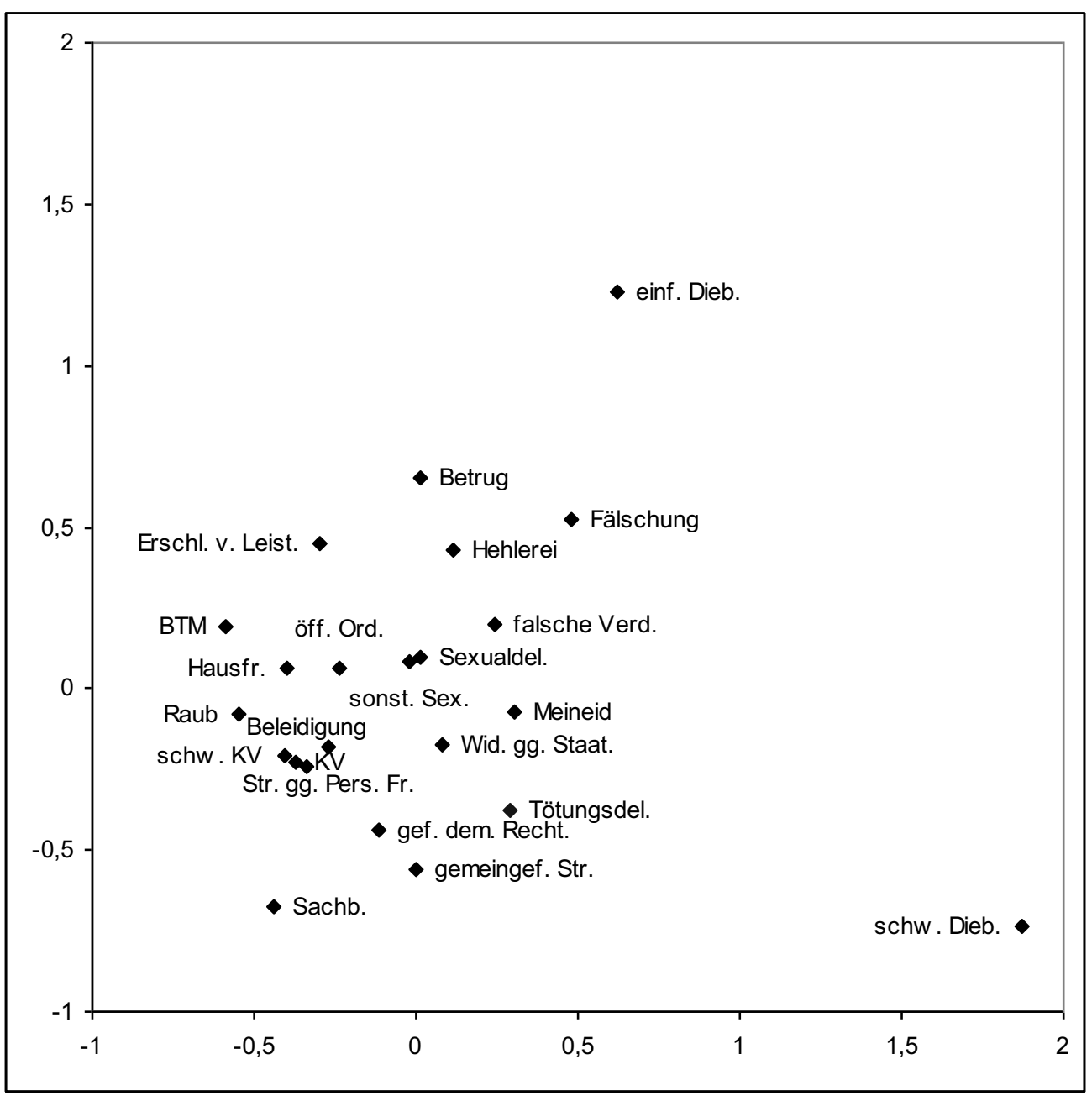

Abbildung 20 zeigt die Deliktskonfiguration der Heranwachsenden. In dieser Altersgruppe gibt es mit 464.000 die meisten Deliktspaare. Der STRESS-Wert der Abbildung ist 0,20. Die Delikte sind bis auf Hehlerei im zweidimensionalen gut dargestellt. Die erklärte Varianz bei Hehlerei beträgt nur $18 \%$, ansonsten ist die

212 Ein Abstand von 0,7 Einheiten entspricht einem ASR von Null. 
erklärte Varianz der einzelnen Delikte gut, das $\mathrm{R}^{2}$ ist bei allen anderen Delikten höher als $60 \%$. Die Deliktskonfiguration der Heranwachsenden stimmt mit der aller Männer großteils überein. Dies hängt wohl hauptsächlich damit zusammen, dass die Gruppe der Heranwachsenden mit 464.000 Deliktspaaren die größte Gruppe ist.

Abbildung 20: Ergebnis der MDS (deutsche Männer PAD, Alter 18 bis 21)213

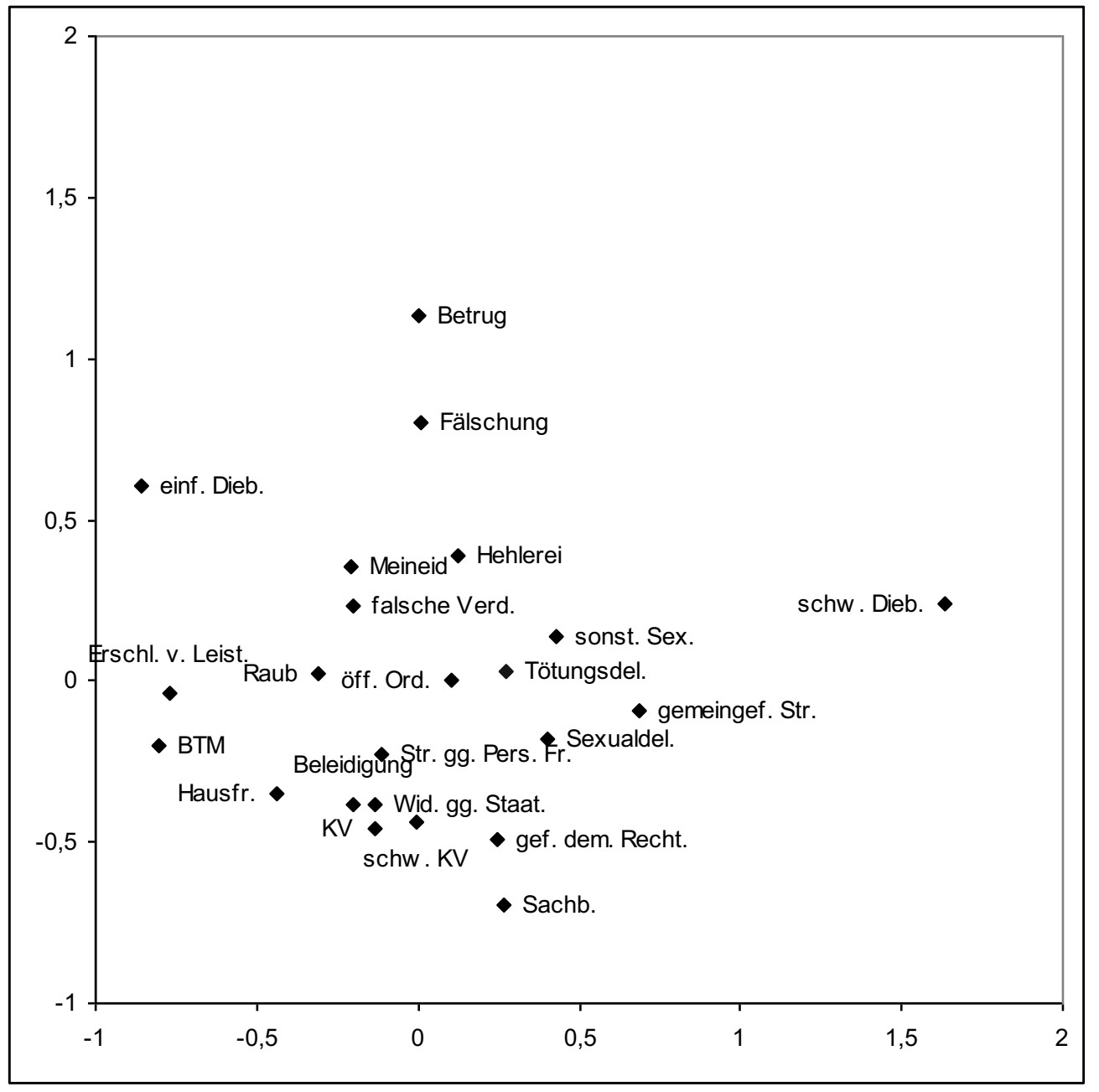

Abbildung 21 zeigt die Deliktskonfiguration der jungen Erwachsenen. Die Anzahl der Deliktspaare (222.000) ist im Vergleich zu den Heranwachsenden deutlich geringer. Der STRESS-Wert der Darstellung ist 0,21. Die erklärte Varianz bei gemeingefährlichen Straftaten (29\%), Hehlerei (47\%) und Meineid (44\%) ist relativ gering. Die Darstellung dieser Delikte ist im zweidimensionalen Raum nicht optimal. Alle anderen Delikte werden gut dargestellt. Die erklärte Varianz der übrigen Delikte liegt zwischen 56\% und 94\%. Die Deliktskonfiguration der jungen Erwachsenen stimmt mit der Deliktskonfiguration aller deutschen Männer überein.

213 Ein Abstand von 0,7 Einheiten entspricht einem ASR von Null. 
Abbildung 21: Ergebnis der MDS (deutsche Männer PAD, Alter 22 bis 25)214

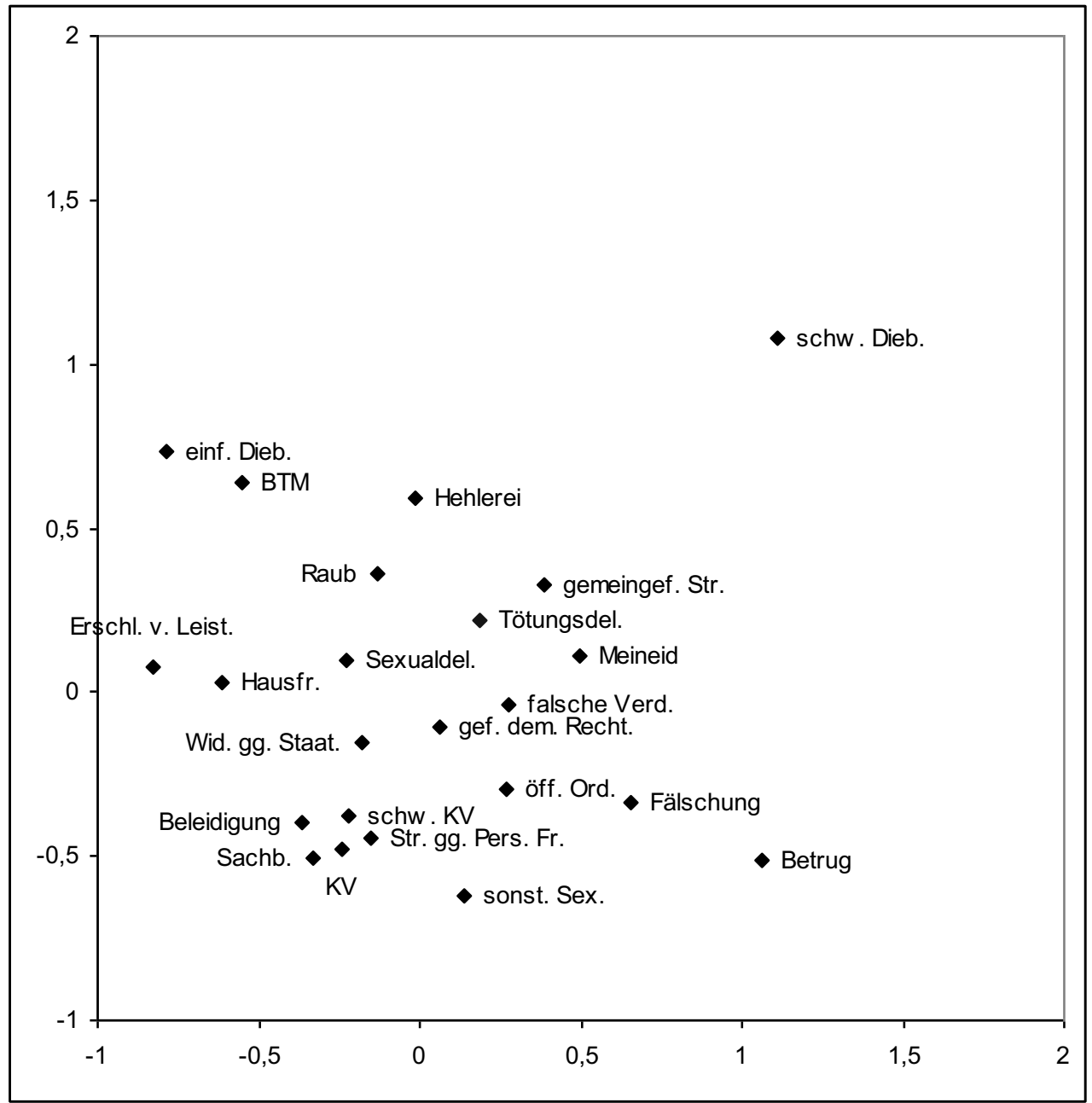

Abbildung 22 zeigt die Deliktskonfiguration der 26- bis 31-Jährigen. Mit 125.000 Deliktspaaren sind es im Vergleich zu den jungen Erwachsenen deutlich weniger. Der STRESS-Wert der Abbildung ist 0,21. Die erklärte Varianz von schwerem Diebstahl (43\%), Meineid (38\%) und Fälschung (36\%) ist relativ klein. Diese Delikte sind in der zweidimensionalen Abbildung nicht optimal dargestellt. Alle anderen Delikte werden in der zweidimensionalen Darstellung gut präsentiert. Die Deliktskonfiguration der Erwachsenen stimmt bis auf die Darstellung von Sexualdelikten mit der Deliktskonfiguration aller deutschen Männer überein. Bei allen deutschen Männern zeigt sich eine Ähnlichkeit von Gewaltdelikten und Sexualdelikten, diese Ähnlichkeit zeigt sich bei den 26- bis 31- Jährigen nicht.

214 Ein Abstand von 0,7 Einheiten entspricht einem ASR von Null. 
Abbildung 22: Ergebnis der MDS (deutsche Männer PAD, Alter 26 bis $31^{215}$

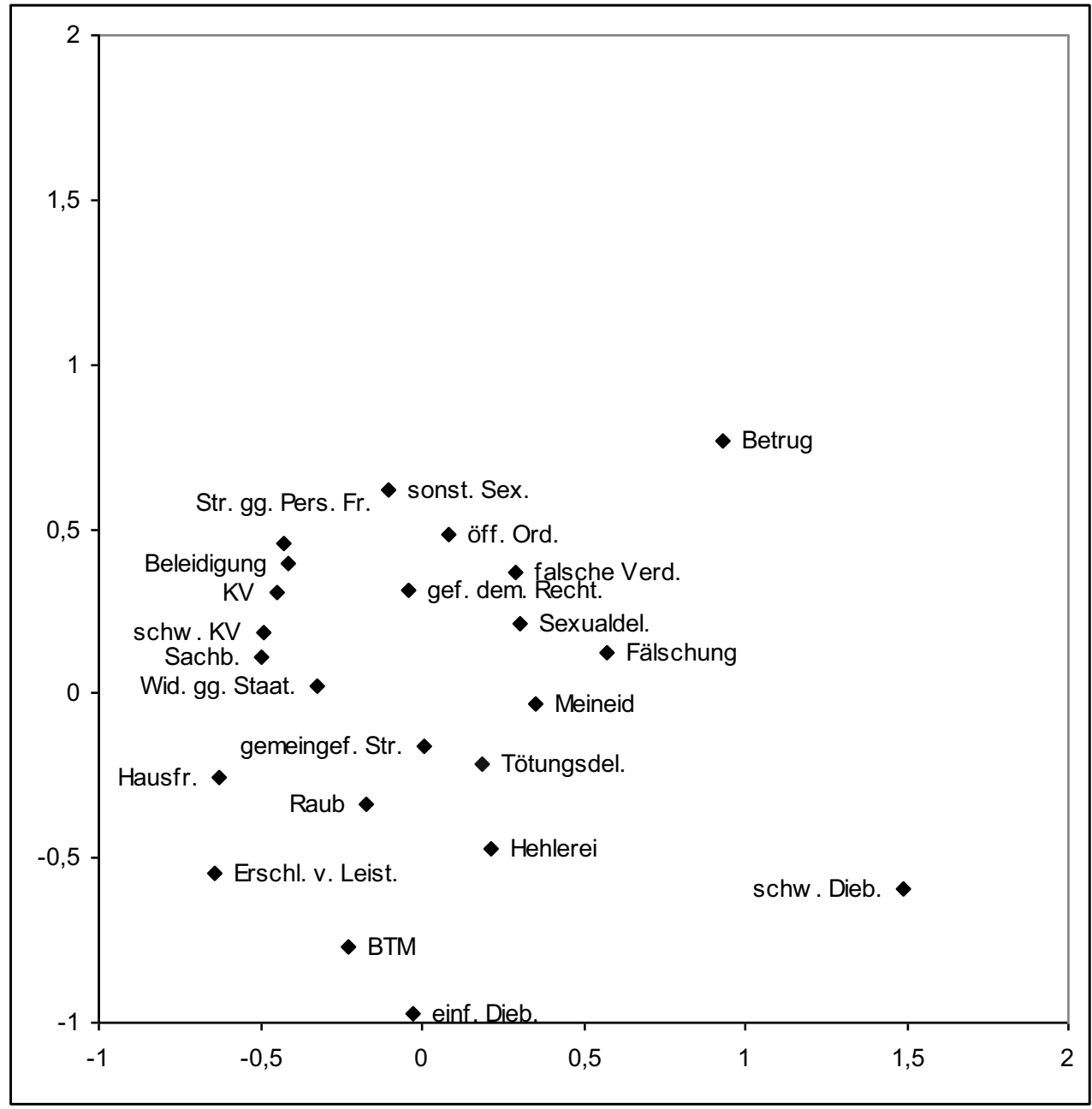

Bei den Erwachsenen und bei allen deutschen Männern zeigt sich die Unähnlichkeit von einfachem Diebstahl und Betrug. Bei Kindern und Jugendlichen ergibt sich eine Ähnlichkeit von einfachem Diebstahl und Betrug.

Bei allen Altersgruppen außer bei den Kindern erweist sich die Ähnlichkeit von Leistungserschleichung und Betäubungsmitteldelikten.

Zusammenfassend ist zu sagen, dass die Deliktskonfiguration der Heranwachsenden und der Jungerwachsenen mit der Deliktskonfiguration aller übereinstimmt. Abweichungen zeigen sich bei der Deliktskonfiguration der übrigen Altersgruppen. Die Ähnlichkeit von Gewaltstraftaten und Sexualstraftaten, die sich bei den jüngeren Altersgruppen zeigt, kann bei den Erwachsenen (Alter 26 bis 31) nicht festgestellt werden.

Auch anhand der justiziellen Registrierungen der deutschen Männer werden die Deliktskonfigurationen der einzelnen Altersgruppen untersucht. Die Deliktskombi-

215 Ein Abstand von 0,7 Einheiten entspricht einem ASR von Null. 
nationen werden aus Delikten aller gerichtlichen Entscheidungen der Täter gebildet. Wenn innerhalb einer Entscheidung mehrere Delikte registriert sind, wird pro Deliktskategorie nur ein Delikt in die Auswertung einbezogen. Die Delikte sind in 27 Deliktskategorien unterteilt, vier mehr als bei den polizeilichen Registrierungen. Im Einzelnen sind dies Verkehrsdelikte, eine große Gruppe innerhalb der justiziellen Registrierungen, die leider in den Polizeidaten nicht registriert werden. Des Weiteren fahrlässige Körperverletzung/fahrlässige Tötung, Vollrausch und gefährlicher Eingriff in den Straßenverkehr, die zwar von der Polizei registriert werden, aber sehr selten, da diese Delikte meist in Zusammenhang mit einem Verkehrsdelikt vorkommen.

Abbildung 23: Ergebnis der MDS (deutsche Männer BZR, Alter 14 bis 17)216

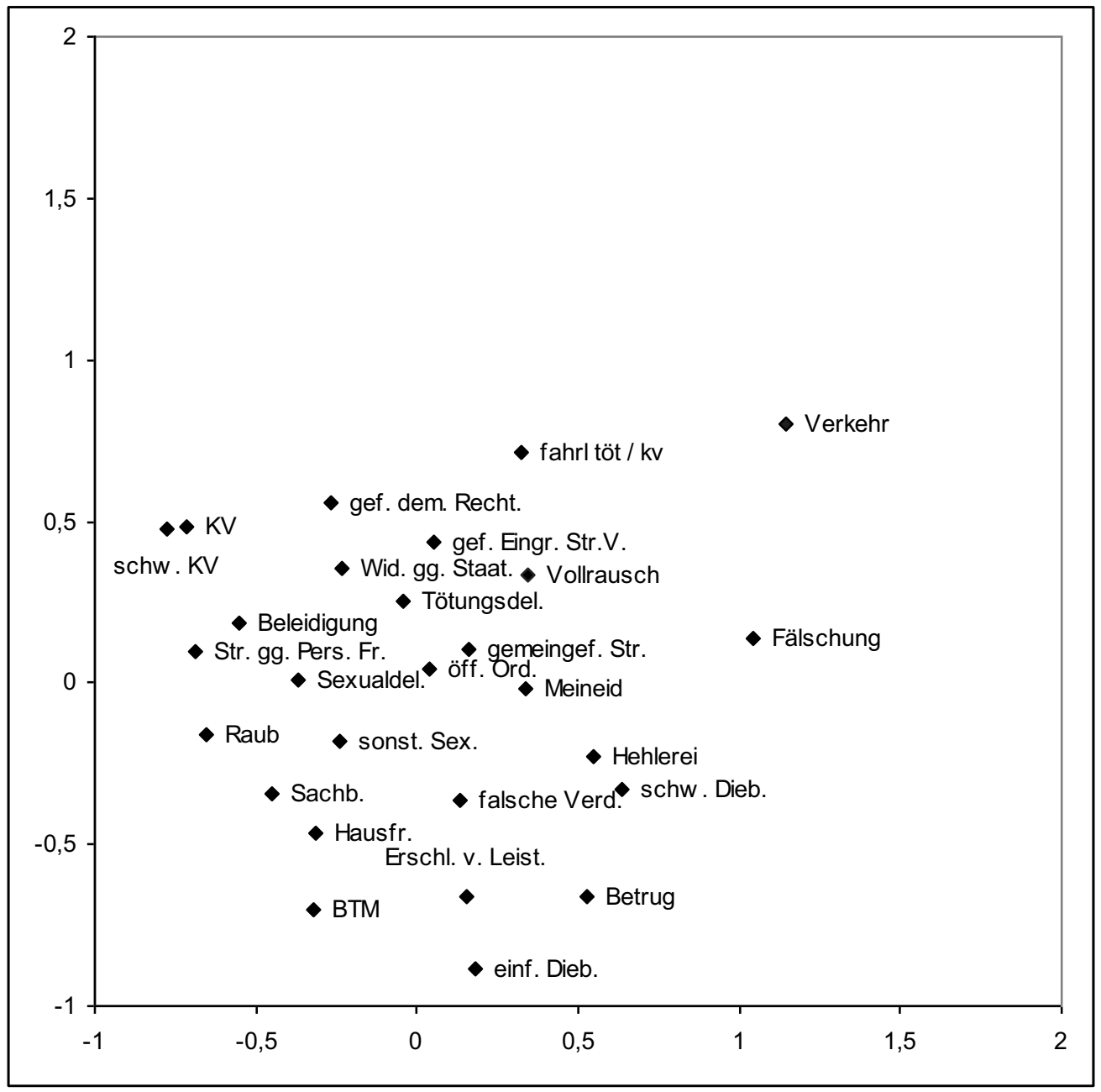

Bei der Einteilung der Altersgruppen wird bei den justiziellen Registrierungen das Alter zum Zeitpunkt der Entscheidung benutzt. Die Altersgruppe der Kinder, die es bei polizeilichen Registrierungen gibt, gibt es bei justiziellen Registrierungen nicht, da es zu gerichtlichen Entscheidungen erst ab der Strafmündigkeit von 14 Jahren kommen kann.

216 Ein Abstand von 0,7 Einheiten entspricht einem ASR von Null. 
Die Abbildungen 23 bis 26 zeigen die Ergebnisse der Multidimensionalen Skalierung der justiziell registrierten deutschen Männer der einzelnen Altersgruppen.

Bei den Jugendlichen (Abbildung 23) gibt es 83.000 Deliktspaare. Der STRESS-Wert ist mit 0,25 relativ schlecht. (Der STRESS-Wert der zweidimensionalen Darstellung der Delikte aller deutschen Männer ist 0,21.) Dennoch sind die meisten Delikte gut dargestellt, nur bei Straftaten gegen die öffentliche Ordnung, Meineid, gefährlicher Eingriff in den Straßenverkehr, Vollrausch und fahrlässiger Tötung/Körperverletzung und damit bei 5 von 27 Deliktskategorien ist die erklärte Varianz kleiner als $40 \%$. Sehr gut dargestellt sind die Deliktskategorien schwerer Körperverletzung, Körperverletzung, Straftaten gegen die persönliche Freiheit, Tötungsdelikte, einfacher Diebstahl, Widerstand gegen die Staatsgewalt, Beleidigung, Fälschung, falsche Verdächtigung, Betäubungsmitteldelikte und Verkehrsdelikte.

Abbildung 24: Ergebnis der MDS (deutsche Männer BZR, Alter 18 bis 21)217

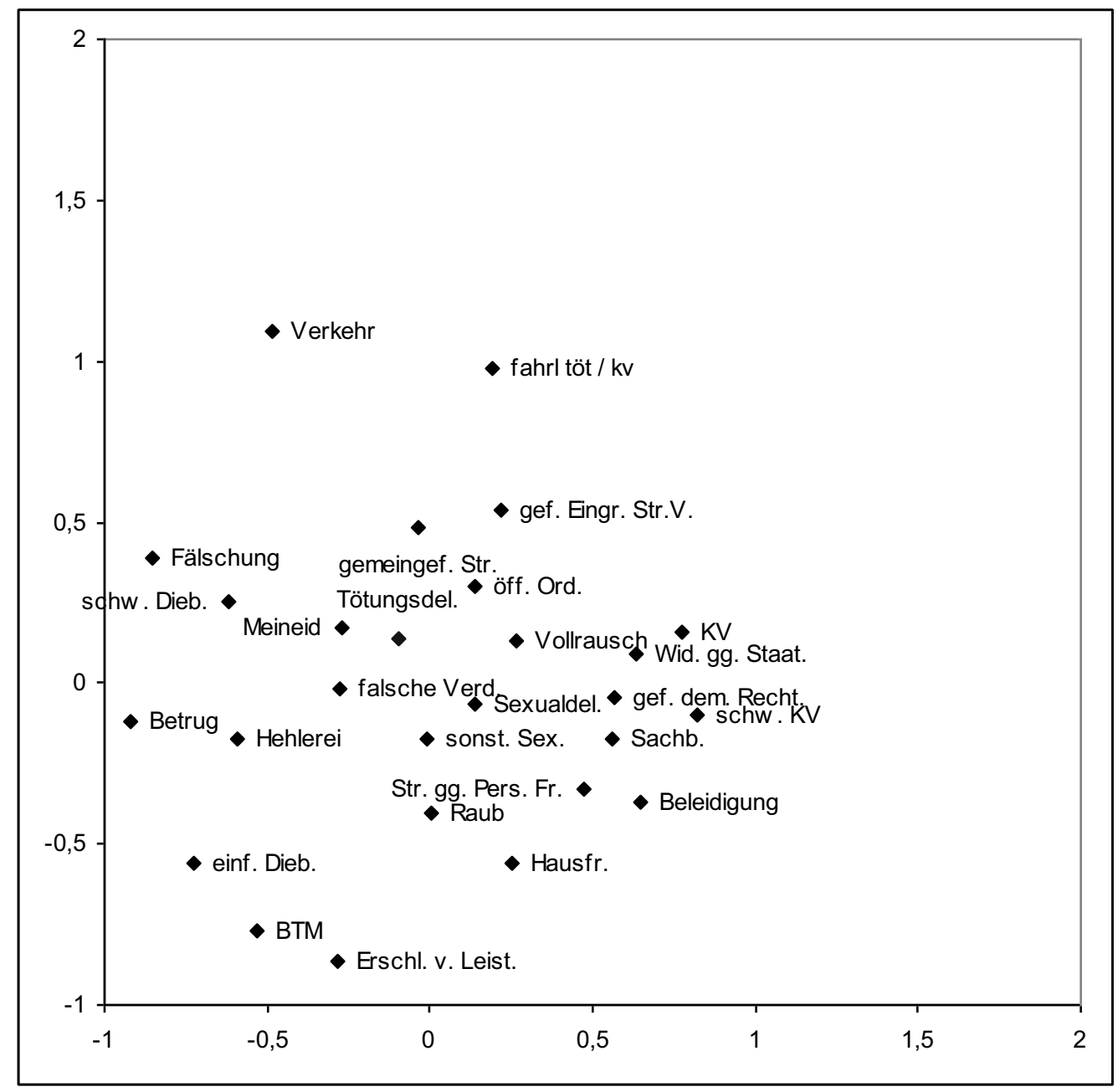

217 Ein Abstand von 0,7 Einheiten entspricht einem ASR von Null. 
Die zweidimensionale Darstellung der Deliktskonfiguration der Jugendlichen stimmt größtenteils mit der Darstellung aller Altersgruppen überein. Ein Unterschied offenbart sich aber bei Betrug und Leistungserschleichung. Hier zeigt sich bei den Jugendlichen eine Ähnlichkeit, die sich bei der Darstellung aller Altersgruppen nicht ergibt.

Bei den Heranwachsenden (Abbildung 24) gibt es 120.000 Deliktspaare. Damit bilden die Heranwachsenden wie auch bei den polizeilichen Registrierungen die größte Altersgruppe bei der Betrachtung der Deliktskombinationen. Der STRESSWert der Abbildung ist 0,22. Die Delikte sind abgesehen von Tötungsdelikten Straftaten gegen die öffentliche Ordnung, falsche Verdächtigung und Meineid (erklärte Varianz kleiner als 40\%) gut dargestellt. Die Deliktskonfiguration der Heranwachsenden entspricht der Deliktskonfiguration aller Männer.

Abbildung 25: Ergebnis der MDS (deutsche Männer BZR, Alter 22 bis 25)218

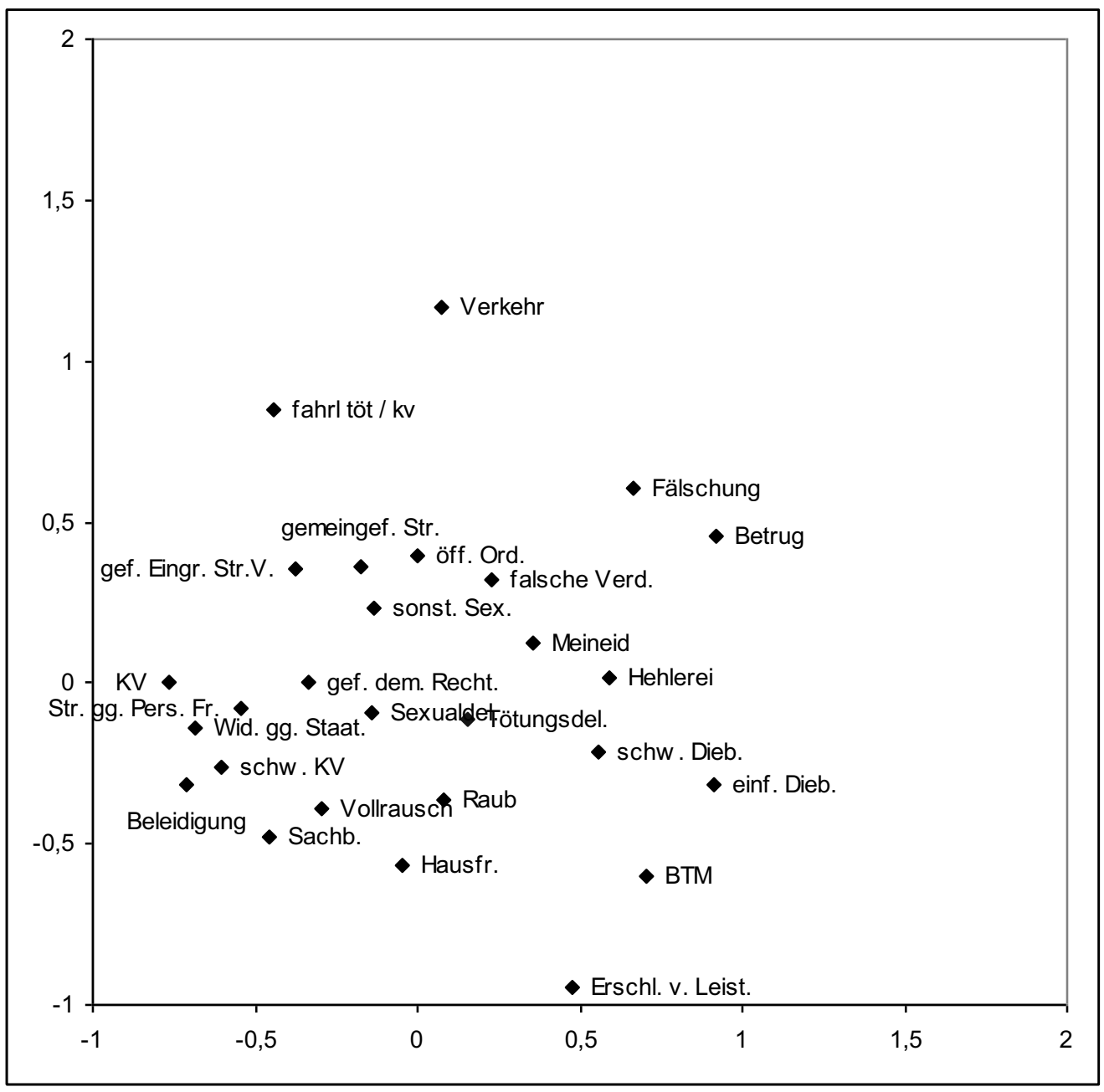

Auch die jungen Erwachsenen (Abbildung 25) bilden mit 89.000 Deliktspaaren eine relativ große Gruppe. Der STRESS-Wert ist 0,21. Die Darstellung der Delikte

218 Ein Abstand von 0,7 Einheiten entspricht einem ASR von Null. 
ist gut, bis auf die Darstellung von Straftaten gegen die öffentliche Ordnung; hier beträgt die erklärte Varianz 36\%. Auch die Deliktskonfiguration der jungen Erwachsenen entspricht der Deliktskonfiguration aller Männer.

Bei den 26- bis 33-Jährigen gibt es 65.600 Deliktspaare. Diese Altersgruppe umfasst zwei Jahre mehr als die vergleichbare Altersgruppe der polizeilich Registrierten. Der STRESS-Wert der zweidimensionalen Darstellung beträgt 0,21. Die erklärte Varianz ist schlechter als $40 \%$ bei Raub (39\%), Straftaten gegen die öffentliche Ordnung (36\%) und Meineid (34\%). Alle anderen Deliktskategorien, insbesondere schwere Körperverletzung, Körperverletzung, Straftaten gegen die persönliche Freiheit, einfacher Diebstahl, Sachbeschädigung, Hehlerei, Betrug, Widerstand gegen die Staatsgewalt, Beleidigung, Fälschung, Betäubungsmitteldelikte, gefährlicher Eingriff in den Straßenverkehr, fahrlässige Tötung/Körperverletzung und Verkehrsdelikte, sind gut dargestellt.

Abbildung 26: Ergebnis der MDS (deutsche Männer BZR, Alter 26 bis 33)219

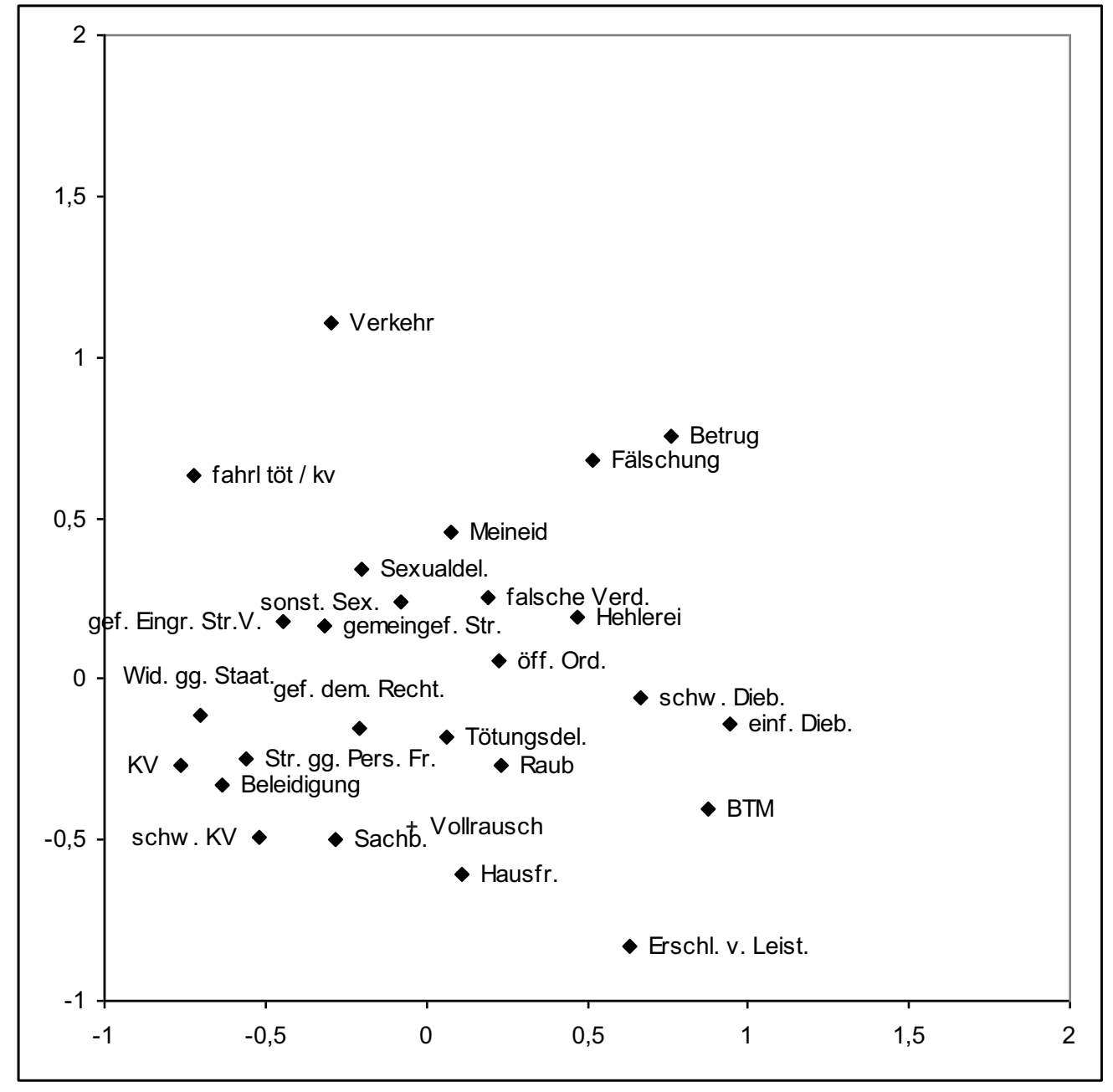

219 Ein Abstand von 0,7 Einheiten entspricht einem ASR von Null. 
Bei der Deliktskonfiguration der 26- bis 33-Jährigen zeigen sich Unterschiede zur Deliktskonfiguration der anderen Altersgruppen. Insbesondere zeigt sich bei den Erwachsenen kein Zusammenhang zwischen Sexualdelikten und Gewaltdelikten, im Gegensatz zu den jüngeren Altersgruppen, bei denen sich ein schwacher Zusammenhang zeigt.

Auf den ersten Blick weisen die zweidimensionalen Darstellungen der Deliktskonfiguration der verschiedenen Altersgruppen der justiziell registrierten deutschen Männer keine offensichtlichen Unterschiede auf. Aber im Detail zeigen sich kleine Differenzen.

Bei allen Altersgruppen übereinstimmend zeigt sich die Randlage von Verkehrsdelikten und damit die Unähnlichkeit von Verkehrsdelikten zu den meisten anderen Delikten. Ähnlichkeiten ergeben sich zwischen Verkehrsdelikten und fahrlässiger Tötung/Körperverletzung und zwischen Verkehrsdelikten und „Fälschung“. Urkundenfälschung, genauer gesagt Fälschung der Autopapiere, ist bei einer Person häufig registriert, wenn ein Verkehrsdelikt registriert ist.

Ähnlichkeiten treten bei allen Altersgruppen der justiziell registrierten deutschen Männer zwischen Leistungserschleichung, einfachem Diebstahl, Hausfriedensbruch und Betäubungsmitteldelikten hervor.

Bei allen Altersgruppen übereinstimmend lässt sich die Ähnlichkeit von Körperverletzung, schwerer Körperverletzung, Straftaten gegen die persönliche Freiheit, Widerstand gegen die Staatsgewalt, Beleidigung und Sachbeschädigung feststellen. Außer bei den Jugendlichen ist auch Vollrausch ähnlich mit diesen Delikten. Auch bei den Sexualdelikten zeigt sich eine schwache Ähnlichkeit zu dieser Gruppe von Gewaltdelikten in allen Altersgruppen, außer bei den Erwachsenen.

Auch die Delikte schwerer Diebstahl und Hehlerei kommen in allen Altersgruppen häufig gemeinsam bei Personen vor. Die Unähnlichkeit von schwerem Diebstahl zu allen anderen Delikten, wie es die Deliktskonfiguration der polizeilichen Registrierungen präsentiert, zeigt sich bei den justiziellen Registrierungen in keiner Altersgruppe. Das hängt damit zusammen, dass schwerer Diebstahl häufig in Serie begangen wird und demzufolge häufig mehrfach bei einer Person polizeilich registriert wird. Diese Seriendiebstähle werden justiziell in einer gerichtlichen Entscheidung abgeurteilt und werden somit justiziell nur einmal gezählt.

Die Deliktskategorien Betrug und Fälschung sind außer bei den Jugendlichen in allen Altersgruppen ähnlich.

Zusammenfassend ist zu sagen, dass bei den polizeilich Registrierten die Deliktskonfiguration der Heranwachsenden und der Jungerwachsenen mit der Deliktskonfiguration aller übereinstimmt. Abweichungen zeigen sich bei den polizeilich Registrierten bei der Deliktskonfiguration der Kinder, Jugendlichen und 26bis 31-Jährigen. Bei den justiziell Registrierten zeigen sich sowohl bei den Jugendlichen wie bei den 26- bis 33-Jährigen Abweichungen zur Deliktskonfiguration 
aller justiziell Registrierten. Veränderungen mit dem Alter stellen sich bei Betrug und auch bei Sexualdelikten dar. Bei Sexualdelikten zeigen sich bei Jugendlichen, Heranwachsenden und Jungerwachsenen Ähnlichkeiten zu Gewaltdelikten, bei den älteren Erwachsenen findet man diese Ähnlichkeit nicht.

\subsection{Hierarchische Clusteranalyse}

Mit einer Clusteranalyse ist es möglich, aus einer Vielzahl von Personen bzw. Objekten Gruppen zu bilden, die ähnliche Objekte enthalten, wobei die einzelnen Gruppen möglichst unähnlich zueinander sein sollen. ${ }^{220}$ Hier ist das Ziel, unterschiedliche Straftaten, Delikte, in Gruppen von ähnlichen Delikten zusammenzufassen. Mit der Stata Prozedur clustermat kann auf der Basis der auch bei der Multidimensionalen Skalierung verwendeten Ähnlichkeitsmaße ${ }^{221}$ eine Clusteranalyse durchgeführt werden. ${ }^{222}$ Mit den vorhandenen Daten sind drei verschiedene Berechnungsmethoden möglich: single linkage, complete linkage und average linkage. ${ }^{223}$ Bei single linkage, auch nearest-neighbor method oder minimum method genannt, wird jeweils der kleinste Wert der Dissimilaritäten zur Berechnung der Cluster benutzt. Bei complete linkage, auch furthest-neighbor method oder maximum method genannt, wird jeweils der größte Wert zur Berechnung der Cluster benutzt. Die Prozedur average linkage, arithmetic-average clustering, benutzt den jeweiligen Mittelwert der Dissimilaritäten zur Bildung der Cluster. Die Methode average linkage liefert hier die besten Ergebnisse, weil alle Dissimilaritäten in die Berechnung eingehen.

Abbildung 27 zeigt das Ergebnis der Clusteranalyse für die deutschen Männer (polizeiliche Registrierungen) mit der Prozedur average linkage. Die y-Achse ist die Achse des Mittelwerts der Dissimilaritäten. Die Dissimilaritäten sind die negativen ASR addiert mit 100 damit alle Werte größer Null sind. Somit ist bei 100 die Stelle an der ASR 0 ist, unter 100 zeigen sich Ähnlichkeiten, über 100 Unähnlich-

${ }^{220}$ Vgl. Backhaus, Erichson, Plinke \& Weiber 2003, S. 480 ff.

221 Als Ähnlichkeitsmaße werden die in Abschnitt 5.1 beschriebenen Adjusted Standardized Residuals benutzt. Eine Voraussetzung bei der Clusteranalyse mit Stata ist, dass das Ähnlichkeitsmaß eine Dissimilarität und positiv ist. Das ASR ist ein Similaritätsmaß. Deshalb werden die ASR mit -1 multipliziert und eine Konstante dazu addiert, hier 100, damit alle Werte positiv sind. Zu den Mindestanforderungen der Distanzen, hier Dissimilaritätsmaße, gehört: „Die Distanz zwischen zwei verschiedenen Punkten ist positiv“ Ahrens S. 74. Die Dissimilaritäten dürfen aber verändert werden, solange ihre Rangordnung nicht verändert wird. Vgl. Borg \& Gronen S. 24. Insbesondere darf also eine Konstante zu den Dissimilaritäten dazu addiert werden. Diese Veränderung bei den Ausgangsdaten wird bei SPSS (PROXSCAL) automatisch durchgeführt, bei Stata muss diese Datenanpassung vorher durchgeführt werden.

222 Vgl. Stata [MV] S.83 ff., zur Erzeugung der Dissimilaritätsmatrix siehe Stata [P] matrix mkmat S. 264 ff. und zur Clusterberechnung mit Stata siehe Stata [MV] cluster S. $71 \mathrm{ff}$.

${ }^{223} \mathrm{Vgl}$. Stata [MV] S. 79, S.83 ff. 
keiten. Damit ist der schwere Diebstahl unähnlich zu allen anderen Delikten, weil die Verknüpfung mit den anderen Delikten über 100 liegt. Der Wert von 150 beim schweren Diebstahl gibt an, dass das mittlere ASR von schwerem Diebstahl -50 ist. $^{224}$

Je kleiner der Mittelwert der Dissimilaritäten, desto ähnlicher sind die Delikte. Am ähnlichsten sind schwere Körperverletzung (schw_kv) und Körperverletzung (kv), hier ist der Dissimilaritätswert am kleinsten. Am unähnlichsten zu allen anderen Delikten ist schwerer Diebstahl (sd).

Abbildung 27: Arithmetic-average clustering (deutsche Männer PAD)

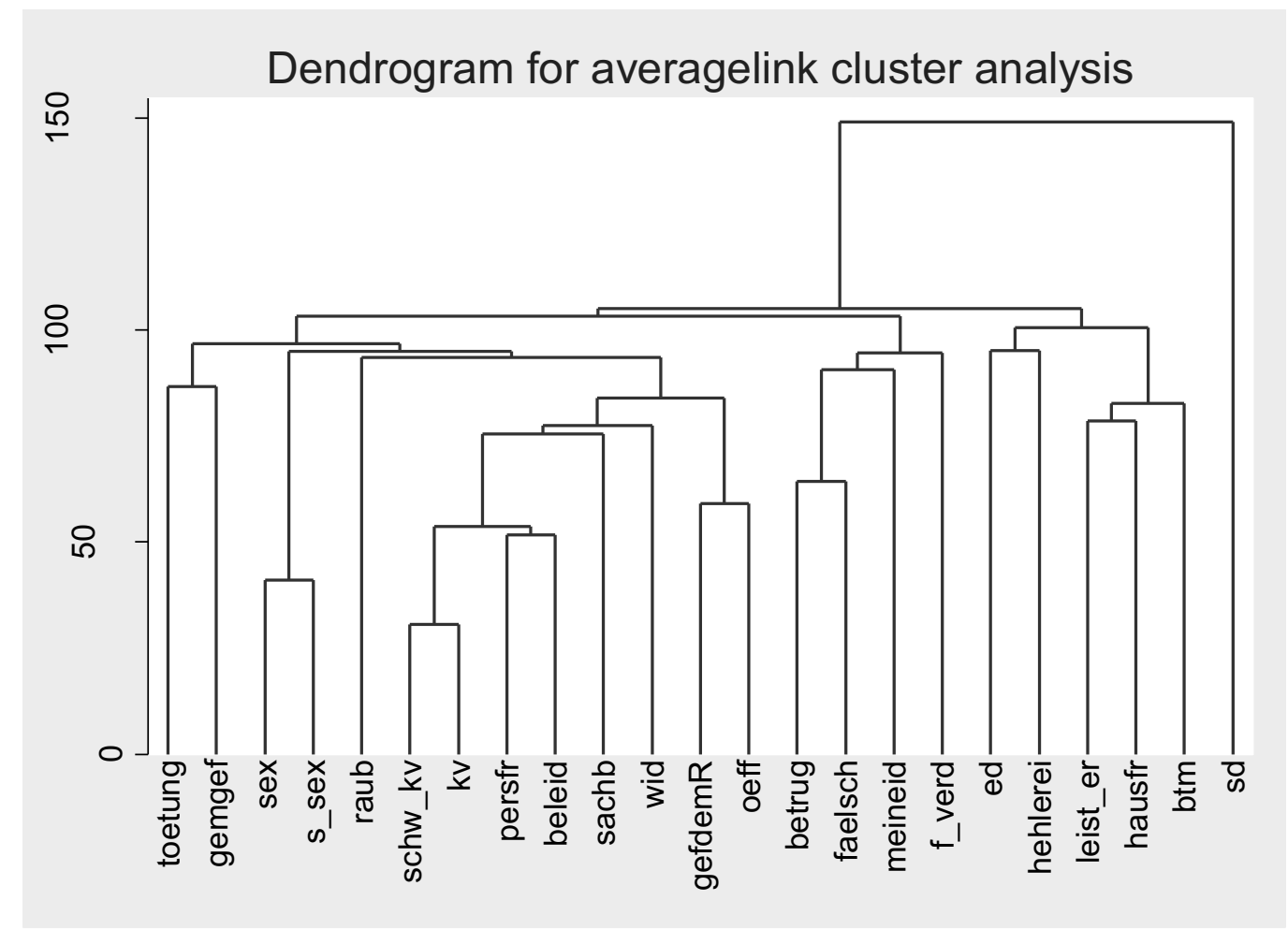

Teilt man die Delikte in vier Cluster ergibt sich folgende Aufteilung:

1. Gewaltdelikte plus gemeingefährliche Straftaten, Beleidigung, Sachbeschädigung, Widerstand gegen die Staatsgewalt, Gefährdung des demokratischen Rechtsstaates und Straftaten gegen die öffentliche Ordnung. Hier sind die Delikte zusammengefasst, die links in der Abbildung stehen, von Tötungsdelikte (toetung) bis Straftaten gegen die öffentliche Ordnung (oeff). Dieses Cluster kann weiter in vier Gruppen unterteilt werden:

$224150-100=50$, somit ist der Mittelwert aller Paarungen mit schwerem Diebstahl -50 (ASR). Würde mit der Methode single linkage gerechnet werden, würde nicht der Mittelwert benutzt werden sondern das kleinste Dissimilaritätsmaß, umgekehrt bei der Methode complete linkage das größte Dissimilaritätsmaß. 
Tötungsdelikte und gemeingefährliche Straftaten,

Sexualdelikte,

Raub,

Rest mit Körperverletzung, Straftaten gegen die persönliche Freiheit, Beleidigung, Sachbeschädigung,.., Straftaten gegen die öffentliche Ordnung.

2. Betrug, Fälschung (faelsch), Meineid, falsche Verdächtigung (f_verd),

3. Einfacher Diebstahl (ed), Hehlerei, Leistungserschleichung (leist_er), Hausfriedensbruch (hausfr), Betäubungsmitteldelikte (btm),

4. Schwerer Diebstahl (sd).

Das Ergebnis der Clusteranalyse entspricht dem Ergebnis der Multidimensionalen Skalierung mit unterschiedlicher grafischer Darstellung. Beide Darstellungen basieren auf dem ASR als Ähnlichkeitsmaß für die Delikte. Die Delikte, die ein Cluster bilden, liegen in der zweidimensionalen Darstellung der Multidimensionalen Skalierung nah beieinander. Schwerer Diebstahl bildet ein eigenes Cluster und befindet sich beim Ergebnis der MDS abseits aller anderen Deliktskategorien.

Dieselbe Analyse wird auch für die polizeilich registrierten Frauen in BadenWürttemberg durchgeführt. In Abbildung 28 ist das Ergebnis der Clusteranalyse dargestellt. Am ähnlichsten ist das Deliktspaar Körperverletzung und Beleidigung (kv, beleid). Weiter ist der Abbildung zu entnehmen, dass die Deliktspaare ,sonstige Sexualdelikte“ (hauptsächlich verbotene Prostitution) und Betäubungsmitteldelikte (s_sex, btm), sowie Betrug und Meineid ähnlich sind. Das mittlere Dissimilaritätsmaß ist relativ klein verglichen mit dem anderer Deliktspaare. Außerdem zeigt sich die Ähnlichkeit von schwerer Körperverletzung (schw_kv), Körperverletzung $(\mathrm{kv})$, Beleidigung (beleid) und Straftaten gegen die persönliche Freiheit (persfr). Die Einteilung in fünf Cluster ergibt folgende Aufgliederung:

1. Gewaltdelikte, dazu gehören Tötungsdelikte (toetung), Raub, schwere Körperverletzung (schw_kv), Körperverletzung, Straftaten gegen die persönliche Freiheit (persfr) (,sonstige Sexualdelikte“ gehören bei den Frauen nicht zu den Gewaltdelikten und Sexualdelikte kommen kaum vor), des Weiteren gehört zu diesem Cluster Beleidigung (beleid), Sachbeschädigung (sachb) und Widerstand gegen die Staatsgewalt (wid). Außerdem gemeingefährliche Straftaten (gemgef), Gefährdung des demokratischen Rechtsstaates (gefdemR), Straftaten gegen die öffentliche Ordnung (oeff) und falsche Verdächtigung (f_verd). Hier sind die Delikte zusammengefasst, die links in der Abbildung stehen, von Tötungsdelikte (toetung) bis falsche Verdächtigung (f_verd).

2. Schwerer Diebstahl (sd) und Hehlerei,

3. Sonstige Sexualdelikte (verbotene Prostitution), Betäubungsmitteldelikte, Leistungserschleichung (leist_er) und Hausfriedensbruch (hausfr),

4. Einfacher Diebstahl (ed), 
5. Betrug, Fälschung, Meineid.

Abbildung 28: Arithmetic-average clustering (deutsche Frauen PAD)

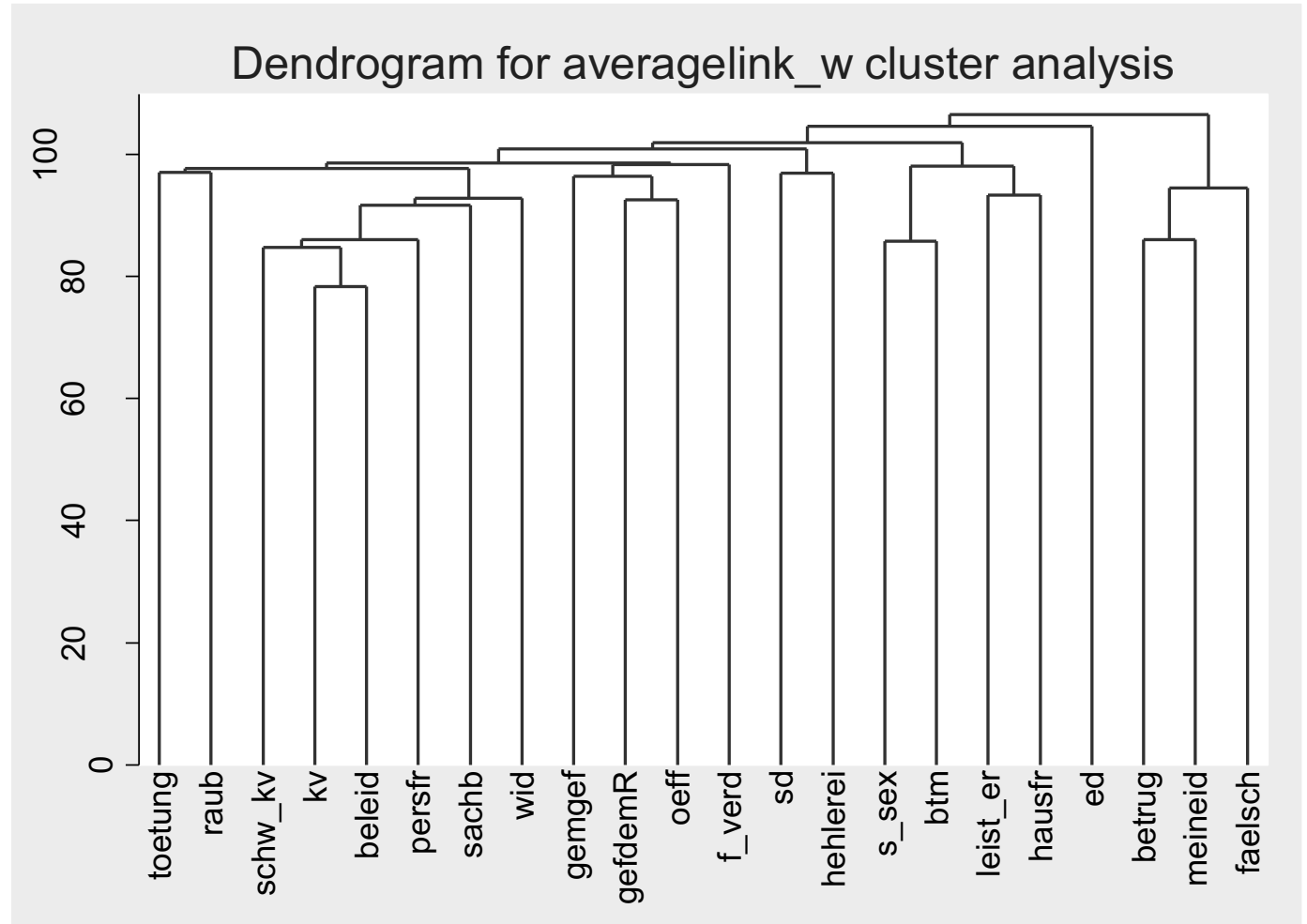

Im Gegensatz zu den Männern bildet der einfache Diebstahl bei den Frauen ein eigenes Cluster. Die Unähnlichkeit von schwerem Diebstahl zu anderen Delikten zeigt sich bei den Frauen nicht. „sonstige Sexualdelikte“ befinden sich bei den Frauen nicht wie bei den Männern im Gewaltcluster, sondern liegen zusammen mit Betäubungsmitteldelikten in einem Cluster. Hier zeigt sich die Verbindung von verbotener Prostitution und Betäubungsmitteldelikten bei den Frauen und von sexuellem Missbrauch und Vergewaltigung bei den Männern.

Die Ergebnisse der Clusteranalyse der polizeilichen Registrierungen entsprechen den Ergebnissen, die die Multidimensionale Skalierung ergibt. Die Delikte, die hier Gruppen bilden, liegen in der Abbildung der Multidimensionalen Skalierung nah beieinander und sind somit ähnlich.

Ebenso wie für die polizeilich Registrierten wird auch für die justiziell Registrierten eine Clusteranalyse durchgeführt. Abbildung 29 zeigt das Ergebnis der Clusteranalyse der deutschen Männer mit justiziellen Registrierungen. Besonders ähnlich sind, wie auch bei den polizeilichen Registrierungen, einfache und schwere Körperverletzung (kv, schw_kv). Auch die Ähnlichkeit von Betrug und Fälschung (faelsch) sowie von Verkehrsdelikten und fahrlässiger Tötung/Körperverletzung (fahrl_t_kv) ist zu sehen. 


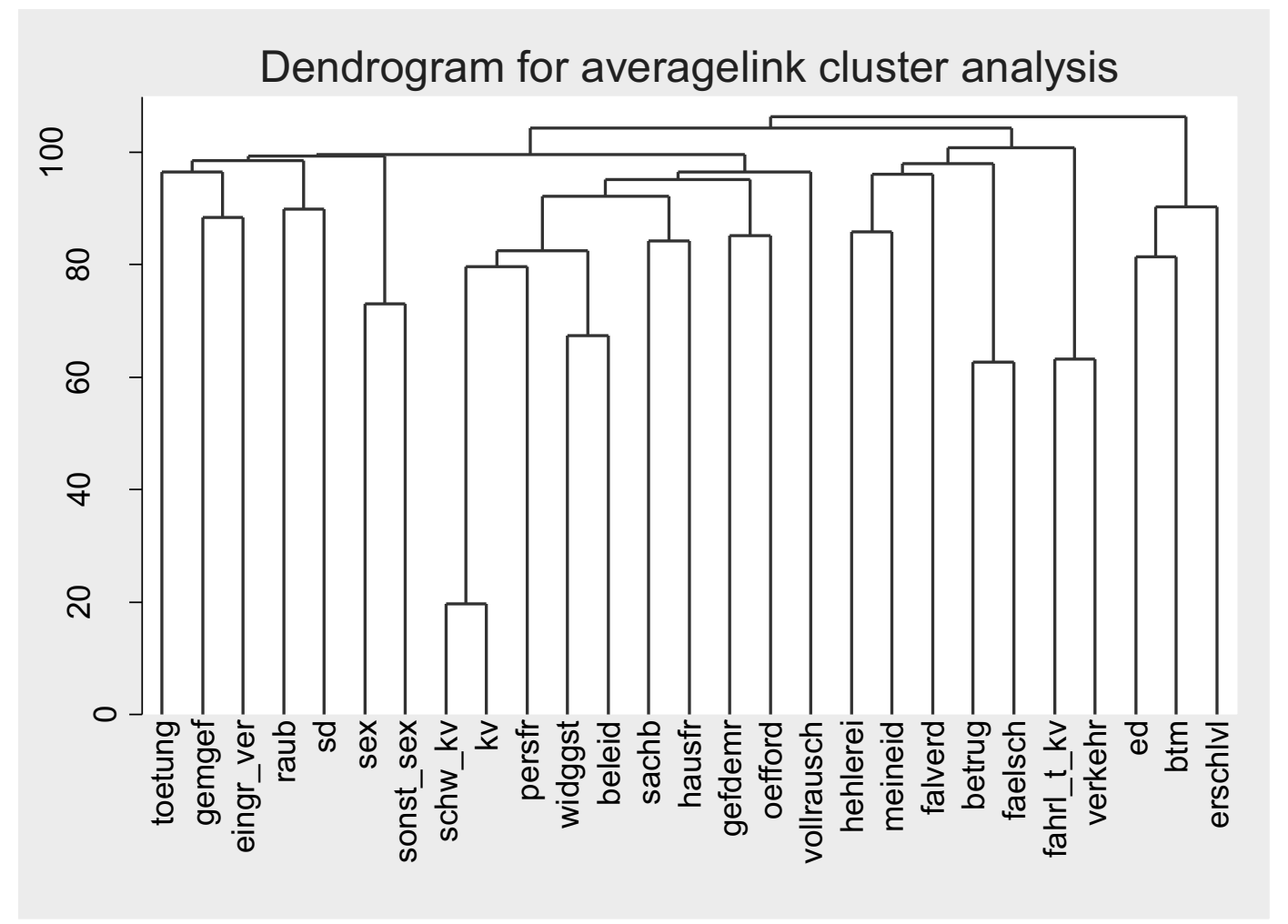

Teilt man die Delikte in fünf Cluster ergibt sich folgende Aufteilung:

1. Tötungsdelikte (toetung), gemeingefährliche Straftaten (gemgef) und gefährlicher Eingriff in den Straßenverkehr (eingr_ver), des Weiteren Raub, schwerer Diebstahl (sd) und auch Sexualdelikte (sex und sonst_sex).

2. Schwere Körperverletzung (schw_kv), Körperverletzung (kv), Straftaten gegen die persönliche Freiheit (persfr), Beleidigung (beleid), Widerstand gegen die Staatsgewalt (widggst), Sachbeschädigung (sachb), Hausfriedensbruch (hausfr), Gefährdung des demokratischen Rechtsstaates (gefdemr), Straftaten gegen die öffentliche Ordnung (oefford) und Vollrausch.

3. Hehlerei, Meineid, falsche Verdächtigung (falverd), Betrug, Fälschung (faelsch).

4. Verkehrsdelikte, fahrlässige Tötung/Körperverletzung (fahrl_t_kv),

5. Einfacher Diebstahl (ed), Betäubungsmitteldelikte (btm), Leistungserschleichung (erschlvl).

Die Clusteraufteilung stimmt teilweise mit der Clusteraufteilung der polizeilichen Registrierungen der deutschen Männer überein, es gibt aber auch deutliche Abweichungen. Das Cluster der Gewaltdelikte ist bei den justiziellen Registrierun- 
gen zweigeteilt, wobei zu der Gruppe mit Raub auch der schwere Diebstahl gehört. Schwerer Diebstahl bildet bei den polizeilichen Registrierungen ein eigenes Cluster. Cluster 3 (Betrug, Fälschung, Meineid, falsche Verdächtigung) bildet auch bei den polizeilichen Registrierungen ein eigenes Cluster, aber bei den justiziellen Registrierungen gehört auch Hehlerei diesem Cluster an. Cluster 5 existiert auch bei den polizeilichen Registrierungen, wobei hier aber zusätzlich zu den Delikten einfacher Diebstahl, Betäubungsmitteldelikte und Leistungserschleichung noch die Delikte Hehlerei und Hausfriedensbruch zum Cluster gehören. Einen deutlichen Unterschied gibt es beim schweren Diebstahl. Bei den polizeilichen Registrierungen bildet der schwere Diebstahl eine eigene Gruppe. Polizeiliche Registrierungen wegen schweren Diebstahls gibt es deutlich mehr als justizielle Registrierungen. Verkehrsdelikte, die polizeilich nicht registriert werden, bilden mit fahrlässiger Tötung/Körperverletzung ein Cluster (4).

Abbildung 30: Arithmetic-average clustering (deutsche Frauen BZR)

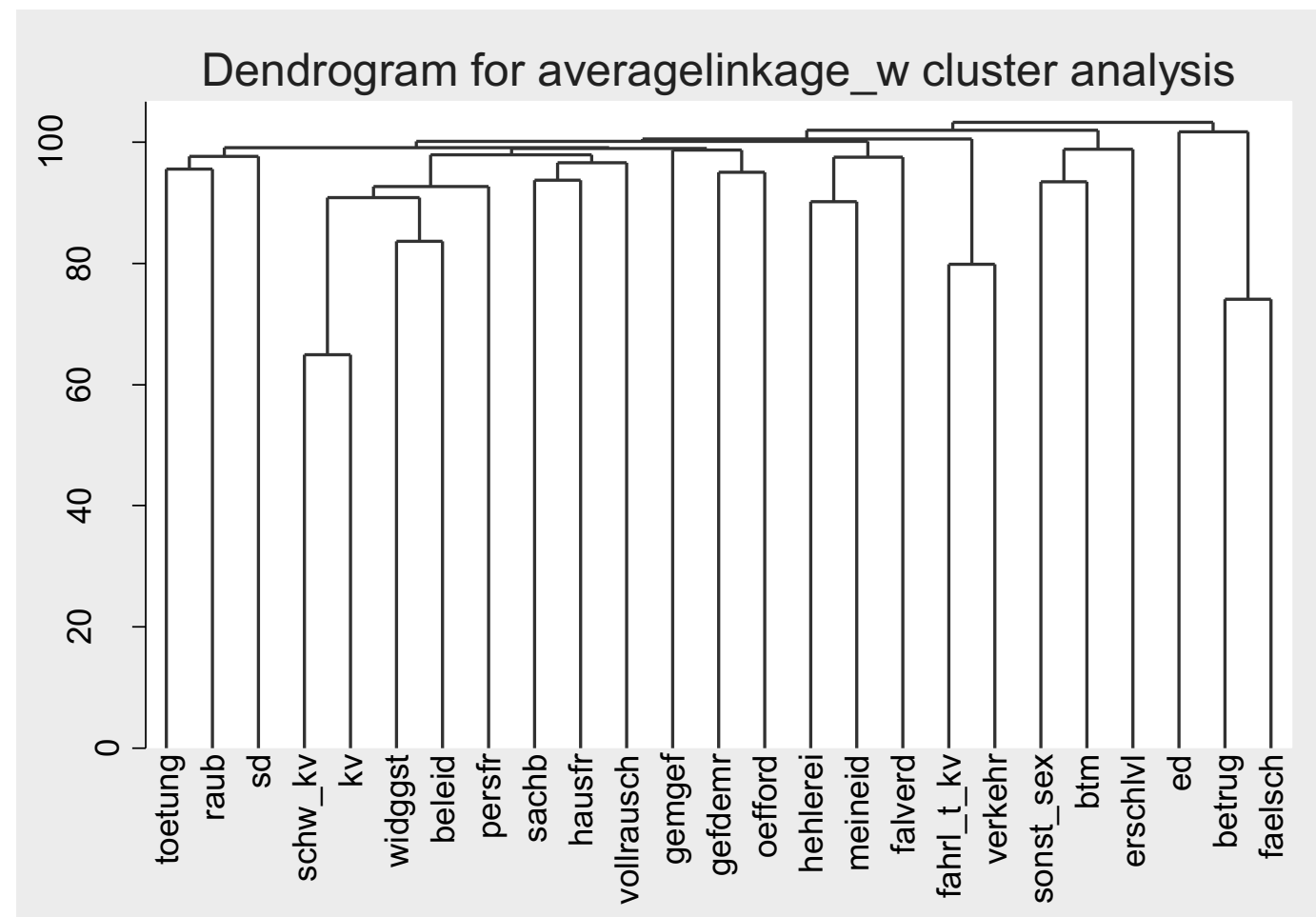

Abbildung 30 zeigt das Ergebnis der Clusteranalyse der justiziell registrierten deutschen Frauen. Besonders ähnlich sind die Deliktskategorien Betrug und Fälschung und ebenso wie bei den Männern Körperverletzung und schwere Körperverletzung. 
Teilt man die Delikte in fünf Cluster ergibt sich folgende Aufteilung:

1. Gewaltdelikte,

Dieses Cluster kann in drei Gruppen unterteilt werden:

- Tötungsdelikte, Raub und schwerer Diebstahl (sd),

- Gewaltdelikte (schwere Körperverletzung (schw_kv), Körperverletzung (kv), Straftaten gegen die persönliche Freiheit (persfr)), Widerstand gegen die Staatsgewalt (widggst), Beleidigung, Sachbeschädigung (sachb), Hausfriedensbruch (hausfr), Vollrausch,

- Gemeingefährliche Straftaten (gemgef), Gefährdung des demokratischen Rechtsstaates (gefdemr), Straftaten gegen die öffentliche Ordnung (oefford),

2. Hehlerei, Meineid, falsche Verdächtigung (falverd),

3. Verkehrsdelikte, fahrlässige Körperverletzung (Tötung),

4. Betäubungsmitteldelikte (btm), sonstige Sexualdelikte (sonst_sex) (hauptsächlich verbotene Prostitution) und Leistungserschleichung (erschlvl),

5. Einfacher Diebstahl (ed), Betrug und Fälschung.

Die Clusteraufteilung entspricht in vielen Teilen der Clusteraufteilung der polizeilichen Registrierungen. Abgesehen von der Clusterzuordnung von einfachem und schwerem Diebstahl stimmt die grundsätzliche Struktur der Cluster bei den polizeilichen und justiziellen Registrierungen überein. Sowohl bei den justiziellen wie bei den polizeilichen Registrierungen gibt es ein Cluster Gewaltdelikte (1), ein Cluster Hehlerei (2), ein Cluster Betäubungsmitteldelikte, Leistungserschleichung und sonstige Sexualdelikte (4) und ein Cluster Betrug und Fälschung (5).

Bei den justiziellen Registrierungen befindet sich der einfache Diebstahl im Betrugscluster, bei den polizeilichen Registrierungen bildet der einfache Diebstahl ein eigenes Cluster und befindet sich auch auf der nächsten Ebene nicht im Betrugscluster. Die polizeilichen und justiziellen Gewaltcluster unterscheiden sich darin, dass bei den justiziellen Registrierungen auch schwerer Diebstahl und Hausfriedensbruch dem Gewaltcluster zugeordnet sind. Schwerer Diebstahl bildet bei den polizeilichen Registrierungen gemeinsam mit Hehlerei ein Cluster. Hausfriedensbruch ist bei den polizeilichen Registrierungen im Cluster der Betäubungsmitteldelikte und sonstigen Sexualdelikten. Bei den polizeilichen Registrierungen befindet sich auch falsche Verdächtigung im Cluster der Gewaltdelikte. Falsche Verdächtigung tritt bei den justiziellen Registrierungen im Cluster Hehlerei auf. Bei den justiziellen Registrierungen gehört auch Vollrausch dem Gewalt-cluster an. Vollrausch wird polizeilich sehr selten registriert und ist deshalb keine Kategorie bei den polizeilichen Registrierungen.

Wie schon erwähnt, werden einfacher und schwerer Diebstahl polizeilich und justiziell unterschiedlich registriert. Einfacher Diebstahl ist eine leichte Straftat und 
das Verfahren wird dementsprechend häufig eingestellt. Einfacher Diebstahl wird dann justiziell registriert, wenn weitere Registrierungen vorliegen. Eine Straftat, die polizeilich als schwerer Diebstahl registriert wird, wird justiziell häufig als einfacher Diebstahl registriert. Vergleicht man die Cluster der justiziell registrierten Männer mit denen der Frauen, so zeigen sich sowohl Gemeinsamkeiten wie Unterschiede. Bei den Männern ist das Gewaltcluster zweigeteilt und Sexualdelikte und auch „sonstige Sexualdelikte“ befinden sich im Cluster mit Tötungsdelikten und Raub. Bei den Frauen sind Sexualdelikte kaum vorhanden und unter „sonstige Sexualdelikte" fällt hauptsächlich verbotene Prostitution. Verbotene Prostitution liegt im Cluster der Betäubungsmitteldelikte, worin sich auch Leistungserschleichung befindet. Bei den Männern bilden die Deliktskategorien Betrug, Fälschung, Hehlerei, Meineid und falsche Verdächtigung ein Cluster. Dies ist bei den Frauen zweigeteilt und einfacher Diebstahl befindet sich im Cluster von Betrug und Fälschung. Sowohl bei den Männern wie bei den Frauen bilden die Verkehrsdelikte gemeinsam mit fahrlässiger Tötung/Körperverletzung ein Cluster.

Die Ergebnisse der Clusteranalyse bestätigen die Ergebnisse der Multidimensionalen Skalierung. Beide Darstellungen basieren auf dem ASR als Ähnlichkeitsmaß für die Delikte. Bei den justiziellen Registrierungen der Männer liegen in der Abbildung der zweidimensionalen Multidimensionalen Skalierung die Deliktskategorien einfacher Diebstahl, Erschleichung von Leistungen und Betäubungsmitteldelikte relativ nah beieinander. Diese Deliktskategorien befinden sich innerhalb eines Clusters und sind somit ähnlich. Sie kommen häufig innerhalb der kriminellen Karriere eines Mannes vor. Gleiches gilt für die Deliktskategorien fahrlässige Körperverletzung/Tötung und Straßenverkehrsdelikte, und ebenso für die Deliktskategorien Fälschung und Betrug.

Besondere Deliktsähnlichkeiten bei den Frauen zeigen sich sowohl bei der Auswertung mit Multidimensionaler Skalierung, wie bei der Clusteranalyse, bei den Delikten verbotene Prostitution (sonst_sex) und Betäubungsmitteldelikte, sowie bei Betrug und „Fälschung““.

Sowohl bei den Männern wie bei den Frauen erweisen sich die Gewaltdelikte (schwere Körperverletzung, Körperverletzung, Straftaten gegen die persönliche Freiheit) einschließlich Beleidigung, Sachbeschädigung, Vollrausch und Widerstand gegen die Staatsgewalt als ähnlich.

\subsection{Zusammenfassung der Ergebnisse}

Bei allen Gruppen, sowohl bei Frauen wie Männern, bei Deutschen wie Nichtdeutschen, bei polizeilich Registrierten wie justiziell Registrierten, zeigt sich die Ähnlichkeit der Gewaltdelikte Körperverletzung, schwere Körperverletzung und Straftaten gegen die persönliche Freiheit untereinander sowie auch mit Beleidigung, Sachbeschädigung und Widerstand gegen die Staatsgewalt. 
Wenn nicht nach Altersgruppen unterschieden wird, bilden bei Männern Sexualdelikte mit Gewaltdelikten eine Gruppe, „sonstige Sexualdelikte“ sind ähnlich mit Sexualdelikten. Bei Frauen sind Sexualdelikte sehr selten registriert und deshalb nicht in die Analyse mit einbezogen. ,sonstige Sexualdelikte“ gehören bei Frauen nicht zu den Gewaltdelikten. Bei den Männern umfasst die Deliktskategorie ,sonstige Sexualdelikte" hauptsächlich sexuellen Missbrauch, bei den Frauen beinhaltet diese Deliktskategorie hauptsächlich verbotene Prostitution.

Bei Betäubungsmitteldelikten zeigen sich bei den Männern Ähnlichkeiten zu Leistungserschleichung, Raub, Hausfriedensbruch und bei justiziellen Registrierungen auch zu einfachem Diebstahl. Damit wird der Zusammenhang zwischen Drogendelikten und Beschaffungskriminalität sichtbar. Bei den Frauen zeigt sich die Ähnlichkeit von verbotener Prostitution (sonstige Sexualdelikte) und Betäubungsmitteldelikten, was auf den Zusammenhang zwischen Betäubungsmitteldelikten und Beschaffungsprostitution hinweist.

Bei allen Gruppen sind die Deliktskategorien Betrug und Fälschung ähnlich.

Schwerer Diebstahl ist bei polizeilichen Registrierungen der Männer unähnlich $\mathrm{zu}$ allen anderen Delikten. Bei justiziellen Registrierungen ist schwerer Diebstahl ähnlich mit Raub. Was die Ähnlichkeiten von schwerem Diebstahl betrifft, so zeigen sich deutliche Registrierungsunterschiede bei Polizei und Justiz. Schwerer Diebstahl wird wesentlich häufiger polizeilich registriert als justiziell. Schwerer Diebstahl (Einbruch) ist ein Delikt, das häufig in Serie begangen wird und dementsprechend häufig mehrfach bei Personen polizeilich registriert wird. Justiziell wird diese „Einbruchserie“ innerhalb einer Entscheidung abgehandelt und als ein schwerer Diebstahl gezählt.

Ebenso zeigen sich bei der polizeilichen und justiziellen Registrierung von einfachem Diebstahl deutliche Unterschiede. Bei polizeilich Registrierten zeigt sich im Gegensatz zu den justiziell Registrierten eine deutliche Unähnlichkeit von Vermögens- und Eigentumsdelikten (Betrug und einfacher Diebstahl). Dies hängt ebenso wie beim schweren Diebstahl damit zusammen, dass einfacher Diebstahl polizeilich häufig mehrfach registriert wird, justiziell aber nur einmal.

Die Deliktskategorie Vergehen gegen Ausländergesetze ist ähnlich zu Fälschung, ansonsten unähnlich zu allen anderen Deliktskategorien. Wobei es sich bei der Deliktskategorie Fälschung sowohl um Urkundenfälschung wie auch um Wertzeichenfälschung (Fälschung von Fahrkarten) handelt.

Bei justiziellen Registrierungen zeigt sich die Ähnlichkeit des Delikts Vollrausch mit Gewaltdelikten und die Ähnlichkeit von Verkehrsdelikten mit fahrlässiger Tötung/Körperverletzung.

Kontrollrechnungen ergeben, dass zwar bei bestimmten Deliktspaaren gehäuft Kombinationen an einem Tag vorkommen, dass das Ergebnis aber durch tateinheitlich begangene Straftaten nicht verfälscht wird. Die Deliktskonfiguration verändert sich nicht deutlich, wenn bei der Analyse statt aller Delikte jeweils nur das schwerste eines Tages oder einer Entscheidung, einbezogen wird. 
Die Einbeziehung der Dauer zwischen den Straftaten einer kriminellen Laufbahn beeinflusst das Ergebnis der Deliktsähnlichkeiten nicht. Die Untersuchung der Deliktskonfiguration einzelner Altersgruppen der deutschen Männer ergibt, dass Sexualdelikte bei Jugendlichen, Heranwachsenden und Jungerwachsenen ähnlich sind zu Gewaltdelikten, nicht aber bei den Älteren. Bei den polizeilich Registrierten 26bis 31-Jährigen und bei den justiziell Registrierten 26- bis 33-Jährigen deutschen Männern wird kein Zusammenhang zwischen Sexualdelikten und Gewaltdelikten sichtbar. 



\section{Probabilistische Clusteranalyse}

Individuen begehen $\mathrm{zu}$ verschiedenen Zeitpunkten verschiedene kriminelle Handlungen. Wenn die Strukturen dieser Handlungen bekannt wären, wäre es möglich, Zusammenhänge unterschiedlicher krimineller Handlungen zu erkennen und des Weiteren könnten Prognosen über folgende kriminelle Handlungen getroffen werden. Mit der Methode Multidimensionale Skalierung ist es möglich, Deliktsähnlichkeiten und damit Strukturen grafisch darzustellen. In diesem Abschnitt werden Muster von kriminellem Verhalten weiter analysiert. Typische Verläufe sollen erkannt werden. Untersucht wird, ob sich typische Verläufe mit dem Alter ändern. Erkenntnisse über typische Deliktsmuster werden mit Hilfe eines probabilistischen Clusteranalyseverfahrens bestimmt. Dafür werden die Registrierungen einer Person in Altersabschnitte aufgeteilt. Mit einem Clusteranalyseverfahren werden die einzelnen Altersabschnitte der Personen, entsprechend der Deliktszusammensetzung, Clustern zugeordnet. Bei einer probabilistischen Clusteranalyse wird jedes Objekt, hier Altersabschnitt, der sich aus verschiedenen Deliktskategorien zusammensetzt, mit einer bestimmten Wahrscheinlichkeit einem Cluster zugeordnet. ${ }^{225}$ Die Wahrscheinlichkeiten ,werden als Maximum-Likelihood-Schätzer berechnet. Das heißt, sie werden so bestimmt, dass die empirische Verteilung der Objekte bestmöglich durch das Modell reproduziert wird.“226

Das hier benutzte Verfahren wurde von Francis, Soothill und Fligelstone angewendet. ${ }^{227}$ Die kriminelle Karriere einer Person wird dabei in Altersabschnitte eingeteilt. Innerhalb dieser Abschnitte wird das Auftreten verschiedener Delikte 0/1 kodiert, d.h. wenn z.B. das Delikt Ladendiebstahl bei einer Person in einem Altersabschnitt auftritt wird eine 1 notiert, andernfalls eine Null. Die Anzahl der Delikte spielt also keine Rolle, es ist nur von Bedeutung, ob ein Delikt bei einer Person in einem Altersabschnitt vorkommt oder nicht. Die Auswertung wird nach Geschlecht differenziert durchgeführt. Die Altersabschnitte werden in Cluster eingeteilt, wobei das Kriterium für die Einordnung die Deliktszusammensetzung ist.

Die Wahrscheinlichkeiten der Clusterzugehörigkeit werden wie folgt berechnet:228 Für alle Altersabschnitte i und alle Deliktskategorien j gibt es eine Matrix $\mathrm{O}_{\mathrm{ij}}$, wobei $\mathrm{O}_{\mathrm{ij}}=1$, wenn das Delikt $\mathrm{j}$ im Altersabschnitt $\mathrm{i}$ vorkommt, ansonsten ist $\mathrm{O}_{\mathrm{ij}}=0 . \mathrm{O}_{\mathrm{i}}$ sind die Zeilen der Matrix. Angenommen es gibt K Cluster, dann gibt es für jedes Cluster $\mathrm{k}(\mathrm{k}=1, \ldots, \mathrm{K})$ eine Wahrscheinlichkeit $\pi(\mathrm{k})$. Wenn man $\mathrm{p}_{\mathrm{jk}}$ als die Wahrscheinlichkeit definiert, dass ein Altersabschnitt in Cluster $\mathrm{k}$ mindestens eine

\footnotetext{
225 Vgl. Bacher 1996, S. 353 ff. Im Gegensatz zu einem probabilistischen Clusteranalyseverfahren werden bei einem deterministischen Clusteranalyseverfahren die Objekte mit der Wahrscheinlichkeit 1 oder 0 den Clustern zugeordnet (vgl. Bacher 1996, S. 4 f.).

226 Bacher 1996, S. 353 f.

${ }^{227}$ Francis, Soothill \& Fligelstone 2004. Ergebnisse von Francis, Soothill \& Fligelstone befinden sich im Anhang.

228 Vgl. Francis, Soothill \& Fligelstone 2004, S.56.
} 
Registrierung von Delikt j enthält, dann sieht die Schätzfunktion LL folgendermaBen aus:

$$
\mathrm{LL}(\mathrm{K})=\prod_{\mathrm{i}} \sum_{\mathrm{k}} \pi(\mathrm{k}) \mathrm{p}\left(\mathrm{O}_{\mathrm{i}} \mid \mathrm{k}\right)
$$

wobei

$\mathrm{p}\left(\mathrm{O}_{\mathrm{i}} \mid \mathrm{k}\right)=\prod_{\mathrm{j}} \mathrm{p}_{\mathrm{jk}}^{\mathrm{O}_{\mathrm{ij}}}\left(1-\mathrm{p}_{\mathrm{jk}}\right)^{1-\mathrm{O}_{\mathrm{j}}}$, die bedingte Wahrscheinlichkeit ist, dass sich der Altersabschnitt i mit dem Delikt j im Cluster k befindet.

Die Schätzung der Modellparameter $\mathrm{p}_{\mathrm{jk}}$ und $\pi(\mathrm{k})$ erfolgt über ein Iterationsverfahren. Das Ziel ist, LL(k) zu maximieren. Bezeichnet wird dieses Verfahren als EM-Algorithmus (Expected-Maximum-Likelihood-Estimator). ${ }^{229}$ Um lokale Maxima zu vermeiden wird mit vielen verschiedenen zufälligen Startwerten gerechnet.

Mit dem probabilistischen Clusteranalyseverfahren werden die einzelnen Altersabschnitte der Personen Clustern zugeordnet. Für jeden Altersabschnitt wird in Abhängigkeit vom Delikt die Wahrscheinlichkeit der Clusterzugehörigkeit berechnet. Umgekehrt wird auch berechnet, mit welcher Wahrscheinlichkeit ein Delikt zu welchem Cluster gehört.

Des Weiteren wird untersucht, ob die gefundenen Cluster in Abhängigkeit vom Alter unterschiedlich häufig auftreten.

Eine Person kann auf verschiedene Cluster verteilt sein, wenn die Deliktszusammensetzung der einzelnen Altersabschnitte verschieden ist.

Das Ergebnis der Clusteranalyse wird für weitere Auswertungen zum Verlauf der Kriminalität, speziell zur Untersuchung von Spezialisierung, benutzt. Der Begriff Spezialisierung wird hier im Sinne von Francis, Soothill und Fligelstone verwendet. ${ }^{230}$ Spezialisierung bedeutet somit, dass bei einer Person in einem späteren Altersabschnitt Delikte registriert sind, so dass dieser Altersabschnitt demselben Cluster zugeordnet werden kann wie ein früherer Altersabschnitt. Die Personen werden in diesem Sinne in drei Gruppen eingeteilt: Abbrecher, Spezialisierte und Wechsler. Einige Untersuchungen zu Spezialisierung werden ohne die Abbrecher durchgeführt. Des Weiteren wird der Verlauf der Cluster mit dem Alter untersucht. Francis, Soothill und Fligelstone sind der Ansicht, dass es, wenn die kriminelle Aktivität der vorhergehenden fünf Jahre bekannt ist, möglich ist, die Qualität und die Art des Delikts in den folgenden fünf Jahren vorherzusagen. ${ }^{231}$

229 Vgl. Bacher 1996, S. 354.

230 Vgl. Francis, Soothill \& Fligelstone 2004, S. 73.

231 Vgl. Francis, Soothill \& Fligelstone 2004, S. 82. 


\subsection{Probabilistische Clusteranalyse für justiziell Registrierte des Geburtsjahrgangs 1970}

Im Folgenden werden die justiziellen Registrierungen deutscher Männer und Frauen in Baden-Württemberg des Geburtsjahrgangs 1970 anhand der probabilistischen Clusteranalyse analysiert. Die Daten des Geburtsjahrgangs 1970 werden für die Clusteranalyse benutzt, weil hier der längste Altersbereich zur Verfügung steht. Die Registrierungen der Personen werden vier Altersgruppen zugeordnet: 14-18, 19-23, 24-28, 29-33.232 Es werden nur die Daten von Personen benutzt, die wegen mindestens zwei Straftaten registriert sind. D.h. entweder sind bei einer Person innerhalb einer Entscheidung mindestens zwei Straftaten eingetragen oder die Person ist mehrfach registriert. Da es um die Untersuchung von Deliktsmustern geht, werden Einfachtäter in diese Untersuchung nicht mit einbezogen. Insgesamt werden die Altersabschnitte von 12.101 Männern und von 2.089 Frauen analysiert. Die einzelnen Altersabschnitte enthalten mindestens eine Straftat. In Abbildung 31 sind die Häufigkeiten der Altersgruppen der deutschen Männer und Frauen der Kohorte 1970 dargestellt. Die größte Altersgruppe bildet sowohl bei den Männern wie bei den Frauen die Gruppe der 19- bis 23-Jährigen, mit 7.734 Personen bei den Männern und 1.081 Personen bei den Frauen. Bei den Männern nimmt die Anzahl der Täter mit dem Alter deutlicher ab als bei den Frauen.

\section{Abbildung 31: Häufigkeiten der Altersgruppen (justizielle Registrierung, deutsche Männer und Frauen, Kohorte 1970)}
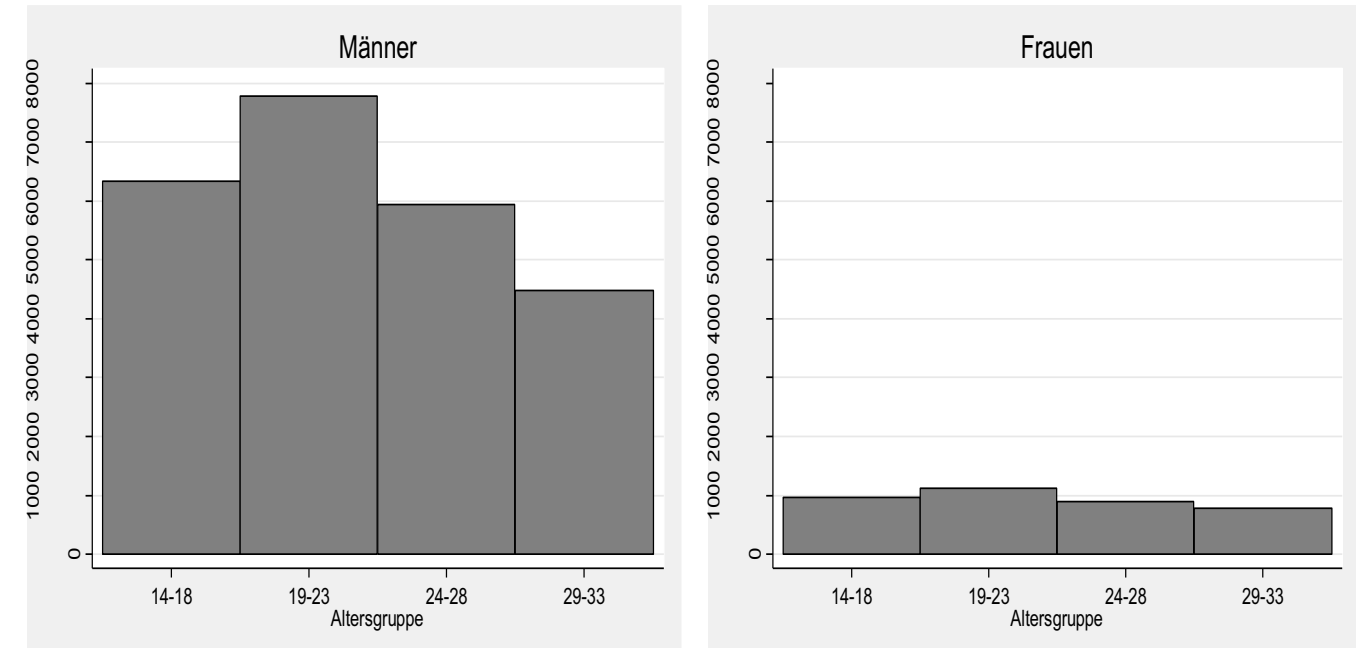

232 Die Altersabschnitte sind nicht identisch mit den Abschnitten von Francis, Soothill und Fligelstone, da die erste Eintragung im Bundeszentralregister mit 14 Jahren möglich ist. 
Für die Clusteranalyse werden die Deliktskategorien benutzt, die auch für die Analyse mit Multidimensionaler Skalierung benutzt wurden, 27 Deliktskategorien bei den Männern und 25 Deliktskategorien bei den Frauen (Sexualdelikte und gefährlicher Eingriff in den Straßenverkehr kommen bei Frauen sehr selten vor und werden deshalb bei den Frauen nicht berücksichtigt). Gerechnet wird die probabilistische Clusteranalyse mit dem Programm Latent Gold, das auch Francis, Soothill und Fligelstone zur Bestimmung der Cluster verwendet haben. ${ }^{233}$

\subsubsection{Clusteranalyse justizieller Registrierungen deutscher Männer}

Zuerst wird die optimale Anzahl der Cluster bestimmt. Dazu werden verschiedene Modellprüfgrößen 234 berechnet in Abhängigkeit von der Anzahl der Cluster, die in Tabelle 38 dargestellt sind. Eine Prüfgröße für die Güte des Modells ist der BICWert $^{235}$. Francis, Soothill und Fligelstone bestimmen mit dem BIC-Wert die Clusteranzahl, wobei die Clusteranzahl des kleinsten BIC-Wertes als optimal gilt. ${ }^{236}$ Das Minimum des BIC-Wertes liegt für die justiziellen Daten aus BadenWürttemberg bei den deutschen Männern der Kohorte 1970 bei 13 Clustern.

233 Zuerst habe ich versucht, die Cluster mit dem Statistikprogramm SPSS und der Funktion "TwoStep" zu bestimmen. Dies ergab aber keine stabilen Ergebnisse. Je nachdem wie der Ausgangsdatensatz sortiert ist, ergeben sich unterschiedliche Clusterlösungen. Mit dem Programm Latent Gold erhält man auch bei großen Datensätzen stabile Clusterlösungen.

${ }^{234}$ Siehe hierzu Vermunt \& Magidson 2005, Latent GOLD User's Guide, S. 107 f., S. 170 f., Vermunt \& Magidson 2005, Technical Guide for Latent GOLD, S. 57 ff., Bacher 1996, S. 385 f. kann.

${ }^{235}$ BIC ist ein Informationskriterium mit dem das optimale Modell ausgewählt werden

$\operatorname{BIC}(\mathrm{k})=-2 \cdot \log \mathrm{LL}(\mathrm{k})+\log (\mathrm{n}) \cdot \mathrm{Npar}$

$\mathrm{k}$ Anzahl Cluster

LL(k) Wert der log-Likelihood Funktion bei k Clustern

n Anzahl der Beobachtungen

Npar Anzahl der Parameter

Vgl. Vermunt \& Magidson 2005, Technical Guide for Latent GOLD, S. 61.

236 Vgl. Francis, Soothill \& Fligelstone 2004, S. 57. 
Tabelle 38: Modellprüfgrößen zur Bestimmung der Clusteranzahl (deutsche Männer, Kohorte 1970, justizielle Registrierungen) ${ }^{237}$

\begin{tabular}{lccrrrrr}
\hline $\begin{array}{l}\text { Cluster- } \\
\text { zahl }\end{array}$ & LL & BIC(LL) & Npar & L $^{2}$ & df & p-Wert & Class.Err. \\
\hline 2 & -131370 & 263315 & 57 & 33235 & 24362 & $1,80 \mathrm{E}-286$ & 0,056 \\
3 & -128069 & 257007 & 86 & 26634 & 24333 & $2,20 \mathrm{E}-24$ & 0,103 \\
4 & -127062 & 255285 & 115 & 24619 & 24304 & 0,077 & 0,130 \\
5 & -126364 & 254183 & 144 & 23224 & 24275 & 1 & 0,136 \\
6 & -125686 & 253120 & 173 & 21868 & 24246 & 1 & 0,134 \\
7 & -125073 & 252187 & 202 & 20642 & 24217 & 1 & 0,125 \\
8 & -124474 & 251282 & 231 & 19444 & 24188 & 1 & 0,111 \\
9 & -124016 & 250659 & 260 & 18528 & 24159 & 1 & 0,169 \\
10 & -123634 & 250188 & 289 & 17764 & 24130 & 1 & 0,163 \\
11 & -123397 & 250007 & 318 & 17290 & 24101 & 1 & 0,154 \\
12 & -123122 & 249750 & 347 & 16740 & 24072 & 1 & 0,153 \\
13 & -122907 & 249612 & 376 & 16310 & 24043 & 1 & 0,151 \\
14 & -122789 & 249669 & 405 & 16073 & 24014 & 1 & 0,155 \\
\hline
\end{tabular}

LL Wert der Log-Likelihood-Funktion

BIC Bayesian Information Criterion siehe Fußnote 235

Npar Anzahl der Parameter

$\mathrm{L}^{2} \quad$ Likelihood ratio chi-squared statistic

df Freiheitsgrade

p-Wert Signifikanzniveau

Class.Err. Classification Errors

Eine weitere Modellprüfgröße ist $\mathrm{L}^{2} .{ }^{238} \mathrm{~L}^{2}$ ist ein Statistikmaß mit dem abgeschätzt werden kann, wie passend das Modell für die Daten ist oder genauer wie ähnlich sich die geschätzten und die beobachteten Häufigkeiten sind. In Bezug zur probabilistischen Clusteranalyse kann $\mathrm{L}^{2}$ interpretiert werden als ein Indikator dafür, wie der beobachtete Zusammenhang zwischen den Variabeln mit dem Modell erklärt wird. Je größer L ${ }^{2}$, desto schlechter ist das Modell. Der dazugehörende pWert ist ein Schätzwert der Güte des Modells (die Null-Hypothese ist, dass das Modell in der Population gilt). Wenn $\mathrm{p}<0,05$ ist das Modell schlecht. ${ }^{239}$

Eine Daumenregel für ein gutes Modell ist, dass der Wert von $\mathrm{L}^{2}$ nicht größer als die Anzahl der Freiheitsgrade ist. 240

Ein weiteres Gütemaß ist der Classification Error. Dieser Wert gibt den Anteil der Fälle an, die im falschen Cluster liegen. Je kleiner dieser Fehlerwert ist, desto geringer ist der Anteil an falschen Klassifikationen im Modell.241

\footnotetext{
237 Die Werte der Tabelle wurden mit dem Programm Latent GOLD berechnet.

${ }^{238} \mathrm{~L}^{2}$ basiert auf dem Chi-Quadrat-Test.

239 Vgl. Vermunt \& Magidson 2005, Latent GOLD User's Guide, S. 107 f.

240 Vgl. Vermunt \& Magidson 2005, Latent GOLD User's Guide, S. 108.

241 Vgl. Vermunt \& Magidson 2005, Latent GOLD User's Guide, S. 112.
} 
Die Gütekriterien zur Bestimmung der Clusteranzahl werden im Folgenden gegeneinander abgewogen. Der BIC-Wert ist minimal bei 13 Clustern. Der p-Wert ist größer als 0,05 bei 4 Clustern, aber noch sehr niedrig. 1 ist der p-Wert ab 5 Clustern. Die Daumenregel, dass $\mathrm{L}^{2}$ nicht größer als die Anzahl der Freiheitsgrade sein soll, trifft das erste Mal bei 5 Clustern zu. Die Aufteilung in 13 Cluster ist nicht sinnvoll, weil sich dabei zu viele Cluster mit einzelnen Delikten ergeben (siehe hierzu Tabelle 42). Cluster mit einem einzelnen Delikt entstehen immer dann, wenn viele Altersabschnitte vorhanden sind, die nur aus diesem einzelnen Delikt bestehen. Schon bei einer Aufteilung in 9 Cluster ergibt sich ein eigenes Cluster einfacher Diebstahl und ein eigenes Cluster Betäubungsmitteldelikte mit jeweils nur durchschnittlich 1,2 bzw. 1,3 Delikten pro Altersabschnitt. ${ }^{242}$ Aussagen über Deliktsähnlichkeiten wären bei einer zu hohen Anzahl von Clustern nicht möglich.

Deshalb werden die Altersabschnitte in 6 Cluster aufgeteilt. Bei 6 Clustern sind kaum Cluster mit einem einzelnen Delikt vorhanden. Außerdem gelten die Gütekriterien. Es gilt die Daumenregel, dass $\mathrm{L}^{2}$ kleiner als die Anzahl der Freiheitsgrade ist. Der p-Wert ist 1. Bei den anderen Gruppierungen, deutsche Frauen mit justiziellen Registrierungen, deutsche Männer und Frauen mit polizeilichen Registrierungen, ist jeweils auch bei 6 Clustern die Daumenregel bzgl. $\mathrm{L}^{2}$ erfüllt und der pWert 1. Bei den Frauen ist, sowohl bei den polizeilich Registrierten wie bei den justiziell Registrierten, der BIC-Wert bei 6 Clustern minimal. Das Ergebnis der Clusteranalyse für die 6-Clusterlösung ist in Tabelle 39 dargestellt. Es sind die Cluster von 24.419 Altersabschnitten die zu 12.101 Tätern gehören.

Die Clustergröße reicht von 5,2\% bis 35,6\% aller Altersabschnitte. Die Tabelle gibt die Wahrscheinlichkeiten der Clusterzusammensetzung wieder. Hierzu ein Beispiel: 68,7\% der Altersabschnitte aus Cluster C enthalten das Delikt Körperverletzung und 25,4\% das Delikt Beleidigung. Die Prozentwerte sind spaltenweise, wobei die Summe größer 100 ist, weil ein Altersabschnitt mehrere Delikte enthalten kann. Im Durchschnitt enthält ein Altersabschnitt zwei Delikte. Die Spalte rechts außen gibt die Verteilung aller Altersabschnitte wieder. Hier sieht man, dass 53\% aller Altersabschnitte ein Verkehrsdelikt enthalten und 27,9\% einfachen Diebstahl. Die Deliktsanteile, die mindestens 1,5-mal so groß sind wie der durchschnittliche Deliktsanteil, sind fett gedruckt.

242 Siehe Tabelle 76 im Anhang. 
Tabelle 39: Deliktscluster justizieller Registrierungen deutscher Männer der Kohorte 1970: Wahrscheinlichkeiten der Deliktskategorien in den Clustern (in \%)

\begin{tabular}{|c|c|c|c|c|c|c|c|}
\hline \multirow[b]{3}{*}{$\begin{array}{l}\text { Clusterwahrscheinlichkeit } \pi(\mathrm{k}) \\
\text { in } \%\end{array}$} & \multicolumn{7}{|c|}{ Cluster } \\
\hline & A & $\mathrm{B}$ & $\mathrm{C}$ & $\mathrm{D}$ & $\mathrm{E}$ & $\mathrm{F}$ & gesamt \\
\hline & 35,6 & 32,9 & 12,6 & 8,4 & 5,2 & 5,2 & 100 \\
\hline \multicolumn{8}{|l|}{ Wahrscheinlichkeiten $\mathrm{p}_{\mathrm{jk}}$ in \% } \\
\hline Tötungsdelikte & 0,2 & 0,0 & 0,4 & 0,1 & 0,0 & $\mathbf{0 , 8}$ & 0,2 \\
\hline Sexualdelikte & 0,7 & 0,0 & 2,0 & 0,1 & 0,0 & 2,4 & 0,7 \\
\hline sonst. Sexualdelikte & 1,6 & 0,1 & 1,1 & 0,4 & 0,3 & 1,3 & 0,9 \\
\hline Raub & 2,5 & 0,3 & 5,4 & 0,8 & 0,0 & 20,0 & 2,8 \\
\hline schw. Körperverletzung & 0,0 & 0,0 & 35,0 & 0,2 & 0,0 & 33,1 & 6,2 \\
\hline Körperverletzung & 0,7 & 0,6 & 68,7 & 0,7 & 33,4 & 60,9 & 14,1 \\
\hline Str. gg. Pers. Freiheit & 2,2 & 0,6 & 20,6 & 1,4 & 1,1 & 21,4 & 4,9 \\
\hline Einfacher Diebstahl & 48,9 & 6,4 & 14,5 & 34,1 & 3,3 & 67,5 & 27,9 \\
\hline Schwerer Diebstahl & 17,6 & 3,8 & 5,6 & 8,4 & 0,8 & 48,2 & 11,5 \\
\hline Sachbeschädigung & 10,7 & 1,3 & 14,9 & 0,5 & 0,7 & 32,6 & 7,9 \\
\hline gemeingef. Straftaten & 1,5 & 0,2 & 1,0 & 0,1 & 1,6 & 3,5 & 1,0 \\
\hline Hehlerei & 7,0 & 0,8 & 1,1 & 2,1 & 0,7 & 12,7 & 3,7 \\
\hline Betrug & 4,0 & 0,2 & 3,8 & 99,8 & 2,4 & 33,0 & 12,2 \\
\hline Erschl. v. Leistungen & 11,0 & 0,6 & 5,1 & 6,0 & 0,1 & 14,9 & 6,0 \\
\hline gef. dem. Rechtsstaat & 0,4 & 0,1 & 1,4 & 0,0 & 0,0 & $\mathbf{3 , 0}$ & 0,5 \\
\hline Wid. gg. Staatsgewalt & 0,4 & 0,2 & 13,3 & 0,1 & 1,0 & 15,0 & 2,8 \\
\hline Öffentliche Ordnung & 2,4 & 1,2 & 2,2 & 3,8 & 0,6 & 10,2 & 2,4 \\
\hline Hausfriedensbruch & 2,7 & 0,1 & 3,8 & 0,0 & 0,2 & 12,2 & 2,1 \\
\hline Beleidigung & 3,7 & 0,8 & 25,4 & 1,8 & 0,8 & 24,8 & 6,3 \\
\hline Fälschung & 4,4 & 4,8 & 0,6 & 23,5 & 0,7 & 26,6 & 6,6 \\
\hline Meineid & 1,2 & 0,2 & 0,6 & 1,5 & 0,2 & 2,0 & 0,8 \\
\hline falsche Verdächtigung & 0,8 & 0,2 & 0,4 & 0,6 & 0,3 & 2,1 & 0,6 \\
\hline BTM-Delikte & 21,4 & 3,6 & 8,4 & 4,2 & 2,2 & 23,5 & 11,5 \\
\hline gef. Eingr. Str.V. & 0,5 & 0,0 & 2,8 & 0,1 & 1,2 & 3,1 & 0,8 \\
\hline Vollrausch & 1,6 & 0,4 & 2,4 & 0,2 & 0,0 & 4,5 & 1,3 \\
\hline fahrl. Tötung & 0,2 & 0,5 & 0,4 & 0,1 & 4,1 & 0,0 & 0,5 \\
\hline fahrl. Körperverletzung & 2,0 & 10,0 & 5,8 & 1,9 & 99,9 & 13,2 & 10,8 \\
\hline Verkehrsdelikte & 22,4 & 100,0 & 28,1 & 31,9 & 46,7 & 65,2 & 53,0 \\
\hline $\begin{array}{l}\text { Durchschnittliche Anzahl } \\
\text { Delikte }\end{array}$ & 1,7 & 1,4 & 2,9 & 2,2 & 2,0 & 6,1 & 2,0 \\
\hline
\end{tabular}

Das größte Cluster, Cluster A, setzt sich hauptsächlich zusammen aus einfachem Diebstahl, Betäubungsmitteldelikten, Leistungserschleichung, schwerem Diebstahl und Hehlerei. Diese Delikte sind überdurchschnittlich häufig vertreten. $49 \%$ der Altersabschnitte dieses Clusters enthalten einfachen Diebstahl, 21\% Betäubungsmitteldelikte, 11\% Leistungserschleichung, 18\% schwerer Diebstahl und 7\% Hehlerei. Bei den Eigentumsdelikten und auch bei „sonstigen Sexualdelikten“ kann es sich um Beschaffungskriminalität für die Drogensucht handeln. 95\% der Altersabschnitte aus Cluster A, die 
„sonstige Sexualdelikte“ enthalten, enthalten auch ein Betäubungsmitteldelikt. Cluster A ist das Cluster mit dem geringsten Anteil von Verkehrsdelikten, aber dennoch enthalten $22 \%$ aller Altersabschnitte ein Verkehrsdelikt.

Das zweitgrößte Cluster, Cluster B, das Cluster der Verkehrsdelikte umfasst 32,9\% aller Altersabschnitte. Alle Altersabschnitte, die zu diesem Cluster gehören, enthalten ein Verkehrsdelikt. Der Anteil der übrigen Delikte ist sehr gering. Eine Ausnahme bildet fahrlässige Körperverletzung mit einem Anteil von 10\%. Es ist anzunehmen, dass die neben den Verkehrsdelikten auftretenden zusätzlichen Delikte in engem Zusammenhang mit Verkehrsdelikten stehen. Dies ist für die fahrlässige Körperverletzung im Rahmen eines Verkehrsunfalls offensichtlich. Aber auch Betäubungsmitteldelikte können in diesem Rahmen auffällig geworden sein, Gleiches gilt für Fälschung von Fahrzeugpapieren.

Cluster C (12,6\%) ist das Cluster der Gewaltdelikte (Tötungsdelikte, Sexualdelikte, Raub, schwere Körperverletzung, Körperverletzung, Straftaten gegen die persönliche Freiheit) einschließlich Beleidigung, Sachbeschädigung, Hausfriedensbruch, Widerstand gegen die Staatsgewalt und Vollrausch. Die durchschnittliche Anzahl von Delikten pro Altersabschnitt ist mit 2,9 höher als die durchschnittliche Anzahl von 2,0.

Cluster D (8,4\%) umfasst Betrug und „Fälschung“.

Fast alle Altersabschnitte des Cluster E (5,4\%) enthalten fahrlässige Körperverletzung und auch fahrlässige Tötung ist überdurchschnittlich häufig vertreten. Knapp die Hälfte der Altersabschnitte enthält ein Verkehrsdelikt.

Cluster F $(5,4 \%)$ ist das Cluster der verschiedenen Straftaten. Alle Delikte kommen überdurchschnittlich häufig vor. Hier versammeln sich die chronischen Straftäter, die, die viele verschiedene Straftaten begehen und häufig Straftaten begehen. Die durchschnittliche Anzahl von Delikten pro Altersabschnitt ist in diesem Cluster deutlich größer als in den übrigen Clustern, im Durchschnitt enthält jeder Altersabschnitt 6,1 Delikte.

Umgekehrt kann auch ermittelt werden, mit welcher Wahrscheinlichkeit $\Theta_{\mathrm{kj}}$ ein Altersabschnitt mit Delikt j zu einem Cluster k gehört. ${ }^{243}$

$$
\Theta_{\mathrm{kj}}=\frac{\pi(\mathrm{k}) \mathrm{p}_{\mathrm{jk}}}{\sum_{\mathrm{k}} \pi(\mathrm{k}) \mathrm{p}_{\mathrm{jk}}}
$$

Die Wahrscheinlichkeiten der Clusterzugehörigkeiten nach Deliktsvorkommen zeigt Tabelle 40. Enthält der Altersabschnitt einer Person das Delikt schwere Körperverletzung, dann befindet sich dieser Abschnitt mit 72\%iger Wahrscheinlichkeit im Cluster $\mathrm{C}$, aber umgekehrt ist der Anteil an Cluster $\mathrm{C}$ mit Abschnitten des De-

243 Vgl. Francis, Soothill \& Fligelstone 2004, S.62. 
likts schwere Körperverletzung 35\% (siehe Tabelle 39). In Tabelle 40 addieren sich die Prozente einer Zeile immer zu 100\%. In Tabelle 39, bei der spaltenweisen Betrachtung, ergibt sich in der Summe ein höherer Prozentsatz als Hundert, weil die Altersabschnitte der Personen mehrere Delikte enthalten können. Fett gedruckt sind alle Prozentanteile größer als 30.

Tabelle 40: Wahrscheinlichkeit der Clusterzugehörigkeit bei Deliktsvorkommen in \% (Justizielle Registr., deutsche Männer, Kohorte 1970, Baden-Württemberg)

\begin{tabular}{|c|c|c|c|c|c|c|}
\hline \multirow[b]{3}{*}{$\begin{array}{l}\text { Clusterwahrscheinlichkeiten } \pi(\mathrm{k}) \text { in } \\
\%\end{array}$} & \multicolumn{6}{|c|}{ Cluster* } \\
\hline & A & $\mathrm{B}$ & $\mathrm{C}$ & $\mathrm{D}$ & $\mathrm{E}$ & $\mathrm{F}$ \\
\hline & 35,6 & 32,9 & 12,6 & 8,4 & 5,2 & 5,2 \\
\hline \multicolumn{7}{|l|}{ Wahrscheinlichkeiten $\Theta_{\mathrm{kj}}$ in \% } \\
\hline Tötungsdelikte & 35,8 & 0,0 & 34,6 & 3,5 & 0,0 & 26,1 \\
\hline Sexualdelikte & 39,6 & 1,9 & 37,9 & 1,3 & 0,0 & 19,4 \\
\hline Sonstige Sexualdelikte & 67,6 & 2,2 & 16,8 & 3,9 & 1,9 & 7,7 \\
\hline Raub & 32,0 & 3,1 & 24,9 & 2,3 & 0,0 & 37,8 \\
\hline Schwere Körperverletzung & 0,0 & 0,0 & 71,9 & 0,2 & 0,0 & 27,9 \\
\hline Körperverletzung & 1,8 & 1,4 & 61,6 & 0,4 & 12,4 & 22,4 \\
\hline Str. gegen die persönliche Freiheit & 15,9 & 4,2 & 53,4 & 2,4 & 1,2 & 22,8 \\
\hline Einfacher Diebstahl & 62,4 & 7,6 & 6,6 & 10,3 & 0,6 & 12,6 \\
\hline Schwerer Diebstahl & 54,6 & 10,9 & 6,2 & 6,2 & 0,4 & 21,8 \\
\hline Sachbeschädigung & 48,2 & 5,3 & 23,9 & 0,6 & 0,5 & 21,5 \\
\hline Gemeingefährliche Straftaten & 53,4 & 6,2 & 12,7 & 1,0 & 8,5 & 18,2 \\
\hline Hehlerei & 66,3 & 6,7 & 3,8 & 4,7 & 1,0 & 17,6 \\
\hline Betrug & 11,6 & 0,6 & 3,9 & 68,8 & 1,0 & 14,0 \\
\hline Erschleichen von Leistungen & 64,8 & 3,3 & 10,8 & 8,4 & 0,1 & 12,8 \\
\hline Gefährdung dem. Rechtsstaates & 29,6 & 3,1 & 36,1 & 0,0 & 0,0 & 31,2 \\
\hline Widerstand gegen die Staatsgewalt & 5,6 & 2,8 & 60,9 & 0,4 & 2,0 & 28,4 \\
\hline Öffentliche Ordnung & 35,3 & 17,0 & 11,3 & 13,2 & 1,3 & 22,0 \\
\hline Hausfriedensbruch & 44,9 & 1,7 & 22,9 & 0,0 & 0,4 & 30,1 \\
\hline Beleidigung & 21,2 & 4,3 & 51,1 & 2,4 & 0,6 & 20,5 \\
\hline Fälschung & 23,6 & 23,9 & 1,1 & 30,0 & 0,5 & 20,9 \\
\hline Meineid & 53,4 & 9,0 & 9,2 & 15,1 & 0,9 & 12,4 \\
\hline falsche Verdächtigung & 50,0 & 8,9 & 10,0 & 9,3 & 2,4 & 19,5 \\
\hline BTM-Delikte & 66,0 & 10,2 & 9,2 & 3,1 & 1,0 & 10,6 \\
\hline gefährlicher Eingriff Straßenverkehr & 23,8 & 1,1 & 45,5 & 1,2 & 7,7 & 20,7 \\
\hline Vollrausch & 45,1 & 11,3 & 23,9 & 1,6 & 0,0 & 18,2 \\
\hline fahrlässige Tötung & 11,2 & 32,3 & 10,4 & 1,1 & 45,1 & 0,0 \\
\hline fahrlässige Körperverletzung & 6,7 & 30,4 & 6,8 & 1,5 & 48,3 & 6,3 \\
\hline Verkehrsdelikte & 15,0 & 62,2 & 6,7 & 5,1 & 4,6 & 6,4 \\
\hline
\end{tabular}

* Cluster: A Diebstahl, B Verkehrsdelikte, C Gewaltdelikte, D Betrug, E fahrlässige Körperverletzung, F verschiedene Straftaten

Zwei Drittel aller Altersabschnitte mit Registrierungen wegen ,sonstiger Sexualdelikte“, Betäubungsmitteldelikten, Leistungserschleichung und Hehlerei befinden 
sich in Cluster A. Auch der Anteil von einfachem Diebstahl ist über 60\%. Des Weiteren sind die Altersabschnitte mit schwerem Diebstahl, gemeingefährliche Straftaten, Meineid und falsche Verdächtigung mit einer Wahrscheinlichkeit von über $50 \%$ in Cluster $\mathrm{A}$.

In Cluster B, in dem alle Altersabschnitte ein Verkehrsdelikt enthalten (siehe Tabelle 39), liegen $62 \%$ aller Verkehrsdelikte. Außerdem befinden sich im Cluster B über 30\% der Delikte fahrlässige Körperverletzung und fahrlässige Tötung, was zeigt, dass diese Delikte häufig in Zusammenhang mit einem Verkehrsdelikt auftreten.

$72 \%$ aller Altersabschnitte mit schwerer Körperverletzung und $62 \%$ aller Altersabschnitte mit Körperverletzung befinden sich in Cluster C. Auch Straftaten gegen die persönliche Freiheit und die übrigen Gewaltdelikte mit Ausnahme von ,,sonstigen Sexualdelikten“ befinden sich zu einem großen Teil in Cluster C. 53\% der Abschnitte mit dem Delikt Straftaten gegen die persönliche Freiheit sind in Cluster C. Auch 61\% aller Abschnitte mit Widerstand gegen die Staatsgewalt und 51\% aller Altersabschnitte mit Beleidigung liegen in Cluster C.

In Cluster D befinden sich knapp 70\% aller Altersabschnitte mit Betrug und 30\% mit „Fälschung“. In Cluster E befinden sich knapp 50\% aller Altersabschnitte mit fahrlässiger Körperverletzung und fahrlässiger Tötung. Im Cluster F, in dem alle Delikte außer fahrlässiger Tötung überdurchschnittlich häufig vorkommen, sind alle Delikte vertreten. Dies ist das Cluster der chronischen Straftäter. Dies sieht man auch, wenn man die Cluster bzgl. ihrer durchschnittlichen Deliktshäufigkeiten je Altersabschnitt vergleicht. Alle Altersabschnitte enthalten im Schnitt zwei Delikte. Im Cluster der verschiedenen Straftaten enthalten die Altersabschnitte im Schnitt mehr als 6 Delikte (siehe Tabelle 41). Im Gewaltcluster liegt der Durchschnitt bei knapp drei Delikten pro Altersabschnitt. Am niedrigsten ist der durchschnittliche Deliktsanteil im Cluster der Verkehrsdelikte, hier gibt es im Schnitt pro Altersabschnitt 1,4 Delikte.

In den dargestellten Ergebnissen der probabilistischen Clusteranalyse wurde bis hierher das Alter nicht berücksichtigt. Jetzt soll das Alter in die Analyse einbezogen werden. Untersucht wird, welche Deliktskonfigurationen (Cluster) für welches Alter besonders typisch sind. Des Weiteren werden die Übergänge der Altersgruppen in Bezug zur Clusterzugehörigkeit untersucht. Kriminelle Aktivitäten verändern sich mit dem Alter. ${ }^{244}$ Einerseits nehmen die kriminellen Aktivitäten mit dem Alter $\mathrm{ab}$, auf jeden Fall die offiziell registrierten, andererseits ändern sich mit dem Alter die typischen Delikte (Abbildung 3). Mit den Ergebnissen der Clusteranalyse soll untersucht werden, wie sich delinquentes Verhalten mit dem Alter ändert und wie typisches kriminelles Verhalten innerhalb verschiedener Altersgruppen aussieht. 245

244 Vgl. Francis, Soothill \& Fligelstone 2004, S.64.

245 Vgl. Francis, Soothill \& Fligelstone 2004, S.64. 
Bei einer deterministischen Clusteranalyse wird jedes Objekt mit einer Wahrscheinlichkeit von 0 oder 1 einem oder mehreren Clustern zugeordnet. ${ }^{246}$ Bei der probabilistischen Clusteranalyse wird jedes Objekt mit einer Wahrscheinlichkeit zwischen 0 und 1 einem Cluster zugeordnet. Um Analysen bzgl. der Altersgruppen durchführen zu können, wird jeder Altersabschnitt einem Cluster zugeordnet. Die a posteriori Wahrscheinlichkeit $\mathrm{q}_{\mathrm{ik}}$, dass ein Altersabschnitt i zu einem Cluster k gehört, kann wie folgt berechnet werden:

$$
\mathrm{q}_{\mathrm{ik}}=\frac{\pi(\mathrm{k}) \prod_{\mathrm{j}}\left(\mathrm{p}_{\mathrm{jk}}\right)^{O_{i j}}\left(1-\mathrm{p}_{\mathrm{jk}}\right)^{1-O_{i j}}}{\sum_{k=1}^{K} \pi(\mathrm{k}) \prod_{\mathrm{j}}\left(\mathrm{p}_{\mathrm{jk}}\right)^{O_{i j}}\left(1-\mathrm{p}_{\mathrm{jk}}\right)^{1-O_{i j}}} 247
$$

i Altersabschnitt

j Delikt

k Cluster

$\mathrm{O}_{\mathrm{ij}} \quad$ 1, wenn Delikt $\mathrm{j}$ im Altersabschnitt i vorkommt, sonst 0

$\pi(\mathrm{k}) \quad$ Cluster $\mathrm{k}$ enthält $\pi(\mathrm{k}) \%$ aller Altersabschnitte

$\mathrm{p}_{\mathrm{jk}} \quad$ Wahrscheinlichkeit, dass ein Altersabschnitt im Cluster k das Delikt $\mathrm{j}$ enthält.

Jeder Altersabschnitt gehört somit mit einer gewissen Wahrscheinlichkeit zu einem Cluster. Um weitere Analysen durchführen zu können, wird jeder Altersabschnitt dem Cluster mit der größten a posteriori Wahrscheinlichkeit $\mathrm{q}_{\mathrm{ik}}$ zugeordnet.

98,7\% der Altersabschnitte haben eine a posteriori Wahrscheinlichkeit größer als $50 \%$ und damit ist eine Zuordnung mit absolutem Mehrheitsentscheid möglich. Bei $81,2 \%$ ist die a posteriori Wahrscheinlichkeit größer $80 \%$. D.h. die Clusterzuordnung ist sehr gut. Im Cluster „,verschiedene Straftaten“ ist die Zuordnung etwas schlechter. Hier haben 5,5\% eine Zuordnungswahrscheinlichkeit kleiner als 50\%.

Abbildung 32 zeigt die Altersverteilung der Cluster. Auffällig ist die unterschiedliche Altersverteilung der einzelnen Cluster. Die Altersverteilung der Cluster lassen sich in vier Gruppen einteilen. Das Cluster einfacher Diebstahl (A) hat ein deutliches Altersmaximum im Alter von 14 bis 18 Jahren und ist dann sehr stark abfallend auf weniger als die Hälfte.

246 Vgl. Bacher 1996, S. 141.

247 Francis, Soothill \& Fligelstone 2004, S.66 
Abbildung 32: Verteilung der männlichen Täter auf Cluster nach Altersgruppen

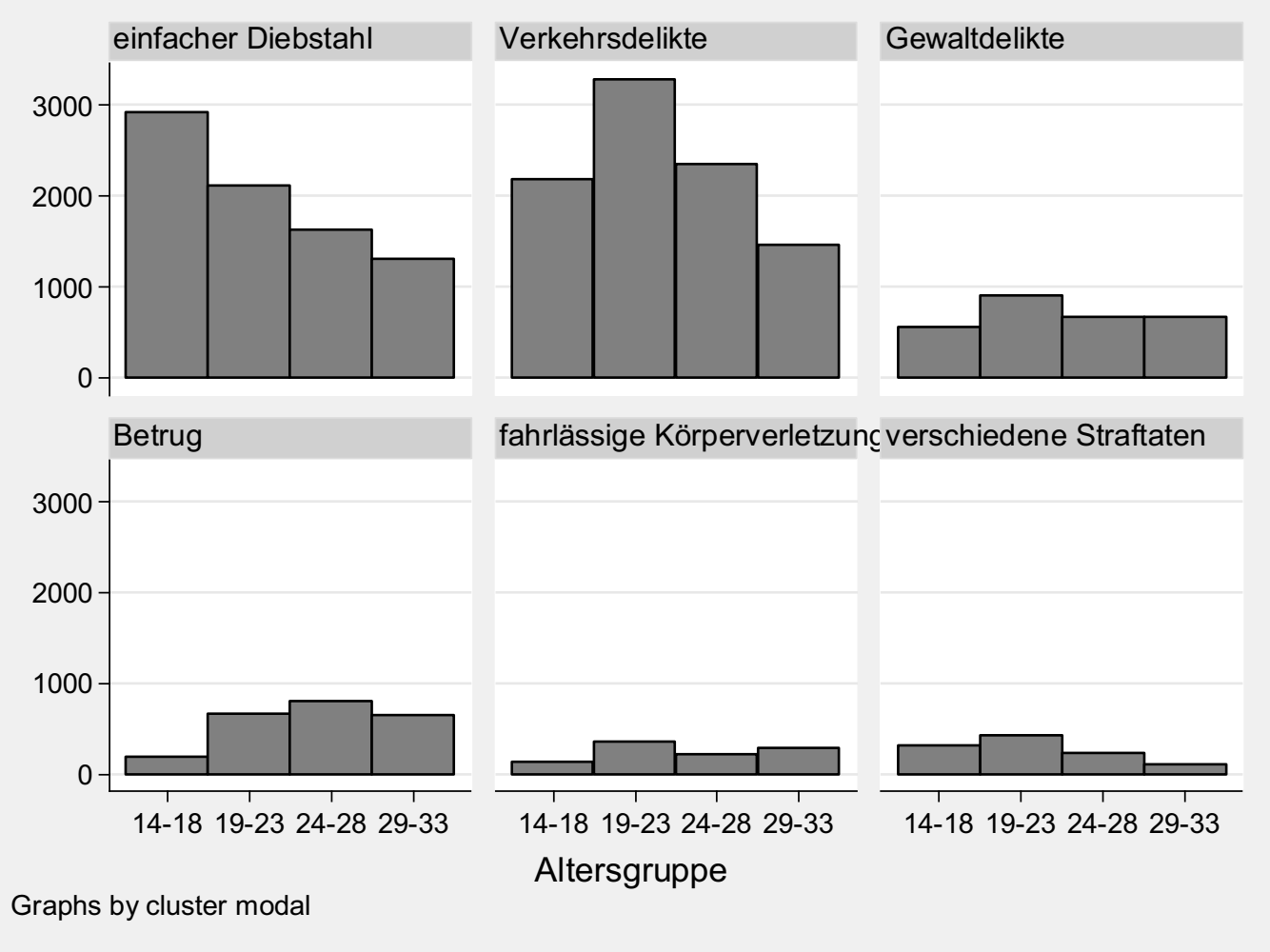

Die Cluster Verkehrsdelikte (B) und verschiedene Straftaten (F) haben ihr Maximum im Alter von 19 bis 23. Dies entspricht der Altersverteilung der justiziell registrierten Männer ohne Berücksichtigung der Deliktsgruppe. Verkehrsdelikte bilden die größte Deliktsgruppe bei den justiziellen Registrierungen der Männer. Verkehrsdelikte kommen ab dem Alter 18 häufiger vor, weil in Deutschland erst mit 18 der Autoführerschein erhältlich ist.

Eine andere Altersverteilung haben das Cluster der Gewaltdelikte (C) und das Cluster fahrlässige Körperverletzung (E). Hier befinden sich die Altersmaxima zwar auch bei den 19- bis 23-Jährigen, aber 24-28-Jährige sind nur etwas seltener vertreten und 29- bis 33-Jährige ebenso häufig bzw. im Cluster der fahrlässigen Körperverletzung sogar wieder etwas häufiger.

Die vierte Gruppe der Altersverteilungen zeigt sich beim Cluster Betrug (D). Hier nimmt das Alter bis zu den 24- bis 28-Jährigen zu und erst danach ab. Das Altersmaximum ist später als bei den anderen Clustern.

Zusammenfassend gibt Tabelle 41 nochmals einen Überblick über die Zusammensetzung der Cluster, die jeweilige Altersverteilung und die durchschnittliche Anzahl von Delikten pro Altersabschnitt. 
Tabelle 41: Deliktszusammensetzung der Cluster deutscher Männer der Kohorte 1970 (6 Cluster)

\begin{tabular}{|c|c|}
\hline Cluster & Zusammensetzung \\
\hline $\begin{array}{l}\text { A }(35,6 \%) \\
\text { Diebstahl und } \\
\text { BTM-Delikte }\end{array}$ & $\begin{array}{l}\text { Einfacher Diebstahl, schwerer Diebstahl, Hehlerei, Leistungserschlei- } \\
\text { chung, Betäubungsmitteldelikte und sonstige Sexualdelikte. } \\
\text { Altersmaximum bei 14-18, dann abnehmend. } \\
\text { Im Durchschnitt 1,7 Delikte pro Altersabschnitt. }\end{array}$ \\
\hline $\begin{array}{l}\text { B }(32,9 \%) \\
\text { Verkehrsdelikte }\end{array}$ & $\begin{array}{l}\text { Verkehrsdelikte. } \\
\text { Alter hauptsächlich 19-28, Maximum bei 19-23. } \\
\text { Im Durchschnitt 1,4 Delikte pro Altersabschnitt. }\end{array}$ \\
\hline $\begin{array}{l}\mathrm{C}(12,6 \%) \\
\text { Gewaltdelikte }\end{array}$ & $\begin{array}{l}\text { Gewaltdelikte (Tötungsdelikte, Sexualdelikte, Raub, schwere Körperverlet- } \\
\text { zung, Körperverletzung, Straftaten gegen die persönliche Freiheit), Wider- } \\
\text { stand gegen die Staatsgewalt, Hausfriedensbruch, Sachbeschädigung, Belei- } \\
\text { digung und Vollrausch. Altersmaximum bei den 19- bis 23-Jährigen, an- } \\
\text { sonsten relativ gleichmäßig verteilt über alle Altersgruppen. } \\
\text { Im Durchschnitt 2,9 Delikte pro Altersabschnitt. }\end{array}$ \\
\hline $\begin{array}{l}\mathrm{D}(8,4 \%) \\
\text { Betrug }\end{array}$ & $\begin{array}{l}\text { Betrug und Fälschung. } \\
\text { Alter hauptsächlich 19-33, Maximum bei den 24- bis 28-Jährigen. } \\
\text { Im Durchschnitt 2,2 Delikte pro Altersabschnitt. }\end{array}$ \\
\hline $\begin{array}{l}\text { E }(5,2 \%) \\
\text { Fahrlässige Kör- } \\
\text { perverletzung }\end{array}$ & $\begin{array}{l}\text { Fahrlässige Körperverletzung und Tötung. } \\
\text { Alter 19-33, Altersmaximum bei den 19- bis 23-Jährigen. } \\
\text { Im Durchschnitt 2,0 Delikte pro Altersabschnitt. }\end{array}$ \\
\hline $\begin{array}{l}\text { F }(5,2 \%) \\
\text { Verschiedene } \\
\text { Straftaten }\end{array}$ & $\begin{array}{l}\text { Alle Straftaten kommen überdurchschnittlich häufig vor. } \\
\text { Altersverteilung wie über alle Delikte, Maximum bei den 19- bis 23- } \\
\text { Jährigen. } \\
\text { Im Durchschnitt 6,1 Delikte pro Altersabschnitt. }\end{array}$ \\
\hline
\end{tabular}

Bei der Sechsclusterlösung ergeben sich die Cluster: Diebstahl, Betäubungsmitteldelikte, sonstige Sexualdelikte, Leistungserschleichung (A), Verkehrsdelikte (B), Gewaltdelikte (C), Betrug (D), fahrlässige Körperverletzung (E) und verschiedene Straftaten (F).

Im Folgenden wird die Deliktsstruktur der Sechsclusterlösung mit anderen Clusterlösungen verglichen. Tabelle 42 gibt einen Überblick über die Clusterzusammensetzung je nach Anzahl der Cluster. Teilt man die Altersabschnitte auf 5 Cluster auf, vereinigt sich im Vergleich zur 6-Cluster Lösung Cluster D (Betrug) mit Cluster A (einfacher Diebstahl, Betäubungsmitteldelikte, sonstige Sexualdelikte, Leistungserschleichung). Bei einer Aufteilung auf 7 Cluster bildet einfacher Diebstahl aus Cluster A ein eigenes Cluster. ${ }^{248} 52 \%$ der Altersabschnitte aus Cluster A (6 Cluster) befinden sich bei der 7Clusterlösung im Cluster der Betäubungsmitteldelikte, 44\% im Cluster einfacher Diebstahl. Damit sind 92\% der Altersabschnitte des Betäubungsmittelclusters der 7-Clusterlösung aus Cluster A (6 Cluster), die restlichen 8\% kommen aus dem Gewaltcluster. 82\% der Altersabschnitte des Clusters einfacher Diebstahl (7 Cluster) sind bei der 6Clusterlösung in Cluster A, die restlichen 18\% des Clusters einfacher Diebstahl (7 Clus-

\footnotetext{
248 Siehe Tabelle $77 \mathrm{im}$ Anhang.
} 
ter) sind bei der 6-Clusterlösung im Cluster der Verkehrsdelikte. Bei 8 Clustern bilden auch die Betäubungsmitteldelikte aus Cluster A (6 Cluster) ein eigenes Cluster. Bei 9 Clustern teilt sich das Cluster der Gewaltdelikte (Cluster C) in zwei Cluster auf. Bei 13 Clustern wird das Gewaltcluster weiter aufgeteilt, Leistungserschleichung und schwerer Diebstahl bilden jeweils ein eigenständiges Cluster. Dafür gehören ,sonstige Sexualdelikte“ zum Cluster ,verschiedene Straftaten“. Neu ist auch das Cluster „schwerer Diebstahl“ und „Fälschung“. Bei jeder hier aufgeführten Anzahl von Clustern existieren die Cluster „Verkehrsdelikte“, „fahrlässige Körperverletzung“ und „verschiedene Straftaten“. Das Cluster ,verschiedene Straftaten“ und damit das Cluster der chronischen Straftäter existiert unabhängig von der Anzahl der Cluster, ebenso die Cluster „Verkehrsdelikte“ und „fahrlässige Körperverletzung“.

Tabelle 42: Überblick über die Clusterzusammensetzung je nach Anzahl von Clustern (deutsche Männer Kohorte 1970)

\begin{tabular}{|c|c|c|c|c|c|}
\hline 5 & 6 & 7 & 8 & 9 & 13 \\
\hline & & Einf. Diebst. & Einf. Diebst. & Einf. Diebst. & Einf. Diebstahl \\
\hline & & & & & Schw. Diebstahl \\
\hline & & & & & Leistungserschl. \\
\hline \multirow{14}{*}{$\begin{array}{l}\text { Einf. Diebstahl } \\
\text { Schw. Diebstahl } \\
\text { Leistungserschl. } \\
\text { BTM-Del. } \\
\text { sonstige Sexualdel. } \\
\text { Betrug }\end{array}$} & Einf. Diebstahl & Schwerer & Schwerer & Schwerer & \\
\hline & Schw. Diebstahl & Diebstahl, & Diebstahl, & Diebstahl, & \\
\hline & Leistungserschl. & Leistungser- & Leistungser- & Leistungser- & \\
\hline & BTM-Del. & schleichung & schleichung & schleichung & \\
\hline & $\begin{array}{l}\text { sonst. Sexual- } \\
\text { del. }\end{array}$ & $\begin{array}{l}\text { BTM-Del. } \\
\text { sonstige Se- } \\
\text { xuldel }\end{array}$ & $\begin{array}{l}\text { sonstige Se- } \\
\text { xualdel. }\end{array}$ & $\begin{array}{l}\text { sonstige Se- } \\
\text { xualdel. }\end{array}$ & \\
\hline & & & BTM-Delikte & BTM-Delikte & BTM-Delikte \\
\hline & Betrug & Betrug & Betrug & Betrug & Betrug \\
\hline & & & & & Tötungsdel. \\
\hline & & & & & Sexualdel. \\
\hline & & & & & Sonst. Sex. \\
\hline & & & & & Raub \\
\hline & & & & & Vollrausch \\
\hline & & & & & Hehlerei \\
\hline & & & & & Öff. Ord. \\
\hline \multirow[t]{14}{*}{ Gewalt } & Gewalt & Gewalt & Gewalt & Gewalt 1 & Sexualdel. \\
\hline & & & & (Körperver- & Körperverlet- \\
\hline & & & & letzung, Tö- & zung \\
\hline & & & & tungsdel., & \\
\hline & & & & Sexualdel., & \\
\hline & & & & Raub) & \\
\hline & & & & Gewalt 2 (Be- & Persfr. \\
\hline & & & & leidg., Persfr., & Beleidigung \\
\hline & & & & Sachb., Wid. & Wid. gg. St. \\
\hline & & & & Gg. St.) & \\
\hline & & & & & Sachbesch. \\
\hline & & & & & Hausfr. \\
\hline & & & & & Schw. Diebstahl \\
\hline & & & & & Fälschung \\
\hline Versch. Str. & Versch. Str. & Versch. Str. & Versch. Str. & Versch. Str. & Versch. Str. \\
\hline Verkehrsdel. & Verkehrsdel. & Verkehrsdel. & Verkehrsdel. & Verkehrsdel. & Verkehrsdel. \\
\hline Fahrl. KV & Fahrl. KV & Fahrl. KV & Fahrl. KV & Fahrl. KV & Fahrl. KV \\
\hline
\end{tabular}


Vergleicht man die Clusteranalyse der englischen Kohorte 1953 mit der Clusteranalyse aus Baden-Württemberg der Kohorte 1970, so ist festzustellen, dass das Verhältnis von Altersabschnitten und Tätern bei den beiden Kohorten unterschiedlich ist. Die Anzahl der Täter der deutschen 1970er Kohorte (12.101) ist um ein Drittel größer als die der englischen 53er Kohorte (9.232). Bei den Deutschen der Kohorte 1970 gibt es etwa doppelt soviel Altersabschnitte wie Täter. Bei der englischen Kohorte ist das Verhältnis von Altersabschnitten und Tätern 1,7. Dies ist einerseits erstaunlich, weil die Altersspanne, die von der englischen Kohorte umfasst wird, größer ist als die der deutschen Kohorte. Es gibt davor und danach jeweils eine weitere Altersgruppe, die es in der deutschen Kohorte nicht gibt. Trotzdem gibt es in der englischen Kohorte in Relation zu den Tätern weniger Altersabschnitte, d.h. die Täter sind seltener in verschiedenen Altersabschnitten registriert. Der Unterschied lässt sich einerseits damit erklären, dass hier bei der Auswertung nur Täter analysiert werden, die mit mindestens zwei Straftaten registriert sind. Bei der Analyse der englischen Kohorte gab es diese Bedingung nicht. Ein weiterer Unterschied zeigt sich in den registrierten Straftaten. Die größte Deliktsgruppe der justiziellen Registrierungen der deutschen Männer bilden die Verkehrsdelikte. 27\% der Registrierungen von deutschen Männern mit zwei oder mehr Straftaten sind Verkehrsdelikte. In den englischen Daten ist die Anzahl der registrierten Verkehrsdelikte sehr gering.

Der Vergleich der 9-Clusterlösung der englischen Kohorte mit der 9-Clusterlösung der deutschen Kohorte ergibt teilweise eine Übereinstimmung bei den Clustern Betrug, verschiedene Straftaten, einfacher Diebstahl, schwerer Diebstahl und bei den Gewaltclustern. ${ }^{249}$ Die Cluster Verkehrsdelikte, fahrlässige Körperverletzung und Betäubungsmitteldelikte ergeben sich bei der Clusteranalyse der englischen Kohorte nicht. Stattdessen ergibt die Clusteranalyse der englischen Kohorte noch drei weitere Cluster unterschiedlicher Diebstahlsdelikte und anderen Eigentumsdelikten. Die große Häufung von Clustern mit differierenden Diebstahlsdelikten, die die Clusteranalyse der englischen Kohorte ergibt, hängt damit zusammen, dass bei der Analyse der englischen Kohorte 71 Deliktskategorien mit sehr vielen unterschiedlichen Diebstahlskategorien in die Untersuchung einbezogen sind ${ }^{250}$, bei den deutschen Daten jedoch nur 28 Deliktskategorien mit zwei verschiedenen Diebstahlskategorien. Werden mit den deutschen Daten 8 Cluster berechnet, vereinigen sich die beiden Gewaltcluster zu einem. Die Cluster, die es bei der Analyse der englischen Daten nicht gibt, Verkehrsdelikte, Betäubungsmitteldelikte und fahrlässige Körperverletzung, sind weiterhin deutliche Cluster, die jeweils 100\%

249 Im Anhang befinden sich die Tabellen der 9-Clusterlösung der englischen Kohorte (Tabelle 70, S. 254) und der deutschen Kohorte (Tabelle 76, S. 261).

250 Wie im Anhang beschrieben, kommen von den 71 Deliktskategorien 26 häufiger vor und darunter befinden sich 11 verschiedene Diebstahlskategorien. Die Deliktscluster der Altersabschnitte der Männer der englischen Kohorte sind in Tabelle 70 im Anhang dargestellt. 
ihres Delikts enthalten. Das Nichtvorhandensein der Cluster Verkehrsdelikte und fahrlässige Körperverletzung bei der Analyse der englischen Kohorte hängt damit zusammen, dass in England in den Justizdaten lange nicht so viele Verkehrsdelikte registriert sind wie in Deutschland.

Die Cluster einfacher Diebstahl, schwerer Diebstahl und Betrug ergibt sowohl die Analyse der englischen Kohorte wie die Analyse der deutschen Kohorte. Auch die Altersverteilung dieser Cluster stimmt überein. ${ }^{251}$ Die Diebstahlcluster haben ihr Maximum bei den Jugendlichen und die Anteile der Altersabschnitte nehmen mit dem Alter ab. Im Betrugscluster befinden sich hauptsächlich Ältere.

Zwei Gewaltcluster ergibt die Analyse sowohl für die englische Kohorte wie für die Kohorte aus Baden-Württemberg, aber die Altersverteilung der Cluster ist unterschiedlich. Im Cluster Gewalt der deutschen Kohorte befinden sich gleich viele 19- bis 23-Jährige und 24- bis 28-Jährige und sogar etwas mehr 29- bis 33-Jährige. Im Cluster Gewalt der englischen Kohorte befinden sich hauptsächlich 16- bis 25Jährige, Jüngere und Ältere sind kaum vertreten. Der Unterschied der Gewaltcluster wird somit hauptsächlich bei der Altersgruppe 26 bis 33 sichtbar, von dieser Altersgruppe befinden sich in Baden-Württemberg deutlich mehr Altersabschnitte im Gewaltcluster als in England. Die Altersverteilung des Clusters Körperverletzung der deutschen Kohorte entspricht der gesamten Altersverteilung. Den größten Anteil bilden die 19- bis 23-Jährigen. Anders sieht es hier im Cluster Körperverletzung der englischen Kohorte aus. Hier sind die 16- bis 30-Jährigen gleich stark vertreten, erst bei den 31- bis 35-Jährigen zeigt sich eine Abnahme.

Sowohl die Clusteranalyse der englischen Kohorte wie die Clusteranalyse der deutschen Kohorte ergibt ein Cluster mit verschiedenen Straftaten. Sowohl die relativ geringe Größe dieser Cluster wie die große Anzahl jeweils registrierter Straftaten lassen vermuten, dass es sich hier um chronische Straftäter handelt. Aber auch hier unterscheidet sich die Altersverteilung der Cluster. Im englischen Cluster sind die 16- bis 30-Jährigen gleich stark vertreten, erst bei den 31- bis 35-Jährigen zeigt sich eine Abnahme. Das deutsche Cluster hat die Verteilung der Gesamtverteilung. Die meisten Altersabschnitte stammen von 19- bis 23-Jährigen, danach nehmen die Altersabschnitte im Cluster mit dem Alter ab.

Der Vergleich der Clusteranalyse der englischen Kohorte mit der Clusteranalyse der deutschen Kohorte ist nur bedingt möglich, weil sich die Registrierung der justiziellen Daten unterscheidet. Die Delikte, speziell die Diebstahldelikte, werden in den englischen Daten feiner unterschieden. Im Bundeszentralregister gibt es auBer den $\S \S 242,243$ (StGB) keine weiteren Angaben. Außerdem werden in den englischen Daten auch die Einstellungen der Verfahren von Erwachsenen registriert. ${ }^{252}$ Dies ist im Bundeszentralregister nicht der Fall und könnte damit ein Grund für die unterschiedliche Altersverteilung der Cluster sein.

${ }^{251}$ Die Alterstruktur der 9-Clusterlösung der deutschen Kohorte ist in Abbildung 66 im Anhang dargestellt.

${ }^{252}$ Siehe http://homeoffice.gov.uk/justice/what-happens-at-court/sentencing 
Einige Ergebnisse, die sich schon bei der Multidimensionalen Skalierung (Abbildung 11) zeigten, bestätigen sich hier. Die Deliktskategorien Fälschung und Betrug liegen im Ergebnis der Multidimensionalen Skalierung nah beieinander und befinden sich auch hier häufig in einem Cluster. Ebenso die Delikte einfacher Diebstahl, Betäubungsmitteldelikte und Erschleichen von Leistungen. Auch die Gewaltdelikte bilden ein Cluster und liegen im Ergebnis der Multidimensionalen Skalierung nah beieinander.

\subsubsection{Spezialisierung, Wechsel und Abbruch im Lebenslauf der Männer}

Mit der Clusteranalyse ist es möglich, Deliktsstrukturen zu identifizieren. Des Weiteren ist es auch möglich, Veränderungen der Deliktskonfigurationen über den Lebenslauf zu erforschen. Das kriminelle Verhalten der offiziell Registrierten wird untersucht, indem sowohl der Grad der Spezialisierung als auch Fragen des Abbruchs und Verhaltensänderungen analysiert werden. ${ }^{253}$ Anhand der vorliegenden Daten kann die offiziell registrierte kriminelle Karriere untersucht werden. Hierzu werden Ergebnisse zur Spezialisierung und zur kriminellen Laufbahn dargestellt. Spezialisierung bedeutet hier, dass es von einer Altersgruppe zur nächsten keinen Wechsel des Clusters gibt. Spezialisierung bedeutet hier also nicht, dass eine Person immer das gleiche Delikt begeht, sondern Spezialisierung bedeutet, dass die Deliktskonfiguration einer Altersspanne, der Deliktskonfiguration der nächsten Altersspanne entspricht.

Tabelle 43: Spezialisierungsanteile in \%: Deutsche Männer der Kohorte 1970 (justizielle Registrierungen) 254

\begin{tabular}{|c|c|c|c|c|c|c|c|c|}
\hline \multirow{2}{*}{\multicolumn{2}{|c|}{ Altersgruppen }} & & \multicolumn{6}{|c|}{ Cluster } \\
\hline & & \multirow[b]{2}{*}{$\begin{array}{l}\mathrm{N} \text { ("von" } \\
\text { ohne Ab- } \\
\text { brecher) }\end{array}$} & \multirow{2}{*}{$\begin{array}{c}\text { A } \\
\text { Diebstahl } \\
(\mathrm{N})\end{array}$} & \multirow{2}{*}{$\begin{array}{c}\text { B } \\
\text { Verkehrs- } \\
\text { delikte }(\mathrm{N})\end{array}$} & \multirow{2}{*}{$\begin{array}{c}\mathrm{C} \\
\text { Gewalt (N) }\end{array}$} & \multirow{2}{*}{$\begin{array}{c}\text { D } \\
\text { Betrug (N) }\end{array}$} & \multirow{2}{*}{$\begin{array}{c}\mathrm{E} \\
\text { Fahrlässige } \\
\text { Körperver- } \\
\text { letzung (N) }\end{array}$} & \multirow{2}{*}{$\begin{array}{c}\mathrm{F} \\
\text { Verschie- } \\
\text { dene Straf } \\
\text { taten }(\mathrm{N})\end{array}$} \\
\hline von & bis & & & & & & & \\
\hline $14-18$ & $19-23$ & 4892 & $29(2447)$ & $39(1488)$ & $24(431)$ & $18(136)$ & $6(105)$ & $19(285)$ \\
\hline $19-23$ & $24-28$ & 4762 & $34(1371)$ & $43(1815)$ & $24(575)$ & $29(451)$ & $4(177)$ & $18(373)$ \\
\hline $24-28$ & $29-33$ & 2664 & $51 \quad(797)$ & $46(886)$ & $36(334)$ & $37(417)$ & $8(50)$ & $17(180)$ \\
\hline
\end{tabular}

253 Vgl. Francis, Soothill \& Fligelstone 2004, S. 71

${ }^{254}$ Spezialisierung wird hier benutzt im Sinne von Francis, Soothill und Fligelstone (vgl. Francis, Soothill \& Fligelstone 2004, S. 73 ff.). Ein Täter ist spezialisiert, wenn er sich im folgenden Altersabschnitt im selben Cluster befindet. Die Prozente wurden hier ohne Abbrecher berechnet, d.h. in der „,von“ Altersgruppe befinden sich nur Straftäter, die mindestens einmal in einer späteren Altersgruppe (nicht unbedingt in der folgenden) registriert sind. Berechnet wurden die Prozente von einer Altersgruppe zur folgenden. 
Tabelle 43 zeigt die Spezialisierungsanteile bezogen auf die Clusterzugehörigkeit. Betrachtet werden hier nur Personen, die in einer späteren Altersspanne wieder eine Registrierung haben; Abbrecher, bzw. Personen, die nur innerhalb einer Altersspanne registriert sind, werden hier nicht mit einbezogen. Die prozentualen Anteile sind berechnet von einer Altersgruppe zur nächsten, d.h. es ist angegeben, wie groß der Anteil der Ausgangsgruppe ist, die in der nächsten Altersspanne wieder Delikte aus demselben Cluster verrichtet. Das N in der dritten Spalte von links gibt an, wie viele Personen in der ,von“ Altersgruppe sind und in einer späteren Altersgruppe wieder registriert sind. Die Häufigkeiten in Klammern $(\mathrm{N})$ geben an, wie viele Personen im jeweiligen Cluster in der „von“ Altersgruppe sind und in einer späteren Altersgruppe wieder eine Registrierung haben.

2447 Männer im Alter von 14 bis 18 Jahren befinden sich im Cluster A (Diebstahl) und haben als Ältere eine weitere Registrierung. 29\% davon gehören im Alter von 19 bis 23 auch zu Cluster A. Von den 24- bis 28-Jährigen sind 51\% auch mit 29 bis 33 Jahren im Cluster Diebstahl. Die Spezialisierungsanteile nehmen im Cluster A mit dem Alter zu. Auch im Cluster B (Verkehrsdelikte) nimmt der Spezialisierungsgrad mit dem Alter zu, hier aber nur sehr leicht. Der Spezialisierungsgrad ist bei den 14- bis 18-Jährigen $39 \%$ und bei den 24- bis 28 -Jährigen $46 \%$. Auch im Cluster Betrug (D) nimmt der Spezialisierungsanteil mit dem Alter zu. In der Altersgruppe 14 bis 18 ist der Spezialisierungsanteil 18\%, in der Altersgruppe 24 bis 28 37\%. Im Cluster der Gewaltdelikte ist der Spezialisierungsanteil der 14bis 18-Jährigen so groß wie der der 19- bis 23-Jährigen (24\%). Die Spezialisierungsrate der 24- bis 28-Jährigen ist höher als die der Jüngeren. Im Cluster der fahrlässigen Körperverletzung sind die Spezialisierungsanteile am Niedrigsten. Zu beachten ist beim Cluster fahrlässige Körperverletzung aber, dass wegen der kleinen Häufigkeiten die Fehler ${ }^{255}$ der Prozentangaben groß sind. In der Altersgruppe 14 bis 18 und 19 bis 23 ist der Fehler 2\%, in der Altersgruppe 24 bis 28 sogar 4\%. Die Fehler sind in Relation zu den Prozentwerten sehr groß, deshalb ist hier keine Aussage über einen Anstieg des Spezialisierungsgrades möglich. Genauso ist die Situation im Cluster F (verschiedene Straftaten), hier liegt der Fehler bei 2 bis 3\% und somit sind keine Aussagen über eine Veränderung der Spezialisierungsraten möglich. Bei den anderen Clustern (Diebstahl, Verkehrsdelikte, Gewaltdelikte, Betrug) ist der Fehler der Prozentwerte klein. Generell ist eine Zunahme der Spezialisierungsrate mit dem Alter zu beobachten. Am deutlichsten im Cluster A (Diebstahl). Allerdings unter der Voraussetzung, dass, wie schon erwähnt, kein Abbruch der kriminellen Karriere stattfand.

Das Ergebnis der Clusteranalyse kann gleichfalls dazu benutzt werden, kriminelle Laufbahnen zu identifizieren, d.h. es kann untersucht werden, welche Clusterzu-

255 Der Fehler $\mathrm{f}$ der Prozentangaben wird folgendermaßen berechnet:

$f=\sqrt{\frac{p^{*} q}{N}}$ mit p Prozentangabe (z.B. $\left.29 \%=0,29\right), \mathrm{q}=1-\mathrm{p}$. 
gehörigkeit Täter einer Altersgruppe und eines Clusters in der vorhergehenden Altersgruppe und in der folgenden Altersgruppe haben bzw. ob sie vorher oder nachher überhaupt eine Registrierung haben. Somit wird je nach Clusterzugehörigkeit das Verhältnis von spezialisierten Tätern, deliktswechselnden Tätern oder Abbrechern betrachtet. ${ }^{256}$

Abbildung 33: Deliktsübergänge männlicher Täter der Altersgruppe 19-23

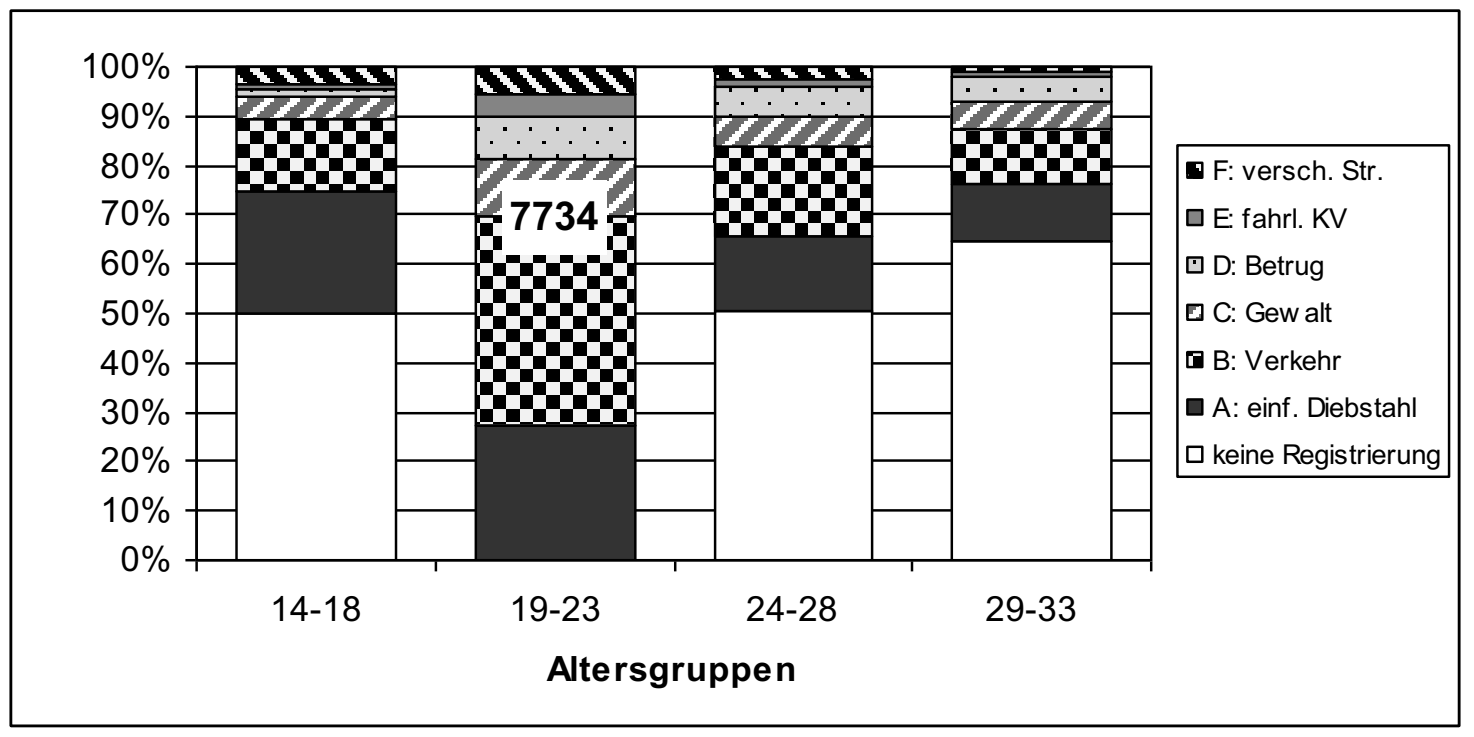

Abbildung 33 gibt einen Überblick über die Deliktsübergänge der männlichen Täter der Altersgruppe 19-23. 7734 männliche Täter haben mindestens eine Registrierung in der Altersspanne 19-23. Diese teilen sich auf in 27\%, die zum Cluster Diebstahl gehören, 43\% gehören zum Cluster Verkehrsdelikte, 12\% Cluster Gewaltdelikte, 9\% Cluster Betrug, 5\% Cluster fahrlässige Körperverletzung und 6\% Cluster verschiedene Straftaten. Von den männlichen Tätern, die im Alter von 19 bis 23 mindestens eine Registrierung haben, haben 50\% im Alter von 14 bis 18 und im Alter von 24 bis 28 keine Registrierung, 65\% haben im Alter von 29 bis 33 keine Registrierung. 25\% der 19- bis 23-Jährigen mit Registrierung gehören im Alter 14 bis 18 zum Cluster Diebstahl und 15\% zum Cluster Verkehrsdelikte. 15\% der 19- bis 23-Jährigen mit Registrierung gehören im Alter 24-28 zum Cluster Diebstahl und 18\% zum Cluster Verkehrsdelikte. In der Altersgruppe 29-33 gehören noch 12\% zum Cluster Diebstahl und 11\% zum Cluster Verkehrsdelikte.

256 Vgl. Francis, Soothill \& Fligelstone 2004, S.76 f. 
Die Abbildungen 34 bis 39 zeigen die Laufbahn der 19- bis 23-Jährigen Registrierten der sechs Cluster. Dazu wird jeweils bei den 19- bis 23-Jährigen die Zahl der Täter angegeben, die zu diesem Cluster gehören. Der linke Balken gibt die prozentualen Anteile der Cluster der 14- bis 18-Jährigen an, unter der Bedingung einer bestimmten Clusterzugehörigkeit im Alter von 19 bis 23 Jahren. Die rechten Balken geben diese Zugehörigkeit für die 24- bis 28-Jährigen und 29- bis 33-Jährigen an. Am wahrscheinlichsten ist, dass in der vorhergehenden wie in der nachfolgenden Altersspanne keine Registrierung vorliegt. In fünf der sechs Cluster ist der Anteil der Gruppe ,keine Registrierung“ sowohl in der Altersgruppe vorher wie nachher am größten. Nur im Cluster der verschiedenen Straftaten ist die Gruppe „keine Registrierung" in der Altersgruppe davor und in den Altersgruppen danach nicht besonders ausgeprägt. Dies bestätigt die Vermutung, dass sich in Cluster F die chronischen Straftäter befinden. Am höchsten ist der Anteil von „keiner Registrierung" vor und nach der Clusterzugehörigkeit der 19- bis 23-Jährigen des Clusters Verkehrsdelikte (Abbildung 35), jeweils etwa 60\% der 14- bis 18-Jährigen und der 24- bis 28-Jährigen. Von den 29- bis 33-Jährigen, die sich mit 19-23 im Verkehrscluster befanden, sind über $70 \%$ ohne Registrierung. Dies spricht dafür, dass es sich bei Verkehrsdelikten überwiegend um Einzelfälle handelt. Dennoch finden sich $24 \%$ in der Altersgruppe 24-28 wieder im Cluster Verkehrsdelikte, was für einen, wenn auch geringen Anteil von Spezialisierung bei dieser Deliktskonfiguration spricht. Alle anderen Cluster sind kaum vertreten, d.h. es gibt fast keinen Wechsel in andere Deliktsbereiche.

Von den 19- bis 23-Jährigen des Gewaltclusters (Abbildung 36) gehören 12\% vorher, $16 \%$ in der Altersgruppe 24-28 und 13\% in der Altersgruppe 29-33 auch zum Gewaltcluster. Die Anteile des Gewaltclusters in den Altersgruppen 14-18, 24-28 und 29-33, bei einer Zugehörigkeit zum Gewaltcluster im Alter 19-23, sind deutlich höher als die Anteile des Gewaltclusters der gesamten Altersgruppe 19-23 (siehe Abbildung 33). Damit zeigt sich, dass zum Gewaltcluster Spezialisierte gehören. Auch bei den anderen Clustern ist davor und danach der Anteil desselben Clusters im Vergleich zu den anderen Clustern und abgesehen von der Gruppe „keine Registrierung“ am höchsten. Eine Ausnahme bildet das Cluster fahrlässige Körperverletzung (Abbildung 38). Bei diesem Cluster gibt es keine „Spezialisierung" und auch bei den übrigen Clustern tritt davor und danach das Cluster fahrlässige Körperverletzung kaum auf. Auffällig ist aber bei den 19- bis 23-Jährigen, die zum Cluster „fahrlässige Körperverletzung“ gehören, der relativ hohe Anteil von Verkehrsdelikten der 14- bis 18-Jährigen (25\%). Auch in den folgenden Altersgruppen ist der Anteil an Verkehrsdelikten relativ hoch. 
Abbildung 34: Deliktsübergänge männlicher Täter des Clusters A (Diebstahl) im Alter 19 bis 23

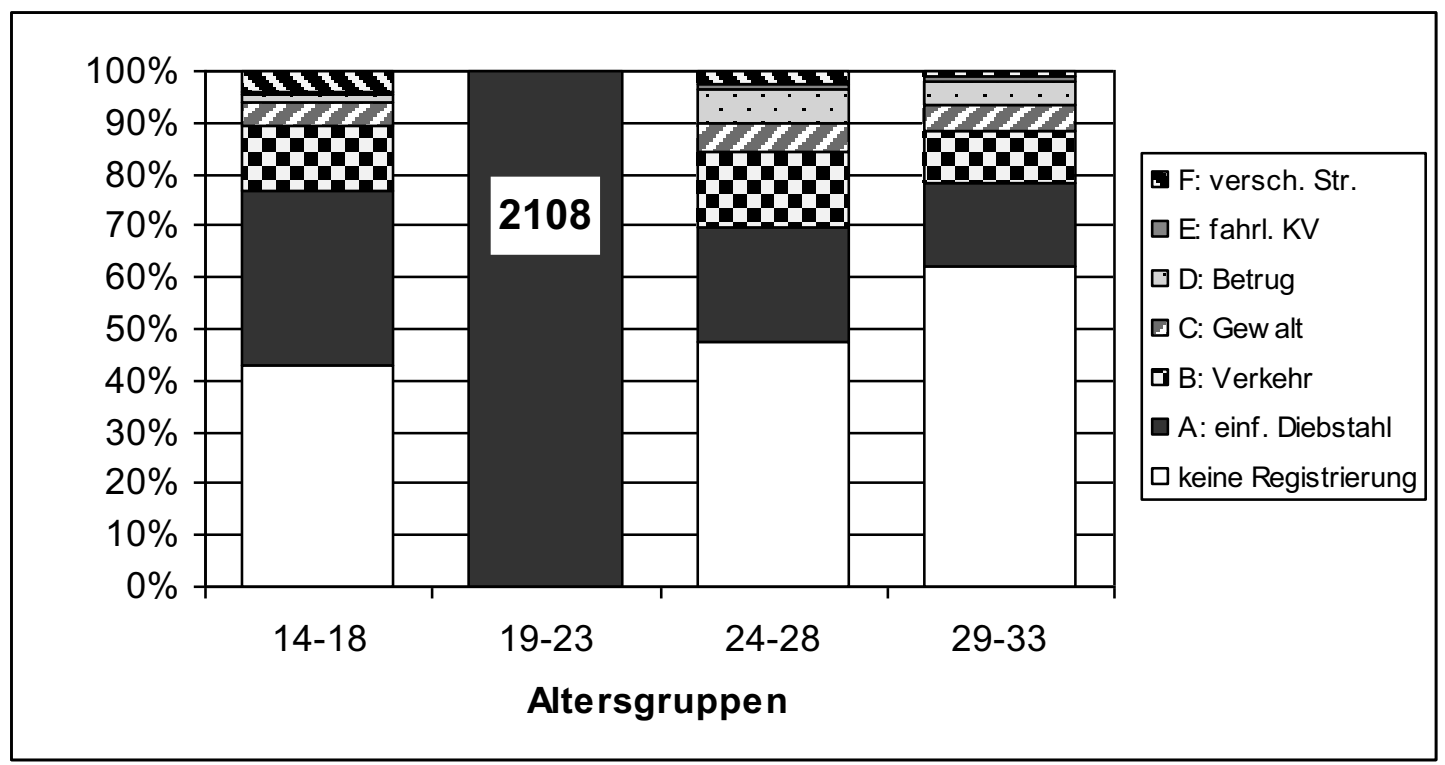

Abbildung 35: Deliktsübergänge männlicher Täter des Clusters B (Verkehrsdelikte) im Alter 19 bis 23

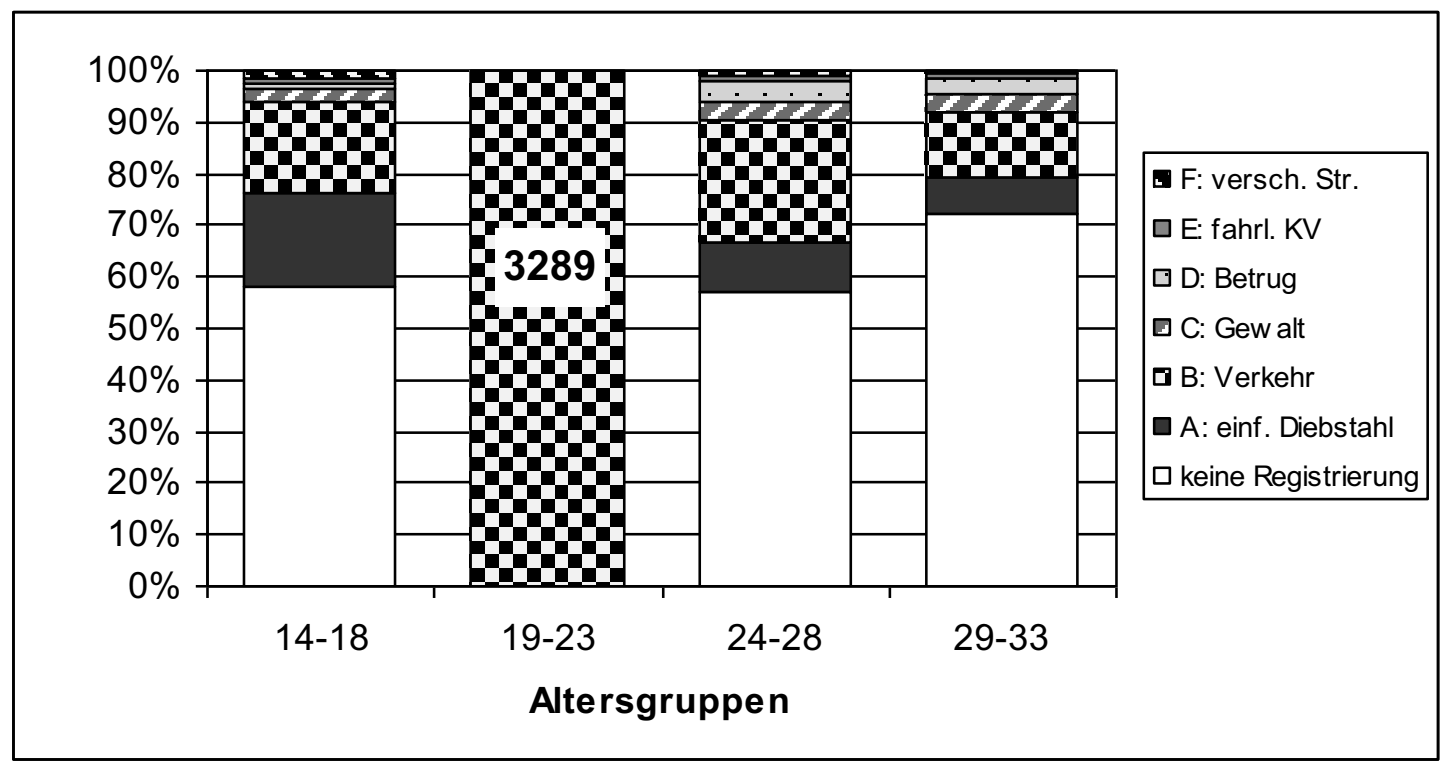


Abbildung 36: Deliktsübergänge männlicher Täter des Clusters C (Gewaltdelikte) im Alter 19 bis 23

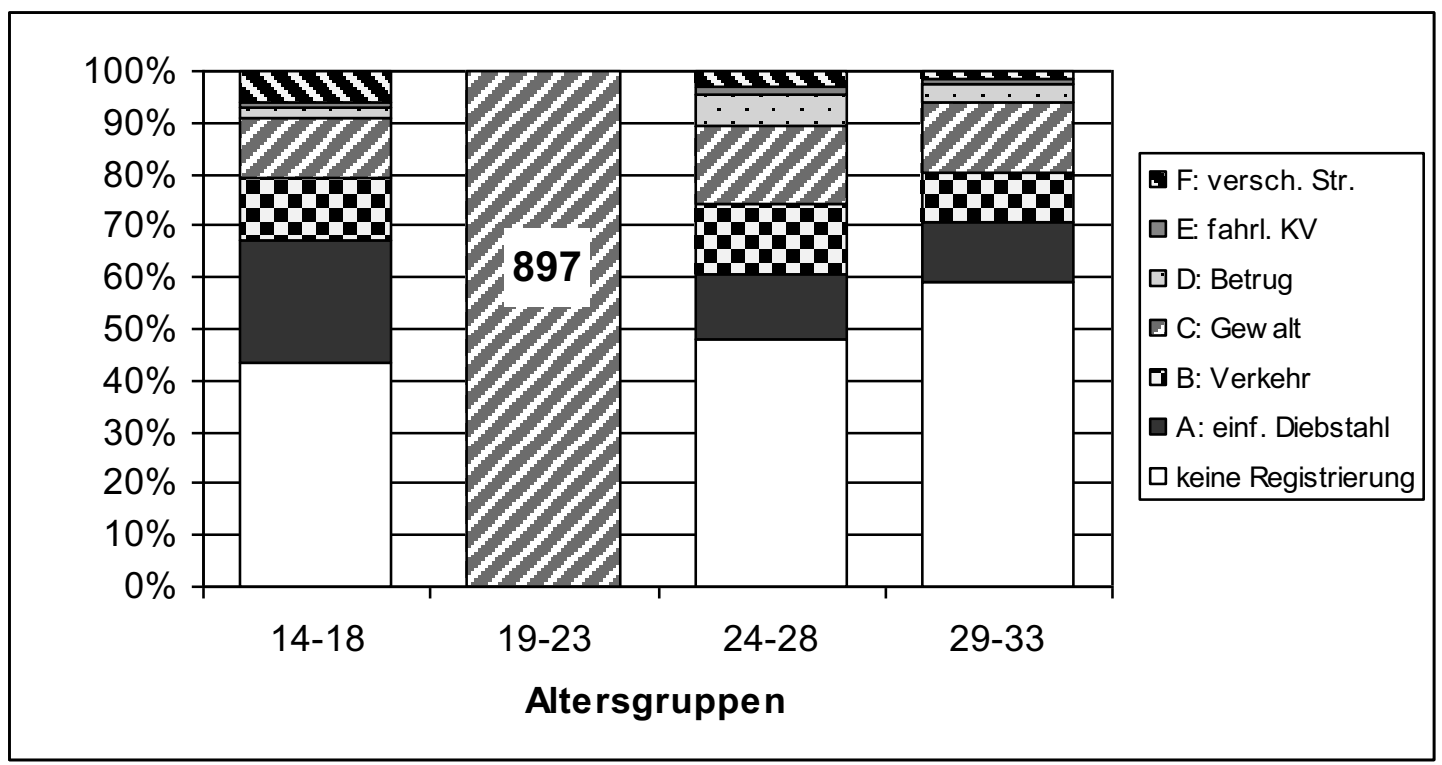

Abbildung 37: Deliktsübergänge männlicher Täter des Clusters D (Betrug) im Alter 19 bis 23

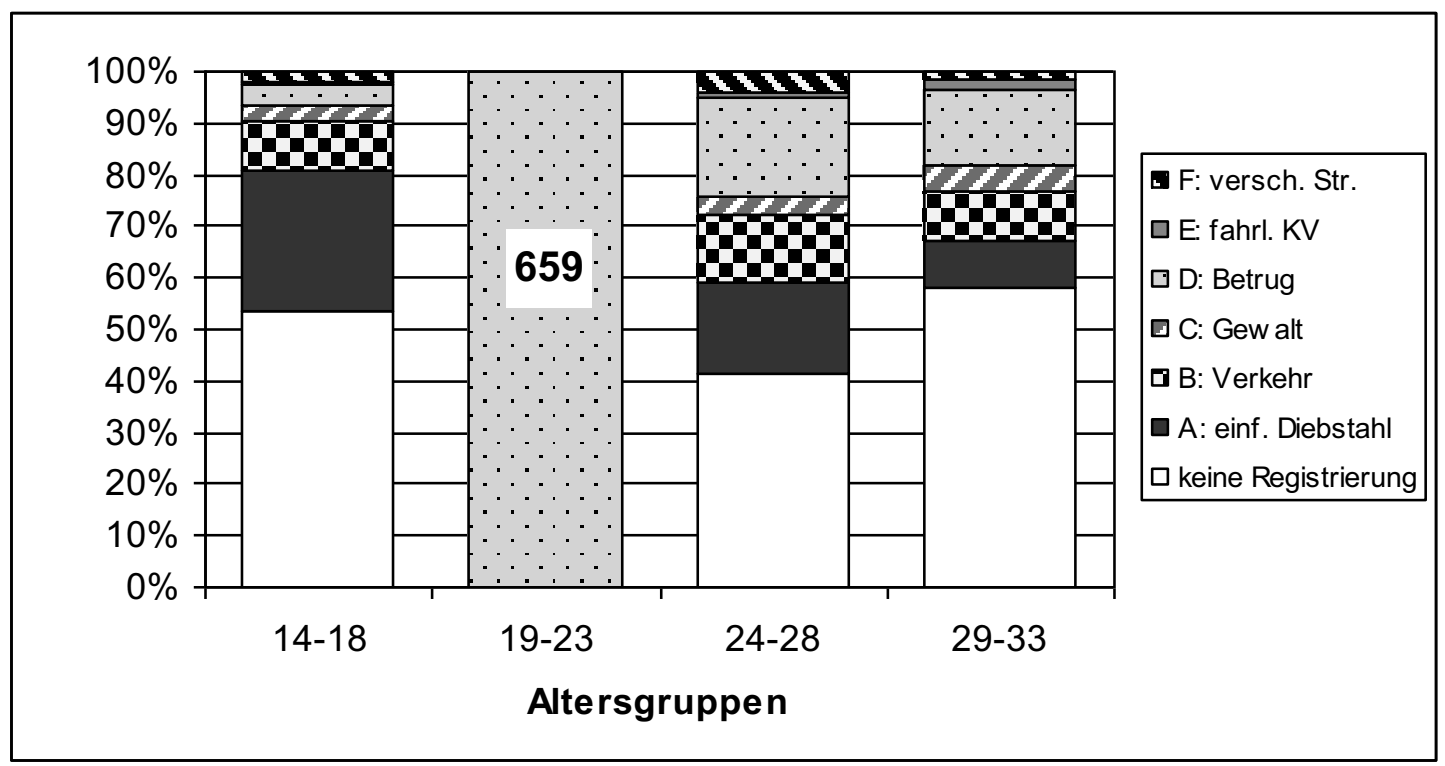


Abbildung 38: Deliktsübergänge männlicher Täter des Clusters E (fahrlässige Körperverletzung) im Alter 19 bis 23

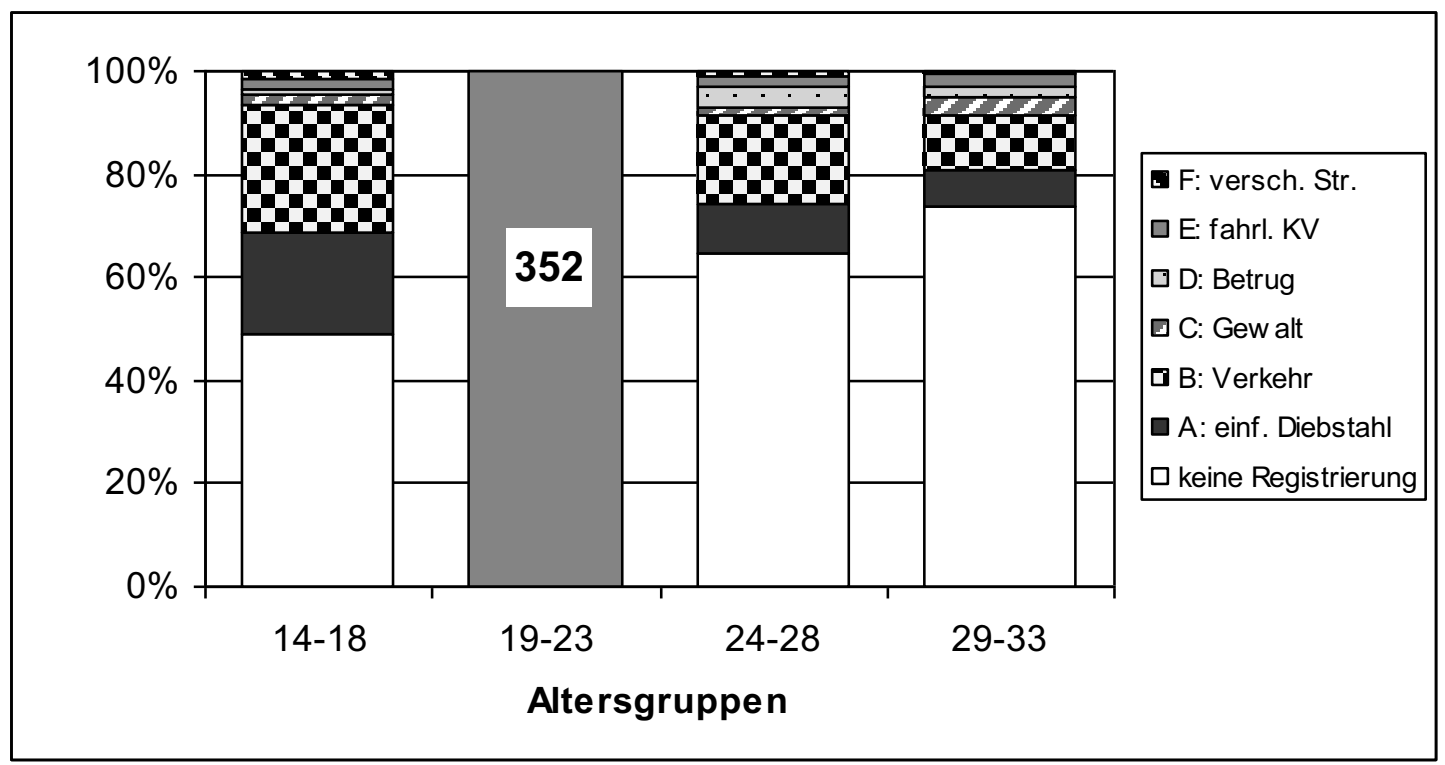

Abbildung 39: Deliktsübergänge männlicher Täter des Clusters F (verschiedene Straftaten) im Alter 19 bis 23

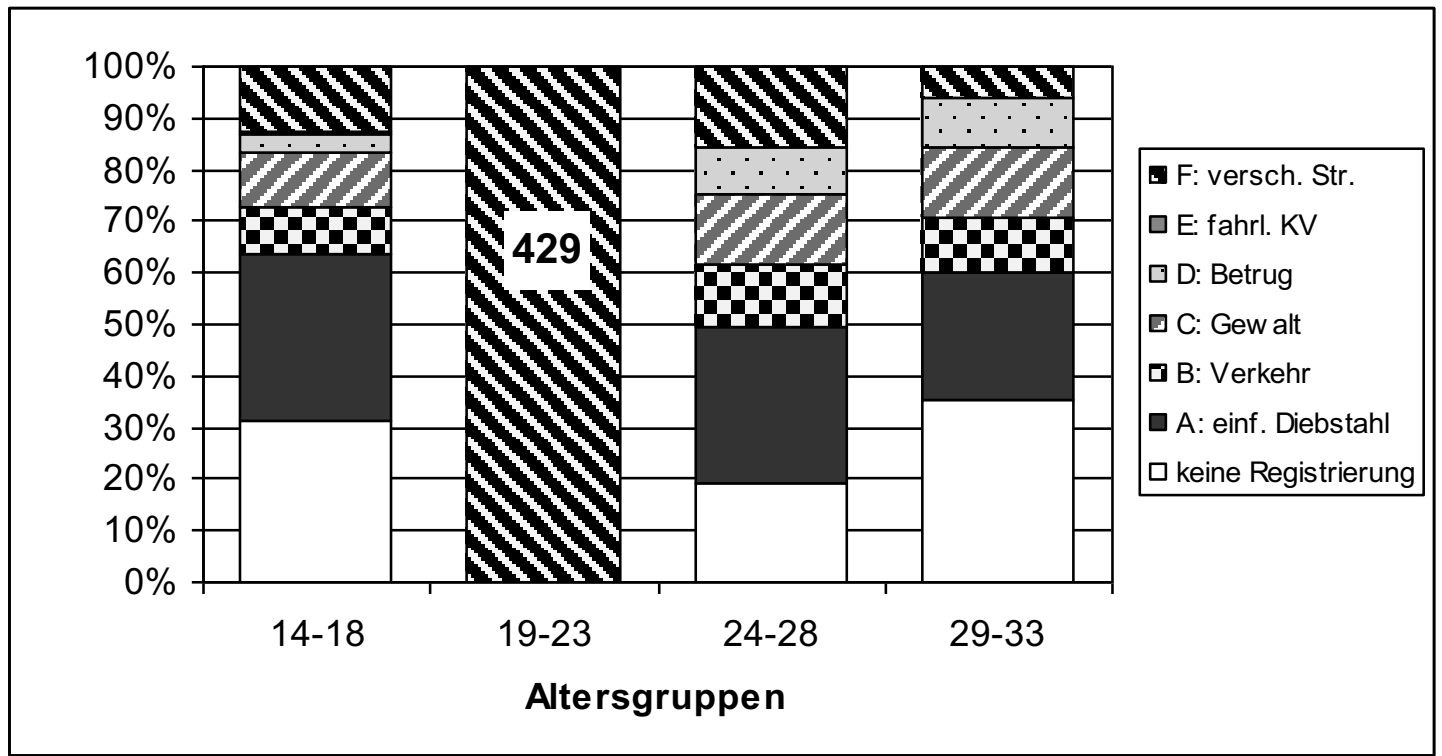

Bei den 14- bis 18-Jährigen ist bei allen Clustern der Anteil der Eigentumsdelikte relativ groß. Überdurchschnittlich groß ist der Anteil bei den Gruppen, die im Alter 19-23 zum Cluster Diebstahl (Abbildung 34), Betrug (Abbildung 37) und verschiedene Straftaten (Abbildung 39) gehören. 
Nur im Cluster der verschiedenen Straftaten und damit bei den chronischen Straftätern sind die Anteile von „keine Registrierung“ bei den Jüngeren und Älteren nicht hoch. Der Großteil ist auch als Jugendlicher und Erwachsener registriert. Das zeigt auch, dass chronische Straftäter sehr viele verschiedene Straftaten begehen.

Der hohe Anteil Nichtregistrierter beim Übergang vom Cluster Verkehrsdelikten (Abbildung 35) und der geringe Anteil anderer Cluster deutet darauf hin, dass es keinen Zusammenhang zwischen ,normalen“ Straftaten, klassischen oder konventionellen Rechtsbrüchen, und Verkehrsdelikten gibt. ${ }^{257}$ Die meisten Verkehrsdelikte werden fahrlässig und nicht vorsätzlich begangen. ${ }^{258}$ Umgekehrt ist aber zu sagen, dass Männer mit Registrierungen der klassischen Kriminalität auch häufig wegen Verkehrsdelikten registriert werden. ${ }^{259}$ Im Cluster Verkehrsdelikte befinden sich hauptsächlich Altersabschnitte, die nur ein Verkehrsdelikt enthalten. Zwei Drittel der Altersabschnitte des Clusters verschiedene Straftaten enthalten ein Verkehrsdelikt. Die Verkehrsdelikte im Cluster verschiedener Straftaten sind immer in Verbindung mit anderen Straftaten. Bei Verkehrsdelikten muss daher unterschieden werden zwischen fahrlässigen Straftaten (Cluster Verkehrsdelikte) und Straftaten von chronischen Straftätern, die häufig auch wegen Verkehrsdelikten registriert sind (Cluster verschiedene Straftaten).

Zusammenfassend ist zur Spezialisierung bei justiziellen Registrierungen der Männer anzumerken, dass Spezialisierung mit dem Alter zunimmt, unter der Voraussetzung, dass kein Abbruch der kriminellen Karriere stattfand. Wobei Spezialisierung hier bedeutet, dass es von einer Altersgruppe zur nächsten keine Veränderung der Deliktskonfiguration gibt.

Untersuchungen zu spezialisierten Tätern, deliktswechselnden Tätern und Abbrechern offenbarten eine große Gruppe Abbrecher und ergaben einzelne Hinweise auf Spezialisierung und auf eine kleine Gruppe chronischer Straftäter. Ausgehend von der Altersgruppe der 19- bis 23-Jährigen ist sowohl in der Altersgruppe davor wie in den nachfolgenden Altersgruppen der Anteil der Nichtregistrierten am größten. Die Ausnahme bilden hier die 19- bis 23-Jährigen des Clusters ,verschiedene Straftaten“, was ein weiterer Hinweis darauf ist, dass es sich hier um chronische Straftäter handelt.

\subsubsection{Clusteranalyse justizieller Registrierungen deutscher Frauen}

Wie schon im Abschnitt der deutschen Männer beschrieben, wird für die Clusteranalyse der Frauen zuerst die Anzahl der Cluster bestimmt, d.h. es wird das optimale Verhältnis von einerseits möglichst allgemeinen und andererseits ausreichend differenzierten Deliktskonfigurationen ermittelt. Zur Bestimmung der optimalen

\footnotetext{
257 Vgl. Kaiser 1997, S. 179 f.

258 Vgl. Schöch 1993, S. 577.

259 Vgl. Schöch 1993, S. 580.
} 
Clusterzahl werden die Modellprüfgrößen aus Tabelle 44 herangezogen. ${ }^{260}$ Der BIC-Wert ist minimal bei 6 Clustern und auch weitere Kriterien zur Bestimmung der Anzahl der Cluster treffen bei 6 Clustern zu. Die Daumenregel, dass L ${ }^{2}$ kleiner als die Anzahl der Freiheitsgrade ist, ist erfüllt, wie bei den Männern trifft dieses Kriterium auch schon bei 5 Clustern zu. Der p-Wert ist 1 , auch dies ist schon bei 5 Clustern der Fall. Da der BIC-Wert bei 6 Clustern minimal ist, wird die 6Clusterlösung verwendet.

Tabelle 44: Modellprüfgrößen zur Bestimmung der Clusteranzahl (deutsche Frauen, Kohorte 1970, justizielle Registrierungen) ${ }^{261}$

\begin{tabular}{|c|c|c|c|c|c|c|c|}
\hline $\begin{array}{l}\text { Cluster- } \\
\text { zahl }\end{array}$ & LL & BIC(LL) & Npar & $\mathrm{L}^{2}$ & $\mathrm{df}$ & $\mathrm{p}$-Wert & Class.Err. \\
\hline 2 & -17140 & 34716 & 53 & 5340 & 3626 & $7,50 \mathrm{E}-70$ & 0,061 \\
\hline 3 & -16625 & 33906 & 80 & 4309 & 3599 & $1,60 \mathrm{E}-15$ & 0,063 \\
\hline 4 & -16322 & 33523 & 107 & 3704 & 3572 & 0,061 & 0,057 \\
\hline 5 & -16076 & 33253 & 134 & 3212 & 3545 & 1 & 0,054 \\
\hline 6 & -15862 & 33046 & 161 & 2784 & 3518 & 1 & 0,071 \\
\hline 7 & -15802 & 33148 & 188 & 2664 & 3491 & 1 & 0,094 \\
\hline $\begin{array}{l}\text { LL } \\
\text { BIC } \\
\text { Npar } \\
\text { L }^{2} \\
\text { df } \\
\text { p-Wert } \\
\text { Class.Err. }\end{array}$ & $\begin{array}{l}\text { Wert der Log } \\
\text { Bayesian Info } \\
\text { Anzahl der P } \\
\text { Likelihood ra } \\
\text { Freiheitsgrad } \\
\text { Signifikanzn } \\
\text { Classification }\end{array}$ & $\begin{array}{l}\text { ikelihood-Fu } \\
\text { nation Criter } \\
\text { ameter } \\
0 \text { chi-squared } \\
\text { eau } \\
\text { Errors }\end{array}$ & $\begin{array}{l}\text { aktion } \\
\text { on siehe Fy } \\
\text { statistic }\end{array}$ & ote 235 & & & \\
\hline
\end{tabular}

Welche Delikte mit welchen Wahrscheinlichkeiten in den 6 Clustern dargestellt sind, zeigt Tabelle 45 für die justiziellen Registrierungen deutscher Frauen der Kohorte 1970. Insgesamt werden 3.679 Altersabschnitte, die zu 2.089 justiziell registrierten Frauen gehören, 6 Clustern zugeordnet. Wie schon bei den Männern beschrieben, ist auch jede Frau entweder mindestens zweimal registriert oder innerhalb einer Entscheidung sind mindestens zwei verschiedene Delikte registriert.

Das größte Cluster ist Cluster A mit 27,7\% aller Altersabschnitte. Alle Altersabschnitte dieses Clusters enthalten einfachen Diebstahl, andere Delikte sind sehr selten. So ist neben einfachem Diebstahl nur bei $40 \%$ der Altersabschnitte ein weiteres Delikt registriert, wobei es sich dabei meist um Leistungserschleichung, ein Betäubungsmitteldelikt oder ein Verkehrsdelikt handelt.

\footnotetext{
${ }^{260}$ Die Modellprüfgrößen sind im Abschnitt 6.1.1 Clusteranalyse justizieller Registrierungen deutscher Männer beschrieben.

261 Die Werte der Tabelle sind mit Latent GOLD berechnet.
} 
Tabelle 45: Deliktscluster justizieller Registrierungen deutscher Frauen der Kohorte 1970: Wahrscheinlichkeiten der Deliktskategorien in den Clustern (in \%) 262

\begin{tabular}{|c|c|c|c|c|c|c|c|}
\hline \multirow[b]{3}{*}{$\begin{array}{l}\text { Clusterwahrscheinlichkeiten } \pi(\mathrm{k}) \\
\text { in } \%\end{array}$} & \multicolumn{7}{|c|}{ Cluster } \\
\hline & A & B & $\mathrm{C}$ & $\mathrm{D}$ & E & $\mathrm{F}$ & ges. \\
\hline & 27,7 & 21,7 & 20,1 & 19,1 & 6,0 & 5,4 & 100 \\
\hline \multicolumn{8}{|l|}{ Wahrscheinlichkeiten $p_{k j}$ in $\%$} \\
\hline Tötungsdelikte & 0,0 & 0,2 & 0,4 & 0,1 & 0,6 & 0,0 & 0,2 \\
\hline sonstige Sexualdelikte & 0,3 & 0,6 & 3,3 & 0,4 & 2,3 & 0,0 & 1,1 \\
\hline Raub & 0,5 & 0,3 & 2,2 & 0,0 & 11,3 & 0,0 & 1,3 \\
\hline Schwere Körperverletzung & 0,1 & 0,0 & 0,3 & 0,1 & 46,0 & 1,6 & 2,9 \\
\hline Körperverletzung & 0,5 & 0,8 & 0,0 & 0,9 & 82,2 & 55,5 & 8,4 \\
\hline Str. gg. persönliche Freiheit & 0,5 & 0,7 & 2,3 & 0,4 & 11,4 & 0,0 & 1,5 \\
\hline Einfacher Diebstahl & 100,0 & 40,8 & 21,5 & 2,1 & 39,0 & 2,9 & 43,8 \\
\hline Schwerer Diebstahl & 2,0 & 3,2 & 8,1 & 0,4 & 3,8 & 0,5 & 3,2 \\
\hline Sachbeschädigung & 2,0 & 0,4 & 5,7 & 0,5 & 13,3 & 0,0 & 2,7 \\
\hline Gemeingefährliche Straftaten & 0,2 & 0,1 & 0,5 & $\mathbf{0 , 4}$ & 0,0 & 0,0 & 0,2 \\
\hline Hehlerei & 2,6 & 1,9 & 7,9 & 1,8 & 4,2 & 0,4 & 3,3 \\
\hline Betrug & 0,1 & 99,8 & 0,5 & 2,1 & 12,7 & 1,6 & 23,0 \\
\hline Erschleichen von Leistungen & 7,2 & 7,8 & 27,8 & 1,3 & 14,7 & 1,0 & 10,5 \\
\hline Gefährdung dem. Rechtsstaat & 0,0 & 0,0 & 0,8 & 0,0 & 0,0 & 0,0 & 0,2 \\
\hline Widerstand gg. Staatsgewalt & 0,0 & 0,0 & 1,2 & 0,0 & 16,6 & 0,0 & 1,3 \\
\hline Öffentliche Ordnung & 2,0 & 1,6 & 5,3 & 2,1 & 1,5 & 0,0 & 2,5 \\
\hline Hausfriedensbruch & 1,2 & 0,8 & 3,4 & 0,2 & 5,8 & 0,5 & 1,6 \\
\hline Beleidigung & 1,2 & 1,7 & 7,4 & 0,5 & 32,5 & 0,5 & 4,3 \\
\hline Fälschung & 1,4 & 25,8 & 5,7 & 1,5 & 6,8 & 0,5 & 7,9 \\
\hline Meineid & 0,2 & 2,8 & 4,8 & 0,9 & 1,0 & 0,0 & 1,9 \\
\hline falsche Verdächtigung & 1,0 & 0,5 & 3,5 & 1,2 & 1,6 & 0,0 & 1,4 \\
\hline BTM-Delikte & 6,2 & 5,8 & 33,6 & 3,5 & 11,2 & 1,6 & 11,1 \\
\hline Vollrausch & 0,1 & 0,0 & 0,9 & 0,0 & 1,0 & 0,0 & 0,3 \\
\hline Fahrlässige Tötung & 0,0 & 0,0 & 0,2 & 0,9 & 0,0 & 3,2 & 0,4 \\
\hline fahrlässige Körperverletzung & 0,9 & 0,8 & 0,1 & 15,1 & 5,2 & 99,7 & 9,0 \\
\hline Verkehrsdelikte & 8,3 & 14,8 & 8,9 & 100,0 & 13,7 & 21,7 & 28,4 \\
\hline $\begin{array}{l}\text { Durchschnittliche Anzahl } \\
\text { der Delikte }\end{array}$ & 1,4 & 2,1 & 1,6 & 1,4 & 3,5 & 1,9 & 1,7 \\
\hline
\end{tabular}

262 Die Prozentanteile, die mindestens 1,5-mal so groß sind wie der durchschnittliche Anteil (ganz rechte Spalte), sind in der Tabelle fett gekennzeichnet. 
Cluster B ist mit 21,7\% aller Altersabschnitte das zweitgrößte Cluster. Fast alle Altersabschnitte dieses Clusters enthalten Betrug und jeder vierte Altersabschnitt enthält zusätzlich ein Fälschungsdelikt. Damit ist „Fälschung“ in diesem Cluster mehr als dreimal häufiger vertreten als im Durchschnitt. 41\% der Altersabschnitte dieses Clusters enthalten zusätzlich einfachen Diebstahl, dies entspricht etwa der durchschnittlichen Verteilung von einfachem Diebstahl.

Cluster C enthält 20,1\% aller Altersabschnitte. In diesem Cluster sind mehrere Deliktskategorien überdurchschnittlich häufig vertreten. Die größte Kategorie sind die Betäubungsmitteldelikte. Jeder dritte Altersabschnitt dieses Clusters enthält ein Betäubungsmitteldelikt. 27,8\% der Alterabschnitte enthalten Leistungserschleichung. "Sonstige Sexualdelikte" (verbotene Prostitution) sind in diesem Cluster mit $3,3 \%$ dreimal häufiger als im Durchschnitt vertreten. Überproportional häufig sind auch die Deliktskategorien schwerer Diebstahl, Hehlerei, Straftaten gegen die öffentliche Ordnung und Vollrausch vorhanden. Es lässt sich vermuten, dass es sich bei diesem Cluster um Drogendelikte und Beschaffungskriminalität handelt.

Cluster D ist typischerweise durch Verkehrsdelikte geprägt. Alle Altersabschnitte dieses Clusters enthalten ein Verkehrsdelikt, außerdem sind die Delikte fahrlässige Körperverletzung und Tötung überdurchschnittlich häufig vertreten, während alle anderen Delikte kaum vertreten sind. 19,1\% aller Altersabschnitte befinden sich in diesem Cluster.

Cluster E, mit 6\% aller Altersabschnitte, kann als das Cluster der Gewaltdelikte bezeichnet werden. Hier finden sich neben den "normalen" Gewaltdelikten auch Widerstand gegen die Staatsgewalt, Sachbeschädigung, Hausfriedensbruch, Vollrausch und Beleidigung überdurchschnittlich häufig. Die durchschnittliche Anzahl der Delikte pro Altersabschnitt $(3,5)$ ist in diesem Cluster mehr als doppelt hoch als im Durchschnitt. In diesem Cluster befinden sich die Frauen mit den meisten Registrierungen pro Altersabschnitt.

Cluster F, mit 5,4\% aller Altersabschnitte enthält hauptsächlich fahrlässige Körperverletzung, dazu auch fahrlässige Tötung, Verkehrsdelikte und Körperverletzung.

Tabelle 46 gibt die Wahrscheinlichkeiten an, mit denen ein Delikt zu einem Cluster gehört. Etwa 63\% aller Altersabschnitte mit einfachem Diebstahl befinden sich im Cluster A. Umgekehrt enthalten alle Altersabschnitte des Clusters A einfachen Diebstahl (Tabelle 45). Im Betrugscluster (B) befinden sich 94\% der Altersabschnitte mit Betrug und 71\% der Altersabschnitte mit „Fälschung“. Das Cluster $\mathrm{C}$ enthält über $60 \%$ der Altersabschnitte mit Betäubungsmitteldelikten und ebenso viele mit ,sonstigen Sexualdelikten“, fast 70\% der Altersabschnitte mit Vollrausch, alle Altersabschnitte mit Gefährdung des demokratischen Rechtsstaates und um die $50 \%$ der Altersabschnitte mit einfachem Diebstahl, Hehlerei, Leistungserschleichung, Meineid und falsche Verdächtigung. In Cluster D befinden sich 67\% aller Altersabschnitte mit Verkehrsdelikten, 47\% aller Altersabschnitte mit fahrlässiger Tötung und 32\% aller Altersabschnitte mit fahrlässiger Körperverletzung. Das Ge- 
waltcluster (E) enthält 94\% aller Altersabschnitte mit schwerer Körperverletzung und $46 \%$ aller Altersabschnitte mit Beleidigung. Im Cluster $\mathrm{F}$ befinden sich $60 \%$ der Altersabschnitte mit fahrlässiger Körperverletzung, 46\% der Altersabschnitte mit fahrlässiger Tötung, aber auch 36\% der Altersabschnitte mit vorsätzlicher Körperverletzung.

Tabelle 46: Wahrscheinlichkeit der Clusterzugehörigkeit bei Deliktsvorkommen in \% (Justizielle Registrierung, deutsche Frauen, Kohorte 1970, BadenWürttemberg) $)^{263}$

\begin{tabular}{|c|c|c|c|c|c|c|}
\hline \multirow[b]{3}{*}{$\begin{array}{l}\text { Clusterwahrscheinlichkeiten } \pi(\mathrm{k}) \\
\text { in } \%\end{array}$} & \multicolumn{6}{|c|}{ Cluster* } \\
\hline & A & $\mathrm{B}$ & $\mathrm{C}$ & $\mathrm{D}$ & E & $\mathrm{F}$ \\
\hline & 27,7 & 21,7 & 20,1 & 19,1 & 6,0 & 5,4 \\
\hline \multicolumn{7}{|l|}{ Wahrscheinlichkeiten $\Theta_{\mathrm{kj}}$ in \% } \\
\hline Tötungsdelikte & 0,0 & 27,2 & 42,3 & 13,2 & 17,3 & 0,0 \\
\hline Sonstige Sexualdelikte & 7,5 & 11,6 & 60,7 & 7,6 & 12,7 & 0,0 \\
\hline Raub & 9,7 & 4,3 & 34,2 & 0,0 & 51,8 & 0,0 \\
\hline Schwere Köperverletzung & 0,5 & 0,0 & 2,1 & 0,5 & 93,9 & 3,0 \\
\hline Körperverletzung & 1,6 & 2,1 & 0,0 & 2,1 & $\mathbf{5 8 , 7}$ & 35,5 \\
\hline Str. gg. persönliche Freiheit & 8,8 & 9,9 & 31,3 & 4,6 & 45,6 & 0,0 \\
\hline Einfacher Diebstahl & 63,3 & 20,2 & 9,9 & 0,9 & 5,3 & 0,4 \\
\hline Schwerer Diebstahl & 17,3 & 21,5 & 50,9 & 2,4 & 7,2 & 0,8 \\
\hline Sachbeschädigung & 20,8 & 3,6 & 42,8 & 3,4 & 29,5 & 0,0 \\
\hline Gemeingefährliche Str. & 19,6 & 11,0 & 36,7 & 32,7 & 0,0 & 0,0 \\
\hline Hehlerei & 21,5 & 12,2 & 47,8 & 10,5 & 7,4 & 0,7 \\
\hline Betrug & 0,1 & 94,2 & 0,4 & 1,7 & 3,3 & 0,4 \\
\hline Erschleichen von Leistungen & 19,0 & 16,2 & 53,5 & 2,4 & 8,4 & 0,5 \\
\hline Gefährdung dem. Rechtsstaat & 0,0 & 0,0 & 100,0 & 0,0 & 0,0 & 0,0 \\
\hline Widerstand gg. Staatsgewalt & 0,3 & 0,0 & 19,8 & 0,0 & 79,9 & 0,0 \\
\hline Öffentliche Ordnung & 22,7 & 14,1 & 43,2 & 16,4 & 3,6 & 0,0 \\
\hline Hausfriedensbruch & 20,9 & 10,6 & 42,3 & 2,6 & 21,9 & 1,8 \\
\hline Beleidigung & 7,9 & 8,6 & 35,1 & 2,1 & 45,7 & 0,7 \\
\hline Fälschung & 4,9 & 71,2 & 14,6 & 3,7 & 5,2 & 0,3 \\
\hline Meineid & 3,4 & 32,7 & 51,7 & 9,2 & 3,1 & 0,0 \\
\hline falsche Verdächtigung & 20,2 & 7,4 & 49,3 & 16,5 & 6,6 & 0,0 \\
\hline BTM-Delikte & 15,4 & 11,3 & 60,5 & 6,0 & 6,0 & 0,7 \\
\hline Vollrausch & 10,1 & 0,0 & 68,5 & 0,0 & 21,5 & 0,0 \\
\hline Fahrlässige Tötung & 0,0 & 0,0 & 7,7 & 46,6 & 0,0 & 45,8 \\
\hline Fahrlässige Körperverletzung & 2,8 & 2,0 & 0,2 & 32,0 & 3,5 & 59,6 \\
\hline Verkehrsdelikte & 8,1 & 11,3 & 6,3 & 67,3 & 2,9 & 4,1 \\
\hline
\end{tabular}

* Cluster: A einfacher Diebstahl, B Betrug, C Betäubungsmitteldelikte, D Verkehrsdelikte, E Gewaltdelikte, F fahrlässige Körperverletzung

263 Die Prozentanteile größer 30\% sind fett gedruckt. 
Bei den Frauen ist die eindeutige Zuordnung der Altersabschnitte zu Clustern nochmals deutlich besser als bei den Männern. Fast alle Altersabschnitte haben eine a posteriori Wahrscheinlichkeit größer 50\%, 88\% der Altersabschnitte haben eine a posteriori Wahrscheinlichkeit größer $80 \%$.

Abbildung 40: Verteilung der weiblichen Täter auf Cluster nach Altersgruppen

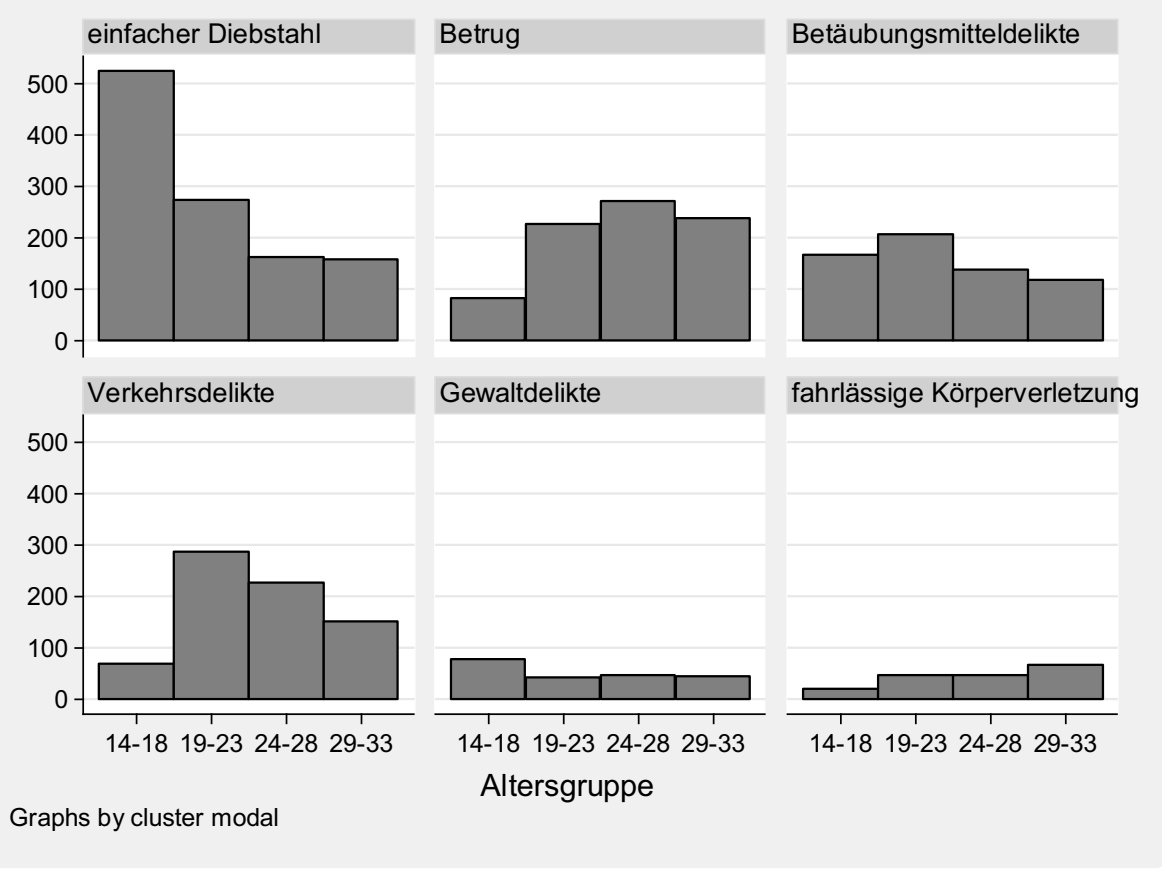

Die Altersverteilung der sechs Cluster ist in Abbildung 40 dargestellt. Das Cluster des einfachen Diebstahls (A) enthält mehrheitlich Altersabschnitte von 14 bis 18 Jahren. Im Cluster mit Betrug und „Fälschung“ (B) nimmt die Anzahl der Altersabschnitte bis zu den 24- bis 28-Jährigen mit dem Alter der Frauen zu und auch Altersabschnitte von 29- bis 33-Jährigen sind zahlreich vorhanden. Im Cluster der Betäubungsmitteldelikte (C) befinden sich hauptsächlich 14- bis 18-Jährige und 19- bis 23-Jährige, dann nimmt die Häufigkeit der Altersabschnitte mit dem Alter ab. Den Hauptanteil im Cluster der Verkehrsdelikte (D) bilden die 19- bis 23-Jährigen, danach nimmt der Anteil der Altersabschnitte mit dem Alter langsam ab. Im Cluster der Gewaltdelikte (E) ist das Alter relativ gleichmäßig verteilt mit einem Maximum bei den 14- bis 18-Jährigen. Im Cluster mit fahrlässiger Körperverletzung (F) ist der Anteil der Altersgruppe 14-18 am geringsten, 19- bis 23-Jährige und 24- bis 28-Jährige sind gleichstark vertreten und der Anteil der 29- bis 33-Jährigen ist am höchsten.

Die Clusterzusammensetzung, die dazugehörende Altersstruktur und die durchschnittliche Anzahl der Delikte pro Altersabschnitt beschreibt Tabelle 47. Alle Altersabschnitte der Frauen enthalten im Durchschnitt 1,7 Delikte. Besonders viele 
Delikte pro Altersabschnitt gibt es im Gewaltcluster mit einem Durchschnitt von 3,5 Delikten pro Abschnitt.

Tabelle 47: Deliktszusammensetzung der Cluster deutscher Frauen der Kohorte 1970

\begin{tabular}{ll}
\hline Cluster & Zusammensetzung \\
\hline A (27,7\%) & Einfacher Diebstahl \\
Einfacher Diebstahl & Alter hauptsächlich 14-18 \\
& Im Durchschnitt 1,4 Delikte pro Altersabschnitt. \\
\hline B (21,7\%) & Betrug, Fälschung \\
Betrug & Alter zunehmend bis 24-28 \\
& Im Durchschnitt 2,1 Delikte pro Altersabschnitt. \\
\hline & Betäubungsmitteldelikte, sonstige Sexualdelikte (verbotene \\
& Prostitution), Leistungserschleichung, Vollrausch, schwerer \\
& Diebstahl, Raub, Sachbeschädigung, Hausfriedensbruch, Heh- \\
Cerei, Meineid, falsche Verdächtigung, Gefährdung des demo- \\
Betäubungsmitteldelikte & kratischen Rechtsstaates und Straftaten gegen die öffentliche \\
& Ordnung \\
& Alter hauptsächlich 14-23, dann abnehmend. \\
& Im Durchschnitt 1,6 Delikte pro Altersabschnitt. \\
\hline D (19,1\%) & Verkehrsdelikte, fahrlässige Körperverletzung und Tötung \\
Verkehrsdelikte & Altersmaximum 19-23, dann abnehmend \\
& Im Durchschnitt 1,4 Delikte pro Altersabschnitt. \\
\hline & Gewaltdelikte (Tötungsdelikte, sonstige Sexualdelikte, Raub, \\
& schwere Körperverletzung, Körperverletzung, Straftaten gegen \\
die persönliche Freiheit), Sachbeschädigung, Widerstand gegen \\
die Staatsgewalt, Hausfriedensbruch, Beleidigung und Voll- \\
E (6,0\%) & rausch \\
Gewaltdelikte & Alter hauptsächlich 14-18, von 19-33 konstant \\
& Im Durchschnitt 3,5 Delikte pro Altersabschnitt. \\
Fahrlässige Körperver- & Fahrlässige Körperverletzung, fahrlässige Tötung und Körper- \\
letzung & verletzung \\
& Alter hauptsächlich 19-33, Maximum bei 29-33, kaum 14-18 \\
& Im Durchschnitt 1,9 Delikte pro Altersabschnitt. \\
\hline
\end{tabular}

Vergleicht man bei den Frauen die Clusteranalyse der englischen Kohorte 1953 (siehe Anhang Seite 257 f.) mit der Clusteranalyse aus Baden-Württemberg der Kohorte 1970, so ist festzustellen, dass die Anzahl der Täter in etwa übereinstimmt, die Anzahl der Altersabschnitte bei den deutschen Frauen aber größer ist. Bei der englischen Kohorte ist die Altersspanne größer, es gibt davor und danach jeweils eine Altersgruppe, die bei der deutschen Kohorte nicht vorhanden ist. Wie schon im Abschnitt der Männer erwähnt, hängt der Unterschied in der Anzahl der Altersabschnitte damit zusammen, dass bei der Clusteranalyse der Frauen aus BadenWürttemberg nur Frauen mit mindestens zwei Straftaten in die Untersuchung einbezogen sind. Dieses Kriterium wird bei der Analyse der englischen Daten nicht 
benutzt. Deshalb gibt es bei der Analyse hier, im Vergleich zur englischen, mehr Altersabschnitte pro Person. Auch innerhalb der Altersabschnitte ergibt sich dadurch ein Unterschied. Die Altersabschnitte der englischen Kohorte enthalten 1,2 Delikte pro Abschnitt, die Altersabschnitte der deutschen Kohorte 1,7. Da es hier um die Untersuchung von Deliktsmustern geht, sind Einfachtäter in diesem $\mathrm{Zu}$ sammenhang nicht relevant.

Die Anzahl der Deliktskategorien stimmt bei den justiziellen Registrierungen der Frauen in England und Deutschland in etwa überein, es sind 29 bei den englischen Daten und 26 bei den deutschen Daten. Wie schon bei den Männern beschrieben, sind die Diebstahldelikte in der englischen Analyse deutlich feiner aufgeteilt. Es gibt sehr viele verschiedene Deliktskategorien unterschiedlicher Diebstahldelikte. Bei der Clusteranalyse der englischen Kohorte ergibt die optimale Lösung drei Cluster $^{264}$, die eine sehr unterschiedliche Größe haben. Das Cluster der verschiedenen Straftaten enthält knapp 60\% aller Altersabschnitte. Das Cluster des Ladendiebstahls etwas mehr als ein Drittel der Altersabschnitte und dazu gibt es noch ein sehr kleines Cluster (Untreue). Die drei Cluster der englischen Kohorte haben alle ihr Maximum im Alter von 16 bis 20 Jahren und mit dem Alter nimmt die Häufigkeit der Altersabschnitte in den Clustern ab. Die Altersverteilung der Clusteranalyse der deutschen Kohorte ist wie oben beschrieben nicht identisch bei den sechs Clustern. Die Clusteranalyse der englischen Kohorte lässt sich schlecht mit dem Ergebnis der deutschen Kohorte vergleichen, da bei der englischen Untersuchung nur drei Cluster dargestellt sind. Dennoch zeigt der Vergleich zumindest die Bedeutung der Cluster Betrug (darin enthalten ist Untreue) und einfacher Diebstahl. Diese Cluster ergeben sich sowohl bei der Analyse der deutschen wie der englischen Kohorte. Die Cluster einfacher Diebstahl und Betrug sind die größten Cluster der Altersabschnitte der deutschen Frauen. Das Cluster einfacher Diebstahl umfasst $28 \%$ der Altersabschnitte, das Cluster Betrug 22\%.

Werden die Altersabschnitte der Frauen der deutschen Kohorte in fünf Cluster zusammengefasst, wird aus dem Cluster des einfachen Diebstahls und dem Cluster der Betäubungsmitteldelikte ein Cluster. Bei vier Clustern wird aus dem Cluster Verkehrsdelikte und fahrlässige Körperverletzung ein Cluster. Damit ist die Clusterstruktur abgesehen vom Verkehrscluster sehr ähnlich dem Ergebnis der englischen Kohorte.

264 Die Clusteranalyse wurde hier mit dem Programm Latent Gold berechnet. Das Kriterium für die optimale Clusteranzahl ist dabei der minimale BIC Wert. 
Das Ergebnis der Clusteranalyse bestätigt das Ergebnis der Multidimensionalen Skalierung in Bezug auf die Ähnlichkeit von den Delikten:

- $\quad$ Betrug - Fälschung

- Betäubungsmitteldelikte - schwerer Diebstahl - falsche Verdächtigung - Hehlerei - Meineid - Straftaten gegen die öffentliche Ordnung - sonstige Sexualdelikte

- Gewaltdelikte.

Diese Delikte liegen in der zweidimensionalen Darstellung der Multidimensionalen Skalierung nah beieinander und befinden sich im Ergebnis der Clusteranalyse innerhalb eines Clusters. Dies weist auf die Ähnlichkeit dieser Deliktspaarungen und Deliktskategorien hin.

\subsubsection{Spezialisierung, Wechsel und Abbruch im Lebenslauf der Frauen}

Im Weiteren wird mittels der Ergebnisse der Clusteranalyse die „kriminelle Karriere" der justiziell registrierten Frauen untersucht. Als Erstes wird hierzu ein Ergebnis zum Grad der Spezialisierung bezüglich der Cluster dargestellt, danach folgen Betrachtungen zu den Clusterübergängen.

Die Spezialisierungsanteile bezogen auf die Clusterzugehörigkeit sind in Tabelle 48 dargestellt. Die prozentualen Anteile sind von einer Altersgruppe zur nächsten innerhalb desselben Cluster berechnet, wobei Abbrecher nicht berücksichtigt sind. Die Häufigkeiten (N) sind bei den Frauen jeweils klein. 652 Frauen sind im Alter von 14 bis 18 justiziell registriert und haben in späteren Jahren, nicht unbedingt im Alter von 19 bis 23, eine weitere Registrierung. 387 Frauen sind im Alter von 14 bis 18 im Cluster einfacher Diebstahl. Von diesen sind 19\% auch im Alter von 19 bis 23 im Cluster einfacher Diebstahl. Im Cluster der fahrlässigen Körperverletzung sind die Häufigkeiten sehr gering. Es gibt kaum Frauen, die wegen fahrlässiger Körperverletzung registriert sind und die in einer späteren Altersgruppe nochmals registriert wurden. Fast alle Frauen, die sich im Cluster fahrlässige Körperverletzung befinden, brechen ihre „kriminelle Karriere“ vor dem Erreichen der nächsten Altersgruppe wieder ab. Im Cluster Gewaltdelikte und fahrlässige Körperverletzung sind keine Aussagen über Spezialisierung möglich, da das N sehr klein ist und deshalb der Fehler der Spezialisierungsrate sehr hoch ist. Im Cluster der Gewaltdelikte ist der Fehler der Spezialisierungsrate zwischen 4 und 7\% und damit fast so hoch wie die Raten. Im Cluster fahrlässige Körperverletzung ist der Fehler der Spezialisierungsrate 9\% und damit wesentlich höher als die Raten selbst. Aber auch in den übrigen Clustern ist die Anzahl der Frauen, die in späteren Altersgruppen wieder registriert sind, sehr gering. Hier aber bleibt der Fehler innerhalb vertretbarer Grenzen und das Ergebnis ist damit statistisch signifikant. Bei den Clustern einfacher Diebstahl, Betrug, Betäubungsmitteldelikte und Verkehrsdelikte nimmt die Spezialisierungsrate mit dem Alter zu. Je älter, desto mehr Frauen gibt es prozentual, die in der nächsten Altersgruppe demselben Cluster zugeordnet sind. 
Tabelle 48: Spezialisierungsanteile in \%: Deutsche Frauen der Kohorte 1970 (justizielle Registrierungen) ${ }^{265}$

\begin{tabular}{|c|c|c|c|c|c|c|c|c|}
\hline \multirow{2}{*}{\multicolumn{2}{|c|}{ Altersgruppen }} & & \multicolumn{6}{|c|}{ Cluster } \\
\hline & & \multirow[b]{2}{*}{$\mathrm{N}$} & \multirow{2}{*}{$\begin{array}{c}\text { A } \\
\text { Einfacher } \\
\text { Diebstahl } \\
(\mathrm{N})\end{array}$} & \multirow{2}{*}{$\begin{array}{c}\text { B } \\
\text { Betrug } \\
(\mathrm{N})\end{array}$} & \multirow{2}{*}{$\begin{array}{c}\text { C } \\
\text { BTM- } \\
\text { Delikte } \\
(\mathrm{N})\end{array}$} & \multirow{2}{*}{$\begin{array}{c}\text { D } \\
\text { Verkehrs- } \\
\text { delikte } \\
\text { (N) }\end{array}$} & \multirow{2}{*}{$\begin{array}{c}\mathrm{E} \\
\text { Gewalt- } \\
\text { delikte } \\
(\mathrm{N})\end{array}$} & \multirow{2}{*}{$\begin{array}{c}\mathrm{F} \\
\text { Fahrlässige } \\
\text { Körperver- } \\
\text { letzung }(\mathrm{N})\end{array}$} \\
\hline Von & bis & & & & & & & \\
\hline $14-18$ & $19-23$ & 652 & $19(387)$ & $21(34)$ & $24(136)$ & $18(38)$ & $9 *(44)$ & $0 *(13)$ \\
\hline $19-23$ & $24-28$ & 573 & $24(161)$ & $40(124)$ & $25(131)$ & $30(115)$ & $8^{*}(25)$ & $18 *(17)$ \\
\hline $24-28$ & $29-33$ & 365 & $48(71)$ & $47(126)$ & $37(62)$ & $33(78)$ & $10 *(20)$ & $0^{*}(8)$ \\
\hline
\end{tabular}

* nicht signifikant

Die größte Gruppe bildet mit 387 die Gruppe der 14- bis 18-Jährigen aus Cluster A (einfacher Diebstahl). Die Gruppe ist deutlich kleiner als bei den Männern. Bei den Männern war die Häufigkeit dieser Gruppe über 2000. Die Spezialisierungsanteile bei den Frauen nehmen im Cluster A (Einfacher Diebstahl), im Cluster B (Betrug), im Cluster C (Betäubungsmitteldelikte) und im Cluster D (Verkehrsdelikte) mit dem Alter zu. 19\% der 14- bis 18-Jährigen aus Cluster A, die später eine Registrierung haben, sind im Alter von 19 bis 23 auch im Cluster A registriert. Bei den 24- bis 28-Jährigen, die im Cluster A sind, sind 48\% auch mit 29 bis 33 Jahren im Cluster des einfachen Diebstahls. Die Hauptaussage, die getroffen werden kann, ist, dass es sehr wenige Frauen mit justiziell registrierter krimineller Karriere gibt. Von diesen befinden sich aber relativ viele in der nächsten Altersgruppe im selben Cluster und die Spezialisierungsrate nimmt mit dem Alter zu.

Abbildung 41: Deliktsübergänge weiblicher Täter der Altersgruppe 19-23

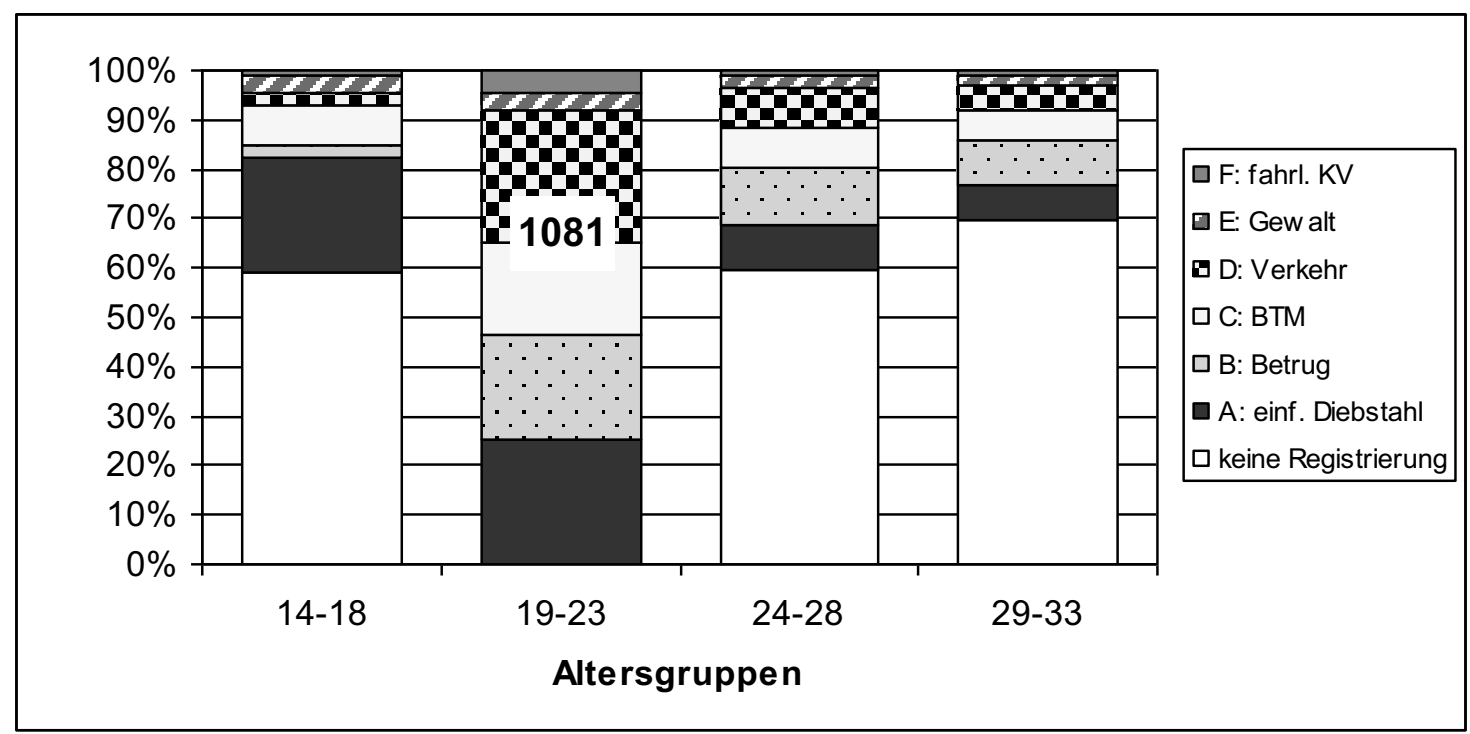

265 Siehe Fußnote 254. 
Abbildung 41 gibt einen Überblick über die Deliktsübergänge der Altersgruppe 19 bis 23. 1081 weibliche Täter haben mindestens eine Registrierung in der Altersspanne 19 bis 23. Diese teilen sich auf in 25\%, die zum Cluster einfacher Diebstahl gehören, 27\% gehören zum Cluster Verkehrsdelikte, 4\% Cluster Gewaltdelikte, 21\% Cluster Betrug, 19\% Betäubungsmitteldelikte und 4\% Cluster fahrlässige Körperverletzung. Von den weiblichen Tätern, die im Alter von 19 bis 23 mindestens eine Registrierung haben, haben 59\% im Alter von 14 bis 18 und im Alter von 24 bis 28 keine Registrierung, 70\% haben im Alter von 29 bis 33 keine Registrierung. 23\% der 19- bis 23-Jährigen mit Registrierung gehören im Alter von 14 bis 18 zum Cluster einfacher Diebstahl.

Die folgenden Abbildungen 42 bis 47 geben ein Bild über die kriminellen Laufbahnen der justiziell registrierten Frauen und geben also an, was Täter einer Altersgruppe und eines Clusters in der vorhergehenden Altersgruppe und in der folgenden Altersgruppe für eine Clusterzugehörigkeit haben bzw. ob sie vorher oder nachher überhaupt eine Registrierung haben. Die Abbildungen zeigen die Entwicklung der 19- bis 23-Jährigen der sechs Cluster. In der Altersgruppe 19 bis 23 ist jeweils die Zahl der Täter angegeben, die in dieser Altersgruppe zu diesem Cluster gehören. Der linke Balken gibt die prozentualen Anteile der Cluster bei den 14- bis 18-Jährigen an, unter der Bedingung einer bestimmten Clusterzugehörigkeit im Alter von 19 bis 23 Jahren, ebenso die beiden rechten Balken für die Altersgruppen 24 bis 28 und 29 bis 33 .

Die Mehrheit, sowohl in der vorhergehenden wie in den folgenden Altersgruppen, bildet bei allen Clustern die Gruppe „keine Registrierung“. Beim Cluster des einfachen Diebstahls (Abbildung 42) haben 56\% in der Altersgruppe 14 bis 18, $59 \%$ in der Altersgruppe 24 bis 28 und 63\% in der Altersgruppe 29 bis 33 keine Registrierung. 28\% der Frauen, die sich in der Altersgruppe 19 bis 23 im Cluster des einfachen Diebstahls befinden, sind auch im Alter von 14 bis 18 im Cluster des einfachen Diebstahls. Dieser Anteil liegt über dem durchschnittlichen Anteil von 23\%. 14\% sind auch in der nächsten Altersgruppe und 8\% im Alter von 29 bis 33 im Cluster des einfachen Diebstahls, auch diese Anteile liegen über dem Durchschnitt von $9 \%$ bzw. $7 \%$.

Beim Cluster Betrug (Abbildung 43) ist der Anteil der Nichtregistrierten in der Altersgruppe 14 bis 18 mit 64\% sehr hoch. Dies zeigt, dass Betrug ein Erwachsenendelikt ist. $22 \%$ der Frauen, die mit 19 bis 23 im Cluster Betrug sind, sind auch mit 24 bis 28 im Cluster Betrug und 15\% auch mit 29 bis 33. Diese Anteile liegen deutlich über dem durchschnittlichen Anteil von 12\% bzw. 9\%. Damit ist die Spezialisierung bei Betrug relativ hoch.

16\% der Frauen und damit deutlich mehr als im Durchschnitt (8\%), die im Alter von 19 bis 23 zum Cluster Betäubungsmitteldelikte (Abbildung 44) gehören, befinden sich auch schon im Alter von 14 bis 18 im Betäubungsmittelcluster. 20\% sind mit 14 bis 18 im Cluster des einfachen Diebstahls und 51\% sind nicht registriert. In 
der Altersgruppe danach (24-28) ist die Zahl der Nichtregistrierten im Vergleich zu den anderen Clustern am kleinsten (46\%). Der Anteil des Betäubungsmittelclusters liegt mit 15,5\% deutlich über dem durchschnittlichen Anteil von 8\%. In der Altersgruppe 29 bis 33 gibt es 69\% Nichtregistrierte, was dem Durchschnitt entspricht, aber mit $9 \%$ auch überdurchschnittlich viele, die zum Betäubungsmittelcluster gehören.

Beim Cluster Verkehrsdelikte (Abbildung 45) ist der Anteil der Nichtregistrierten in der vorhergehenden Altersgruppe mit 68\% am höchsten von allen Clustern. Auch in der Altersgruppe 24 bis 28 (71\%) und 29 bis 33 (79\%) ist der Anteil der Nichtregistrierten beim Cluster Verkehrsdelikte sehr hoch. Der Anteil der Nichtregistrierten liegt somit beim Übergang des Clusters Verkehrsdelikte deutlich über dem Durchschnitt (siehe Abbildung 41). Der hohe Anteil der Nichtregistrierten bei den Altersgruppen vor und nach Verkehrsdelikten deutet darauf hin, dass es auch bei Frauen keinen Zusammenhang zwischen ,normalen“ Straftaten, klassischen oder konventionellen Rechtsbrüchen, und Verkehrsdelikten gibt. ${ }^{266}$ Die meisten Verkehrsdelikte werden fahrlässig und nicht vorsätzlich begangen. ${ }^{267}$

In den späteren Altersgruppen ist auch beim Cluster fahrlässige Körperverletzung (Abbildung 47) der Anteil Nichtregistrierte sehr hoch, in der Altersgruppe 24 bis $2872 \%$ und in der Altersgruppe 29 bis 33 85\% und somit auch hier deutlich über dem Durchschnitt. In der Altersgruppe 14 bis 18 gibt es beim Cluster fahrlässige Körperverletzung nicht überdurchschnittlich viele Nichtregistrierte. Hier ist der Anteil Nichtregistrierte ebenso hoch wie im Cluster einfacher Diebstahl und im Cluster Betäubungsmitteldelikte (Abbildung 44). Dies weist darauf hin, dass eine fahrlässige Körperverletzung bei Frauen eher justiziell registriert wird, wenn es davor schon eine justizielle Registrierung gab.

266 Vgl. Kaiser 1997, S. 179 f.

267 Vgl. Schöch 1993, S. 577. 
Abbildung 42: Deliktsübergänge weiblicher Täter des Clusters A (einfacher Diebstahl) im Alter 19 bis 23

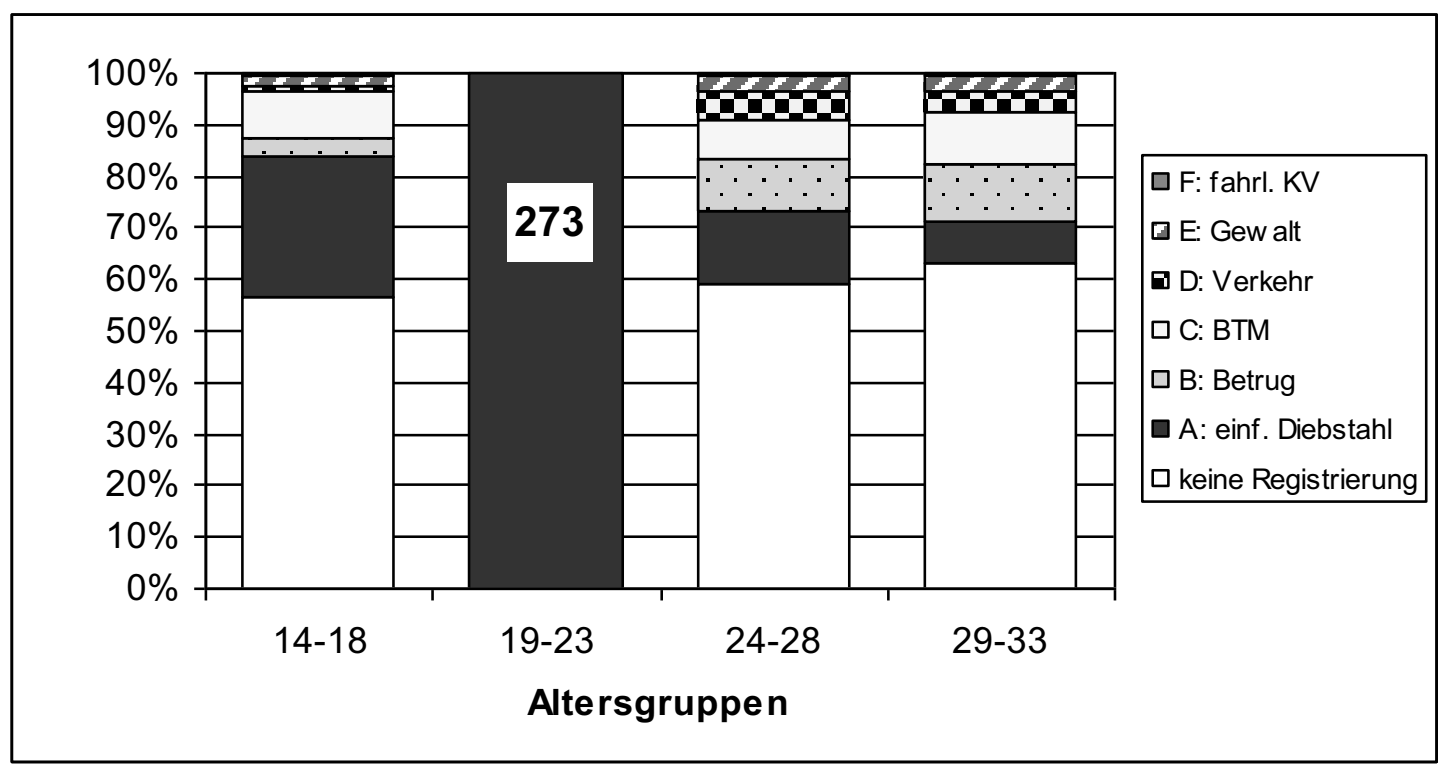

Abbildung 43: Deliktsübergänge weiblicher Täter des Clusters B (Betrug) im Alter 19 bis 23

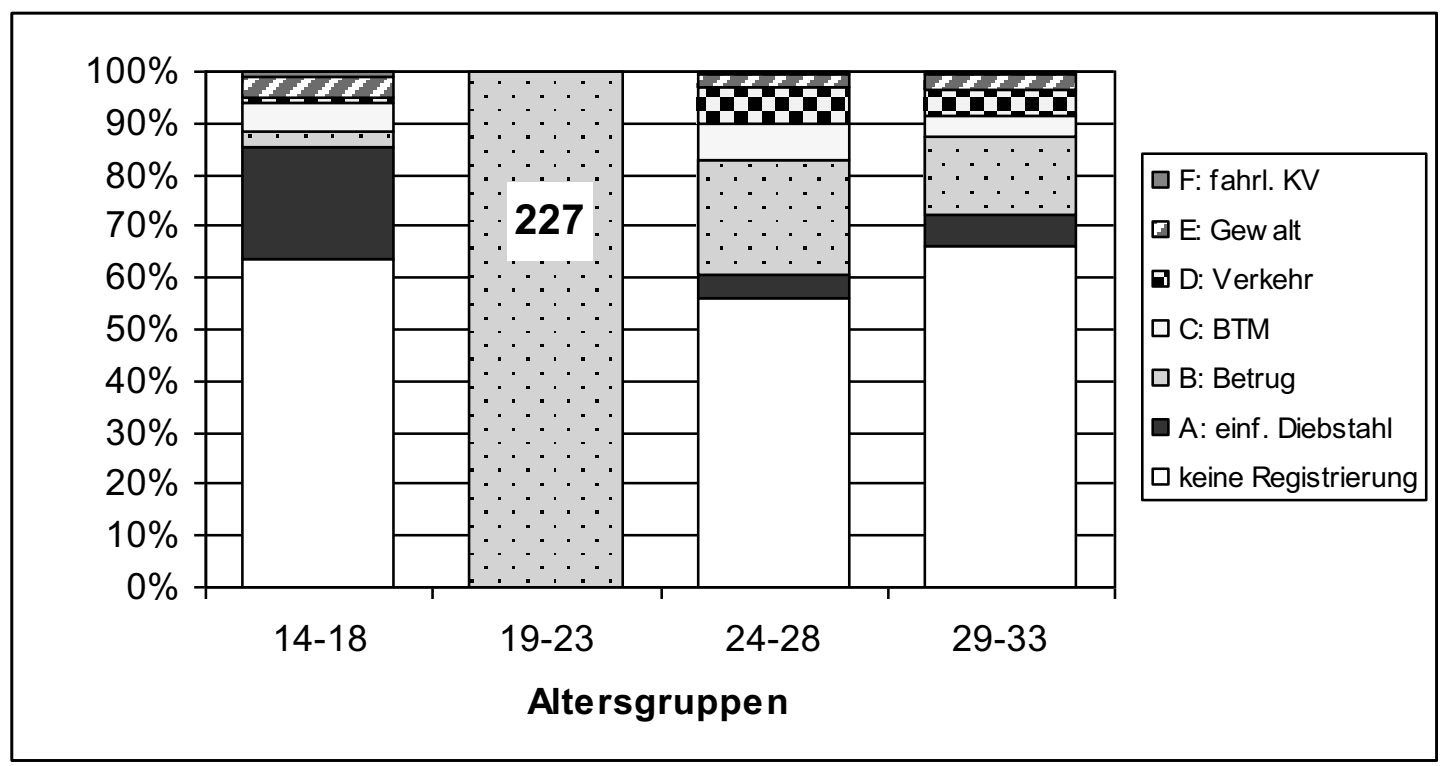


Abbildung 44: Deliktsübergänge weiblicher Täter des Clusters C (Betäubungsmitteldelikte) im Alter 19 bis 23

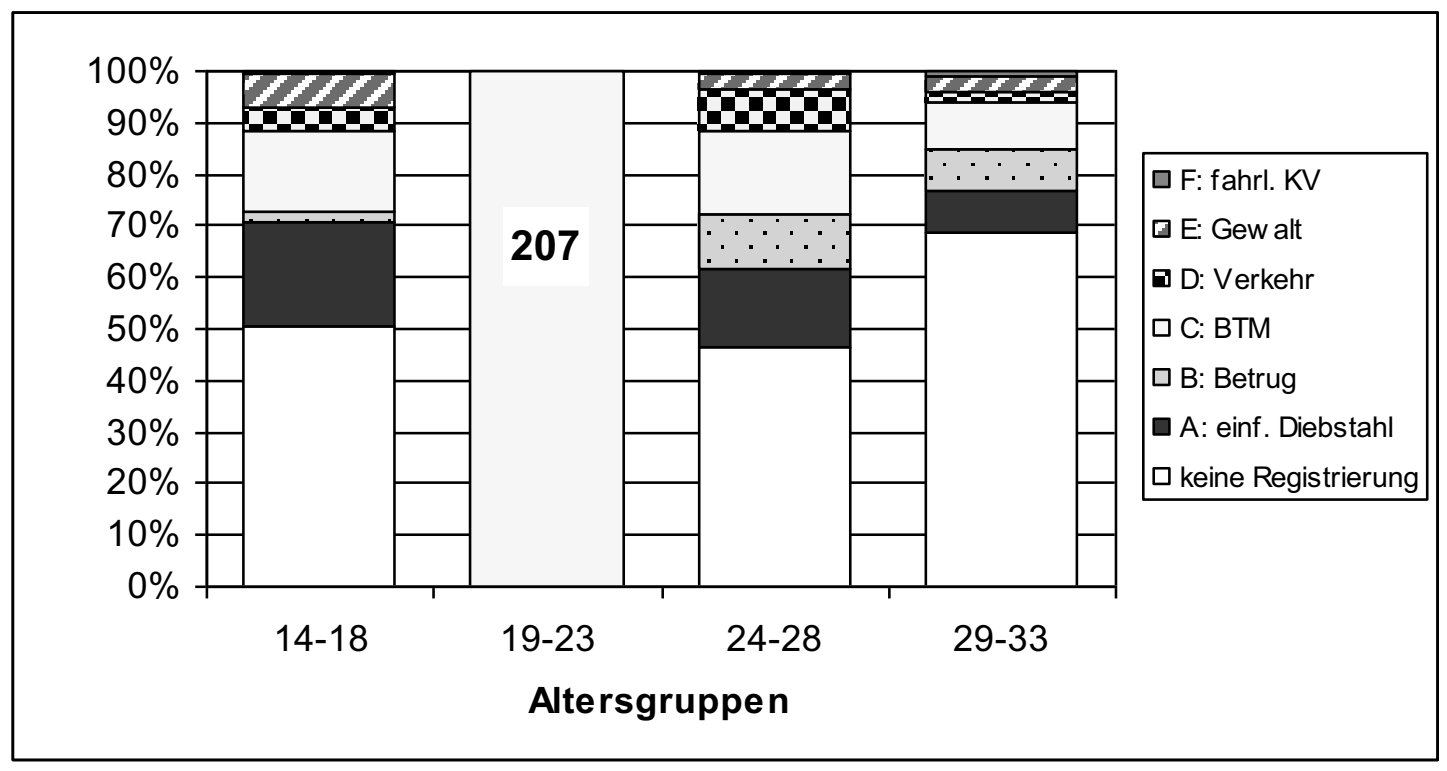

Abbildung 45: Deliktsübergänge weiblicher Täter des Clusters D (Verkehrsdelikte) im Alter 19 bis 23

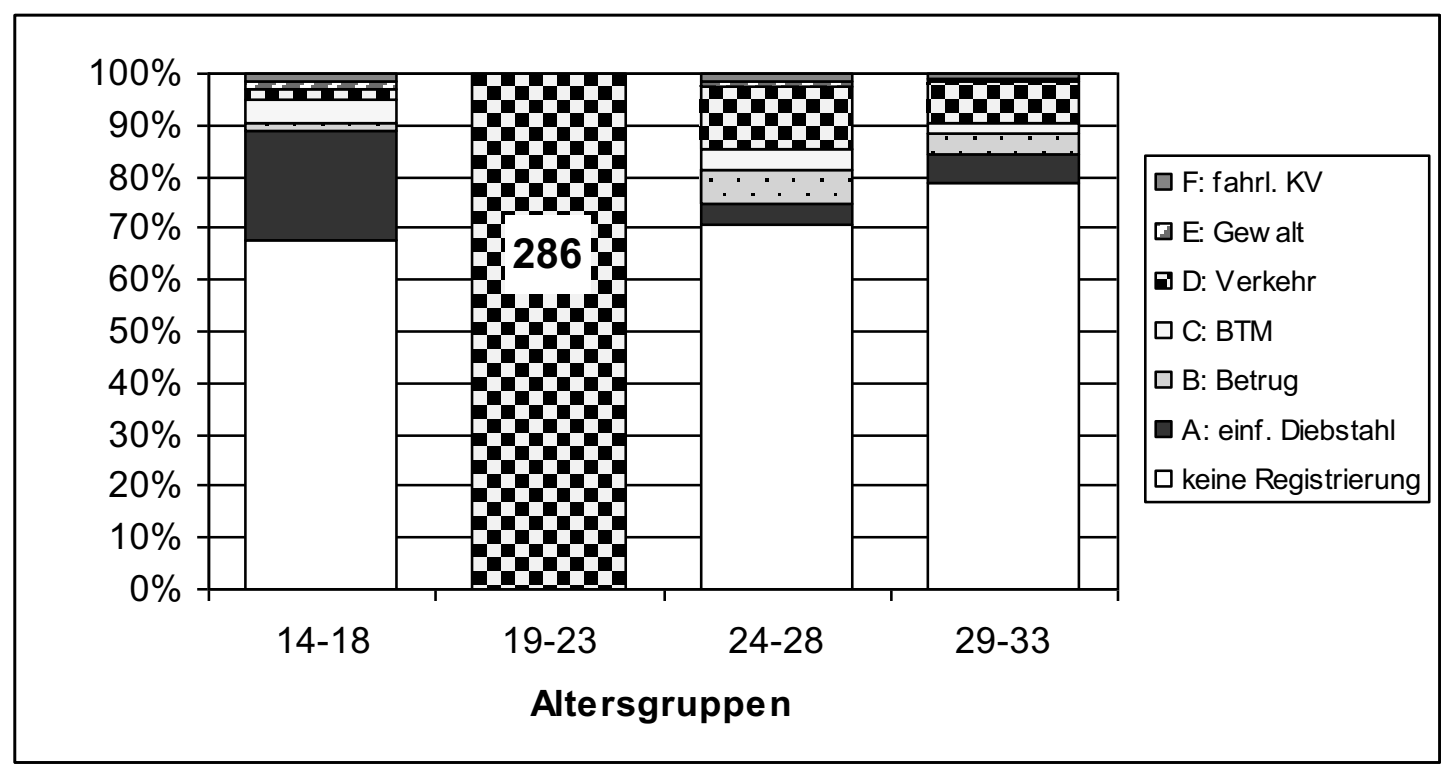


Abbildung 46: Deliktsübergänge weiblicher Täter des Clusters E (Gewaltdelikte) im Alter 19 bis 23

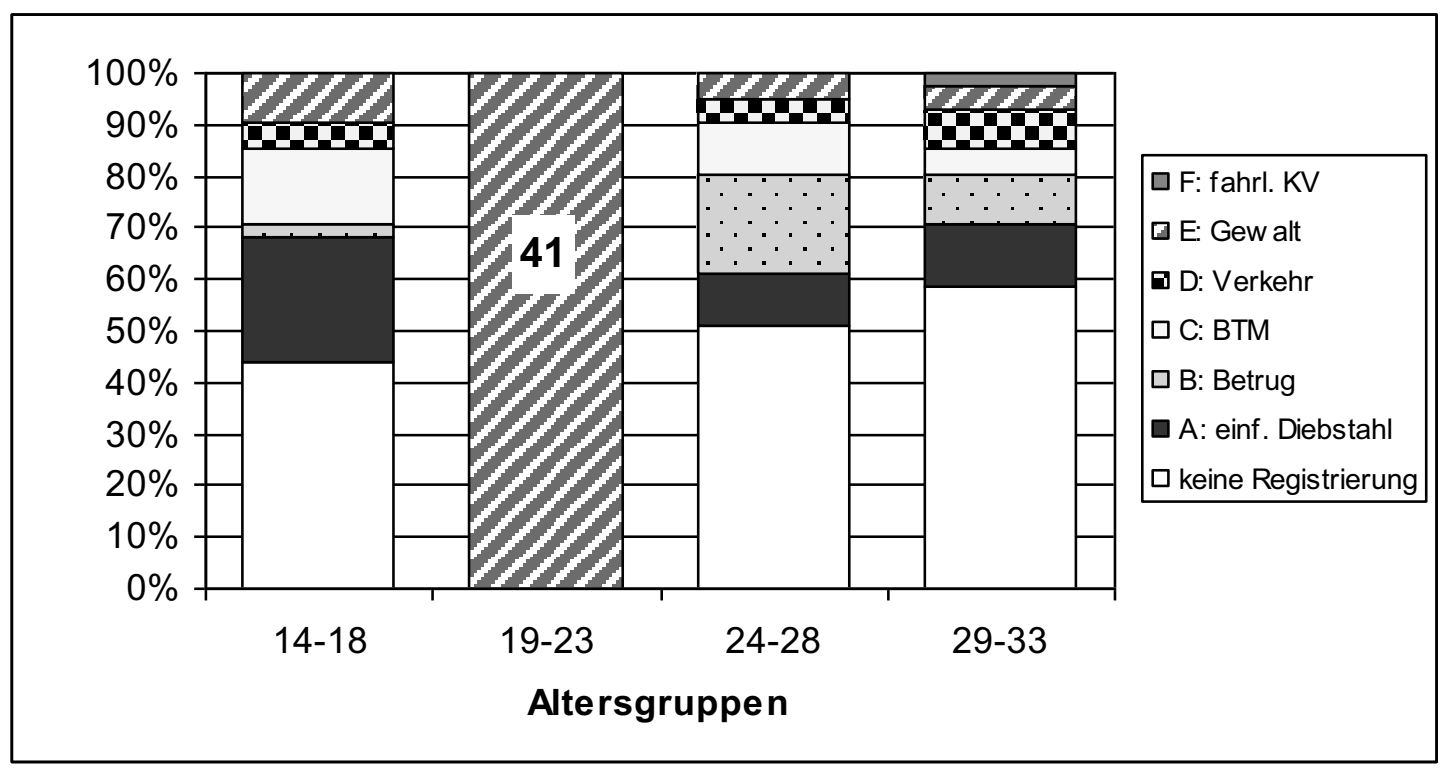

Abbildung 47: Deliktsübergänge weiblicher Täter des Clusters F (fahrlässige Körperverletzung) im Alter 19 bis 23

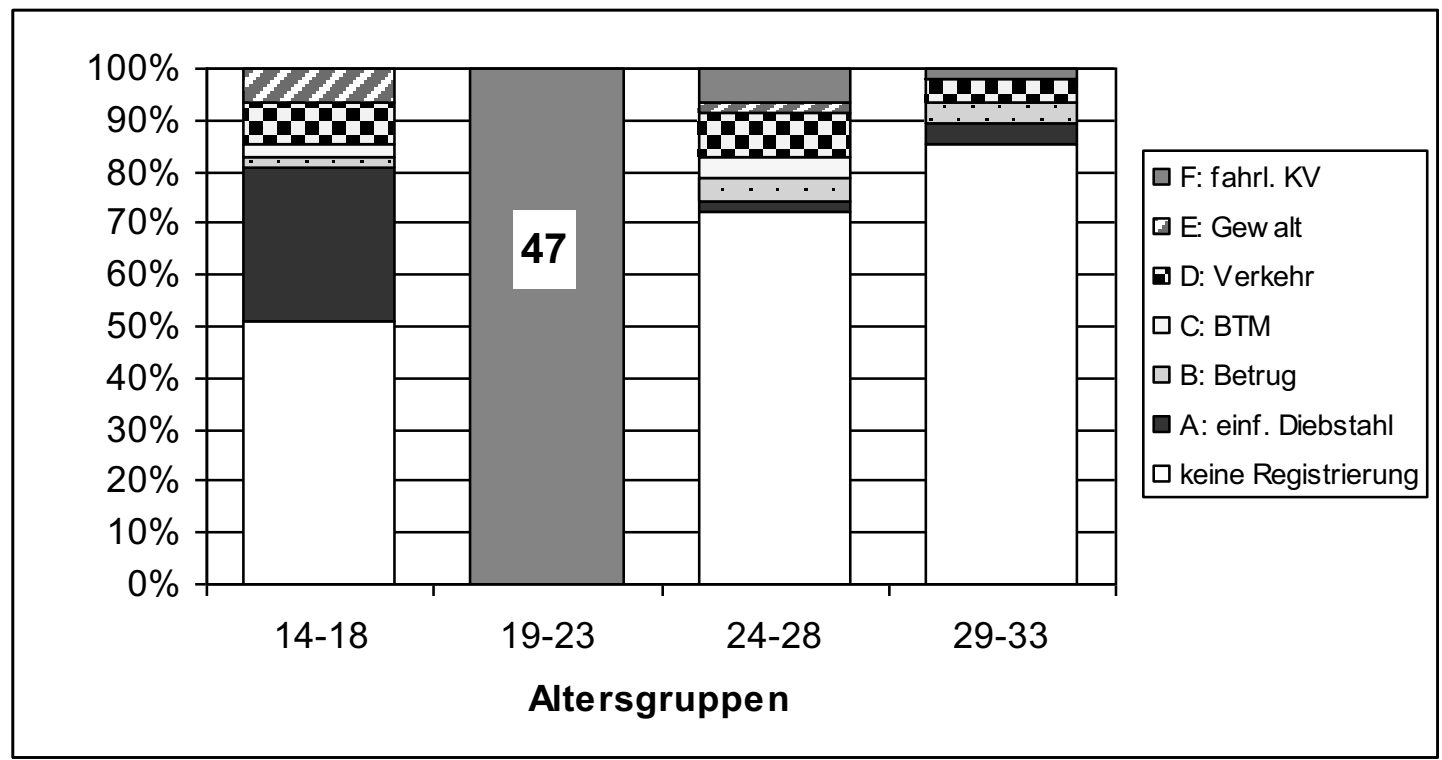

Das Cluster der Gewaltdelikte (Abbildung 46) ist bei den Frauen ein sehr kleines Cluster. 41 Frauen befinden sich im Alter von 19 bis 23 im Cluster der Gewaltdelikte. Von diesen Frauen ist der Anteil Nichtregistrierte in der Altersgruppe davor (14- bis 18-Jährige) relativ niedrig mit 44\%. 24\% sind im Alter von 14 bis 18 im Cluster einfacher Diebstahl, 15\% im Cluster der BTM-Delikte und 10\% im Cluster der Gewaltdelikte. Das Gewaltcluster ist in den Altersgruppen davor und danach 
überdurchschnittlich häufig vertreten. Wie schon erwähnt, beinhaltet bei den justiziell registrierten Frauen das Cluster der Gewaltdelikte die Mehrfachtäter.

Bei allen Clustern zeigen sich insofern Hinweise auf Spezialisierung, dass diese Cluster in den anderen Altersgruppen überdurchschnittlich häufig vertreten sind.

Ein Vergleich des Ergebnisses der Clusteranalyse der Männer mit dem der Frauen ergibt, dass vier der sechs Cluster bei Männern und Frauen übereinstimmen. Die Cluster Betrug, Verkehrsdelikte, fahrlässige Körperverletzung und Gewaltdelikte ergeben sich sowohl bei den Männern wie bei den Frauen. Die Altersverteilung stimmt bei den Clustern Betrug, Verkehrsdelikte und fahrlässige Körperverletzung bei Männern und Frauen überein. Im Gegensatz zu den Männern ist im Cluster Gewaltdelikte bei den Frauen schon im Alter von 14 bis 18 das Maximum erreicht, bei den Männern erst im Alter von 19 bis 23. Das Gewaltcluster umfasst bei den Männern 12,6\% aller Altersabschnitte, bei den Frauen nur 6\%. Im Gewaltcluster der Frauen sind die Altersabschnitte mit dem durchschnittlich größten Deliktsanteil enthalten. Die Zahl mehrfach justiziell registrierter Frauen ist sehr gering.

Bei den Männern ist mit 33\% das Cluster der Verkehrsdelikte sehr groß. Das Cluster der Verkehrsdelikte umfasst bei den Frauen nur 19\% der Altersabschnitte. Deutlich größer proportional als bei den Männern ist bei den Frauen das Cluster Betrug. Das Cluster Betrug enthält 22\% der Altersabschnitte der Frauen und 8\% der Abschnitte der Männer. Das Cluster fahrlässige Körperverletzung ist sowohl bei Männern wie bei den Frauen klein (5\%). In absoluten Zahlen sind natürlich alle Cluster der Männer deutlich größer als die der Frauen.

Das Cluster einfacher Diebstahl bzw. Diebstahl ist sowohl bei den Männern wie bei den Frauen bei der 6-Clusterlösung vorhanden und auch die Altersverteilung stimmt überein. Dennoch unterscheiden sich diese Cluster bei Männern und Frauen. Die Cluster bilden sowohl bei den Männern wie bei den Frauen jeweils das größte Cluster. Aber dieses Cluster umfasst bei den Männern mit 36\% noch einen deutlich größeren Anteil der Altersabschnitte als bei den Frauen (28\%). Bei den Frauen enthalten alle Altersabschnitte dieses Clusters das Delikt einfacher Diebstahl, andere Delikte sind nicht überdurchschnittlich häufig vertreten. Bei den Männern enthalten knapp 50\% der Altersabschnitte das Delikt einfacher Diebstahl und auch die Delikte schwerer Diebstahl, Leistungserschleichung, sonstige Sexualdelikte, Hehlerei und Betäubungsmitteldelikte sind überdurchschnittlich häufig registriert. Dies zeigt, dass das Cluster der Betäubungsmitteldelikte, welches nur bei den Frauen existiert, bei der 6-Clusterlösung der Männer zum Cluster des Diebstahls gehört. Wie im Abschnitt der Männer beschrieben, ergibt sich bei der 7Clusterlösung der Männer auch ein Cluster mit Betäubungsmitteldelikten. Zum Cluster Betäubungsmitteldelikte gehören auch Delikte, die zur Beschaffungskriminalität gezählt werden können, wie Leistungserschleichung, schwerer Diebstahl, Raub und verbotene Prostitution.

Das Cluster der verschiedenen Straftaten, welches bei den Männern die chronischen Straftäter umfasst, existiert bei den Frauen nicht. Mehrfachstraftäter sind bei 
den Frauen im Gewaltcluster enthalten, aber ein Cluster, in dem alle Delikte überdurchschnittlich häufig auftreten und in dem die durchschnittliche Anzahl der Delikte pro Altersabschnitt doppelt so hoch ist wie im Gewaltcluster, wie es bei den Männern vorkommt, existiert bei den Frauen nicht.

Ergebnisse, die sich bei der Multidimensionalen Skalierung (Abbildung 12) zeigen, treten auch hier auf. Die Deliktskategorien Fälschung und Betrug liegen im Ergebnis der Multidimensionalen Skalierung nah beieinander und befinden sich auch hier häufig in einem Cluster. Auch die Gewaltdelikte bilden ein Cluster und liegen im Ergebnis der Multidimensionalen Skalierung nah beieinander. Die Delikte einfacher Diebstahl, Verkehrsdelikte und fahrlässige Körperverletzung befinden sich am Rand der zweidimensionalen Darstellung, zeigen somit keine Ähnlichkeiten mit anderen Delikten und bilden jeweils ein eigenes Cluster. Bei den Frauen ergibt sowohl das Ergebnis der Multidimensionalen Skalierung wie die Clusteranalyse einen Zusammenhang zwischen den Delikten Betäubungsmitteldelikte, Leistungserschleichung, verbotene Prostitution (sonstige Sexualdelikte), Sachbeschädigung, Raub, Hausfriedensbruch, schwerer Diebstahl und Vollrausch.

\subsection{Probabilistische Clusteranalyse für polizeilich Registrierte des Geburtsjahrgangs 1970}

Wie schon bei den justiziell Registrierten beschrieben, sollen mit Hilfe eines probabilistischen Clusteranalyseverfahrens die Deliktskonfigurationen der polizeilich Registrierten bestimmt werden. Die Fragen, die sich stellen, sind: Gibt es Tätertypen, und wenn ja, welche Typen lassen sich identifizieren? Dafür werden mit Hilfe der Clusteranalyse die Altersabschnitte der Tatverdächtigen anhand ihrer Straftaten gruppiert. Im Folgenden werden die Ergebnisse vorgestellt, die sich mit der probabilistischen Clusteranalyse bei polizeilich Registrierten des Geburtsjahrgangs 1970 in Baden-Württemberg ergeben. Die Registrierungen der Tatverdächtigen werden in 5-Jahresschritte eingeteilt. Eine polizeiliche Registrierung ist bereits bei 7-Jährigen möglich. Die Daten sind vorhanden bis 2001 und damit bei 1970 Geborenen bis zum Alter von 31 Jahren. Es werden auch hier nur die Daten von Personen benutzt, die mindestens zweimal registriert sind. Es werden die Altersgruppen 10-15, 16-20, 21-25 und 26-30 gebildet. Dies entspricht den Altersgruppen in der Untersuchung einer englischen Kohorte von Francis, Soothill und Fligelstone. Die Altersgruppen wurden nicht ebenso eingeteilt wie bei den justiziellen Daten, da diese eine Altersspanne von 14 bis 33 Jahren umfassen, die Polizeidaten aber von 7 bis 31 Jahren vorhanden sind. Die 7- bis 9-Jährigen werden nicht mit einbezogen, weil es in diesem Alter sehr wenige Registrierungen gibt. Die Häufigkeiten der Altersgruppen zeigt Abbildung 48.

Der zahlenmäßige Unterschied zwischen den Frauen und den Männern ist deutlich erkennbar, aber nicht so groß wie bei den justiziellen Registrierungen. 
Die größte Altersgruppe ist sowohl bei den Männern wie bei den Frauen die Gruppe der 16- bis 20-Jährigen. Die Altersgruppe der 21- bis 25-Jährigen ist etwas kleiner und die der 26- bis 30-Jährigen deutlich kleiner. Am kleinsten ist die Gruppe der 10- bis 15-Jährigen.

Abbildung 48: Häufigkeiten der Altersgruppen (polizeiliche Registrierung, deutsche Männer und Frauen, Kohorte 1970)
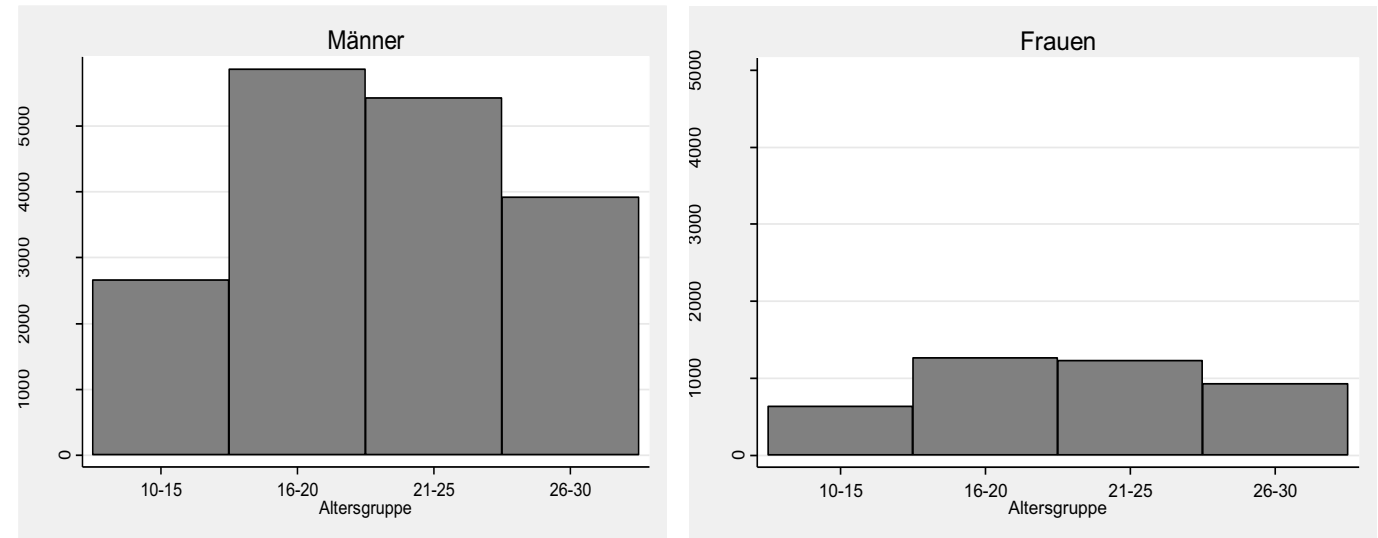

Für die Clusteranalyse werden die Deliktskategorien benutzt, die auch für die Analyse mit Multidimensionaler Skalierung verwendet wurden: 23 Deliktskategorien bei den Männern und 22 Deliktskategorien bei den Frauen. (Sexualdelikte kommen bei Frauen sehr selten vor und Verkehrsdelikte werden in der PAD nicht registriert.)

\subsubsection{Clusteranalyse polizeilicher Registrierungen deutscher Männer}

Analysiert werden 17.863 Altersabschnitte von 9.260 Tatverdächtigen. Mit Hilfe der Modellprüfgrößen aus Tabelle 49 wird die optimale Clusterzahl bestimmt. ${ }^{268}$ Der BIC-Wert ist minimal bei 14 Clustern. Die Aufteilung in 14 Cluster ist aber nicht sinnvoll, weil sich dabei zu viele Cluster mit einzelnen Delikten ergeben.

Wie schon bei den justiziellen Registrierungen wird auch hier die 6-Clusterlösung verwendet. Denn auch hier gelten die Kriterien, die für die Bestimmung der optimalen Anzahl der Cluster bei den justiziellen Registrierungen der Männer benutzt werden. Es gilt die Daumenregel, dass $\mathrm{L}^{2}$ kleiner als die Anzahl der Freiheitsgrade ist. Der p-Wert ist 1.

268 Die Modellprüfgrößen sind im Abschnitt 6.1.1 Clusteranalyse justizieller Registrierungen deutscher Männer (justizielle Registrierungen) beschrieben. 
Tabelle 49: Modellprüfgrößen zur Bestimmung der Clusteranzahl (deutsche Männer, Kohorte 1970, polizeiliche Registrierungen) ${ }^{269}$

\begin{tabular}{|c|c|c|c|c|c|c|c|}
\hline $\begin{array}{l}\text { Cluster- } \\
\text { zahl }\end{array}$ & LL & $\mathrm{BIC}(\mathrm{LL})$ & Npar & $\mathrm{L}^{2}$ & $\mathrm{df}$ & $\mathrm{p}$-Wert & Class.Err. \\
\hline 2 & -103002 & 206463 & 47 & 23227 & 17816 & $1,60 \mathrm{E}-151$ & 0,033 \\
\hline 3 & -101551 & 203796 & 71 & 20325 & 17792 & $4,70 \mathrm{E}-38$ & 0,153 \\
\hline 4 & -100854 & 202638 & 95 & 18932 & 17768 & $7,20 \mathrm{E}-10$ & 0,161 \\
\hline 5 & -100231 & 201628 & 119 & 17687 & 17744 & 0,62 & 0,171 \\
\hline 6 & -99738 & 200876 & 143 & 16700 & 17720 & 1 & 0,205 \\
\hline 7 & -99429 & 200494 & 167 & 16083 & 17696 & 1 & 0,129 \\
\hline 8 & -99167 & 200203 & 191 & 15558 & 17672 & 1 & 0,129 \\
\hline 9 & -98937 & 199979 & 215 & 15098 & 17648 & 1 & 0,135 \\
\hline 10 & -98724 & 199789 & 239 & 14673 & 17624 & 1 & 0,169 \\
\hline 11 & -98477 & 199528 & 263 & 14177 & 17600 & 1 & 0,116 \\
\hline 12 & -98213 & 199235 & 287 & 13650 & 17576 & 1 & 0,104 \\
\hline 13 & -98045 & 199134 & 311 & 13314 & 17552 & 1 & 0,104 \\
\hline 14 & -97914 & 199107 & 335 & 13052 & 17528 & 1 & 0,116 \\
\hline 15 & -97798 & 199111 & 359 & 12821 & 17504 & 1 & 0,123 \\
\hline $\begin{array}{l}\text { LL } \\
\text { BIC } \\
\text { Npar } \\
\text { L }^{2} \\
\text { df } \\
\text { p-Wert } \\
\text { Class.Err. }\end{array}$ & $\begin{array}{l}\text { Wert der L } \\
\text { Bayesian In } \\
\text { Anzahl der } \\
\text { Likelihood } \\
\text { Freiheitsgra } \\
\text { Signifikanz } \\
\text { Classificati }\end{array}$ & $\begin{array}{l}\text {-Likelihood- } \\
\text { prmation Crit } \\
\text { arameter } \\
\text { tio chi-squar } \\
\text { e } \\
\text { veau } \\
\text { Errors }\end{array}$ & $\begin{array}{l}\text { aktion } \\
\text { on siehe } \\
\text { statistic }\end{array}$ & te 235 & & & \\
\hline
\end{tabular}

Das Ergebnis der Clusteranalyse für die 6-Clusterlösung ist in Tabelle 50 dargestellt. Die Clustergröße reicht von 6,7\% aller Altersabschnitte bis 31,1\%. Die Deliktsanteile der einzelnen Cluster, die mindestens 1,5-mal so groß sind wie der durchschnittliche Deliktsanteil (rechte Spalte), sind fett gedruckt.

Das größte Cluster ist Cluster A. 31,1\% aller Altersabschnitte befinden sich in diesem Cluster. Cluster A enthält hauptsächlich Gewaltdelikte (Sexualdelikte, sonstige Sexualdelikte, schwere Körperverletzung, Körperverletzung, Straftaten gegen die persönliche Freiheit) und des Weiteren sind auch Sachbeschädigung, Hausfriedensbruch, Widerstand gegen die Staatsgewalt und Beleidigung überdurchschnittlich häufig vertreten.

269 Die Werte der Tabelle sind mit Latent GOLD berechnet. 
Tabelle 50: Deliktscluster polizeilicher Registrierungen deutscher Männer der Kohorte 1970: Wahrscheinlichkeiten der Deliktskategorien in den Clustern (in \%)

\begin{tabular}{|c|c|c|c|c|c|c|c|}
\hline \multirow[b]{3}{*}{ Clusterwahrscheinlichk. $\pi(\mathrm{k})$ in \% } & \multicolumn{6}{|c|}{ Cluster } & \multirow[b]{2}{*}{ gesamt } \\
\hline & A & B & $\mathrm{C}$ & $\mathrm{D}$ & E & $\mathrm{F}$ & \\
\hline & 31,1 & 29,2 & 15,0 & 9,2 & 8,7 & 6,7 & 100 \\
\hline \multicolumn{8}{|l|}{ Wahrscheinlichkeiten $p_{\mathrm{kj}}$ in \% } \\
\hline Tötungsdelikte & 0,4 & 0,1 & 0,0 & 0,1 & 0,0 & 2,5 & 0,3 \\
\hline Sexualdelikte & 2,5 & 0,6 & 0,0 & 0,2 & 0,3 & 4,0 & 1,3 \\
\hline sonstige Sexualdelikte & 3,9 & 1,1 & 0,5 & 0,2 & 0,6 & 2,5 & 1,8 \\
\hline Raub & 3,1 & 5,6 & 0,2 & 2,0 & 1,0 & 27,3 & 4,7 \\
\hline Schwere Körperverletzung & 22,2 & 4,8 & 1,2 & 3,2 & 1,3 & 42,3 & 11,7 \\
\hline Körperverletzung & 29,8 & 4,3 & 2,3 & 2,7 & 2,8 & 45,3 & 14,4 \\
\hline Str. gg. persönliche Freiheit & 15,5 & 1,1 & 1,3 & 1,6 & 1,6 & 32,7 & 7,8 \\
\hline Einfacher Diebstahl & 12,0 & 51,9 & 100,0 & 14,8 & 26,1 & 83,4 & 43,1 \\
\hline Schwerer Diebstahl & 3,9 & 51,6 & 8,8 & 0,4 & 0,0 & 63,8 & 21,9 \\
\hline Sachbeschädigung & 25,2 & 15,9 & 6,0 & 2,1 & 0,1 & 52,0 & 17,1 \\
\hline gemeingefährliche Straftaten & 2,0 & 2,4 & 0,2 & 0,0 & 0,0 & 3,8 & 1,6 \\
\hline Hehlerei & 3,4 & 12,7 & 3,1 & 1,1 & 1,2 & 18,2 & 6,7 \\
\hline Betrug & 5,1 & 15,0 & 0,0 & 1,6 & 100,0 & 36,5 & 17,3 \\
\hline Erschleichen von Leistungen & 6,6 & 11,3 & 2,8 & 4,9 & 5,2 & 27,1 & 8,5 \\
\hline Gefährdung dem. Rechtsstaat & 2,1 & 0,2 & 0,1 & 0,0 & 0,1 & 3,7 & 1,0 \\
\hline Widerstand gg. Staatsgewalt & 5,0 & 0,4 & 0,0 & 0,9 & 0,0 & 18,3 & 3,0 \\
\hline öffentliche Ordnung & 5,8 & 4,0 & 0,8 & 0,9 & 5,9 & 12,6 & 4,6 \\
\hline Hausfriedensbruch & 6,8 & 2,4 & 0,6 & 0,7 & 0,3 & 22,8 & 4,5 \\
\hline Beleidigung & 18,6 & 1,5 & 0,9 & 1,9 & 1,9 & 33,3 & 8,9 \\
\hline Fälschung & 2,5 & 11,0 & 1,0 & 1,3 & 9,6 & 16,8 & 6,2 \\
\hline Meineid & 1,1 & 1,5 & 0,1 & 0,3 & 2,2 & 1,7 & 1,1 \\
\hline falsche Verdächtigung & 0,7 & 1,4 & 0,0 & 0,4 & 0,8 & 4,2 & 1,0 \\
\hline BTM-Delikte & 7,0 & 17,1 & 0,0 & 100,0 & 6,4 & 45,7 & 20,0 \\
\hline Durchschn. Anzahl der Delikte & 1,8 & 2,5 & 1,2 & 1,4 & 1,7 & 6,5 & 2,1 \\
\hline
\end{tabular}

Cluster B ist das zweitgrößte Cluster (29,2\%). Außer insbesondere schwerem Diebstahl befinden sich auch die Deliktskategorien Hehlerei und Fälschung überdurchschnittlich häufig in diesem Cluster. Die durchschnittliche Anzahl von Delikten pro Altersabschnitt ist in Cluster B mit 2,5 überdurchschnittlich hoch.

Cluster C enthält 15\% aller Altersabschnitte. Alle zugehörigen Altersabschnitte enthalten das Delikt einfacher Diebstahl. Alle anderen Delikte sind sehr selten in diesem Cluster vertreten. Um wie viele Registrierungen von einfachem Diebstahl es sich handelt, kann den Altersabschnitten nicht entnommen werden. Im Altersabschnitt wird eine 1 notiert, wenn mindestens ein einfacher Diebstahl in dieser Altersspanne registriert wird. 
Cluster D enthält 9,2\% aller Altersabschnitte. Alle Altersabschnitte des Cluster D enthalten Betäubungsmitteldelikte, andere Delikte sind selten in diesem Cluster.

Cluster E ist das Cluster der Betrugsdelikte (8,7\%). Außer Betrug befinden sich in diesem Cluster die Deliktskategorien Fälschung und Meineid überdurchschnittlich häufig.

In Cluster F (6,7\%) sind alle Delikte überdurchschnittlich häufig vertreten. Cluster F ist somit das Cluster der verschiedenen Straftaten. Die durchschnittliche Anzahl von Straftaten pro Altersabschnitt ist im Cluster der verschiedenen Straftaten mit 6,5 besonders hoch. Im Durchschnitt sind pro Altersabschnitt 2,1 Straftaten registriert.

Tabelle 51: Wahrscheinlichkeit der Clusterzugehörigkeit bei Deliktsvorkommen in \% (Polizeiliche Registrierung, deutsche Männer, Kohorte 1970, Baden.-Württemberg)

\begin{tabular}{|c|c|c|c|c|c|c|}
\hline \multirow[b]{3}{*}{ Clusterwahrscheinlichkeiten $\pi(\mathrm{k})$ in $\%$} & \multicolumn{6}{|c|}{ Cluster* } \\
\hline & A & $\mathrm{B}$ & $\mathrm{C}$ & $\mathrm{D}$ & $\mathrm{E}$ & $\mathrm{F}$ \\
\hline & 31,1 & 29,2 & 15,0 & 9,2 & 8,7 & 6,7 \\
\hline \multicolumn{7}{|l|}{ Wahrscheinlichkeiten $\Theta_{\mathrm{kj}}$ in \% } \\
\hline Tötungsdelikte & 34,6 & 11,9 & 1,5 & 2,6 & 0,9 & 48,6 \\
\hline Sexualdelikte & 61,2 & 14,0 & 0,1 & 1,4 & 1,9 & 21,4 \\
\hline sonstige Sexualdelikte & 65,9 & 17,3 & 3,9 & 1,2 & 2,7 & 9,0 \\
\hline Raub & 20,7 & 34,4 & 0,5 & 4,0 & 1,8 & 38,6 \\
\hline schwere Körperverletzung & 59,0 & 12,0 & 1,5 & 2,5 & 1,0 & 24,1 \\
\hline Körperverletzung & 64,4 & 8,7 & 2,4 & 1,7 & 1,7 & 21,0 \\
\hline Str. gg. persönliche Freiheit & 61,8 & 4,0 & 2,5 & 1,9 & 1,8 & 27,9 \\
\hline Einfacher Diebstahl & 8,6 & 35,2 & 34,8 & 3,2 & 5,3 & 12,9 \\
\hline Schwerer Diebstahl & 5,6 & 68,9 & 6,0 & 0,2 & 0,0 & 19,4 \\
\hline Sachbeschädigung & 46,0 & 27,3 & 5,3 & 1,1 & 0,0 & 20,3 \\
\hline gemeingef. Straftaten & 38,7 & 43,2 & 2,3 & 0,0 & 0,0 & 15,9 \\
\hline Hehlerei & 16,0 & 55,7 & 6,9 & 1,6 & 1,6 & 18,3 \\
\hline Betrug & 9,2 & 25,4 & 0,0 & 0,9 & 50,4 & 14,1 \\
\hline Erschleichen von Leistungen & 24,3 & 38,9 & 4,9 & 5,3 & 5,3 & 21,3 \\
\hline Gefährdung dem. Rechtsstaat & 66,8 & 5,6 & 1,3 & 0,2 & 0,9 & 25,1 \\
\hline Widerstand gg. Staatsgewalt & 52,0 & 4,2 & 0,0 & 2,9 & 0,0 & 40,9 \\
\hline Öffentliche Ordnung & 39,9 & 25,9 & 2,6 & 1,7 & 11,4 & 18,5 \\
\hline Hausfriedensbruch & 46,8 & 15,8 & 1,9 & 1,4 & 0,6 & 33,5 \\
\hline Beleidigung & 64,9 & 4,9 & 1,6 & 2,0 & 1,8 & 24,9 \\
\hline Fälschung & 12,6 & 51,6 & 2,5 & 1,9 & 13,4 & 18,0 \\
\hline Meineid & 31,4 & 37,6 & 1,4 & 2,4 & 17,2 & 10,1 \\
\hline falsche Verdächtigung & 21,9 & 40,1 & 0,6 & 3,2 & 7,0 & 27,2 \\
\hline BTM-Delikte & 10,9 & 25,0 & 0,0 & 46,1 & 2,8 & 15,2 \\
\hline
\end{tabular}

* Cluster: A Gewalt, B schwerer Diebstahl, C einfacher Diebstahl, D BTM, E Betrug, F verschiedene Straftaten

Tabelle 51 ist zu entnehmen, mit welcher Wahrscheinlichkeit ein Delikt in ein Cluster gehört. 68,9\% aller Altersabschnitte mit schwerem Diebstahl und 35,2\% 
aller Altersabschnitte mit einfachem Diebstahl befinden sich in Cluster B (schwerer Diebstahl). Auch viele Altersabschnitte mit Raub (34,4\%), Hehlerei (55,7\%), Leistungserschleichung (38,9\%), Fälschung (51,6\%), Meineid (37,6\%) und falsche Verdächtigung (40,1\%) befinden sich in Cluster B. 34,8\% der Altersabschnitte mit einfachem Diebstahl liegen in Cluster C (einfacher Diebstahl). Im Unterschied da$\mathrm{zu}$ ist Tabelle $50 \mathrm{zu}$ entnehmen, dass alle Altersabschnitte aus Cluster $\mathrm{C}$ einfachen Diebstahl enthalten. Wie Tabelle 51 zeigt, liegen 46,1\% aller Altersabschnitte mit Betäubungsmitteldelikten in Cluster D, andererseits enthalten alle zu Cluster D gehörenden Altersabschnitte ein Betäubungsmitteldelikt (Tabelle 50). 50\% aller Altersabschnitte mit Betrug befinden sich in Cluster E (Tabelle 51), umgekehrt enthalten alle Altersabschnitte aus Cluster E ein Betrugsdelikt (Tabelle 50).

Die eindeutige Zuordnung der Altersabschnitte $\mathrm{zu}$ den Clustern ist etwas schlechter als bei den justiziellen Registrierungen. 94,3\% der Altersabschnitte haben eine a posteriori Wahrscheinlichkeit größer als 50\%, 62,7\% haben eine a posteriori Wahrscheinlichkeit größer $80 \%$. Aber auch diese Zuordnung kann man als sehr gut bezeichnen.

Abbildung 49: Verteilung der männlichen Tatverdächtigen auf Cluster nach Altersgruppen

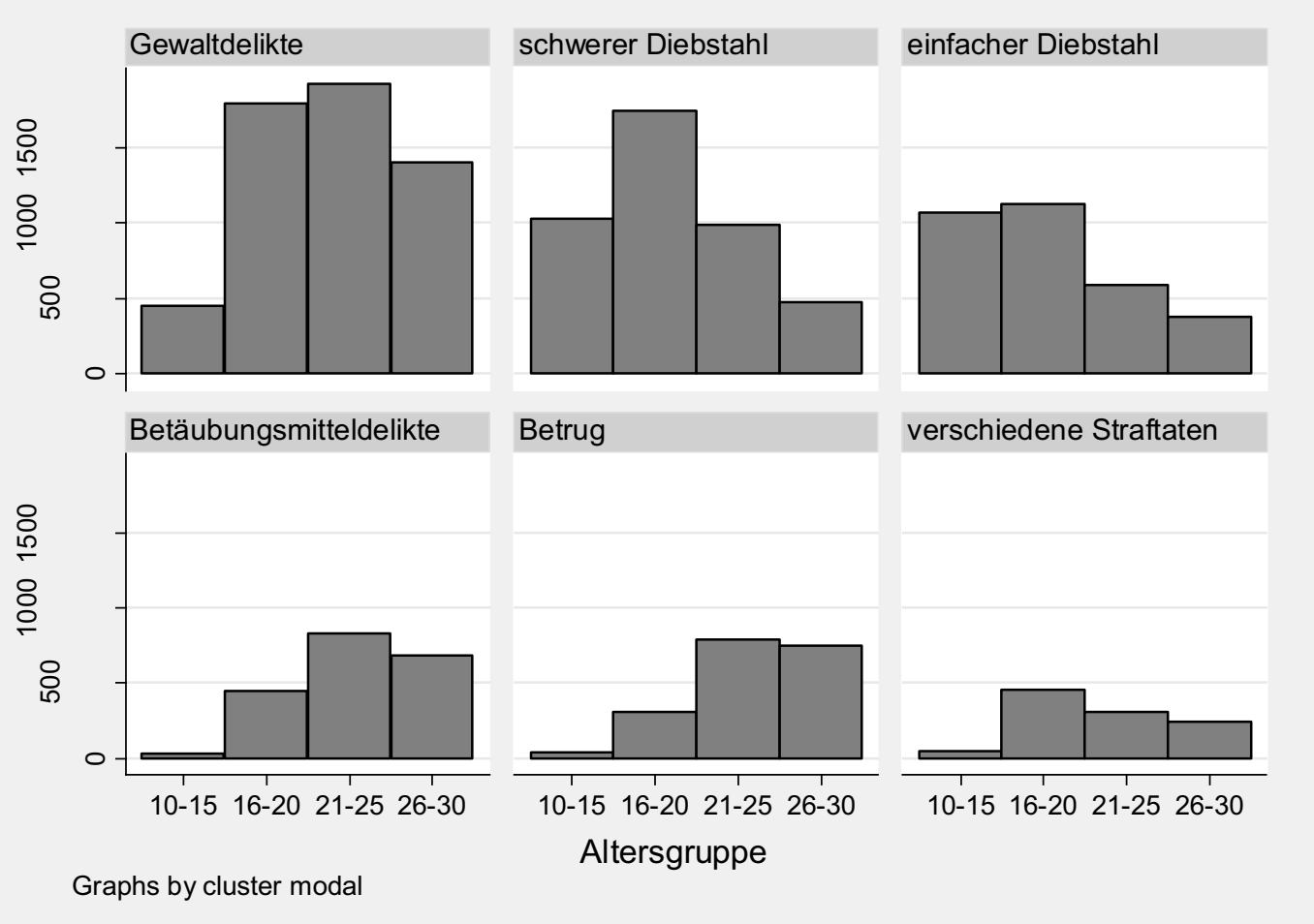

Abbildung 49 gibt die Altersverteilung der Cluster wieder. Deutlich zu sehen sind die unterschiedlichen Alterszusammensetzungen der Cluster. In den Clustern Gewaltdelikte, Betrug und Betäubungsmitteldelikte ist jeweils der größte Anteil der 
Altersabschnitte der der 21- bis 25-Jährigen. Im Cluster der Gewaltdelikte ist der Anteil der 16- bis 20-Jährigen fast genauso groß wie der der 21- bis 25-Jährigen. Im Cluster schwerer Diebstahl gibt es ein deutliches Altersmaximum in der Altersgruppe 16-20. Auch im Cluster der verschiedenen Straftaten liegt das Altersmaximum in der Altersgruppe 16-20. Im Cluster der verschiedenen Straftaten befinden sich nur sehr wenige Altersabschnitte von 10-15. Ab dem Altersabschnitt 16-20 entspricht die Altersverteilung des Clusters verschiedene Straftaten in etwa der Verteilung aller polizeilich Registrierten (vgl.

Abbildung 48). Die Altersabschnitte 10-15 befinden sich hauptsächlich in den Diebstahlclustern und auch im Gewaltcluster. Im Cluster des einfachen Diebstahls treten hauptsächlich Altersabschnitte von 10- bis 15-Jährigen und 16- bis 20Jährigen auf. Die Häufigkeit nimmt mit dem Alter ab.

Tabelle 52: Deliktszusammensetzung der Cluster deutscher Männer der Kohorte 1970 (6 Cluster)

\begin{tabular}{ll}
\hline Cluster & Zusammensetzung \\
\hline \multirow{3}{*}{ A $(31,1 \%)$} & Sexualdelikte, Körperverletzung, Straftaten gegen die persönliche \\
Gewalt & Freiheit, Beleidigung, Hausfriedensbruch, Sachbeschädigung und \\
& Widerstand gegen die Staatsgewalt. \\
& Alter hauptsächlich 16-30. \\
& Im Durchschnitt 1,8 Delikte pro Altersabschnitt. \\
\hline B (29,2\%) & Schwerer Diebstahl, Hehlerei und Fälschung. \\
Schwerer Diebstahl & $\begin{array}{l}\text { Altersmaximum bei 16-20. } \\
\text { Im Durchschnitt 2,5 Delikte pro Altersabschnitt. }\end{array}$ \\
\hline C (15,0\%) & Einfacher Diebstahl. \\
Einfacher Diebstahl & Alter hauptsächlich 10-20. \\
\hline D (9,2\%) & Im Durchschnitt 1,2 Delikte pro Altersabschnitt. \\
Betäubungsmitteldelikte & Betäubungsmitteldelikte. \\
\hline E $(8,7 \%)$ & Alter hauptsächlich 21-30. \\
Betrug & Betrug, Fälschung und Meineid. \\
\hline F (6,7\%) & Alter hauptsächlich 21-30. \\
Verschiedene Straftaten & Im Durchschnitt 1,7 Delikte pro Altersabschnitt. \\
\hline
\end{tabular}

Tabelle 52 gibt einen Überblick über die Deliktszusammensetzung der Cluster, die jeweilige Alterszusammensetzung und die durchschnittliche Anzahl von Delikten pro Altersabschnitt. Alle Altersabschnitte beinhalten im Schnitt 2,1 Delikte. Besonders auffällig ist das Cluster verschiedene Straftaten (F) mit 6,5 Delikten pro Altersabschnitt. Damit wird deutlich, dass dies das Cluster der chronischen Straftäter ist. In diesem Cluster sind alle Delikte überdurchschnittlich häufig vertreten. 
Vergleicht man die Ergebnisse der Clusteranalyse der polizeilichen Registrierungen mit denen der justiziellen Registrierungen, so ist zuerst darauf hinzuweisen, dass Verkehrsdelikte und somit auch zahlreiche fahrlässige Körperverletzungen/Tötungen polizeilich nicht registriert sind. Somit ist das sehr große Cluster Verkehrsdelikte und das Cluster fahrlässige Körperverletzung nicht unter den Clustern der Altersabschnitte der polizeilichen Registrierung. Die Güte der Lösungen stimmt aber bei den jeweiligen 6-Clusterlösungen überein. Deshalb wird das Ergebnis der Clusteranalyse der polizeilichen Registrierungen mit der 6-Clusterlösung der justiziellen Registrierungen verglichen, aber auch mit der 8-Clusterlösung der justiziellen Registrierungen (siehe im Anhang Tabelle 75 und Abbildung 65).

Sowohl die 6-Clusterlösung der justiziellen Registrierungen der Männer ${ }^{270}$ als auch die 6-Clusterlösung der polizeilichen Registrierungen ${ }^{271}$ ergab die Cluster Gewaltdelikte, Betrug und verschiedene Straftaten. Eine klare Übereinstimmung ist sichtbar beim Cluster Betrug und verschiedene Straftaten. Das Cluster verschiedene Straftaten ist sowohl bei den justiziellen Registrierungen wie auch bei den polizeilichen Registrierungen das Cluster der chronischen Straftäter. Die einzelnen Altersabschnitte enthalten viele Delikte, im Durchschnitt mehr als sechs, und alle Delikte sind überdurchschnittlich häufig vorhanden. Auch die Altersverteilung der Cluster stimmt in etwa überein. Beim Vergleich der Altersverteilung müssen die unterschiedlichen Alterseinteilungen beachtet werden. Der erste Altersabschnitt der Polizeidaten umfasst die 10- bis 15-Jährigen, der erste Abschnitt der justiziellen Daten die 14- bis 18-Jährigen. Vergleicht man die Abbildungen der Altersverteilung, so muss der Vergleich ohne die ersten Balken der polizeilich Registrierten durchgeführt werden und auch bei den anderen Altersgruppen muss bedacht werden, dass sie nicht exakt übereinstimmen.

Beim Gewaltcluster der polizeilichen Registrierungen gibt es Unterschiede in der Deliktszusammensetzung im Vergleich zum Gewaltcluster der justiziellen Registrierungen. Im Gewaltcluster der polizeilichen Registrierungen sind ,sonstige Sexualdelikte" überdurchschnittlich häufig vertreten, im Gewaltcluster der justiziellen Registrierungen dagegen nicht. Raub ist bei den polizeilichen Registrierungen kaum im Cluster Gewaltdelikte, bei den justiziellen Registrierungen ist Raub im Gewaltcluster überdurchschnittlich häufig vertreten. Mehr als zwei Drittel der Altersabschnitte des Gewaltclusters der justiziellen Registrierungen enthalten Körperverletzung. Bei den polizeilichen Registrierungen liegt der Anteil von Körperverletzung unter einem Drittel. Der Anteil von Körperverletzung stimmt aber bei den justiziellen und polizeilichen Registrierungen überein (jeweils 14\%). Vergleicht man aber die Größe der Gewaltcluster, so stimmt der Anteil von Körperverletzung bei justiziellen und polizeilichen Registrierungen wieder überein. Das Ge-

270 Siehe Tabelle 39, Abbildung 32 und Tabelle 41.

271 Siehe Tabelle 50, Abbildung 49 und Tabelle 52. 
waltcluster der polizeilichen Registrierungen ist mit 31,1\% aller Altersabschnitte sehr groß. Das Gewaltcluster der justiziellen Registrierungen enthält 12,6\% aller Altersabschnitte. Betrachtet man den Anteil von Körperverletzung im Gewaltcluster bezogen auf alle Altersabschnitte, dann sind es sowohl bei polizeilichen wie bei justiziellen Registrierungen 9\%.

Die Cluster Diebstahl bzw. einfacher Diebstahl sind zwar sowohl bei der 6-Clusterlösung der polizeilichen wie justiziellen Registrierungen vorhanden, sie unterscheiden sich aber. Das Cluster einfacher Diebstahl der polizeilichen Registrierungen enthält fast ausnahmslos das Delikt einfacher Diebstahl. Alle Altersabschnitte dieses Clusters enthalten einfachen Diebstahl, alle anderen Delikte sind sehr selten, bzw. gar nicht in diesem Cluster. Das Cluster des einfachen Diebstahls der justiziellen Registrierungen enthält auch überdurchschnittlich häufig Betäubungsmitteldelikte, schweren Diebstahl, Leistungserschleichung, Hehlerei und sonstige Sexualdelikte. Insofern könnte dieses Cluster auch als Betäubungsmittel-cluster mit Beschaffungskriminalität bezeichnet werden. Hierin sind also auch die beiden fehlenden Cluster schwerer Diebstahl und Betäubungsmitteldelikte enthalten.

Vergleicht man die Clusterlösungen der polizeilichen Registrierungen mit der 8Clusterlösung der justiziellen Registrierungen ${ }^{272}$, zeigen sich viele Gemeinsamkeiten. In der 8-Clusterlösung sind auch die in der 6-Clusterlösung der justiziellen Registrierungen fehlenden Cluster Betäubungsmitteldelikte und schwerer Diebstahl enthalten. Auch das Cluster des einfachen Diebstahls stimmt bei der 8-Clusterlösung mit dem Cluster einfacher Diebstahl der polizeilichen Registrierungen überein. Unterschiede zeigen sich nur im Cluster schwerer Diebstahl. Bei den polizeilichen Registrierungen enthält das Cluster „schwerer Diebstahl“ zusätzlich überdurchschnittlich häufig nur die Delikte Hehlerei und „Fälschung“. Das Cluster „schwerer Diebstahl“ der justiziellen Registrierungen enthält viele Delikte; unter anderem ist hier der höchste Anteil von ,sonstigen Sexualdelikten“. Auch Sexualdelikte, Tötungsdelikte und Straftaten gegen die persönliche Freiheit kommen überdurchschnittlich häufig vor. Ebenso die Delikte Leistungserschleichung und Sachbeschädigung. Hier zeigt sich, wie auch schon das Ergebnis der Multidimensionalen Skalierung ergab, die unterschiedliche Registrierung von schwerem Diebstahl bei Polizei und Justiz.

\subsubsection{Spezialisierung, Wechsel und Abbruch im Lebenslauf der Männer}

Des Weiteren wird mit den Ergebnissen der Clusteranalyse die kriminelle Karriere der offiziell Registrierten untersucht. Dazu werden hier Ergebnisse zur Spezialisierung und zur kriminellen Laufbahn dargestellt. Spezialisierung bedeutet hier, dass es von einer Altersgruppe zur nächsten keinen Clusterwechsel gibt. Spezialisierung bedeutet hier also nicht, dass eine Person immer das gleiche Delikt begeht,

272 Siehe Tabelle 75 und Abbildung 65 im Anhang. 
sondern dass die Deliktsstruktur einer Altersgruppe der Deliktsstruktur der nächsten Altersgruppe entspricht. Tabelle 53 zeigt die Spezialisierungsanteile bezogen auf die Clusterzugehörigkeit. Betrachtet werden hier nur Personen, die in einer späteren Altersgruppe wieder eine Registrierung haben, d.h. Abbrecher bzw. Personen, die nur innerhalb einer Altersgruppe registriert sind, werden hier nicht mit einbezogen. Die prozentualen Anteile sind berechnet von einer Altersgruppe zur nächsten. Das N in der dritten Spalte von links gibt an, wie viele Personen in der ,von“ Altersgruppe sind und in einer späteren Altersgruppe wieder registriert sind. Die Häufigkeiten in Klammern $(\mathrm{N})$ geben an, wie viele Personen im jeweiligen Cluster in der ,von“ Altersgruppe sind und in einer späteren Altersgruppe wieder eine Registrierung haben.

Tabelle 53: Spezialisierungsanteile in \%: Deutsche Männer der Kohorte 1970 (polizeiliche Registrierungen) ${ }^{273}$

\begin{tabular}{|c|c|c|c|c|c|c|c|c|}
\hline \multirow{2}{*}{\multicolumn{2}{|c|}{ Altersgruppen }} & & \multicolumn{6}{|c|}{ Cluster } \\
\hline & & & A & B & $\mathrm{C}$ & $\mathrm{D}$ & E & $F$ \\
\hline von & bis & $\mathrm{N}$ & $\begin{array}{l}\text { Gewalt- } \\
\text { delikte } \\
\text { (N) }\end{array}$ & $\begin{array}{c}\text { Schwerer } \\
\text { Diebstahl } \\
\text { (N) }\end{array}$ & $\begin{array}{c}\text { Einfacher } \\
\text { Diebstahl } \\
\text { (N) }\end{array}$ & $\begin{array}{c}\text { BTM- } \\
\text { Delikte } \\
\text { (N) }\end{array}$ & $\begin{array}{c}\text { Betrug } \\
\text { (N) }\end{array}$ & $\begin{array}{l}\text { Veschie- } \\
\text { dene } \\
\text { Straftaten } \\
\text { (N) }\end{array}$ \\
\hline $10-15$ & $16-20$ & 2187 & $26(389)$ & $30(812)$ & $15(890)$ & $20(25)$ & $7 *(30)$ & $56(41)$ \\
\hline $16-20$ & $21-25$ & 3691 & $41(1130)$ & $23(1028)$ & $12(676)$ & $39(301)$ & $27(172)$ & $25(384)$ \\
\hline $21-25$ & $26-30$ & 2365 & $56(760)$ & $23(492)$ & $20(217)$ & $47(366)$ & 39 (297) & $31(233)$ \\
\hline
\end{tabular}

* nicht signifikant

Eine Zunahme der Spezialisierungsanteile mit dem Alter zeigt sich bei den Clustern Gewaltdelikte, Betäubungsmitteldelikte und Betrug. 760 Personen sind im Alter von 21 bis $25 \mathrm{im}$ Gewaltcluster und haben im Alter von 26 bis 30 mindestens eine weitere Registrierung. Davon befinden sich 56\% im Alter von 26 bis 30 wieder im Gewaltcluster. Beim Cluster schwerer Diebstahl ist der Spezialisierungsanteil bei den 10 - bis 15 -Jährigen $30 \%$, bei den Älteren 23\%. Im Cluster des einfachen Diebstahls ist erstaunlicherweise der Spezialisierungsanteil der 16- bis 20Jährigen mit $12 \%$ am niedrigsten. Im Cluster der Betäubungsmitteldelikte gibt es nur sehr wenige 10- bis 15-Jährige (25), die in einer folgenden Altersgruppe wieder registriert sind. Das gleiche gilt für das Cluster Betrug. Deshalb ist im Cluster Betrug auch der Prozentwert der 10- bis 15-Jährigen nicht signifikant, da der Fehler $\mathrm{zu}$ groß ist. Alle anderen Spezialisierungsraten sind signifikant. 56\% der 10 - bis 15-Jährigen des Clusters verschiedene Straftaten sind auch im Alter von 16 bis 20 im Cluster verschiedene Straftaten.

Als nächstes wird das Ergebnis der Clusteranalyse dazu benutzt, kriminelle Laufbahnen zu identifizieren. Abbildung 50 gibt einen Überblick über die Delikts-

273 Siehe Fußnote 254. 
übergänge der männlichen Tatverdächtigen der Altersgruppe 21-25. 5421 männliche Tatverdächtige haben mindestens eine Registrierung in der Altersspanne 21-25. Diese teilen sich auf in 36\%, die zum Cluster Gewaltdelikte gehören; $18 \%$ gehören zum Cluster schwerer Diebstahl, 11\% Cluster einfacher Diebstahl, 15\% Cluster Betrug, 15\% Cluster Betäubungsmitteldelikte und 6\% Cluster verschiedene Straftaten. Von den männlichen Tatverdächtigen, die im Alter von 21 bis 25 mindestens eine Registrierung haben, haben $76 \%$ im Alter von 10 bis 15 keine Registrierung, $43 \%$ haben im Alter von 16 bis 20 und 56\% im Alter von 26 bis 30 keine Registrierung. $17 \%$ der 21 - bis 25 -Jährigen mit Registrierung gehören im Alter von 16 bis 20 zum Cluster Gewaltdelikte, 16\% zum Cluster schwerer Diebstahl und 10\% zum Cluster einfacher Diebstahl. 15\% der 21- bis 25-Jährigen mit Registrierung gehören in der Altersgruppe 26-30 zum Cluster Gewaltdelikte.

Abbildung 50: Deliktsübergänge männlicher Tatverdächtiger der Altersgruppe 21-25

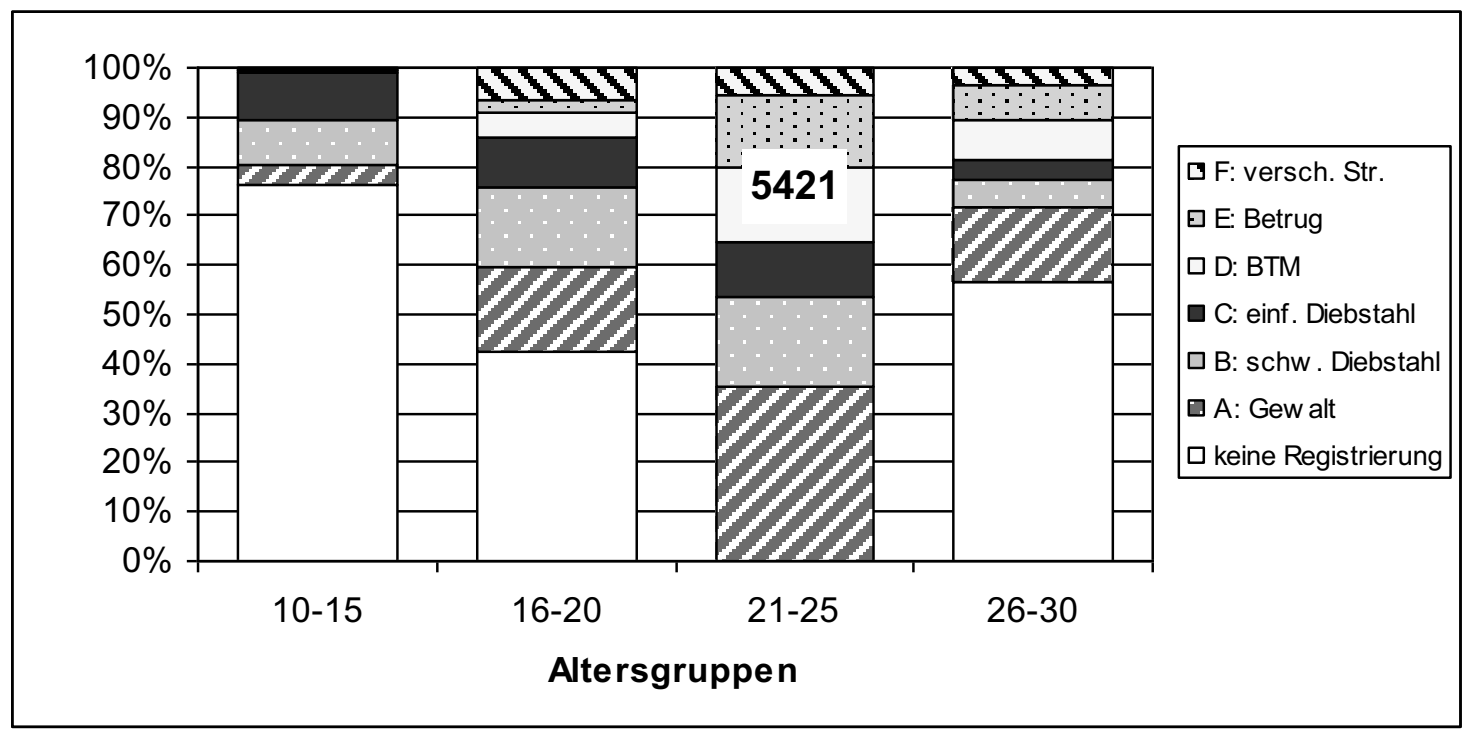

Die Abbildungen 51 bis 56 zeigen die Laufbahn getrennt nach der Clusterzugehörigkeit der 21- bis 25-Jährigen. Angegeben ist jeweils die Clusterzugehörigkeit der Altersgruppen davor und die der Altersgruppe danach bzw. die Angabe, dass es keine Registrierung gab. Im Balken der Altersgruppe 21-25 ist jeweils die Zahl der Tatverdächtigen angegeben, die zu diesem Cluster gehören. Die linken Balken geben dazu die prozentualen Anteile der Clusterzugehörigkeit der 10- bis 15-Jährigen und der 16- bis 20-Jährigen an, der rechte Balken die prozentualen Anteile der 26bis 30-Jährigen. Der größte Anteil vorher und nachher ist jeweils der Anteil ,keine Registrierung“. Eine Ausnahme bilden hier die Personen des Clusters F (verschiedene Straftaten, Abbildung 56). Von den 21- bis 25-Jährigen, die zu diesem Cluster gehören, sind nur 19\% im Alter von 16 bis 20 nicht registriert und 25\% sind mit 26 bis 30 Jahren nicht registriert. Cluster F beinhaltet, wie schon beschrieben, chronische Straftäter. Hier ist auch der Anteil der Spezialisierten im Vergleich zu den anderen Clustern am höchsten. Spezialisiert heißt, wie schon gesagt, hier nicht, 
dass immer das gleiche Delikt registriert ist, sondern dass sich die Clusterzugehörigkeit von einer Altersgruppe zur nächsten nicht ändert. 31\% der Gruppe, die mit 21 bis 25 Jahren zum Cluster F gehören, gehören auch schon im Alter von 16 bis $20 \mathrm{zu}$ Cluster F, dies sind deutlich mehr als im Durchschnitt (siehe Abbildung 50) und 24\% dieser Gruppe gehören auch im Alter von 26 bis 30 Cluster F an und damit deutlich mehr als im Durchschnitt.

Abbildung 51: Deliktsübergänge männlicher Tatverdächtiger des Clusters A

(Gewaltdelikte) im Alter 21 bis 25

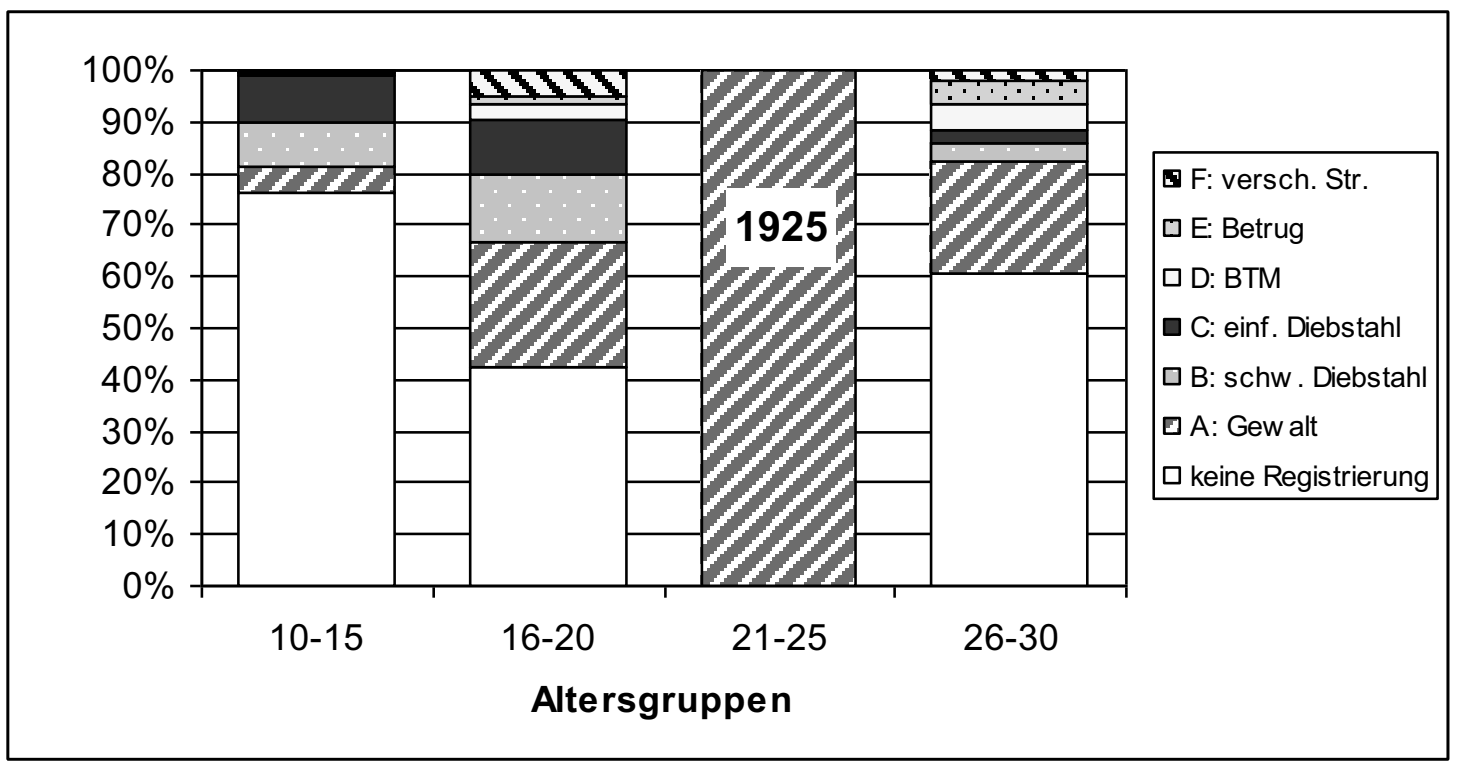

Abbildung 52: Deliktsübergänge männlicher Tatverdächtiger des Clusters B (schwerer Diebstahl) im Alter 21 bis 25

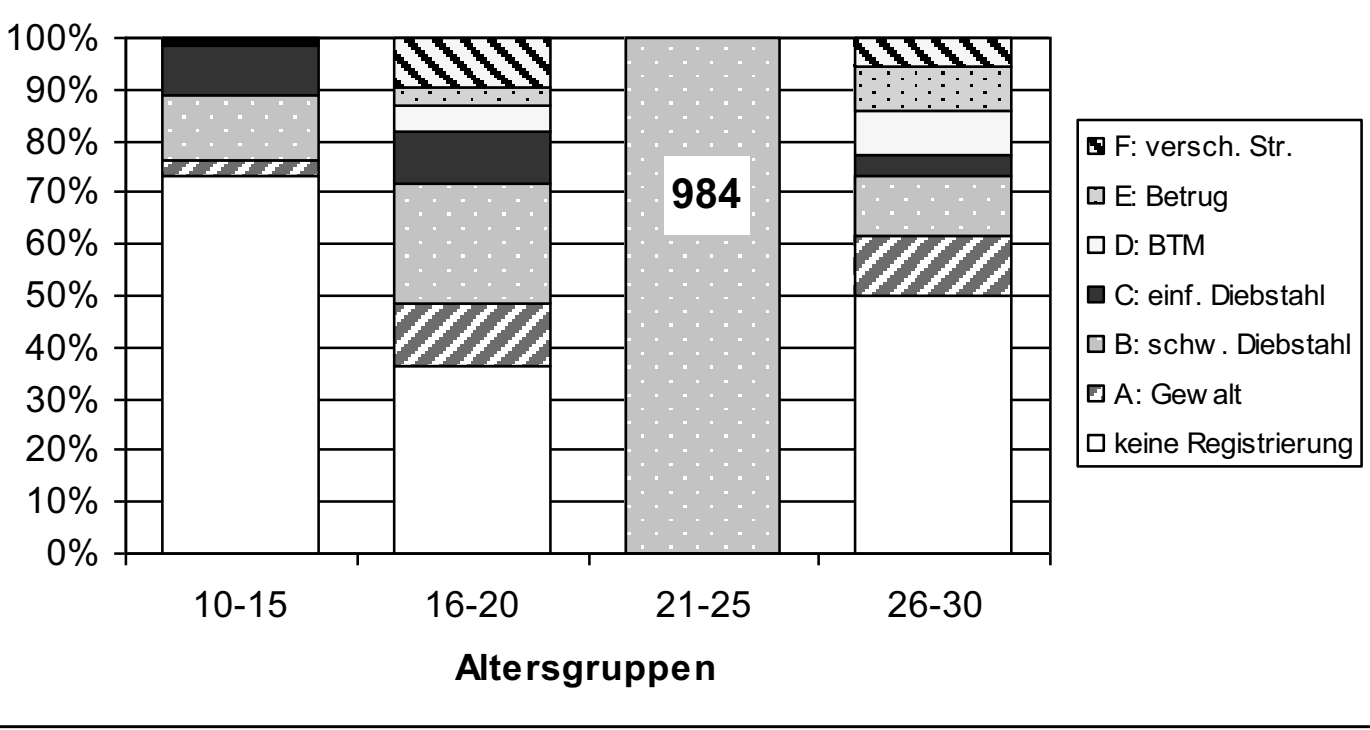


Abbildung 53: Deliktsübergänge männlicher Tatverdächtiger des Clusters C (einfacher Diebstahl) im Alter 21 bis 25

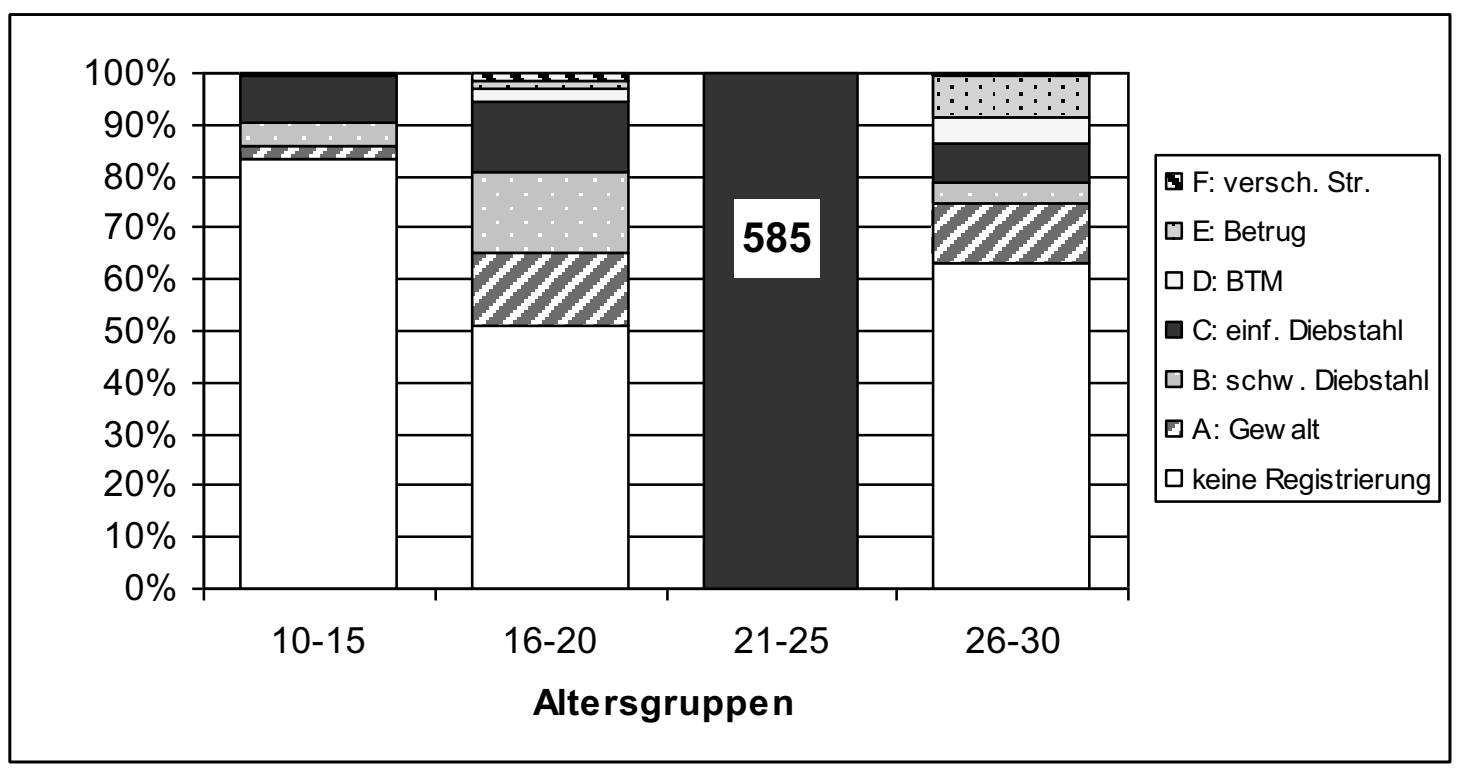

Abbildung 54: Deliktsübergänge männlicher Tatverdächtiger des Clusters D (Betäubungsmitteldelikte) im Alter 21 bis 25

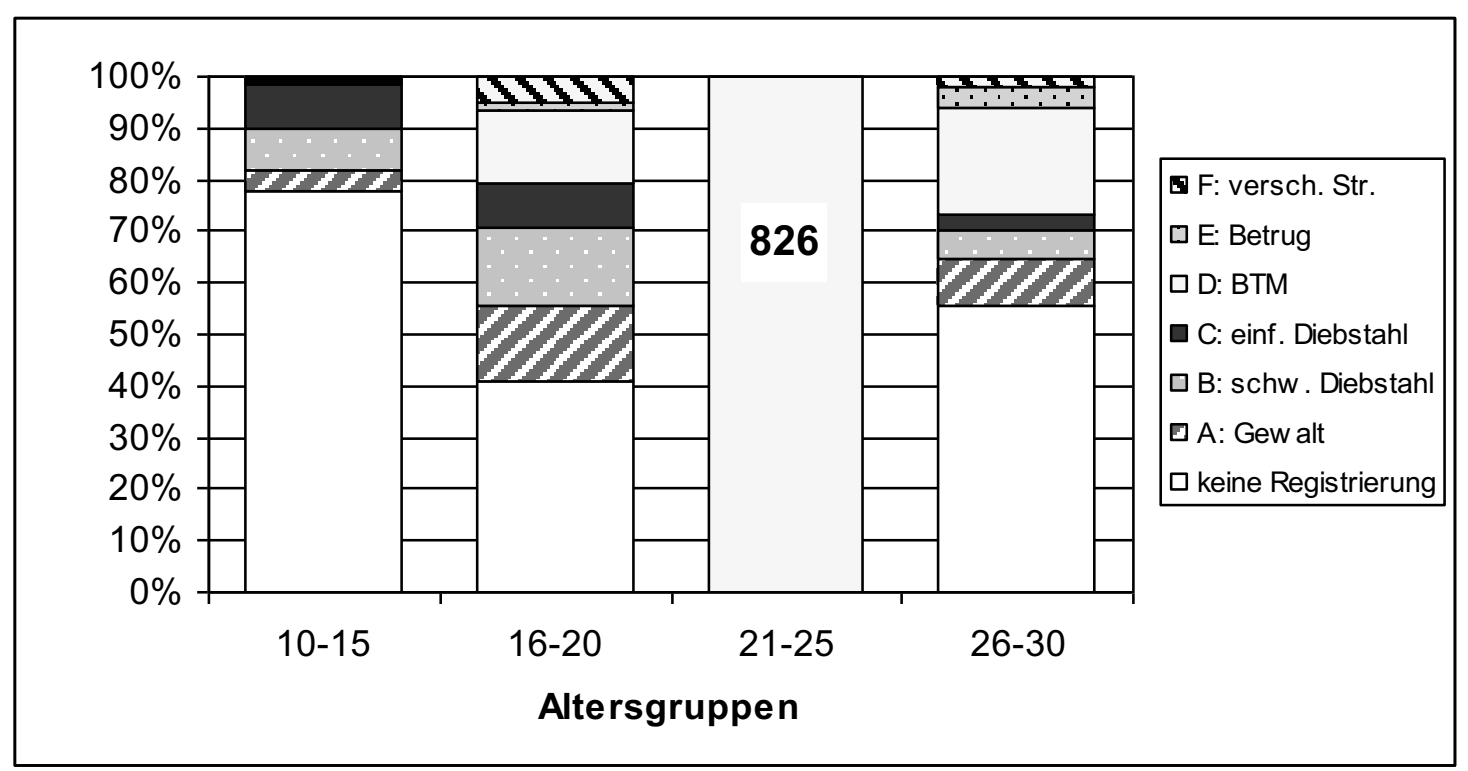


Abbildung 55: Deliktsübergänge männlicher Tatverdächtiger des Clusters E (Betrug) im Alter 21 bis 25

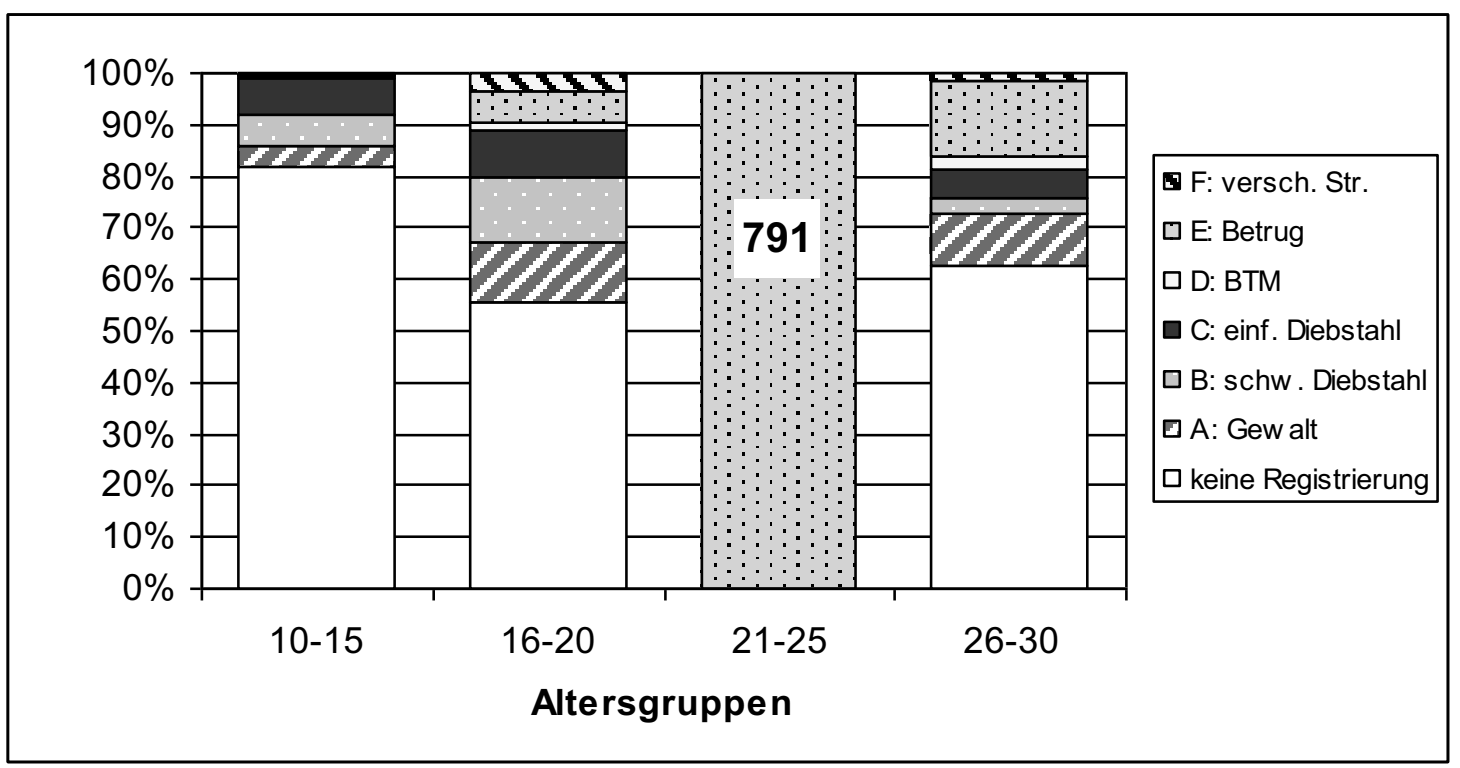

Abbildung 56: Deliktsübergänge männlicher Tatverdächtiger des Clusters F (verschiedene Straftaten) im Alter 21 bis 25

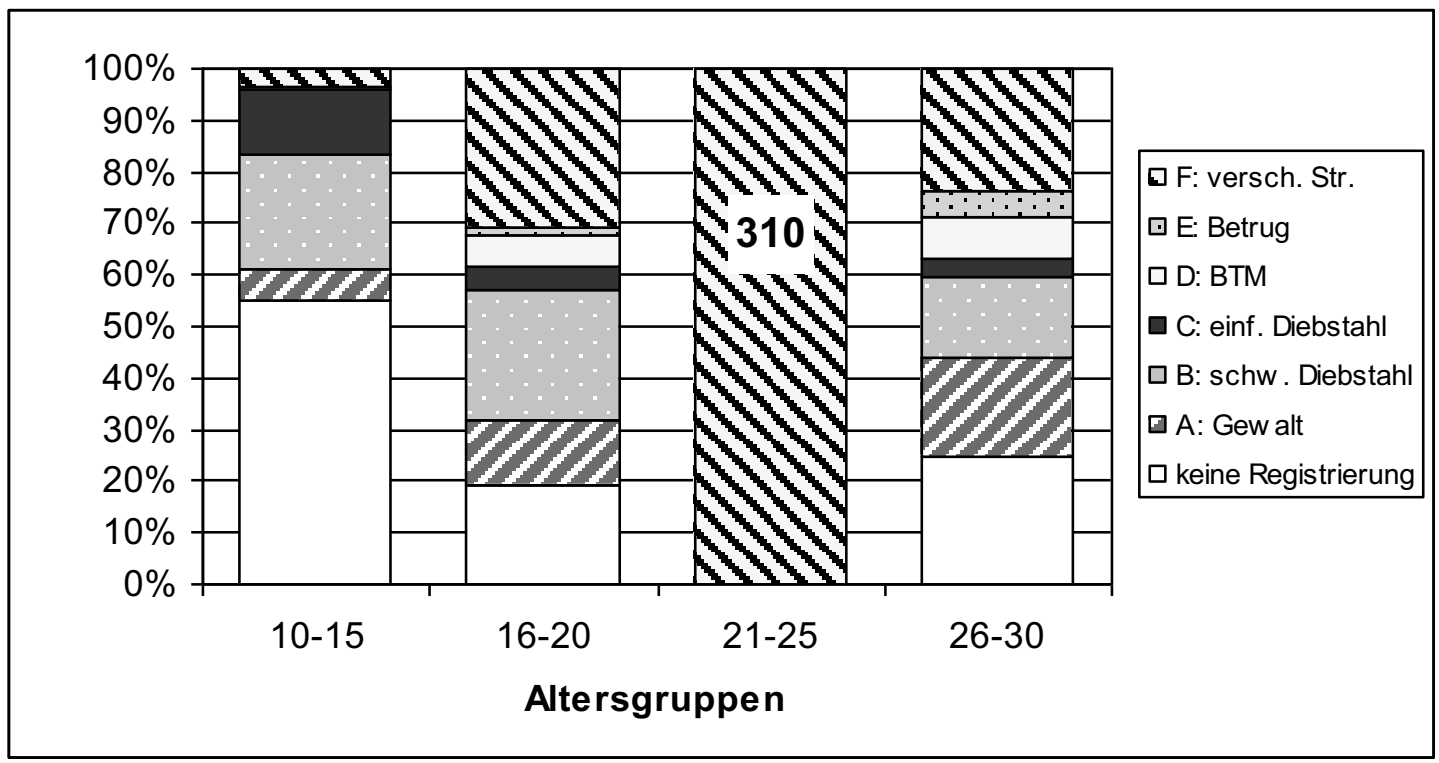

Die meisten Nichtregistrierten im Alter von 10 bis 20 und von 26 bis 30 sind bei den Tatverdächtigen vorhanden, die im Alter von 21 bis 25 zum Cluster Betrug (Abbildung 55) oder zum Cluster einfacher Diebstahl (Abbildung 53) gehören. Über 50\% derjenigen, die im Alter von 21 bis 25 zu diesen Clustern gehören, haben im Alter von 16 bis 20 keine Registrierung. In der Altersgruppe 10 bis 15 haben über $80 \%$ keine Registrierung und über $60 \%$ in der nachfolgenden Altersgruppe (26- bis 30-Jährige). Von den 585, die im Alter von 21 bis 25 dem Cluster ein- 
facher Diebstahl angehören, sind 14\% schon mit 16 bis 20 im Cluster einfacher Diebstahl (im Durchschnitt sind es 9\%). Im Cluster Betrug gibt es einen relativ großen Anteil Spezialisierte in der Altersgruppe danach. 15\% derjenigen, die im Alter von 21 bis $25 \mathrm{im}$ Betrugscluster sind, sind auch im Alter von 26 bis $30 \mathrm{im}$ Betrugscluster; dies ist deutlich mehr als im Durchschnitt (7\%).

Bei den 21- bis 25-Jährigen des Gewaltclusters (Abbildung 51) haben 42\% im Alter von 16 bis 20 keine polizeiliche Registrierung und $61 \%$ im Alter von 26 bis 30 keine Registrierung. 22\% befinden sich auch im Alter von 26 bis 30 im Gewaltcluster $(\varnothing 15 \%)$ und 24\% waren schon im Alter von 16 bis 20 im Gewaltcluster (Ø 17\%). Auch hier zeigt sich im Vergleich zu den Deliktsübergängen der ganzen Altersgruppe, dass es im Gewalt-cluster Spezialisierte gibt. Der Anteil des Gewaltcluster liegt hier bei den 16- bis 20-Jährigen und bei den 26- bis 30-Jährigen deutlich über dem durchschnittlichen Anteil.

Im Cluster schwerer Diebstahl (Abbildung 52) ist der Anteil Nichtregistrierte in der Altersgruppe 16-20 relativ gering (36\%) und der Anteil Spezialisierte mit 24\% sehr hoch (Ø 16\%).

Auch im Cluster der Betäubungsmitteldelikte (Abbildung 54) ist der Anteil Spezialisierter sehr hoch. 21\% der Tatverdächtigen, die im Alter von 21 bis 25 Jahren diesem Cluster angehören, gehören auch im Alter von 26 bis 30 diesem Cluster an (Ø 8\%).

Die Ergebnisse der Clusteranalyse bestätigen die Ergebnisse der Multidimensionalen Skalierung (Abbildung 7). Beim Ergebnis der Multidimensionalen Skalierung liegen die Deliktskategorien Betäubungsmitteldelikte, schwerer Diebstahl und Betrug jeweils weit auseinander am Rand der Abbildung. D.h. die Kombinationen dieser Delikte kommen innerhalb der kriminellen Karriere von Personen selten vor. Beim Ergebnis der Clusteranalyse bilden diese Delikte (Betäubungsmitteldelikte, schwerer Diebstahl und Betrug) jeweils ein eigenes Cluster. Die Gewaltdelikte liegen beim Ergebnis der Multidimensionalen Skalierung eng zusammen und bilden auch ein eigenes Cluster.

\subsubsection{Clusteranalyse polizeilicher Registrierungen deutscher Frauen}

Bei den polizeilich registrierten Frauen in Baden-Württemberg des Geburtsjahrgangs 1970 wurden 4.066 Altersabschnitte von 2.421 Tatverdächtigen untersucht. Mit Hilfe der Modellprüfgrößen aus Tabelle 54 wird die optimale Clusterzahl bestimmt. ${ }^{274}$ Der BIC-Wert ist minimal bei 6 Clustern und auch das bei allen anderen Gruppen gewählte Kriterium trifft hier bei 6 Clustern zu. Hier gilt zum zweiten Mal die Daumenregel, dass $L^{2}$ kleiner als die Anzahl der Freiheitsgrade ist. Der pWert ist 1 .

${ }^{274}$ Die Modellprüfgrößen sind im Abschnitt 6.1.1 Clusteranalyse justizieller Registrierungen deutscher Männer beschrieben. 
In Tabelle 55 sind die Wahrscheinlichkeiten der 6-Clusterlösung dargestellt. Die Clustergröße reicht von $6,8 \%$ bis $39,3 \%$ aller Altersabschnitte. Die Wahrscheinlichkeiten, die mehr als 1,5-mal so groß sind wie die durchschnittlichen Anteile, sind fett gedruckt. Das größte Cluster ist Cluster A mit 39,3\% aller Altersabschnitte. Alle Altersabschnitte dieses Cluster enthalten das Delikt einfacher Diebstahl, andere Delikte sind kaum vorhanden. Gewaltdelikte (Tötungsdelikte, sonstige Sexualdelikte, Raub, schwere Körperverletzung, Körperverletzung, Straftaten gegen die persönliche Freiheit) befinden sich hauptsächlich in Cluster B $(21,2 \%)$, des Weiteren die Delikte Sachbeschädigung, Widerstand gegen die Staatsgewalt, Beleidigung und Hausfriedensbruch. Auch die Delikte Gemeingefährliche Straftaten, Hehlerei, Gefährdung des demokratischen Rechtsstaates, Straftaten gegen die öffentliche Ordnung, Meineid und falsche Verdächtigung kommen in Cluster B überdurchschnittlich häufig vor. Alle zu Cluster C gehörenden Altersabschnitte (17,5\% aller Altersabschnitte) enthalten Betrug. Auch „Fälschung“ und Meineid befinden sich häufig in diesem Cluster. Alle Altersabschnitte des Cluster D (8,3\% aller Altersabschnitte) enthalten ein Betäubungsmitteldelikt, alle anderen Delikte sind kaum vertreten. Cluster E (6,8\%) ist das Cluster mit verschiedenen Straftaten. In diesem Cluster sind alle Delikte überdurchschnittlich häufig vertreten. Die durchschnittliche Anzahl der Delikte pro Altersabschnitt ist in diesem Cluster mit 5 besonders hoch. Alle Altersabschnitte aus Cluster F, enthalten das Delikt Leistungserschleichung. Außerdem befinden sich in diesem Cluster überdurchschnittlich viele Altersabschnitte mit der Deliktskategorie ,sonstige Sexualdelikte“ (hauptsächlich verbotene Prostitution, § 184a).

Tabelle 54: Modellprüfgrößen zur Bestimmung der Clusteranzahl (deutsche Frauen, Kohorte 1970, polizeiliche Registrierungen) 275

\begin{tabular}{lccccccc}
\hline $\begin{array}{l}\text { Cluster- } \\
\text { zahl }\end{array}$ & LL & BIC(LL) & Npar & $\mathrm{L}^{2}$ & $\mathrm{df}$ & $\mathrm{p}$-Wert & $\begin{array}{c}\text { Class. } \\
\text { Err. }\end{array}$ \\
\hline 2 & -18162 & 36699 & 45 & 4984 & 4021 & $8,50 \mathrm{E}-24$ & 0,098 \\
3 & -17872 & 36309 & 68 & 4402 & 3998 & $5,80 \mathrm{E}-06$ & 0,102 \\
4 & -17668 & 36091 & 91 & 3994 & 3975 & 0,41 & 0,103 \\
5 & -17469 & 35885 & 114 & 3597 & 3952 & 1 & 0,094 \\
6 & -17335 & 35809 & 137 & 3330 & 3929 & 1 & 0,093 \\
7 & -17254 & 35839 & 160 & 3168 & 3906 & 1 & 0,149 \\
\hline
\end{tabular}

LL Wert der Log-Likelihood-Funktion

BIC Bayesian Information Criterion siehe Fußnote 235

Npar Anzahl der Parameter

$\mathrm{L}^{2} \quad$ Likelihood ratio chi-squared statistic

df Freiheitsgrade

p-Wert Signifikanzniveau

Class.Err. Classification Errors

275 Die Werte der Tabelle sind mit Latent GOLD berechnet. 
Tabelle 55: Deliktscluster polizeilicher Registrierungen deutscher Frauen der Kohorte 1970: Wahrscheinlichkeiten der Deliktskategorien in den Clustern (in \%)

\begin{tabular}{|c|c|c|c|c|c|c|c|}
\hline \multirow[b]{3}{*}{$\begin{array}{l}\text { Clusterwahrscheinlichkeiten } \pi(\mathrm{k}) \\
\text { in } \%\end{array}$} & \multicolumn{7}{|c|}{ Cluster } \\
\hline & A & $\mathrm{B}$ & $\mathrm{C}$ & $\mathrm{D}$ & E & $\mathrm{F}$ & ges. \\
\hline & 39,3 & 21,2 & 17,5 & 8,3 & 7,0 & 6,8 & 100 \\
\hline \multicolumn{8}{|l|}{$\begin{array}{l}\text { Wahrscheinlichkeiten } \mathbf{p}_{\mathrm{kj}} \\
\text { in } \%\end{array}$} \\
\hline Tötungsdelikte & 0,1 & 0,8 & 0,2 & 0,0 & 1,7 & 0,6 & 0,4 \\
\hline Sonstige Sexualdelikte & 0,2 & 3,1 & 0,0 & 0,6 & 10,8 & 2,9 & 1,7 \\
\hline Raub & 0,4 & 2,4 & 0,0 & 0,9 & 11,7 & 0,4 & 1,6 \\
\hline Schwere Köperverletzung & 1,0 & 15,4 & 1,3 & 0,0 & 18,4 & 1,7 & 5,3 \\
\hline Körperverletzung & 1,6 & 21,4 & 0,9 & 1,7 & 21,7 & 1,9 & 7,1 \\
\hline Str. gg. persönliche Freiheit & 0,7 & 8,3 & 0,8 & 0,4 & 9,8 & 0,3 & 2,9 \\
\hline Einfacher Diebstahl & 100,0 & 8,3 & 28,1 & 2,4 & 86,2 & 22,5 & 53,7 \\
\hline Schwerer Diebstahl & 5,6 & 9,0 & 3,6 & 3,2 & 29,7 & 1,7 & 7,2 \\
\hline Sachbeschädigung & 2,5 & 13,5 & 0,0 & 1,4 & 19,0 & 0,7 & 5,3 \\
\hline Gemeingefährliche Straftaten & 0,1 & 2,6 & 0,1 & 0,3 & 1,2 & 0,0 & 0,7 \\
\hline Hehlerei & 3,7 & 8,6 & 2,1 & 3,9 & 15,2 & 0,1 & 5,0 \\
\hline Betrug & 5,9 & 7,8 & 99,9 & 1,6 & 46,9 & 17,6 & 26,0 \\
\hline Erschleichen von Leistungen & 3,3 & 2,2 & 0,1 & 0,1 & 37,4 & 99,9 & 11,2 \\
\hline Gefährdung dem. Rechtsstaat & 0,0 & 1,1 & 0,0 & 0,0 & 0,5 & 0,0 & 0,3 \\
\hline Widerstand gg. Staatsgewalt & 0,0 & 1,8 & 0,0 & 1,1 & 8,3 & 1,0 & 1,1 \\
\hline Öffentliche Ordnung & 1,7 & 7,6 & 3,3 & 0,7 & 8,1 & 0,8 & 3,5 \\
\hline Hausfriedensbruch & 0,8 & 4,7 & 0,0 & 0,2 & 14,0 & 3,5 & 2,5 \\
\hline Beleidigung & 1,6 & 25,9 & 2,4 & 0,5 & 21,6 & 2,4 & 8,3 \\
\hline Fälschung & 0,9 & 5,1 & 10,5 & 1,7 & 15,2 & 1,7 & 4,6 \\
\hline Meineid & 0,2 & 4,5 & 3,0 & 1,1 & 2,7 & 0,0 & 1,8 \\
\hline falsche Verdächtigung & 1,0 & 5,4 & 1,3 & 0,8 & 7,8 & 0,3 & 2,4 \\
\hline Betäubungsmitteldelikte & 5,8 & 2,3 & 3,7 & 99,9 & 50,1 & 12,3 & 16,0 \\
\hline Durchschnittl. Anzahl der Delikte & 1,3 & 1,6 & 1,7 & 1,2 & 5,0 & 1,7 & 1,7 \\
\hline
\end{tabular}

In Tabelle 56 ist zu sehen, mit welcher Wahrscheinlichkeit ein Delikt einem Cluster angehört. 67,2\% aller Altersabschnitte, die das Delikt Betrug enthalten, befinden sich in Cluster C (Betrug). 12,5\% der Altersabschnitte mit Betrug liegen in Cluster E (verschiedene Straftaten). 99,9\% der Altersabschnitte, die Cluster C angehören, enthalten das Delikt Betrug (vgl. Tabelle 55). In Cluster B (Gewalt) befinden sich 63,8\% der Altersabschnitte mit Körperverletzung und fast ebenso viele mit schwerer Körperverletzung $(61,8 \%)$, Straftaten gegen die persönliche Freiheit (61\%) und 66,5\% der Altersabschnitte mit Beleidigung. Cluster D (BTMDelikte) enthält 52\% aller Altersstreifen mit BTM-Delikten. Umgekehrt enthalten aber alle Altersabschnitte dieses Clusters ein Betäubungsmitteldelikt (vgl. Tabelle 55). In Cluster A (einfacher Diebstahl) befinden sich ebenso nur Altersabschnitte, die einfachen Diebstahl enthalten (vgl. Tabelle 55). 73\% aller Altersabschnitte mit 
einfachem Diebstahl liegen in Cluster A, aber auch 31\% aller Altersabschnitte mit schwerem Diebstahl befinden sich in Cluster A. Cluster E (verschiedene Straftaten) enthält 51\% aller Altersabschnitte mit Raub und ebenso viele mit Widerstand gegen die Staatsgewalt.

Tabelle 56: Wahrscheinlichkeit der Clusterzugehörigkeit bei Deliktsvorkommen in \% (Polizeiliche Registrierung, deutsche Frauen, Kohorte 1970, Baden-Württemberg)

\begin{tabular}{|c|c|c|c|c|c|c|}
\hline \multirow[b]{3}{*}{$\begin{array}{l}\text { Clusterwahrscheinlichkeiten } \pi(\mathrm{k}) \\
\text { in \% }\end{array}$} & \multicolumn{6}{|c|}{ Cluster* } \\
\hline & A & $\mathrm{B}$ & $\mathrm{C}$ & $\mathrm{D}$ & $\mathrm{E}$ & $\mathrm{F}$ \\
\hline & 39,3 & 21,2 & 17,5 & 8,3 & 7,0 & 6,8 \\
\hline \multicolumn{7}{|l|}{ Wahrscheinlichkeiten $\Theta_{\mathrm{kj}}$ in \% } \\
\hline Tötungsdelikte & 10,3 & 41,9 & 9,6 & 0,0 & 27,9 & 10,3 \\
\hline Sonstige Sexualdelikte & 3,8 & 38,6 & 0,0 & 2,7 & 43,5 & 11,3 \\
\hline Raub & 10,8 & 32,3 & 0,0 & 4,7 & $\mathbf{5 0 , 7}$ & 1,6 \\
\hline Schwere Körperverletzung & 7,3 & 61,8 & 4,4 & 0,1 & 24,2 & 2,2 \\
\hline Körperverletzung & 9,0 & 63,8 & 2,3 & 1,9 & 21,3 & 1,8 \\
\hline Str. gg. persönliche Freiheit & 8,8 & 61,0 & 4,8 & 1,1 & 23,8 & 0,6 \\
\hline Einfacher Diebstahl & 73,2 & 3,3 & 9,2 & 0,4 & 11,2 & 2,9 \\
\hline Schwerer Diebstahl & $\mathbf{3 0 , 7}$ & 26,6 & 8,7 & 3,7 & 28,8 & 1,6 \\
\hline Sachbeschädigung & 18,1 & 54,0 & 0,1 & 2,2 & 24,8 & 0,8 \\
\hline Gemeingefährliche Straftaten & 6,2 & 76,8 & 2,4 & 3,2 & 11,5 & 0,0 \\
\hline Hehlerei & 28,7 & 36,3 & 7,3 & 6,4 & 21,1 & 0,2 \\
\hline Betrug & 8,8 & 6,3 & 67,2 & 0,5 & 12,5 & 4,6 \\
\hline Erschleichen von Leistungen & 11,7 & 4,1 & 0,1 & 0,0 & 23,3 & 60,8 \\
\hline Gefährdung dem. Rechtsstaat & 6,1 & 82,1 & 0,0 & 0,0 & 11,9 & 0,0 \\
\hline Widerstand gg. Staatsgewalt & 0,0 & 34,2 & 0,0 & 8,3 & 51,2 & 6,3 \\
\hline Öffentliche Ordnung & 18,8 & 45,7 & 16,3 & 1,8 & 16,0 & 1,5 \\
\hline Hausfriedensbruch & 12,9 & 38,8 & 0,0 & 0,7 & 38,3 & 9,3 \\
\hline Beleidigung & 7,7 & 66,5 & 5,2 & 0,5 & 18,1 & 2,0 \\
\hline Fälschung & 8,0 & 23,4 & 40,1 & 3,0 & 23,0 & 2,6 \\
\hline Meineid & 3,6 & 52,3 & 29,0 & 4,9 & 10,3 & 0,0 \\
\hline falsche Verdächtigung & 16,7 & 47,7 & 9,2 & 2,9 & 22,8 & 0,7 \\
\hline BTM-Delikte & 14,1 & 3,0 & 4,0 & 51,9 & 21,8 & 5,2 \\
\hline
\end{tabular}

* Cluster: A einfacher Diebstahl, B Gewalt, C Betrug, D BTM, E verschiedene Straftaten, F Leistungserschleichung

Wie ,gut" die Clusterlösung ist, d.h. wie gut die Altersabschnitte in die jeweiligen Cluster passen, gibt die A-posteriori-Wahrscheinlichkeit $\mathrm{q}_{\mathrm{ik}}$ (i Altersabschnitt, k Cluster) an. ${ }^{276}$ Die Clusterzuordnung ist sehr gut, sogar nochmals etwas besser als bei den Männern. Nur 4,1\% aller Altersabschnitte haben eine Wahrscheinlichkeit kleiner 50\% bei der Zuordnung zu ihrem optimalen Cluster. Bei $82 \%$ aller Al-

276 Die Berechnung der A-posteriori-Wahrscheinlichkeit ist auf Seite 145 beschrieben. 
tersabschnitte ist die a posteriori Wahrscheinlichkeit größer als $80 \%$. Die Zuordnung ist am besten im Cluster D (Betäubungsmitteldelikte), hier ist die a posteriori Wahrscheinlichkeit bei $90 \%$ aller Altersabschnitte größer als $80 \%$. Fast ebenso gut ist die Zuordnung im Cluster B (Gewalt). Auch bei den anderen Clustern ist die Zuordnung gut.

Abbildung 57 zeigt die Altersverteilung der Cluster. Deutlich zu sehen sind die unterschiedlichen Alterszusammensetzungen der Cluster. Die Altersverteilung der Cluster lässt sich in vier Gruppen einteilen:

- Cluster C (Betrug) hat das Maximum bei den 21- bis 25-Jährigen und die Abnahme hin zu den 26- bis 30-Jährigen ist nur schwach.

- Cluster F (Leistungserschleichung) hat das Maximum bei den 16- bis 20Jährigen.

- Im Cluster A (einfacher Diebstahl) liegt das Maximum bei den 16- bis 20Jährigen, aber auch der Anteil der Altersgruppe 10-15 ist sehr hoch. Mit dem Alter nehmen die Anteile ab.

- Im Cluster B (Gewaltdelikte), D (Betäubungsmitteldelikte) und E (verschiedene Straftaten) sind die Altersabschnitte 16-20, 21-25 und 26-30 relativ gleich stark vertreten. Altersabschnitte von 10- bis 15-Jährigen sind hingegen nur wenige vorhanden. Die Altersverteilung des Gewaltclusters entspricht der A1tersverteilung aller polizeilich registrierten Frauen Baden-Württembergs des Geburtsjahrgangs 1970 (vgl. Abbildung 48).

Abbildung 57: Verteilung der weiblichen Tatverdächtigen auf Cluster nach Altersgruppen

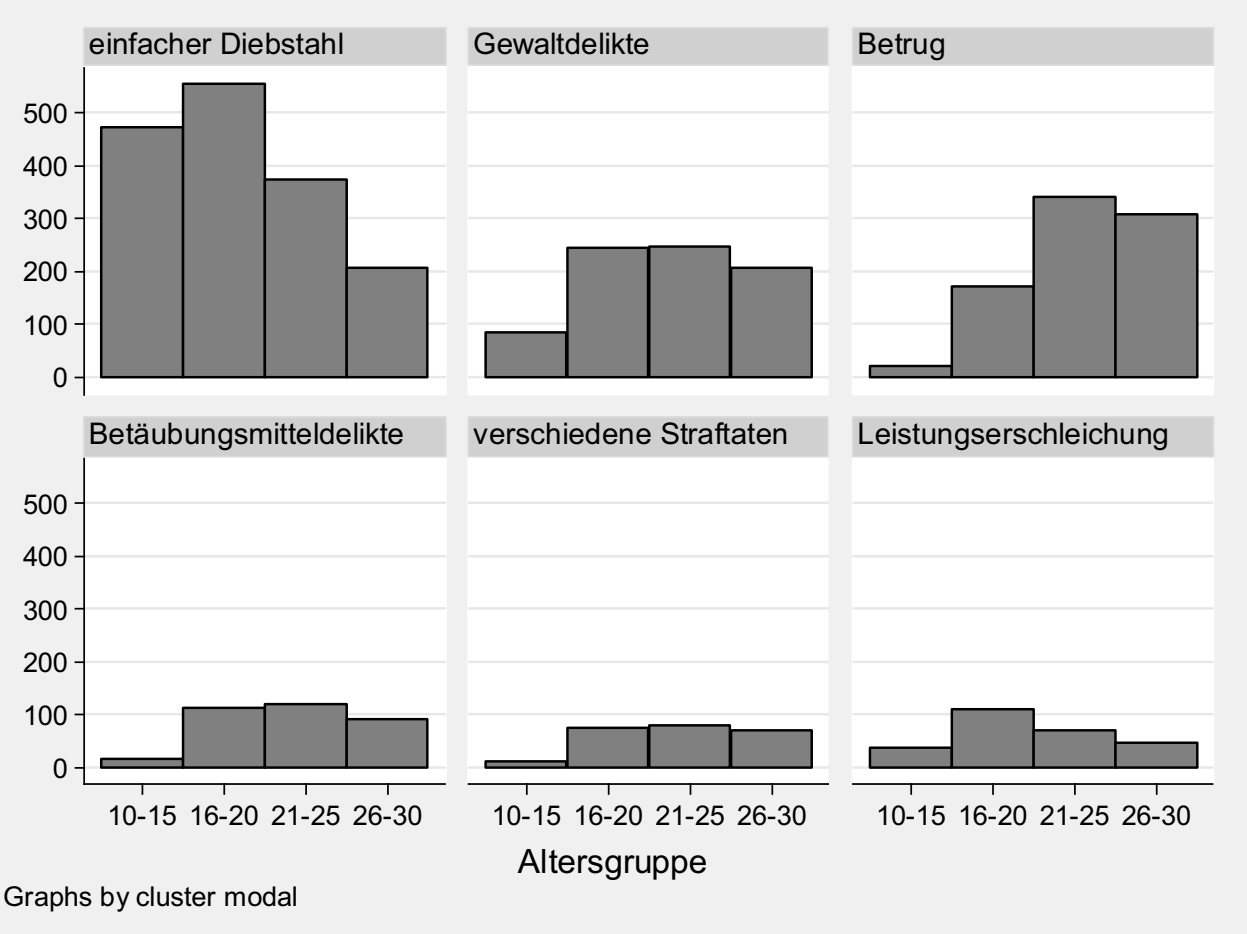


Tabelle 57 gibt einen Überblick über die Deliktszusammensetzung der Cluster, die jeweilige Alterszusammensetzung und die durchschnittliche Anzahl der Delikte pro Altersabschnitt. Alle Altersabschnitte beinhalten im Schnitt 1,7 Delikte pro Altersabschnitt. Besonders auffällig ist auch hier wie schon bei den Männern das Cluster verschiedene Straftaten (E). Es enthält im Schnitt 5 Straftaten. Es gibt also auch bei den Frauen ein Cluster von chronischen Tatverdächtigen. Den größten Anteil in Cluster A (einfacher Diebstahl) bilden die Altersabschnitte von 10- bis 15-Jährigen und von 16- bis 20-Jährigen. Danach nimmt mit dem Alter die Häufigkeit ab. Cluster A ist das einzige Cluster mit einem großen Anteil von Altersabschnitten der Altersgruppe 10 bis 15.

Tabelle 57: Deliktszusammensetzung der Cluster deutscher Frauen der Kohorte 1970

\begin{tabular}{|c|c|}
\hline Cluster & Zusammensetzung \\
\hline \multirow{3}{*}{$\begin{array}{l}\text { A }(31,1 \%) \\
\text { Einfacher Diebstahl }\end{array}$} & Einfacher Diebstahl. \\
\hline & Alter hauptsächlich 10-20 dann abnehmend. \\
\hline & Im Durchschnitt 1,3 Delikte pro Altersabschnitt. \\
\hline \multirow[t]{3}{*}{$\begin{array}{l}\text { B }(21,2 \%) \\
\text { Gewalt }\end{array}$} & $\begin{array}{l}\text { Tötungsdelikte, sonstige Sexualdelikte, Raub, Körperverlet- } \\
\text { zung, Straftaten gegen die persönliche Freiheit, Beleidigung, } \\
\text { Hausfriedensbruch, Sachbeschädigung, Widerstand gegen die } \\
\text { Staatsgewalt, Straftaten gegen die öffentliche Ordnung, Hehle- } \\
\text { rei, Meineid und falsche Verdächtigung. }\end{array}$ \\
\hline & Alter hauptsächlich 16-30. \\
\hline & Im Durchschnitt 1,6 Delikte pro Altersabschnitt. \\
\hline \multirow{3}{*}{$\begin{array}{l}\text { C }(17,5 \%) \\
\text { Betrug }\end{array}$} & Betrug, Fälschung. \\
\hline & Alter hauptsächlich 21-30. \\
\hline & Im Durchschnitt 1,7 Delikte pro Altersabschnitt. \\
\hline \multirow{3}{*}{$\begin{array}{l}\mathrm{D}(8,3 \%) \\
\text { Betäubungsmitteldelikte }\end{array}$} & Betäubungsmitteldelikte. \\
\hline & Alter hauptsächlich 16-30. \\
\hline & Im Durchschnitt 1,2 Delikte pro Altersabschnitt. \\
\hline \multirow{3}{*}{$\begin{array}{l}\text { E }(7,0 \%) \\
\text { Verschiedene Straftaten }\end{array}$} & Alle Delikte kommen überdurchschnittlich häufig vor. \\
\hline & Alter hauptsächlich 16-30. \\
\hline & Im Durchschnitt 5,0 Delikte pro Altersabschnitt. \\
\hline \multirow{3}{*}{$\begin{array}{l}\mathrm{F}(6,8 \%) \\
\text { Erschleichen von } \\
\text { Leistungen }\end{array}$} & $\begin{array}{l}\text { Erschleichen von Leistungen und sonstige Sexualdelikte } \\
\text { (verbotene Prostitution). }\end{array}$ \\
\hline & Alter hauptsächlich 16-20, dann abnehmend. \\
\hline & Im Durchschnitt 1,7 Delikte pro Altersabschnitt. \\
\hline
\end{tabular}

Vergleicht man die Ergebnisse der Clusteranalyse der Frauen mit den Ergebnissen der Männer, zeigt sich, dass fünf Cluster der Männer auch bei den Frauen vorhanden sind, nur das Cluster Leistungserschleichung fehlt bei den Männern. Dafür gibt es bei den Männern ein Cluster schwerer Diebstahl. Die Altersverteilung der Cluster einfacher Diebstahl, Gewaltdelikte und Betrug stimmen bei Männern und Frauen überein. Im Cluster Betäubungsmitteldelikte und im Cluster verschiedene Straftaten unterscheiden sich die Altersverteilungen bei Männern und Frauen. Im 
Cluster Betäubungsmitteldelikte ist bei den Frauen die Altersgruppe 16-20 gleich stark wie die Altersgruppe 21-25. Bei den Männern ist die Altersgruppe 21-25 deutlich stärker vertreten als die Altersgruppe 16-20. Im Cluster verschiedene Straftaten ist bei den Männern ein Maximum in der Altersgruppe 16-20 sichtbar. Dies gibt es bei den Frauen nicht. Hier sind die Altersgruppen 16-20 und 21-25 gleich stark vertreten. Aber sowohl bei den Männern wie bei den Frauen ist anhand der großen durchschnittlichen Anzahl der Delikte pro Altersabschnitt im Cluster verschiedene Straftaten sichtbar, dass sich hier die chronischen Straftäter versammeln. Unterschiede zwischen Männern und Frauen gibt es im Cluster Gewaltdelikte insofern, dass bei den Frauen deutlich mehr Delikte überdurchschnittlich häufig auftreten, als bei den Männern. Darunter sind auch Delikte, bei denen ansonsten kein Zusammenhang mit Gewaltdelikten besteht. Der Hauptunterschied ist im Cluster des schweren Diebstahls bei den Männern zu sehen. Schwerer Diebstahl ist, wie auch schon in Tabelle $14 \mathrm{zu}$ sehen war, ein „Männerdelikt“.

Vergleicht man die Ergebnisse der polizeilichen Registrierungen mit den Ergebnissen der justiziellen Registrierungen, ist darauf hinzuweisen, dass Verkehrsdelikte in den Polizeidaten nicht registriert sind und damit auch deutlich weniger fahrlässige Körperverletzungen, da dieses Delikt sehr häufig in Zusammenhang mit einem Verkehrsunfall auftritt. Somit ist das große Cluster Verkehrsdelikte und das Cluster fahrlässige Körperverletzung nur bei justiziellen Registrierungen möglich. Übrig bleiben vier Cluster bei den justiziellen Registrierungen, die mit den Clustern der polizeilichen Registrierungen verglichen werden können. Die Güte der polizeilichen und justiziellen 6-Clusterlösung stimmt überein. Deshalb ist es sinnvoll diese beiden Lösungen zu vergleichen. Zusätzlich wird aber auch die polizeiliche 4-Clusterlösung betrachtet und mit der justiziellen Lösung verglichen. Die polizeiliche 4-Clusterlösung ergibt ein Cluster mit verschiedenen Straftaten, ein Cluster in dem alle Altersabschnitte das Delikt einfacher Diebstahl enthalten, ein Cluster in dem alle Abschnitte Betrug enthalten, überdurchschnittlich auch „Fälschung" und ein Cluster in dem alle Abschnitte mindestens ein Betäubungsmitteldelikt aufweisen und überdurchschnittlich auch „sonstige Sexualdelikte“ (verbotene Prostitution). Somit stimmen bei polizeilichen und justiziellen Registrierungen die Cluster einfacher Diebstahl und Betrug überein. Das Cluster Betäubungsmitteldelikte besteht bei den polizeilichen Registrierungen hauptsächlich aus Betäubungsmitteldelikten. Bei den justiziellen Registrierungen setzt sich dieses Cluster aus weiteren Delikten zusammen. Im justiziellen Cluster sind Delikte, die zur Beschaffungskriminalität zu rechnen sind, im selben Cluster. Das vierte Cluster der justiziellen Registrierungen ist das Cluster der Gewaltdelikte.

Vergleicht man die polizeiliche mit der justiziellen 6-Clusterlösung ${ }^{277}$ der Frauen, so zeigt sich, dass die drei Cluster einfacher Diebstahl, Betrug und Gewalt übereinstimmen, wobei bei den polizeilichen Registrierungen im Gewaltcluster

277 Siehe Tabelle 45, Abbildung 40 und Tabelle 47. 
mehr Delikte überdurchschnittlich häufig vorkommen. Im Unterschied zum Gewaltcluster der justiziellen Registrierungen enthält das polizeiliche Gewaltcluster auch die Delikte Hehlerei, Straftaten gegen die öffentliche Ordnung, Meineid und falsche Verdächtigung überdurchschnittlich häufig. Das Cluster Betäubungsmitteldelikte ist, wie oben beschrieben, bei den polizeilichen Registrierungen ein reines Betäubungsmittelcluster, bei den justiziellen Registrierungen jedoch ein Betäubungsmittelcluster in Kombination mit Beschaffungskriminalität. Die Cluster verschiedene Straftaten und Leistungserschleichung ergeben sich bei der optimalen Lösung der polizeilichen Registrierungen, nicht aber bei der Lösung der justiziellen Registrierungen. Das polizeiliche Cluster verschiedene Straftaten, welches die chronischen Straftäter enthält, entspricht insofern dem justiziellen Gewaltcluster, da dieses Cluster bei den justiziellen Registrierungen die Mehrfachtäter umfasst.

Beim Vergleich der Altersverteilung der Cluster sind die unterschiedlichen Altersgruppierungen zu beachten. ${ }^{278}$ Die Altersverteilung der Cluster stimmt überein bei Berücksichtigung der unterschiedlichen Alterseinteilung.

\subsubsection{Spezialisierung, Wechsel und Abbruch im Lebenslauf der Frauen}

Des Weiteren wird mit den Ergebnissen der Clusteranalyse die kriminelle Karriere der polizeilich registrierten Frauen untersucht. Dazu werden Ergebnisse zur Spezialisierung und zur kriminellen Laufbahn vorgestellt. Spezialisierung bezieht sich hier auf die Clusterzugehörigkeit. Eine Person ist spezialisiert, wenn es von einem Altersabschnitt zum nächsten keinen Clusterwechsel gibt, d.h. wenn die Deliktsstruktur einer Altersgruppe der Deliktsstruktur der nächsten Altersgruppe entspricht. In Tabelle 58 sind die Spezialisierungsanteile der Frauen, bezogen auf die Clusterzugehörigkeit, dargestellt. Betrachtet werden hier nur Frauen, die in einer späteren Altersgruppe erneut polizeilich registriert sind. Die prozentualen Anteile sind von einer Altersgruppe zur nächsten berechnet. Das N in der dritten Spalte von links gibt an, wie viele Personen in der ,,von“ Altersgruppe sind und in einer späteren Altersgruppe wieder registriert sind. Die Häufigkeiten in Klammern (N) geben an, wie viele Personen im jeweiligen Cluster in der „,von“ Altersgruppe sind und in einer späteren Altersgruppe wieder eine Registrierung haben. Das $\mathrm{N}$ ist hier, wie auch schon bei den justiziell registrierten Frauen, relativ klein. Die Spezialisierungsanteile im Cluster A (einfacher Diebstahl) sind konstant bei etwa 30\%. Im Cluster B (Gewaltdelikte) sind die Spezialisierungsanteile in den Altersgruppen 1015 und $16-20$ bei $20 \%$, in der Altersgruppe 21-25 aber mit $38 \%$ deutlich höher. In den Clustern C (Betrug), D (Betäubungsmitteldelikte), E (verschiedene Straftaten) und $\mathrm{F}$ (Leistungserschleichung) sind in der Altersgruppe 10 bis 15 die Häufigkeit (N) sehr klein und deshalb die Spezialisierungsrate nicht signifikant, da der Fehler zu groß ist. Im Cluster F ist auch die Spezialisierungsrate der Altersgruppe 21-25 nicht signifikant. Im Cluster C (Betrug) ist die Spezialisierungsrate in der Alters-

278 Alterseinteilung bei den polizeilichen Registrierungen: 10-15, 16-20, 21-25, 26-30 Jahre, bei den justiziellen Registrierungen: 14-18, 19-23, 24-28, 29-33 Jahre. 
gruppe der 16- bis 20-Jährigen und der 21-25-Jährigen bei 50\% und damit sehr hoch. Im Cluster D (Betäubungsmitteldelikte) liegt die Spezialisierungsrate in der Altersgruppe 16-20 und 21-25 zwischen 30\% und 40\%. Von einer Zunahme der Spezialisierungsrate kann nicht gesprochen werden, da der Fehler der Spezialisierungsrate hier $6 \%$ beträgt. Im Cluster E (verschiedene Straftaten) ist der Spezialisierungsgrad in der Altersgruppe 16-20 und 21-25 knapp unter 40\%. Den höchsten Spezialisierungsanteil gibt es im Cluster C (Betrug), 56\% aller 21- bis 25-Jährigen aus Cluster $\mathrm{C}$, die im Alter von 26 bis 30 wieder polizeilich registriert sind, sind wieder im Cluster C. Eine Zunahme der Spezialisierungsrate mit dem Alter, wie sie bei den Männern beobachtet werden kann, zeigt sich bei den Frauen nicht. Nur im Cluster Gewaltdelikte steigt die Spezialisierungsrate von 22\% bei der Altersgruppe der 16- bis 20 -Jährigen auf $38 \%$ bei den 21 - bis 25 -Jährigen.

Tabelle 58: Spezialisierungsanteile in \%: Deutsche Frauen der Kohorte 1970 (polizeiliche Registrierungen) ${ }^{279}$

\begin{tabular}{|c|c|c|c|c|c|c|c|c|}
\hline & & & \multicolumn{6}{|c|}{ Cluster } \\
\hline \multicolumn{2}{|c|}{ Altersgruppen } & & $\mathrm{A}$ & $\mathrm{B}$ & $\mathrm{C}$ & $\mathrm{D}$ & $\mathrm{E}$ & $\mathrm{F}$ \\
\hline von & bis & $\begin{array}{c}\mathrm{N}(,, \text { Von“ } \\
\text { ohne } \\
\text { Abbrecher) }\end{array}$ & $\begin{array}{l}\text { Einfacher } \\
\text { Diebstahl } \\
\text { (N) }\end{array}$ & Gewalt & $\begin{array}{l}\text { Betrug } \\
\text { (N) }\end{array}$ & $\begin{array}{l}\text { Betäu- } \\
\text { bungsmit- } \\
\text { teldel. } \\
\text { (N) }\end{array}$ & $\begin{array}{l}\text { Verschie- } \\
\text { dene } \\
\text { Straftaten } \\
\text { (N) }\end{array}$ & $\begin{array}{l}\text { Leistung- } \\
\text { serschlei- } \\
\text { chung } \\
\text { (N) }\end{array}$ \\
\hline $10-15$ & $16-20$ & 456 & $31(320)$ & $20(70)$ & $8^{*}(12)$ & $15 *(13)$ & $29 *(7)$ & $27 *(34)$ \\
\hline $16-20$ & $21-25$ & 705 & $29(278)$ & $22(130)$ & 49 ( 98) & $30(79)$ & $38(53)$ & $24(67)$ \\
\hline $21-25$ & $26-30$ & 484 & 33 (128) & $38(87)$ & $56(136)$ & $41(56)$ & 37 (49) & $18 *(28)$ \\
\hline
\end{tabular}

* nicht signifikant

Im Folgenden wird das Ergebnis der Clusteranalyse dazu benutzt, kriminelle Laufbahnen zu identifizieren. Abbildung 58 gibt einen Überblick über die Deliktsübergänge der weiblichen Tatverdächtigen der Altersgruppe 21-25. 1231 weibliche Tatverdächtige haben mindestens eine Registrierung in der Altersspanne 21-25. Diese teilen sich auf in 31\%, die zum Cluster einfacher Diebstahl gehören, 20\% gehören zum Cluster Gewaltdelikte, 28\% zum Cluster Betrug, 10\% zum Cluster Betäubungsmitteldelikte und jeweils $6 \%$ zu den Clustern verschiedene Straftaten und Leistungserschleichung. Von den weiblichen Tatverdächtigen die im Alter von 21 bis 25 mindestens einmal polizeilich registriert sind, haben $84 \%$ im Alter von 10 bis 15 keine Registrierung, 53\% im Alter von 16 bis 20 und 61\% haben im Alter von 26 bis 30 keine Registrierung. 17\% der 21- bis 25-Jährigen mit Registrierung gehören im Alter 16 bis 20 zum Cluster einfacher Diebstahl, 9\% zum Cluster Gewaltdelikte und 7\% zum Cluster Betrug. 12\% der 21 - bis 25-Jährigen mit Registrierung gehören in der Altersgruppe 26-30 zum Cluster Betrug, 9\% zum Cluster einfacher Diebstahl und 8\% zum Cluster Gewaltdelikte.

279 Siehe Fußnote 254. 
Abbildung 58: Deliktsübergänge weiblicher Tatverdächtiger der Altersgruppe 21-25

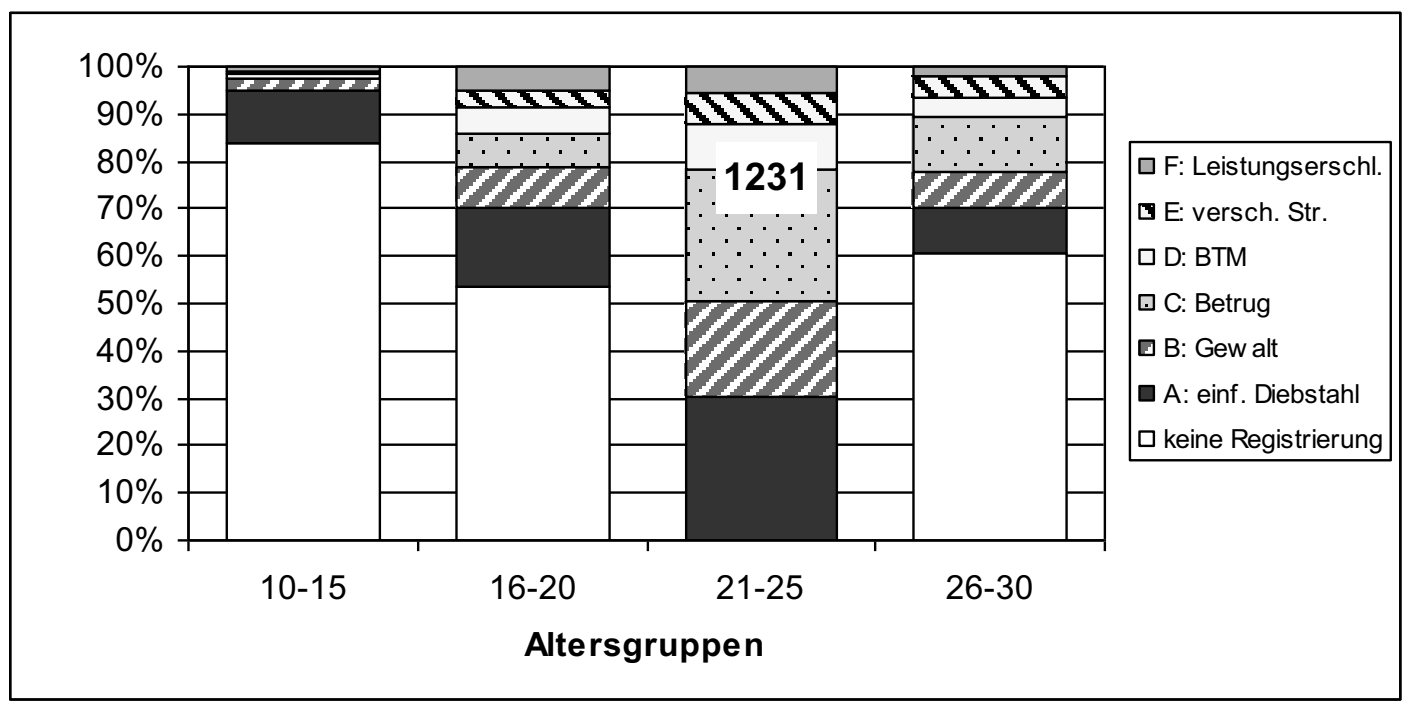

Die Abbildungen 59 bis 64 zeigen die Laufbahn der Frauen getrennt nach der Clusterzugehörigkeit. Im Balken der Altersgruppe 21 bis 25 ist jeweils die Zahl der Frauen angegeben, die dem jeweiligen Cluster zugeordnet sind. Die linken Balken geben, ausgehend von der Gruppe der 21- bis 25-Jährigen, die Clusterzugehörigkeit der 10- bis 15-Jährigen und der 16- bis 20-Jährigen an bzw. die Angabe, dass es im jeweiligen Alter keine Registrierung gab. Der rechte Balken enthält dieselben Informationen für die nachfolgende Altersgruppe. Besonders groß, sowohl in den Altersgruppen davor wie in der Altersgruppe danach, ist der Anteil derjenigen Frauen, die keine Registrierung haben. In der Altersgruppe der 10- bis 15-Jährigen liegt der Anteil der „Nichtregistrierten“ je nach Clusterzugehörigkeit im Alter von 21 bis 25 , zwischen $75 \%$ und $88 \%$, in der Altersgruppe 16 bis 20 zwischen $32 \%$ und $62 \%$ und in der Altersgruppe danach, bei den 26- bis 30-Jährigen, liegt der Anteil der „Nichtregistrierten“ zwischen 38\% und 66\%. Überdurchschnittlich viele „Nichtregistrierte“ in der Altersgruppe danach gibt es bei denjenigen Frauen, die im Alter von 21 bis 25 dem Cluster A (einfacher Diebstahl, Abbildung 59) zugeordnet sind, zwei Drittel der Frauen sind in der Altersphase 26 bis 30 nicht registriert. Vorher, im Alter von 16 bis 20, sind 54\% nicht polizeilich registriert, das entspricht dem Durchschnitt. Auch beim Cluster B (Gewaltdelikte, Abbildung 60) sind in der Altersgruppe 26 bis 30 überdurchschnittlich viele nichtregistriert. Beim Cluster E (verschiedene Straftaten, Abbildung 63) gibt es in den Altersgruppen davor und danach deutlich weniger Nichtregistrierte als im Durchschnitt. Große Anteile von „Spezialisierten“, d. h. von Frauen, die während ihrer kriminellen Karriere nicht das Cluster wechseln, zeigen sich beim Cluster $\mathrm{C}$ (Betrug, Abbildung 61), Cluster D (Betäubungsmitteldelikte, Abbildung 62), Cluster E (verschiedene Straftaten, Abbildung 63) und Cluster F (Leistungserschleichung, Abbildung 64). Von denjenigen Frauen, die im Alter von 21 bis 25 dem Cluster Betäubungsmitteldelikte zugeordnet sind (120 Frauen), sind im Alter von 16 bis $2020 \%$ auch dem Cluster Betäubungsmitteldelikte zugeordnet, im Alter von 26 bis 30 19\%. Diese 
Anteile liegen deutlich über dem durchschnittlichen Anteil vom BetäubungsmittelCluster (siehe Abbildung 58). Dagegen ist in der Altersgruppe 10 bis 15 das Betäubungsmittelcluster nicht vertreten. Dasselbe gilt für das Cluster Betrug. 14\% der Frauen, die im Alter 21 bis 25 dem Betrugscluster angehörten, gehörten schon im Alter von 16 bis 20 dem Betrugscluster an, 22\% in der Altersgruppe danach. Auch hier ist das Betrugscluster in der Altersgruppe 10 bis 15 nicht vorhanden. Bei den Deliktsübergängen des Clusters Leistungserschleichung zeigt sich, dass der Anteil des Clusters Leistungserschleichung in der Altersgruppe 16 bis 20 groß ist (23\%). Das Cluster der verschiedenen Straftaten, welches auch bei den Frauen, wie schon beschrieben, das Cluster der chronischen Straftäter ist, hat einen großen Anteil „Spezialisierte“. 25\% der Frauen die im Alter von 21 bis 25 dem Cluster verschiedene Straftaten angehören, waren schon im Alter von 16 bis 20 in diesem Cluster. 23\% sind auch im Alter 26 bis 30 im Cluster verschiedene Straftaten. Hier zeigt sich, wie auch schon bei den Männern, dass die Wahrscheinlichkeit groß ist, dass chronische Straftäter chronische Straftäter bleiben. Beim Cluster einfacher Diebstahl entsprechen die Clusteranteile von einfachem Diebstahl in der Altersgruppe davor und danach dem Durchschnitt.

Abbildung 59: Deliktsübergänge weiblicher Tatverdächtiger des Clusters A (einfacher Diebstahl) im Alter 21 bis 25

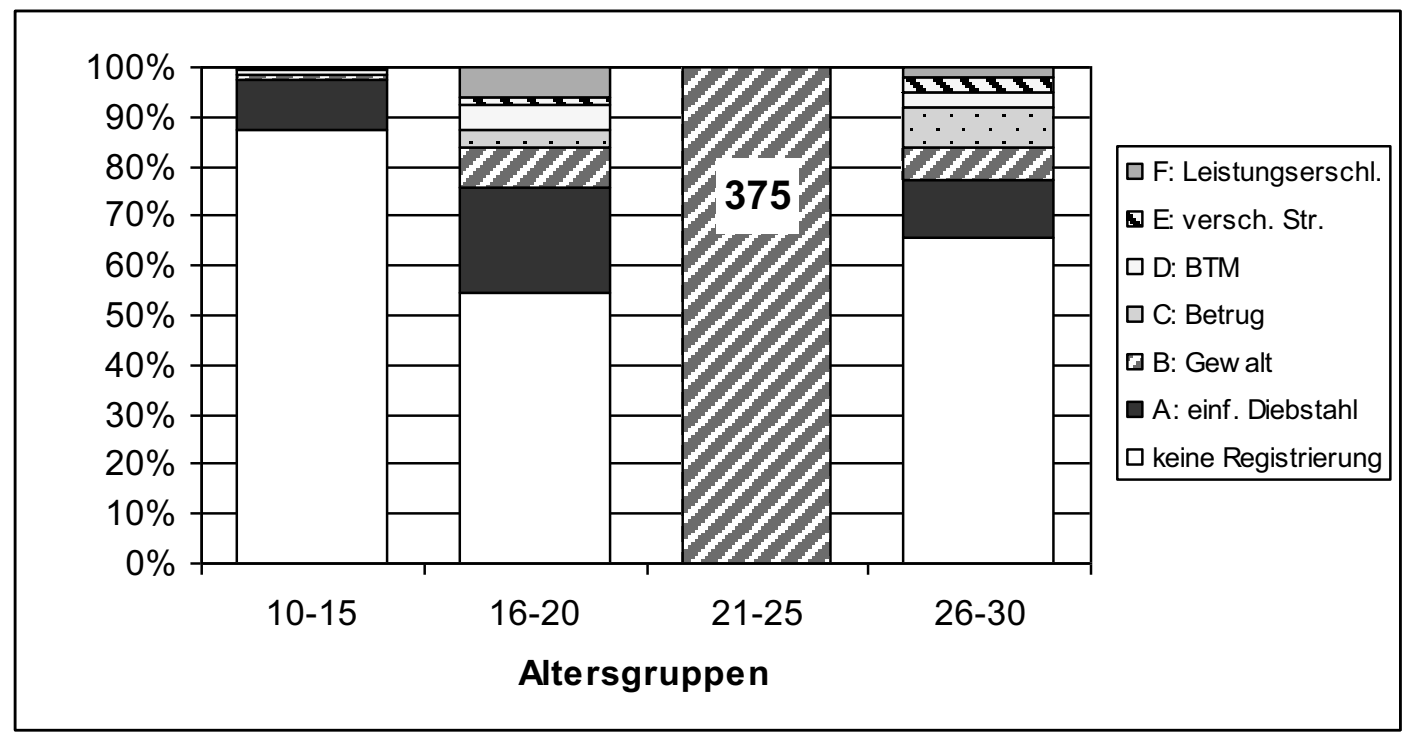

Die Ergebnisse der Clusteranalyse bestätigen großteils die Ergebnisse der Multidimensionalen Skalierung (Abbildung 8). Das Ergebnis der Multidimensionalen Skalierung zeigt, dass die Delikte Betrug, BTM-Delikte, Leistungserschleichung und einfacher Diebstahl weit auseinander liegen und damit unähnlich sind. Die Delikte einfacher Diebstahl, BTM-Delikte, Leistungserschleichung und Betrug kommen selten gleichzeitig innerhalb der polizeilich registrierten kriminellen Karriere von Frauen vor. Diese Delikte bilden auch eigene Cluster. Die Gewaltdelikte liegen eng beieinander, sind damit ähnlich und bilden auch ein Cluster. 
Im Gegensatz zu den justiziell registrierten Frauen ergibt sich bei der 6-Clusterlösung der polizeilich registrierten Frauen wie bei den Männern ein Cluster der chronisch delinquenten Frauen.

Abbildung 60: Deliktsübergänge weiblicher Tatverdächtiger des Clusters B (Gewaltdelikte) im Alter 21 bis 25

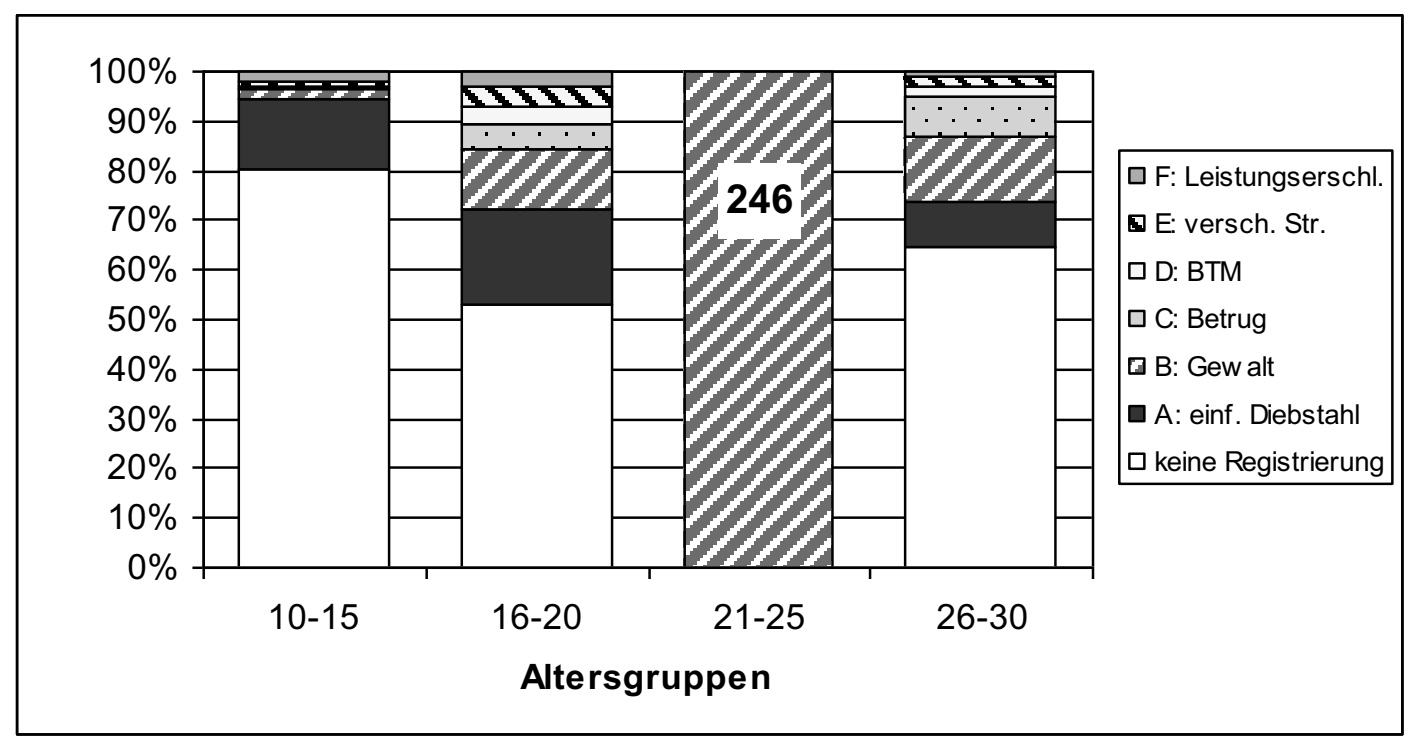

Abbildung 61: Deliktsübergänge weiblicher Tatverdächtiger des Clusters $C$ (Betrug) im Alter 21 bis 25

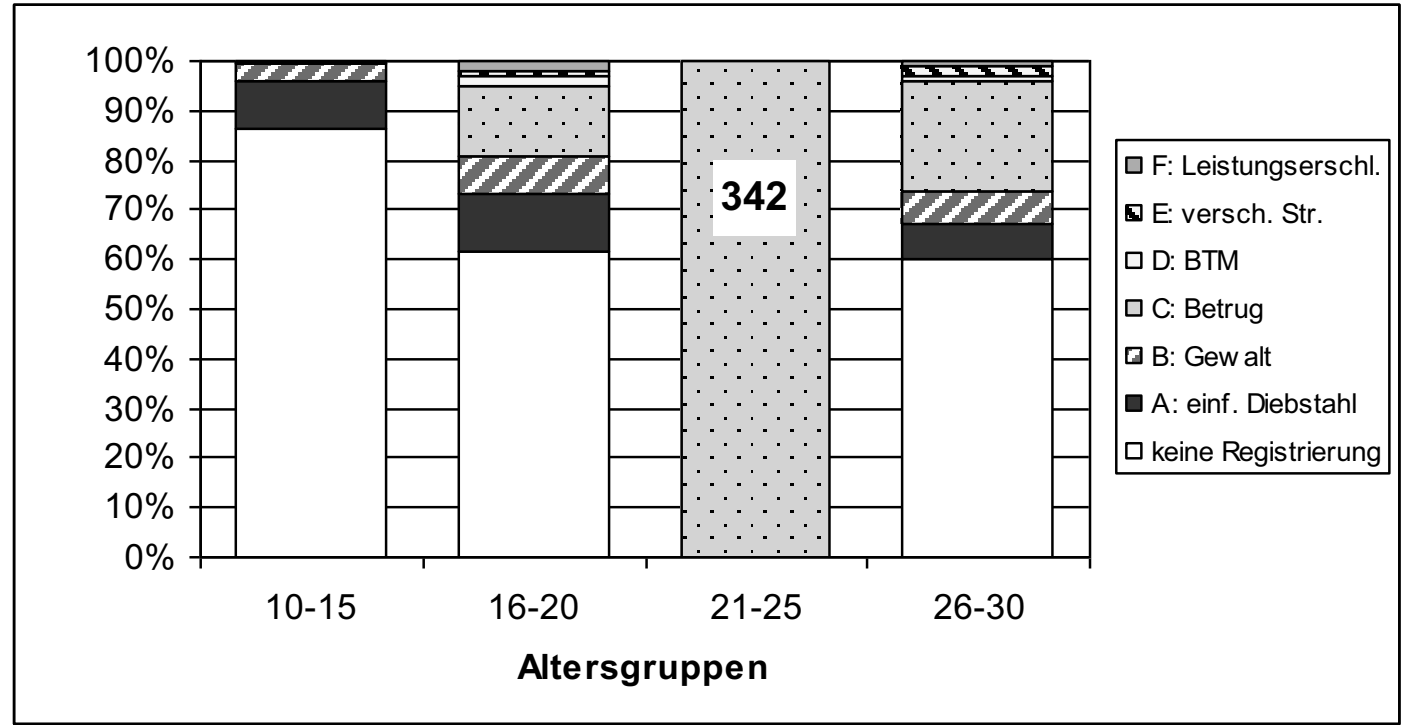


Abbildung 62: Deliktsübergänge weiblicher Tatverdächtiger des Clusters D (Betäubungsmitteldelikte) im Alter 21 bis 25

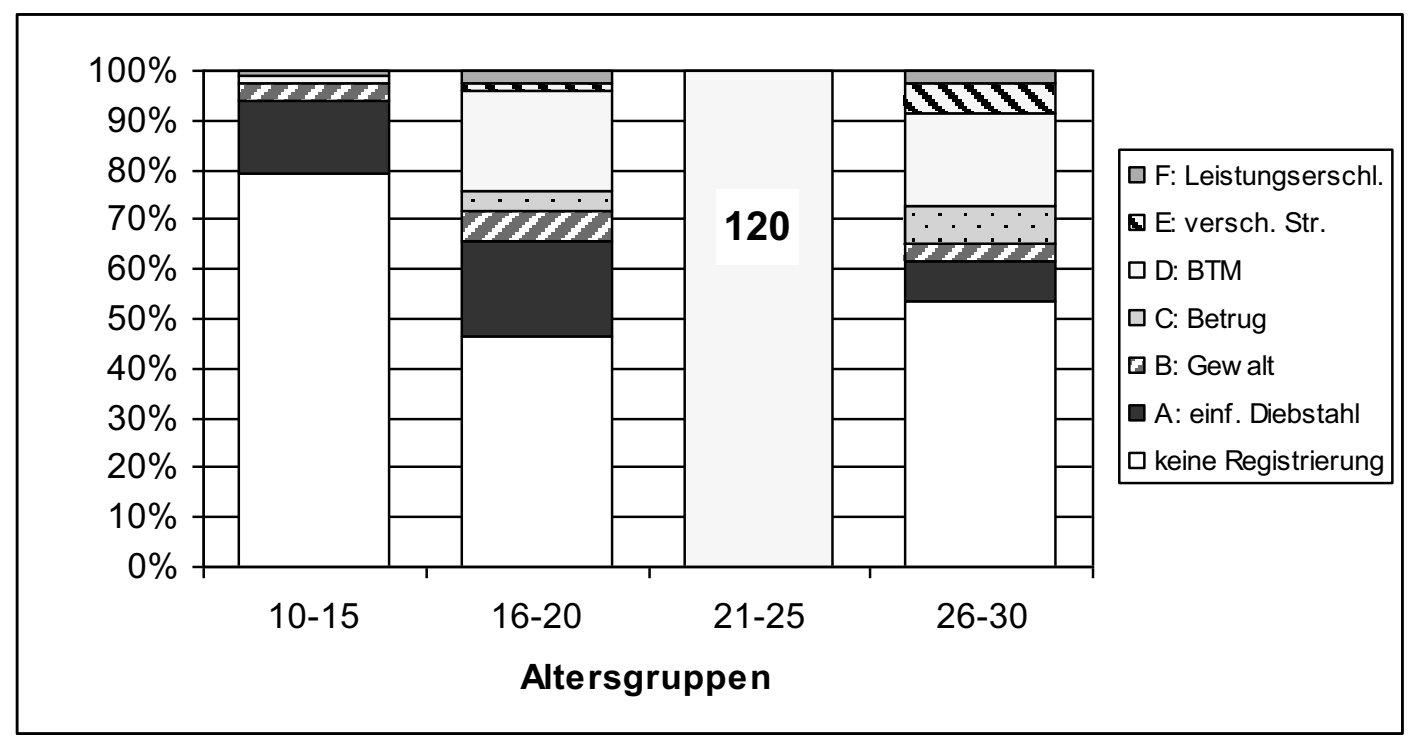

Abbildung 63: Deliktsübergänge weiblicher Tatverdächtiger des Clusters E (verschiedene Straftaten) im Alter 21 bis 25

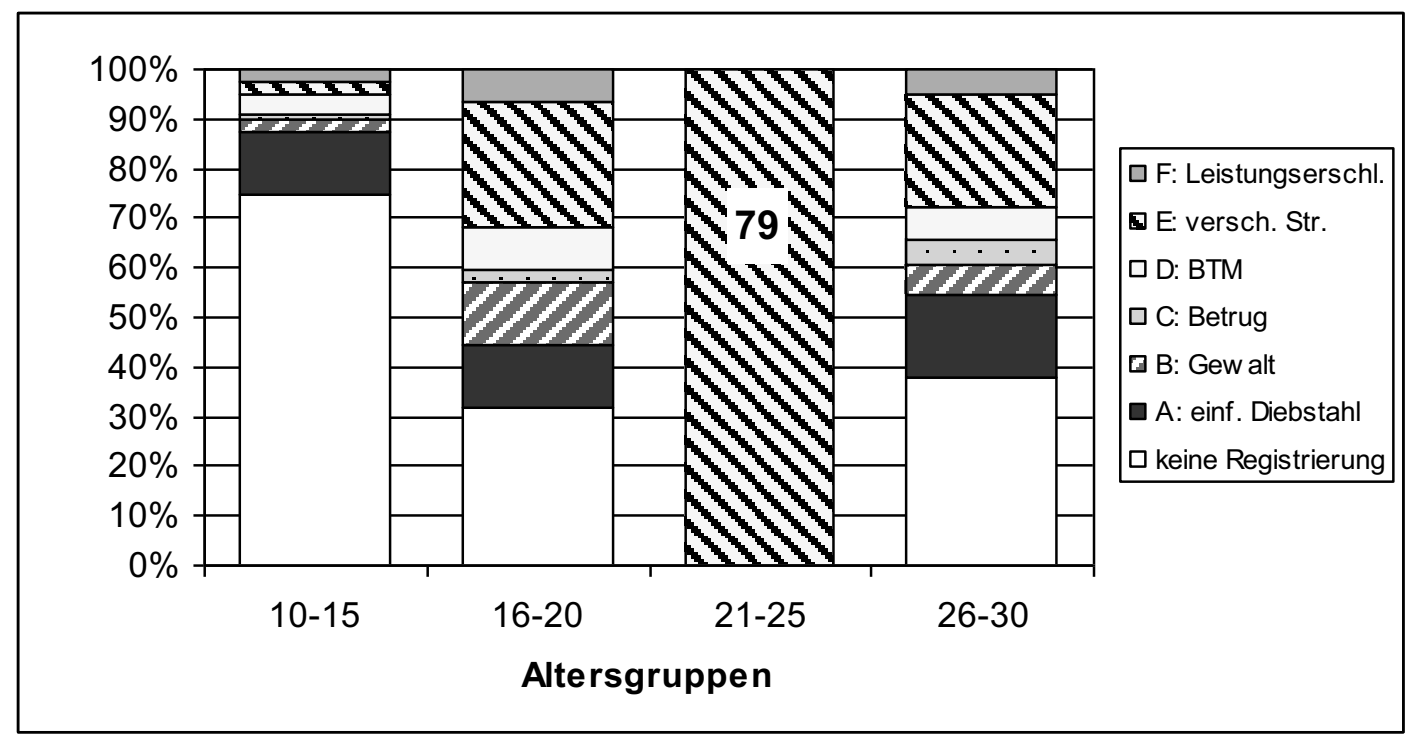


Abbildung 64: Deliktsübergänge weiblicher Tatverdächtiger des Clusters F (Erschleichen von Leistungen) im Alter 21 bis 25

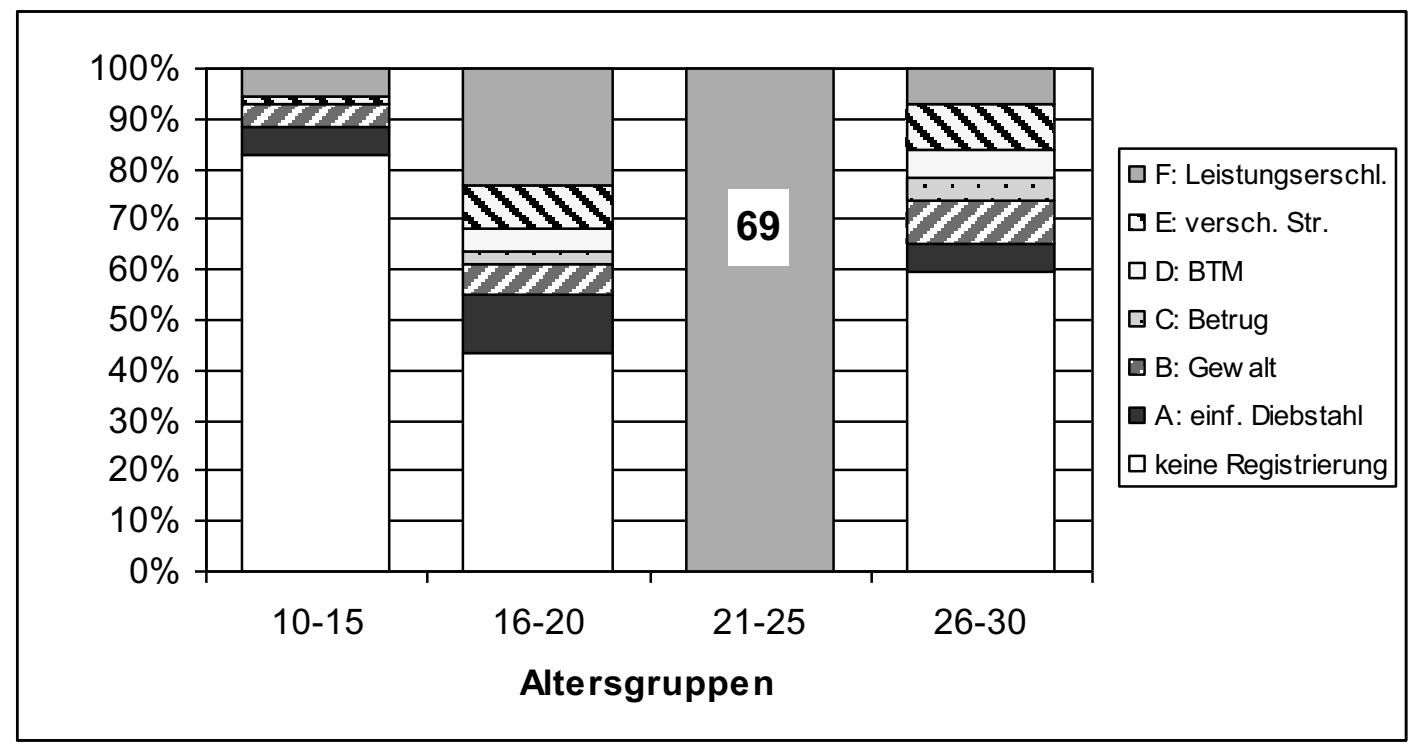

\subsection{Zusammenfassung der Ergebnisse}

In allen vier Gruppen des Geburtsjahrgangs 1970, sowohl bei polizeilich wie bei justiziell registrierten deutschen Männern und Frauen, stimmt die Güte der 6-Clusterlösung überein. In Tabelle 59 ist der Vergleich der 6-Clusterlösung der vier Gruppen dargestellt. In allen vier Gruppen ergaben sich bei der 6-Clusterlösung die Cluster ,einfacher Diebstahl“, „Gewaltdelikte“ und „Betrug“. Am besten stimmen die Cluster „Betrug“ bei den vier Gruppen überein. Alle Altersabschnitte der Betrugscluster enthalten Betrug und außerdem sind „Fälschung“ und Meineid überzufällig häufig vertreten. Die Deliktskategorien Betrug, Fälschung und Meineid sind ähnlich, d.h. Delikte dieser Kategorien sind häufig zusammen bei Personen offiziell registriert. Auch das Gewaltcluster war unter der 6-Clusterlösung aller vier Gruppen. Dies bestätigt die Ähnlichkeit von Gewaltdelikten zusammen mit Beleidigung, Sachbeschädigung, Widerstand gegen die Staatsgewalt und Hausfriedensbruch.

Bei den polizeilich registrierten Männern und Frauen und bei den justiziell registrierten Frauen enthalten alle Altersabschnitte des Clusters „einfacher Diebstahl“ das Delikt einfacher Diebstahl, während andere Delikte sehr selten in diesem Cluster vorkommen. Bei den justiziell registrierten Männern enthalten die Hälfte der Altersabschnitte des Clusters ,einfacher Diebstahl“ das Delikt einfacher Diebstahl, aber überdurchschnittlich häufig kommen auch Betäubungsmitteldelikte, schwerer Diebstahl, Hehlerei, Leistungserschleichung und „sonstige Sexualdelikte“ in diesem Cluster vor. Insofern ist dieses Cluster eine Mischung der Cluster ,einfacher Diebstahl“" und dem in dieser Gruppe nicht vorhandenem Betäubungsmittelcluster. Das Cluster „einfacher Diebstahl“ der justiziell registrierten deutschen Männer kann auch als Betäubungsmittelcluster mit Beschaffungskriminalität bezeichnet werden. 
Bei den polizeilich Registrierten ist das Betäubungsmittelcluster hingegen ein reines Betäubungsmittelcluster. Bei den justiziell registrierten Frauen besteht das Betäubungsmittelcluster aus einer Mischung aus Betäubungsmitteldelikten und Delikten, die zur Beschaffungskriminalität zu zählen sind.

Tabelle 59: Vergleich der 6-Clusterlösungen

\begin{tabular}{|c|c|c|c|c|c|c|c|c|c|}
\hline & & \multicolumn{8}{|c|}{ Cluster } \\
\hline \multirow{2}{*}{$\begin{array}{l}\text { Deutsche } \\
\text { Männer } \\
\text { Kohorte } \\
1970\end{array}$} & \multirow{2}{*}{$\begin{array}{l}\text { BZR } \\
\text { PAD }\end{array}$} & $\begin{array}{l}\mathrm{Be}- \\
\text { trug }\end{array}$ & $\begin{array}{l}\text { Ge- } \\
\text { walt }\end{array}$ & $\begin{array}{l}\text { Einf. } \\
\text { Diebst. }^{\text {a }}\end{array}$ & \multicolumn{3}{|c|}{$\begin{array}{l}\text { Versch. } \\
\text { Str. }\end{array}$} & \multirow[t]{2}{*}{$\begin{array}{l}\text { Ver- } \\
\text { kehr }\end{array}$} & \multirow[t]{2}{*}{$\begin{array}{l}\text { Fahrl. } \\
\text { KV }\end{array}$} \\
\hline & & $\begin{array}{l}\mathrm{Be}- \\
\text { trug }\end{array}$ & $\begin{array}{l}\text { Ge- } \\
\text { walt }\end{array}$ & $\begin{array}{l}\text { Einf. } \\
\text { Diebst. }\end{array}$ & BTM & $\begin{array}{l}\text { Versch. } \\
\text { Str. }\end{array}$ & $\begin{array}{l}\text { Schw. } \\
\text { Diebst. }\end{array}$ & & \\
\hline $\begin{array}{l}\text { Deutsche } \\
\text { Frauen }\end{array}$ & BZR & $\begin{array}{l}\mathrm{Be}- \\
\text { trug }\end{array}$ & $\begin{array}{l}\text { Ge- } \\
\text { walt }\end{array}$ & $\begin{array}{l}\text { Einf. } \\
\text { Diebst. }\end{array}$ & $\mathrm{BTM}^{\mathrm{b}}$ & & & $\begin{array}{l}\text { Ver- } \\
\text { kehr }\end{array}$ & $\begin{array}{l}\text { Fahrl. } \\
\text { KV }\end{array}$ \\
\hline $\begin{array}{l}\text { Kohorte } \\
1970\end{array}$ & PAD & $\begin{array}{l}\mathrm{Be}- \\
\text { trug }\end{array}$ & $\begin{array}{l}\text { Ge- } \\
\text { walt }\end{array}$ & $\begin{array}{l}\text { Einf. } \\
\text { Diebst. }\end{array}$ & BTM & $\begin{array}{l}\text { Versch. } \\
\text { Str. }\end{array}$ & $\begin{array}{l}\text { Leistung- } \\
\text { serschl. }\end{array}$ & & \\
\hline
\end{tabular}

a auch sonstige Sexualdelikte, schwerer Diebstahl, Hehlerei, Leistungserschleichung und Betäubungsmitteldelikte sind überdurchschnittlich häufig in diesem Cluster

b auch sonstige Sexualdelikte, schwerer Diebstahl, Hehlerei, Leistungserschleichung, Sachbeschädigung, gemeingefährliche Straftaten, Gefährdung des demokratischen Rechtsstaates, Straftaten gegen die öffentliche Ordnung, Hausfriedensbruch, Meineid, falsche Verdächtigung und Vollrausch sind überdurchschnittlich häufig in diesem Cluster

Die Cluster „verschiedene Straftaten“ der Männer und der polizeilich registrierten Frauen enthalten chronische Straftäter. Alle Delikte sind überzufällig häufig vertreten. Die durchschnittliche Anzahl der Delikte pro Altersabschnitt ist in diesem Cluster deutlich überdurchschnittlich. Bei den justiziell registrierten Frauen ist das Cluster verschiedene Straftaten nicht vorhanden und es gibt damit kein eindeutiges Cluster von chronischen Straftätern. Die durchschnittliche Anzahl von Delikten pro Altersabschnitt ist bei den justiziell registrierten Frauen im Cluster der Gewaltdelikte am höchsten.

Bei den justiziell Registrierten bildet die große Gruppe der Verkehrsdelikte ein eigenes Cluster. Verkehrsdelikte werden polizeilich nicht registriert. Erstaunlich ist, dass auch die fahrlässige Körperverletzung in einem eigenen Cluster zusammengefasst wird. Somit gibt es jeweils ein Cluster, in dem jeder Altersabschnitt ein Verkehrsdelikt enthält. Außerdem gibt es ein Cluster fahrlässige Körperverletzung, hier enthält jeder Altersabschnitt das Delikt fahrlässige Körperverletzung und relativ häufig auch ein Verkehrsdelikt. D.h. es gibt ein Cluster von Verkehrsdelikten mit Personenschäden und ein Cluster von Verkehrsdelikten ohne Personenschäden.

Bei den polizeilich registrierten Männern ergibt die 6-Clusterlösung noch das Cluster „schwerer Diebstahl“. Jeder zweite Altersabschnitt dieses Clusters enthält das Delikt schweren Diebstahl. Außerdem sind die Delikte Hehlerei und „Fälschung" überdurchschnittlich häufig vertreten. Bei den Frauen ergibt sich das Cluster „Leistungserschleichung“, in dem alle Altersabschnitte dieses Clusters das Delikt Leistungserschleichung enthalten.

Schwerer Diebstahl wird polizeilich anders registriert als justiziell und ist besonders ein „Männerdelikt“. Leistungserschleichung ist eher ein „Frauendelikt“. 


\section{Spezialisierung auf einzelne Deliktskategorien}

Bis jetzt wurde hauptsächlich die Ähnlichkeit von verschiedenen Delikten untersucht. Dabei wurde auch schon auf die besondere Ähnlichkeit von gleichen Delikten hingewiesen. Das Adjusted Standardized Residual gleicher Deliktskategorien miteinander gab einen Hinweis auf Spezialisierung. In diesem Abschnitt wird das Auftreten von Delikten einer Deliktskategorie innerhalb der kriminellen Karriere untersucht. Dafür werden Berechnungen zum Grad der Spezialisierung durchgeführt.

Ein Spezialist ist ein Individuum, das dieselbe Straftat mehrmals auf dieselbe Art und Weise begeht. ${ }^{280}$ In den Medien wird von den „Einbrechern“, „Vergewaltigern“, „Gewalttätern“ gesprochen. Dieser Sprachgebrauch deutet auf Spezialisierung hin.

In diesem Abschnitt wird von Spezialisierung gesprochen, wenn eine Person mehrmals wegen Straftaten polizeilich oder justiziell registriert wurde, die in eine bestimmte Deliktskategorie fallen. Dabei stellte sich dasselbe Problem wie auch schon bei der Untersuchung von Deliktsähnlichkeiten. Wenn die Straftaten in sehr viele Kategorien eingeteilt werden, nimmt die Wahrscheinlichkeit von Spezialisierung stark ab. Werden sehr wenige Kategorien gebildet, kann nicht mehr von Spezialisierung gesprochen werden, da sehr unterschiedliche Delikte zu einer Gruppe zusammengefasst wurden. „Spezialisten“, die zwar verschiedene Straftaten begehen, aber z.B. immer dieselben drei Straftaten aus unterschiedlichen Deliktskategorien, werden in diesem Abschnitt nicht betrachtet. Mit einer Ausnahme werden dieselben Deliktskategorien verwendet, die auch schon für die Untersuchung der Deliktsähnlichkeiten gewählt wurden. Die neue Deliktskategorie ist die Kategorie Gewaltdelikte. Das Ergebnis der Multidimensionalen Skalierung und auch die Ergebnisse der Clusteranalysen zeigen, dass die Gewaltdelikte und auch Beleidigung demselben Deliktstyp angehören. Deshalb werden Tötungsdelikte, Sexualdelikte, Körperverletzung, schwere Körperverletzung, Straftaten gegen die persönliche Freiheit und Beleidigung zur Deliktskategorie Gewaltdelikte zusammengefasst. Somit sind die Straftaten der polizeilich registrierten Deutschen in 17 Deliktskategorien eingeteilt, die der Nichtdeutschen, bei denen Vergehen gegen Ausländergesetze hinzukommen, in 18 Kategorien. Die Delikte der justiziell registrierten Deutschen sind in 20 Deliktskategorien eingeteilt, die der Nichtdeutschen in 21.

Der Grad der Spezialisierung wird mit der von Farrington vorgeschlagenen Methode berechnet. Farrington empfiehlt den Forward Specialization Coefficient (FSC). ${ }^{281}$

280 Vgl. Kyvsgaard 2003, S. 147.

281 Farrington 1986, S. 227, Kyvsgaard 2003, S. 154. 


$$
\begin{aligned}
& \mathrm{FSC}=\frac{\mathrm{N}-\mathrm{E}}{\mathrm{Z}-\mathrm{E}} \\
& \mathrm{N} \text { beobachtete Häufigkeit einer Eigenkombination } \\
& \mathrm{E} \text { Erwartungswert der Eigenkombination } \\
& \mathrm{Z} \text { Zeilensumme }
\end{aligned}
$$

Berechnet wird der FSC für die Diagonale der Transitionsmatrix, d.h. die Eigenkombinationen. Betrachtet werden zeitlich unmittelbar aufeinander folgende Delikte. Der Koeffizient nimmt Werte zwischen 0 und 1 an, in seltenen Fällen auch kleiner als 0, wenn die beobachtete Häufigkeit kleiner als die erwartete Häufigkeit ist. Ein Wert von 1 gibt eine Spezialisierung von $100 \%$ an. ${ }^{282}$ Der FSC hängt von der Größe des Erwartungswertes ab, d.h. vom Anteil den diese Deliktskategorie an allen Deliktskategorien hat. ${ }^{283}$ Bei großen Deliktsgruppen ist der FSC relativ gröBer als bei kleinen Deliktsgruppen.

Bei den polizeilichen Registrierungen wird dieselbe Straftat einer Person, die mehrfach zu einem Tatzeitpunkt vorkommt, nur einmal in die Analyse miteinbezogen. D.h. wenn ein Tatverdächtiger mit 10 Diebstählen an einem Tag registriert ist, wird davon nur ein Diebstahl gezählt.

Tabelle 60 zeigt die Spezialisierungskoeffizienten für polizeilich Registrierte. FSC größer als der Durchschnittswert von 0,16 (ohne AuslG) sind hervorgehoben. Die letzte Spalte gibt den prozentualen Anteil der Deliktspaare mit dem jeweiligen Ausgangsdelikt der insgesamt knapp 193.000 Deliktspaare an.

282 Vgl. Farrington 1986, S. 227.

283 Dazu zwei Beispiele: $\mathrm{N}=20, \mathrm{E}=10, \mathrm{Z}=100$ ergibt $\mathrm{FSC}=0,11$. Hier ist der Anteil der Deliktsgruppe $10 \%$ von allen Deliktsgruppen, in der Annahme, dass die Gruppengröße der Ausgangsdelikte der Gruppengröße der Enddelikte entspricht (Zeilensumme = Spaltensumme). Die Differenz von N und $\mathrm{E}$ ist 10, aber $\mathrm{N}$ ist doppelt so groß wie $\mathrm{E}$.

$\mathrm{N}=60, \mathrm{E}=50, \mathrm{Z}=100$ ergibt $\mathrm{FSC}=0,20$. Hier ist der Anteil der Deliktsgruppe $50 \%$ von allen Deliktsgruppen. Die Differenz von $\mathrm{N}$ und $\mathrm{E}$ ist auch 10 wie im ersten Beispiel, $\mathrm{N}$ ist nur $20 \%$ größer als E, aber der FSC ist fast doppelt so groß wie im ersten Beispiel. 
Tabelle 60: Forward Specialization Coefficients für den Übergang innerhalb derselben Deliktskategorie von einer Registrierung zur nächsten getrennt nach Geschlecht und Nationalität (polizeiliche Registrierungen)

\begin{tabular}{lcccccc} 
& \multicolumn{3}{c}{ deutsch } & \multicolumn{2}{c}{ nichtdeutsch } & $\begin{array}{c}\text { Anteile Aus- } \\
\text { gangsdeilkt } \\
\text { gesamt in \% }\end{array}$ \\
\hline mënnlich & weiblich. & männlich & weiblich. & gesamt & & \\
sonst. Sexualdel. & 0,26 & 0,23 & 0,29 & 0,30 & 0,28 & 14,9 \\
Raub & 0,27 & 0,39 & 0,17 & 0,39 & 0,28 & 0,6 \\
einf. Diebstahl & 0,08 & 0,03 & 0,10 & 0,11 & 0,09 & 2,0 \\
schw. Diebstahl & 0,18 & 0,24 & 0,24 & 0,28 & 0,22 & 26,0 \\
Sachbeschädigung & 0,26 & 0,17 & 0,30 & 0,26 & 0,28 & 12,5 \\
gemeingef. Str. & 0,14 & 0,05 & 0,06 & 0,05 & 0,12 & 3,8 \\
Hehlerei & 0,16 & 0,21 & 0,05 & 0,00 & 0,14 & 0,3 \\
Betrug & 0,04 & 0,06 & 0,04 & 0,08 & 0,04 & 1,8 \\
Erschl. v. Leistungen & 0,34 & 0,44 & 0,24 & 0,37 & 0,33 & 6,8 \\
Gef. dem. Rechtsst. & 0,31 & 0,34 & 0,19 & 0,25 & 0,28 & 5,8 \\
Öffentliche Ordnung & 0,12 & 0,00 & 0,00 & & 0,11 & 0,2 \\
Hausfriedensbruch & 0,07 & 0,05 & 0,04 & 0,03 & 0,06 & 1,0 \\
Fälschung & 0,09 & 0,09 & 0,12 & 0,01 & 0,10 & 1,1 \\
Meineid & 0,07 & 0,08 & 0,09 & 0,11 & 0,08 & 2,1 \\
falsche Verdächtigung & 0,04 & 0,07 & 0,03 & 0,07 & 0,04 & 0,3 \\
BTM-Delikte & 0,02 & 0,04 & 0,01 & 0,02 & 0,02 & 0,2 \\
AuslG & 0,35 & 0,34 & 0,36 & 0,31 & 0,35 & 14,4 \\
\hline
\end{tabular}

* Gewalt $=$ Tötungsdelikte, Sexualdelikte, Körperverletzung, schwere Körperverletzung, Straftaten gegen die persönliche Freiheit, Beleidigung

Hohe Spezialisierungskoeffizienten ergeben sich bei den Nichtdeutschen bei Verstößen gegen Ausländergesetze und dabei besonders hoch bei den Männern $(0,49)$. Bei Frauen ist der FSC bei Betrug sehr hoch. Der FSC beträgt 0,44 bei deutschen Frauen. Auch bei den Männern zeigt sich, dass Betrug häufig hintereinander registriert wird, der FSC ist bei den deutschen Männern 0,34. „Sonstige Sexualdelikte“, insbesondere bei Frauen, wo es sich hauptsächlich um verbotene Prostitution handelt, weisen einen hohen Spezialisierungsgrad auf (0,39). Dies ist ein deutliches Zeichen für Spezialisierung, da diese Deliktsgruppe mit einem Anteil von 0,6\% sehr klein ist. Des Weiteren zeigt sich bei Betäubungsmitteldelikten eine Tendenz zur Spezialisierung (FSC über alle Gruppen 0,35). Spezialisierung bei Betäubungsmitteldelikten dürfte auf Drogenkonsum und Drogenabhängigkeit zurückzuführen sein. ${ }^{284}$ Bei Gewaltdelikten ist der Spezialisierungskoeffizient zwar groß (über alle Gruppen 0,28), aber wegen der großen Deliktskategorie wären noch höhere Werte zu erwarten gewesen. Nach einem Gewaltdelikt folgt häufig ein Gewaltdelikt, aber häufig auch ein anderes Delikt. Bei Männern und auch bei nichtdeutschen Frauen ist der FSC bei schwerem Diebstahl mit 0,26 bei den deutschen

284 Vgl. Landeskriminalamt Nordrhein-Westfalen 2005, S. 38. 
Männern und bei den nichtdeutschen Frauen und 0,30 bei den nichtdeutschen Männern hoch. Bei den deutschen Frauen ist der FSC bei schwerem Diebstahl mit 0,17 zwar auch überdurchschnittlich hoch, aber doch deutlich niedriger als bei den Männern und den nichtdeutschen Frauen.

Ebenso wie für die polizeilichen Registrierungen wird auch für die justiziellen Registrierungen der FSC berechnet. Bei den justiziellen Registrierungen werden die Übergänge von einer Entscheidung zur nächsten betrachtet. In jeder Entscheidung wird nur ein Delikt miteinbezogen, und zwar das schwerste. ${ }^{285}$ Tabelle 61 zeigt den Forward Specialization Coefficient für justiziell Registrierte. Die letzte Spalte zeigt die Deliktskategoriengrößen der insgesamt etwas mehr als 192.000 Deliktspaare. Die FSC, die größer als der Durchschnitt von 0.11 sind (ohne AuslG), sind grau markiert. Wie bei den polizeilich registrierten zeigt sich auch bei den justiziell registrierten Nichtdeutschen eine Tendenz zur Spezialisierung bei Straftaten gegen Ausländergesetze. Bei den Männern beträgt der FSC 0,42 und bei den Frauen 0,30. Auch bei Betrug zeigt sich insbesondere bei Frauen (deutsche Frauen $\mathrm{FSC}=0,35$ ), aber auch bei Männern (deutsche Männer $\mathrm{FSC}=0,25$ ), eine Tendenz zur Spezialisierung. Ebenso wie bei polizeilich Registrierten wird Spezialisierung bei „sonstigen Sexualdelikten“ (verbotene Prostitution) bei Frauen (deutsche Frauen FSC=0,29) deutlich sichtbar, da die Deliktskategorie relativ klein ist. Auch bei Betäubungsmitteldelikten zeigt sich ein hoher FSC, im Schnitt über alle Gruppen 0,24. Verkehrsdelikte, die polizeilich nicht registriert werden, weisen mit 0,23 auch einen hohen FSC auf.

Außer bei Leistungserschleichung sind die Spezialisierungskoeffizienten der justiziellen Registrierungen niedriger als die der polizeilichen Registrierungen. Deutlich niedriger sind die FSC von schwerem Diebstahl bei justiziellen Registrierungen im Vergleich zu polizeilichen Registrierungen. Die unterschiedliche Registrierung von schwerem Diebstahl in Polizei- und Justizdaten war schon bei der Berechnung der Adjusted Standardized Residuals und bei der Multidimensionalen Skalierung aufgefallen. ${ }^{286}$

285 Zum schwersten Delikt siehe Fußnote 200.

286 Siehe Abschnitt 5. 
Tabelle 61: Forward Specialization Coefficients für den Übergang innerhalb derselben Deliktskategorie von einer Registrierung zur nächsten getrennt nach Geschlecht und Nationalität (justizielle Registrierungen)

\begin{tabular}{lcccccc} 
& \multicolumn{2}{c}{ deutsch } & \multicolumn{2}{c}{ nichtdeutsch } & $\begin{array}{c}\text { Anteile Aus- } \\
\text { gangsdelikt } \\
\text { gesamt in \% }\end{array}$ \\
\hline Gewalt* & 0,17 & 0,18 & 0,20 & 0,23 & 0,19 & 11,8 \\
sonst. Sexualdel. & 0,17 & 0,29 & 0,10 & 0,32 & 0,18 & 0,4 \\
Raub & 0,02 & 0,02 & 0,03 & 0,00 & 0,03 & 0,8 \\
einf. Diebstahl & 0,13 & 0,18 & 0,21 & 0,25 & 0,18 & 27,4 \\
schw. Diebstahl & 0,12 & 0,08 & 0,14 & 0,12 & 0,13 & 6,5 \\
Sachbeschädigung & 0,05 & 0,05 & 0,04 & 0,02 & 0,06 & 2,9 \\
gemeingef. Str. & 0,03 & 0,00 & 0,03 & 0,00 & 0,02 & 0,2 \\
Hehlerei & 0,02 & 0,02 & 0,03 & 0,06 & 0,02 & 1,2 \\
Betrug & 0,25 & 0,35 & 0,16 & 0,31 & 0,25 & 3,8 \\
Erschl. v. Leistungen & 0,31 & 0,35 & 0,21 & 0,32 & 0,29 & 5,4 \\
Gef. dem. Rechtsst. & 0,06 & 0,00 & 0,00 & & 0,06 & 0,1 \\
Öffentliche Ordnung & 0,02 & 0,03 & 0,03 & 0,04 & 0,02 & 1,0 \\
Hausfriedensbruch & 0,06 & 0,05 & 0,06 & 0,12 & 0,06 & 0,8 \\
Fälschung & 0,05 & 0,05 & 0,07 & 0,09 & 0,06 & 3,6 \\
Meineid & 0,03 & 0,03 & 0,04 & 0,14 & 0,03 & 0,2 \\
falsche Verdächtigung & 0,00 & $-0,01$ & 0,00 & 0,00 & 0,00 & 0,2 \\
BTM-Delikte & 0,23 & 0,19 & 0,26 & 0,24 & 0,24 & 7,8 \\
AuslG & & & 0,42 & 0,30 & & 4,6 \\
Vollrausch & 0,08 & 0,07 & 0,03 & 0,00 & 0,07 & 0,2 \\
Fahrl. KV/Tötung & 0,05 & 0,09 & 0,05 & 0,01 & 0,05 & 2,6 \\
Verkehrsdelikte & 0,21 & 0,27 & 0,21 & 0,31 & 0,23 & 18,5 \\
\hline
\end{tabular}

* Gewalt $=$ Tötungsdelikte, Sexualdelikte, Körperverletzung, schwere Körperverletzung, Straftaten gegen die persönliche Freiheit, Beleidigung

Die Spezialisierungskoeffizienten, die sich mit den Daten der Freiburger Kohortenstudie ergaben, sollen im Folgenden mit den Spezialisierungskoeffizienten früherer Studien verglichen werden.

Farrington berechnete 1986 Spezialisierungskoeffizienten mit Daten, die Phillpotts und Lancucki 1979 in ihrer Studie benutzten. ${ }^{287}$ Die Daten umfassten 5.000 Personen, die im Januar 1971 in England oder Wales gerichtlich verurteilt wurden und von diesen Personen alle Verurteilungen zwischen 1963 und 1976. Es handelte sich um Männer und Frauen ab dem Alter von zehn Jahren. Die Delikte waren in drei Deliktskategorien eingeteilt:

287 Vgl. Farrington 1986, S. 226 und Phillpotts \& Lancucki 1979. 
Gewalt- und Sexualdelikte;

Eigentumsdelikte: Diebstahl, Raub und Hehlerei;

Sonstiges: Betrug, Fälschung, Sachbeschädigung, Verkehrsdelikte, u.a. ${ }^{288}$

Farrington unterteilte die Personen in zwei Gruppen (die unter 21-Jährigen und die 21-Jährigen oder Älteren) und berechnete den FSC. Farringtons Ergebnis war, dass Ältere spezialisierter waren als Jüngere. ${ }^{289}$ Der FSC war bei Gewalt- und Sexualdelikten bei den Jüngeren 0,15 und bei den Älteren 0,26. Damit stimmt der FSC bei Gewaltdelikten mit denen der justiziellen Registrierungen dieser Arbeit in etwa überein $(0,19)$. Die Spezialisierungskoeffizienten von Eigentumsdelikten ( 0,06 bei den Jüngeren und 0,23 bei den Älteren) und sonstigen Delikten $(0,14$ bei den Jüngeren und 0,23 bei den Älteren) waren niedriger als die dieser Arbeit, was erstaunlich ist, da die Deliktskategorien mehr Delikte umfassen.

Auch Blumstein, Cohen, Das und Moitra berechneten 1988 Spezialisierungskoeffizienten. Sie benutzten die Daten von mehr als 32.000 erwachsener Strafgefangenen in den USA, die zwischen 1974 und 1977 wegen schwerer Straftaten inhaftiert waren. ${ }^{290}$ Die Delikte wurden zur Berechnung des FSC in 10 Kategorien eingeteilt. ${ }^{291}$ Die Deliktskategorien waren: Tötungsdelikte, Sexualdelikte, Raub, Körperverletzung, Betäubungsmitteldelikte, schwerer Diebstahl, einfacher Diebstahl, Autodiebstahl, Waffendelikte und Betrug. Ein Ergebnis der Studie war, dass sich bzgl. der Deliktskategorien Unterschiede im Grad der Spezialisierung zeigten. Der Spezialisierungsgrad war bei Drogendelikten, Betrug und Autodiebstahl am höchsten. Bei Gewaltkriminalität war der Spezialisierungsgrad deutlich geringer. ${ }^{292} \mathrm{Al}$ lerdings waren die Gewaltdelikte nicht zu einer Deliktskategorie zusammengefasst. Die FSC sind höher als die der justiziell Registrierten dieser Studie. Der FSC war bei Betäubungsmitteldelikten knapp unter 0,4, bei Betrug etwa 0,34 und bei Körperverletzung etwa 0,18. Der relativ hohe Wert bei Körperverletzung lässt darauf schließen, dass der Spezialisierungsgrad von Gewaltdelikten sehr hoch wäre, wenn alle Gewaltdelikte in einer Kategorie zusammengefasst worden wären. Der Unterschied im Grad der Spezialisierung in der Studie von Blumstein, Cohen, Das und Moitra und dieser Untersuchung hier hängt einerseits damit zusammen, dass Blumstein, Cohen, Das und Moitra 10 Deliktskategorien verwenden und damit deutlich weniger als hier und andererseits kann der Unterschied damit zusammenhängen, dass in der Studie von Blumstein, Cohen, Das und Moitra nur Strafgefangene mit schweren Straftaten analysiert wurden. Damit sind schwere Straftaten überrepräsentiert.

\footnotetext{
288 Vgl. Phillpotts \& Lancucki 1979, S. 33.

289 Vgl. Farrington 1986, S. 227.

290 Vgl. Blumstein, Cohen, Das \& Moitra 1988, S. 307 f.

291 Vgl. Blumstein, Cohen, Das \& Moitra 1988, S. 322.

292 Vgl. Blumstein, Cohen, Das \& Moitra 1988, S. 341 f.
} 
In der Studie von Stander, Farrington, Hill und Altham 1989 wurden 698 englische männliche Strafgefangene im Alter von 21 Jahren oder älter untersucht. Die Delikte waren in sechs Deliktskategorien eingeteilt. ${ }^{293}$ Es ergaben sich hohe Spezialisierungskoeffizienten bei Sexualdelikten $(0,45)$ und bei Betrug $(0,27)$. Deutlich niedrigere Spezialisierungskoeffizienten zeigten sich bei Gewaltdelikten $(0,15)$ und Diebstahl $(0,14) .{ }^{294}$ Die FSC der beiden anderen Deliktskategorien waren 0,21 bei schwerem Diebstahl und 0,15 in der Kategorie sonstige Delikte. Damit stimmen die Spezialisierungskoeffizienten, bis auf den Koeffizienten bei Sexualdelikten, mit den Ergebnissen der justiziellen Registrierungen dieser Studie überein. Erstaunlich hoch war der FSC bei Sexualdelikten. Eventuell hängt der hohe Spezialisierungskoeffizient bei Sexualdelikten mit dem Alter und mit der Gruppe der Strafgefangenen zusammen.

Gottfredson und Gottfredson veröffentlichten 1994 die Ergebnisse einer Studie über mehr als 6000 Männer, die in den frühen 60er Jahren in Kalifornien inhaftiert waren. ${ }^{295} 1988$ wurden die weiteren Inhaftierungen dieser Männer erfasst und damit eine Spanne von über 25 Jahren betrachtet. Die Delikte waren in sechs Deliktskategorien eingeteilt (Nuisance ${ }^{296}$, Gewaltdelikte, Eigentumsdelikte, Betrug, schwere Betäubungsmitteldelikte, Sonstiges), wobei es beim Übergang der Transitionsmatrix auch die Gruppe „,keine Straftat“ gab. ${ }^{297}$ Auch Gottfredson und Gottfredson berechneten den FSC mit ihren Daten. Die FSC mit den Daten von Gottfredson und Gottfredson waren sehr niedrig. Der höchste Wert war bei Betrug mit 0,13 , bei Gewaltdelikten war der FSC mit 0,05 sehr niedrig und auch bei Betäubungsmitteldelikten mit 0,02. Damit unterscheidet sich diese Studie deutlich von den Ergebnissen dieser Studie und auch von allen anderen hier beschriebenen Studien. Auch wenn die Berechnung des FSC ohne die Gruppe der „keine Straftat“ durchgeführt wird, was Gottfredson und Gottfredson nicht taten, sind die FSC's deutlich niedriger als die der anderen Studien. ${ }^{298}$

293 Vgl. Stander, Farrington, Hill \& Altham 1989, S. 321.

294 Vgl. Stander, Farrington, Hill \& Altham 1989, S. 327.

295 Gottfredson \& Gottfredson 1994.

296 "Nuisance" umfasst die Delikte leichte Betäubungsmitteldelikte, Trunkenheit im Verkehr und Verstöße gegen Bewährungsauflagen, siehe Gottfredson \& Gottfredson 1994, S. 451.

297 Vgl. Gottfredson \& Gottfredson 1994, S. 461.

298 Berechnet man den FSC ohne die Gruppe „keine Straftat“ ergibt sich für Betrug ein Wert von 0,15 , für Gewaltdelikte 0,08 und für BTM 0,02 . 
Kyvsgaard untersuchte 2003 die kriminelle Karriere mit dänischen Längsschnittdaten. Die Daten umfassten die justiziellen Registrierungen von knapp 45.000 Personen im Alter von 15 Jahren und älter. Auch Kyvsgaard berechnete Spezialisierungsgrade mit dem Forward Specialization Coefficient. ${ }^{299}$ Sie unterteilte die Delikte in 11 Deliktskategorien (Sexualdelikte, Gewaltdelikte, schwerer Diebstahl, einfacher Diebstahl, Autodiebstahl, Betrug/ Fälschung/ Leistungserschleichung, Betäubungsmitteldelikte, sonstige Delikte, Trunkenheit im Verkehr/ fahrlässige Tötung, sonstige Verkehrsdelikte, Verstöße gegen Steuer-, Umwelt-, Ausländeroder sonstige Gesetze). ${ }^{300}$ Kyvsgaard errechnete sehr hohe Spezialisierungskoeffizienten. Bei Sexualdelikten war der FSC bei Männern 0,66 und bei Frauen 0,86, bei Gewaltdelikten bei Männern und Frauen 0,36. Bei schwerem Diebstahl ergab sich bei Männern ein FSC von 0,54 und bei Frauen von 0,60, bei Betäubungsmitteldelikten war der FSC bei Männern 0,42 und bei Frauen 0,57, bei Verkehrsdelikten war der FSC bei Männern 0,44 und bei Frauen 0,53. Damit waren die FSC der dänischen Studie insgesamt sehr hoch. Besonders auffällig ist der hohe Wert bei Sexualdelikten. Ein weiteres außergewöhnliches Ergebnis von Kyvsgaard war, dass Frauen häufiger spezialisiert sind als Männer. ${ }^{301}$ Dieses Ergebnis hat sich in dieser Studie nicht bestätigen. Frauen sind häufiger spezialisiert bei verbotener Prostitution und bei Betrug, aber nicht grundsätzlich. Weitere Ergebnisse von Kyvsgaard waren, dass die Dauer zwischen den Straftaten die Spezialisierungsrate beeinflusst. Je kürzer das Zeitintervall zwischen den einzelnen Delikten ist, desto höher ist der Spezialisierungsgrad. Wie auch schon in anderen erwähnten Studien kam sie zum Ergebnis, dass Ältere einen höheren Spezialisierungsgrad aufweisen als Jüngere. ${ }^{302}$

Das Landeskriminalamt Nordrhein-Westfalen führte 2005 eine Untersuchung von jungen Mehrfachtatverdächtigen in Nordrhein-Westfalen durch. ${ }^{303}$ Mehrfachtatverdächtige sind Personen, die innerhalb eines Kalenderjahres fünf oder mehr Straftaten begehen. ${ }^{304}$ Ausgewertet wurde die Polizeiliche Kriminalstatistik von 1994 bis 2003. Mit den Daten der Geburtskohorte 1983, Alter 11 bis 19 Jahre, wurde der Spezialisierungsgrad für Mehrfachtatverdächtige und für alle Tatverdächtigen dieser Kohorte mit dem FSC berechnet. ${ }^{305}$ Tatverdächtige mit zwei oder mehr Straftaten gab es in diesem Zeitraum 29.172, darunter befanden sich 9.542 Mehrfachtatverdächtige. ${ }^{306}$ Die Delikte wurden in 10 Deliktskategorien eingeteilt (Tötungsdelikte/ Sexualdelikte/ Straftaten gegen die persönliche Freiheit, Raub,

299 Kyvsgaard 2003, S. 154. Es handelt sich um eine dänische Längsschnittstudie, die justizielle Registrierungen von 1979 bis 1991 umfasst.

300 Vgl. Kyvsgaard 2003, S. 150.

301 Vgl. Kyvsgaard 2003, S. 165.

302 Vgl. Kyvsgaard 2003, S. 242.

303 Landeskriminalamt Nordrhein-Westfalen 2005.

304 Vgl. Landeskriminalamt Nordrhein-Westfalen 2005, S. 1.

305 Vgl. Landeskriminalamt Nordrhein-Westfalen 2005, S. 9.

306 Vgl. Landeskriminalamt Nordrhein-Westfalen 2005, S. 23. 
schwere Körperverletzung, leichte Körperverletzung, einfacher Diebstahl, schwerer Diebstahl, Betrug, Sachbeschädigung, Betäubungsmitteldelikte, sonstige Delikte). ${ }^{307}$ Den höchsten Spezialisierungsgrade gab es bei den Deliktskategorien Betrug $(\mathrm{FSC}=0,20)$ und Betäubungsmitteldelikte $(\mathrm{FSC}=0,27)$. Die Deliktskategorie Tötungsdelikte/ Sexualdelikte/ Straftaten gegen die persönliche Freiheit hat einen FSC von 0,05, Raub 0,08, einfacher Diebstahl 0,09, schwerer Diebstahl 0,08 und Sachbeschädigung 0,05.308 Auch bei den polizeilichen Registrierungen dieser Arbeit sind die FSC von Betäubungsmitteldelikten $(0,35)$ und Betrug $(0,33)$ am höchsten. Die höheren Werte erklären sich mit der breiteren Altersspanne. Die Deliktskategorie mit Sexualdelikten hat einen FSC von 0,05 und damit einen deutlich niedrigeren Wert als der in der dänischen Studie von Kyvsgaard ermittelten.

Deutlich abweichende Ergebnisse beim Spezialisierungsgrad ergaben sich in der dänischen Studie von Kyvsgaard, alle Spezialisierungskoeffizienten sind deutlich höher als in den anderen Studien, insbesondere der Wert bei Sexualdelikten ist sehr hoch. Niedrigere Werte als in allen Studien ergaben sich bei Gottfredson und Gottfredson. Dies ist insbesondere auffällig, da es sich um eine Untersuchung von Erwachsenen handelt, die im Allgemeinen höhere Spezialisierungsraten aufweisen als Jugendliche. Ansonsten sind eindeutige Gemeinsamkeiten in der Spezialisierungstendenz festzustellen. Trotz sehr unterschiedlicher Deliktskategorien sind gemeinsame Aussagen zur Spezialisierung möglich. Die Deliktskategorien mit dem höchsten Spezialisierungskoeffizient sind sonstige Sexualdelikte, Betrug und Betäubungsmitteldelikte.

Der Koeffizient ist abhängig von der Wahl der Deliktskategorien. Größe, Anzahl und Zusammensetzung der Deliktskategorien beeinflusst die „Spezialisierungswahrscheinlichkeit“".

Je größer die Deliktskategorie, desto breiter das Deliktsspektrum und desto höher ist die Wahrscheinlichkeit, dass die nächste Straftat derselben Deliktskategorie angehört wie die vorhergehende.

307 Vgl. Landeskriminalamt Nordrhein-Westfalen 2005, S. 10.

308 Vgl. Landeskriminalamt Nordrhein-Westfalen 2005, S. 38. 



\section{Zusammenfassung der Ergebnisse und Schlussfolgerungen}

In vorliegender Arbeit wurde untersucht, welche Kombinationen von Delikten innerhalb von kriminellen Karrieren besonders häufig auftreten. D.h., es wurden Analysen zu Spezialisierung in mehrfachem Sinne durchgeführt. Einerseits wurde die Spezialisierung innerhalb einzelner Deliktskategorien untersucht, andererseits wurde aber auch der Zusammenhang verschiedener Deliktskategorien erforscht, mit dem Ziel, typische Deliktskonfigurationen darzustellen. Die Untersuchung basiert auf offiziellen polizeilichen und justiziellen Registrierungen, wobei nur Personen mit mindestens zwei Tatbegehungen bzw. bei justiziellen Registrierungen mit mindestens zwei justiziellen Entscheidungen oder mit Delikten aus zwei Deliktskategorien innerhalb einer Entscheidung berücksichtigt wurden.

Bei der Einteilung der Deliktskategorien bestand die Problematik, dass es einerseits gut wäre, alle registrierten Delikte möglichst detailliert zu betrachten, andererseits aber nicht zu viele zu kleine Kategorien entstehen sollten. Einzelfälle sind für eine statistische Analyse unbrauchbar. Generell soll die Zusammenfassung von Delikten zu Kategorien das Ziel der Analyse sein, gleichwohl sind Deliktskategorien auch Ausgangspunkt der Analyse. Eine zu große Zahl von Deliktskategorien würde dazu führen, dass es sehr viele einzelne Deliktskategorien mit sehr wenigen Fällen geben würde und es damit viel zu viele Kombinationen gäbe, was eine sinnvolle Auswertung nicht mehr gestatten würde. So wurden die Delikte für die polizeilich Registrierten in 25 und für die justiziell Registrierten in 29 Kategorien eingeteilt. ${ }^{309}$ Die Deliktskategorien entsprechen großteils den Abschnitten im besonderen Teil des StGB, soweit nicht einzelne Delikte speziell betrachtet wurden. Des Weiteren wurden alle Betäubungsmitteldelikte sowie alle Vergehen gegen das Ausländergesetz und Asylverfahrensgesetz zu jeweils einer Kategorie zusammengefasst.

Die Deliktsähnlichkeiten wurden mit der Methode der Multidimensionalen Skalierung grafisch dargestellt. Für die Multidimensionale Skalierung wurde ein Ähnlichkeitsmaß für Delikte benötigt. Dafür wurden zunächst die Kombinationen von Straftaten einer Person betrachtet. Die chronologische Reihenfolge der Delikte wurde hierbei nicht mit einbezogen, sondern es wurde untersucht, welche Deliktskombinationen häufig innerhalb der kriminellen Karriere auftraten. Als Ähnlichkeitsmaß wurde, wie auch schon in früheren Studien, das Adjusted Standardized Residual (ASR) verwendet.

309 Die justiziellen Deliktskategorien umfassen die polizeilichen Deliktskategorien plus „Verkehr“ (Verkehrsdelikte nach StGB, StVG, PflVG), „Vollrausch“, „fahrlässige Körperverletzung“ und „fahrlässige Tötung“. In manchen Auswertungen wurde „fahrlässige Körperverletzung“ und „fahrlässige Tötung“ zu einer Kategorie zusammengefasst wegen der geringen Fallzahlen in der Kategorie „fahrlässige Tötung“ und wegen der Typgleichheit dieser beiden Delikte insofern, dass beides Delikte ohne Vorsatz sind. 
Das ASR zeigt, welche Deliktspaare innerhalb der kriminellen Karrieren überdurchschnittlich häufig bzw. selten auftreten. Betrachtet man zunächst Spezialisierung auf einzelne Deliktskategorien, so ergab sich folgendes Bild: Das ASR aller Deliktskategorien mit sich selbst war in allen Gruppen positiv. D.h., bei allen Deliktskategorien zeigte sich ein gewisser Grad von Spezialisierung. Bei allen Gruppen, sowohl bei Männern wie Frauen, bei Deutschen wie Nichtdeutschen, bei polizeilich Registrierten wie justiziell Registrierten, zeigte sich ein relativ hoher Grad an Spezialisierung in den Kategorien Betrug und Betäubungsmitteldelikte. Auch bei „sonstigen Sexualdelikten“ 310 wurde eine starke Tendenz zur Spezialisierung sichtbar. Bei Männern zeigte sich auch bei Sexualdelikten eine Tendenz zur Spezialisierung, aber nicht so stark wie bei ,sonstigen Sexualdelikten“.

Bei schwerem Diebstahl zeigte sich bei polizeilich Registrierten, nicht aber bei justiziell Registrierten, ein hoher Spezialisierungsgrad. Schwerer Diebstahl wird deutlich häufiger polizeilich registriert als justiziell. Schwere Diebstähle (Einbrüche) werden häufig in Serie begangen und dementsprechend häufig polizeilich registriert. Justiziell wird diese „Einbruchserie“ innerhalb einer Entscheidung abgehandelt und wird als ein schwerer Diebstahl gezählt.

Häufig mehrfach registriert wurden Verkehrsdelikte innerhalb einer kriminellen Karriere, diese werden jedoch nur justiziell registriert. Bei Nichtdeutschen waren überdurchschnittlich häufig Mehrfach-Vergehen gegen Ausländergesetze innerhalb der kriminellen Karriere registriert.

Die Spezialisierung auf einzelne Deliktskategorien wurde auch mit dem Forward Specialization Coefficient (FSC) von Farrington bestimmt. Der Koeffizient ist ein Maß dafür, wie häufig Straftaten derselben Deliktskategorie bei Personen unmittelbar aufeinander folgend registriert sind. Der FSC nimmt normalerweise Werte zwischen 0 und 1 an, wobei ein Wert von 1 eine hundertprozentige Spezialisierung bedeutet. Der Wert kann auch kleiner als 0 sein, wenn definitiv keine Tendenz zur Spezialisierung vorhanden ist. ${ }^{311}$ Bei Frauen stärker als bei Männern zeigte sich Spezialisierung bei „sonstigen Sexualdelikten“ und bei Betrug. Bei Männern und Frauen gab es hohe Spezialisierungskoeffizienten bei Leistungserschleichung. Auch bei Betäubungsmitteldelikten zeigte sich eine Tendenz zur Spezialisierung, was auf Drogenkonsum und Drogenabhängigkeit zurückzuführen sein dürfte. ${ }^{312}$ Bei Gewaltdelikten zeigte sich eine Tendenz zur Spezialisierung, die aber kleiner war als erwartet. Auch bei einfachem Diebstahl war der Spezialisierungskoeffizient überdurchschnittlich hoch. Bei polizeilich Registrierten war der FSC bei schwerem Diebstahl hoch. Hohe Spezialisierungskoeffizienten zeigten sich bei Nichtdeut-

310 Die Zusammensetzung der Deliktskategorie „sonstige Sexualdelikte“ unterscheidet sich bei Männern und Frauen. Bei Frauen enthält diese Kategorie hauptsächlich verbotene Prostitution, bei Männern hauptsächlich sexuellen Missbrauch.

311 Vgl. Kyvsgaard 2003, S. 154.

312 Vgl. Landeskriminalamt Nordrhein-Westfalen 2005, S. 38. 
schen bei Vergehen gegen Ausländergesetze. Verkehrsdelikte, die polizeilich nicht registriert werden, wiesen auch einen hohen FSC auf. Außer bei Leistungserschleichung waren die Spezialisierungskoeffizienten der justiziellen Registrierungen niedriger als die der polizeilichen Registrierungen. Dies hängt auch damit zusammen, dass bei justiziellen Registrierungen mehr Deliktskategorien vorhanden sind als bei polizeilichen Registrierungen.

Beide Maße, sowohl das Adjusted Standardized Residual als auch der Forward Specialization Coefficient, führten zum selben Ergebnis bzgl. Spezialisierung auf einzelne Deliktskategorien. Spezialisierung auf einzelne Deliktskategorien zeigte sich hauptsächlich bei Betrug, Betäubungsmitteldelikten, sonstigen Sexualdelikten, insbesondere bei polizeilich Registrierten bei schwerem Diebstahl, bei justiziell Registrierten bei Verkehrsdelikten und bei Nichtdeutschen bei Vergehen gegen Ausländergesetze.

Zur Bestimmung weiterer Deliktskonfigurationen, d.h. zur Analyse des überzufällig häufigen Auftretens von Deliktskombinationen verschiedener Deliktskategorien, waren nur die ASR verschiedener Deliktskategorien von Bedeutung. Bei polizeilich und justiziell Registrierten war bei allen Gruppen, sowohl bei Männern wie bei Frauen, bei Deutschen wie bei Nichtdeutschen, das ASR der Deliktskombinationen Körperverletzung - schwere Körperverletzung, Körperverletzung - Beleidigung und Körperverletzung - Straftaten gegen die persönliche Freiheit hoch positiv. Diese Deliktskombinationen waren überzufällig häufig registriert. Bei justiziellen Registrierungen zeigte sich auch der Zusammenhang der Kategorien Betrug und Fälschung sowie von Verkehrsdelikten und fahrlässiger Körperverletzung/Tötung. Der Zusammenhang zwischen Verkehrsdelikten und fahrlässiger Körperverletzung/Tötung kommt durch Verkehrsunfälle mit Personenschäden zustande. In diesen Fällen kommt es zum Delikt fahrlässiger Körperverletzung bzw. fahrlässige Tötung.

Bei polizeilichen Registrierungen zeigten sich beim schweren Diebstahl bei allen Gruppen Unähnlichkeiten zu allen anderen Deliktskategorien, d.h. Kombinationen anderer Delikte mit schwerem Diebstahl traten seltener auf als erwartet. Sowohl bei polizeilich wie bei justiziell Registrierten zeigte sich die Unähnlichkeit von Betrug und Betäubungsmitteldelikten.

Anhand der justiziellen Registrierungen zeigte sich auch die Unähnlichkeit von Verkehrsdelikten zu vielen anderen Delikten. D.h., es gibt keinen Zusammenhang zwischen „normalen“ Straftaten, klassischen oder konventionellen Rechtsbrüchen, und Verkehrsdelikten. Besonders selten gemeinsam innerhalb einer kriminellen Karriere waren die Delikte Leistungserschleichung und Verkehrsdelikte.

Bei den Nichtdeutschen zeigte sich, dass es zwischen Vergehen gegen das Ausländergesetz oder Asylverfahrensgesetz und fast allen anderen Delikten keinen Zusammenhang gab. Nur die Kombinationen von „Fälschung“ bzw. von Leistungserschleichung mit Vergehen gegen das Ausländergesetz oder Asylverfahrens- 
gesetz kamen überzufällig häufig vor. Vergehen gegen das Ausländergesetz oder Asylverfahrensgesetz gehen häufig mit Fälschung von Ausweispapieren einher (Urkundenfälschung). Eine häufige Straftat von Asylbewerbern ist Schwarzfahren (Leistungserschleichung) und Graufahren (Fahren mit gefälschten Karten und somit Wertzeichenfälschung). ${ }^{313}$

Die Ergebnisse der zweidimensionalen Darstellung der Multidimensionalen Skalierung zeigten, dass insbesondere die Gewaltdelikte Körperverletzung, schwere Körperverletzung, Straftaten gegen die persönliche Freiheit und auch Beleidigung und Sachbeschädigung sowie Widerstand gegen die Staatsgewalt sich durch hohe Ähnlichkeiten auszeichnen, d.h. sie kamen häufig als Deliktskombination vor. Bei Männern, wenn nicht nach Altersgruppen unterschieden wurde, bildeten Sexualdelikte eine Gruppe mit Gewaltdelikten, wobei Sexualdelikte und ,sonstige Sexualdelikte" einander ähnlich waren. Bei der Unterscheidung nach Altersgruppen zeigte sich die Ähnlichkeit von Sexualdelikten und Gewaltdelikten bei Erwachsenen nicht.

Bei Tötungsdelikten ergab sich kein spezieller Zusammenhang mit anderen Delikten. Dazu waren Tötungsdelikte zu selten registriert, und wenn sie registriert waren, dann mit unterschiedlichen Delikten.

Bei Betäubungsmitteldelikten gab es bei den Männern Ähnlichkeiten zu Leistungserschleichung, Raub, Hausfriedensbruch und bei justiziellen Registrierungen auch zu einfachem Diebstahl. Damit war der Zusammenhang zwischen Drogendelikten und Beschaffungskriminalität sichtbar. Bei den Frauen erwies sich die Ähnlichkeit von verbotener Prostitution (sonstige Sexualdelikte) und Betäubungsmitteldelikten. Dies weist auf den Zusammenhang zwischen Betäubungsmitteldelikten und Beschaffungsprostitution hin.

Bei allen Gruppen wurde die Ähnlichkeit der Deliktskategorien Betrug und Fälschung sichtbar. Besonders unähnlich waren die Deliktskategorien Verkehrsdelikte und Erschleichen von Leistungen, was wohl damit zusammenhängt, dass Autofahrer nicht mit öffentlichen Verkehrsmitteln unterwegs sind und somit auch keine Leistungserschleichung (Beförderungserschleichung) begehen.

Kombinationen von schwerem Diebstahl mit anderen Delikten gab es bei polizeilichen registrierten Männern selten. Bei justiziellen Registrierungen gab es überdurchschnittlich häufig die Kombination von schwerem Diebstahl und Raub. Beim schweren Diebstahl zeigten sich deutliche Registrierungsunterschiede bei Polizei und Justiz. Schwerer Diebstahl wurde wesentlich häufiger polizeilich registriert als justiziell.

Ebenso traten bei der polizeilichen und justiziellen Registrierung von einfachem Diebstahl deutliche Unterschiede auf. Bei polizeilichen Registrierungen zeigte sich

313 Vgl. Althoff \& de Haan 2004, S. 445. 
eine deutliche Unähnlichkeit von Vermögens- und Eigentumsdelikten (Betrug und einfacher Diebstahl), was bei justiziellen Registrierungen nicht der Fall war. Dies hängt damit zusammen, dass der Anteil von einfachem Diebstahl bei polizeilichen Registrierungen deutlich höher ist als bei justiziellen Registrierungen. Dies hängt wiederum einerseits damit zusammen, dass einfacher Diebstahl eine leichte Straftat ist und dementsprechend häufig das Verfahren eingestellt wird, wenn keine weiteren Straftaten vorliegen. Andererseits wird, wie schon beim schweren Diebstahl beschrieben, auch der einfache Diebstahl häufig in Serie begangen. Diese Seriendiebstähle werden mehrfach polizeilich registriert, aber justiziell in einer Verhandlung abgeurteilt. Somit zeigt sich bei Betrachtung der ASR bei polizeilich registriertem einfachem Diebstahl eine hohe Selbstähnlichkeit und eine Unähnlichkeit von einfachem Diebstahl und anderen Delikten. Bei justiziell registriertem einfachem Diebstahl ist die Selbstähnlichkeit nicht so groß und die Unähnlichkeit zu anderen Delikten wird nicht sichtbar.

Bei den nichtdeutschen Männern und Frauen war deutlich zu sehen, dass es zwischen Vergehen gegen das Ausländergesetz oder Asylverfahrensgesetz und den meisten anderen Delikten keinen Zusammenhang gab, insbesondere gab es keine Gemeinsamkeit mit Gewaltdelikten. Ähnlichkeiten traten zwischen Vergehen gegen das Ausländergesetz oder Asylverfahrensgesetz und „Fälschung“ (Urkundenfälschung und auch Wertzeichenfälschung) auf.

Bei justiziellen Registrierungen fiel die Ähnlichkeit des Delikts Vollrausch mit Gewaltdelikten und die Ähnlichkeit von Verkehrsdelikten mit fahrlässiger Tötung/Körperverletzung auf.

Kontrollrechnungen zeigten, dass das Ergebnis nicht durch tateinheitlich begangene Straftaten verfälscht wurde, obgleich bei bestimmten Deliktspaaren gehäuft Kombinationen an einem Tag vorkamen. Die Deliktskonfiguration veränderte sich nicht deutlich, wenn bei der Analyse statt aller Delikte jeweils nur das schwerste eines Tages bzw. einer Entscheidung einbezogen wurde.

Die Berücksichtigung der Dauer zwischen den Straftaten einer kriminellen Laufbahn beeinflusste das Ergebnis der Deliktsähnlichkeiten nicht.

Die Untersuchung der Deliktskonfiguration einzelner Altersgruppen der deutschen Männer ergab, dass Sexualdelikte bei Jugendlichen, Heranwachsenden und Jungerwachsenen ähnlich zu Gewaltdelikten waren, nicht aber bei den Älteren. Bei den polizeilich registrierten 26- bis 31-Jährigen und bei den justiziell registrierten 26- bis 33-Jährigen deutschen Männern wird kein Zusammenhang zwischen Sexualdelikten und Gewaltdelikten sichtbar.

Weiter wurden die Zusammenhänge der Deliktskategorien mit einem probabilistischen Clusteranalyseverfahren untersucht. Die Registrierungen jeder Person wurden in Altersabschnitte aufgeteilt. Die Altersabschnitte wurden entsprechend ihrer Deliktszusammensetzung Clustern zugeordnet. Hierzu wurden die Daten des Geburtsjahrgangs 1970 verwendet, da diese die größte Altersspanne umfassen. Die 
Analyse wurde sowohl für polizeilich wie für justiziell registrierte deutsche Männer und Frauen durchgeführt. In allen vier Gruppen ergaben sich bei der 6Clusterlösung die Cluster „einfacher Diebstahl“, „Gewaltdelikte“ und „Betrug“. Deutlich wurde die Ähnlichkeit der Gewaltdelikte (Sexualdelikte, Körperverletzung, Straftaten gegen die persönliche Freiheit) untereinander sowie mit Beleidigung, Sachbeschädigung, Widerstand gegen die Staatsgewalt und Hausfriedensbruch. Außerdem zeigte sich die Ähnlichkeit der Deliktskategorien Betrug, Fälschung und Meineid. Bei den polizeilich registrierten Männern und Frauen und bei den justiziell registrierten Frauen enthielten alle Altersabschnitte des Clusters ,einfacher Diebstahl“" ausschließlich das Delikt einfacher Diebstahl, andere Delikte sind sehr selten in diesem Cluster. Bei den justiziell registrierten Männern enthielt die Hälfte der Altersabschnitte des Clusters „einfacher Diebstahl“ zusätzlich zu einfachem Diebstahl ein weiteres Delikt wie Betäubungsmitteldelikt, schwerer Diebstahl, Hehlerei, Leistungserschleichung oder sonstige Sexualdelikte. Insofern ist dieses Cluster eine Mischung der Cluster ,einfacher Diebstahl“ und dem in dieser Gruppe nicht vorhandenem Betäubungsmittelcluster. Das Cluster „einfacher Diebstahl" der justiziell registrierten deutschen Männer kann somit auch als Betäubungsmittelcluster mit Beschaffungskriminalität bezeichnet werden. Bei allen anderen Gruppen außer den justiziell registrierten Männern ergab sich ein Cluster mit Betäubungsmitteldelikten. Bei den polizeilich Registrierten ist das Betäubungsmittelcluster ein reines Betäubungsmittelcluster. Bei den justiziell registrierten Frauen besteht das Betäubungsmittelcluster aus einer Mischung aus Betäubungsmitteldelikten und Delikten, die zur Beschaffungskriminalität zu zählen sind.

Bei allen Gruppen, außer den justiziell registrierten Frauen, ergab sich ein Cluster von „chronischen Straftätern“ mit verschiedenen Straftaten. Alle Delikte waren in diesem Cluster überzufällig häufig vertreten. Die durchschnittliche Anzahl der Delikte pro Altersabschnitt war in diesem Cluster deutlich überdurchschnittlich. Bei den justiziell registrierten Frauen ergab die 6-Clusterlösung kein Cluster „,verschiedene Straftaten“ und damit kein eindeutiges Cluster von „,chronischen Straftätern“. Die durchschnittliche Anzahl von Delikten pro Altersabschnitt ist bei den justiziell registrierten Frauen im Cluster der Gewaltdelikte am höchsten, d.h. wenn man bei Frauen von chronischen Straftätern sprechen kann, befinden sich diese im Cluster der Gewaltdelikte.

Bei den justiziell Registrierten bildete die große Gruppe der Verkehrsdelikte ein eigenes Cluster. ${ }^{314}$ Jeder Altersabschnitt dieses Clusters enthält ein Verkehrsdelikt. Außerdem gab es ein Cluster „fahrlässige Körperverletzung“; hier enthält jeder Altersabschnitt das Delikt fahrlässige Körperverletzung und relativ häufig auch ein Verkehrsdelikt. D.h. es gab ein Cluster von Verkehrsdelikten mit Personenschäden (Cluster „fahrlässige Körperverletzung“) und ein Cluster von Verkehrsdelikten ohne Personenschäden (Cluster „Verkehrsdelikte“).

314 Verkehrsdelikte werden polizeilich nicht registriert. 
Bei den polizeilich registrierten Männern ergab die 6-Clusterlösung zusätzlich noch das Cluster „schwerer Diebstahl“. Jeder zweite Altersabschnitt dieses Clusters enthält schweren Diebstahl. Außerdem sind die Delikte der Deliktskategorien Hehlerei und Fälschung überdurchschnittlich häufig vertreten. Bei den Frauen ergab sich das Cluster „Leistungserschleichung“. Alle Altersabschnitte dieses Clusters enthalten Leistungserschleichung.

Auch hieran sieht man, dass schwerer Diebstahl polizeilich anders registriert wird als justiziell und besonders ein „Männerdelikt“ ist. Leistungserschleichung ist eher ein „Frauendelikt“".

Ähnlichkeiten von Sexualdelikten zu Gewaltdelikten zeigten sich zwar, daraus kann aber nicht geschlossen werden, dass jeder Gewaltstraftäter auch zu einem Sexualdelikt fähig ist. Umgekehrt kann aber gesagt werden, dass Sexualstraftäter auch häufig wegen anderer Gewaltstraftaten registriert sind.

Die probabilistische Clusteranalyse ergab, dass es eine kleine Gruppe von chronischen Straftätern gibt, die alle Delikte begeht. Insofern kann bzgl. Sexualstraftätern gesagt werden, dass ein Teil von ihnen zu den chronischen Tätern gehört, ein weiterer Teil zum Cluster der Gewaltstraftäter und eine kleine Gruppe auf Sexualdelikte spezialisiert ist.

Zusammenfassend ist festzuhalten, dass innerhalb aller Deliktskategorien mehr oder weniger starke Tendenzen zur Spezialisierung vorhanden sind. Besonders häufig kommt es innerhalb der Deliktskategorien Betrug, Betäubungsmitteldelikte und sonstige Sexualdelikte zu Spezialisierung. Deliktsähnlichkeiten konnten insbesondere bei Gewaltdelikten zusammen mit Beleidigung und Sachbeschädigung festgestellt werden. Ein Zusammenhang zwischen Sexualdelikten und Gewaltdelikten zeigte sich bei den Jugendlichen, Heranwachsenden und Jungerwachsenen, nicht aber in der Altersgruppe ab 26 Jahre. Die Deliktskategorien Betrug und Fälschung sind ähnlich. Bei Betäubungsmitteldelikten zeigte sich ein Zusammenhang zur Beschaffungskriminalität. Bei Frauen wurde der Zusammenhang von Drogendelikten und Beschaffungsprostitution (sonstige Sexualdelikte) sichtbar.

Es gibt eine kleine Gruppe chronische Straftäter, die Straftaten jeder Deliktskategorie begehen.

Kriminelle Karriere kann weder durch totale Spezialisierung noch durch völlige Vielfältigkeit charakterisiert werden. Spezialisierung sollte, wie auch Kyvsgaard feststellte, nicht überinterpretiert werden, denn dies könnte bei der Aufklärung von Verbrechen $\mathrm{zu}$ falschen Verdächtigungen führen. ${ }^{315}$ Trotz des festgestellten $\mathrm{Zu}$ sammenhangs von Gewaltstraftaten kann daraus nicht gefolgert werden, dass jeder Gewaltstraftäter wieder eine Gewaltstraftat begeht.

315 Vgl. Kyvsgaard 2003, S. 243. 



\section{Literaturverzeichnis}

Ahrens, H. J., Multidimensionale Skalierung: Methodik, Theorie und empirische Gültigkeit mit Anwendungen aus der differentiellen Psychologie und Sozialpsychologie. Weinheim und Basel 1974.

Albrecht, H.-J./Moitra, S., Escalation and Specialization: A comparative Analysis of Patterns in Criminal Careers. In: Kaiser, G. \& Geissler, I. (Eds.), Crime and Criminal Justice Band 36 (1988). Freiburg: Kriminologische Forschungsberichte aus dem MaxPlanck-Institut für ausländisches und internationales Strafrecht, S. 115-136.

Althoff, M. \& Haan, W. de, Sind Asylbewerber krimineller? In: Monatsschrift für Kriminologie und Strafrechtsreform 87. Jahrgang Heft 6. Köln 2004, S. 436-450.

Bacher, J., Clusteranalyse: anwendungsorientierte Einführung. München 1996.

Bacher, J./Wenzig, K../Vogler, M. SPSS TwoStep Cluster - A first Evaluation. Arbeitsund Diskussionspapiere des Lehrstuhls für Soziologie. Friedrich-Alexander-Universität Erlangen-Nürnberg 2004.

Backhaus, K./Erichson, B./Plinke, W./Weiber, R., Multivariate Analysemethoden: eine anwendungsorientierte Einführung. Berlin 2003.

Blanc, M. Le/Fréchette, M., Male Criminal Activity from Childhood Through Youth. Multilevel and Developmental Perspectives. New York 1989.

Blumstein, A./Cohen, J., Estimation of Individual Crime Rates from Arrest Records. In: The Journal of Criminal Law \& Criminology, Vol. 70, No. 4 (1979), S. 561-585.

Blumstein, A./Cohen, J./Das, S./Moitra, S. D., Specialization and Seriousness During Adult Criminal Careers. In: Journal of Quantitative Criminology, Vol. 4, No. 4 (1988), S. 303345 .

Blumstein, A./Cohen, J./Roth, J. A./Visher, C A. (eds.), Criminal Careers and "Career Criminals", vol. 1. Washington D.C. 1986.

Borg, I./Groenen, P., Modern Multidimensional Scaling: Theory and Applications. New York 1997.

Budd, T., Sharp, C./Mayhew, P., Offending in England and Wales: First results from the 2003 Crime and Justice Survey. Home Office Research Study 275. London 2005.

Bundesministerium des Innern und Bundesministerium der Justiz (Hrsg.), Erster Periodischer Sicherheitsbericht. Berlin 2001.

Bundesministerium des Innern und Bundesministerium der Justiz (Hrsg.), Zweiter Periodischer Sicherheitsbericht. Berlin 2006.

Chaiken, J. M./Chaiken M. R., Varieties of Criminal Behavior. Summary and Policy Implications. Santa Monica, Californien 1982.

Chaiken, J. M./Chaiken M. R., Offender Types and Public Policy. In: Crime \& Delinquency, Vol. 30 No. 2 (1984), S. 195-226.

Clarke, S. H., Getting 'em out of Circulation: Does Incarceration of Juvenile Offenders Reduce Crime? In: The Journal of Criminal Law \& Criminology, Vol. 65, No. 4 (1975), S. 528-535. 
Clinard, M. B./Quinney, R./Wildeman, J., Criminal Behavior Systems: A Typology. Cincinnati, OH 1994.

Coxon, A. P. M., The User's Guide to Multidimensional Scaling. London 1982.

Davies, P. M./Coxon, A. P. M. (eds.), Key Texts in Multidimensional Scaling. London 1982.

Duden „Fremdwörterbuch“, Mannheim:1982.

Engel, S. W./Engelhardt, D. von, Kriminalität und Verlauf. Heidelberg 1978.

Farrington, D. P., Age and Crime. In: Tonry, M., Morris, N. (ed), Crime and Justice. An Annual Review of Research. Chicago 1986, S. 189-250.

Farrington, D. P., Criminal Career Research: Lessons for Crime Prevention. In: Studies on Crime \& Crime Prevention, Annual Review Vol. 1 No. 1 (1992), S. 7-29.

Farrington, D. P./Snyder, H. N./Finnegan, T. A., Specialization in Juvenile Court Careers. In: Criminology Volume 26 Number 3 (1988), S. 461-487.

Ferdinand, T. N., Typologies of Delinquency. A critical Analysis. New York 1966.

Figlio, R. M., Delinquency Careers as a Simple Markov Process. In: Fox, J. A. (ed), Models in Quantitative Criminology. New York 1981, S. 25-37.

Francis, B./Soothill, K./Fligelstone, R., Identifying Patterns and Pathways of Offending Behaviour. A New Approach to Typologies of Crime. In: European Journal of Criminology (ed by Smith, D., J.) Vol. 1, No. 1 (2004), S. 47-87.

Gibbons, D. C., Offender Typologies-Two Decades Later. In: British Journal of Criminology, Vol. 15, No. 2 (1975), S. 140-156.

Gibbons, D. C., Society, crime, and criminal behavior. Englewood Cliffs, New Jersey 1992.

Gottfredson, S. D./Gottfredson D. M., Behavioral Prediction and the Problem of Incapacitation. In: Criminology, Vol. 32, No. 3 (1994), S. 441-474.

Götz, A./Tolzmann, G., Bundeszentralregister: Kommentar. Stuttgart 2000.

Götz, A./Tolzmann, G., Bundeszentralregister: Kommentar. Stuttgart 2003.

Grundies, V., The Freiburg Cohort Study. In: Albrecht, H.-J., Kury, H. (ed), Research on Crime and Criminal Justice at the Max-Planck-Institute. Freiburg 1998, S. 29-32.

Grundies, $V$., Verfahrenseinstellungen nach $\S \S 45,47$ Jugendgerichtsgesetz. Arbeitsberichte 1/2004. Freiburg 2004.

Grundies, V./Höfer, S./Tetal, C., Basisdaten der Freiburger Kohortenstudie. Prävalenz und Inzidenz polizeilicher Registrierung. Arbeitsberichte 1/2002. Freiburg 2002.

Grundies, V./Titterington, V. B., An Exploratory Analysis of German and US Youthful Homicide Offendind. In: Homicide Studies 11 (2007), S. 189-212.

Haapanen, $R$. A., Selective Incapacitation and the Serious Offender. A Longitudinal Study of Criminal Career Patterns. New York 1990.

Höfer, S., Sanktionskarrieren. Freiburg 2003. 
Home office, Sentencing. http://www.homeoffice.gov.uk/justice/what-happens-at-court /sentencing/?view=Standard (Stand: 26. Januar 2007)

Kaiser, G., Kriminalität. In: Kaiser, G., Kerner, H.-J., Sack, F. \& Schellhoss, H. (Hrsg.), Kleines Kriminologisches Wörterbuch. Heidelberg 1993, S. 238-246.

Kaiser, G., Kriminologie. Ein Lehrbuch. Heidelberg 1996.

Kaiser, G., Kriminologie. Eine Einführung in die Grundlagen. Heidelberg 1997.

Kaiser, G./Kerner, H.-J./Sack, F./Schellhoss, H. (Hrsg.), Kleines Kriminologisches Wörterbuch. Heidelberg 1993.

Kempf, K. L., Specialization and the Criminal Career. In: Criminology, Vol. 25, No. 2 (1987), S. 399-420.

Klein, M. W., Offence Specialization and Versatility among Juveniles. In: British Journal of Crminology Vol. 24 No. 2 (1984), S. 185-194.

Kobrin, S./Hellum, F. R./Peterson, J. W., Offence Patterns of Status Offenders. In: Shichor, D. \& Kelly, D. H. (eds.), Critical Issues in Juvenile Delinquency. Lexington, MA: Lexington 1980, S. 203-235.

Kreuzer, A./Görgen, T./Krüger, R./Münch, V./Schneider, H., Jugenddelinquenz in Ost und West. (Schriftenreihe der Deutschen Vereinigung für Jugendgerichte und Jugendgerichtshilfen ; Bd. 22) Bonn 1993.

Kruskal, J. B./Wish, M., Multidimensional Scaling. Beverly Hills 1978.

Kunz, K.-L., Kriminologie. Berlin, Stuttgart, Wien 2004.

Kürzinger, J., Gewaltkriminalität. In: Kaiser, G., Kerner, H.-J., Sack, F. \& Schellhoss, H. (Hrsg.), Kleines Kriminologisches Wörterbuch. Heidelberg 1993, S. 171-177.

Kyvsgaard, B., The Criminal Career: The Danish Longitudinal Study. Cambridge 2003.

Landeskriminalamt Baden-Württemberg, INPOL-BW. Informationssystem der Polizei in Baden-Württemberg.

Landeskriminalamt Baden-Württemberg, Polizeiliche Kriminalstatistik Baden-Württemberg 2001. Stuttgart 2002.

Landeskriminalamt Nordrhein-Westfalen (Hrsg.), Junge Mehrfachtäter in NRW. Eine Auswertung der Polizeilichen Kriminalstatistik 1994-2003. Düsseldorf: Forschungsbericht Nr. 1/2005.

Lombroso, C., Criminal Man. Durham and London 2006.

Lynam, D. R./Piquero, A. R./Moffitt, T. E., Specialization and the Propensity to Violence. Support from Self-Reports but not Official Records. In: Journal of Contemporary Criminal Justice Vol. 20, No. 2 (2004), S. 215-228.

Moffitt, T. E., Adolescence-Limited and Life-Course-Persistent Antisocial Behavior: A Developmental Taxonomy. In: Psychological Review Vol. 100, No. 4 (1993), S. 674701.

Paternoster, R./Dean, C. W./Piquero, A./Mazerolle, P./Brame, R., Generality, Continuity, and Change in Offending. In: Journal of Quantitative Criminology 13 (1997), S. 231266. 
Petersilia, J., Criminal Career Research: A review of recent evidence. In: Morris, N. \& Tonry, M. (eds.), Crime and Justice, Vol. 2. Chicago 1980, S. 321-379.

Petersilia, J./Greenwood, P. W./Lavin, M., Criminal Careers of Habitual Felons. Washington D.C.1978.

Peterson, M. A./Braiker, H. B./Polich, S. M., Who Commits Crimes. A Survey of Prison Inmates. Cambridge, Massachusetts 1981.

Phillpotts, G. J. O./Lancucki, L. B., Previous Convictions, Sentence, and Reconviction. Home Office Research Study No. 53. London 1979.

Piquero, A./Paternoster, R./Mazerolle, P./Brame, R./Dean, C. W., Onset Age and Offense Specialization. In: Journal of Research in Crime and Delinquency 36 (1999), S. $275-$ 299.

Roebuck, J. B., The Negro numbers man as a criminal type: The construction and application of a typology. In: Journal of Criminal Law, Criminology and Police Science 54 (1963), S. 48-60.

Roebuck, J. B., Criminal Typology. The Legalistic, Physical-Constitutional-Hereditary, Psychological-Psychiatric and Sociological Approaches. Springfield, Illinois 1967.

Rojek, D. G./Erickson, M. L., Delinquent Careers. A Test of the Career Escalation Model. In: Criminology Vol. 20 No. 1 (1982), S. 5-28.

Schneider, G./Sutterer, P./Karger, T., Cohort Study on the Development of PoliceRecorded Criminality and Criminal Sanctioning. In: Kaiser, G. \& Geissler, I. (eds.), Crime and Criminal Justice, Criminological Research in the $2^{\text {nd }}$ Decade at the MaxPlanck-Institute. Freiburg 1988, S. 71-88.

Schneider, H. J., Kriminologie. Berlin; New York 1987.

Schöch, H., Klassifikation und Typologie. In: Kaiser, G., Kerner, H.-J., Sack, F. \& Schellhoss, H. (Hrsg.), Kleines Kriminologisches Wörterbuch. Heidelberg (1993), S. 214-217.

Schöch, H., Verkehrsdelikte. In: Kaiser, G., Kerner, H.-J., Sack, F. \& Schellhoss, H. (Hrsg.), Kleines Kriminologisches Wörterbuch. Heidelberg 1993, S. 577-581.

Schwind, H.-D., Kriminologie. Eine praxisorientierte Einführung mit Beispielen, Heidelberg 2007.

Seelig, E./Weindler, W., Die Typen der Kriminellen. Berlin und München 1949.

Smith, D. J., Crime and the Life Course. In: Maguire, M., Morgan, R. \& Reiner, R. (ed), The Oxford Handbook of Criminology 3rd edn. Oxford 2002, S. 702-745.

Smith, D. R./Smith, W. R., Patterns of Delinquent Careers: An Assessment of Three Perspectives. In: Social Science Research 13 (1984), S. 129-158.

SPSS Base 14.0 User's Guide http://sw.cs.uoguelph.ca/dsoft/SPSS/SPSS14Manuals/ Unwrapped/SPSS Base Users Guide 14.0.pdf, (Stand: 8. Januar 2007)

SPSS 14.0 Statistical Algorithms, PROXSCAL.pdf, http://support.spss.com/tech/default.asp, (Stand: 19. Dezember 2006)

Stander, J./Farrington, D. P./Hill, G./Altham, P. M. E., Markov Chain Analysis and Specialization in Criminal Careers, In: The British Journal of Criminology, Vol. 29, No. 4 (1989), S. 317-335. 
Stata Statistical Software, Multivariate Statistics [MV]. Reference Manual, Release 9. College Station, Texas 2005.

Stata Statistical Software, Programming [P]. Reference Manual, Release 9. College Station, Texas 2005.

Svenson, R., Strategic offences in the criminal career context. In: British Journal of Criminology 42, (2002), S. 395-411.

Tracy, P. E./Wolfgang, M. E./Figlio R. M., Delinquency Careers in Two Birth Cohorts. New York and London 1990.

Uhlig, S., Justizregister: Vorschriftensammlung für das Bundeszentralregister mit dem Erziehungsregister, für das Gewerbezentralregister und das Verkehrszentralregister. Köln 1985.

D'Unger, A. V./Land, K. C./McCall, P. L./Nagin, D. S., How Many Latent Classes of Delinquent/Criminal Careers? Results from Mixed Poisson Regression Analyses. In: American Journal of Sociology Volume 103 Number 6 (1998), S. 1593-1630.

Veith, H.-M., Das Bundeszentralregister. In: Bewährungshilfe, 46 (1999), S. 111-133.

Vermunt, J. K./Magidson, J., Technical Guide for Latent GOLD 4.0: Basic and Advanced. Belmont, Massachusetts 2005.

Vermunt, J. K./Magidson, J., Latent GOLD 4.0 User's Guide. Belmont, Massachusetts 2005.

Weis, J. G., Issues in the Measurement of Criminal Careers. In: Blumstein, A., Cohen, J., Roth, J. A. \& Visher, C A. (eds.) (1986). Criminal Careers and "Career Criminals", vol. 2. Washington D.C. 1986, S. 1-51.

Wessels, J./Beulke, W., Strafrecht, allgemeiner Teil: die Straftat und ihr Aufbau. Heidelberg 1999.

West, D. J., Delinquency. Its Roots, Careers and Prospects. London 1982.

Wolfgang, M./Figlio, R./Sellin, T., Delinquency in a Birth Cohort. Chicago 1972.

Zwisler, R., Multidimensionale Skalierung. Skript, Universität Regensburg, Institut für Psychologie 1998. http://www.zwisler.de/scripts/mds/mds.html (Stand: 9. November 2006) 



\section{Abkürzungsverzeichnis}

am

aw

ASR

AsylVG

AuslG

bes.

BTM

BTMG

bzgl.

BZR

BZRG

Del.

$\mathrm{dm}$

dw

Eingr.

Erschl. v. Leist.

gef.

Gef. Dem. Rechtsstaat gemeingef.

gg.

Hausfriedensbr.

JGG

$\mathrm{KV}$

männl.

MDS

NG

nichtdt.

öffentl. Ordnung

PAD

pers.

PflVG

PKS nichtdeutsche Männer

nichtdeutsche Frauen

adjusted Standardized Residuals (korrigiertes standardisiertes Residuum)

Asylverfahrensgesetz

Ausländergesetz

besonderer

Betäubungsmittel

Betäubungsmittelgesetz

bezüglich

Bundeszentralregister

Bundeszentralregistergesetz

Delikt

deutsche Männer

deutsche Frauen

Eingriff

Leistungserschleichung

gefährlich

Gefährdung des demokratischen Rechtsstaates

gemeingefährlich

gegen

Hausfriedensbruch

Jugendgerichtsgesetz

Körperverletzung

männlich

Multidimensionale Skalierung

Nebengesetz

nichtdeutsch

Straftaten gegen die öffentliche Ordnung

Personenauskunftsdatei der Polizei in Baden-Württemberg persönliche

Pflichtversicherungsgesetz

Polizeiliche Kriminalstatistik 
Abkürzungsverzeichnis

Registr. Registrierung

schw. schwer

sonst. $\quad$ sonstige

StGB Strafgesetzbuch

StPo Strafprozessordnung

Straft. Straftaten

Straft. bzgl. Religion Straftaten, welche sich auf Religion und Weltanschauung beziehen

Straßenv. Straßenverkehr

StVG Straßenverkehrsgesetz

TSH Straftatenschlüssel

TV Tatverdächtige

Verletzg. pers. Geheimbereich Verletzung des persönlichen Lebens- und Geheimbereichs weibl. weiblich

Wid. gg. Staatsgew.

Widerstand gegen die Staatsgewalt 


\section{Tabellenverzeichnis}

Tabelle 1: Datenbestand der Freiburger Kohortenstudie (Stand PAD 2003, BZR 2004)

Tabelle 2: $\quad$ Satzaufbau eines BZR-Datensatzes mit den Variablen, die der Freiburger Kohortenstudie geliefert werden

Tabelle 3: Deliktskategorien

Tabelle 4: Häufigkeitsverteilung der Delikte in PAD und BZR (alle

Registrierungen PAD bis 2001, BZR bis 2003)....

Tabelle 5: Datenbestand der Freiburger Kohortenstudie bis 2001 (PAD) ..........25

Tabelle 6: Datenbestand der Freiburger Kohortenstudie bis 2003 (BZR) ..........26

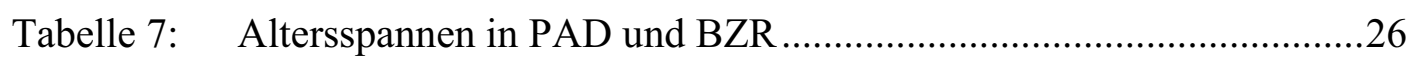

Tabelle 8: Anzahl der Kombinationen je nach Anzahl der Registrierungen .......31

Tabelle 9: PAD: Tatverdächtige und Taten (alle Registrierungen bis 2001)......32

Tabelle 10: BZR: Täter, Registrierungen - männlich - weiblich (alle Reg. bis 2003)

Tabelle 11: BZR: Täter, Delikte - männlich - weiblich (alle Registrierungen bis 2003)

Tabelle 12: Entfernung zwischen 10 Städten in km (Luftlinie) 38

Tabelle 13: Koordinaten

Tabelle 14: Häufigkeitsverteilung der Deliktskategorien bei Tatverdächtigen mit 2 oder mehr Straftaten (Registrierungen bis 2001). .46

Tabelle 15: Anzahl der Deliktspaare (deutsche Männer). 47

Tabelle 16: Adjusted Standardized Residuals der Deliktspaare (deutsche Männer). 49

Tabelle 17: Erklärte Varianz (deutsche Männer) .54

Tabelle 18: Adjusted Standardized Residuals (deutsche Frauen) ..........................55

Tabelle 19: Erklärte Varianz (deutsche Frauen) …...............................................59

Tabelle 20: Adjusted Standardized Residuals (nichtdeutsche Männer)................60

Tabelle 21: Erklärte Varianz (nichtdeutsche Männer) .........................................64

Tabelle 22: Adjusted Standardized Residuals (nichtdeutsche Frauen) .................65

Tabelle 23: Erklärte Varianz (nichtdeutsche Frauen) ...........................................68

Tabelle 24: Delikt mit oder ohne weiteren Delikten am selben Tag.....................70 
Tabelle 25: Anteil Deliktspaare mit gleichem Tatdatum bei einer Person an allen Deliktspaaren in \%

Tabelle 26: Häufigkeitsverteilung der Deliktskategorien bei Tätern mit 2 oder mehr Straftaten (Registrierungen bis 2003)

Tabelle 27: Adjusted Standardized Residuals der Deliktspaare (deutsche Männer)... .78

Tabelle 28: Erklärte Varianz (deutsche Männer) .83

Tabelle 29: Adjusted Standardized Residuals (deutsche Frauen)..... .85

Tabelle 30: Erklärte Varianz (deutsche Frauen) .88

Tabelle 31: Adjusted Standardized Residuals (nichtdeutsche Männer)................90

Tabelle 32: Erklärte Varianz (nichtdeutsche Männer) .......................................93

Tabelle 33: Adjusted Standardized Residuals (nichtdeutsche Frauen) .................94

Tabelle 34: Erklärte Varianz (nichtdeutsche Frauen) ...........................................98

Tabelle 35: Häufigkeit von Delikten ohne weiteres Delikt in selber Registrierung (BZR-Registrierungen bis 2003)

Tabelle 36: Anteil Deliktspaare innerhalb einer Entscheidung an allen Deliktspaaren (BZR) in \%.

Tabelle 37: Anteil Deliktspaare innerhalb einer Entscheidung getrennt nach Deliktskategorien (BZR).

Tabelle 38: Modellprüfgrößen zur Bestimmung der Clusteranzahl (deutsche Männer, Kohorte 1970, justizielle Registrierungen)

Tabelle 39: Deliktscluster justizieller Registrierungen deutscher Männer der Kohorte 1970: Wahrscheinlichkeiten der Deliktskategorien in den Clustern (in \%)

Tabelle 40: Wahrscheinlichkeit der Clusterzugehörigkeit bei Deliktsvorkommen in \% (Justizielle Registrierung, deutsche Männer, Kohorte 1970, Baden-Württemberg)

Tabelle 41: Deliktszusammensetzung der Cluster deutscher Männer der Kohorte 1970 (6 Cluster)

Tabelle 42: Überblick über die Clusterzusammensetzung je nach Anzahl von Clustern (deutsche Männer Kohorte 1970)

Tabelle 43: Spezialisierungsanteile in \%: Deutsche Männer der Kohorte 1970 (justizielle Registrierungen).

Tabelle 44: Modellprüfgrößen zur Bestimmung der Clusteranzahl (deutsche Frauen, Kohorte 1970, justizielle Registrierungen)

Tabelle 45: Deliktscluster justizieller Registrierungen deutscher Frauen der Kohorte 1970: Wahrscheinlichkeiten der Deliktskategorien in den Clustern (in \%) 
Tabelle 46: Wahrscheinlichkeit der Clusterzugehörigkeit bei Deliktsvorkommen in \% (Justizielle Registrierung, deutsche Frauen, Kohorte 1970, Baden-Württemberg)

Tabelle 47: Deliktszusammensetzung der Cluster deutscher Frauen der Kohorte 1970

Tabelle 48: Spezialisierungsanteile in \%: Deutsche Frauen der Kohorte 1970 (justizielle Registrierungen).

Tabelle 49: Modellprüfgrößen zur Bestimmung der Clusteranzahl (deutsche Männer, Kohorte 1970, polizeiliche Registrierungen) ....176

Tabelle 50: Deliktscluster polizeilicher Registrierungen deutscher Männer der Kohorte 1970: Wahrscheinlichkeiten der Deliktskategorien in den Clustern (in \%)

Tabelle 51: Wahrscheinlichkeit der Clusterzugehörigkeit bei Deliktsvorkommen in \% (Polizeiliche Registrierung, deutsche Männer, Kohorte 1970, Baden-Württemberg)

Tabelle 52: Deliktszusammensetzung der Cluster deutscher Männer der Kohorte 1970 (6 Cluster)

Tabelle 53: Spezialisierungsanteile in \%: Deutsche Männer der Kohorte 1970 (polizeiliche Registrierungen)

Tabelle 54: Modellprüfgrößen zur Bestimmung der Clusteranzahl (deutsche Frauen, Kohorte 1970, polizeiliche Registrierungen)

Tabelle 55: Deliktscluster polizeilicherer Registrierungen deutscher Frauen der Kohorte 1970: Wahrscheinlichkeiten der Deliktskategorien in den Clustern (in \%)

Tabelle 56: Wahrscheinlichkeit der Clusterzugehörigkeit bei Deliktsvorkommen in \% (Polizeiliche Registrierung, deutsche Frauen, Kohorte 1970, Baden-Württemberg)

Tabelle 57: Deliktszusammensetzung der Cluster deutscher Frauen der Kohorte 1970

Tabelle 58: Spezialisierungsanteile in \%: Deutsche Frauen der Kohorte 1970 (polizeiliche Registrierungen).

Tabelle 59: Vergleich der 6-Clusterlösungen 202

Tabelle 60: Forward Specialization Coefficients für den Übergang innerhalb derselben Deliktskategorie von einer Registrierung zur nächsten getrennt nach Geschlecht und Nationalität (polizeiliche Registrierungen)

Tabelle 61: Forward Specialization Coefficients für den Übergang innerhalb derselben Deliktskategorie von einer Registrierung zur nächsten getrennt nach Geschlecht und Nationalität (justizielle Registrierungen) 
Tabelle 62: Anzahl der Deliktspaare (PAD deutsche Frauen) ...........................237

Tabelle 63: Anzahl der Deliktspaare (PAD nichtdeutsche Männer)..................239

Tabelle 64: Anzahl der Deliktspaare (PAD nichtdeutsche Frauen) ....................241

Tabelle 65: Anzahl der Deliktspaare (BZR deutsche Männer) ...........................243

Tabelle 66: Anzahl der Deliktspaare (BZR deutsche Frauen) ...........................245

Tabelle 67: Anzahl der Deliktspaare (BZR nichtdeutsche Männer)..................247

Tabelle 68: Anzahl der Deliktspaare (BZR nichtdeutsche Frauen) ....................249

Tabelle 69: Adjusted Standardized Residuals der Deliktspaare (deutsche Männer BZR, nur schwerstes Delikt) ..............................251

Tabelle 70: Male cluster profiles: Probabilities of a strip in each cluster having the specified offence $(\%)$....

Tabelle 71: Deliktszusammensetzung der männlichen Cluster von

Francis, Soothill, Fligelstone

Tabelle 72: Female cluster profiles: Probabilities of a strip in each cluster having the specified offence $(\%)$.

Tabelle 73: Deliktszusammensetzung der weiblichen Cluster von Francis, Soothill, Fligelstone .

Tabelle 74: Deliktscluster justizieller Registrierungen deutscher Männer der Kohorte 1970: Wahrscheinlichkeiten der Deliktskategorien in den Clustern in \% (7-Clusterlösung).

Tabelle 75: Deliktscluster justizieller Registrierungen deutscher Männer der Kohorte 1970: Wahrscheinlichkeiten der Deliktskategorien in den Clustern in \% (8-Clusterlösung).

Tabelle 76: Deliktscluster justizieller Registrierungen deutscher Männer der Kohorte 1970: Wahrscheinlichkeiten der Deliktskategorien in den Clustern in \% (9-Clusterlösung).

Tabelle 77: Vergleich 6-Clusterlösung mit 7-Clusterlösung (justizielle Registrierungen deutsche Männer Kohorte 1970) 262 


\section{Abbildungsverzeichnis}

Abbildung 1: Häufigkeitsverteilung der polizeilichen Registrierungen nach Alter

Abbildung 2: Häufigkeitsverteilung der justiziellen Entscheidungen nach Alter

Abbildung 3: Deliktsverteilung nach Alter bei deutschen Männern (PAD) ........29

Abbildung 4: Deliktsverteilung nach Alter bei deutschen Männern (BZR)..........30

Abbildung 5: Durch MDS gewonnene Konfiguration von 10 Städten (vor Rotation und Spiegelung)

Abbildung 6: Konfiguration der Städte nach Rotation und Spiegelung.... 40

Abbildung 7: Ergebnis der MDS (deutsche Männer) ...........................................53

Abbildung 8: Ergebnis der MDS (deutsche Frauen) …......................................58

Abbildung 9: Ergebnis der MDS (nichtdeutsche Männer)..................................63

Abbildung 10: Ergebnis der MDS (nichtdeutsche Frauen) .................................67

Abbildung 11: Ergebnis der MDS (deutsche Männer).........................................82

Abbildung 12: Ergebnis der MDS (deutsche Frauen) .........................................87

Abbildung 13: Ergebnis der MDS (nichtdeutsche Männer) ..................................92

Abbildung 14: Ergebnis der MDS (nichtdeutsche Frauen) ..................................97

Abbildung 15: Ergebnis der MDS (deutsche Männer, nur schwerstes Delikt) ....106

Abbildung 16: Ergebnis der MDS (deutsche Männer PAD, gewichtet nach Dauer von einer Straftat zur nächsten)

Abbildung 17: Ergebnis der MDS (deutsche Männer BZR, gewichtet nach Dauer von einer Straftat zur nächsten)

Abbildung 18: Ergebnis der MDS (deutsche Männer PAD, Alter 7 bis 13).

Abbildung 19: Ergebnis der MDS (deutsche Männer PAD, Alter 14 bis 17).

Abbildung 20: Ergebnis der MDS (deutsche Männer PAD, Alter 18 bis 21).

Abbildung 21: Ergebnis der MDS (deutsche Männer PAD, Alter 22 bis 25).

Abbildung 22: Ergebnis der MDS (deutsche Männer PAD, Alter 26 bis 31

Abbildung 23: Ergebnis der MDS (deutsche Männer BZR, Alter 14 bis 17) 
Abbildung 24: Ergebnis der MDS (deutsche Männer BZR, Alter 18 bis 21).

Abbildung 25: Ergebnis der MDS (deutsche Männer BZR, Alter 22 bis 25)....

Abbildung 26: Ergebnis der MDS (deutsche Männer BZR, Alter 26 bis 33)..

Abbildung 27: Arithmetic-average clustering (deutsche Männer PAD) 125

Abbildung 28: Arithmetic-average clustering (deutsche Frauen PAD) 127

Abbildung 29: Arithmetic-average clustering (deutsche Männer BZR) 128

Abbildung 30: Arithmetic-average clustering (deutsche Frauen BZR) .129

Abbildung 31: Häufigkeiten der Altersgruppen (justizielle Registrierung, deutsche Männer und Frauen, Kohorte 1970)

Abbildung 32: Verteilung der männlichen Täter auf Cluster nach Altersgruppen 146

Abbildung 33: Deliktsübergänge männlicher Täter der Altersgruppe 19-23 153

Abbildung 34: Deliktsübergänge männlicher Täter des Clusters A (Diebstahl) im Alter 19 bis 23

Abbildung 35: Deliktsübergänge männlicher Täter des Clusters B (Verkehrsdelikte) im Alter 19 bis 23

Abbildung 36: Deliktsübergänge männlicher Täter des Clusters C (Gewaltdelikte) im Alter 19 bis 23.

Abbildung 37: Deliktsübergänge männlicher Täter des Clusters D

(Betrug) im Alter 19 bis 23

Abbildung 38: Deliktsübergänge männlicher Täter des Clusters E (fahrlässige Körperverletzung) im Alter 19 bis 23

Abbildung 39: Deliktsübergänge männlicher Täter des Clusters F (verschiedene Straftaten) im Alter 19 bis 23

Abbildung 40: Verteilung der weiblichen Täter auf Cluster nach Altersgruppen

Abbildung 41: Deliktsübergänge weiblicher Täter der Altersgruppe 19-23.

Abbildung 42: Deliktsübergänge weiblicher Täter des Clusters A (einfacher Diebstahl) im Alter 19 bis 23 .170

Abbildung 43: Deliktsübergänge weiblicher Täter des Clusters B (Betrug) im Alter 19 bis 23 170

Abbildung 44: Deliktsübergänge weiblicher Täter des Clusters C (Betäubungsmitteldelikte) im Alter 19 bis 23 
Abbildung 45: Deliktsübergänge weiblicher Täter des Clusters D

(Verkehrsdelikte) im Alter 19 bis 23.

Abbildung 46: Deliktsübergänge weiblicher Täter des Clusters E

(Gewaltdelikte) im Alter 19 bis 23.

Abbildung 47: Deliktsübergänge weiblicher Täter des Clusters F

(fahrlässige Körperverletzung) im Alter 19 bis 23.

Abbildung 48: Häufigkeiten der Altersgruppen (polizeiliche Registrierung, deutsche Männer und Frauen, Kohorte 1970)

Abbildung 49: Verteilung der männlichen Tatverdächtigen auf Cluster nach Altersgruppen

Abbildung 50: Deliktsübergänge männlicher Tatverdächtiger der Altersgruppe 21-25.

Abbildung 51: Deliktsübergänge männlicher Tatverdächtiger des Clusters A (Gewaltdelikte) im Alter 21 bis 25.

Abbildung 52: Deliktsübergänge männlicher Tatverdächtiger des Clusters B (schwerer Diebstahl) im Alter 21 bis 25

Abbildung 53: Deliktsübergänge männlicher Tatverdächtiger des Clusters C (einfacher Diebstahl) im Alter 21 bis 25.

Abbildung 54: Deliktsübergänge männlicher Tatverdächtiger des Clusters D (Betäubungsmitteldelikte) im Alter 21 bis 25

Abbildung 55: Deliktsübergänge männlicher Tatverdächtiger des Clusters E (Betrug) im Alter 21 bis 25

Abbildung 56: Deliktsübergänge männlicher Tatverdächtiger des Clusters $F$ (verschiedene Straftaten) im Alter 21 bis 25

Abbildung 57: Verteilung der weiblichen Tatverdächtigen auf Cluster nach Altersgruppen

Abbildung 58: Deliktsübergänge weiblicher Tatverdächtiger der Altersgruppe 21-25.

Abbildung 59: Deliktsübergänge weiblicher Tatverdächtiger des Clusters A (einfacher Diebstahl) im Alter 21 bis 25 ....

Abbildung 60: Deliktsübergänge weiblicher Tatverdächtiger des Clusters B (Gewaltdelikte) im Alter 21 bis 25 .

Abbildung 61: Deliktsübergänge weiblicher Tatverdächtiger des Clusters C (Betrug) im Alter 21 bis 25

Abbildung 62: Deliktsübergänge weiblicher Tatverdächtiger des Clusters D (Betäubungsmitteldelikte) im Alter 21 bis 25

Abbildung 63: Deliktsübergänge weiblicher Tatverdächtiger des Clusters E (verschiedene Straftaten) im Alter 21 bis 25 
Abbildung 64: Deliktsübergänge weiblicher Tatverdächtiger des Clusters F (Erschleichen von Leistungen) im Alter 21 bis 25.

Abbildung 65: Altersverteilung der 8-Clusterlösung (deutsche Männer 1970 BZR) 263

Abbildung 66: Altersverteilung der 9-Clusterlösung (deutsche Männer 1970 BZR). 263 


\section{Anhang}

Tabelle 62: Anzahl der Deliktspaare (PAD deutsche Frauen)

\begin{tabular}{|c|c|c|c|c|c|c|c|c|}
\hline & $\begin{array}{c}\text { Tötungs- } \\
\text { delikte }\end{array}$ & $\begin{array}{l}\text { Sexu- } \\
\text { aldel. }\end{array}$ & $\begin{array}{c}\text { sonst. } \\
\text { Sex. }\end{array}$ & Raub & $\begin{array}{c}\text { schw. } \\
\text { KV }\end{array}$ & $\mathrm{KV}$ & $\begin{array}{l}\text { Str. gg. } \\
\text { Pers. Fr. }\end{array}$ & einf. Dieb. \\
\hline Tötungsdelikte & 13 & & & & & & & \\
\hline Sexualdelikte & 0 & 0 & & & & & & \\
\hline sonst. Sexueldel. & 9 & 0 & 1.893 & & & & & \\
\hline Raub & 12 & 0 & 87 & 106 & & & & \\
\hline schw. KV & 17 & 1 & 266 & 145 & 251 & & & \\
\hline Körperverletzung & 26 & 0 & 280 & 139 & 539 & 381 & & \\
\hline Str. gg. Pers. Fr. & 11 & 0 & 169 & 57 & 239 & 269 & 77 & \\
\hline einf. Diebstahl & 104 & 4 & 3.998 & 1.091 & 2.219 & 2.912 & 1.064 & 28.167 \\
\hline schw. Diebstahl & 13 & 16 & 482 & 227 & 265 & 383 & 113 & 6.520 \\
\hline Sachbeschäd. & 17 & 0 & 268 & 89 & 270 & 339 & 159 & 2.198 \\
\hline gemeingef. Str. & 3 & 0 & 0 & 6 & 24 & 25 & 6 & 203 \\
\hline Hehlerei & 4 & 3 & 141 & 47 & 114 & 140 & 67 & 1.640 \\
\hline Betrug & 31 & 4 & 466 & 345 & 595 & 843 & 294 & 13.404 \\
\hline Erschl. v. Leist. & 25 & 0 & 1.741 & 249 & 714 & 745 & 309 & 9.495 \\
\hline Gef. dem. Recht. & 3 & 0 & 1 & 3 & 15 & 18 & 5 & 74 \\
\hline Wid. gg. Staat. & 3 & 0 & 78 & 16 & 92 & 127 & 52 & 505 \\
\hline öff. Ordnung & 10 & 1 & 71 & 38 & 69 & 92 & 44 & 919 \\
\hline Hausfriedensbr. & 8 & 0 & 298 & 53 & 125 & 203 & 86 & 2.527 \\
\hline Beleidigung & 21 & 4 & 362 & 97 & 446 & 715 & 330 & 2.538 \\
\hline Fälschung & 0 & 0 & 89 & 68 & 99 & 126 & 43 & 2.220 \\
\hline Meineid & 1 & 0 & 24 & 6 & 26 & 38 & 16 & 245 \\
\hline falsche Verd. & 1 & 1 & 96 & 16 & 58 & 70 & 43 & 762 \\
\hline BTM-Delikte & 63 & 18 & 3.205 & 661 & 998 & 1.251 & 420 & 16.922 \\
\hline gef. Eingr. Str.V. & 0 & 0 & 0 & 0 & 2 & 8 & 0 & 13 \\
\hline
\end{tabular}




\begin{tabular}{|c|c|c|c|c|c|c|c|c|}
\hline & $\begin{array}{l}\text { schw. } \\
\text { Dieb. }\end{array}$ & Sachb. & $\begin{array}{l}\text { gemein- } \\
\text { gef. Str. }\end{array}$ & Hehlerei & Betrug & $\begin{array}{l}\text { Erschl. v. } \\
\text { Leist. }\end{array}$ & $\begin{array}{l}\text { Gef. } \\
\text { dem. } \\
\text { Recht. }\end{array}$ & $\begin{array}{l}\text { Wid. gg. } \\
\text { Staat. }\end{array}$ \\
\hline schw. Diebstahl & 3.501 & & & & & & & \\
\hline Sachbeschäd. & 403 & 210 & & & & & & \\
\hline gemeingef. Str. & 32 & 27 & 25 & & & & & \\
\hline Hehlerei & 341 & 80 & 6 & 88 & & & & \\
\hline Betrug & 1.872 & 655 & 59 & 449 & 18.684 & & & \\
\hline Erschl. v. Leist. & 995 & 488 & 23 & 291 & 1.726 & 4.375 & & \\
\hline $\begin{array}{l}\text { Gef. dem. } \\
\text { Recht. }\end{array}$ & 32 & 22 & 7 & 3 & 41 & 22 & 12 & \\
\hline Wid. gg. Staat. & 70 & 89 & 4 & 34 & 116 & 182 & 6 & 23 \\
\hline öff. Ordnung & 124 & 80 & 8 & 59 & 794 & 235 & 21 & 25 \\
\hline Hausfriedensbr. & 220 & 199 & 7 & 67 & 284 & 766 & 4 & 65 \\
\hline Beleidigung & 288 & 365 & 12 & 141 & 1.032 & 747 & 21 & 163 \\
\hline Fälschung & 296 & 71 & 8 & 96 & 1.550 & 300 & 4 & 27 \\
\hline Meineid & 27 & 22 & 2 & 22 & 435 & 42 & 1 & 5 \\
\hline falsche Verd. & 106 & 50 & 3 & 50 & 336 & 179 & 0 & 17 \\
\hline BTM-Delikte & 2.489 & 869 & 89 & 808 & 3.074 & 4.453 & 24 & 289 \\
\hline \multirow[t]{2}{*}{$\begin{array}{l}\text { gef. Eingr. } \\
\text { Str.V. }\end{array}$} & 2 & 1 & 1 & 1 & 8 & 14 & 1 & 1 \\
\hline & öff. Ord. & Hausfr. & $\begin{array}{l}\text { Beleidi- } \\
\text { gung }\end{array}$ & $\begin{array}{c}\text { Fäl- } \\
\text { schung }\end{array}$ & Meineid & $\begin{array}{l}\text { falsche } \\
\text { Verd. }\end{array}$ & $\begin{array}{l}\text { BTM- } \\
\text { Delikte }\end{array}$ & $\begin{array}{c}\text { gef. } \\
\text { Eingr. } \\
\text { Str.V. }\end{array}$ \\
\hline öff. Ordnung & 46 & & & & & & & \\
\hline Hausfriedensbr. & 41 & 208 & & & & & & \\
\hline Beleidigung & 104 & 189 & 375 & & & & & \\
\hline Fälschung & 63 & 67 & 117 & 200 & & & & \\
\hline Meineid & 22 & 6 & 25 & 18 & 10 & & & \\
\hline falsche Verd. & 61 & 38 & 105 & 53 & 15 & 46 & & \\
\hline BTM-Delikte & 345 & 1.033 & 1.077 & 723 & 105 & 261 & 9.093 & \\
\hline $\begin{array}{l}\text { gef. Eingr. } \\
\text { Str.V. }\end{array}$ & 2 & 1 & 4 & 0 & 0 & 0 & 13 & 0 \\
\hline
\end{tabular}


Tabelle 63: Anzahl der Deliktspaare (PAD nichtdeutsche Männer)

\begin{tabular}{|c|c|c|c|c|c|c|c|c|}
\hline & $\begin{array}{c}\text { Tötungs- } \\
\text { delikte }\end{array}$ & $\begin{array}{c}\text { Sexual- } \\
\text { del. }\end{array}$ & $\begin{array}{l}\text { sonst. } \\
\text { Sex. }\end{array}$ & Raub & $\begin{array}{l}\text { schw. } \\
\text { KV }\end{array}$ & KV & $\begin{array}{l}\text { Str. gg. } \\
\text { Pers. Fr. }\end{array}$ & $\begin{array}{l}\text { einf. } \\
\text { Dieb. }\end{array}$ \\
\hline Tötungsdelikte & 16 & & & & & & & \\
\hline Sexualdelikte & 14 & 255 & & & & & & \\
\hline $\begin{array}{l}\text { sonst. Sexual- } \\
\text { del. }\end{array}$ & 9 & 164 & 283 & & & & & \\
\hline Raub & 145 & 497 & 213 & 4.890 & & & & \\
\hline schw. KV & 255 & 845 & 385 & 4.942 & 6.247 & & & \\
\hline $\begin{array}{l}\text { Körperverlet- } \\
\text { zung }\end{array}$ & 185 & 782 & 459 & 3.675 & 9.619 & 5.676 & & \\
\hline $\begin{array}{l}\text { Str. gg. Pers. } \\
\text { Fr. }\end{array}$ & 136 & 796 & 272 & 2.615 & 6.072 & 6.393 & 2.484 & \\
\hline einf. Diebstahl & 595 & 2.230 & 1.557 & 18.607 & 23.310 & 21.947 & 13.924 & 137.710 \\
\hline schw. Diebstahl & 570 & 1.925 & 1.210 & 18.700 & 18.879 & 17.293 & 11.478 & 145.700 \\
\hline Sachbeschäd. & 132 & 436 & 302 & 2.522 & 5.481 & 5.064 & 3.142 & 18.932 \\
\hline gemeingef. Str. & 13 & 30 & 23 & 202 & 293 & 265 & 158 & 1.437 \\
\hline Hehlerei & 31 & 172 & 89 & 1.319 & 2.077 & 1.943 & 1.251 & 11.456 \\
\hline Betrug & 119 & 417 & 420 & 3.437 & 4.848 & 5.517 & 4.092 & 31.859 \\
\hline Erschl. v. Leist. & 99 & 332 & 229 & 2.345 & 3.422 & 3.043 & 2.016 & 24.556 \\
\hline $\begin{array}{l}\text { Gef. dem. } \\
\text { Recht. }\end{array}$ & 14 & 23 & 4 & 33 & 85 & 48 & 61 & 165 \\
\hline Wid. gg. Staat. & 40 & 68 & 43 & 464 & 1.284 & 1.432 & 724 & 3.732 \\
\hline öff. Ordnung & 32 & 113 & 75 & 725 & 1.287 & 1.042 & 696 & 3.490 \\
\hline Hausfriedensbr. & 41 & 208 & 93 & 1.163 & 2.230 & 2.085 & 1.566 & 12.653 \\
\hline Beleidigung & 50 & 402 & 271 & 1.505 & 4.454 & 5.032 & 3.551 & 9.819 \\
\hline Fälschung & 54 & 233 & 149 & 1.044 & 1.817 & 1.800 & 1.425 & 13.251 \\
\hline Meineid & 6 & 25 & 16 & 159 & 327 & 307 & 227 & 1.028 \\
\hline falsche Verd. & 14 & 31 & 21 & 155 & 317 & 303 & 261 & 1.417 \\
\hline BTM-Delikte & 297 & 779 & 595 & 9.097 & 11.588 & 10.457 & 6.421 & 61.367 \\
\hline $\begin{array}{l}\text { gef. Eingr. } \\
\text { Str.V. }\end{array}$ & 2 & 3 & 0 & 8 & 33 & 38 & 19 & 135 \\
\hline AuslG & 318 & 671 & 338 & 3.227 & 6.913 & 5.769 & 4.969 & 65.412 \\
\hline
\end{tabular}




\begin{tabular}{|c|c|c|c|c|c|c|c|c|}
\hline & $\begin{array}{l}\text { schw. } \\
\text { Dieb. }\end{array}$ & Sachb. & $\begin{array}{l}\text { gemein- } \\
\text { gef. Str. }\end{array}$ & Hehlerei & Betrug & $\begin{array}{l}\text { Erschl. v. } \\
\text { Leist. }\end{array}$ & $\begin{array}{l}\text { Gef. } \\
\text { dem. } \\
\text { Recht. }\end{array}$ & $\begin{array}{l}\text { Wid. gg. } \\
\text { Staat. }\end{array}$ \\
\hline schw. Diebstahl & 234.691 & & & & & & & \\
\hline Sachbeschäd. & 16.159 & 2.840 & & & & & & \\
\hline gemeingef. Str. & 1.232 & 400 & 148 & & & & & \\
\hline Hehlerei & 10.120 & 1.256 & 98 & 975 & & & & \\
\hline Betrug & 26.555 & 3.376 & 271 & 2.313 & 19.760 & & & \\
\hline Erschl. v. Leist. & 11.994 & 2.501 & 186 & 1.121 & 4.673 & 6.547 & & \\
\hline $\begin{array}{l}\text { Gef. dem. } \\
\text { Recht. }\end{array}$ & 233 & 93 & 6 & 8 & 47 & 20 & 33 & \\
\hline Wid. gg. Staat. & 3.175 & 819 & 48 & 275 & 648 & 737 & 7 & 210 \\
\hline öff. Ordnung & 2.880 & 766 & 69 & 360 & 1.393 & 509 & 45 & 177 \\
\hline Hausfriedensbr. & 7.515 & 1.554 & 93 & 699 & 1.626 & 1.809 & 11 & 495 \\
\hline Beleidigung & 7.595 & 2.501 & 113 & 906 & 2.576 & 1.794 & 34 & 767 \\
\hline Fälschung & 10.921 & 1.205 & 136 & 967 & 5.141 & 1.738 & 27 & 374 \\
\hline Meineid & 1.319 & 147 & 18 & 150 & 607 & 130 & 1 & 40 \\
\hline falsche Verd. & 1.380 & 208 & 19 & 124 & 448 & 152 & 2 & 36 \\
\hline BTM-Delikte & 44.281 & 7.901 & 566 & 5.443 & 11.552 & 11.212 & 62 & 2.321 \\
\hline $\begin{array}{l}\text { gef. Eingr. } \\
\text { Str.V. }\end{array}$ & 118 & 31 & 5 & 4 & 29 & 18 & 0 & 5 \\
\hline AuslG & 22.602 & 3.591 & 237 & 3.032 & 8.010 & 12.916 & 75 & 2.453 \\
\hline
\end{tabular}

\begin{tabular}{|c|c|c|c|c|c|c|c|c|c|}
\hline & öff. Ord. & Hausfr. & $\begin{array}{l}\text { Beleidi- } \\
\text { gung }\end{array}$ & $\begin{array}{c}\text { Fäl- } \\
\text { schung }\end{array}$ & Meineid & $\begin{array}{l}\text { falsche } \\
\text { Verd. }\end{array}$ & $\begin{array}{l}\text { BTM- } \\
\text { Delikte }\end{array}$ & $\begin{array}{c}\text { gef. } \\
\text { Eingr. } \\
\text { Str.V. }\end{array}$ & AuslG \\
\hline $\begin{array}{l}\text { öff. Ord- } \\
\text { nung }\end{array}$ & 191 & & & & & & & & \\
\hline $\begin{array}{l}\text { Hausfrie- } \\
\text { densbr. }\end{array}$ & 247 & 1.616 & & & & & & & \\
\hline Beleidigung & 481 & 1.271 & 1.665 & & & & & & \\
\hline Fälschung & 418 & 706 & 769 & 6.906 & & & & & \\
\hline Meineid & 43 & 74 & 114 & 153 & 23 & & & & \\
\hline $\begin{array}{l}\text { falsche } \\
\text { Verd. }\end{array}$ & 104 & 96 & 153 & 156 & 31 & 25 & & & \\
\hline $\begin{array}{l}\text { BTM- } \\
\text { Delikte }\end{array}$ & 1.784 & 4.662 & 5.197 & 4.750 & 823 & 638 & 37.874 & & \\
\hline $\begin{array}{l}\text { gef. Eingr. } \\
\text { Str.V. }\end{array}$ & 7 & 5 & 19 & 10 & 2 & 1 & 42 & 0 & \\
\hline AuslG & 927 & 5.462 & 2.569 & 11.105 & 316 & 179 & 22.992 & 18 & 86.480 \\
\hline
\end{tabular}


Tabelle 64: Anzahl der Deliktspaare (PAD nichtdeutsche Frauen)

\begin{tabular}{|c|c|c|c|c|c|c|c|c|}
\hline & $\begin{array}{c}\text { Tötungs- } \\
\text { deikte. }\end{array}$ & $\begin{array}{c}\text { Sexual- } \\
\text { del. }\end{array}$ & $\begin{array}{l}\text { sonst. } \\
\text { Sex. }\end{array}$ & Raub & $\begin{array}{l}\text { schw. } \\
\text { KV }\end{array}$ & KV & $\begin{array}{l}\text { Str. gg. } \\
\text { Pers. Fr. }\end{array}$ & $\begin{array}{l}\text { einf. } \\
\text { Dieb. }\end{array}$ \\
\hline Tötungsdelikte & 0 & & & & & & & \\
\hline Sexualdelikte & 0 & 0 & & & & & & \\
\hline $\begin{array}{l}\text { sonst. Sexual- } \\
\text { del. }\end{array}$ & 1 & 0 & 431 & & & & & \\
\hline Raub & 3 & 0 & 14 & 63 & & & & \\
\hline schw. KV & 3 & 0 & 5 & 62 & 66 & & & \\
\hline $\begin{array}{l}\text { Körperverlet- } \\
\text { zung }\end{array}$ & 1 & 0 & 18 & 93 & 180 & 143 & & \\
\hline $\begin{array}{l}\text { Str. gg. Pers. } \\
\text { Fr. }\end{array}$ & 4 & 0 & 4 & 35 & 61 & 131 & 29 & \\
\hline einf. Diebstahl & 12 & 1 & 557 & 657 & 619 & 1.321 & 322 & 15.206 \\
\hline schw. Diebstahl & 0 & 0 & 53 & 164 & 98 & 158 & 55 & 2.910 \\
\hline Sachbeschäd. & 3 & 0 & 14 & 22 & 65 & 118 & 49 & 478 \\
\hline gemeingef. Str. & 0 & 0 & 0 & 0 & 2 & 4 & 1 & 37 \\
\hline Hehlerei & 1 & 0 & 4 & 16 & 29 & 46 & 16 & 608 \\
\hline Betrug & 1 & 0 & 35 & 103 & 166 & 281 & 118 & 3.336 \\
\hline Erschl. v. Leist. & 0 & 0 & 67 & 51 & 111 & 159 & 51 & 2.313 \\
\hline $\begin{array}{l}\text { Gef. dem. } \\
\text { Recht. }\end{array}$ & 0 & 0 & 0 & 0 & 0 & 0 & 2 & 2 \\
\hline Wid. gg. Staat. & 1 & 0 & 14 & 23 & 17 & 38 & 12 & 122 \\
\hline öff. Ordnung & 1 & 0 & 4 & 16 & 16 & 28 & 21 & 326 \\
\hline Hausfriedensbr. & 0 & 0 & 47 & 12 & 34 & 55 & 29 & 984 \\
\hline Beleidigung & 2 & 0 & 4 & 44 & 145 & 294 & 101 & 842 \\
\hline Fälschung & 0 & 0 & 13 & 15 & 46 & 74 & 12 & 939 \\
\hline Meineid & 0 & 0 & 10 & 6 & 9 & 3 & 4 & 89 \\
\hline falsche Verd. & 1 & 0 & 1 & 18 & 18 & 40 & 27 & 195 \\
\hline BTM-Delikte & 1 & 0 & 219 & 83 & 86 & 180 & 42 & 2.557 \\
\hline $\begin{array}{l}\text { gef. Eingr. } \\
\text { Str.V. }\end{array}$ & 0 & 0 & 0 & 0 & 0 & 0 & 1 & 0 \\
\hline AuslG & 0 & 0 & 185 & 155 & 80 & 186 & 62 & 5.365 \\
\hline
\end{tabular}




\begin{tabular}{|c|c|c|c|c|c|c|c|c|c|}
\hline & \multicolumn{2}{|c|}{$\begin{array}{l}\text { schw. } \\
\text { Dieb. }\end{array}$} & Sachb. & $\begin{array}{l}\text { gemein- } \\
\text { gef. Str. }\end{array}$ & Hehlerei & Betrug & $\begin{array}{l}\text { Erschl. v. } \\
\text { Leist. }\end{array}$ & $\begin{array}{l}\text { Gef. } \\
\text { dem. } \\
\text { Recht. }\end{array}$ & $\begin{array}{l}\text { Wid. gg. } \\
\text { Staat. }\end{array}$ \\
\hline schw. Diebstahl & 1.969 & & & & & & & & \\
\hline Sachbeschäd. & 95 & \multicolumn{2}{|r|}{34} & & & & & & \\
\hline gemeingef. Str. & 10 & \multicolumn{2}{|r|}{4} & 0 & & & & & \\
\hline Hehlerei & 156 & \multicolumn{2}{|r|}{28} & 1 & 44 & & & & \\
\hline Betrug & 383 & \multicolumn{2}{|r|}{106} & 6 & 190 & 2.622 & & & \\
\hline Erschl. v. Leist. & 188 & \multicolumn{2}{|r|}{95} & 3 & 52 & 473 & 697 & & \\
\hline $\begin{array}{l}\text { Gef. dem. } \\
\text { Recht. }\end{array}$ & 0 & \multicolumn{2}{|r|}{1} & 0 & 1 & 1 & 0 & 3 & \\
\hline Wid. gg. Staat. & 14 & \multicolumn{2}{|r|}{21} & 0 & 4 & 33 & 15 & 0 & 5 \\
\hline öff. Ordnung & 49 & \multicolumn{2}{|r|}{24} & 4 & 11 & 131 & 42 & 6 & 4 \\
\hline Hausfriedensbr. & 64 & \multicolumn{2}{|r|}{34} & 1 & 17 & 115 & 113 & 2 & 15 \\
\hline Beleidigung & 106 & \multicolumn{2}{|r|}{101} & 3 & 39 & 220 & 157 & 0 & 36 \\
\hline Fälschung & 160 & \multicolumn{2}{|r|}{30} & 0 & 58 & 475 & 106 & 0 & 5 \\
\hline Meineid & 6 & \multicolumn{2}{|r|}{2} & 0 & 10 & 87 & 9 & 0 & 0 \\
\hline falsche Verd. & 26 & \multicolumn{2}{|r|}{21} & 0 & 7 & 83 & 19 & 0 & 5 \\
\hline BTM-Delikte & 414 & \multicolumn{2}{|r|}{110} & 15 & 153 & 474 & 442 & 0 & 32 \\
\hline $\begin{array}{l}\text { gef. Eingr. } \\
\text { Str.V. }\end{array}$ & 0 & \multicolumn{2}{|r|}{0} & 0 & 0 & 0 & 0 & 0 & 1 \\
\hline AuslG & 840 & \multicolumn{2}{|r|}{30} & 7 & 118 & 587 & 451 & 0 & 22 \\
\hline & $\begin{array}{l}\text { öff. } \\
\text { Ord. }\end{array}$ & Hausfr. & $\begin{array}{ll}\text { i. } & \text { Belei- } \\
\text { digung }\end{array}$ & $\begin{array}{c}\text { Fäl- } \\
\text { schung }\end{array}$ & Meineid & $\begin{array}{l}\text { falsche } \\
\text { Verd. }\end{array}$ & $\begin{array}{l}\text { BTM- } \\
\text { Delikte }\end{array}$ & $\begin{array}{c}\text { gef. } \\
\text { Eingr. } \\
\text { Str.V. }\end{array}$ & AuslG \\
\hline öff. Ordnung & 32 & & & & & & & & \\
\hline $\begin{array}{l}\text { Hausfrie- } \\
\text { densbr. }\end{array}$ & 14 & 30 & & & & & & & \\
\hline Beleidigung & 30 & 68 & 112 & & & & & & \\
\hline Fälschung & 25 & 25 & 67 & 151 & & & & & \\
\hline Meineid & 6 & 4 & 7 & 8 & 7 & & & & \\
\hline falsche Verd. & 32 & 3 & 26 & 16 & 4 & 8 & & & \\
\hline BTM-Delikte & 34 & 142 & 176 & 206 & 32 & 21 & 1.076 & & \\
\hline $\begin{array}{l}\text { gef. Eingr. } \\
\text { Str.V. }\end{array}$ & 0 & 0 & 1 & 0 & 0 & 0 & 1 & 0 & \\
\hline AuslG & 36 & 117 & 83 & 734 & 16 & 60 & 137 & 1 & 2.682 \\
\hline
\end{tabular}


Tabelle 65: Anzahl der Deliktspaare (BZR deutsche Männer)

\begin{tabular}{|c|c|c|c|c|c|c|c|c|}
\hline & $\begin{array}{l}\text { Tötungs- } \\
\text { delikte }\end{array}$ & $\begin{array}{l}\text { Sexualde- } \\
\text { likte }\end{array}$ & sonst. Sex. & Raub & schw. KV & $\mathrm{KV}$ & $\begin{array}{l}\text { Str. gg. } \\
\text { Pers. Fr. }\end{array}$ & einf. Dieb. \\
\hline Tötungsdelikte & 0 & & & & & & & \\
\hline Sexualdelikte & 6 & 27 & & & & & & \\
\hline sonst. Sexualdel. & 2 & 134 & 139 & & & & & \\
\hline Raub & 49 & 115 & 75 & 403 & & & & \\
\hline schw. KV & 85 & 227 & 145 & 1.846 & 1.962 & & & \\
\hline Körperverletzung & 109 & 420 & 338 & 3.099 & 12.887 & 7.260 & & \\
\hline Str. gg. Pers. Fr. & 38 & 183 & 138 & 1.011 & 2.520 & 5.145 & 754 & \\
\hline einf. Diebstahl & 147 & 622 & 812 & 4.996 & 8.711 & 17.756 & 5.668 & 32.182 \\
\hline schw. Diebstahl & 65 & 317 & 315 & 2.660 & 3.896 & 7.569 & 2.523 & 20.272 \\
\hline Sachbeschäd. & 49 & 162 & 178 & 1.197 & 3.388 & 6.862 & 2.092 & 11.563 \\
\hline gemeingef. Str. & 20 & 22 & 12 & 97 & 198 & 374 & 146 & 929 \\
\hline Hehlerei & 16 & 54 & 46 & 435 & 829 & 1.602 & 510 & 4.751 \\
\hline Betrug & 37 & 176 & 243 & 1.228 & 1.986 & 4.368 & 1.861 & 18.354 \\
\hline Erschl. v. Leist. & 33 & 101 & 157 & 1.131 & 2.166 & 4.352 & 1.469 & 15.091 \\
\hline Gef. dem. Recht. & 6 & 11 & 14 & 110 & 486 & 760 & 214 & 664 \\
\hline Wid. gg. Staat. & 17 & 41 & 47 & 362 & 1.229 & 2.961 & 744 & 2.867 \\
\hline öff. Ordnung & 14 & 27 & 35 & 259 & 665 & 1.189 & 448 & 2.291 \\
\hline Hausfriedensbr. & 9 & 33 & 45 & 399 & 926 & 1.940 & 707 & 4.381 \\
\hline Beleidigung & 25 & 147 & 182 & 845 & 2.981 & 6.789 & 2.923 & 7.520 \\
\hline Fälschung & 20 & 93 & 95 & 631 & 1.002 & 2.141 & 790 & 7.984 \\
\hline Meineid & 2 & 11 & 17 & 72 & 174 & 336 & 109 & 722 \\
\hline falsche Verd. & 4 & 7 & 14 & 49 & 108 & 245 & 105 & 717 \\
\hline BTM-Delikte & 48 & 181 & 226 & 2.225 & 3.829 & 7.698 & 2.334 & 24.396 \\
\hline gef. Eingr. Str.V. & 4 & 9 & 5 & 73 & 201 & 388 & 252 & 559 \\
\hline Vollrausch & 8 & 17 & 20 & 115 & 399 & 823 & 218 & 1.360 \\
\hline fahrl. Tötung & 0 & 3 & 3 & 12 & 31 & 84 & 22 & 120 \\
\hline Fahrl. KV & 12 & 66 & 91 & 340 & 990 & 4.560 & 752 & 4.383 \\
\hline Verkehrsdel. & 183 & 722 & 891 & 5.093 & 10.711 & 24.419 & 7.453 & $\mathbf{5 7 . 2 8 3}$ \\
\hline & $\begin{array}{l}\text { schw. } \\
\text { Dieb. }\end{array}$ & Sachb. & $\begin{array}{l}\text { gemein- } \\
\text { gef. Str. }\end{array}$ & Hehlerei & Betrug & $\begin{array}{r}\text { Erschl. v. } \\
\text { Leist. }\end{array}$ & $\begin{array}{r}\text { Gef. dem. } \\
\text { Recht. }\end{array}$ & $\begin{array}{r}\text { Wid. gg. } \\
\text { Staat. }\end{array}$ \\
\hline schw. Diebstahl & 6.924 & & & & & & & \\
\hline Sachbeschäd. & 5.437 & 2.063 & & & & & & \\
\hline gemeingef. Str. & 530 & 409 & 46 & & & & & \\
\hline Hehlerei & 2.423 & 870 & 76 & 245 & & & & \\
\hline Betrug & 5.861 & 2.374 & 263 & 1.275 & 6.786 & & & \\
\hline Erschl. v. Leist. & 4.895 & 2.899 & 171 & 789 & 3.915 & 8.107 & & \\
\hline Gef. dem. Recht. & 286 & 579 & 37 & 49 & 183 & 228 & 162 & \\
\hline Wid. gg. Staat. & 1.336 & 1.272 & 78 & 242 & 619 & 835 & 105 & 311 \\
\hline öff. Ordnung & 944 & 750 & 111 & 191 & 1.034 & 580 & 205 & 217 \\
\hline Hausfriedensbr. & 1.456 & 1.583 & 54 & 301 & 804 & 1.242 & 89 & 427 \\
\hline Beleidigung & 2.919 & 3.261 & 168 & 615 & 2.131 & 2.459 & 406 & 1.986 \\
\hline Fälschung & 3.705 & 1.269 & 122 & 747 & 5.175 & 1.611 & 76 & 372 \\
\hline Meineid & 340 & 165 & 16 & 234 & 394 & 113 & 18 & 52 \\
\hline falsche Verd. & 278 & 162 & 26 & 82 & 270 & 162 & 12 & 44 \\
\hline BTM-Delikte & 8.960 & 4.638 & 305 & 2.082 & 4.408 & 6.148 & 209 & 1.375 \\
\hline gef. Eingr. Str.V. & 313 & 224 & 80 & 34 & 202 & 103 & 5 & 90 \\
\hline Vollrausch & 483 & 513 & 36 & 85 & 285 & 330 & 51 & 207 \\
\hline fahrl. Tötung & 50 & 33 & 3 & 14 & 35 & 20 & 1 & 10 \\
\hline fahrl. KV & 1.649 & 1.146 & 148 & 386 & 1.387 & 730 & 76 & 418 \\
\hline Verkehrsdel. & 25.994 & 12.916 & 1.384 & 5.137 & 19.983 & 9.403 & 857 & 4.284 \\
\hline
\end{tabular}


Anhang

\begin{tabular}{lrrrrrrrr}
\hline & öff. Ord. & Hausfr. & $\begin{array}{c}\text { Beleidi- } \\
\text { gung }\end{array}$ & Fälschung & Meineid & $\begin{array}{c}\text { falsche } \\
\text { Verd. }\end{array}$ & $\begin{array}{c}\text { BTM- } \\
\text { Delikte }\end{array}$ & $\begin{array}{c}\text { gef. Eingr. } \\
\text { Str.V. }\end{array}$ \\
\hline öff. Ordnung & 116 & & & & & & & \\
Hausfriedensbr. & 193 & 427 & & & & & & \\
Beleidigung & 579 & 1.152 & 1.749 & & & & & \\
Fälschung & 401 & 368 & 931 & 971 & & & & \\
Meineid & 44 & 48 & 143 & 145 & 19 & & & \\
falsche Verd. & 78 & 40 & 150 & 143 & 38 & 4 & & \\
BTM-Delikte & 743 & 1.769 & 3.348 & 2.641 & 368 & 321 & 10.786 & \\
gef. Eingr. Str.V. & 38 & 30 & 191 & 109 & 7 & 10 & 195 & 13 \\
Vollrausch & 86 & 163 & 428 & 136 & 14 & 19 & 521 & 26 \\
fahrl. Tötung & 8 & 8 & 29 & 31 & 5 & 0 & 42 & 3 \\
fahrl. KV & 243 & 279 & 952 & 783 & 99 & 76 & 1.866 & 185 \\
Verkehrsdel. & 3.421 & 3.334 & 9.536 & 14.754 & 1.137 & 905 & 22.418 & 1.129 \\
\hline
\end{tabular}

\begin{tabular}{lrrrr}
\hline & $\begin{array}{c}\text { Voll- } \\
\text { rausch }\end{array}$ & $\begin{array}{c}\text { fahrl. } \\
\text { Tötung }\end{array}$ & fahrl. KV & $\begin{array}{c}\text { Ver- } \\
\text { kehrsdel. }\end{array}$ \\
\hline Vollrausch & 124 & & & \\
fahrl. Tötung & 3 & 1 & & \\
fahrl. KV & 152 & 181 & 500 & \\
Verkehrsdel. & 1.964 & 443 & 12.233 & $\mathbf{7 2 . 0 8 1}$ \\
\hline
\end{tabular}


Tabelle 66: Anzahl der Deliktspaare (BZR deutsche Frauen)

\begin{tabular}{|c|c|c|c|c|c|c|c|c|}
\hline & $\begin{array}{l}\text { Tötungs- } \\
\text { deikte. }\end{array}$ & $\begin{array}{l}\text { Sexual- } \\
\text { delikte }\end{array}$ & $\begin{array}{l}\text { sonst. } \\
\text { Sex. }\end{array}$ & Raub & $\begin{array}{l}\text { schw. } \\
\text { KV }\end{array}$ & $\mathrm{KV}$ & $\begin{array}{l}\text { Str. gg. } \\
\text { Pers. Fr. }\end{array}$ & $\begin{array}{l}\text { einf. } \\
\text { Dieb. }\end{array}$ \\
\hline Tötungsdelikte & 1 & & & & & & & \\
\hline Sexualdelikte & 0 & 0 & & & & & & \\
\hline $\begin{array}{l}\text { sonst. Sexual- } \\
\text { del. }\end{array}$ & 0 & 0 & 168 & & & & & \\
\hline Raub & 6 & 0 & 22 & 11 & & & & \\
\hline schw. KV & 4 & 1 & 32 & 76 & 53 & & & \\
\hline $\begin{array}{l}\text { Körperverlet- } \\
\text { zung }\end{array}$ & 3 & 0 & 39 & 121 & 739 & 263 & & \\
\hline $\begin{array}{l}\text { Str. gg. Pers. } \\
\text { Fr. }\end{array}$ & 3 & 1 & 19 & 39 & 91 & 189 & 16 & \\
\hline einf. Diebstahl & 16 & 0 & 525 & 399 & 706 & 1.593 & 326 & 9.114 \\
\hline schw. Diebstahl & 3 & 0 & 41 & 53 & 47 & 112 & 30 & 940 \\
\hline Sachbeschäd. & 1 & 0 & 24 & 23 & 66 & 167 & 40 & 565 \\
\hline gemeingef. Str. & 1 & 0 & 0 & 2 & 3 & 7 & 3 & 30 \\
\hline Hehlerei & 1 & 0 & 12 & 13 & 36 & 71 & 12 & 648 \\
\hline Betrug & 11 & 0 & 70 & 64 & 140 & 388 & 104 & 4.503 \\
\hline Erschl. v. Leist. & 4 & 0 & 294 & 99 & 249 & 477 & 80 & 4.448 \\
\hline $\begin{array}{l}\text { Gef. dem. } \\
\text { Recht. }\end{array}$ & 1 & 0 & 0 & 0 & 6 & 9 & 2 & 34 \\
\hline Wid. gg. Staat. & 0 & 0 & 12 & 14 & 51 & 160 & 21 & 222 \\
\hline öff. Ordnung & 2 & 0 & 16 & 17 & 22 & 51 & 27 & 339 \\
\hline Hausfriedensbr. & 0 & 0 & 17 & 13 & 27 & 60 & 22 & 509 \\
\hline Beleidigung & 5 & 0 & 43 & 44 & 179 & 493 & 125 & 1.039 \\
\hline Fälschung & 2 & 0 & 28 & 27 & 54 & 142 & 30 & 1.655 \\
\hline Meineid & 1 & 0 & 14 & 5 & 9 & 19 & 7 & 211 \\
\hline falsche Verd. & 2 & 0 & 15 & 6 & 9 & 33 & 27 & 331 \\
\hline BTM-Delikte & 2 & 0 & 262 & 129 & 172 & 389 & 67 & 3.763 \\
\hline $\begin{array}{l}\text { gef. Eingr. } \\
\text { Str.V. }\end{array}$ & 0 & 0 & 0 & 0 & 7 & 11 & 6 & 13 \\
\hline Vollrausch & 0 & 0 & 0 & 4 & 3 & 18 & 5 & 53 \\
\hline fahrl. Tötung & 0 & 0 & 0 & 2 & 2 & 4 & 0 & 11 \\
\hline fahrl. KV & 0 & 0 & 4 & 7 & 29 & 605 & 12 & 394 \\
\hline Verkehrsdel. & 7 & 0 & 61 & 65 & 158 & 499 & 102 & 3.327 \\
\hline
\end{tabular}


Anhang

\begin{tabular}{|c|c|c|c|c|c|c|c|c|}
\hline & $\begin{array}{l}\text { schw. } \\
\text { Dieb. }\end{array}$ & Sachb. & $\begin{array}{l}\text { gemein- } \\
\text { gef. Str. }\end{array}$ & Hehlerei & Betrug & $\begin{array}{l}\text { Erschl. v. } \\
\text { Leist. }\end{array}$ & $\begin{array}{l}\text { Gef. } \\
\text { dem. } \\
\text { Recht. }\end{array}$ & $\begin{array}{l}\text { Wid. gg. } \\
\text { Staat. }\end{array}$ \\
\hline schw. Diebstahl & 67 & & & & & & & \\
\hline Sachbeschäd. & 91 & 28 & & & & & & \\
\hline gemeingef. Str & 2 & 5 & 1 & & & & & \\
\hline Hehlerei & 65 & 20 & 2 & 16 & & & & \\
\hline Betrug & 242 & 126 & 6 & 162 & 1.718 & & & \\
\hline Erschl. v. Leist. & 236 & 210 & 10 & 156 & 977 & 2.405 & & \\
\hline $\begin{array}{l}\text { Gef. dem. } \\
\text { Recht. }\end{array}$ & 3 & 10 & 1 & 2 & 3 & 7 & 0 & \\
\hline Wid. gg. Staat. & 19 & 32 & 1 & 13 & 44 & 79 & 2 & 12 \\
\hline öff. Ordnung & 28 & 22 & 2 & 17 & 150 & 100 & 8 & 7 \\
\hline Hausfriedensbr. & 23 & 65 & 3 & 32 & 113 & 195 & 2 & 13 \\
\hline Beleidigung & 70 & 139 & 7 & 53 & 250 & 315 & 13 & 160 \\
\hline Fälschung & 98 & 35 & 0 & 62 & 1.340 & 426 & 1 & 20 \\
\hline Meineid & 11 & 5 & 1 & 55 & 126 & 36 & 0 & 2 \\
\hline falsche Verd. & 18 & 14 & 1 & 21 & 104 & 124 & 0 & 12 \\
\hline BTM-Delikte & 285 & 139 & 12 & 224 & 595 & 1.131 & 2 & 76 \\
\hline $\begin{array}{l}\text { gef. Eingr. } \\
\text { Str.V. }\end{array}$ & 1 & 3 & 1 & 0 & 1 & 4 & 0 & 0 \\
\hline Vollrausch & 3 & 15 & 0 & 0 & 6 & 18 & 1 & 8 \\
\hline fahrl. Tötung & 0 & 0 & 0 & 1 & 2 & 0 & 0 & 1 \\
\hline fahrl. KV & 15 & 18 & 3 & 11 & 152 & 66 & 0 & 12 \\
\hline Verkehrsdel. & 211 & 165 & 17 & 184 & 1.377 & 572 & 6 & 90 \\
\hline & öff. Ord. & Hausfr. & $\begin{array}{l}\text { Beleidi- } \\
\text { gung }\end{array}$ & $\begin{array}{l}\text { Fäl- } \\
\text { schung }\end{array}$ & Meineid & $\begin{array}{l}\text { falsche } \\
\text { Verd. }\end{array}$ & $\begin{array}{l}\text { BTM- } \\
\text { Delikte }\end{array}$ & $\begin{array}{l}\text { gef. } \\
\text { Eingr. } \\
\text { Str.V. }\end{array}$ \\
\hline öff. Ordnung & 19 & & & & & & & \\
\hline Hausfriedensbr. & 21 & 24 & & & & & & \\
\hline Beleidigung & 52 & 65 & 119 & & & & & \\
\hline Fälschung & 50 & 37 & 77 & 118 & & & & \\
\hline Meineid & 9 & 7 & 16 & 19 & 4 & & & \\
\hline falsche Verd. & 20 & 15 & 39 & 30 & 18 & 4 & & \\
\hline BTM-Delikte & 67 & 127 & 265 & 315 & 68 & 68 & 1.027 & \\
\hline $\begin{array}{l}\text { gef. Eingr. } \\
\text { Str.V. }\end{array}$ & 0 & 0 & 2 & 1 & 1 & 1 & 5 & 1 \\
\hline Vollrausch & 3 & 6 & 16 & 2 & 0 & 3 & 12 & 0 \\
\hline fahrl. Tötung & 0 & 0 & 1 & 1 & 1 & 1 & 1 & 0 \\
\hline fahrl. KV & 13 & 8 & 40 & 39 & 8 & 5 & 95 & 4 \\
\hline Verkehrsdel. & 154 & 98 & 255 & 458 & 101 & 85 & 888 & 7 \\
\hline
\end{tabular}

\begin{tabular}{lcccc}
\hline & $\begin{array}{c}\text { Voll- } \\
\text { rausch }\end{array}$ & $\begin{array}{c}\text { fahrl. } \\
\text { Tötung }\end{array}$ & fahrl. KV & $\begin{array}{c}\text { Ver- } \\
\text { kehrsdel. }\end{array}$ \\
\hline Vollrausch & 1 & & & \\
fahrl. Tötung & 0 & 0 & & \\
fahrl. KV & 2 & 24 & 28 & \\
Verkehrsdel. & 17 & 17 & 602 & 1.876 \\
\hline
\end{tabular}


Tabelle 67: Anzahl der Deliktspaare (BZR nichtdeutsche Männer)

\begin{tabular}{|c|c|c|c|c|c|c|c|c|}
\hline & $\begin{array}{l}\text { Tötungs- } \\
\text { delikte }\end{array}$ & $\begin{array}{l}\text { Sexual- } \\
\text { delikte }\end{array}$ & $\begin{array}{l}\text { sonst. } \\
\text { Sex. }\end{array}$ & Raub & $\begin{array}{l}\text { schw. } \\
\text { KV }\end{array}$ & $\mathrm{KV}$ & $\begin{array}{l}\text { Str. gg. } \\
\text { Pers. Fr. }\end{array}$ & $\begin{array}{l}\text { einf. } \\
\text { Dieb. }\end{array}$ \\
\hline Tötungsdelikte & 3 & & & & & & & \\
\hline Sexualdelikte & 7 & 6 & & & & & & \\
\hline $\begin{array}{l}\text { sonst. Sexual- } \\
\text { del. }\end{array}$ & 3 & 53 & 31 & & & & & \\
\hline Raub & 36 & 65 & 39 & 279 & & & & \\
\hline schw. KV & 72 & 130 & 93 & 1.254 & 916 & & & \\
\hline $\begin{array}{l}\text { Körperverlet- } \\
\text { zung }\end{array}$ & 87 & 287 & 190 & 1.970 & 6.922 & 3.257 & & \\
\hline $\begin{array}{l}\text { Str. gg. Pers. } \\
\text { Fr. }\end{array}$ & 22 & 148 & 95 & 664 & 1.625 & 3.319 & 540 & \\
\hline einf. Diebstahl & 88 & 332 & 324 & 2.552 & 4.124 & 8.125 & 3.126 & 18.448 \\
\hline schw. Diebstahl & 31 & 130 & 86 & 1.224 & 1.540 & 2.837 & 996 & 6.916 \\
\hline Sachbeschäd. & 15 & 52 & 44 & 367 & 1.021 & 2.001 & 789 & 2.720 \\
\hline gemeingef. Str. & 5 & 6 & 1 & 43 & 81 & 138 & 55 & 185 \\
\hline Hehlerei & 9 & 34 & 32 & 312 & 532 & 966 & 346 & 2.759 \\
\hline Betrug & 15 & 92 & 73 & 608 & 981 & 2.136 & 913 & 6.605 \\
\hline Erschl. v. Leist. & 19 & 60 & 71 & 537 & 902 & 1.872 & 742 & 6.899 \\
\hline $\begin{array}{l}\text { Gef. dem. } \\
\text { Recht. }\end{array}$ & 0 & 1 & 0 & 3 & 8 & 17 & 5 & 10 \\
\hline Wid. gg. Staat. & 7 & 25 & 13 & 151 & 455 & 1.181 & 396 & 1.119 \\
\hline öff. Ordnung & 5 & 10 & 10 & 57 & 194 & 332 & 154 & 542 \\
\hline Hausfriedensbr. & 5 & 26 & 34 & 170 & 399 & 824 & 426 & 2.111 \\
\hline Beleidigung & 16 & 62 & 75 & 430 & 1.214 & 2.863 & 1.515 & 2.717 \\
\hline Fälschung & 15 & 59 & 43 & 361 & 573 & 1.211 & 560 & 4.845 \\
\hline Meineid & 0 & 5 & 6 & 42 & 134 & 243 & 84 & 366 \\
\hline falsche Verd. & 0 & 2 & 5 & 36 & 70 & 143 & 79 & 235 \\
\hline BTM-Delikte & 42 & 120 & 122 & 1.324 & 1.922 & 3.518 & 1.250 & 8.672 \\
\hline $\begin{array}{l}\text { gef. Eingr. } \\
\text { Str.V. }\end{array}$ & 0 & 9 & 5 & 45 & 107 & 196 & 143 & 207 \\
\hline AuslG & 37 & 91 & 73 & 393 & 989 & 2.046 & 1.002 & 12.809 \\
\hline Vollrausch & 0 & 5 & 2 & 14 & 64 & 146 & 43 & 190 \\
\hline fahrl. Tötung & 0 & 1 & 0 & 6 & 23 & 38 & 16 & 42 \\
\hline fahrl. KV & 14 & 35 & 38 & 160 & 430 & 1.595 & 347 & 1.497 \\
\hline Verkehrsdel. & 88 & 307 & 254 & 1.840 & 4.099 & 8.408 & 3.390 & 18.218 \\
\hline
\end{tabular}


Anhang

\begin{tabular}{|c|c|c|c|c|c|c|c|c|}
\hline & $\begin{array}{l}\text { schw. } \\
\text { Dieb. }\end{array}$ & Sachb. & $\begin{array}{l}\text { Gemein- } \\
\text { gef. Str. }\end{array}$ & Hehlerei & Betrug & $\begin{array}{l}\text { Erschl. v. } \\
\text { Leist. }\end{array}$ & $\begin{array}{l}\text { Gef. dem. } \\
\text { Recht. }\end{array}$ & $\begin{array}{l}\text { Wid. gg. } \\
\text { Staat. }\end{array}$ \\
\hline schw. Diebstahl & 2.328 & & & & & & & \\
\hline Sachbeschäd. & 1.104 & 333 & & & & & & \\
\hline gemeingef. Str & 141 & 72 & 2 & & & & & \\
\hline Hehlerei & 1.181 & 293 & 27 & 178 & & & & \\
\hline Betrug & 1.769 & 676 & 63 & 617 & 1.710 & & & \\
\hline Erschl. v. Leist. & 1.526 & 651 & 49 & 420 & 1.681 & 2.560 & & \\
\hline Gef. dem. Recht. & 5 & 21 & 0 & 0 & 8 & 6 & 1 & \\
\hline Wid. gg. Staat. & 387 & 337 & 34 & 100 & 257 & 354 & 2 & 119 \\
\hline öff. Ordnung & 181 & 125 & 13 & 63 & 249 & 135 & 3 & 55 \\
\hline Hausfriedensbr. & 472 & 391 & 11 & 141 & 285 & 505 & 3 & 155 \\
\hline Beleidigung & 896 & 768 & 39 & 298 & 771 & 844 & 9 & 624 \\
\hline Fälschung & 1.436 & 397 & 55 & 454 & 2.455 & 1.011 & 4 & 224 \\
\hline Meineid & 149 & 62 & 6 & 157 & 185 & 69 & 0 & 13 \\
\hline falsche Verd. & 106 & 45 & 5 & 48 & 106 & 47 & 0 & 9 \\
\hline BTM-Delikte & 3.074 & 1.138 & 84 & 1.097 & 1.960 & 2.608 & 11 & 634 \\
\hline gef. Eingr. Str.V. & 114 & 72 & 45 & 29 & 83 & 26 & 0 & 38 \\
\hline AuslG & 1.825 & 567 & 46 & 650 & 1.689 & 2.525 & 7 & 528 \\
\hline Vollrausch & 54 & 52 & 2 & 17 & 37 & 35 & 1 & 30 \\
\hline fahrl. Tötung & 14 & 7 & 0 & 5 & 12 & 7 & 0 & 2 \\
\hline fahrl. KV & 459 & 219 & 36 & 179 & 519 & 286 & 3 & 123 \\
\hline Verkehrsdel. & 6.651 & 2.450 & 267 & 2.109 & 5.990 & 3.056 & 25 & 1.150 \\
\hline
\end{tabular}

\begin{tabular}{lrrrrrrrr}
\hline & öff. Ord. & Hausfr. & $\begin{array}{c}\text { Beleidi- } \\
\text { gung }\end{array}$ & $\begin{array}{c}\text { Fäl- } \\
\text { schung }\end{array}$ & Meineid & $\begin{array}{c}\text { falsche } \\
\text { Verd. }\end{array}$ & $\begin{array}{c}\text { BTM- } \\
\text { Delikte }\end{array}$ & $\begin{array}{c}\text { gef. } \\
\text { Eingr. } \\
\text { Str.V. }\end{array}$ \\
\hline off. Ordnung & 18 & & & & & & & \\
Hausfriedensbr. & 42 & 185 & & & & & & \\
Beleidigung & 106 & 394 & 531 & & & & & \\
Fälschung & 126 & 228 & 422 & 633 & & & & \\
Meineid & 14 & 16 & 58 & 93 & 11 & & & \\
falsche Verd. & 22 & 16 & 48 & 63 & 24 & 6 & & \\
BTM-Delikte & 221 & 665 & 1.358 & 1.476 & 233 & 119 & 4.658 & \\
gef. Eingr. & 12 & 15 & 69 & 62 & 6 & 9 & 78 & 3 \\
Str.V. & 121 & 647 & 698 & 3.181 & 117 & 56 & 2.954 & 52 \\
AuslG & 5 & 20 & 51 & 26 & 1 & 1 & 73 & 2 \\
Vollrausch & 1 & 7 & 13 & 9 & 2 & 0 & 13 & 0 \\
fahrl. Tötung & 73 & 98 & 321 & 304 & 58 & 33 & 611 & 52 \\
fahrl. KV & 703 & 1.112 & 2.950 & 5.858 & 473 & 326 & 6.819 & 446 \\
Verkehrsdel. & & & & & & & & \\
\hline
\end{tabular}

\begin{tabular}{lrrrrr}
\hline & AuslG & $\begin{array}{c}\text { Voll- } \\
\text { rausch }\end{array}$ & $\begin{array}{c}\text { fahrl. } \\
\text { Tötung }\end{array}$ & fahrl. KV & $\begin{array}{c}\text { Ver- } \\
\text { kehrsdel. }\end{array}$ \\
\hline AuslG & 10.381 & & & & \\
Vollrausch & 40 & 4 & & & \\
fahrl. Tötung & 10 & 0 & 0 & & \\
fahrl. KV & 565 & 10 & 49 & 148 & \\
Verkehrsdel. & 7.970 & 214 & 82 & 3.305 & $\mathbf{1 9 . 5 1 1}$ \\
\hline
\end{tabular}


Tabelle 68: Anzahl der Deliktspaare (BZR nichtdeutsche Frauen)

\begin{tabular}{|c|c|c|c|c|c|c|c|c|}
\hline & $\begin{array}{l}\text { Tötungs- } \\
\text { delikte }\end{array}$ & $\begin{array}{c}\text { Sexual- } \\
\text { delikte }\end{array}$ & $\begin{array}{l}\text { sonst. } \\
\text { Sex. }\end{array}$ & Raub & $\begin{array}{l}\text { schw. } \\
\text { KV }\end{array}$ & $\mathrm{KV}$ & $\begin{array}{l}\text { Str. gg. } \\
\text { Pers. Fr. }\end{array}$ & $\begin{array}{l}\text { einf. } \\
\text { Dieb. }\end{array}$ \\
\hline Tötungsdelikte & 0 & & & & & & & \\
\hline Sexualdelikte & 0 & 0 & & & & & & \\
\hline $\begin{array}{l}\text { sonst. Sexual- } \\
\text { del. }\end{array}$ & 0 & 0 & 24 & & & & & \\
\hline Raub & 1 & 0 & 7 & 5 & & & & \\
\hline schw. KV & 1 & 1 & 0 & 30 & 15 & & & \\
\hline $\begin{array}{l}\text { Köperverlet- } \\
\text { zung }\end{array}$ & 2 & 0 & 0 & 32 & 214 & 64 & & \\
\hline $\begin{array}{l}\text { Str. gg. Pers. } \\
\text { Fr. }\end{array}$ & 0 & 1 & 0 & 8 & 37 & 66 & 4 & \\
\hline einf. Diebstahl & 0 & 1 & 19 & 81 & 164 & 302 & 122 & 3.550 \\
\hline schw. Diebstahl & 1 & 0 & 3 & 9 & 12 & 17 & 4 & 387 \\
\hline Sachbeschäd. & 0 & 0 & 1 & 3 & 18 & 41 & 17 & 92 \\
\hline gemeingef. Str & 0 & 0 & 0 & 0 & 2 & 3 & 2 & 9 \\
\hline Hehlerei & 0 & 0 & 0 & 4 & 9 & 19 & 3 & 218 \\
\hline Betrug & 2 & 0 & 1 & 16 & 20 & 75 & 34 & 1.237 \\
\hline Erschl. v. Leist. & 0 & 0 & 8 & 30 & 54 & 98 & 24 & 1.094 \\
\hline $\begin{array}{l}\text { Gef. dem. } \\
\text { Recht. }\end{array}$ & 0 & 0 & 0 & 0 & 0 & 0 & 0 & 0 \\
\hline Wid. gg. Staat. & 0 & 0 & 0 & 4 & 20 & 61 & 9 & 35 \\
\hline öff. Ordnung & 0 & 0 & 0 & 3 & 9 & 15 & 5 & 96 \\
\hline Hausfriedensbr. & 0 & 0 & 2 & 1 & 10 & 24 & 8 & 241 \\
\hline Beleidigung & 0 & 0 & 1 & 8 & 69 & 169 & 42 & 233 \\
\hline Fälschung & 0 & 0 & 3 & 10 & 12 & 36 & 11 & 511 \\
\hline Meineid & 0 & 0 & 2 & 1 & 1 & 0 & 2 & 43 \\
\hline falsche Verd. & 0 & 0 & 0 & 1 & 2 & 6 & 10 & 69 \\
\hline BTM-Delikte & 0 & 0 & 28 & 16 & 8 & 45 & 10 & 441 \\
\hline $\begin{array}{l}\text { gef. Eingr. } \\
\text { Str.V. }\end{array}$ & 0 & 0 & 0 & 0 & 3 & 4 & 4 & 3 \\
\hline AuslG & 0 & 0 & 12 & 20 & 24 & 47 & 5 & 925 \\
\hline Vollrausch & 0 & 0 & 0 & 0 & 1 & 1 & 0 & 12 \\
\hline fahrl. Tötung & 0 & 0 & 0 & 0 & 1 & 1 & 0 & 1 \\
\hline fahrl. KV & 0 & 0 & 0 & 2 & 10 & 119 & 6 & 73 \\
\hline Verkehrsdel. & 0 & 0 & 5 & 14 & 29 & 110 & 36 & 751 \\
\hline
\end{tabular}


Anhang

\begin{tabular}{|c|c|c|c|c|c|c|c|c|}
\hline & $\begin{array}{l}\text { schw. } \\
\text { Dieb. }\end{array}$ & Sachb. & $\begin{array}{l}\text { gemein- } \\
\text { gef. Str. }\end{array}$ & Hehlerei & Betrug & $\begin{array}{c}\text { Erschl. v. } \\
\text { Leist. }\end{array}$ & $\begin{array}{c}\text { Gef. dem. } \\
\text { Recht. }\end{array}$ & $\begin{array}{l}\text { Wid. gg. } \\
\text { Staat. }\end{array}$ \\
\hline schw. Diebstahl & 37 & & & & & & & \\
\hline Sachbeschäd. & 17 & 3 & & & & & & \\
\hline gemeingef. Str. & 2 & 1 & 0 & & & & & \\
\hline Hehlerei & 20 & 10 & 0 & 6 & & & & \\
\hline Betrug & 57 & 26 & 3 & 34 & 262 & & & \\
\hline Erschl. v. Leist. & 38 & 25 & 1 & 24 & 278 & 405 & & \\
\hline $\begin{array}{l}\text { Gef. dem. } \\
\text { Recht. }\end{array}$ & 0 & 0 & 0 & 0 & 0 & 0 & 0 & \\
\hline Wid. gg. Staat. & 2 & 8 & 2 & 4 & 12 & 18 & 0 & 10 \\
\hline öff. Ordnung & 8 & 5 & 1 & 3 & 36 & 19 & 1 & 4 \\
\hline Hausfriedensbr. & 44 & 15 & 0 & 9 & 25 & 37 & 0 & 3 \\
\hline Beleidigung & 10 & 25 & 2 & 10 & 58 & 50 & 0 & 59 \\
\hline Fälschung & 34 & 10 & 1 & 19 & 378 & 103 & 0 & 5 \\
\hline Meineid & 1 & 0 & 0 & 10 & 29 & 5 & 0 & 0 \\
\hline falsche Verd. & 4 & 1 & 0 & 8 & 36 & 23 & 0 & 2 \\
\hline BTM-Delikte & 21 & 17 & 0 & 25 & 82 & 117 & 0 & 4 \\
\hline $\begin{array}{l}\text { gef. Eingr. } \\
\text { Str.V. }\end{array}$ & 1 & 1 & 1 & 2 & 1 & 1 & 0 & 2 \\
\hline AuslG & 54 & 5 & 1 & 27 & 118 & 99 & 0 & 14 \\
\hline Vollrausch & 0 & 0 & 0 & 1 & 2 & 3 & 0 & 0 \\
\hline fahrl. Tötung & 0 & 0 & 1 & 0 & 1 & 0 & 0 & 0 \\
\hline fahrl. KV & 0 & 0 & 2 & 7 & 20 & 14 & 0 & 3 \\
\hline \multirow[t]{2}{*}{ Verkehrsdel. } & 29 & 23 & 0 & 38 & 249 & 151 & 0 & 22 \\
\hline & öff. Ord. & Hausfr. & $\begin{array}{l}\text { Beleidi- } \\
\text { gung }\end{array}$ & $\begin{array}{c}\text { Fäl- } \\
\text { schung }\end{array}$ & Meineid & $\begin{array}{l}\text { falsche } \\
\text { Verd. }\end{array}$ & $\begin{array}{l}\text { BTM- } \\
\text { Delikte }\end{array}$ & $\begin{array}{c}\text { gef. Eingr. } \\
\text { Str.V. }\end{array}$ \\
\hline öff. Ordnung & 4 & & & & & & & \\
\hline Hausfriedensbr. & 3 & 29 & & & & & & \\
\hline Beleidigung & 5 & 11 & 31 & & & & & \\
\hline Fälschung & 10 & 7 & 26 & 46 & & & & \\
\hline Meineid & 0 & 1 & 2 & 6 & 1 & & & \\
\hline falsche Verd. & 10 & 1 & 10 & 13 & 9 & 2 & & \\
\hline BTM-Delikte & 4 & 14 & 27 & 53 & 10 & 9 & 138 & \\
\hline $\begin{array}{l}\text { gef. Eingr. } \\
\text { Str.V. }\end{array}$ & 0 & 0 & 3 & 1 & 0 & 0 & 0 & 0 \\
\hline AuslG & 7 & 17 & 32 & 234 & 8 & 8 & 26 & 0 \\
\hline Vollrausch & 0 & 0 & 1 & 0 & 0 & 0 & 2 & 0 \\
\hline fahrl. Tötung & 0 & 0 & 0 & 0 & 0 & 0 & 0 & 0 \\
\hline fahrl. KV & 5 & 1 & 8 & 4 & 0 & 2 & 14 & 0 \\
\hline \multirow[t]{7}{*}{ Verkehrsdel. } & 17 & 3 & 76 & 122 & 10 & 23 & 135 & 2 \\
\hline & & & AuslG & $\begin{array}{l}\text { Voll- } \\
\text { rausch }\end{array}$ & $\begin{array}{l}\text { fahrl. fahr } \\
\text { Tötung }\end{array}$ & 1. $\mathrm{KV} \quad \mathrm{Ve}$ & $\begin{array}{l}\text { rkehrs- } \\
\text { del. }\end{array}$ & \\
\hline & AuslG & & 31 & & & & & \\
\hline & Vollrausch & & 0 & 0 & & & & \\
\hline & fahrl. Tötung & & 0 & 0 & 0 & & & \\
\hline & fahrl. KV & & 15 & 0 & 2 & 1 & & \\
\hline & Verkehrsdel. & & 37 & 2 & 2 & & 433 & \\
\hline
\end{tabular}


Tabelle 69: Adjusted Standardized Residuals der Deliktspaare (deutsche Männer BZR, nur schwerstes Delikt)

\begin{tabular}{|c|c|c|c|c|c|c|c|c|}
\hline & $\begin{array}{r}\text { Tötungs- } \\
\text { delikte }\end{array}$ & $\begin{array}{r}\text { Sexual- } \\
\text { delikte }\end{array}$ & $\begin{array}{l}\text { sonst. } \\
\text { Sex. }\end{array}$ & Raub & $\begin{array}{c}\text { schw. } \\
\text { KV }\end{array}$ & KV & $\begin{array}{l}\text { Str. gg. } \\
\text { Pers. Fr. }\end{array}$ & $\begin{array}{l}\text { einf. } \\
\text { Dieb. }\end{array}$ \\
\hline Tötungsdelikte & $-0,4$ & & & & & & & \\
\hline Sexualdelikte & 1,0 & 12,5 & & & & & & \\
\hline $\begin{array}{l}\text { Sonst. Sexual- } \\
\text { del. }\end{array}$ & $-0,4$ & 4,5 & 57,4 & & & & & \\
\hline Raub & 2,5 & 2,8 & $-0,8$ & 16,0 & & & & \\
\hline Schw. KV & 2,0 & 0,9 & $-2,3$ & 5,0 & 31,6 & & & \\
\hline $\begin{array}{l}\text { Körperverlet- } \\
\text { zung }\end{array}$ & 1,3 & 1,6 & $-1,9$ & 4,7 & 23,4 & 41,6 & & \\
\hline $\begin{array}{l}\text { Str. gg. Pers. } \\
\text { Fr. }\end{array}$ & 1,6 & 0,2 & 0,2 & 1,6 & 3,9 & 6,6 & 11,8 & \\
\hline einf. Diebstahl & 0,0 & $-1,2$ & $-0,3$ & $-0,3$ & $-10,2$ & $-20,2$ & $-8,1$ & 76,8 \\
\hline $\begin{array}{l}\text { Schw. Dieb- } \\
\text { stahl }\end{array}$ & 0,1 & 2,5 & $-0,5$ & 10,1 & $-4,9$ & $-5,7$ & $-2,3$ & 0,2 \\
\hline Sachbeschäd. & $-0,3$ & $-0,6$ & $-0,1$ & 0,1 & 8,0 & 7,8 & 1,8 & $-5,2$ \\
\hline gemeingef. Str & 0,5 & 1,6 & 0,1 & $-1,7$ & 2,3 & 2,2 & $-0,4$ & $-3,1$ \\
\hline Hehlerei & $-1,0$ & 0,0 & $-0,8$ & $-0,7$ & 0,7 & $-0,3$ & $-0,6$ & $-2,7$ \\
\hline Betrug & $-1,6$ & 0,0 & 0,4 & $-6,8$ & $-11,8$ & $-11,6$ & 2,5 & $-14,0$ \\
\hline Erschl. v. Leist. & $-0,8$ & $-1,3$ & $-1,4$ & 0,2 & $-2,7$ & $-3,4$ & $-0,8$ & 14,2 \\
\hline $\begin{array}{l}\text { Gef. dem. } \\
\text { Recht. }\end{array}$ & $-0,3$ & $-0,3$ & $-0,9$ & 2,0 & 8,5 & 5,6 & 2,6 & $-6,5$ \\
\hline Wid. gg. Staat. & 0,3 & $-0,5$ & $-0,5$ & 1,4 & 4,8 & 7,4 & 1,8 & $-5,3$ \\
\hline öff. Ordnung & 0,1 & $-0,8$ & $-0,1$ & 0,1 & 3,9 & 2,8 & 1,6 & $-6,2$ \\
\hline Hausfriedensbr. & $-0,8$ & $-1,2$ & $-1,0$ & 0,0 & 2,9 & 0,7 & 0,5 & 4,3 \\
\hline Beleidigung & $-0,2$ & 2,3 & 1,3 & $-0,4$ & 7,1 & 9,5 & 7,9 & $-9,5$ \\
\hline Fälschung & 0,6 & 0,7 & $-0,8$ & $-2,4$ & $-5,5$ & $-6,7$ & $-0,1$ & $-8,9$ \\
\hline Meineid & 0,1 & $-0,1$ & 0,8 & $-0,6$ & 1,5 & 1,1 & $-0,7$ & $-5,3$ \\
\hline falsche Verd. & 1,2 & 0,5 & 0,5 & $-1,3$ & $-1,0$ & 0,0 & 0,9 & $-0,5$ \\
\hline BTM-Delikte & $-1,7$ & $-3,6$ & $-4,5$ & 0,8 & $-5,6$ & $-11,2$ & $-5,7$ & 1,0 \\
\hline $\begin{array}{l}\text { gef. Eingr. } \\
\text { Str.V. }\end{array}$ & $-0,8$ & $-0,8$ & $-1,3$ & 0,9 & 0,4 & 1,5 & 2,1 & $-3,6$ \\
\hline $\begin{array}{l}\text { fahrl. Tötung / } \\
\text { KV }\end{array}$ & $-0,6$ & $-0,3$ & 1,6 & $-2,9$ & $-1,7$ & 2,8 & 1,8 & $-8,8$ \\
\hline Verkehrsdel. & $-0,4$ & $-2,6$ & $-1,8$ & $-13,3$ & $-11,3$ & $-4,0$ & $-0,7$ & $-46,6$ \\
\hline
\end{tabular}




\begin{tabular}{|c|c|c|c|c|c|c|c|c|}
\hline & $\begin{array}{l}\text { schw. } \\
\text { Dieb. }\end{array}$ & Sachb. & $\begin{array}{l}\text { gemein- } \\
\text { gef. Str. }\end{array}$ & Hehlerei & Betrug & $\begin{array}{l}\text { Erschl. v. } \\
\text { Leist. }\end{array}$ & $\begin{array}{l}\text { Gef. } \\
\text { dem. } \\
\text { Recht. }\end{array}$ & $\begin{array}{l}\text { Wid. gg. } \\
\text { Staat. }\end{array}$ \\
\hline schw. Diebstahl & 55,3 & & & & & & & \\
\hline Sachbeschäd. & $-3,1$ & 21,9 & & & & & & \\
\hline gemeingef. Str & $-1,6$ & 6,0 & 19,2 & & & & & \\
\hline Hehlerei & 3,8 & $-1,1$ & $-1,8$ & 4,5 & & & & \\
\hline Betrug & $-13,3$ & $-10,0$ & $-4,9$ & $-1,5$ & 100,7 & & & \\
\hline Erschl. v. Leist. & $-1,8$ & 0,8 & $-0,9$ & $-2,2$ & $-0,4$ & 32,5 & & \\
\hline $\begin{array}{l}\text { Gef. dem. } \\
\text { Recht. }\end{array}$ & $-2,6$ & 4,4 & 2,5 & $-0,9$ & $-2,2$ & 0,3 & 39,6 & \\
\hline Wid. gg. Staat. & $-0,6$ & 3,9 & 0,6 & $-1,7$ & $-4,9$ & 0,6 & 4,1 & 10,7 \\
\hline öff. Ordnung & $-2,8$ & 2,9 & 2,4 & $-0,8$ & 3,0 & 1,0 & 7,9 & 1,0 \\
\hline Hausfriedensbr. & $-4,1$ & 3,1 & 2,7 & 0,2 & $-5,6$ & 1,2 & 1,1 & 2,4 \\
\hline Beleidigung & $-5,3$ & 6,3 & 3,1 & $-0,3$ & $-2,0$ & $-0,6$ & 3,0 & 6,8 \\
\hline Fälschung & 2,2 & $-5,3$ & $-2,0$ & 1,4 & 15,9 & $-3,8$ & $-2,9$ & $-2,1$ \\
\hline Meineid & 0,2 & $-0,2$ & $-0,8$ & 1,2 & 3,2 & $-2,0$ & 0,2 & $-0,1$ \\
\hline falsche Verd. & $-0,9$ & 0,8 & 1,2 & $-0,1$ & 1,9 & 1,2 & 0,0 & $-0,7$ \\
\hline BTM-Delikte & $-5,4$ & $-2,3$ & $-4,7$ & 1,2 & $-26,6$ & $-3,2$ & $-5,5$ & $-3,1$ \\
\hline $\begin{array}{l}\text { gef. Eingr. } \\
\text { Str.V. }\end{array}$ & 1,0 & 1,0 & 0,3 & $-0,4$ & 0,8 & $-1,1$ & $-0,6$ & $-0,9$ \\
\hline $\begin{array}{l}\text { fahrl. Tötung / } \\
\text { KV }\end{array}$ & $-5,6$ & $-0,7$ & 0,4 & 0,8 & $-0,7$ & $-1,8$ & $-0,6$ & $-0,1$ \\
\hline \multirow[t]{2}{*}{ Verkehrsdel. } & $-20,5$ & $-6,4$ & 0,9 & 0,7 & 1,7 & $-13,1$ & $-3,8$ & $-1,9$ \\
\hline & öff. Ord. & Hausfr. & $\begin{array}{l}\text { Beleidi- } \\
\text { gung }\end{array}$ & $\begin{array}{c}\text { Fäl- } \\
\text { schung }\end{array}$ & Meineid & $\begin{array}{l}\text { falsche } \\
\text { Verd. }\end{array}$ & $\begin{array}{l}\text { BTM- } \\
\text { Delikte }\end{array}$ & $\begin{array}{c}\text { gef. } \\
\text { Eingr. } \\
\text { Str.V. }\end{array}$ \\
\hline öff. Ordnung & 8,8 & & & & & & & \\
\hline Hausfriedensbr. & $-1,0$ & 21,7 & & & & & & \\
\hline Beleidigung & 2,2 & 1,9 & 25,6 & & & & & \\
\hline Fälschung & 0,6 & $-3,1$ & $-2,5$ & 21,4 & & & & \\
\hline Meineid & 0,6 & $-0,5$ & $-0,1$ & 1,6 & 4,1 & & & \\
\hline falsche Verd. & 1,9 & $-1,0$ & 1,2 & 2,4 & $-1,2$ & 0,8 & & \\
\hline BTM-Delikte & $-6,9$ & 3,8 & $-4,4$ & $-10,2$ & $-0,6$ & $-1,4$ & 85,7 & \\
\hline $\begin{array}{l}\text { gef. Eingr. } \\
\text { Str.V. }\end{array}$ & 1,2 & $-0,2$ & 2,5 & $-0,3$ & 0,0 & 0,5 & $-3,7$ & 2,4 \\
\hline $\begin{array}{l}\text { fahrl. Tötung / } \\
\text { KV }\end{array}$ & 1,5 & $-1,1$ & 0,3 & 0,6 & 0,6 & 0,6 & $-5,1$ & 2,7 \\
\hline Verkehrsdel. & 2,2 & $-9,5$ & $-6,2$ & 10,2 & 2,4 & $-0,9$ & $-24,7$ & 2,3 \\
\hline
\end{tabular}

\begin{tabular}{lcc}
\hline & fahrl. Tötung / KV & Verkehrsdel. \\
\hline fahrl. Tötung / KV & $\mathbf{1 2 , 9}$ & \\
Verkehrsdel. & $\mathbf{1 4 , 0}$ & $\mathbf{1 0 0 , 5}$ \\
\hline
\end{tabular}




\section{Studie von Francis, Soothill und Fligelstone}

Francis, Soothill und Fligelstone ${ }^{316}$ analysierten Deliktsmuster und Verläufe von kriminellem Verhalten mit einem probabilistischem Clusteranalyseverfahren. ${ }^{317}$ Für ihre Untersuchung benutzten sie Gerichtsdaten von Personen aus England und Wales, bis zum Alter von 40 Jahren, die in vier bestimmten Wochen des Jahres 1953 geboren waren. Die Daten der Abgeurteilten wurden in sechs verschiedene Altersgruppen eingeteilt: 10-15, 16-20, 21-25, 26-30, 31-35 und 36-40. Innerhalb dieser Abschnitte wurde das Auftreten verschiedener Delikte 0/1 kodiert, d.h. wenn z.B. das Delikt Ladendiebstahl bei einer Person in einem Altersabschnitt auftrat, wurde eine 1 notiert, andernfalls eine Null. Die Anzahl der Delikte spielte also keine Rolle, es war nur von Bedeutung, ob ein Delikt bei einer Person in einem Altersabschnitt vorkam oder nicht. Die Auswertung wurde nach Geschlecht differenziert durchgeführt. Es handelte sich um 9.232 Männer mit 15.353 einzelnen Altersabschnitten und 2.168 Frauen mit 2.596 Altersabschnitten. ${ }^{318}$ Die Altersabschnitte wurden nach Wahrscheinlichkeiten in Cluster eingeteilt, wobei die Deliktszusammensetzung das einzige Kriterium für die Einordnung war. Bei den Männern wurden 71 Deliktskategorien ${ }^{319}$ in die Auswertung mit einbezogen.

316 Francis, Soothill \& Fligelstone 2004.

317 Francis, Soothill \& Fligelstone verwendeten für die binäre latent class analysis das Programm Latent Gold. (vgl. Francis, Soothill \& Fligelstone 2004, S. 57).

318 Es wurden nur Altersabschnitte benutzt, die mindestens einen Eintrag hatten, mindestens ein Delikt war registriert. (vgl. Francis, Soothill \& Fligelstone 2004, S. 55).

319 Die Deliktskategorien stammten aus The Offenders Index: codebook (Research Development and Statistics Directorate 1998). Es wurden die Delikte benutzt, die 10-mal oder häufiger je Gruppe vorkamen. Teilweise wurden selten vorkommende Delikte mit anderen Delikten in einer Gruppe zusammengefasst. (vgl. Francis, Soothill \& Fligelstone 2004, S. 54) Im Ergebnis der Clusteranalyse wurden von den 71 Deliktskategorien nur 26 aufgeführt, die übrigen 45 Deliktskategorien kamen sehr selten vor. Unter den 26 aufgeführten Deliktskategorien befanden sich 11 verschiedene Diebstahldelikte. (vgl. Francis, Soothill \& Fligelstone 2004, S. 61 f.). 
Tabelle 70: Male cluster profiles: Probabilities of a strip in each cluster having the specified offence (\%) 320

\begin{tabular}{|c|c|c|c|c|c|c|c|c|c|}
\hline \multirow[b]{3}{*}{ Cluster proportions $\pi(\mathrm{k})$ in $\%$} & \multicolumn{9}{|c|}{ Cluster } \\
\hline & A & $\mathrm{B}$ & $\mathrm{C}$ & $\mathrm{D}$ & $\mathrm{E}$ & $\mathrm{F}$ & G & $\mathrm{H}$ & I \\
\hline & 18,5 & 16,6 & 12,4 & 11,8 & 9,9 & 8,6 & 8,3 & 7,9 & 6,0 \\
\hline \multicolumn{10}{|l|}{ Probabilities $p_{j k}$ in $\%$} \\
\hline 8. Malicious wounding & 0,3 & 2,7 & 5,6 & 18,6 & 0,0 & 28,6 & 1,2 & 100 & 0,0 \\
\hline 28. Burglary in a dwelling & 2,0 & 11,6 & 8,9 & 3,0 & 0,7 & 34,8 & 3,3 & $\hat{1, \hat{1}}$ & 0,2 \\
\hline 29. Aggravated burglary in a dwell. & 0,0 & 11,4 & 0,0 & 0,2 & 0,9 & 0,7 & 0,0 & 0,3 & 0,0 \\
\hline 30,27. Commercial burglary & 2,5 & 51,0 & 10,0 & 10,4 & 0,0 & 49,9 & 5,4 & 1,7 & 0,0 \\
\hline 33. Going equipped for stealing & 1,1 & 5,2 & 0,3 & 0,4 & 3,0 & 18,8 & 5,7 & 0,2 & 0,0 \\
\hline 34. Robbery \& assault with intent & 1,2 & 1,3 & 1,4 & 1,3 & 0,4 & 7,1 & 0,5 & 2,2 & 0,4 \\
\hline 37,51,53. Fraud \& embezzlement & 0,9 & 0,0 & 43,6 & 1,7 & 0,2 & 21,2 & 2,6 & 0,0 & 0,0 \\
\hline 40. Stealing in a dwelling & 0,5 & 3,3 & 5,6 & 0,4 & 0,3 & 5,6 & 0,7 & 0,0 & 0,0 \\
\hline 41. Stealing by an employee & 10,0 & 0,6 & 4,7 & 0,4 & 0,8 & 2,9 & 1,5 & 0,7 & 0,3 \\
\hline 44. Bicycle theft & 1,6 & 6,3 & 0,9 & 0,2 & 0,5 & 2,3 & 1,2 & 0,3 & 0,0 \\
\hline 45. Stealing from a vehicle & 9,2 & 9,0 & 0,8 & 0,7 & 1,4 & 20,5 & 11,0 & 0,4 & 0,4 \\
\hline 46. Shoplifting & 0,0 & 9,5 & 12,9 & 2,4 & 0,0 & 15,4 & 1,2 & 3,4 & 100,0 \\
\hline $\begin{array}{l}48,130,918 . \text { Theft or unauthorized } \\
\text { taking of motor vehicle }\end{array}$ & 0,1 & 12,9 & 2,7 & 5,6 & 0,0 & 56,9 & 100,0 & 3,0 & 0,3 \\
\hline 49. Petty theft & 0,0 & 31,4 & 30,0 & 7,6 & 100,0 & $\mathbf{5 7 , 0}$ & 11,7 & 7,5 & 5,5 \\
\hline 54. Receiving/handling stolen goods & 14,0 & 7,4 & 20,3 & 2,6 & 2,0 & 26,2 & 2,8 & 1,4 & 1,1 \\
\hline 56. Arson & 0,4 & 1,1 & 0,1 & 1,0 & 0,2 & 1,0 & 0,0 & 0,2 & 0,0 \\
\hline $57,48,59$. Serious criminal damage & 0,5 & 2,7 & 2,0 & 41,3 & 1,2 & 22,6 & 3,3 & 3,3 & 0,0 \\
\hline $58,60,61$ Coining, forgery & 0,6 & 0,3 & 9,6 & 0,3 & 0,3 & 5,5 & 0,0 & 0,1 & 0,0 \\
\hline 77,92,93. More serious drug offence & 14,6 & 0,2 & 5,9 & 0,0 & 0,2 & 6,9 & 0,2 & 1,7 & 1,5 \\
\hline 80,83 Absconding from custody/bail & 3,1 & 0,0 & 9,2 & 0,7 & 0,0 & 6,9 & 0,2 & 2,3 & 2,4 \\
\hline 104. Assault (minor offence) & 1,3 & 0,4 & 2,1 & 21,9 & 0,0 & 8,2 & 0,2 & 0,7 & 0,4 \\
\hline 149. Criminal damage (less serious) & 15,3 & 10,7 & 5,7 & 9,3 & 1,0 & 12,5 & 1,3 & 7,7 & 2,5 \\
\hline 165. Possession of offensive weapon & 1,9 & 0,9 & 0,2 & 7,8 & 0,8 & 4,9 & 1,8 & 1,2 & 0,1 \\
\hline 193. Misuse of drugs (minor offence) & 6,3 & 0,0 & 1,3 & 1,7 & 0,5 & 3,8 & 0,7 & 0,1 & 1,1 \\
\hline 195. Other minor offences & 0,2 & 0,2 & 0,7 & 11,3 & 0,4 & 4,6 & 0,0 & 1,1 & 0,4 \\
\hline $807,814,958$. Driving licence offences & 0,5 & 0,0 & 1,0 & 0,3 & 0,0 & 10,0 & 2,2 & 0,2 & 0,0 \\
\hline
\end{tabular}

Note: Only the 26 offence categories contributing a probability of greater than $50 \%$ to a cluster are included; the remaining 45 are omitted. These probabilities are highlighted in bold. The full list of 71 offences can be found in Soothill et al. (2002a). For each offence category the Home Office offence numbers and shortened offence names are given - the full names can be found in the Offenders Index Codebook (Research, Development and Statistics Directorate, 1998).

320 Francis, Soothill \& Fligelstone 2004, S. 61 f. 
Tabelle 71: Deliktszusammensetzung der männlichen Cluster von Francis, Soothill, Fligelstone $^{321}$

\begin{tabular}{ll}
\hline Cluster & Zusammensetzung \\
\hline A $(18,5 \%)$ & $\begin{array}{l}\text { Sachbeschädigung, Drogendelikte, Hehlerei, Sexualdelikte. } \\
\text { Verschiedene Straftaten } \\
\text { fig vertreten. }\end{array}$ \\
$\begin{array}{l}\text { B }(16,6 \%) \\
\text { Schwerer Diebstahl }\end{array}$ & $\begin{array}{l}\text { Schwerer und einfacher Diebstahl. Kaum Gewaltdelikte. } \\
\text { Alter: jünger als 20. }\end{array}$ \\
C (12,4\%) & Betrug, Fälschung, Diebstahl. \\
Betrug & Alter: älter 15. \\
D $(11,8 \%)$ & Körperverletzung, Sachbeschädigung. \\
Gewaltdelikte & Alter: 16-25 \\
E $(9,9 \%)$ & Diebstahl. Alle Altersgruppen, aber hauptsächlich jünger als \\
Einfacher Diebstahl & 20, Maximum mit 16. \\
F $(8,6 \%)$ & Diebstahl, Gewaltdelikte, Raub, Verkehrsdelikte, Sachbe- \\
Eigentums-, Gewalt- und & schädigung. \\
Verkehrsdelikte & Alter: 16-30 \\
G $(8,3 \%)$ & Autodiebstahl, Diebstahl, keine Gewaltdelikte. Alter: haupt- \\
Autodiebstahl & sächlich 16-20. \\
H (7,9\%) & Körperverletzung. \\
Körperverletzung & Alter: älter 16. \\
I (6,0\%) & Ladendiebstahl. Alle Altersgruppen, Häufigkeit nimmt mit \\
Ladendiebstahl & dem Älterwerden langsam ab. \\
\hline
\end{tabular}

${ }^{321}$ Francis, Soothill \& Fligelstone 2004, S. 65. 
Die optimale Lösung, bestimmt mit dem Bayesian Information Criterion (BIC) $)^{322}$, ergab bei den Männern 9 Cluster, die in Tabelle 70 und 71 dargestellt sind. Die Größe der Cluster war bei den Männern relativ gleichmäßig verteilt und reichte von $6 \%$ bis $18,5 \%$ aller Altersabschnitte. Im größten Cluster, Cluster A, versammelten sich verschiedene Delikte, während dem kleinsten Cluster, Cluster I, fast zu 100\% Altersabschnitte angehörten, die das Delikt Ladendiebstahl enthielten und kaum ein anderes Delikt. Fünf der neun Cluster enthielten hauptsächlich Diebstahldelikte.

Umgekehrt kann angegeben werden, mit welcher Wahrscheinlichkeit ein Delikt zu welchem Cluster gehört. Enthält z.B. ein Altersabschnitt das Delikt Ladendiebstahl, so gehört dieser Altersabschnitt mit 54\%iger Wahrscheinlichkeit zu Cluster I. Weiter enthalten z.B. fast alle Abschnitte des Clusters H Körperverletzung, während umgekehrt 57\% aller Altersabschnitte, die Körperverletzung enthalten, Cluster $\mathrm{H}$ angehören.

Francis, Soothill und Fligelstone untersuchten, ob die gefundenen Cluster in $\mathrm{Ab}$ hängigkeit vom Alter unterschiedlich häufig auftreten. Für die verschiedenen Cluster zeigten sich unterschiedliche Altersschwerpunkte. Cluster B, schwerer Diebstahl, enthielt besonders häufig Altersabschnitte der Altergruppe der unter Sechzehnjährigen. Im Gegensatz dazu befanden sich im Cluster C, Betrug, hauptsächlich Altersabschnitte der älteren Altersgruppen.

Bei den Frauen wurde die Clusteranalyse mit 29 Delikten gerechnet und die optimale Lösung ergab drei Cluster (Tabelle 72 und 73). Bei den Frauen hatten die drei Cluster der Altersabschnitte deutlich unterschiedliche Größen. Das größte Cluster enthielt knapp 60\% aller Altersabschnitte und setzte sich aus verschiedenen Delikten zusammen. Es hatte bei allen Delikten die höchste Wahrscheinlichkeit, außer bei Ladendiebstahl und Untreue. Diese Eigentumsdelikte bildeten die zwei weiteren Cluster. Cluster B enthielt fast ausschließlich Altersabschnitte mit dem Delikt Ladendiebstahl. Fast alle Altersabschnitte, die zum Cluster C gehörten enthielten das Delikt Untreue. Dieses Cluster war mit 4\% aller Altersabschnitte relativ klein. Bei den Frauen hatten alle drei Cluster dieselbe Altersverteilung, das Maximum lag jeweils bei der Altersgruppe 16 bis 20 und nahm danach ab. Das Cluster des Ladendiebstahls gab es auch bei den Männern.

322 BIC ist ein Informationskriterium mit dem das bestmögliche Modell, hier die optimale Anzahl der Cluster, ausgewählt werden kann.

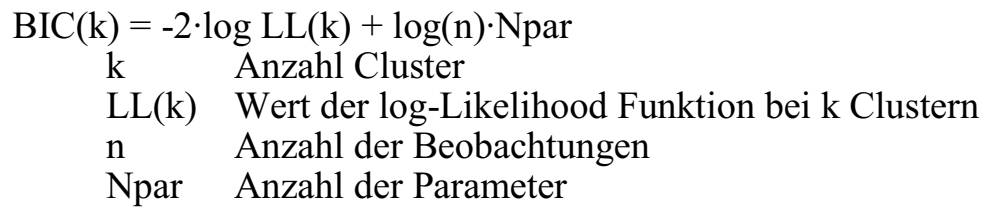

Vgl. Vermunt \& Magidson 2005, Technical Guide for Latent GOLD, S. 61.

Das Minimum des Wertes von BIC ergibt die optimale Anzahl Cluster (vgl. Francis, Soothill \& Fligelstone 2004, S. 57). 
Tabelle 72: Female cluster profiles: Probabilities of a strip in each cluster having the specified offence $(\%)^{323}$

\begin{tabular}{|c|c|c|c|}
\hline \multirow[b]{3}{*}{ Cluster proportions $\pi(\mathrm{k})$ in $\%$} & \multicolumn{3}{|c|}{ Cluster } \\
\hline & A & B & $\mathrm{C}$ \\
\hline & 59,4 & 36,3 & 4,3 \\
\hline \multicolumn{4}{|l|}{ Probabilities $\mathrm{p}_{\mathrm{jk}}$ in \% } \\
\hline 8. Malicious wounding & 10,1 & 0,1 & 0,0 \\
\hline 28. Burglary in a dwelling & 3,0 & 0,3 & 1,0 \\
\hline 29. Aggravated burglary in a dwelling & 0,7 & 0,5 & 0,0 \\
\hline 30,27. Commercial burglary & 4,4 & 0,0 & 0,9 \\
\hline 34. Robbery \& assault with intent & 0,9 & 0,0 & 0,0 \\
\hline 39. Stealing from the person of another & 1,4 & 0,0 & 0,0 \\
\hline 37,51,53. Fraud \& embezzlement & 18,8 & 0,8 & 3,7 \\
\hline 40. Stealing in a dwelling & 3,8 & 0,2 & 2,7 \\
\hline 41. Stealing by an employee & 0,1 & 0,2 & 98,9 \\
\hline 43. Abstracting electricity & 3,1 & 0,1 & 0,0 \\
\hline 44. Bicycle theft & 0,7 & 0,0 & 0,0 \\
\hline 45. Stealing from a vehicle & 1,1 & 0,0 & 0,0 \\
\hline 46. Shoplifting & 10,5 & 100 & 0,5 \\
\hline 47. Stealing from automatic machines / meters & 3,8 & $0, \overline{2}$ & 0,0 \\
\hline $48,130,918$. Theft or unauthorized taking of motor & 3,8 & 0,0 & 0,9 \\
\hline 49. Petty theft & 30,5 & 2,8 & 3,6 \\
\hline 52. Falsifying accounts & 0,6 & 0,2 & 17,4 \\
\hline 54. Receiving/handling stolen goods & 13,6 & 1,3 & 0,0 \\
\hline 56. Arson & 0,7 & 0,0 & 0,0 \\
\hline $57,48,59$. Serious criminal damage & 4,2 & 0,0 & 0,0 \\
\hline $58,60,61$ Coining, forgery & 6,7 & 0,1 & 1,0 \\
\hline 77,92,93. More serious drug offences & 5,4 & 0,3 & 0,0 \\
\hline 80,83 Absconding from custody/bail & 2,9 & 0,3 & 0,0 \\
\hline 99. Other indictable offences & 1,5 & 0,1 & 0,0 \\
\hline 104. Assault (minor offence) & 5,1 & 0,2 & 0,0 \\
\hline 149. Criminal damage (less serious) & 5,5 & 0,3 & 0,9 \\
\hline 193. Misuse of drugs (minor offence) & 2,3 & 0,1 & 0,0 \\
\hline 194. Immigration Act & 0,6 & 0,0 & 0,9 \\
\hline 195. Other minor offences & 1,6 & 0,0 & 0,0 \\
\hline
\end{tabular}

Note: All 29 offence categories are included. Probabilities greater than $50 \%$ are highlighted in bold. For each offence category the Home Office offence numbers and shortened offence names are given - the full names can be found in the Offenders Index Codebook (Research, Development and Statistics Directorate, 1998).

323 Francis, Soothill \& Fligelstone 2004, S. 63 
Tabelle 73: Deliktszusammensetzung der weiblichen Cluster von Francis, Soothill, Fligelstone $^{324}$

\begin{tabular}{ll}
\hline Cluster & Zusammensetzung \\
\hline A (59,4\%) & $\begin{array}{l}\text { Dieses Cluster hat bei allen Delikten die höchste Wahrscheinlichkeit, } \\
\text { Verschiedene Straftaten }\end{array}$ \\
B (36,3\%) & $\begin{array}{l}\text { Ladendiebstahl, alle Altersgruppen, aber ab dem Alter 26-30 stark } \\
\text { abnehmend. }\end{array}$ \\
Ladendiebstahl & Untreue, Unterschlagung. \\
C (4,3\%) & Alter: 16-20. \\
Untreue &
\end{tabular}

Das Ergebnis der Clusteranalyse wurde für weitere Auswertungen zum Verlauf der Kriminalität, speziell zur Untersuchung von Spezialisierung, benutzt. Von Spezialisierung sprachen Francis, Soothill und Fligelstone dann, wenn eine Person in einem späteren Altersabschnitt Delikte begeht, so dass dieser Altersabschnitt demselben Cluster zugeordnet werden kann wie der frühere Altersabschnitt. Diesbezüglich unterteilten sie die Personen in drei Gruppen: Abbrecher, Spezialisierte und Wechsler. Einige Untersuchungen zu Spezialisierung wurden ohne die Abbrecher durchgeführt. Ein Ergebnis war, dass es einen Zusammenhang zwischen Spezialisierung und Alter gibt. So waren z.B. von den 10- bis 15-Jährigen Männern aus Cluster I, Ladendiebstahl, nur 1\% auch mit 16 bis 20 im Cluster I. Aber von den 31- bis 35-Jährigen aus Cluster I, befanden sich fast die Hälfte auch im Alter von 36 bis 40 im Cluster I. Des Weiteren wurde der Verlauf der Cluster mit dem Alter untersucht. Francis, Soothill und Fligelstone waren der Ansicht, dass wenn die kriminelle Aktivität der vorhergehenden fünf Jahre bekannt ist, es möglich ist, die Qualität und die Art des Delikts in den folgenden fünf Jahren vorherzusagen. ${ }^{325}$

324 Francis, Soothill \& Fligelstone 2004, S. 65.

325 Vgl. Francis, Soothill \& Fligelstone 2004, S. 82. 
Tabelle 74: Deliktscluster justizieller Registrierungen deutscher Männer der Kohorte 1970: Wahrscheinlichkeiten der Deliktskategorien in den Clustern in \% (7Clusterlösung)

\begin{tabular}{|c|c|c|c|c|c|c|c|c|}
\hline \multirow[b]{3}{*}{$\begin{array}{l}\text { Clusterwahrscheinlichkeit } \pi(\mathrm{k}) \\
\text { in } \%\end{array}$} & \multicolumn{7}{|c|}{ Cluster } & \multirow[b]{2}{*}{ gesam } \\
\hline & A & B & $\mathrm{C}$ & $\mathrm{D}$ & E & $\mathrm{F}$ & $\mathrm{G}$ & \\
\hline & 32,8 & 21,9 & 14,3 & 10,5 & 9,5 & 5,6 & 5,4 & 100 \\
\hline \multicolumn{9}{|l|}{ Wahrscheinlichkeiten $\mathrm{p}_{\mathrm{jk}}$ in \% } \\
\hline Tötungsdelikte & 0,0 & 0,2 & 0,1 & 0,5 & 0,1 & 0,8 & 0,0 & 0,2 \\
\hline Sexualdelikte & 0,0 & 1,2 & 0,2 & 1,9 & 0,2 & 2,4 & 0,0 & 0,7 \\
\hline sonst. Sexualdelikte & 0,1 & 2,7 & 0,1 & 1,0 & 0,6 & 1,2 & 0,3 & 0,9 \\
\hline Raub & 0,3 & 3,3 & 1,3 & 6,1 & 0,9 & 18,5 & 0,0 & 2,8 \\
\hline schw. Körperverletzung & 0,1 & 0,0 & 0,2 & 43,9 & 0,2 & 26,2 & 0,0 & 6,2 \\
\hline Körperverletzung & 0,6 & 0,1 & 0,3 & 86,4 & 0,6 & 52,5 & 32,6 & 14,1 \\
\hline Str. gg. Pers. Freiheit & 0,6 & 6,7 & 0,2 & 17,2 & 1,5 & 20,7 & 1,5 & 4,9 \\
\hline Einfacher Diebstahl & 3,6 & 14,0 & 100,0 & 16,7 & 36,5 & $\mathbf{7 0 , 7}$ & 2,7 & 27,9 \\
\hline Schwerer Diebstahl & 3,8 & 20,0 & 10,9 & 7,1 & 9,1 & 46,5 & 1,0 & 11,5 \\
\hline Sachbeschädigung & 1,3 & 13,8 & 6,7 & 14,4 & 1,4 & 32,0 & 1,0 & 7,9 \\
\hline gemeingef. Straftaten & 0,2 & 2,2 & 0,5 & 0,7 & 0,3 & 3,6 & 1,6 & 1,0 \\
\hline Hehlerei & 0,8 & 6,6 & 6,0 & 1,5 & 2,5 & 12,8 & 0,8 & 3,7 \\
\hline Betrug & 0,4 & 0,7 & 0,0 & 4,1 & 99,9 & 33,3 & 2,4 & 12,2 \\
\hline Erschl. v. Leistungen & 0,7 & 13,5 & 5,5 & 5,4 & 7,0 & 15,1 & 0,2 & 6,0 \\
\hline Gef. dem. Rechtsstaat & 0,0 & 0,9 & 0,0 & 1,3 & 0,0 & 2,7 & 0,0 & 0,5 \\
\hline Wid. gg. Staatsgewalt & 0,2 & 2,2 & 0,0 & 12,3 & 0,1 & 14,6 & 1,3 & 2,8 \\
\hline Öffentliche Ordnung & 1,2 & 2,9 & 1,5 & 2,2 & 3,7 & $\mathbf{9 , 9}$ & 0,6 & 2,4 \\
\hline Hausfriedensbruch & 0,1 & 3,2 & 1,5 & 3,6 & 0,3 & 12,6 & 0,3 & 2,1 \\
\hline Beleidigung & 0,8 & 9,8 & 0,5 & 20,8 & 1,9 & 24,8 & 1,4 & 6,3 \\
\hline Fälschung & 4,6 & 4,6 & 3,1 & 0,9 & 21,5 & 26,2 & 0,7 & 6,6 \\
\hline Meineid & 0,2 & 1,8 & 0,2 & 0,7 & 1,5 & 2,0 & 0,1 & 0,8 \\
\hline falsche Verdächtigung & 0,2 & 1,0 & 0,3 & 0,3 & 0,6 & 2,5 & 0,3 & 0,6 \\
\hline BTM-Delikte & 3,5 & 29,2 & 7,2 & 8,9 & 6,2 & 23,7 & 2,3 & 11,5 \\
\hline $\begin{array}{l}\text { gef. Eingriff in den Straßenver- } \\
\text { kehr }\end{array}$ & 0,0 & 1,7 & 0,0 & 1,2 & 0,2 & $\mathbf{3 , 3}$ & 1,3 & 0,8 \\
\hline Vollrausch & 0,4 & 2,4 & 0,5 & 2,4 & 0,4 & 4,5 & 0,1 & 1,3 \\
\hline fahrl. Tötung & 0,5 & 0,3 & 0,0 & 0,4 & 0,1 & 0,0 & 4,0 & 0,5 \\
\hline fahrl. Körperverletzung & 9,8 & 1,4 & 2,5 & 5,7 & 1,7 & 13,5 & 100,0 & 10,8 \\
\hline Verkehrsdelikte & 100,0 & 20,8 & 25,0 & 28,1 & 30,3 & 65,9 & 46,6 & 53,0 \\
\hline $\begin{array}{l}\text { Durchschnittliche Anzahl De- } \\
\text { likte }\end{array}$ & 1,3 & 1,7 & 1,8 & 3,0 & 2,3 & 6,0 & 2,1 & 2,0 \\
\hline
\end{tabular}


Tabelle 75: Deliktscluster justizieller Registrierungen deutscher Männer der Kohorte 1970: Wahrscheinlichkeiten d. Deliktskategorien in den Clustern in \% (8-Clusterlösung)

\begin{tabular}{|c|c|c|c|c|c|c|c|c|c|}
\hline \multirow[b]{3}{*}{$\begin{array}{l}\text { Clusterwahrscheinlichkeit } \pi(\mathrm{k}) \text { in } \\
\%\end{array}$} & \multicolumn{8}{|c|}{ Cluster } & \multirow[b]{2}{*}{ gesamt } \\
\hline & $\mathrm{A}$ & $\mathrm{B}$ & $\mathrm{C}$ & $\mathrm{D}$ & $\mathrm{E}$ & $\mathrm{F}$ & G & $\mathrm{H}$ & \\
\hline & 32,4 & 16,7 & 15,4 & 10,0 & 9,4 & 5,7 & 5,4 & 5,0 & 100 \\
\hline \multicolumn{10}{|l|}{ Wahrscheinlichkeiten $\mathrm{p}_{\mathrm{jk}}$ in \% } \\
\hline Tötungsdelikte & 0,0 & 0,1 & $\mathbf{0 , 3}$ & 0,4 & 0,1 & $\mathbf{0 , 8}$ & 0,0 & 0,0 & 0,2 \\
\hline Sexualdelikte & 0,0 & 0,2 & 1,7 & 1,9 & 0,2 & 2,5 & 0,0 & 0,0 & 0,7 \\
\hline sonst. Sexualdelikte & 0,0 & 0,3 & 3,7 & 1,0 & 0,6 & 1,3 & 0,3 & 0,0 & 0,9 \\
\hline Raub & 0,3 & 1,6 & 3,8 & 6,2 & 0,9 & 18,6 & 0,0 & 1,3 & 2,8 \\
\hline schw. Körperverletzung & 0,2 & 0,2 & 0,4 & 45,2 & 0,2 & 26,1 & 0,0 & 0,0 & 6,2 \\
\hline Körperverletzung & 0,6 & 0,2 & 0,0 & 90,4 & 0,6 & 52,4 & 32,8 & 0,3 & 14,1 \\
\hline Str. gg. Pers. Freiheit & 0,5 & 0,8 & 9,5 & 16,6 & 1,6 & 20,9 & 1,5 & 0,9 & 4,9 \\
\hline Einfacher Diebstahl & 3,2 & 100,0 & 5,9 & 16,5 & 35,4 & 72,1 & 2,8 & 0,1 & 27,9 \\
\hline Schwerer Diebstahl & 3,7 & 11,9 & 24,6 & 7,2 & 9,1 & 46,2 & 0,9 & 5,3 & 11,5 \\
\hline Sachbeschädigung & 1,2 & 7,1 & 18,7 & 14,3 & 1,3 & 31,3 & 0,9 & 1,5 & 7,9 \\
\hline gemeingef. Straftaten & 0,2 & 0,6 & 2,9 & 0,6 & 0,3 & 3,3 & 1,6 & 0,4 & 1,0 \\
\hline Hehlerei & 0,8 & 5,9 & 8,2 & 1,5 & 2,4 & 12,6 & 0,7 & 1,9 & 3,7 \\
\hline Betrug & 0,4 & 0,9 & 0,6 & 4,1 & 99,9 & 33,2 & 2,4 & 0,0 & 12,2 \\
\hline Erschl. v. Leistungen & 0,6 & 6,7 & 15,6 & 5,2 & 6,9 & 15,7 & 0,1 & 4,9 & 6,0 \\
\hline Gef. dem. Rechtsstaat & 0,0 & 0,1 & 1,3 & 1,3 & 0,0 & 2,5 & 0,0 & 0,0 & 0,5 \\
\hline Wid. gg. Staatsgewalt & 0,2 & 0,2 & 3,6 & 11,8 & 0,2 & 14,6 & 1,3 & 0,4 & 2,8 \\
\hline Öffentliche Ordnung & 1,2 & 1,6 & 4,0 & 2,2 & 3,7 & 9,6 & 0,6 & 0,0 & 2,4 \\
\hline Hausfriedensbruch & 0,1 & 1,8 & 4,0 & 3,6 & 0,3 & 12,5 & 0,2 & 0,7 & 2,1 \\
\hline Beleidigung & 0,7 & 1,3 & 14,1 & 19,8 & 2,0 & 25,1 & 1,3 & 1,0 & 6,3 \\
\hline Fälschung & 4,6 & 3,4 & 6,0 & 1,0 & 21,5 & 25,3 & 0,7 & 0,9 & 6,6 \\
\hline Meineid & 0,2 & 0,3 & 2,0 & 0,7 & 1,5 & 2,0 & 0,1 & 0,8 & 0,8 \\
\hline falsche Verdächtigung & 0,1 & 0,4 & 1,3 & 0,3 & 0,6 & 2,4 & 0,3 & 0,4 & 0,6 \\
\hline BTM-Delikte & 2,6 & 10,8 & 4,6 & 8,7 & 6,2 & 27,3 & 2,8 & 100,0 & 11,5 \\
\hline gef. Eingriff in den Straßenverkehr & 0,0 & 0,1 & 2,5 & 1,1 & 0,2 & 3,2 & 1,3 & 0,1 & 0,8 \\
\hline Vollrausch & 0,5 & 0,7 & 3,2 & 2,4 & 0,4 & 4,5 & 0,0 & 0,4 & 1,3 \\
\hline fahrl. Tötung & 0,5 & 0,1 & 0,3 & 0,4 & 0,1 & 0,2 & 4,0 & 0,0 & 0,5 \\
\hline fahrl. Körperverletzung & 9,9 & 2,5 & 2,1 & 5,8 & 1,7 & 13,1 & 100,0 & 0,5 & 10,8 \\
\hline Verkehrsdelikte & 100,0 & 25,5 & 23,4 & 28,1 & 30,4 & 64,0 & 46,7 & 18,5 & 53,0 \\
\hline Durchschnittliche Anzahl Delikte & 1,3 & 1,9 & 1,7 & 3,0 & 2,3 & 6,0 & 2,0 & 1,5 & 2,0 \\
\hline
\end{tabular}


Tabelle 76: Deliktscluster justizieller Registrierungen deutscher Männer der Kohorte 1970: Wahrscheinlichkeiten der Deliktskategorien in den Clustern in \% (9-Clusterlösung)

\begin{tabular}{|c|c|c|c|c|c|c|c|c|c|c|}
\hline \multirow[b]{3}{*}{$\begin{array}{l}\text { Clusterwahrscheinlichkeit } \\
\pi(\mathrm{k}) \text { in } \%\end{array}$} & \multicolumn{10}{|c|}{ Cluster } \\
\hline & $\mathrm{A}$ & $\mathrm{B}$ & $\mathrm{C}$ & $\mathrm{D}$ & $\mathrm{E}$ & $\mathrm{F}$ & $\mathrm{G}$ & $\mathrm{H}$ & $\mathrm{I}$ & gesamt \\
\hline & 32,6 & 23,3 & 8,3 & 7,8 & 6,9 & 6,3 & 5,3 & 4,8 & 4,7 & 100 \\
\hline \multicolumn{11}{|c|}{ Wahrscheinlichkeiten $p_{j k}$ in \% } \\
\hline Tötungsdelikte & 0,0 & $\mathbf{0 , 3}$ & 0,1 & 0,0 & 0,6 & 0,1 & 0,0 & 0,0 & $\mathbf{0 , 7}$ & 0,2 \\
\hline Sexualdelikte & 0,0 & 1,2 & 0,1 & 0,0 & 2,1 & 1,5 & 0,0 & 0,0 & 2,5 & 0,7 \\
\hline sonst. Sexualdelikte & 0,0 & 2,4 & 0,3 & 0,0 & 0,8 & 2,5 & 0,3 & 0,0 & 1,1 & 0,9 \\
\hline Raub & 0,2 & 4,3 & 0,7 & 0,3 & 7,4 & 2,0 & 0,0 & 1,1 & 19,6 & 2,8 \\
\hline schw. Körperverletzung & 0,2 & 0,4 & 0,3 & 0,3 & $\mathbf{5 7 , 7}$ & 2,4 & 0,0 & 0,0 & 39,5 & 6,2 \\
\hline Körperverletzung & 0,8 & 0,9 & 1,0 & 0,6 & 92,2 & 29,8 & 33,6 & 1,0 & 73,8 & 14,1 \\
\hline Str. gg. Pers. Freiheit & 0,5 & 2,8 & 1,3 & 0,1 & 12,3 & 28,6 & 1,2 & 1,1 & 25,4 & 4,9 \\
\hline Einfacher Diebstahl & 8,3 & 37,7 & 35,3 & 100,0 & 16,5 & 7,2 & 2,7 & 16,2 & 67,2 & 27,9 \\
\hline Schwerer Diebstahl & 2,5 & 30,7 & 7,1 & 2,1 & 7,6 & 1,5 & 0,7 & 1,7 & 42,9 & 11,5 \\
\hline Sachbeschädigung & 0,9 & 15,2 & 0,1 & 5,0 & 10,4 & 20,1 & 0,8 & 0,2 & 34,4 & 7,9 \\
\hline gemeingef. Straftaten & 0,1 & 2,3 & 0,0 & 0,2 & 0,1 & 2,5 & 1,6 & 0,2 & 2,9 & 1,0 \\
\hline Hehlerei & 0,5 & 10,0 & 1,6 & 4,6 & 1,7 & 0,5 & 0,6 & 1,2 & 10,8 & 3,7 \\
\hline Betrug & 0,1 & 7,8 & 100,0 & 0,0 & 3,0 & 4,3 & 2,4 & 0,0 & 30,2 & 12,2 \\
\hline Erschl. v. Leistungen & 0,3 & 15,3 & 5,7 & 3,2 & 4,0 & 6,2 & 0,1 & 5,3 & 15,2 & 6,0 \\
\hline Gef. dem. Rechtsstaat & 0,0 & 0,7 & 0,0 & 0,0 & 1,0 & 1,8 & 0,0 & 0,0 & 3,3 & 0,5 \\
\hline Wid. gg. Staatsgewalt & 0,2 & 0,6 & 0,1 & 0,0 & 5,4 & 18,5 & 1,1 & 0,5 & 19,8 & 2,8 \\
\hline Öffentliche Ordnung & 1,1 & 4,3 & 3,4 & 0,3 & 2,2 & 1,7 & 0,5 & 0,0 & 9,7 & 2,4 \\
\hline Hausfriedensbruch & 0,0 & 4,3 & 0,0 & 0,4 & 2,7 & 4,6 & 0,2 & 0,6 & 12,0 & 2,1 \\
\hline Beleidigung & 0,5 & 2,8 & 1,7 & 0,8 & 7,4 & 50,6 & 0,3 & 1,0 & 32,5 & 6,3 \\
\hline Fälschung & 4,2 & 9,2 & 22,9 & 0,0 & 1,0 & 0,5 & 0,7 & 0,0 & 21,9 & 6,6 \\
\hline Meineid & 0,2 & 1,9 & 1,4 & 0,0 & 0,8 & 0,4 & 0,2 & 0,7 & 1,6 & 0,8 \\
\hline falsche Verdächtigung & 0,1 & 1,1 & 0,6 & 0,2 & 0,1 & 1,4 & 0,3 & 0,4 & 1,8 & 0,6 \\
\hline BTM-Delikte & 3,2 & 12,8 & 5,1 & 0,0 & 7,9 & 4,9 & 2,7 & 100,0 & 26,8 & 11,5 \\
\hline $\begin{array}{l}\text { gef. Eingriff in den } \\
\text { Straßenverkehr }\end{array}$ & 0,0 & 0,6 & 0,1 & 0,1 & 0,6 & 5,9 & 1,1 & 0,0 & 3,3 & 0,8 \\
\hline Vollrausch & 0,4 & 2,5 & 0,1 & 0,2 & 2,1 & 2,8 & 0,0 & 0,0 & 4,8 & 1,3 \\
\hline fahrl. Tötung & 0,5 & 0,3 & 0,1 & 0,0 & 0,6 & 0,0 & 4,1 & 0,0 & 0,0 & 0,5 \\
\hline fahrl. Körperverletzung & 9,8 & 3,4 & 1,9 & 1,2 & 5,0 & 5,4 & 100,0 & 0,0 & 13,7 & 10,8 \\
\hline Verkehrsdelikte & 100,0 & 35,8 & 30,6 & 0,5 & 25,5 & 28,6 & 47,0 & 10,6 & 60,9 & 53,0 \\
\hline $\begin{array}{l}\text { Durchschnittliche Anzahl } \\
\text { Delikte }\end{array}$ & 1,4 & 2,3 & 2,2 & 1,2 & 2,7 & 2,6 & 2,0 & 1,3 & 6,2 & 2,0 \\
\hline
\end{tabular}


Tabelle 77: Vergleich 6-Clusterlösung mit 7-Clusterlösung (justizielle Registrierungen deutsche Männer Kohorte 1970)

\begin{tabular}{|c|c|c|c|c|c|c|c|c|c|}
\hline Cluster & $\begin{array}{l}\text { Count } \\
\% \text { row } \\
\% \text { column }\end{array}$ & $\begin{array}{r}\text { Verkehr } \\
\text { (A) }\end{array}$ & $\begin{array}{r}\text { BTM } \\
\text { (B) }\end{array}$ & $\begin{array}{r}\text { Einf. } \\
\text { Diebst. } \\
(\mathrm{C}) \\
\end{array}$ & $\begin{array}{r}\text { Gewalt } \\
\text { (D) }\end{array}$ & $\begin{array}{r}\text { Betrug } \\
\text { (E) }\end{array}$ & $\begin{array}{r}\text { Chron. } \\
\text { Straft. } \\
(\mathrm{F})\end{array}$ & $\begin{array}{r}\text { Fahrl. } \\
\text { KV }(G)\end{array}$ & Total \\
\hline \multirow{3}{*}{\multicolumn{2}{|c|}{$\begin{array}{l}\text { Diebstahl, } \\
\text { BTM (A) }\end{array}$}} & 92 & 4127 & 3475 & 8 & 118 & 124 & 24 & 7968 \\
\hline & & $1,2 \%$ & $51,8 \%$ & $43,6 \%$ &, $1 \%$ & $1,5 \%$ & $1,6 \%$ &, $3 \%$ & $100,0 \%$ \\
\hline & & $1,1 \%$ & $91,5 \%$ & $82,4 \%$ & ,3\% & $4,9 \%$ & $10,7 \%$ & $2,3 \%$ & $32,6 \%$ \\
\hline \multirow{3}{*}{\multicolumn{2}{|c|}{ Verkehr (B) }} & 8524 & 7 & 742 & 0 & 0 & 3 & 21 & 9297 \\
\hline & & $91,7 \%$ &, $1 \%$ & $8,0 \%$ &, $0 \%$ &, $0 \%$ &, $0 \%$ &, $2 \%$ & $100,0 \%$ \\
\hline & & $98,9 \%$ &, $2 \%$ & $17,6 \%$ &, $0 \%$ &, $0 \%$ &, $3 \%$ & $2,0 \%$ & $38,1 \%$ \\
\hline \multirow{3}{*}{\multicolumn{2}{|c|}{ Gewalt (C) }} & 2 & 364 & 0 & 2351 & 6 & 50 & 15 & 2788 \\
\hline & &, $1 \%$ & $13,1 \%$ &, $0 \%$ & $84,3 \%$ &, $2 \%$ & $1,8 \%$ &, $5 \%$ & $100,0 \%$ \\
\hline & &, $0 \%$ & $8,1 \%$ &, $0 \%$ & $96,1 \%$ &, $2 \%$ & $4,3 \%$ & $1,4 \%$ & $11,4 \%$ \\
\hline \multirow{3}{*}{\multicolumn{2}{|c|}{ Betrug (D) }} & 0 & 0 & 0 & 1 & 2279 & 6 & 14 & 2300 \\
\hline & &, $0 \%$ &, $0 \%$ &, $0 \%$ &, $0 \%$ & $99,1 \%$ &, $3 \%$ & ,6\% & $100,0 \%$ \\
\hline & &, $0 \%$ &, $0 \%$ &, $0 \%$ &, $0 \%$ & $94,7 \%$ &, $5 \%$ & $1,3 \%$ & $9,4 \%$ \\
\hline \multirow{3}{*}{\multicolumn{2}{|c|}{ Fahrl. KV (E) }} & 0 & 0 & 0 & 0 & 0 & 0 & 979 & 979 \\
\hline & &, $0 \%$ &, $0 \%$ &, $0 \%$ &, $0 \%$ &, $0 \%$ &, $0 \%$ & $100,0 \%$ & $100,0 \%$ \\
\hline & &, $0 \%$ &, $0 \%$ &, $0 \%$ &, $0 \%$ &, $0 \%$ &, $0 \%$ & $93,0 \%$ & $4,0 \%$ \\
\hline \multirow{3}{*}{\multicolumn{2}{|c|}{$\begin{array}{l}\text { Chronische } \\
\text { Straftäter (F) }\end{array}$}} & 0 & 14 & 2 & 87 & 4 & 980 & 0 & 1087 \\
\hline & &, $0 \%$ & $1,3 \%$ &, $2 \%$ & $8,0 \%$ & ,4\% & $90,2 \%$ &, $0 \%$ & $100,0 \%$ \\
\hline & &, $0 \%$ &, $3 \%$ &, $0 \%$ & $3,6 \%$ &, $2 \%$ & $84,3 \%$ &, $0 \%$ & $4,5 \%$ \\
\hline \multirow{3}{*}{\multicolumn{2}{|c|}{ Total }} & 8618 & 4512 & 4219 & 2447 & 2407 & 1163 & 1053 & 24419 \\
\hline & & $35,3 \%$ & $18,5 \%$ & $17,3 \%$ & $10,0 \%$ & $9,9 \%$ & $4,8 \%$ & $4,3 \%$ & $100,0 \%$ \\
\hline & & $100,0 \%$ & $100,0 \%$ & $100,0 \%$ & $100,0 \%$ & $100,0 \%$ & $100,0 \%$ & $100,0 \%$ & $100,0 \%$ \\
\hline
\end{tabular}


Abbildung 65: Altersverteilung der 8-Clusterlösung (deutsche Männer 1970 BZR)

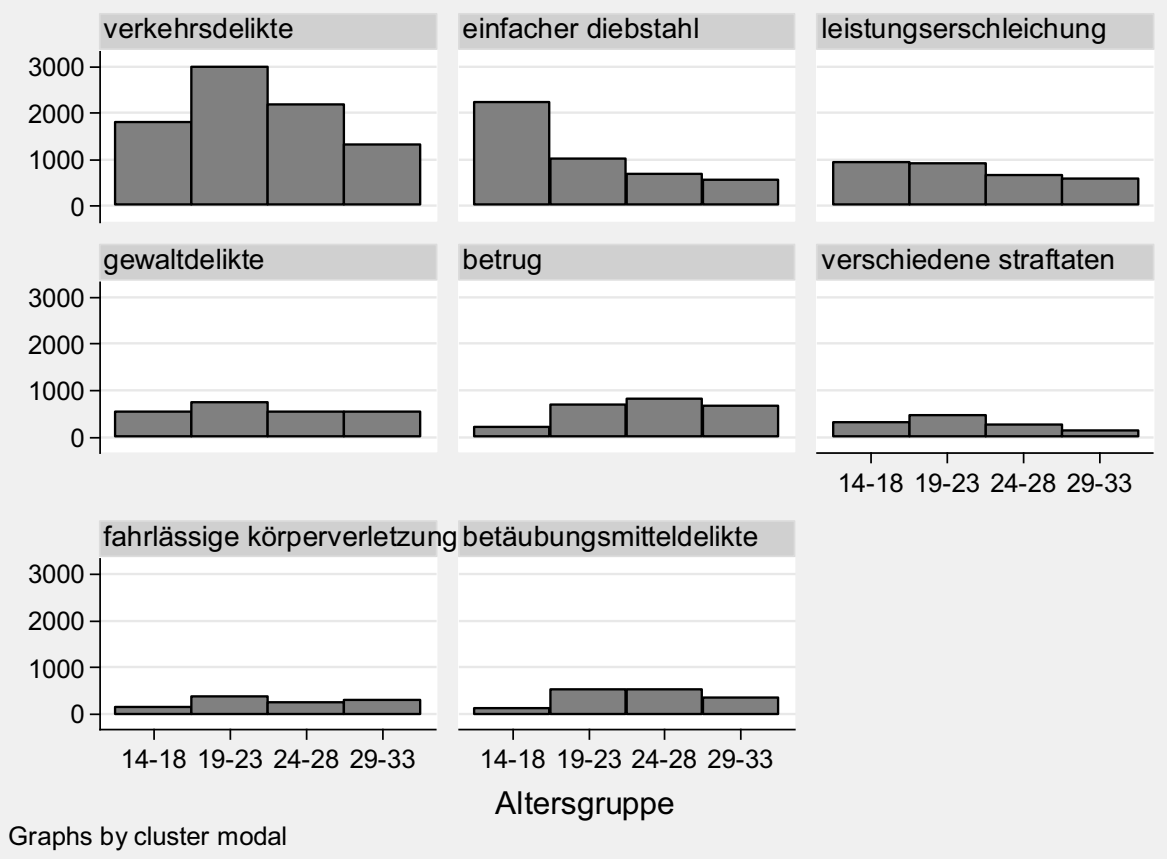

Abbildung 66: Altersverteilung der 9-Clusterlösung (deutsche Männer 1970 BZR)

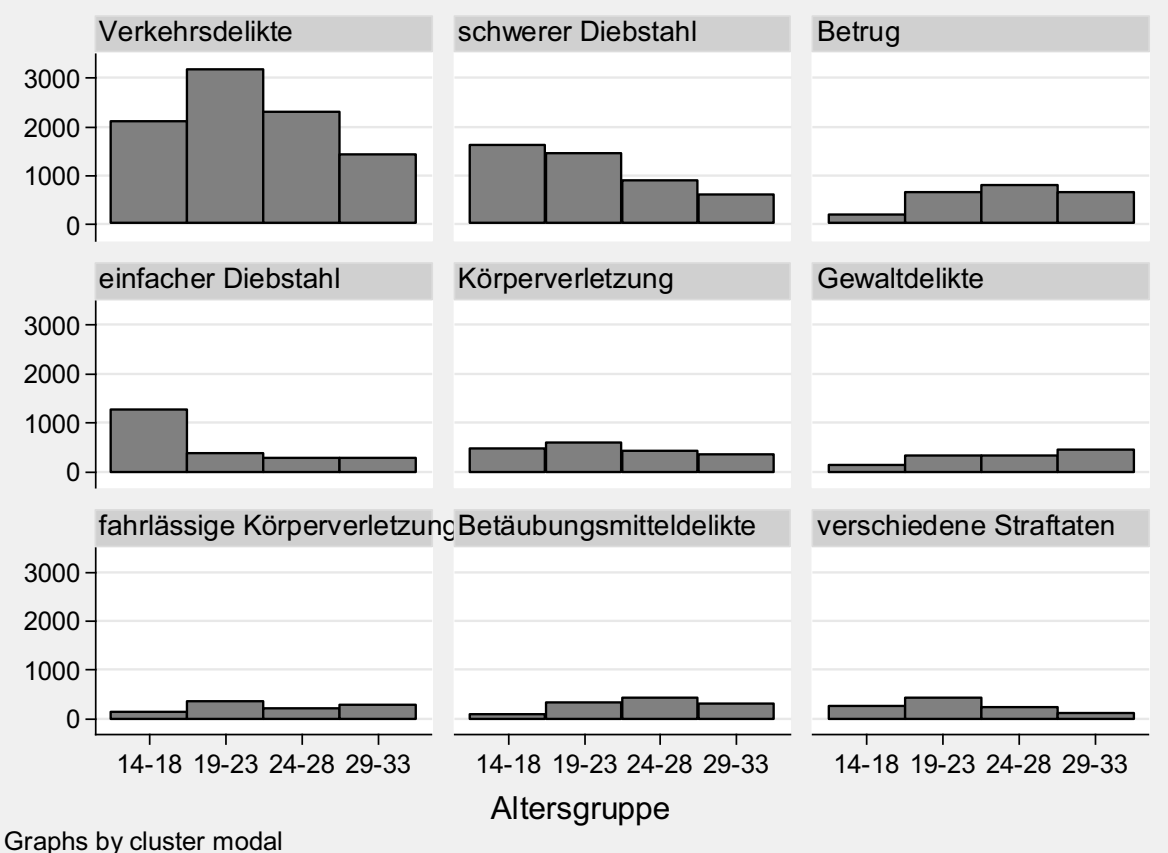




\section{Schriftenreihe des Max-Planck-Instituts für ausländisches und internationales Strafrecht}

Die zentralen Veröffentlichungen des Max-Planck-Instituts für ausländisches und internationales Strafrecht werden in Zusammenarbeit mit dem Verlag Duncker \& Humblot in den folgenden vier Unterreihen der „Schriftenreihe des Max-Planck-Instituts für ausländisches und internationales Strafrecht" vertrieben:

- „Strafrechtliche Forschungsberichte“,

- „Kriminologische Forschungsberichte“,

- „Interdisziplinäre Forschungen aus Strafrecht und Kriminologie“ sowie

- „Sammlung ausländischer Strafgesetzbücher in deutscher Übersetzung“.

Diese Publikationen können direkt über das Max-Planck-Institut unter <www.mpicc.de> oder über den Verlag Duncker \& Humblot unter <www.duncker-humblot.de> erworben werden.

Darüber hinaus erscheinen im Hausverlag des Max-Planck-Instituts in der Unterreihe „research in brief“ zusammenfassende Kurzbeschreibungen von Forschungsergebnissen und in der Unterreihe „Arbeitsberichte“ Veröffentlichungen vorläufiger Forschungsergebnisse. Diese Veröffentlichungen können über das Max-Planck-Institut bezogen werden.

Detaillierte Informationen zu den einzelnen Publikationen des Max-Planck-Instituts für ausländisches und internationales Strafrecht sind unter $<$ www.mpicc.de $>$ abrufbar.

The main research activities of the Max Planck Institute for Foreign and International Criminal Law are published in the following four subseries of the "Schriftenreihe des MaxPlanck-Instituts für ausländisches und internationales Strafrecht" (Research Series of the Max Planck Institute for Foreign and International Criminal Law), which are distributed in cooperation with the publisher Duncker \& Humblot:

- "Strafrechtliche Forschungsberichte" (Reports on Research in Criminal Law),

- "Kriminologische Forschungsberichte" (Reports on Research in Criminology),

- "Interdisziplinäre Forschungen aus Strafrecht und Kriminologie" (Reports on Interdisciplinary Research in Criminal Law and Criminology), and

- "Sammlung ausländischer Strafgesetzbücher in deutscher Übersetzung" (Collection of Foreign Criminal Laws in German Translation).

These publications can be ordered from the Max Planck Institute at <www.mpicc.de> or from Duncker \& Humblot at $<$ www.duncker-humblot.de $>$.

Two additional subseries are published directly by the Max Planck Institute for Foreign and International Criminal Law: "research in brief" contains short reports on results of research activities, and "Arbeitsberichte" (working materials) present preliminary results of research projects. These publications are available at the Max Planck Institute.

Detailed information on all publications of the Max Planck Institute for Foreign and International Criminal Law can be found at $<$ www.mpicc.de $>$. 
Auswahl aus dem strafrechtlichen Veröffentlichungsprogramm:

S 102 Frank Jungfleisch

Fortpflanzungsmedizin als Gegenstand des Strafrechts?

Eine Untersuchung verschiedenartiger Regelungsansätze

aus rechtsvergleichender und rechtspolitischer Perspektive

$2004 \cdot 350$ Seiten • ISBN 978-3-86113-887-7

S 103 Albin Eser/Jörg Arnold/Julie Trappe (Hrsg.)

Strafrechtsentwicklung in Osteuropa

Zwischen Bewältigung und neuen Herausforderungen

2005 • 436 Seiten $・$ ISBN 978-3-86113-877-8

S 104 Roland Michael Kniebühler

Transnationales, ne bis in ideme

Zum Verbot der Mehrfachverfolgung in horizontaler

und vertikaler Dimension

2005 • 461 Seiten • ISBN 978-3-86113-881-5

S 105 Christoph Grammer

Der Straftatbestand des Verschwindenlassens einer Person

Transposition einer völkerrechtlichen Figur ins Strafrecht

2005 • 254 Seiten • ISBN 978-3-86113-880-8

S 106 Clivia von Dewitz

NS-Gedankengut und Strafrecht

Die $\S \S 86,86$ a StGB und $\S 130$ StGB zwischen der Abwehr neonazistischer Gefahren und symbolischem Strafrecht

$2006 \cdot 310$ Seiten $\bullet$ ISBN 978-3-86113-872-3

$€ 31,00$

S 107 Helmut Kreicker

Völkerrechtliche Exemtionen

Grundlagen und Grenzen völkerrechtlicher Immunitäten

und ihre Wirkungen im Strafrecht

2007 • 2 Bände • 1538 Seiten • ISBN 978-3-86113-868-6

$€ 99,00$

S 108 Konstanze Jarvers

Massen- und Kleinkriminalität vor dem italienischen

Friedensrichter

Neue Wege durch alternative Erledigungsmöglichkeiten

und Sanktionsformen

2007 • 308 Seiten • ISBN 978-3-86113-866-2

$€ 31,00$

S 109 Johanna Rinceanu

Völkerstrafrecht in Rumänien

$2008 \cdot 284$ Seiten • ISBN 978-3-86113-864-8

$€ 31,00$ 
Auswahl aus dem kriminologischen Veröffentlichungsprogramm:

K 129 Annette Louise Herz

Menschenhandel

Eine empirische Untersuchung zur Strafverfolgungspraxis

2005 • 404 Seiten • ISBN 978-3-86113-074-1

K 130 Carsten Schäfer/Letizia Paoli

Drogenkonsum und Strafverfolgungspraxis

Eine Untersuchung zur Rechtswirklichkeit der Anwendung des $\S 31$ a BtMG und anderer Opportunitätsvorschriften auf Drogenkonsumentendelikte

2006 • 460 Seiten • ISBN 978-3-86113-075-8

K 132 Gunda Wößner

Typisierung von Sexualstraftätern

Ein empirisches Modell zur Generierung

typenspezifischer Behandlungsansätze

2006 280 Seiten • ISBN 978-3-86113-079-6

K 133 Maria Kafatou-Haeusermann

The Media-Crime Nexus Revisited:

On the Re-Construction of Crime and Law-and-Order

in Crime-Appeal Programming

2007 • 536 Seiten • ISBN 978-3-86113-082-6

K 134 Gundula Schäfer-Vogel

Gewalttätige Jugendkulturen - Symptom der Erosion

kommunikativer Strukturen

$2007 \cdot 591$ Seiten $•$ ISBN 978-3-86113-083-3

K 135 Stefanie Tränkle

Im Schatten des Strafrechts

2007 • 392 Seiten • ISBN 978-3-86113-084-0

$€ 35,00$

K 136 Tim Lukas (ed.)

Crime Prevention in High-Rise Housing

Lessons from the Crime Prevention Carousel

$2007 \cdot 132$ Seiten $・$ ISBN 978-3-86113-085-7

$€ 31,00$

K 137 Evelyn Shea

Why work?

A Study of Prison Labour in England, France and Germany 2007 • 195 Seiten • ISBN 978-3-86113-086-4 